\title{
Soutiche
}

\section{(5) tudentenipradie}

\author{
พxน
}

Srixortrh Hage. 


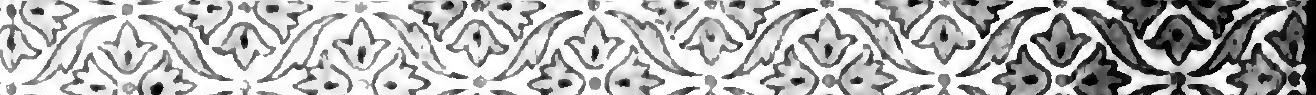

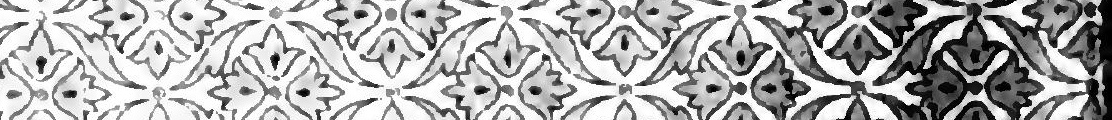

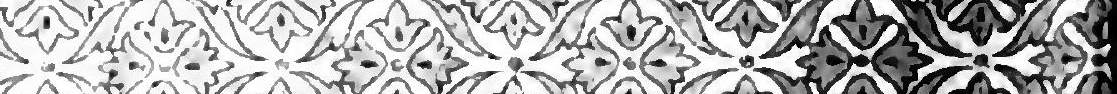
S1) 40 .

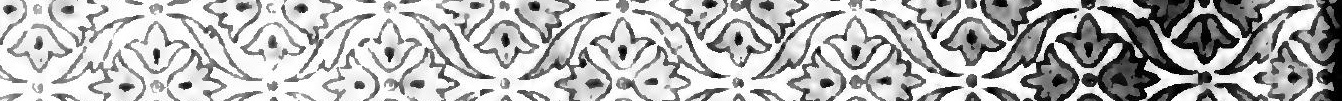
$2 v^{2}-2 / 4,6$

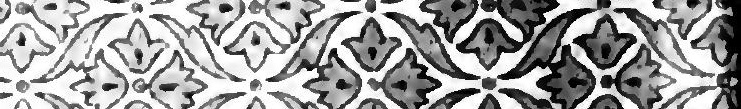

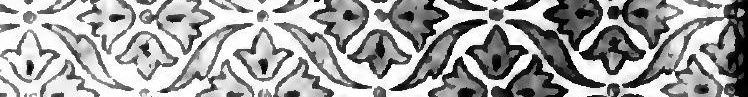

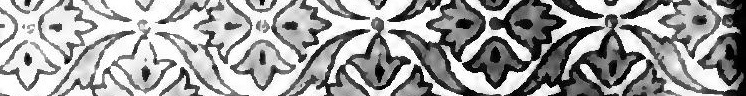

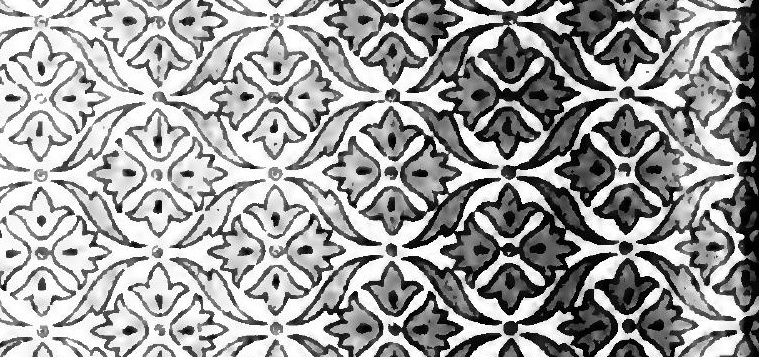

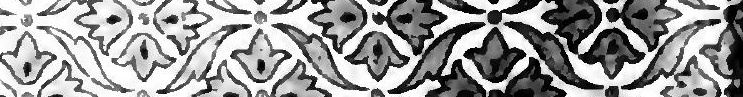

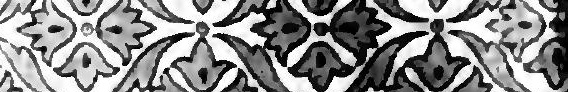

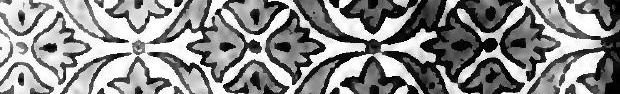

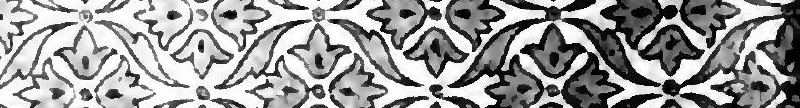

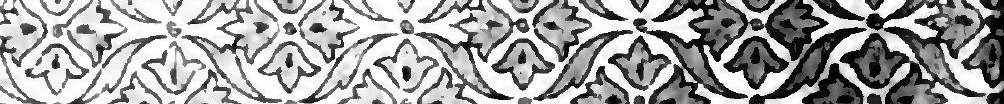

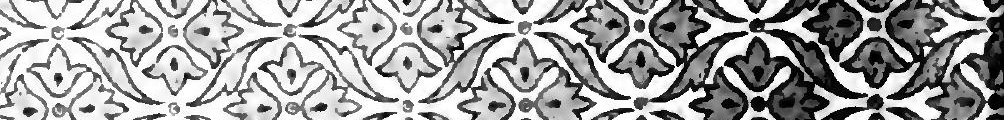

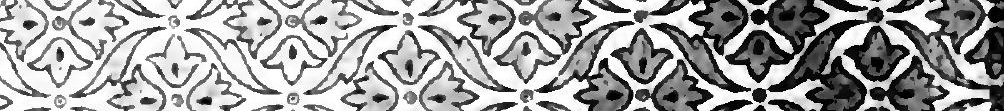

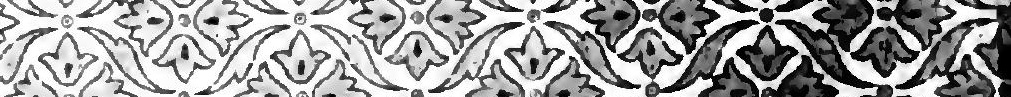

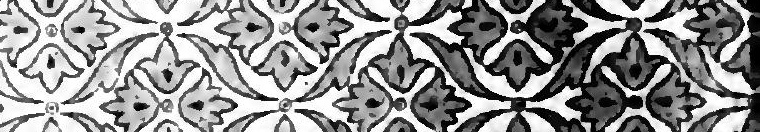

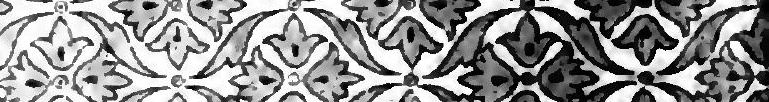

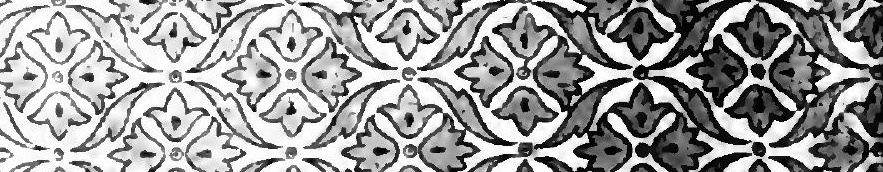

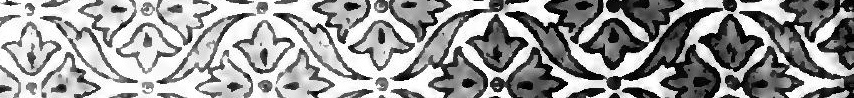

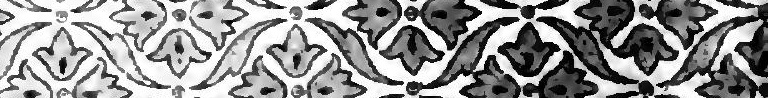

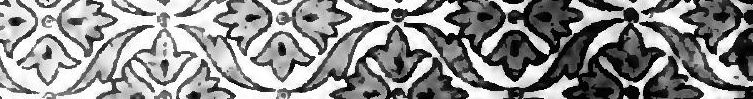
Dif

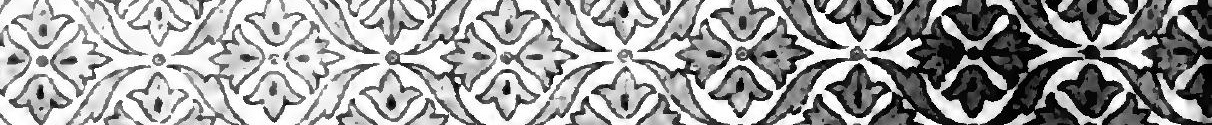

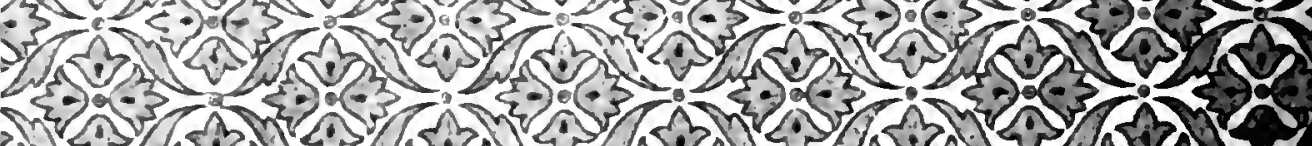

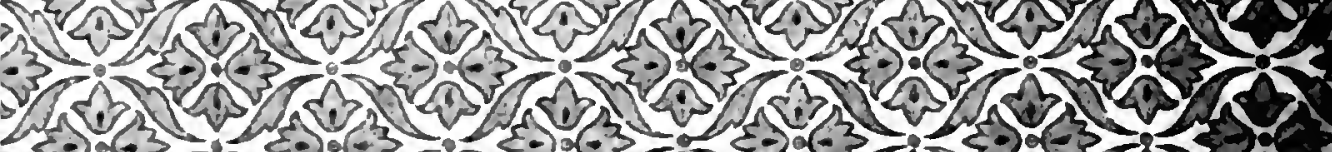





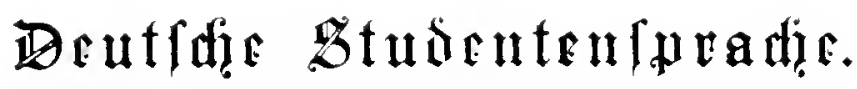




\section{Dentide}

\section{Staterterifirade}

vol1

\section{Frictide Siluge}

Hrofejifor an oce lniverfität Freiburg i. $\mathbb{B}$.

\section{Stronghutrg}

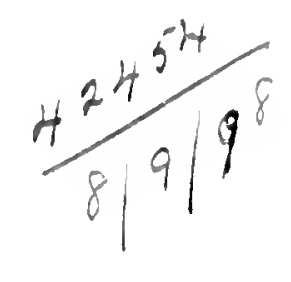

Derlag bon Rarl J. Träbuer

1895 . 


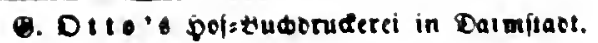




\title{
$\mathfrak{G} \mathfrak{a t t f a t e d ~} \mathfrak{B} \mathfrak{a} \mathfrak{i}$
}

\author{
geviomet.
}




\section{Bormort.}

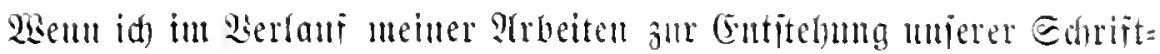

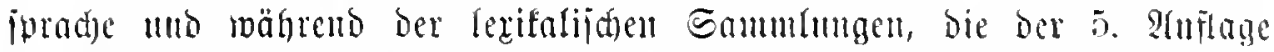

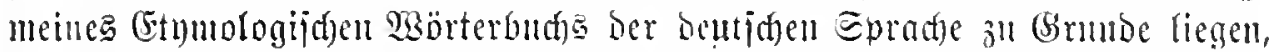

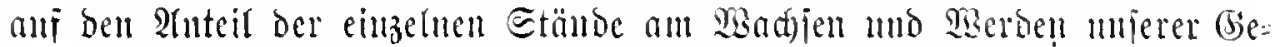

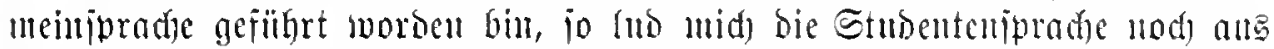
andern Brïnoen żl einer zmanmenfängenben Betradjtung cin. Die Be=

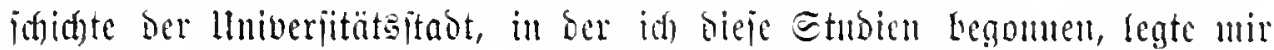

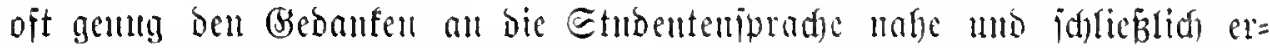

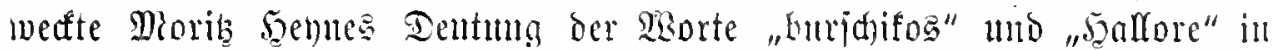

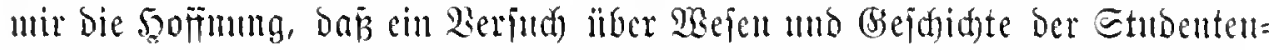

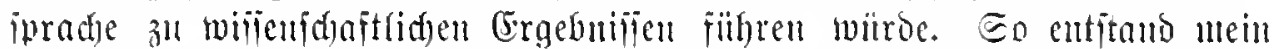
Bortrag "über bentidje Stmbentenipradye", ben bie Beilage Sir. 297 ber

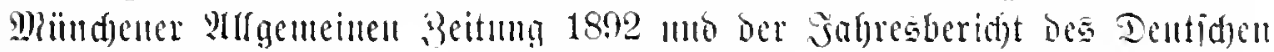

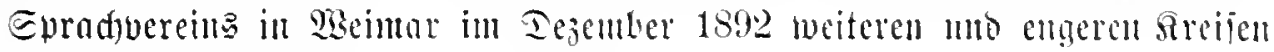
zu!jünglid) mad)te.

Das norliegende Büd)lein bringt ienen Sortran in ertweiterter bejtalt.

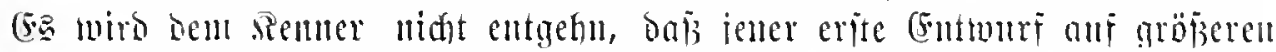
Sammlungen bermft ale ar zur Edtan trïgt. Panjte idh bamals nicles

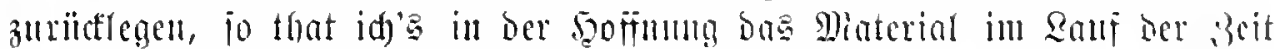

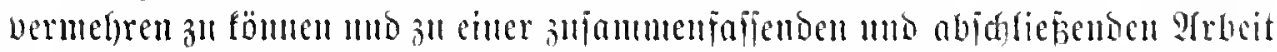

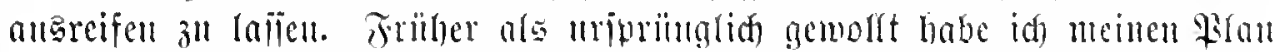

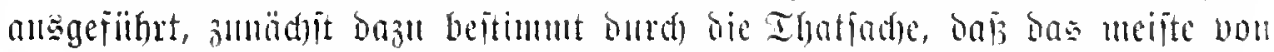

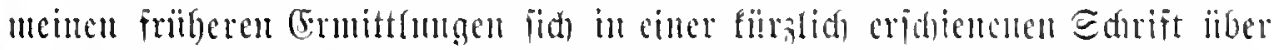

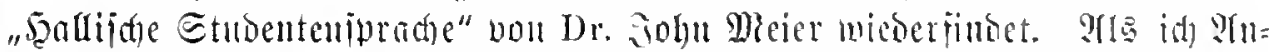

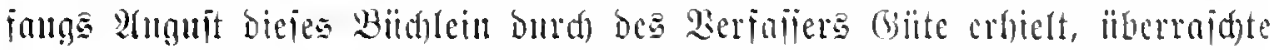

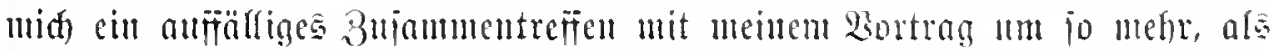

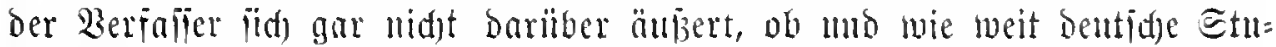

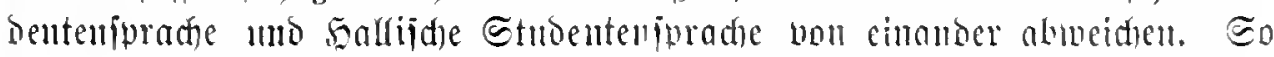

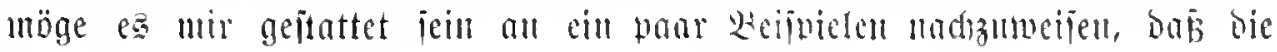


Sallijac Stubenteniprade mit Der allgenteinen beutiden Stubentenipradie iid) jehr oit bect. Id itelle alt bem 3wed Stellen nus Sohn Meiers Bildlein mit iolden nus meilsem Rortrag neben einanber.

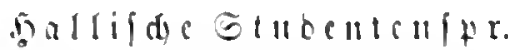

श2an bilocle im 16. Gabrb. ven yrob (5rubität, ins 17. Gabrh. iolgen Mbertät, Filgität, am Ingang be 18. Gabrb. Theben Sdyicfitit wnt bem 19. Gabrf. gebören an Rithlität mo Rnüllität. Wifnumter īi ...

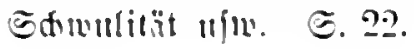

Sdun ill 15. Jabrb. taudt bei Enufgaro Rrififus ani fiic cinen idlunen Mienfiden ... Mabulid ge= biloct ift bas wobl erit in miperem 3abrh. nejdaifene S\$wadmatifuB.. mo Enftifu (and 2uititu). ङ.24.

An Siejer 3eit frammen $\mathfrak{R}$ neipier, Meinfueipier, Zandueipier, Pumpier, Midjier, bamals filoct man pompez,

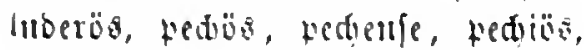
philitrös. ธ. $3 \pi$.
Dentjde Stubentenfpr.

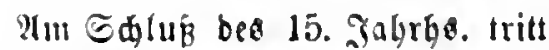
iil afubenifaen Rreifen ber Gro: fianus aui unb in feillem Gefolge bas Mort Grobität, tas 3. B. in Stueid luberieknng von Debefinbe Grobianue 1551 begegnet. 2ui (3robität folgt im 17. Jabrb. Filjität แnb 2Ubertät (ugl. bab DWb.) Da= ran faliebt fid bei einem plubenti= Foien Goriftiteller vom Enbe bes 18. Jabrbs. Gdiefität unt in ber Burfdenfpradje unfers Jabrbs. finbet (id) ncd) Rïblität uno Runillität, iflottität" แno Forjdität. Gin Mit: glied diejer Enotfantilie .. iff ... ç)vulität uin.

Dent gleiden Spradprinzip ent: jtanmen moberne Bilonngen wie

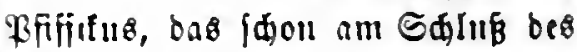
18. Jabrfs. in Eanfbards Romanen bejeugt ift, mo nenerding \&uftifus (munbartliq aud \&uftibus) mie aud) 巨d)แ matifus.

In unferm Gabrb. Irefien wir ir. (stloming in Ruripier 'Ineipmirt', Ridfier 'Gtiefelpuber', Fedtier 'Jedtlebrer', ßumpier 'ßuderer', ad) Zurier unb Edjanjier. $\mathfrak{N m}$

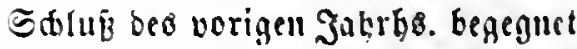
luberös, jeßzt gilt făanberōs unb एe⿻大 
Dhan unterfdie curd nähere 3ujäke Reimplilifiter, Taftphiliter, Gelbuhilifter, Bierwbilititer (Stoppe I, 196. II, 108; I, 6. 69. 172),

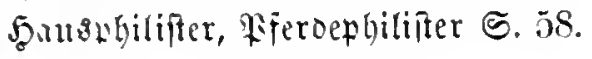

Damals bezeifinete inoentijue Rede mit Bierplitijter ben Biernirt, mit Brelophilifiter bell MSuderer, mit sierbephilifiter den Fierbeverleiher, mit Reimphilitier ben Foptaiter (Stopwe 1728 (5iebidite I, 195. II, 108. 172. I, 69). Эu 19. Эuhrb.

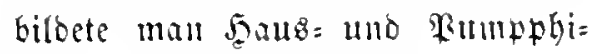
lifter, aud Rreujpkilifiter.

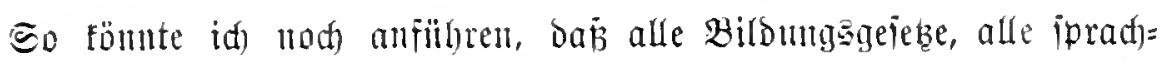
wijienjafattlich bon mir zuerit verwerteten, von unjern Mörterbiufern ïber=

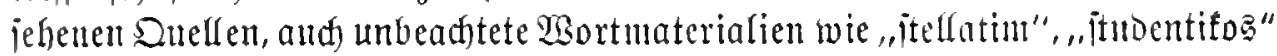

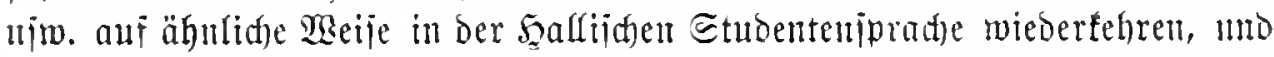
man wird mir wohl gern glauben, baji id) in 2 uguit $\delta$. S. mit einiger lteber=

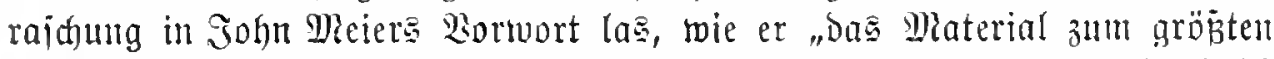

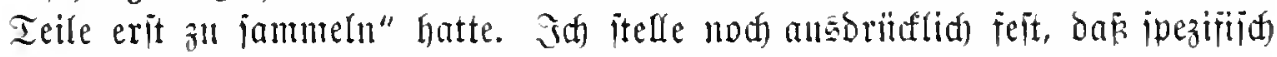

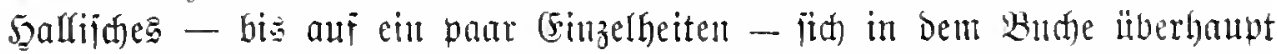
nidgt finbet. (5. hat im wejentliden bie gleidnen gejuidhtlidhen unb morbho= logiiben Sategorien wieberkolt, bie mein Bortrag aufgeitellt hat, mo mur

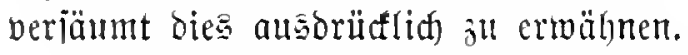

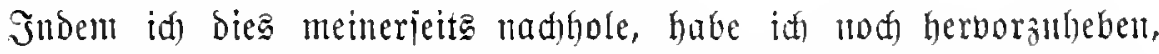

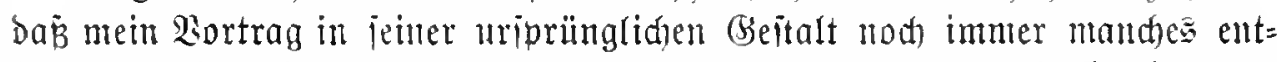

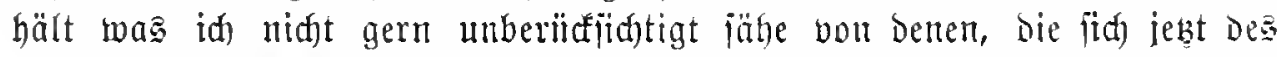

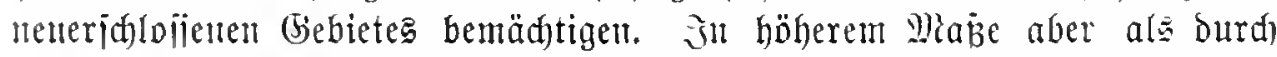

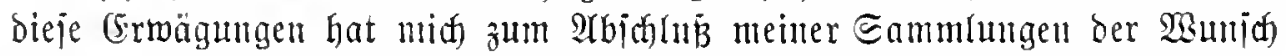
beitimmt, Den Rortrag bon 1892 in ermeiterter Geitalt jelbitänoig ericheinten

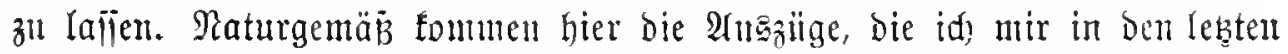
vier Jahren aus ber Sitteratur bes 17. uno 18. Jahrhumberts gemadyt habe, jekt volfer zur (5eltung; biejelben Ientmäler, anf bic jid jenter Dortrag grïndete, find hier unfalfender berwertet worben, mo mandes weitere hat

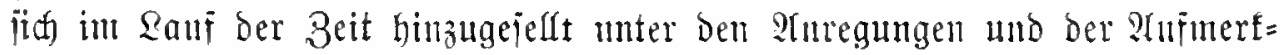
jamteit, wonit Jrennde meiner Beitrebungen nir Silfe geleitet haben. Be:

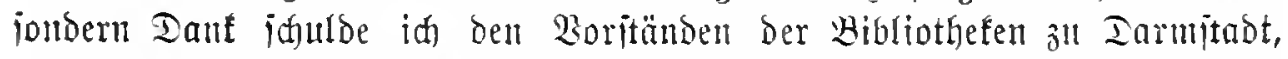

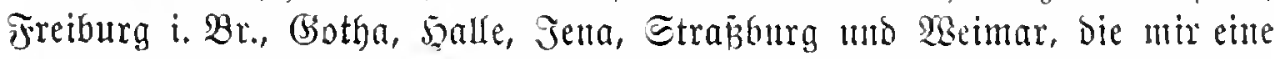

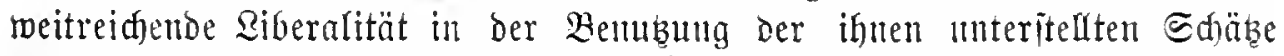

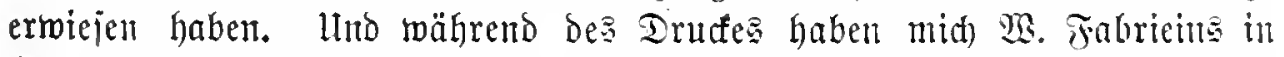

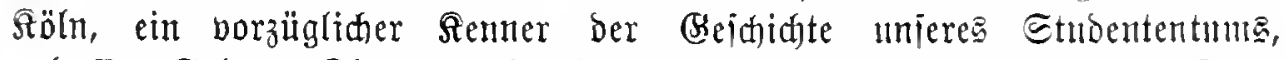

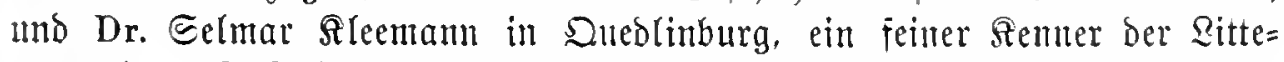

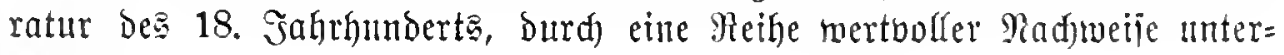
itïnte 


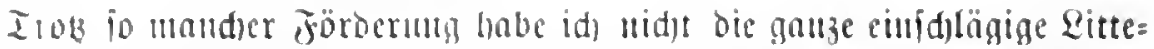
ratur verivertet. Ias Wortmaterial iit żumeift beritedt in entlegetten Duellen,

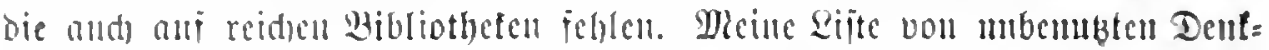

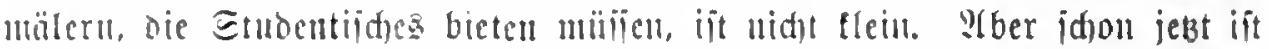

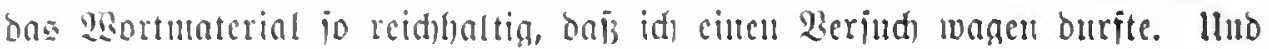

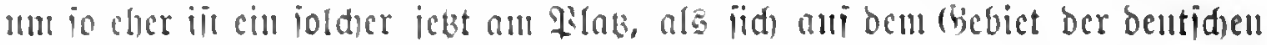
Eerifograbhic gesvidntigne Etimmen hören lafien, bic mit Red)t cin nentes

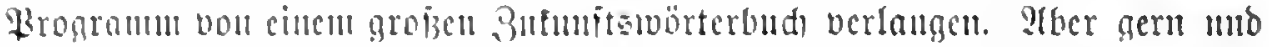
banthar crfente id bic mamnigfatien 2Inregnngen mo gad)weije an, bie in

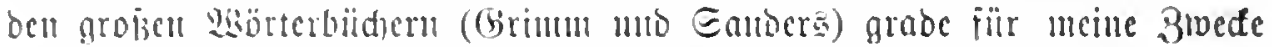
enthallen j̈n - seun and immerhin veriumberbar beibt, wie z. B. bag

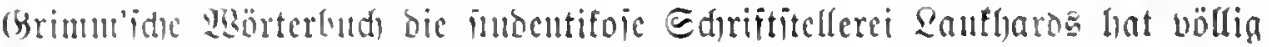

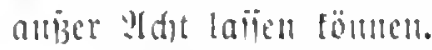

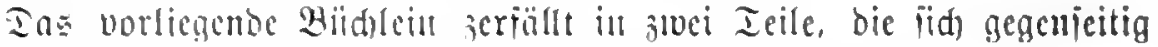

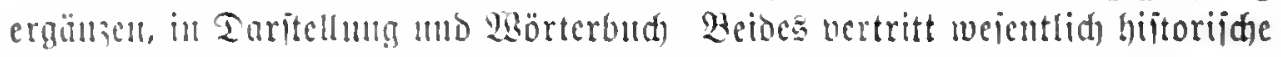
jutereficu, and wo anf (5rid)eimungen ber begentoart Wezug genommen

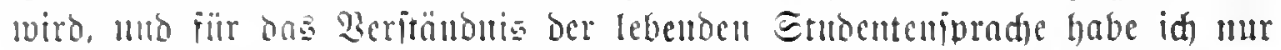
bie allgumene (5rmblage bieten wollen. Ten baritellenten Teil bes vor=

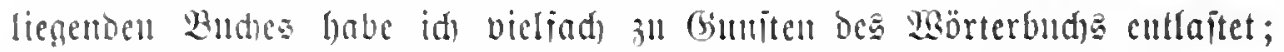

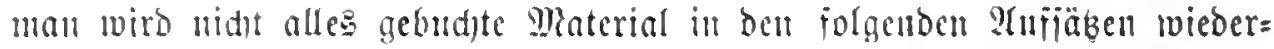

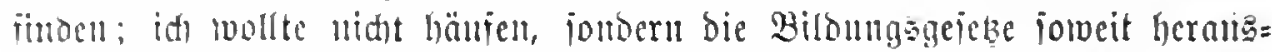
arbeiten, bab ber Sejer mande (sinzelheiten jid) ielkit bequen cinjïgt. llno

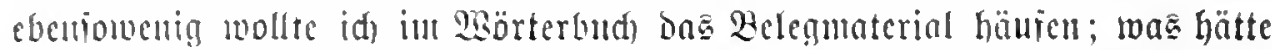

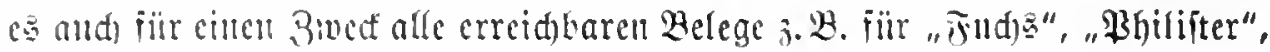

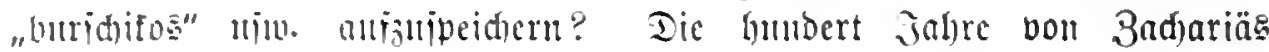
Renommiten $17+4$ an bis ân bem Etndentifoien Sbiotifon bon 1841 jint

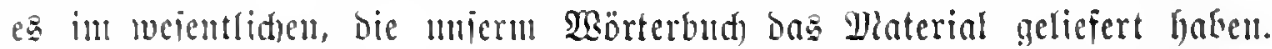
Jd) glaube amebmen ju fönnen, bas mand)e barin wie mandes in ben

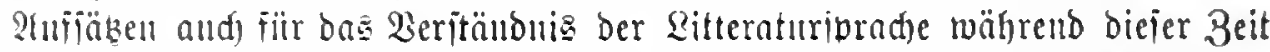
ill Betrad)t toumt; und man wirb zahlreide Morte finden, bie in allen unien neneren mörterbïd)ern jehlen. Go mödte id bas Buđ angejehen svifien als Beitrag zm Deutiden Gpradgeidjidte mo Rexifogranhic.

Treibura i. B., Den 4. Dezember 1894.

\section{Fr. 魯luge.}




\section{Sillialtsolutgabe.}

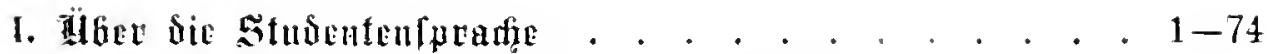

Stubenten mb Whilifter 7-20. - Trmutenlitanei $21-30$.

- 2ntife (Elemente 31--50. - Buridifoic 3oologie 50-55.

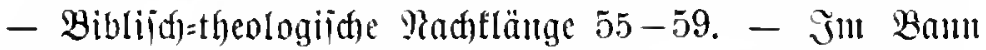

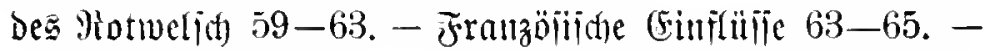

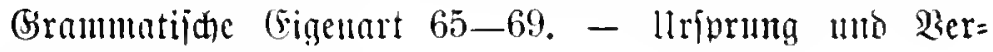
breitung $69-74$.

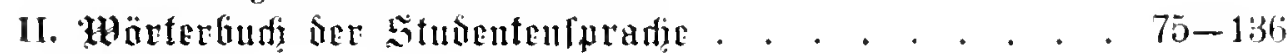




\section{ïther bie Stllbentenipunafise.}

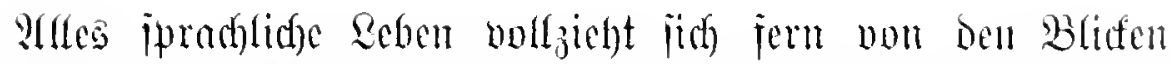

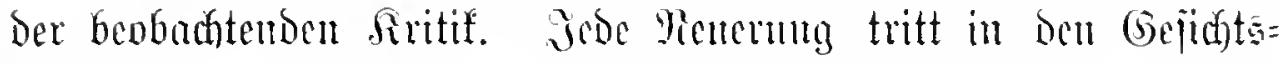

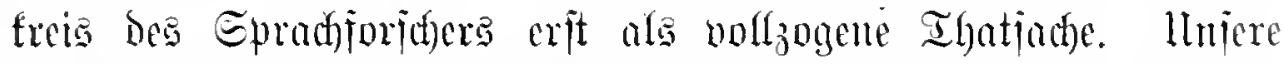

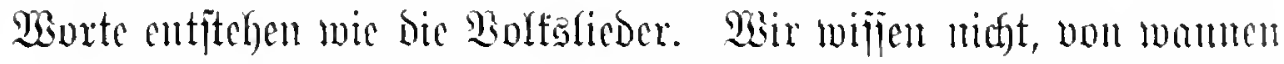

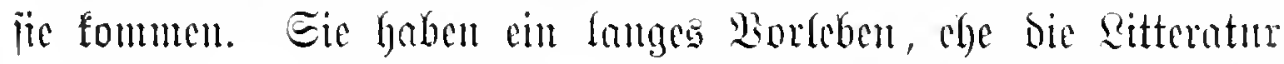

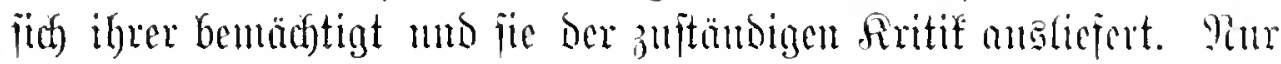

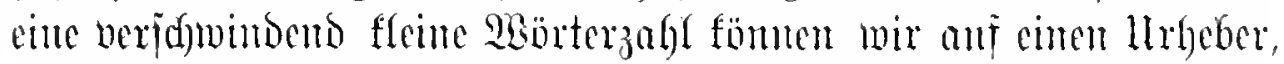

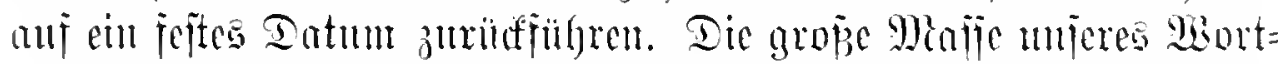

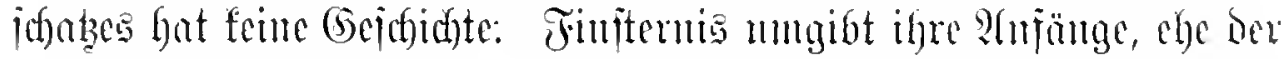

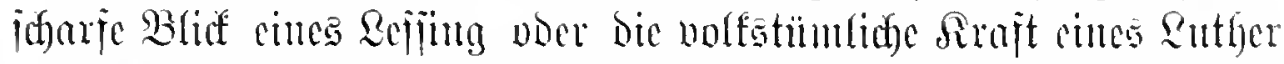
jie uns bem Dutfel herborjieht und afs witroige (blieber in bie

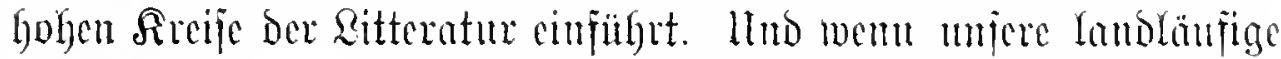
Weisheit jene bentidfejten unter mjeren Didhtem uns Dentern wie

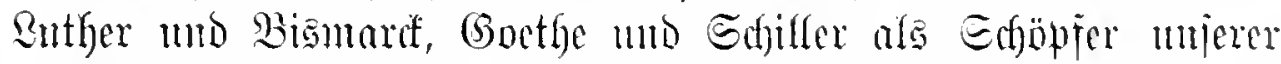

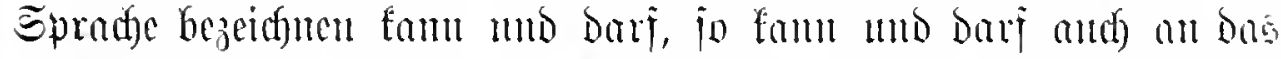

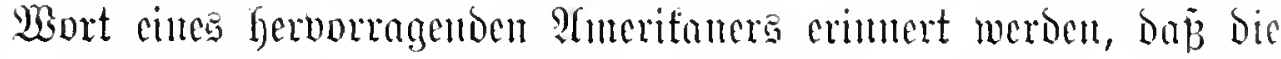

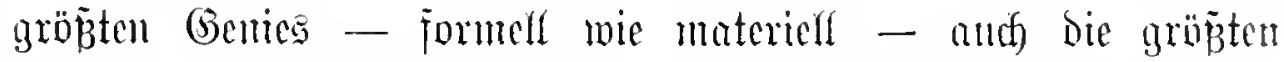

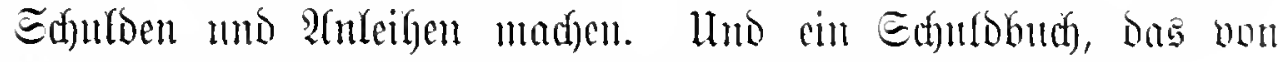

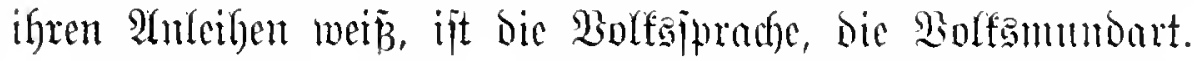

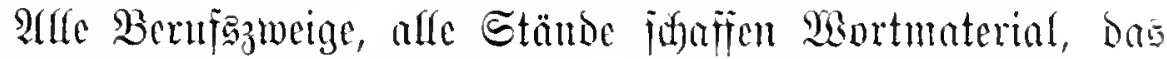
Der Sitteratur zu bienen berufen ift. Arber mosntear genug ent=

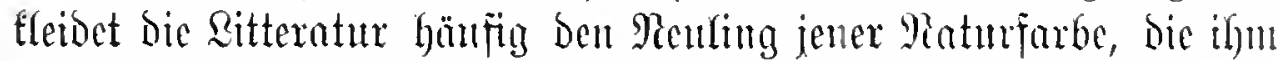

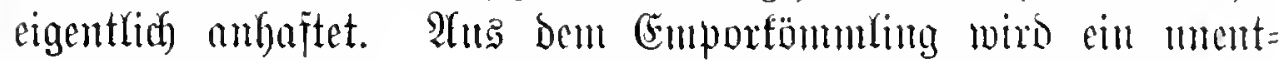

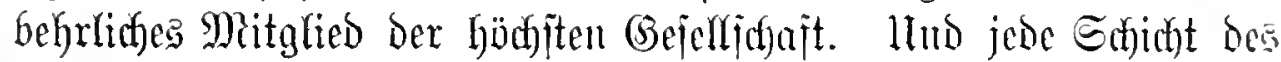




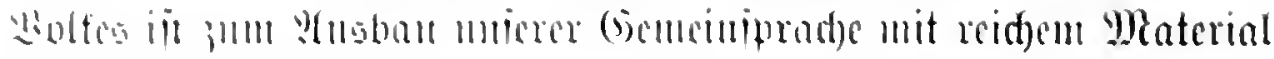
Decmithersit mo bienthor.

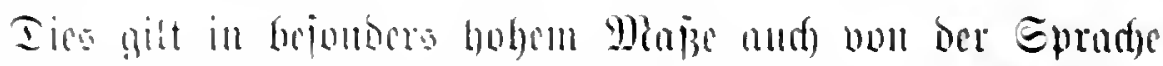

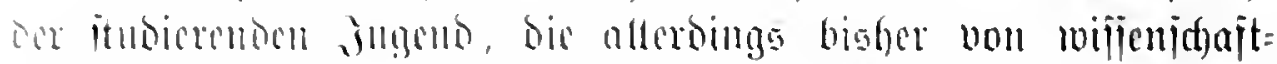

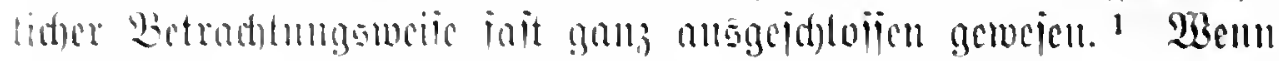

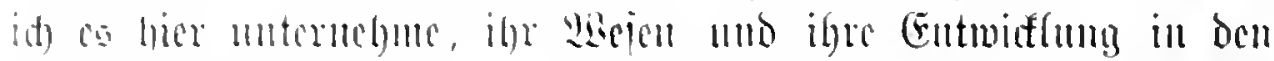

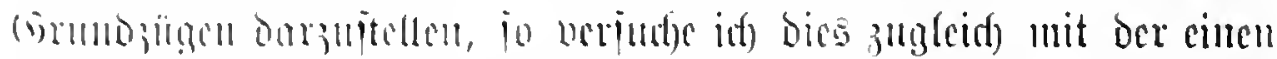

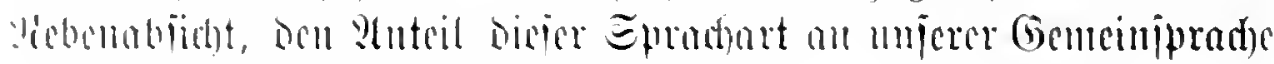

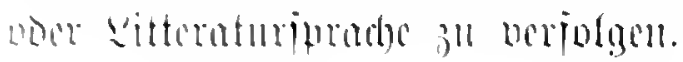

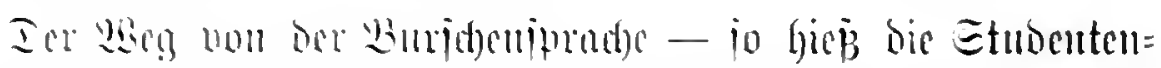

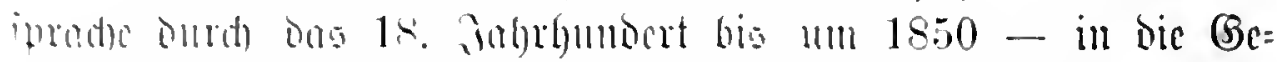

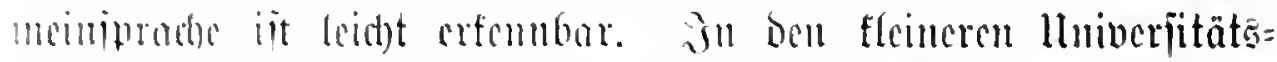

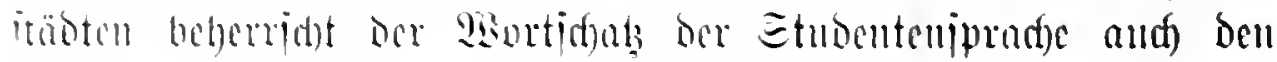

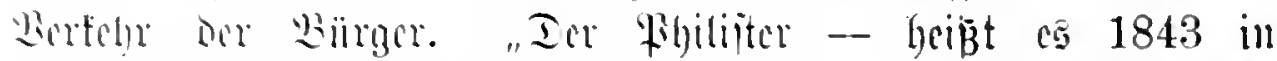

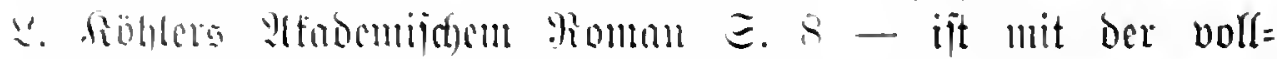

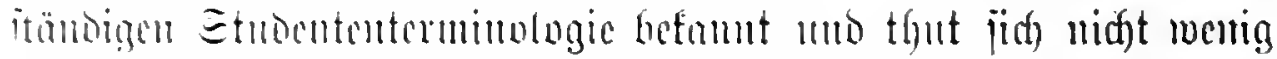

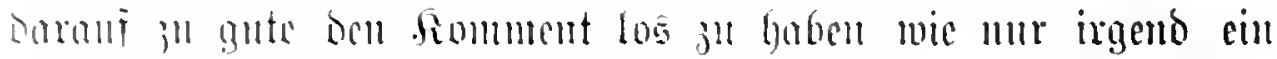

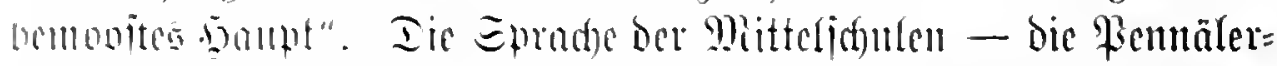

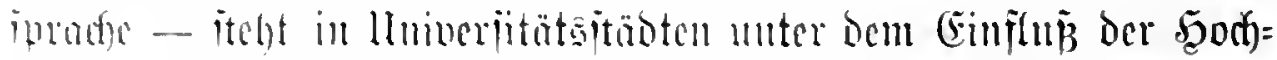

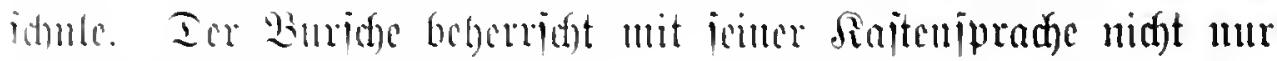

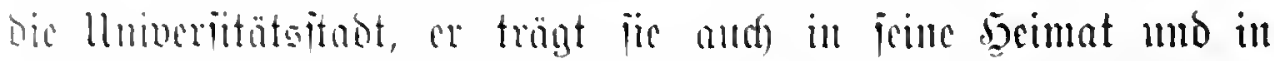

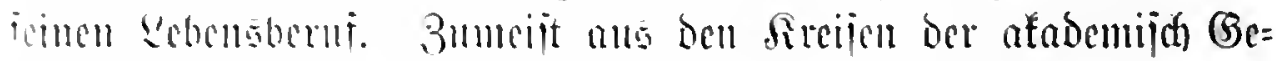

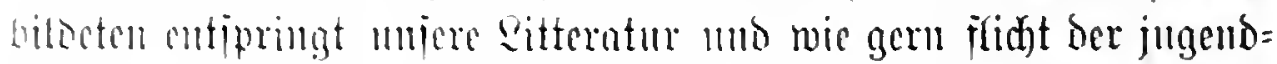

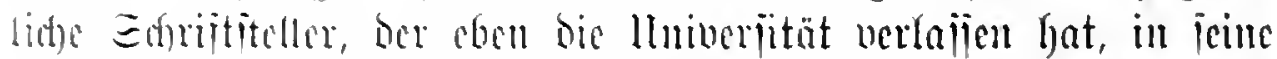

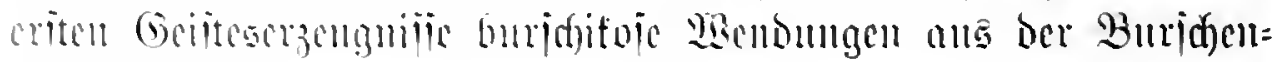

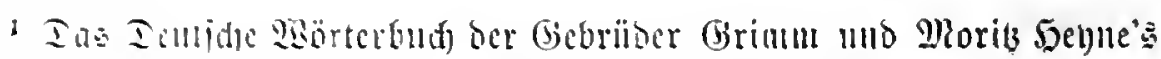

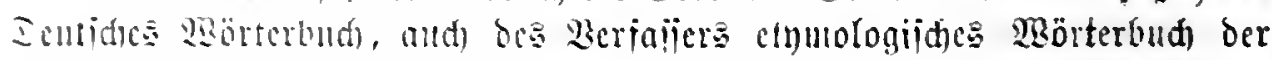

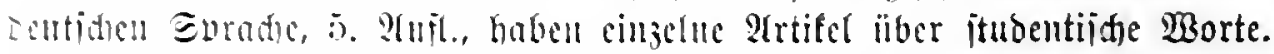

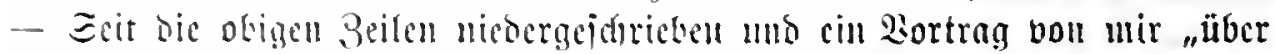

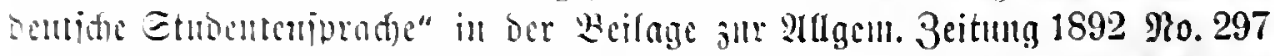

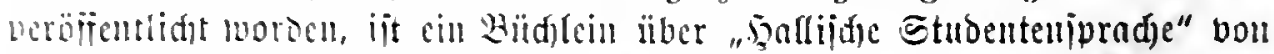

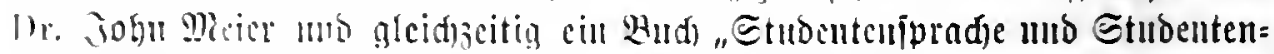

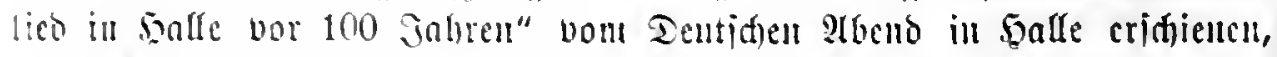

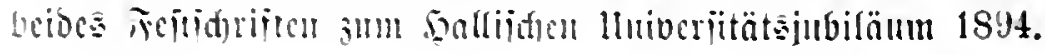


ipradge, bie ibm sime jroh) berlebte Etmoentenzeit nou jeclojt bar= rictet.

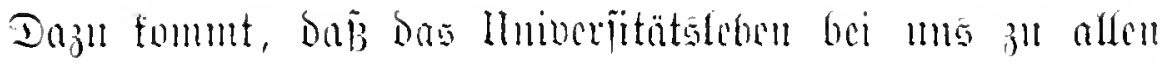

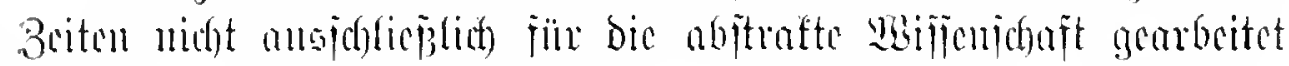

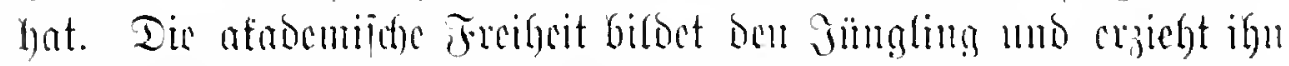

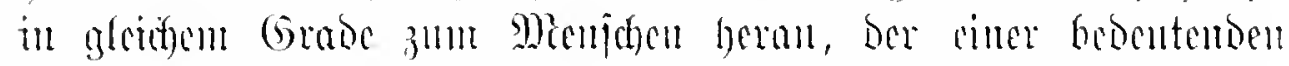

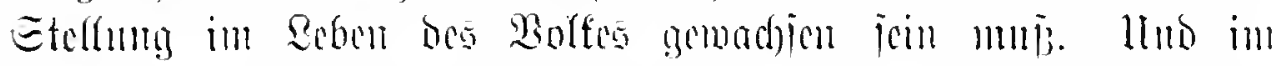

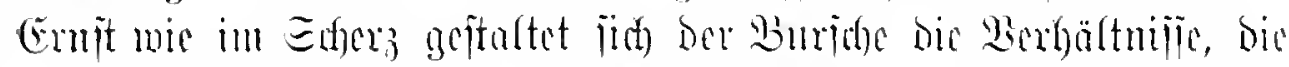

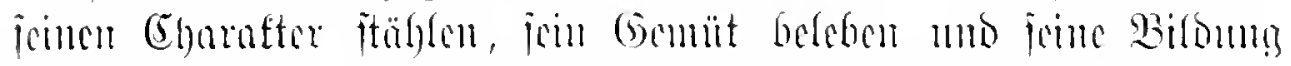

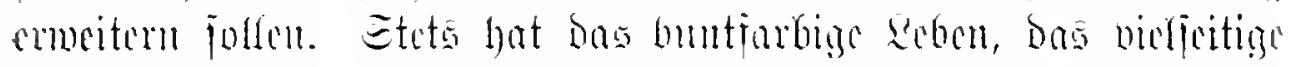

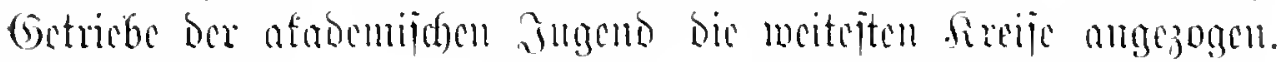

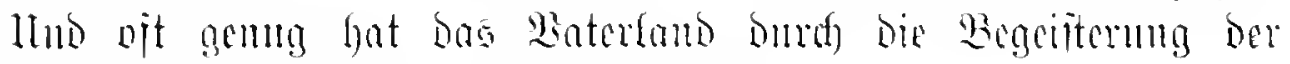
Etudontembelt Esgen mo beil crlsbt!

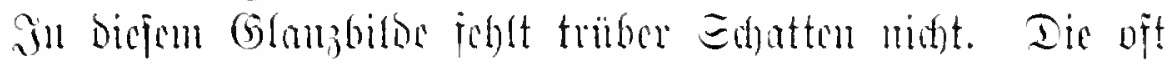

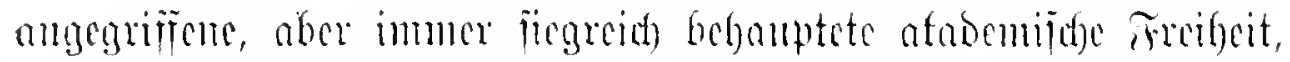

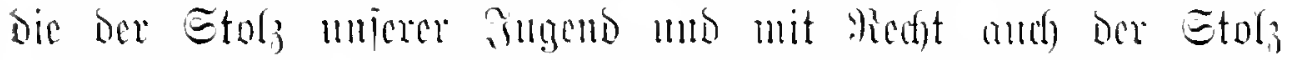

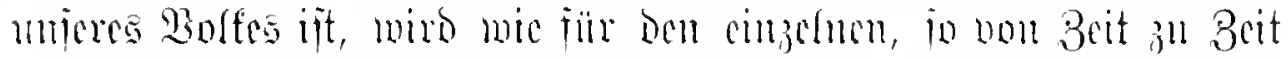

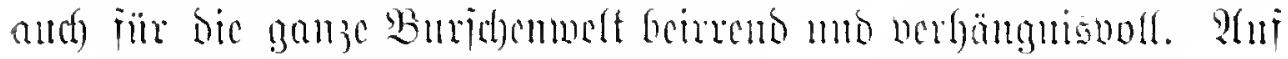

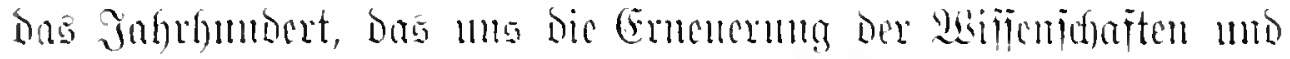

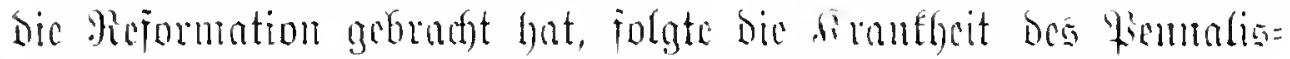

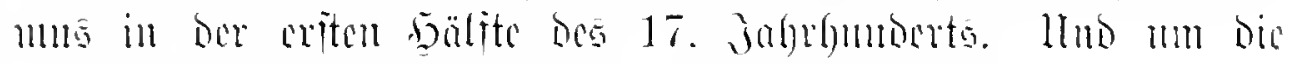

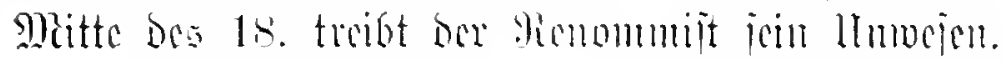

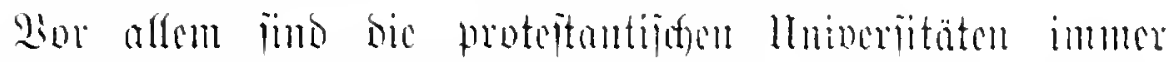

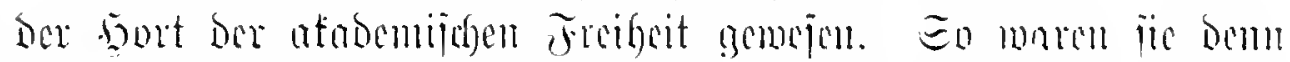

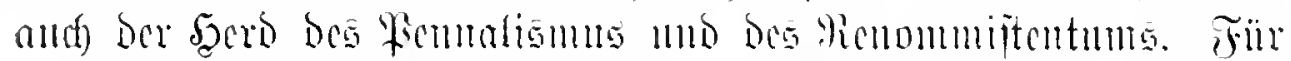

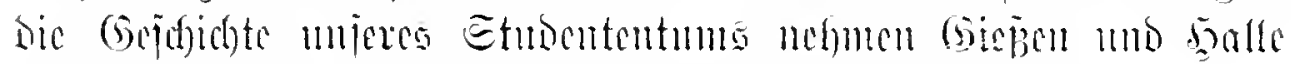

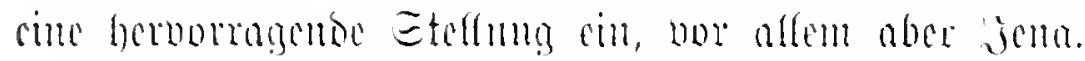

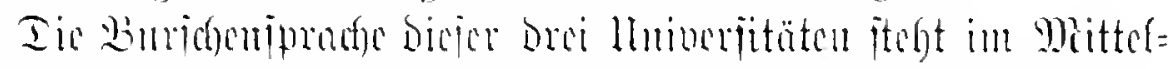

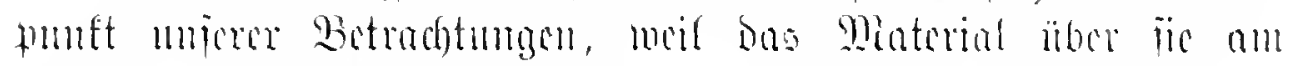

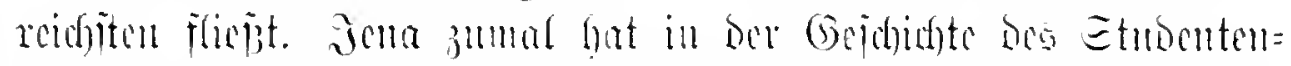

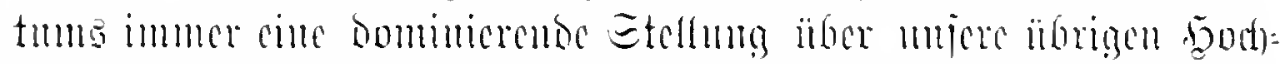

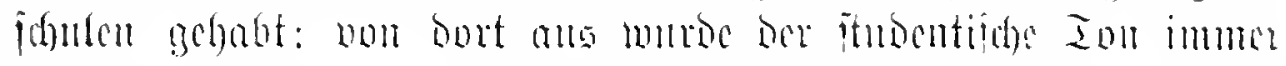

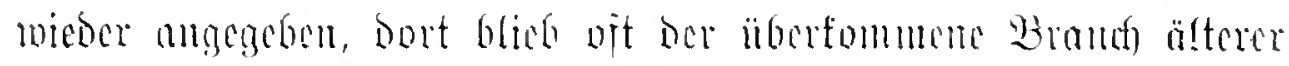
(B) 


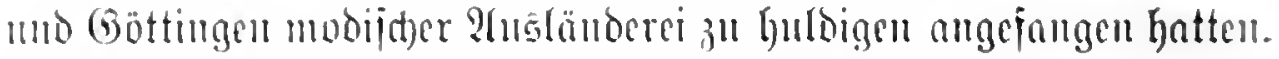

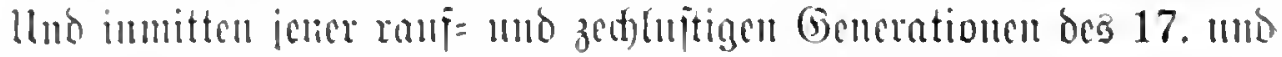

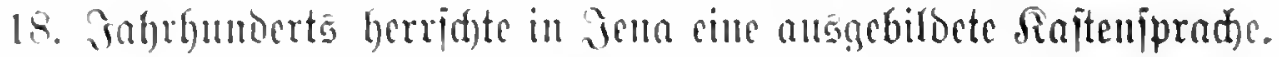
Mir crjabren bas in cince Eative - "Etubentemmoral" betitelt - bie im Jabre 175.4 Jenas Etubententum 3u rejormieren unter=

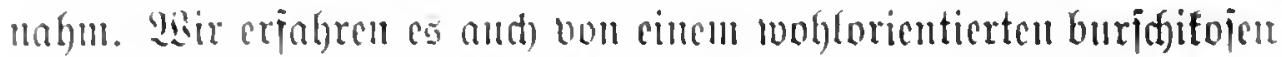
Ed)riftiteller, ber jür bas Etubentenleben jur Zeit bes jumgen Goethe

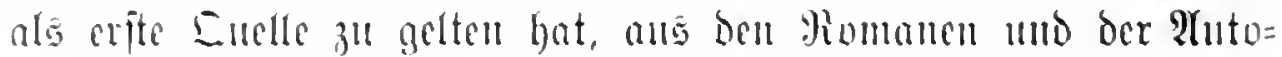

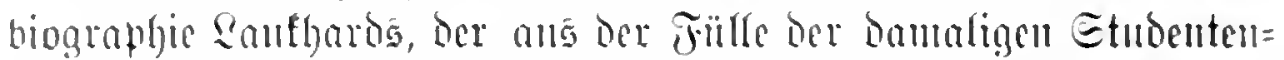

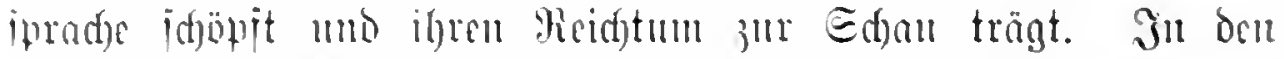
"Wricjen iiter iena" 1793 E. 86 lejen wir, baj bie Epradte

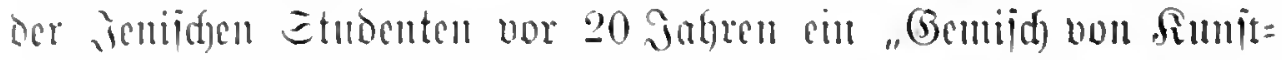

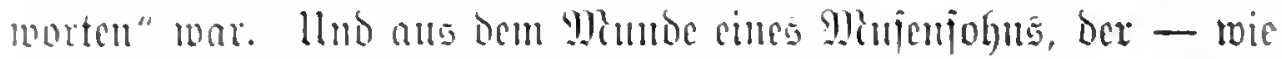

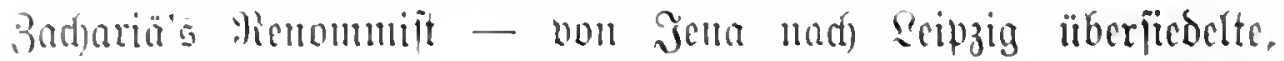

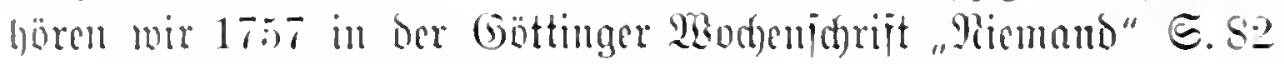

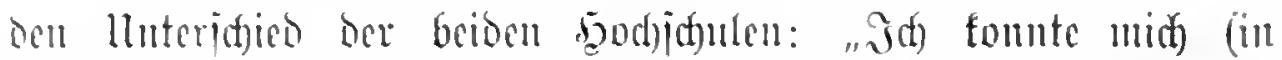

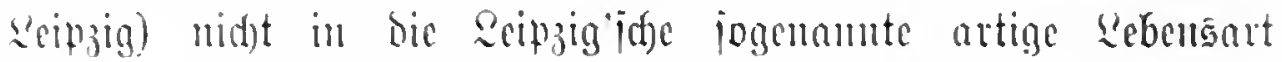
idficter, isf hatte sime ganj anbere Eprathe, mb man veritumo

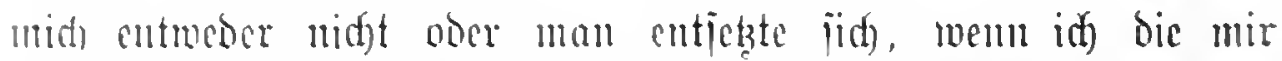

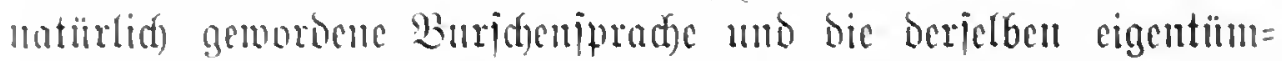

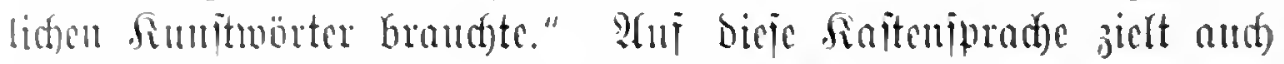

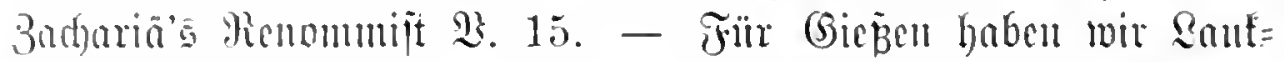

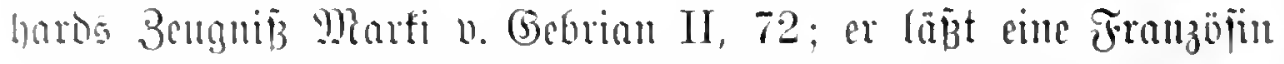

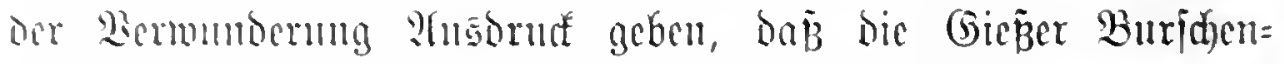

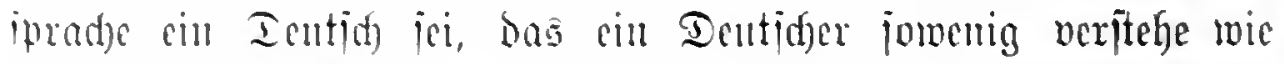

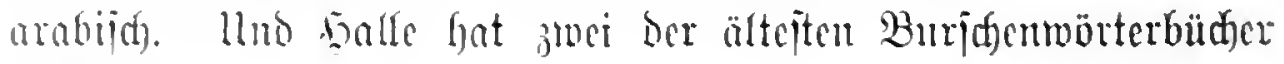
anijumerien.

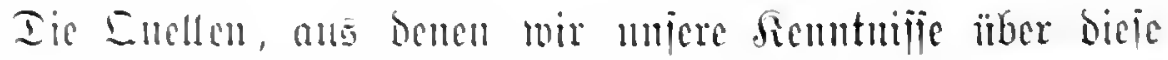

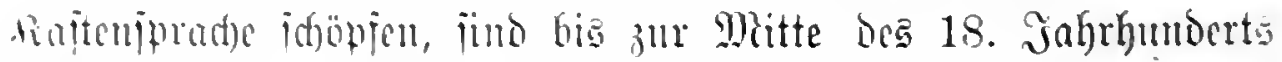

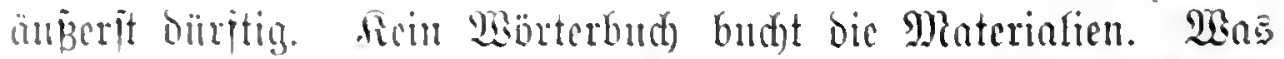
mir crmittelu timmen, bejteld in (Ëmzelbeiten, bie Edriftiteller ge=

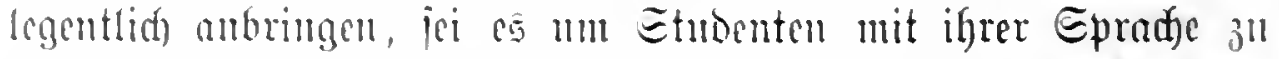
d)arafterijieren mic etwa im Drama, jei cs̄ bei jonjtigen \&nläjien.

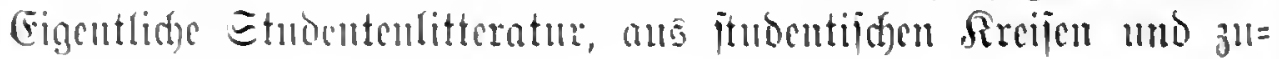




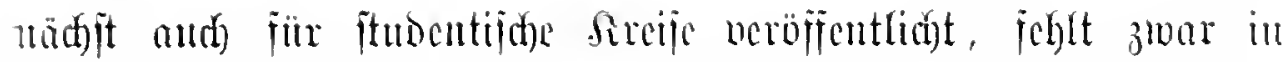

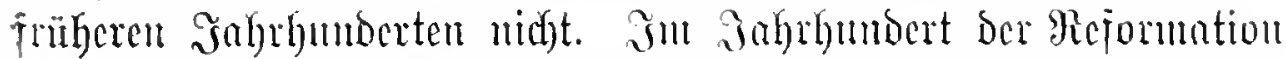

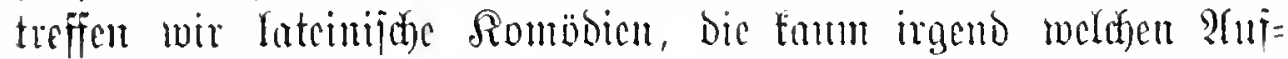

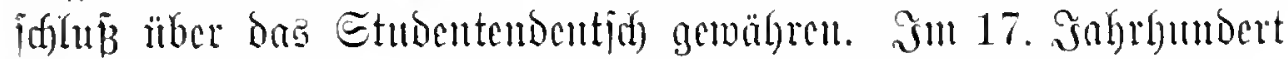

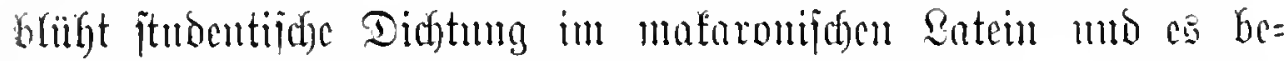

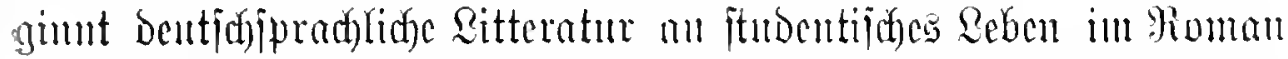

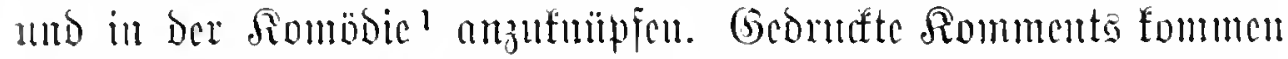

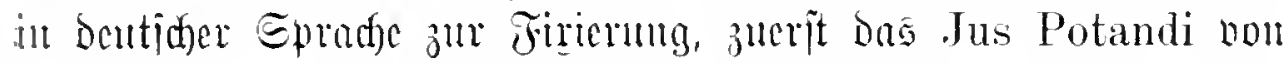
Mrultibibus, Das im 17. Jafrlumbert 8 sfuplagen crlebte, Dam

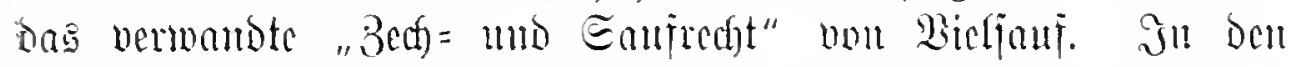
jtudentiichen Etammbücfern begiumen Dentiche Eintragungen 3

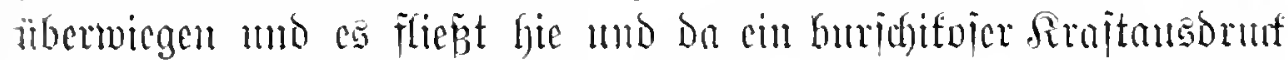

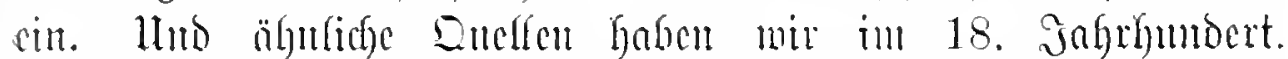

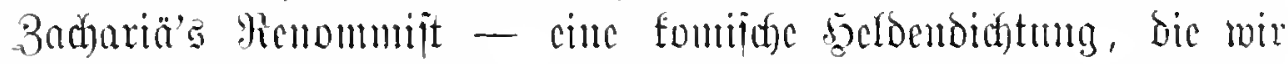

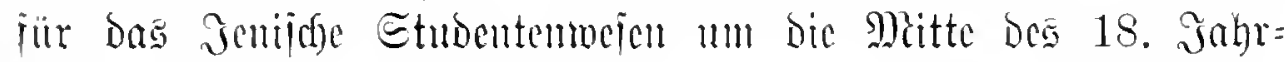

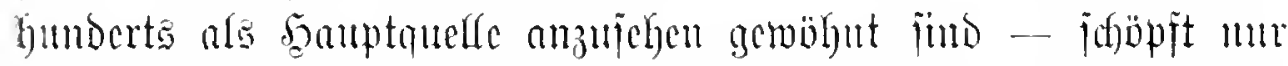

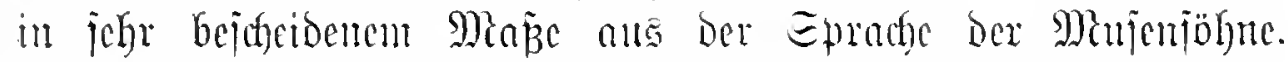

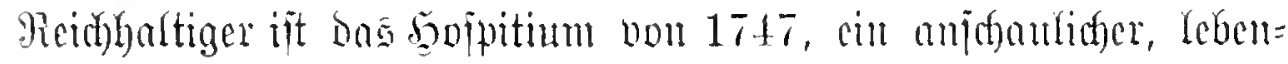
biger Jomment ats bem Beitalter bos Renommiften. Yabe alfes

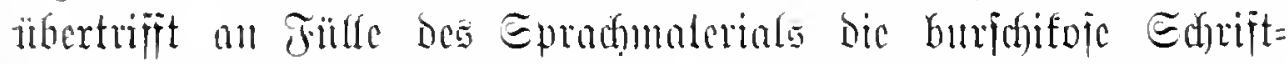

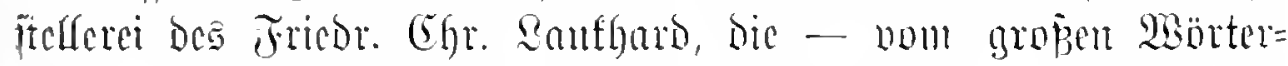
butbe der Gebrïber Grimm zwar ganz ïtherjeben - cinen Giu=

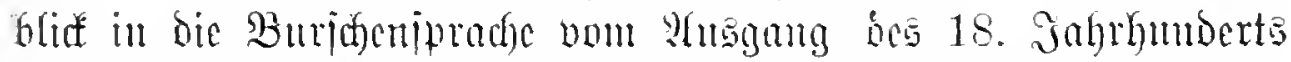

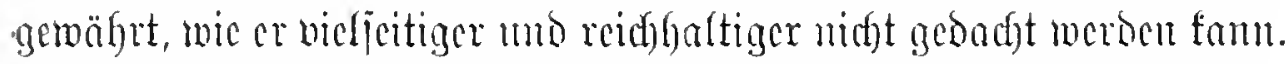
saukford hat cincu Dargänger gebubt an bem berïntigten

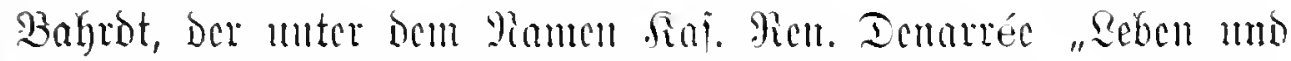

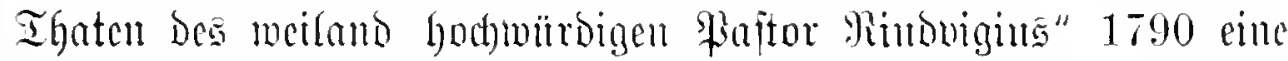

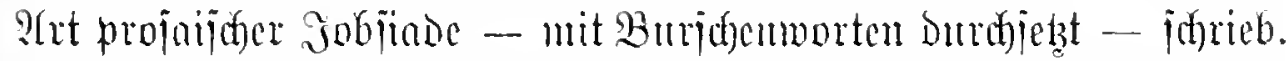

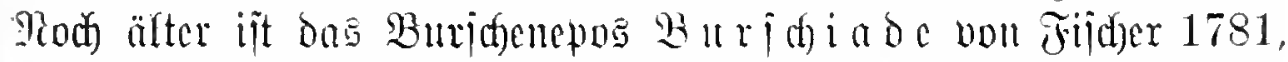

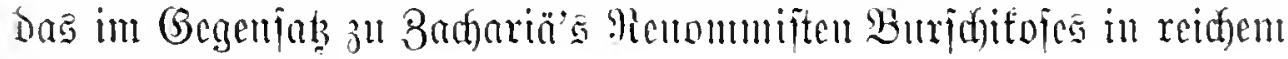

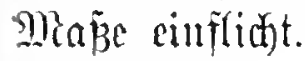

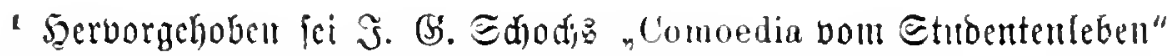

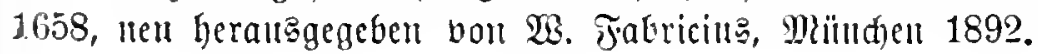




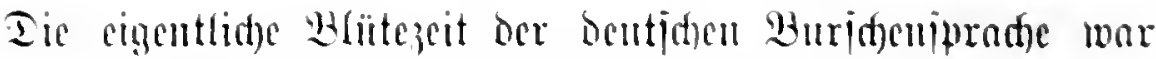

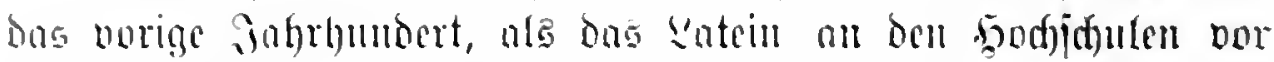

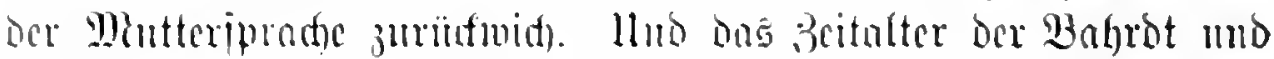

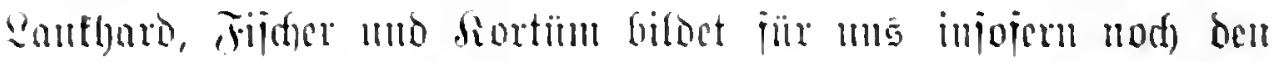

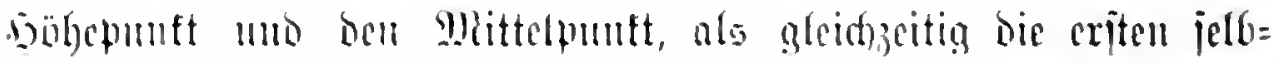

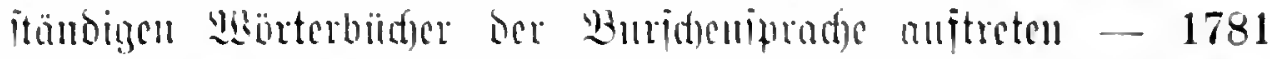

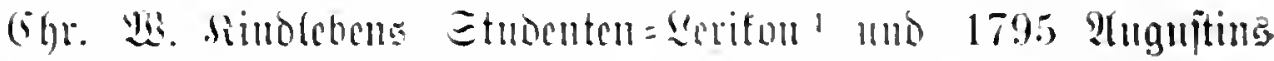

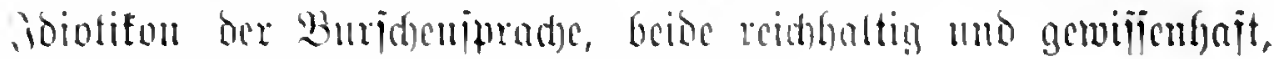

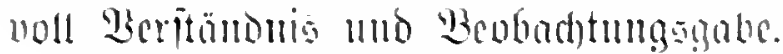

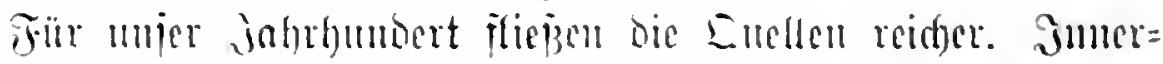

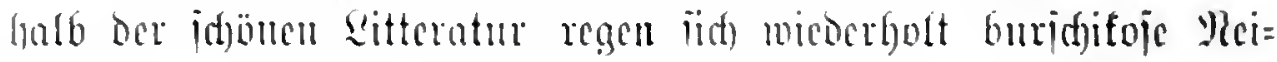

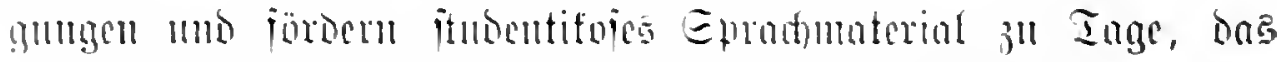

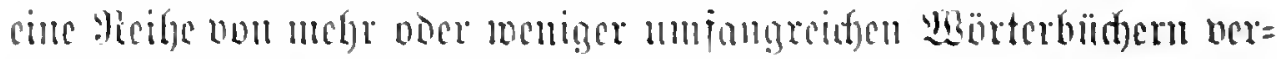

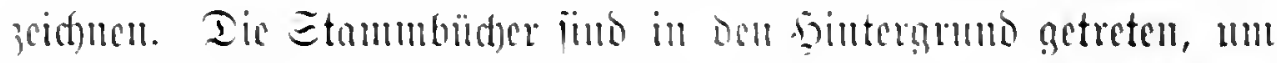

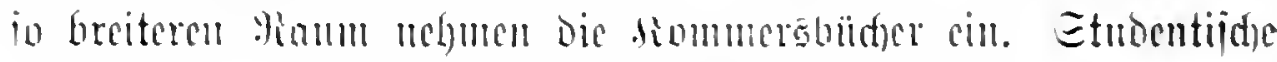

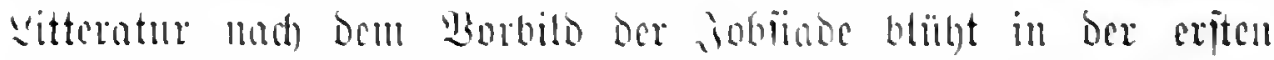

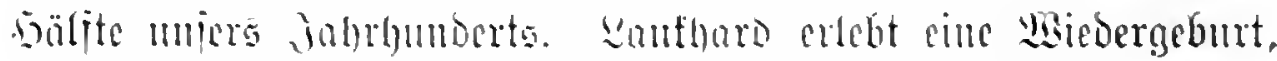

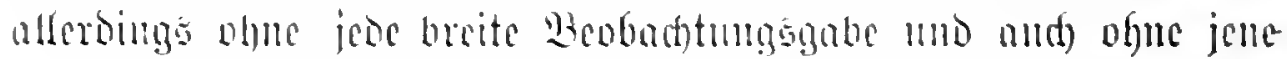

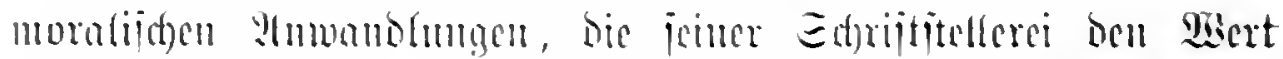

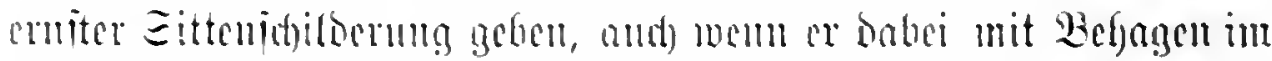

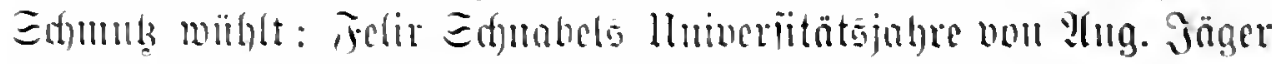

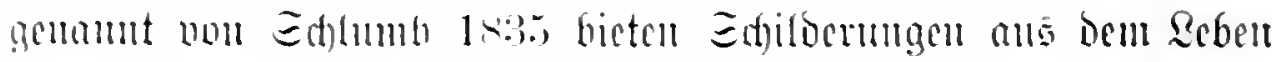

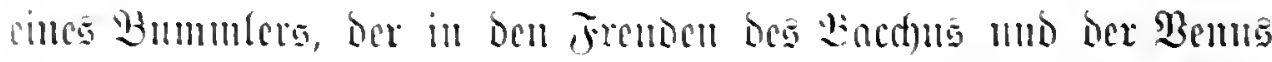

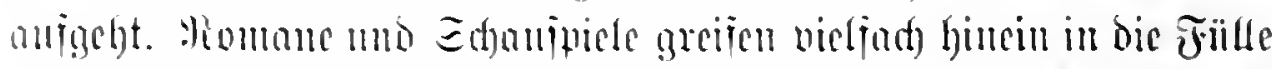

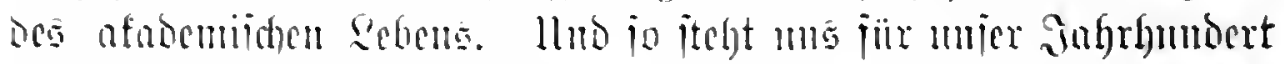

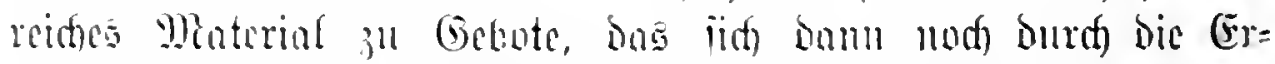

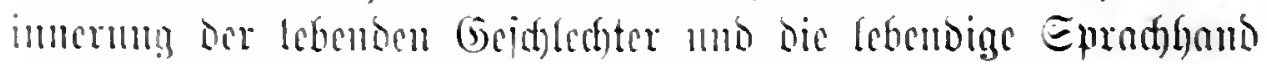

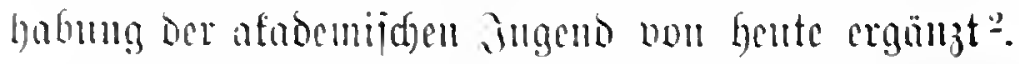

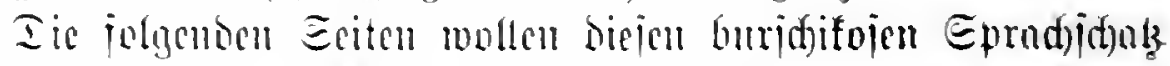

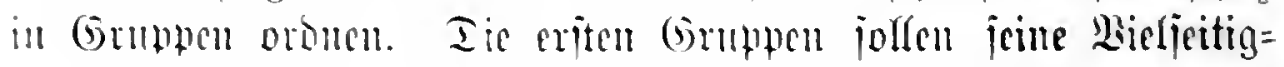

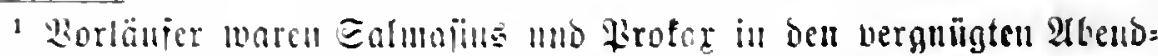

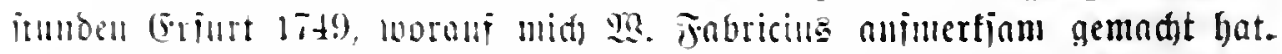

- Eine reidhaltige Wibliographie ber Etmbentenlitteratur bieten

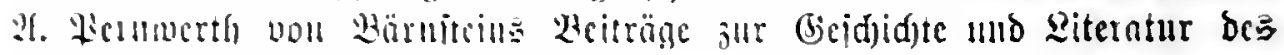

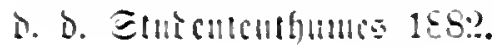




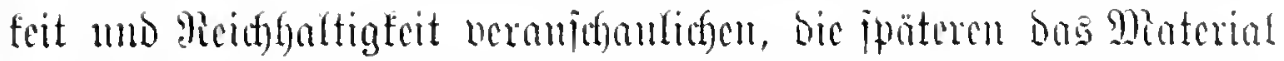

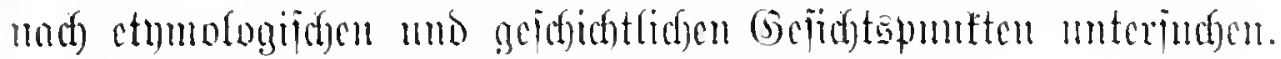

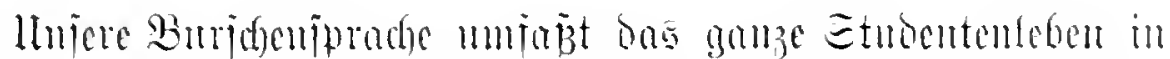

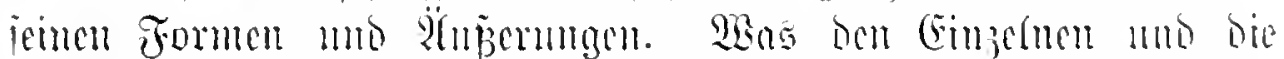
Derbindungen ober bic bejamtheit betrifit, hat cinen itnoentifoien

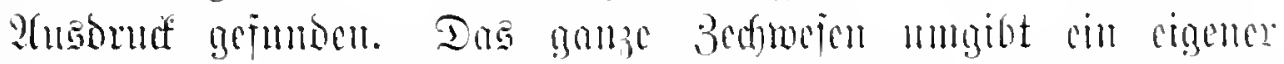

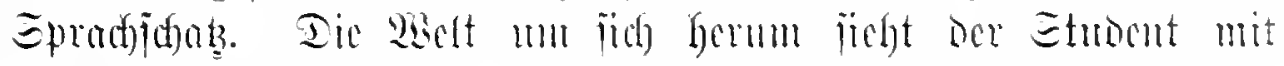

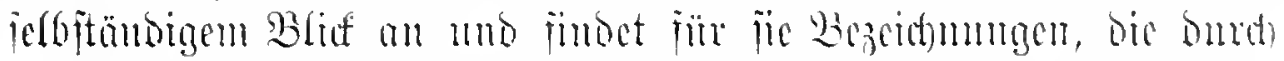

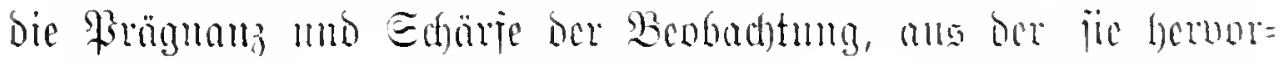

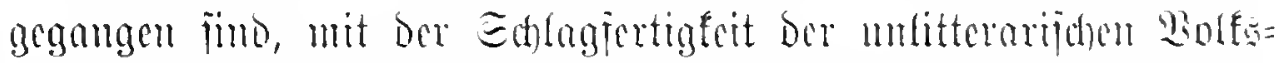

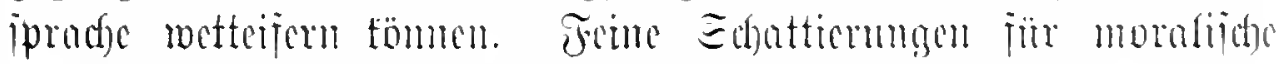

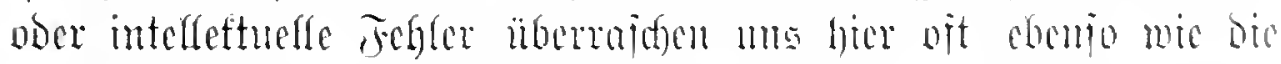

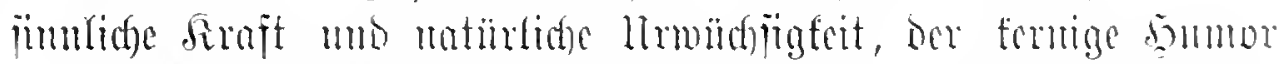

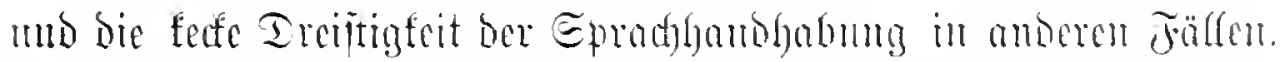

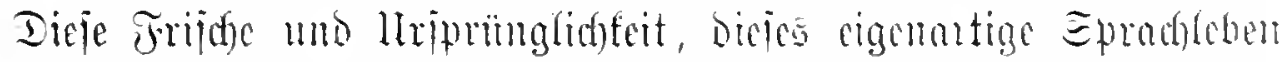

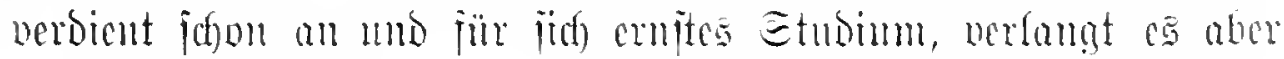

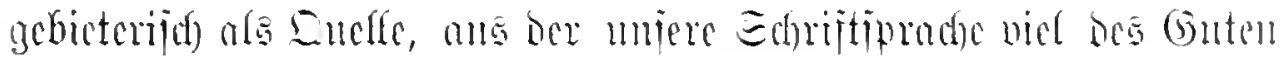
mo bes Deciten ühermommen hat.

\section{Etudenten uno Rifiliter.}

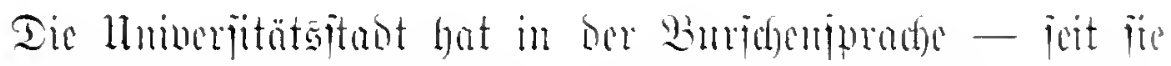

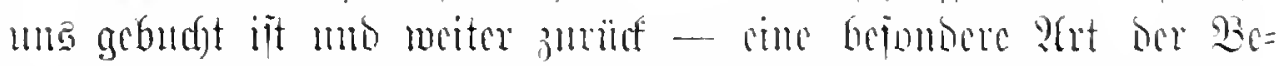

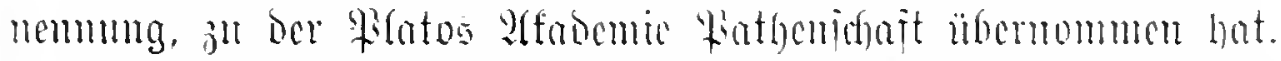

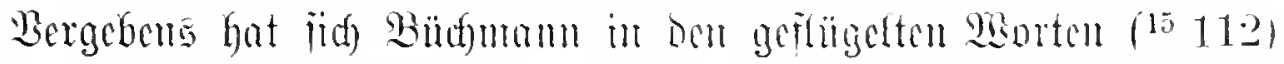

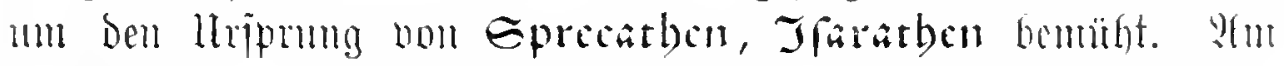

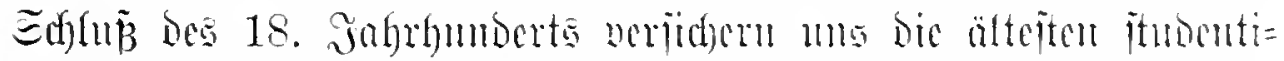

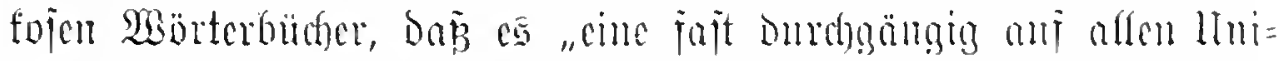

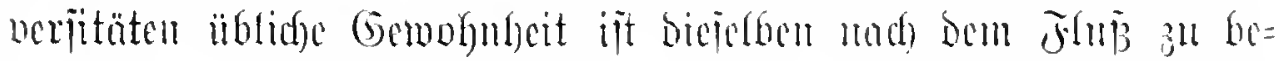

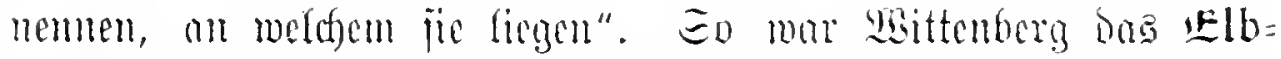

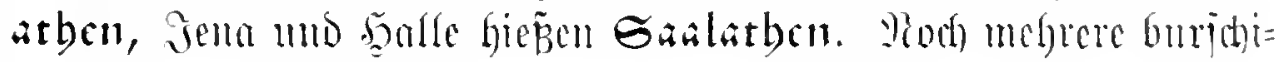




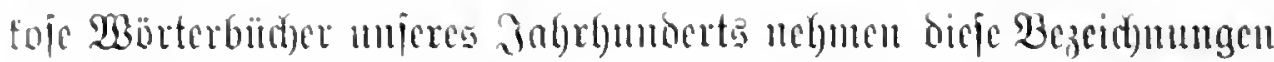

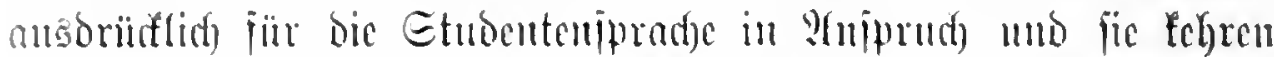

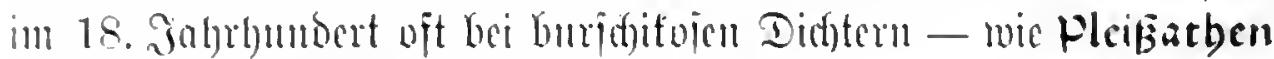
1728 bei bem Estrepier Etoppe 1, 114 - mo in ben Etamm=

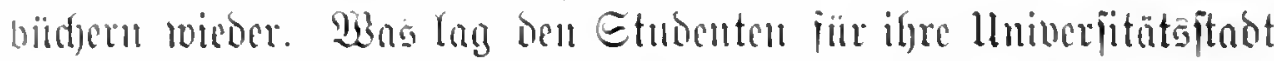

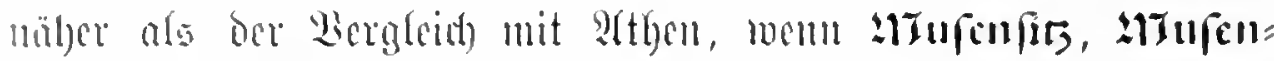

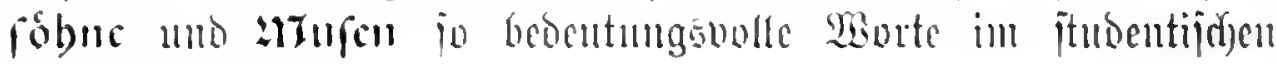

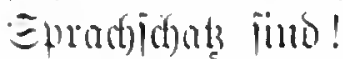

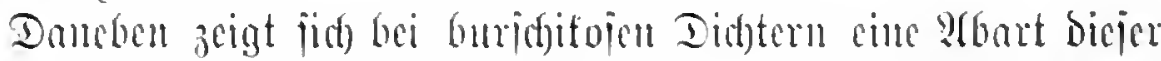
Benenmung. Die betion Enafatben beiben and Gäline (jelten Salana) - Jenu bei (5gr. Sünther uno salle bei fintoleben;

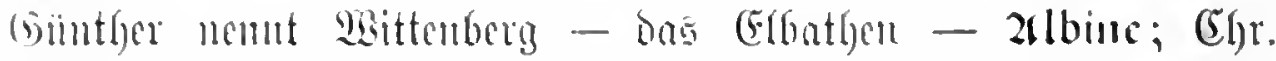

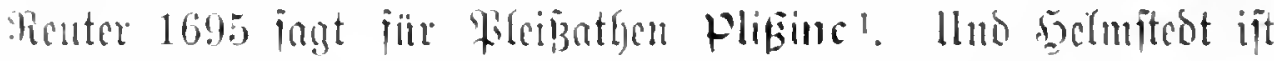

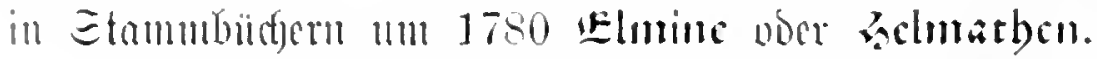

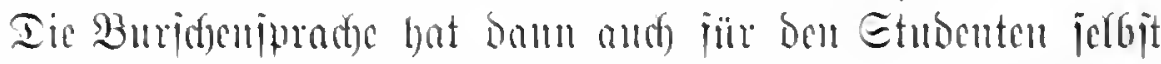

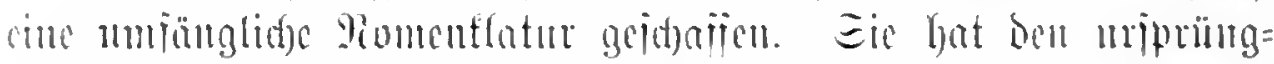

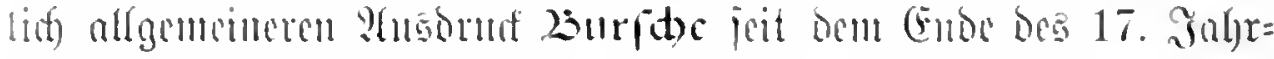

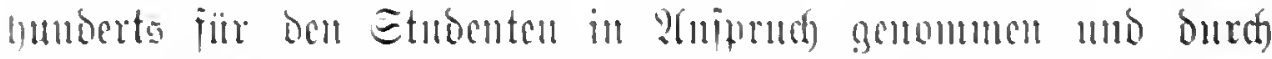

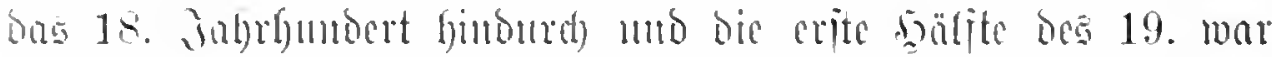

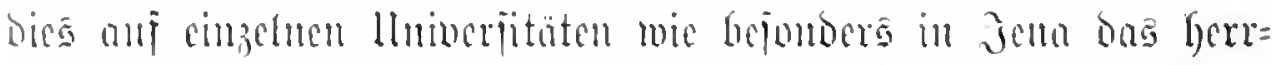

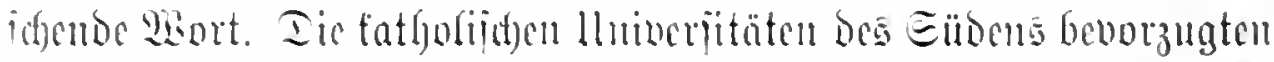

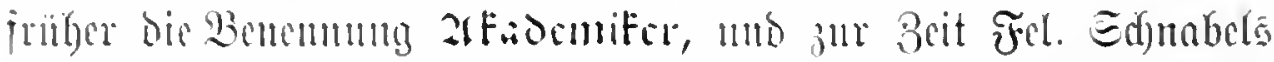

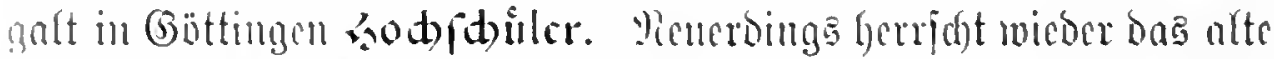

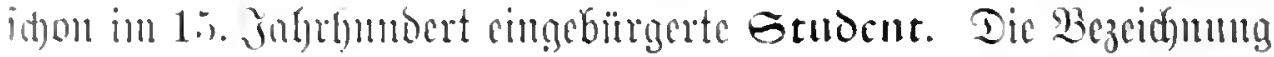

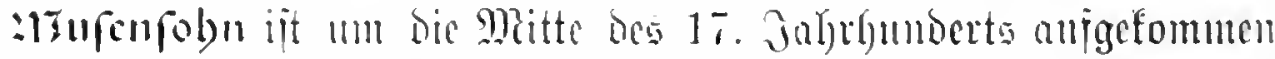

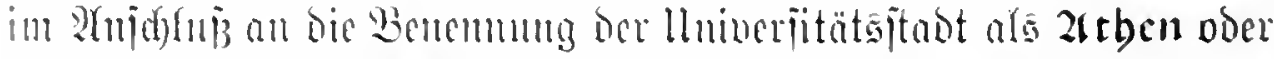

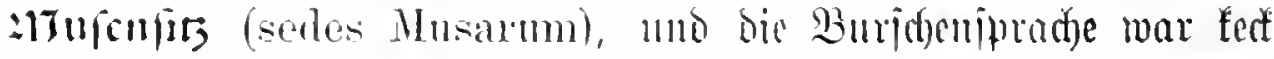

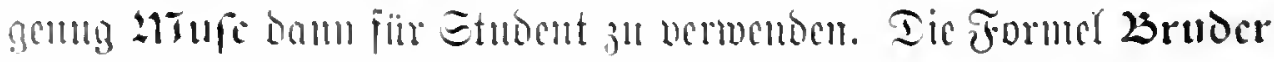

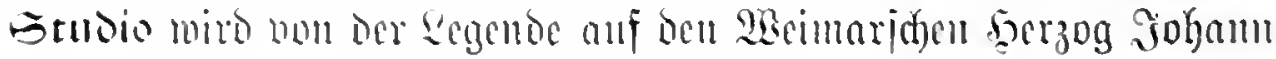

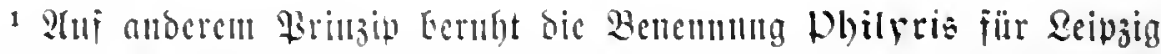

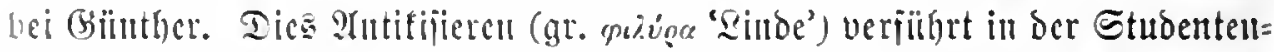
Frad)e aud baju Drten aus ber llungchung ber llniveriität nene Ramen zu

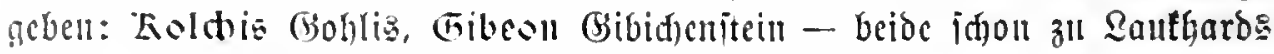
3cit. - Im 1740 gilt aud Elbipolis jïr 2isttenberg. 


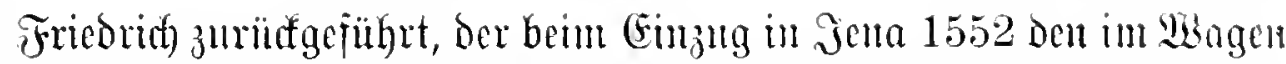

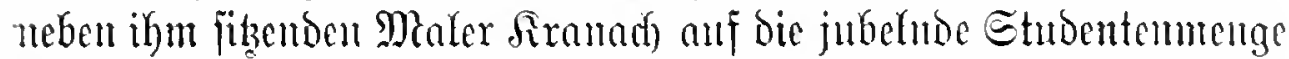

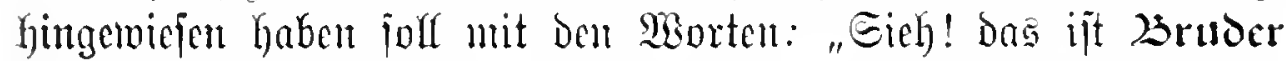
Grubium". In ber That ijt bies die alfere Gejtalt ber Formel, bie aber freilide erift päter, afs bie Regende wilf, anjoutreten iffeint;

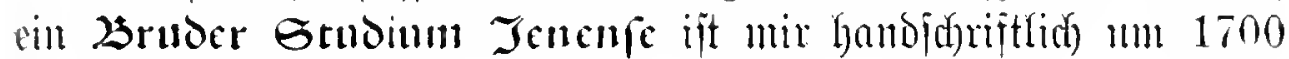
begeguet.

Fiir Den Etnoenten, infujern er in ber llniberijtätşitnot voer

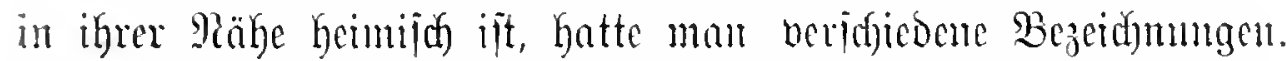
Pflaftertreter namte Der Sommilitone cin ftubierentoses Etant=

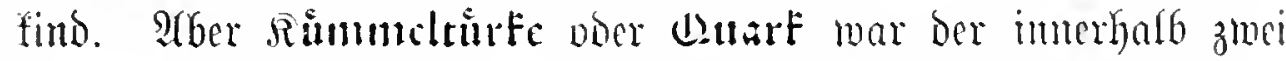

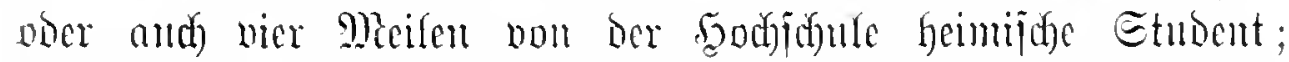

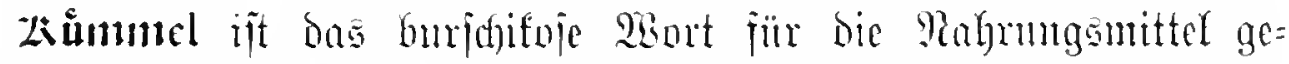

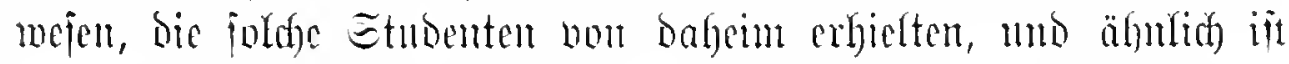

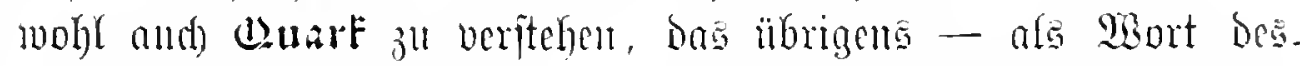

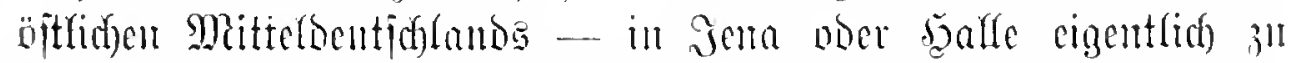

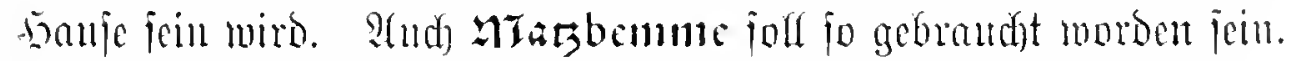

Fïr bie verichiedenen affer Der Etmbentenzeit hat bic

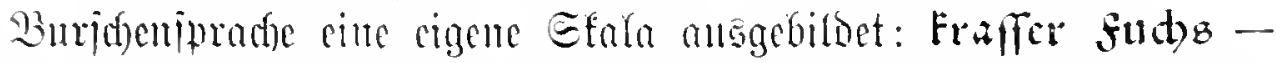

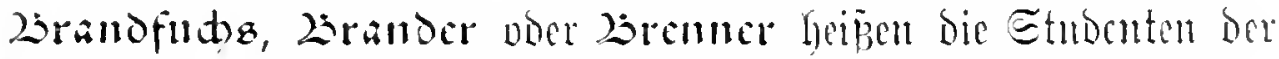
Geiben eriten Eemeiter. Inun folght bic Buriffenzeit: Inugburita,

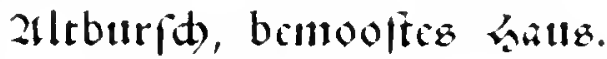

Dicje Sentenmungen haben nidft immer gegurten, mo bis

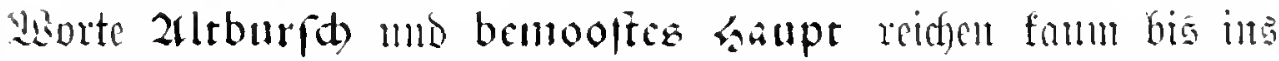

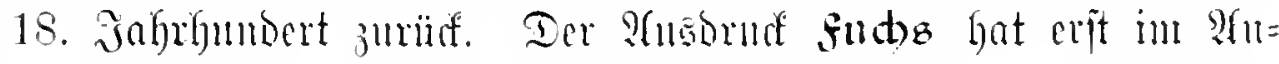
fang bes vorigen Safhrhumberts jeine houtige Bebentung erbalten.

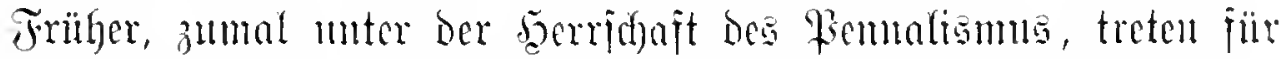

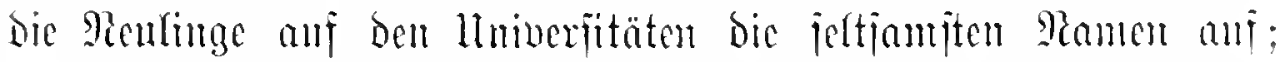

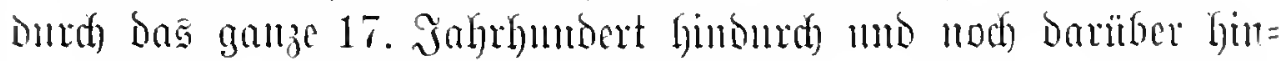
ans hören mir iie Quasimodogeniti, Innocentes, Imperfect, Neovisti memen. 3wijhen 1560-1650 heipt Der Futha foir', modif früber beginut dic Bentunung 25acdant. Der P(usornte

1 Buerit faek ar 'Zaifi, Marr, (Brokian' in Eimon Moth Dictio-

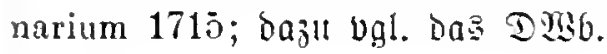




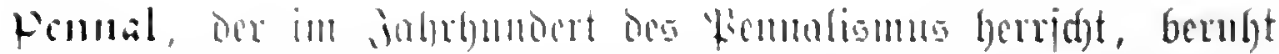

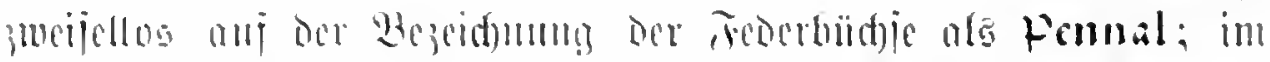

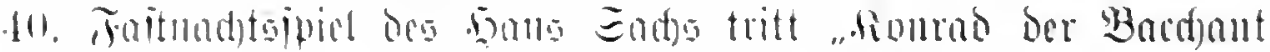

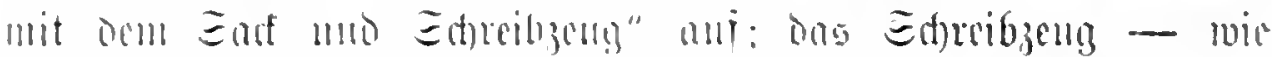

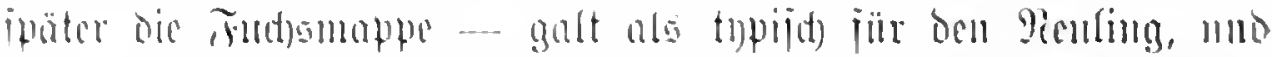

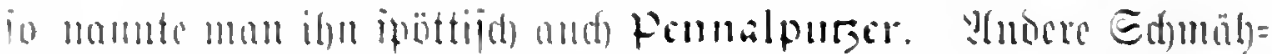

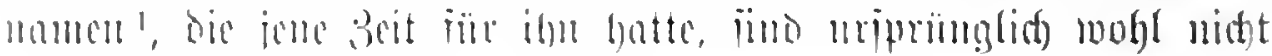

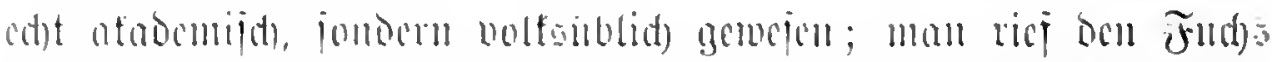

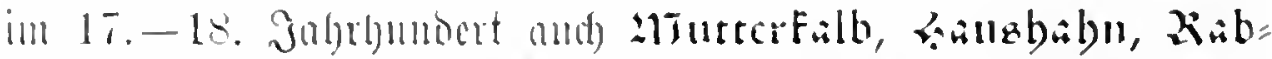

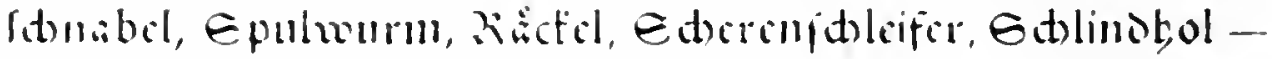

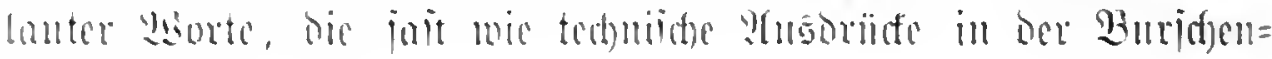
ipration biblict) maren.

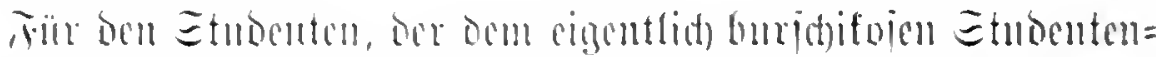

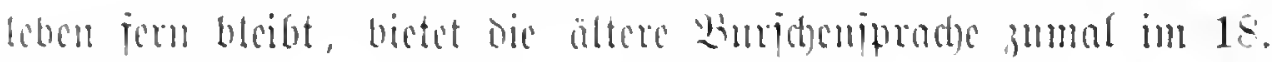

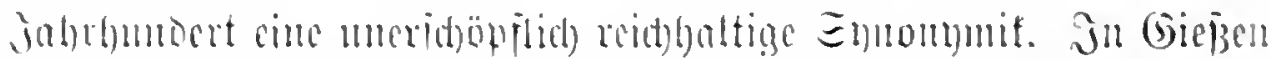

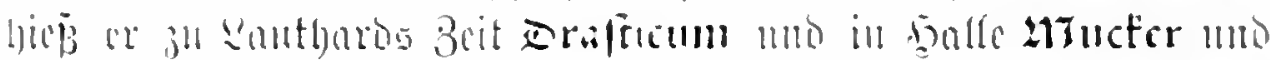

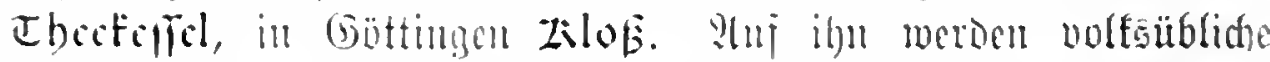

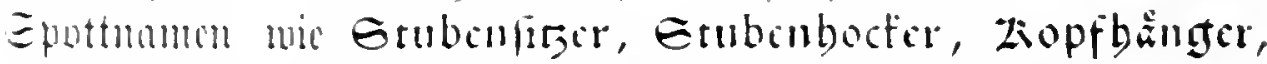

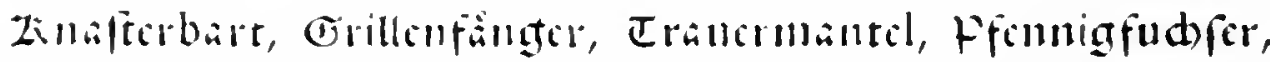

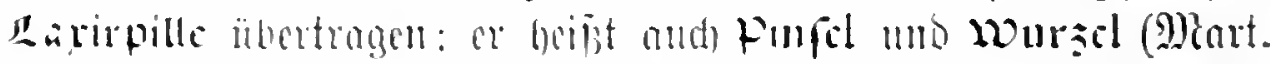

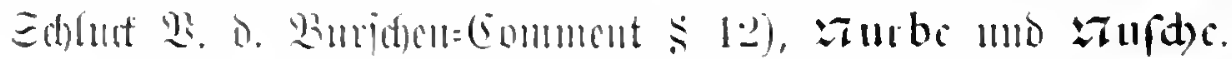

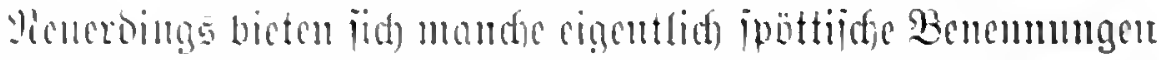

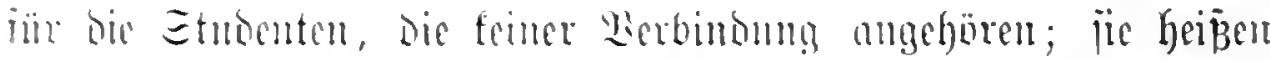

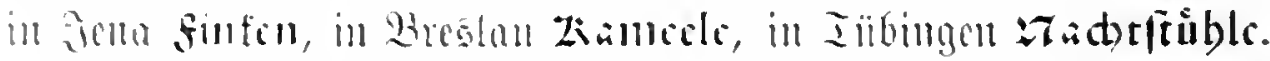

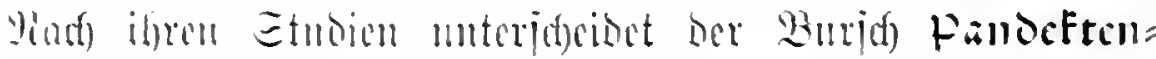

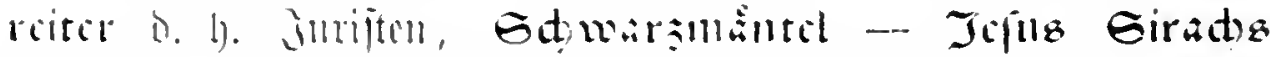

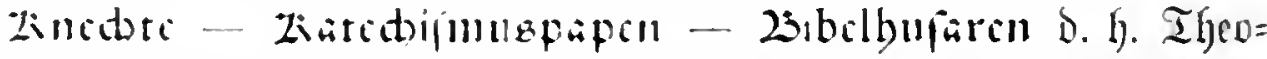

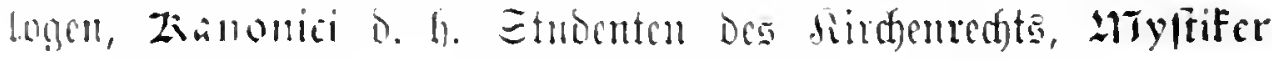

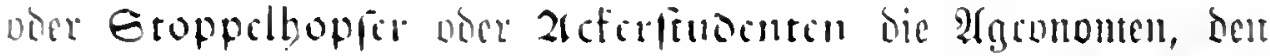

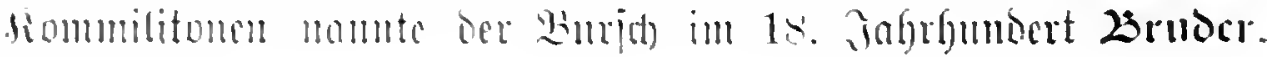

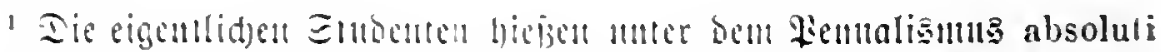

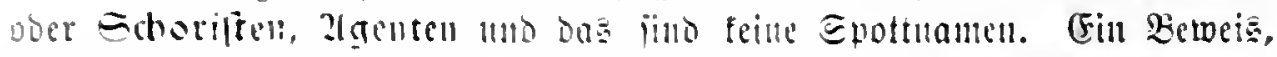

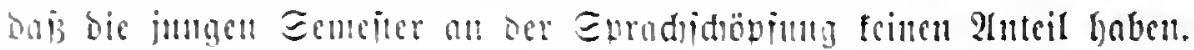


Der Etubengenojie war cin Grubentaball, wopiur uenerdings in

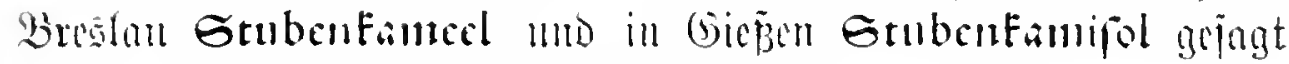
murbs, andermarts and Etubenfmodsen.

Bu biejer jomblabumg bes Epraffunterials jïgte jief) nod

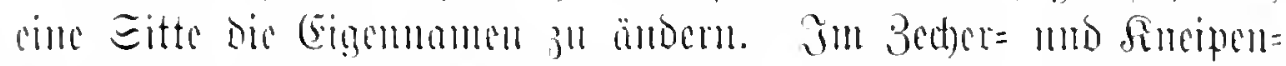

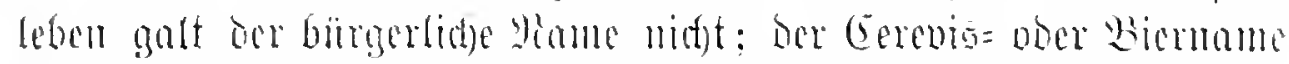

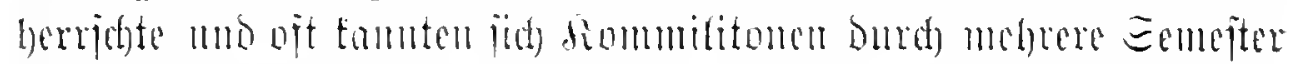

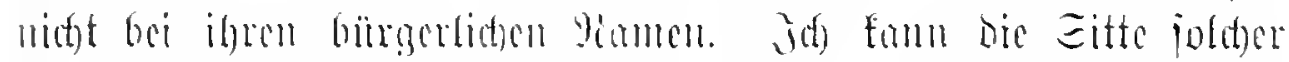

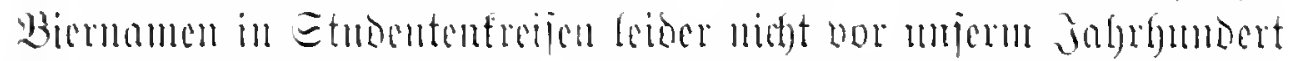

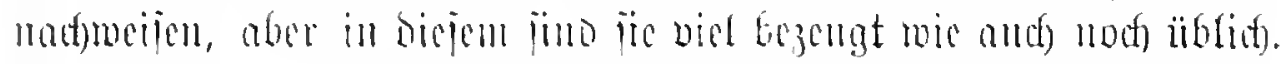

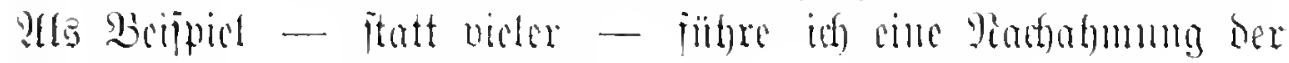

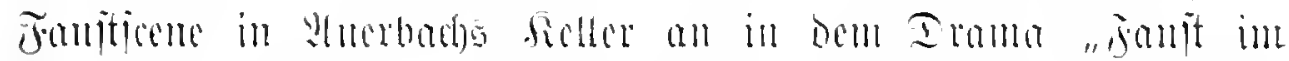
Gerwande ber Beit" non same barring 1831: Da treten ani

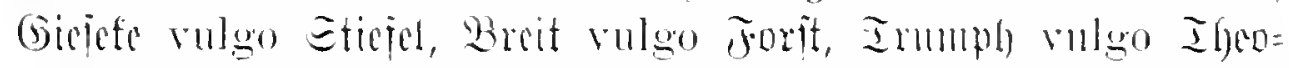

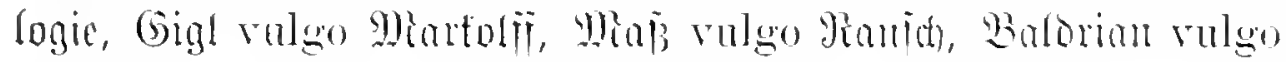

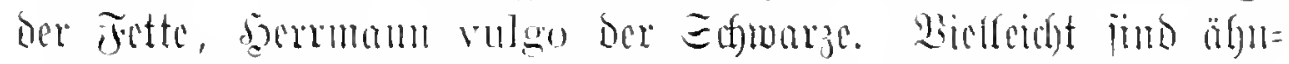

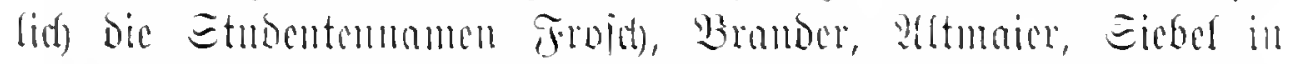

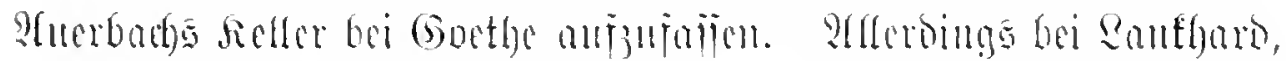

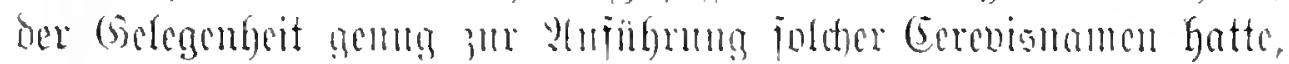

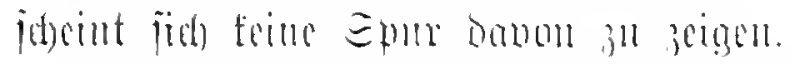

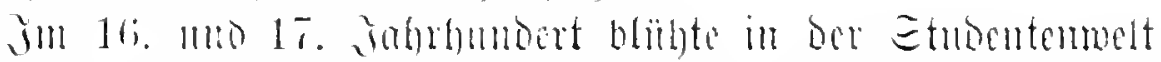

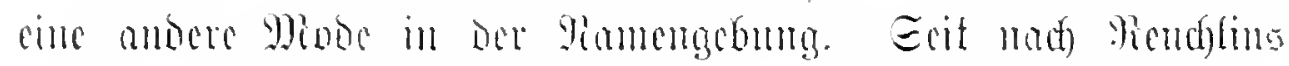

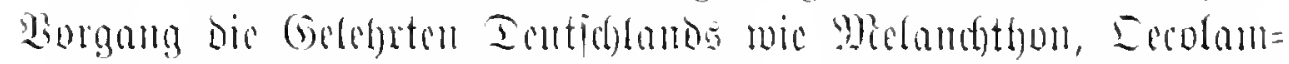

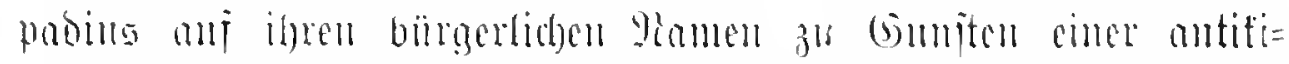

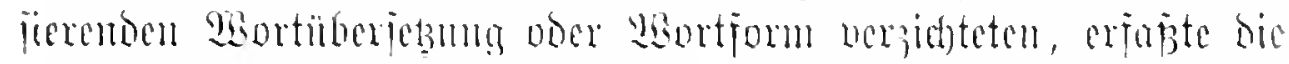

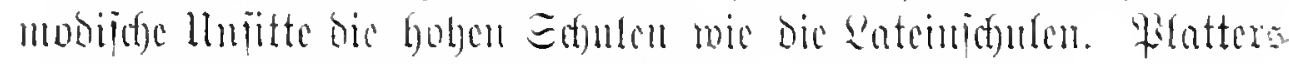

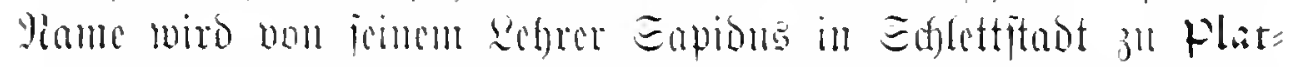

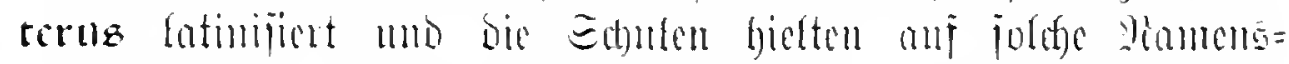

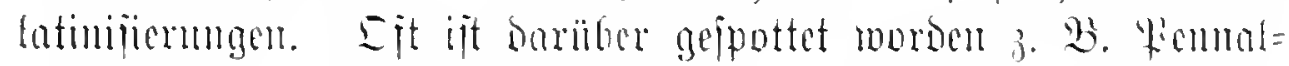

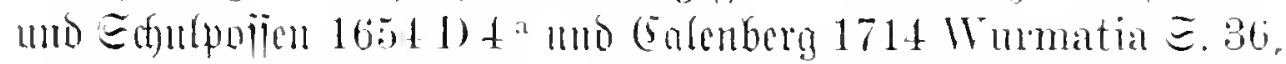
aber immer mieber gört man, baj Etnoenten, Diagijter mo Profejönen an ber Eitte Gejalten baften.

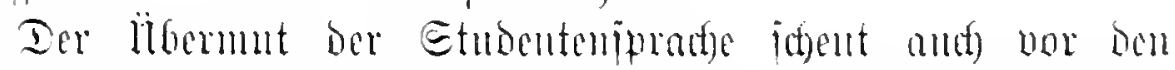

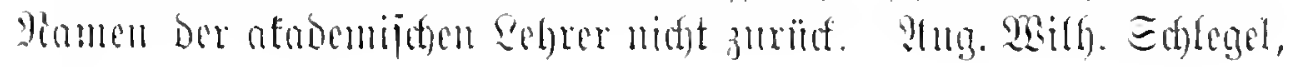


Der 1818-1845 in Boun Wrojejior war, hićs bei ben bortigen

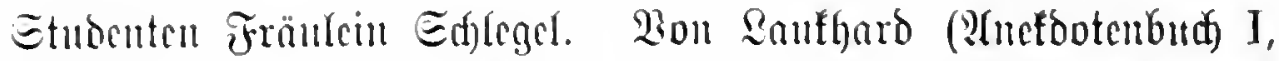

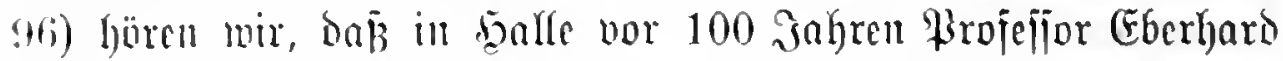

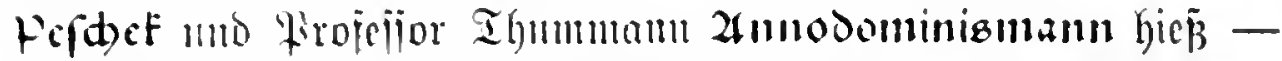
Der Yektere, "meil or bic Juhresjaflen immer io anführte: anno

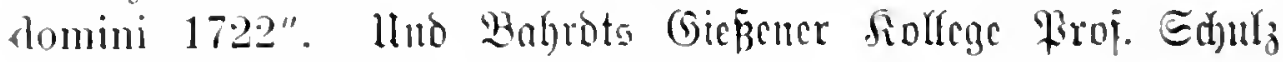
mar Pring Rubba.

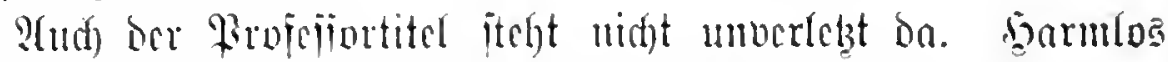

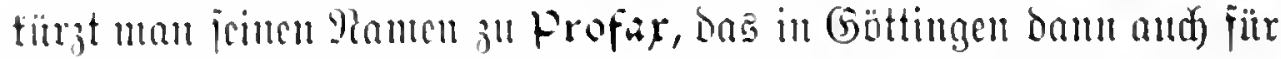

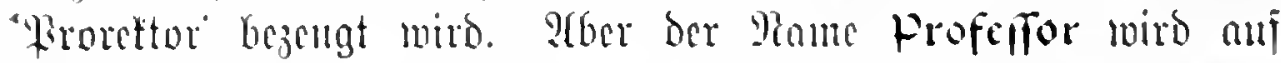
helickige semerbe itbertragen. Der Etuont fanm mit fects=

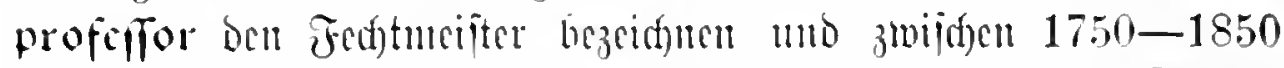

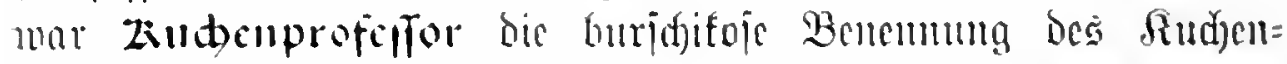
hädfese, bor - wis man an bem boun jungen (socthe gejeierten

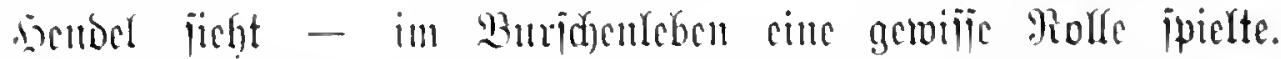
Sicimmalo 1720 nent jid) ielfit cincu 2 beproferfor jür bic Beit

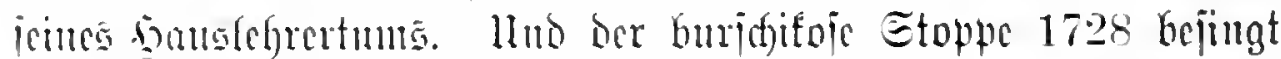

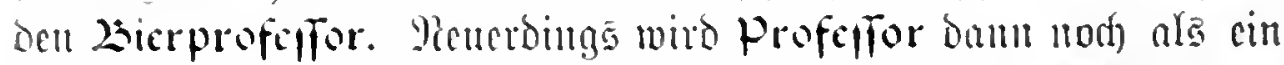

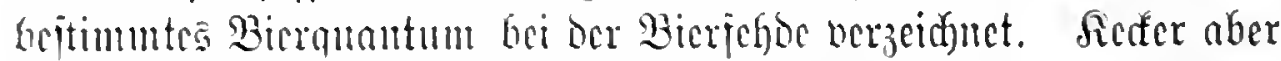
und Drcifter itt bic Bencmung Prinatoocontin für meretrix.

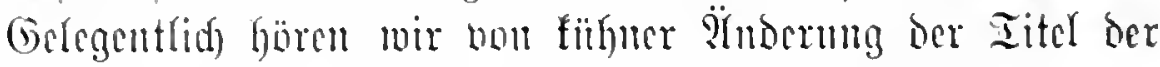

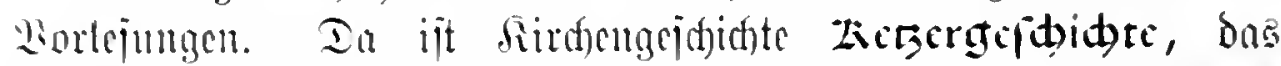
collegium logicum heibt fudefolleg. Int io wiro and bic

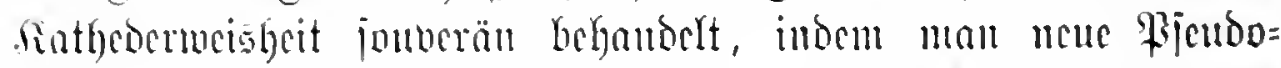

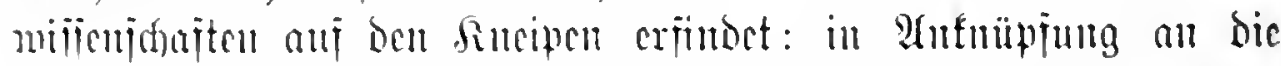

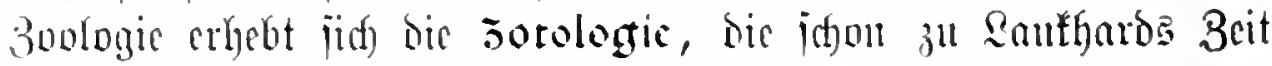

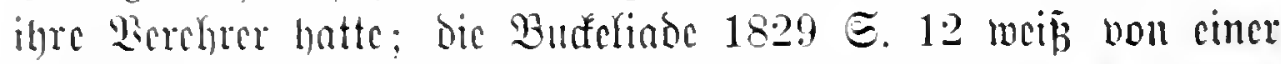
Gdnapicologtic; ill buridjifojen Intefoten von 1831 ijt vou ber zoniffeologic bis Reds; Bolfmann 1846 fennt bic Sdimpfiologie,

1 Ebenjo harmlos iit es, wellu ber \$rofelior beş Sanonijaen Redits

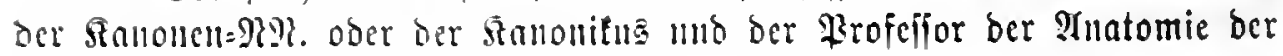

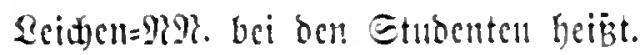


Euffologie, Edfologtic n. a. und nenerbings fört man meben Der Biologie and bon einer sticrologic.

Berfaijen mir bie Rernenden und Refrenten, jo erimem wir uns an bie buridgifoje (Finteilung der Menjhbeit, wie fie uns decine

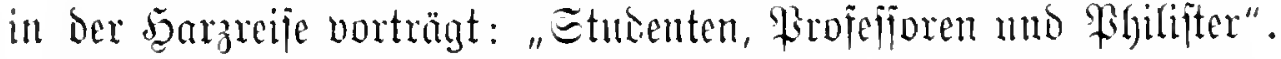
Sanffards Beit unteridjeidet etwas anders - "Burjhen, finoten,

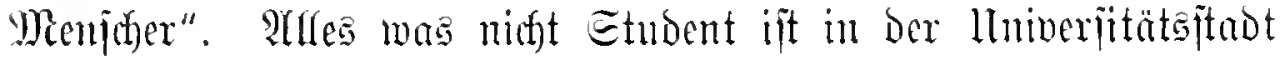
hat Der Etudent ju veridiederten Beiten in veriffiedene Sifhlagmorte

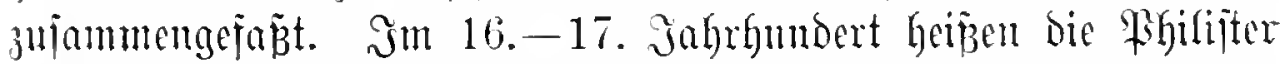

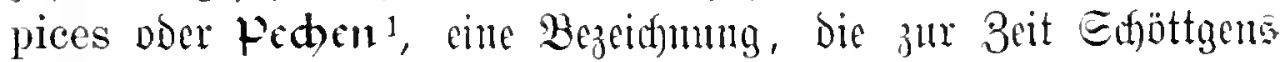
(1747 Şijtorie bes seunalwejens Ð. 26) ansgejtorben war; biel= leidst war es uriprïnglide ein Epottname fïr bie Frubrlente, Der Dann nur nerallgemeinert wäre; Dofür jprifgt anth bie Sangabe Meljarts (1636 (5hrijtl. (Erimterming S. 227, 237), Edmierer mb 23eden jeien Burjugenworte jün bie Bürger. Feimwald 1720

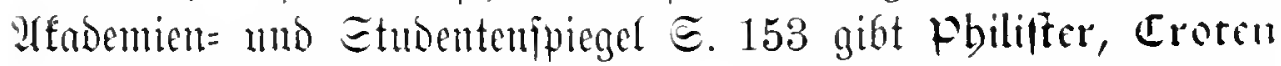
unb Flatter als bie Epottnamen der Burichenipradie für bic Bürger an veriafiedenen şodjidjulen. Croten mo Platter haben iid) bisher jonjt nodh nifgt gefunden; ijt bos eritere als Ziroten

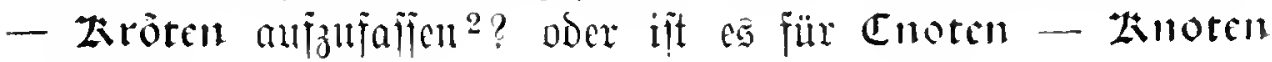
veronutut?

Die Bejeiffunng Fhilifer hat jiat jeit 1700 und - wie es jofeint onn Jena aus - verbreitet. Jgr llriprung mird ums unten હ. 56 beichäjtigen. Eeit 1790 gat jie jict) ans bem jtu= dentipifen Bereidy emporgearbeitet und in Der Ritteraturipracte ein

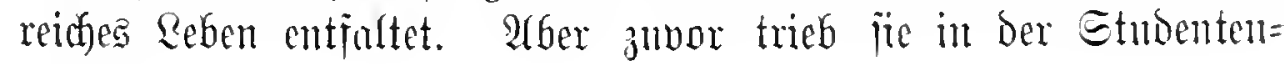

1 Lucas de Penna 1611 De Jure et Natura Pennalium S. T0. Sambers (unter Dhilifter) belegt bie Sdreibutig Degen fïr pecten ans Jablonifiz Iflfgem. 2er. ס. Rünite 787.

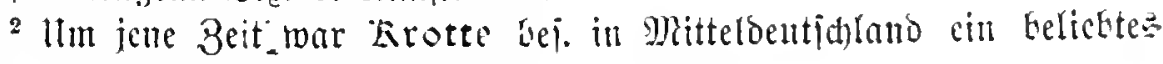
Sdimpitwort; vgl. Sticler Sp. 1043. 


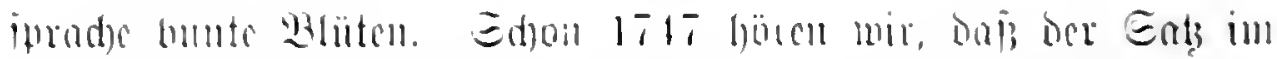

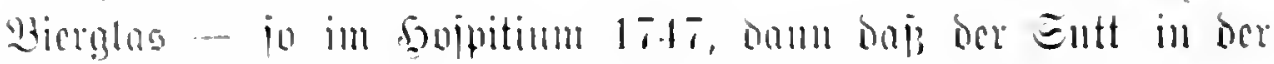

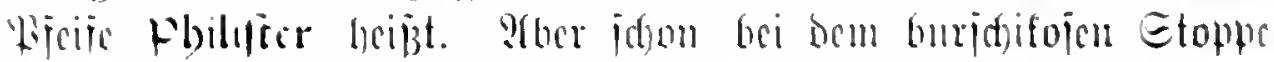

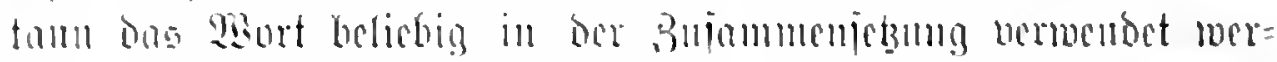

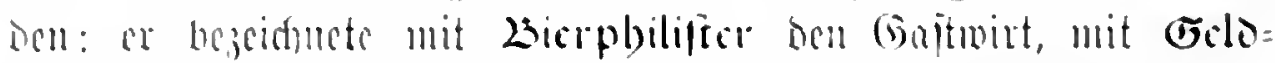

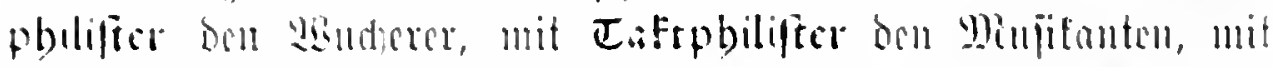

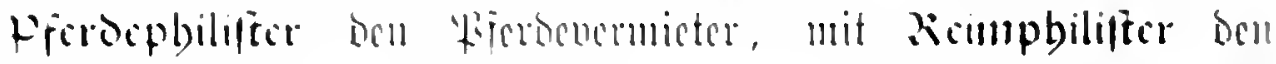

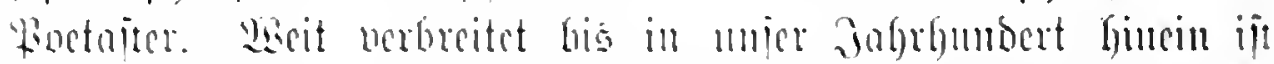

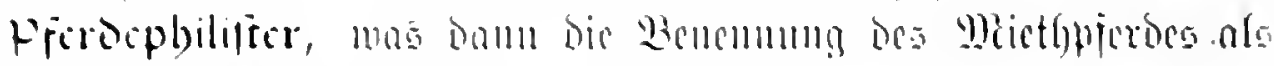

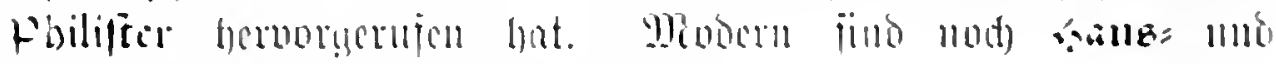
Fumpplilifer, nud zirospbilifer.

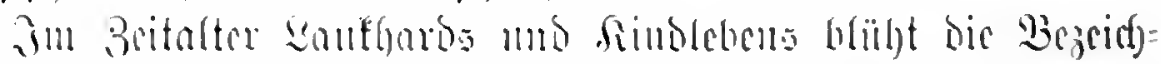

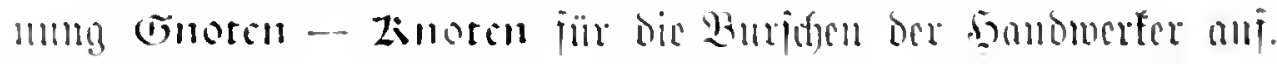

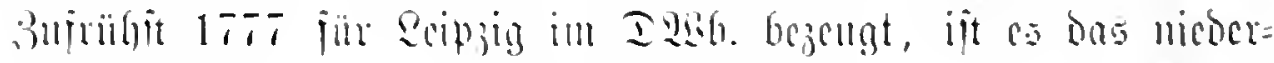

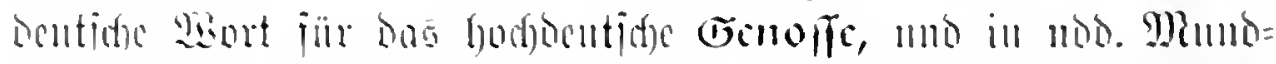

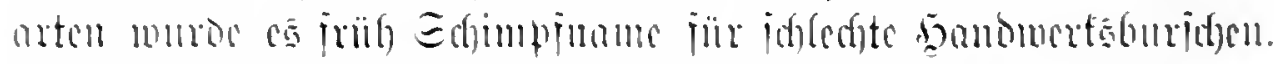

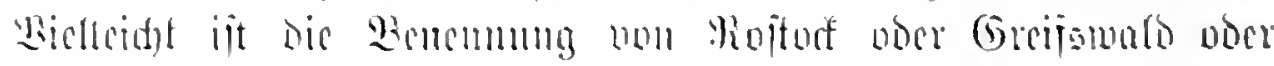

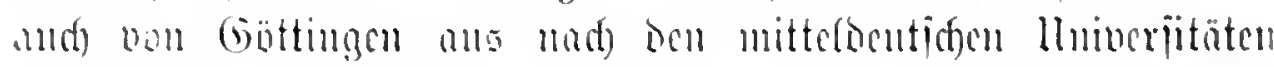

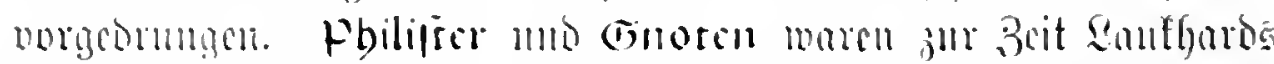

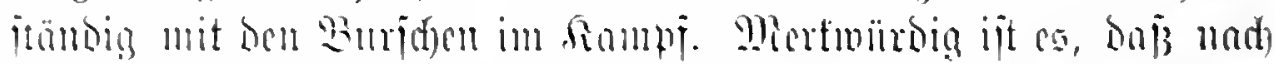

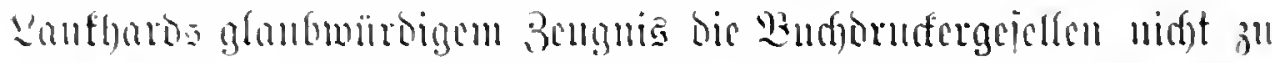

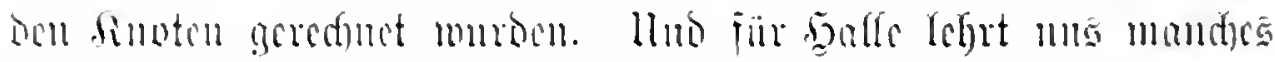

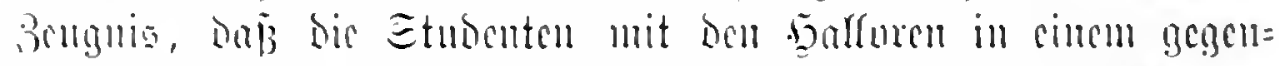

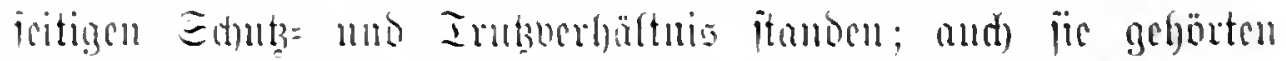

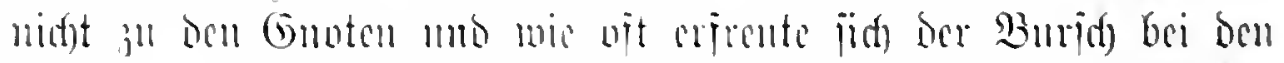

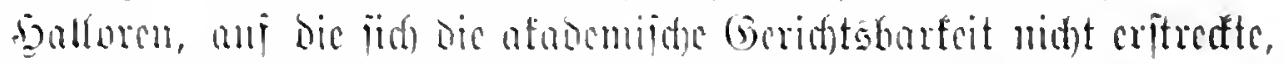

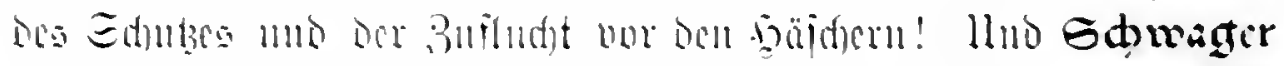

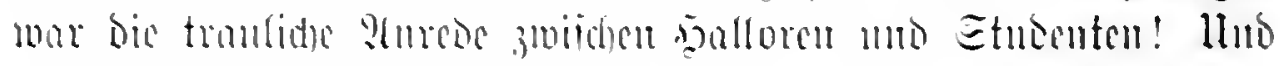

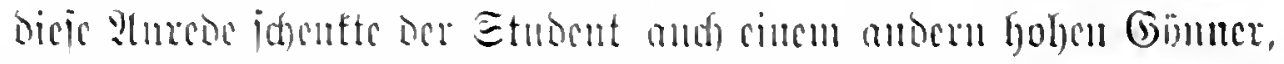

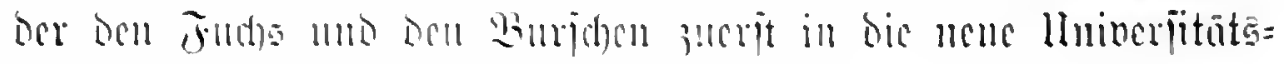

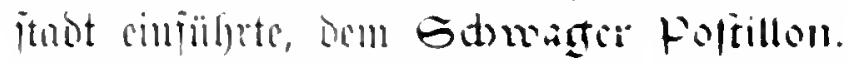

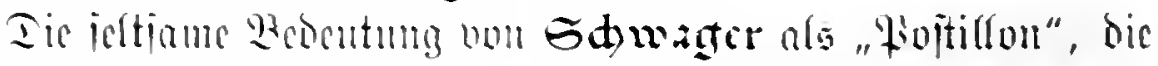

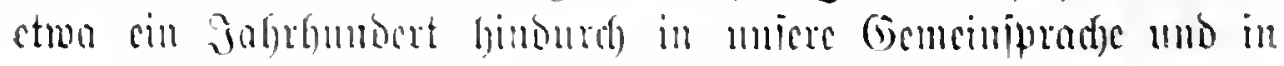

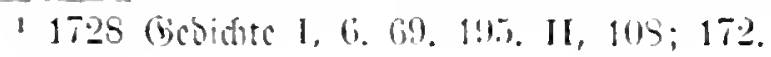




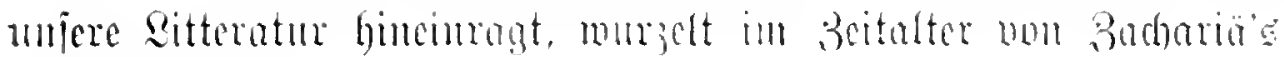
Rentumititen.

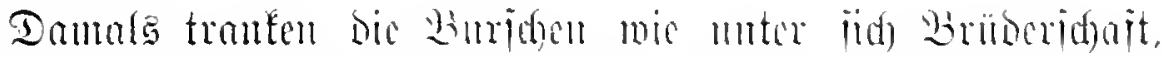

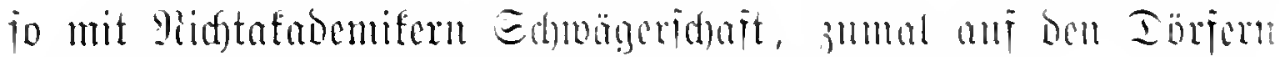

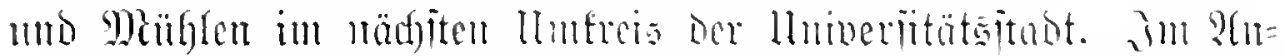

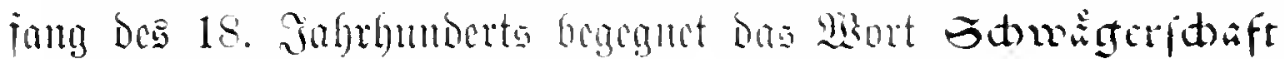
in ciner bitter crmiten Babutum Gei Dem Echlöier Etoppe (II

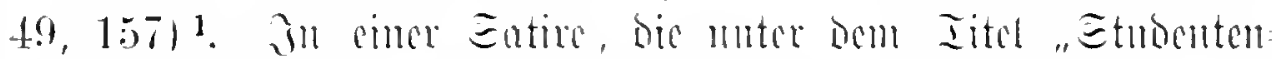
moral" $175 t$ ienas afobemijche imgeno ampüttelu iollte, mire

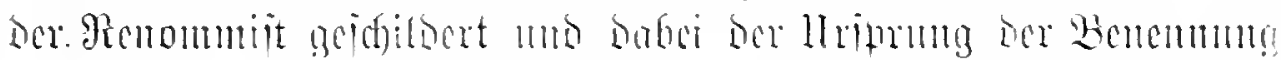

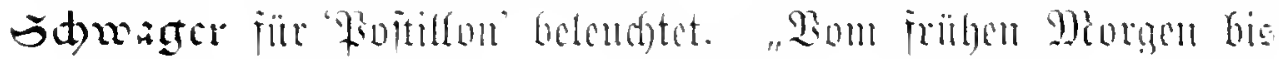

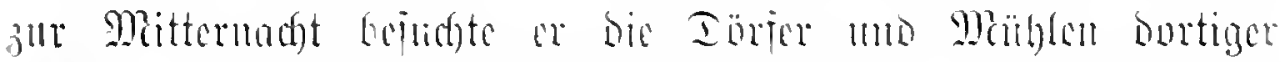

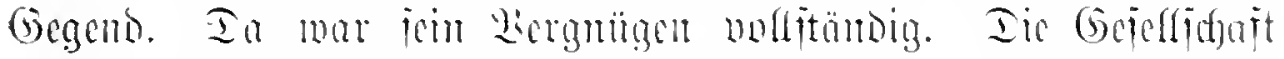

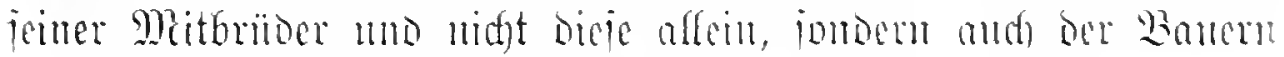
nud Eothaten, dic jeme Echmäger mo Brüber murben - man

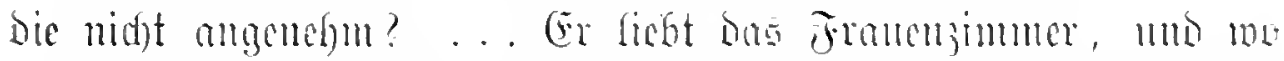

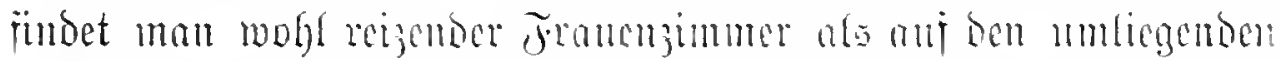

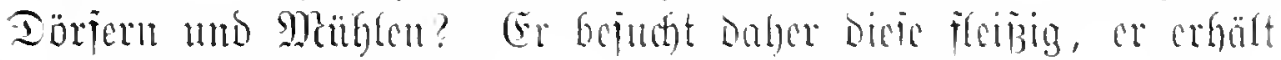

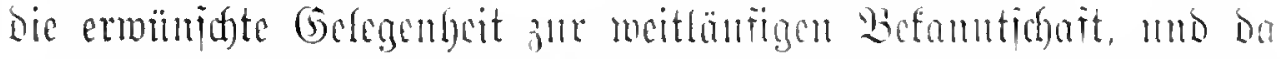

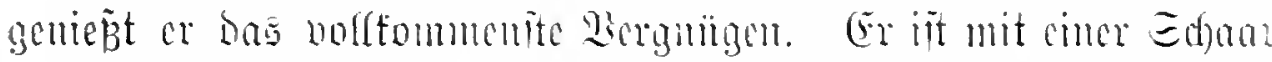

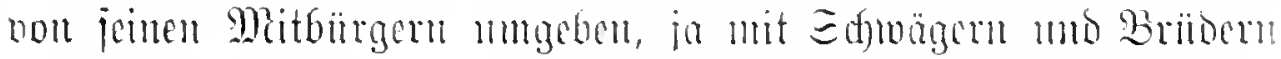

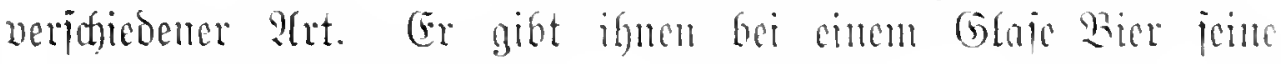

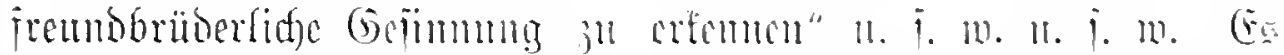

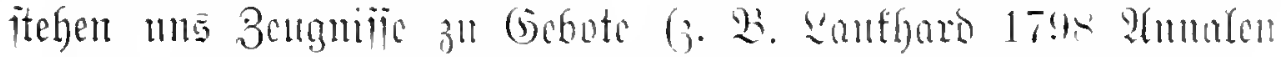

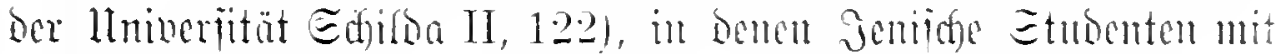

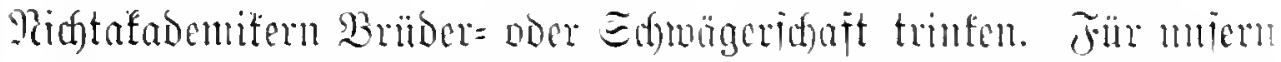

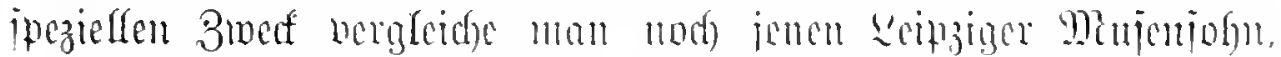

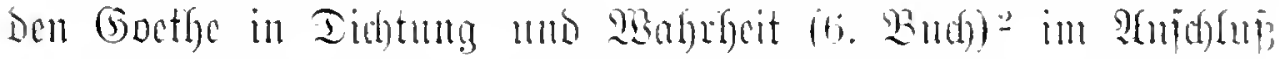

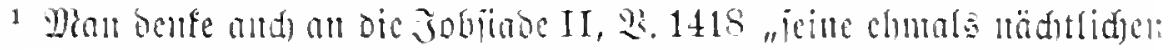

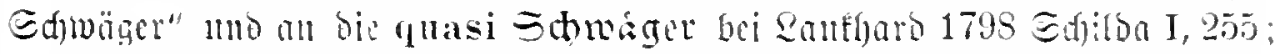
"Der Marfi war mit ieinen lhutertancu mittelfar poer munittelhar ber=

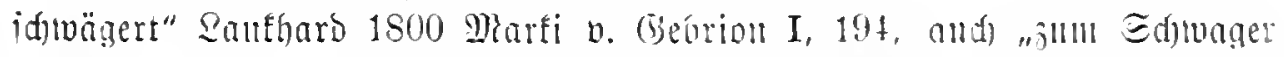
gemad)t werbcu" bei Giïnther. Etieler 1791 bnd Tanfmolthwaget. Ecit

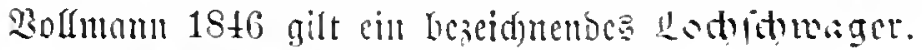

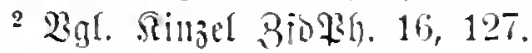




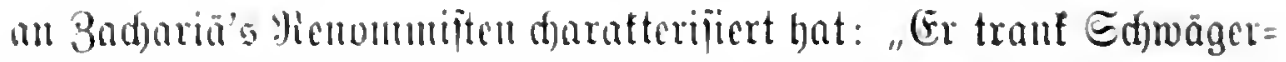

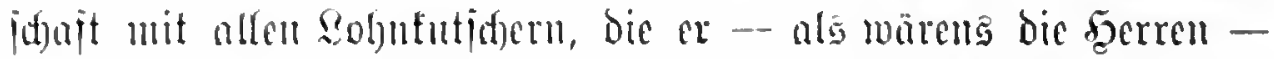

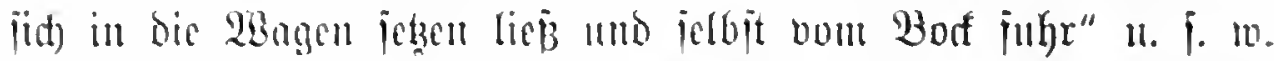
J̌n biejem Bengnis johen mir bis Edymägeringajt auj bic Rohn=

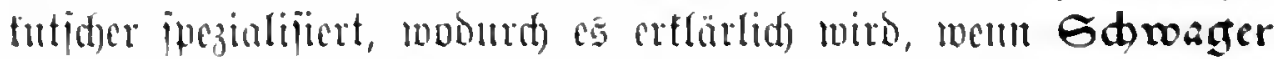

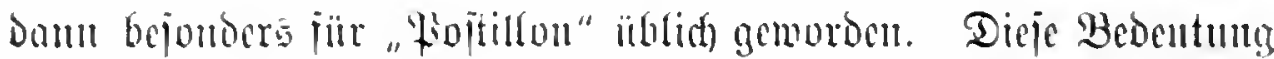

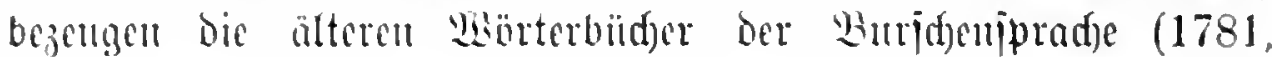

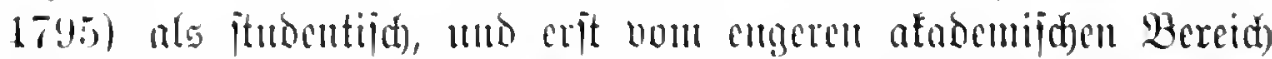

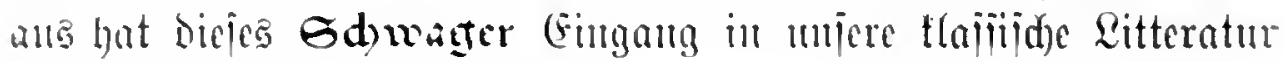
gejuntori.

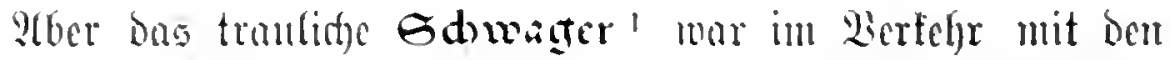

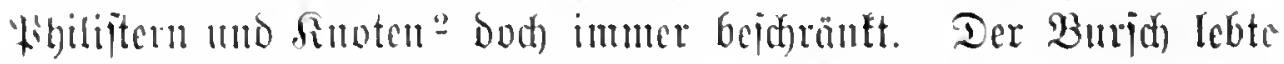
mit ifnen immer in Epanmung mo bie SHmaten mijere llniver=

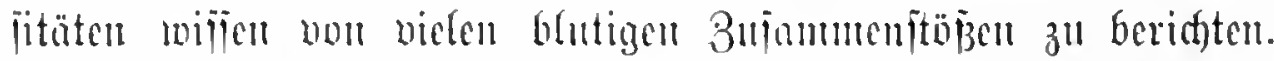

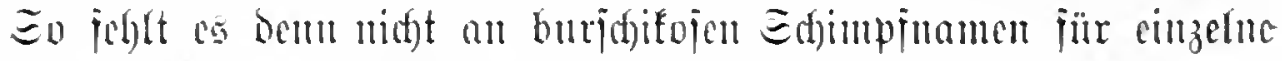

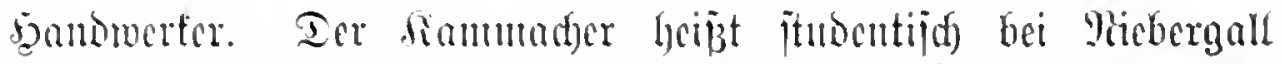

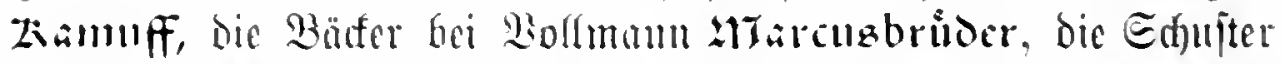

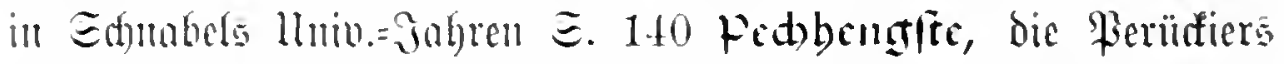
1759 in einem Iraneripiel Perictenbenglte. Jun 16. Jahls=

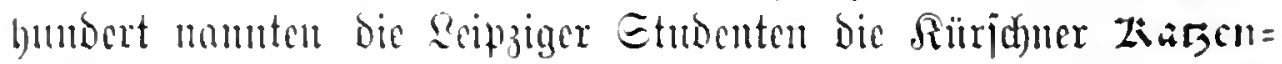

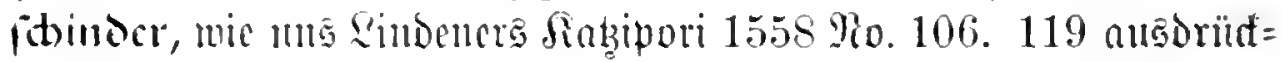

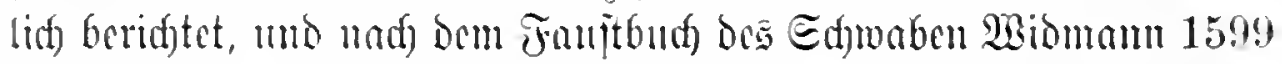

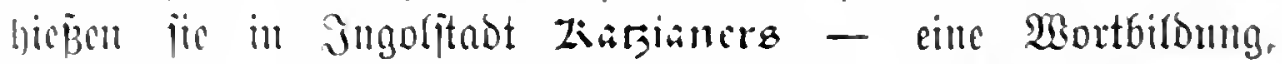

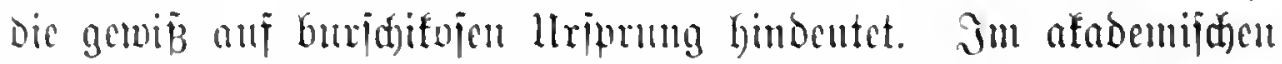

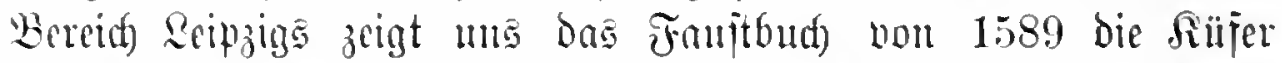

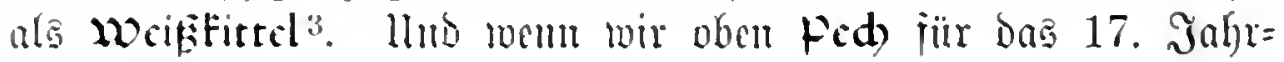

1 Fïr bie obell vorgetragene Bebeltung ipridtt auBer ber bereitz er=

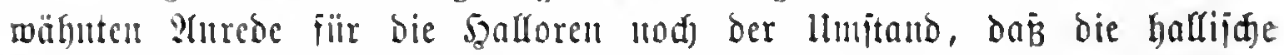

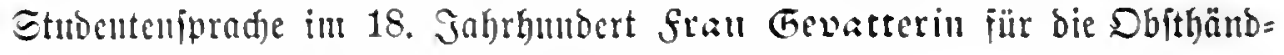

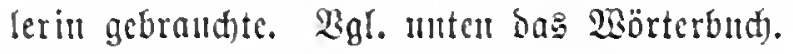

2 Reuersings wirb fïr Tilbingen and Gogen mid für betbelberg

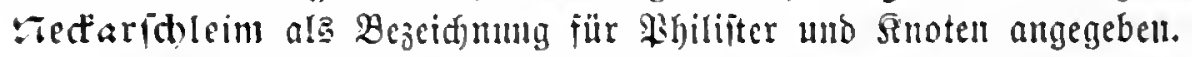

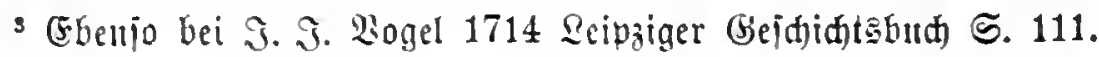




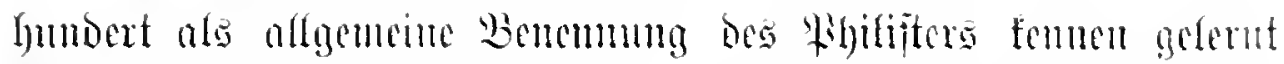

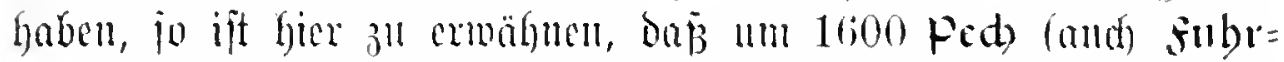

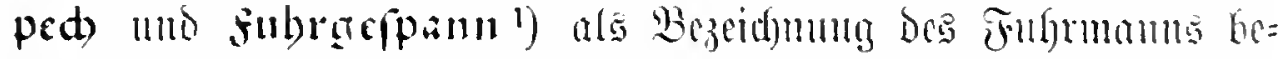

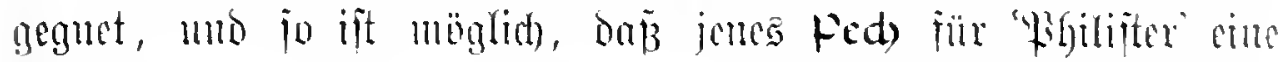

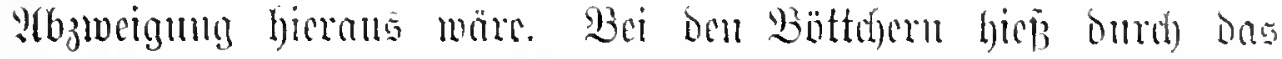

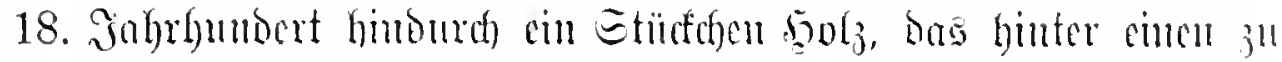
weiten seif gejolangen mird, Pbilifter. Sümte cimmal Pbiluter

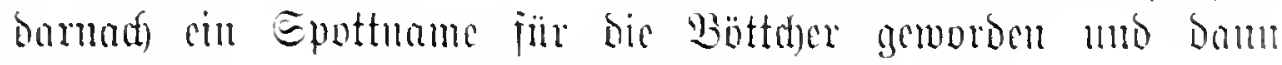

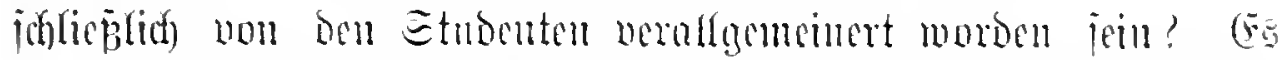

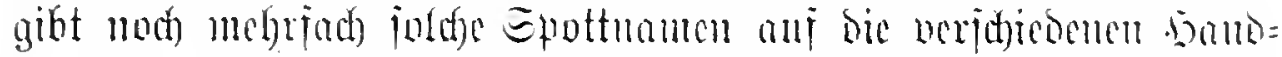

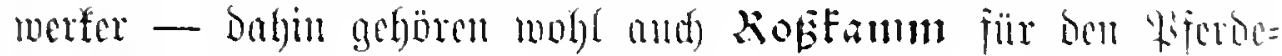

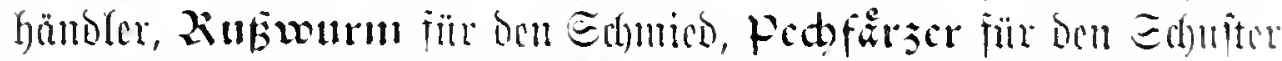

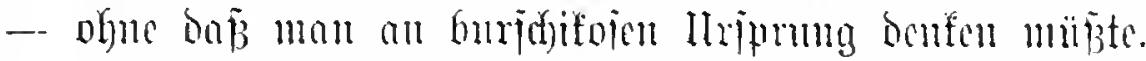

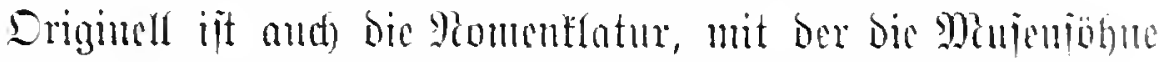

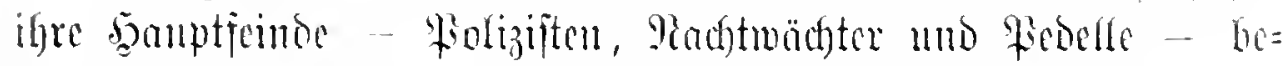

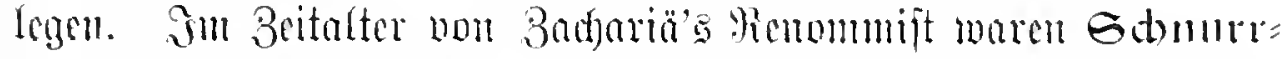

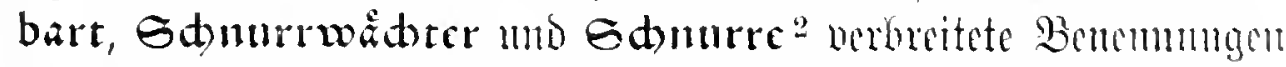

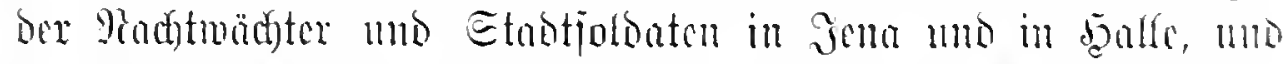

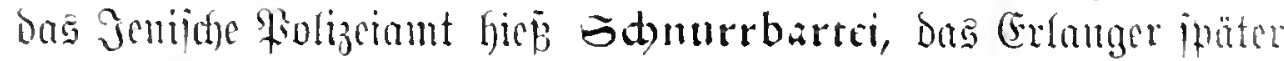

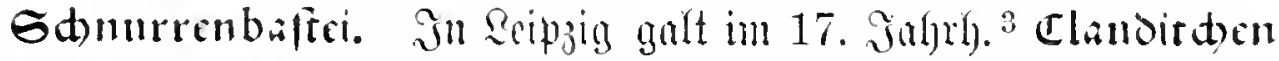

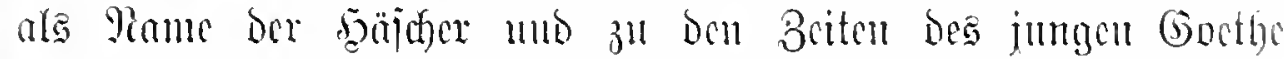
waren MTcifen die Etadtiofonten. Enujt trefien wir im 16. mo 17.

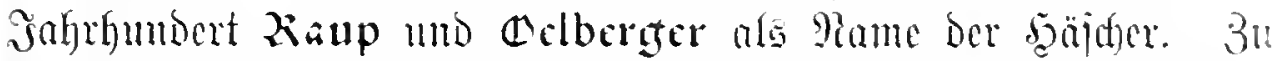

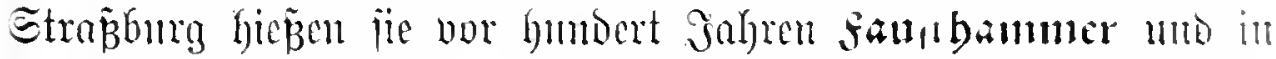

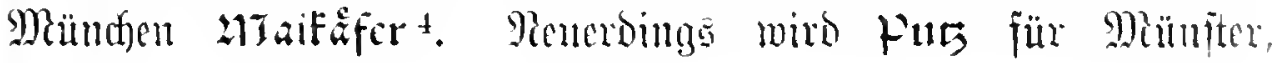

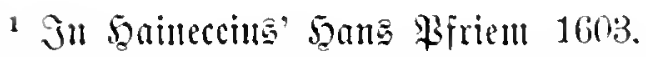

2 Eie fïhrten suarren, mit benen jie jánumrten.

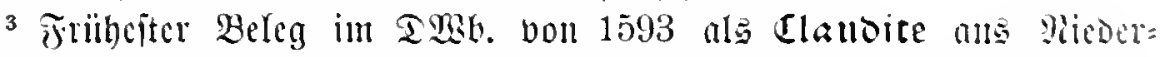

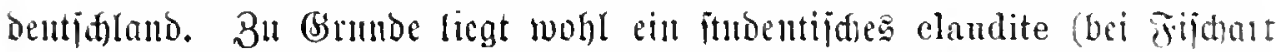

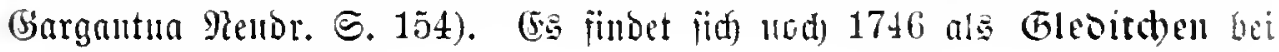
Seber. Blantrumpf.

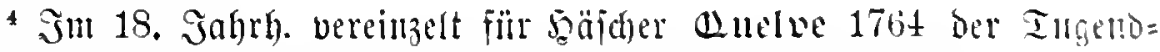
und Rajterbajte Etmbent poetiid) unb moralija) entworfen XII. 


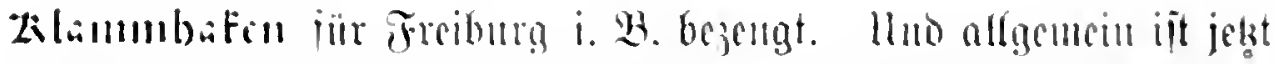

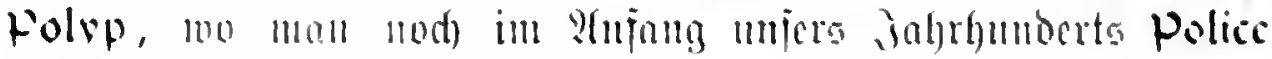

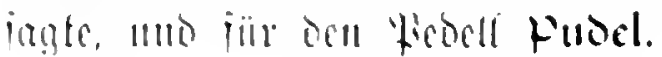

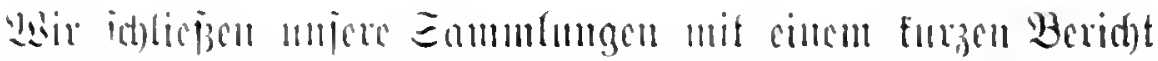

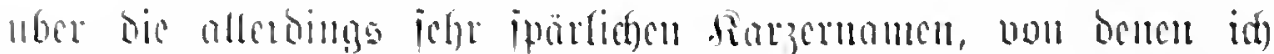

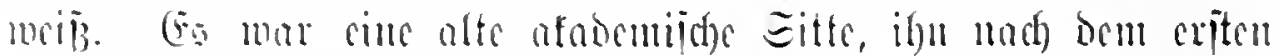

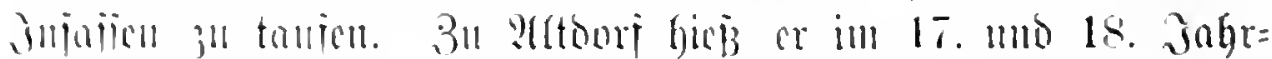

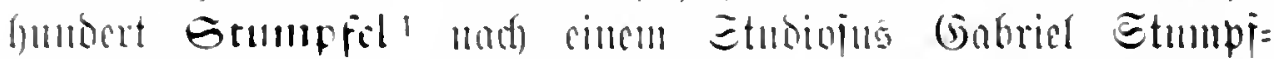

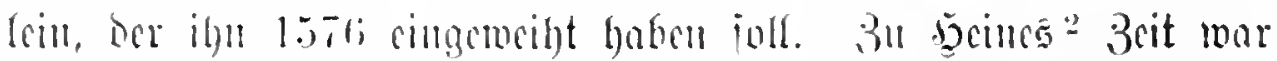

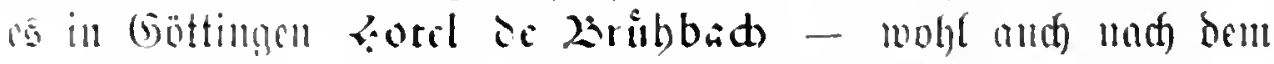

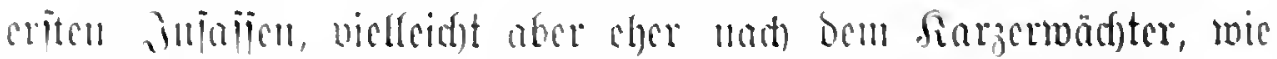

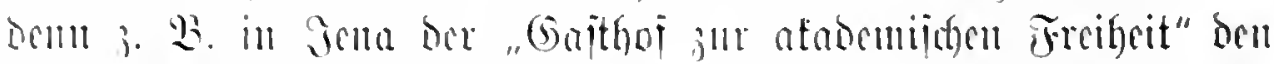

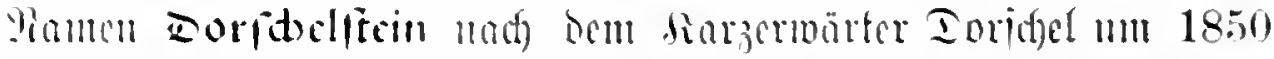

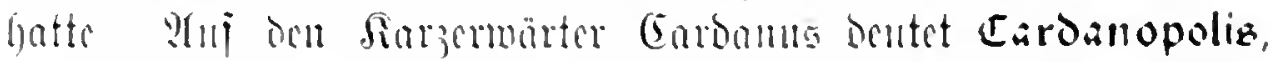

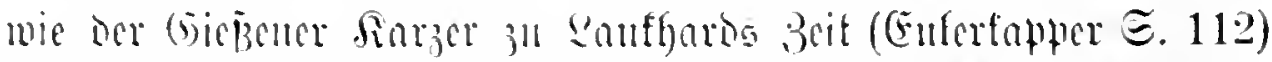

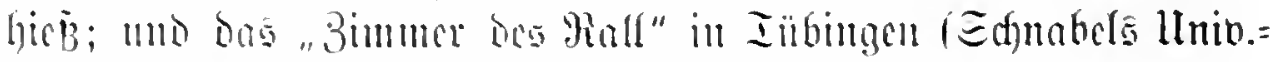
iohre $\Xi$. 3601 it moht chemo ju hemteilen.

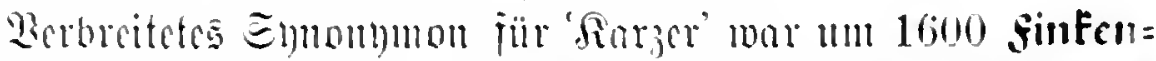

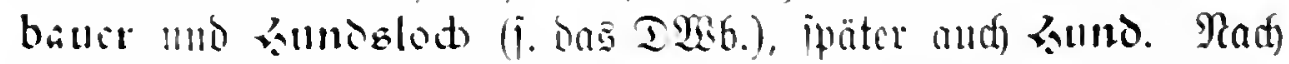

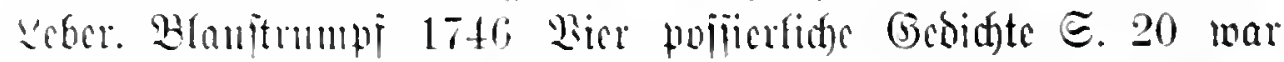
Tabulatgen in jena ber Edyuloturm, wic es im 18. Jabrhunbert

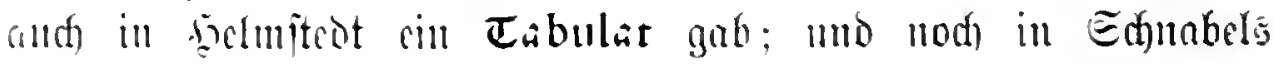

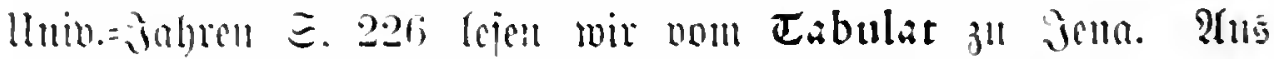

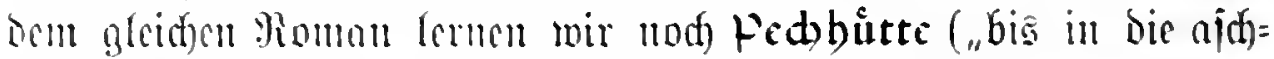

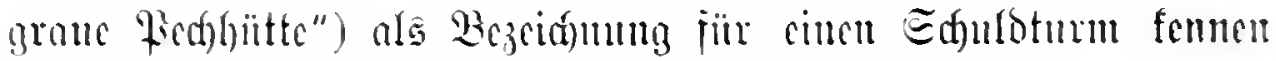

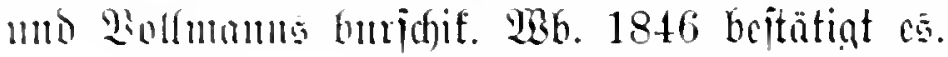

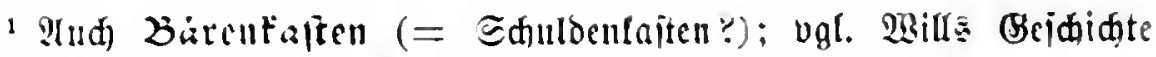

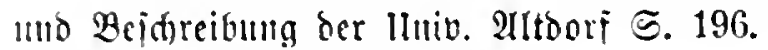

? IEcrke III, 20 celiter. 


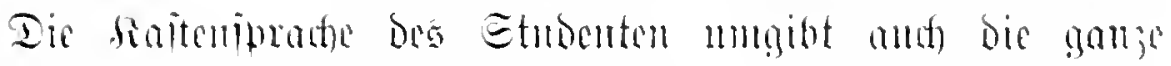

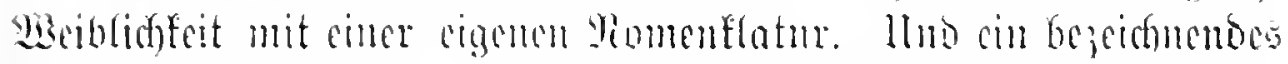

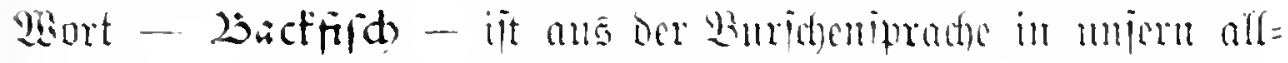

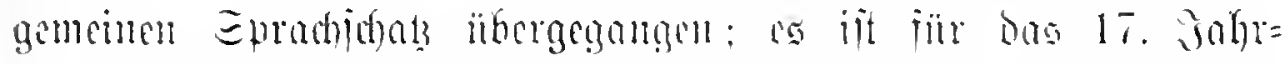

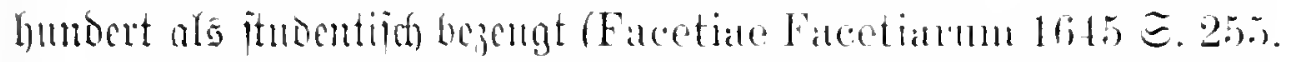

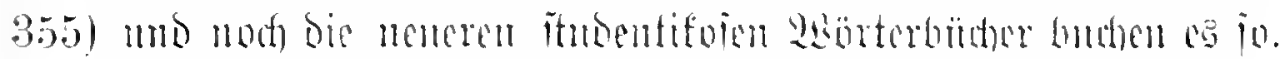

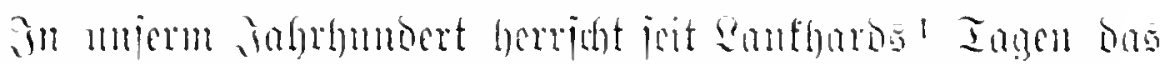

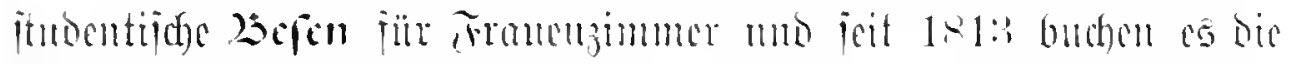

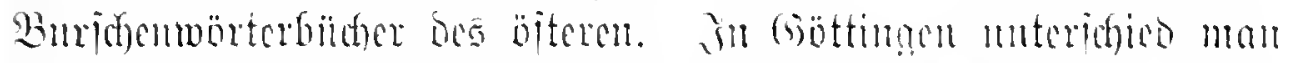

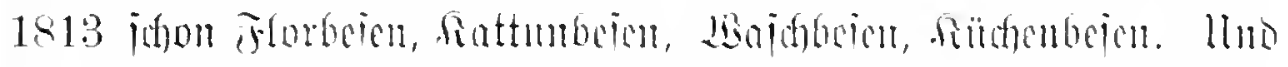

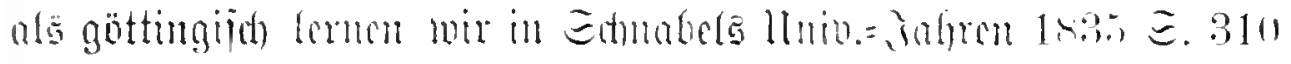

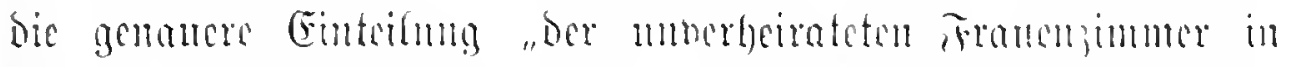

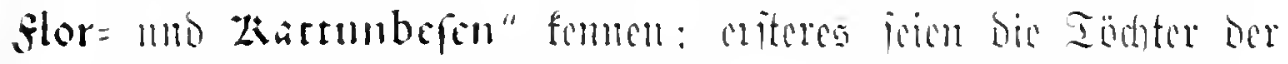

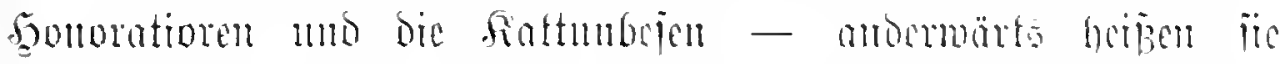

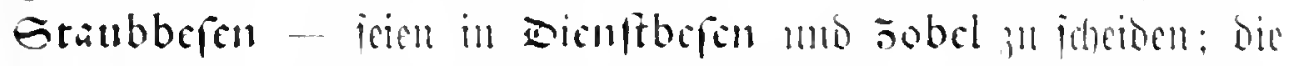

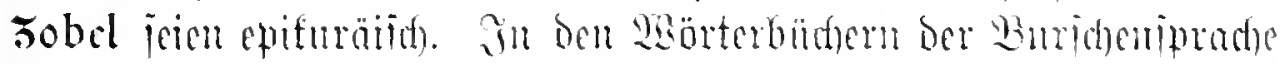

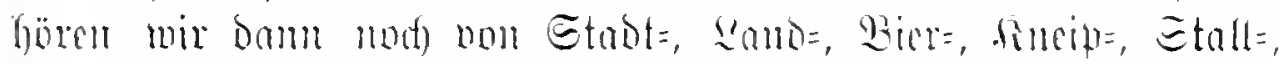

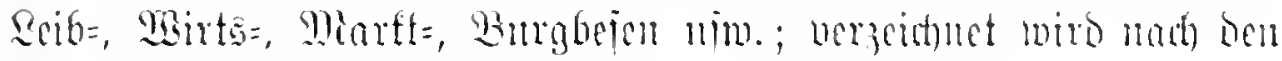

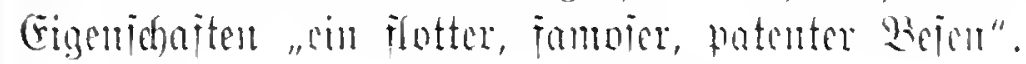

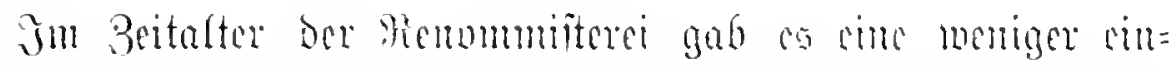

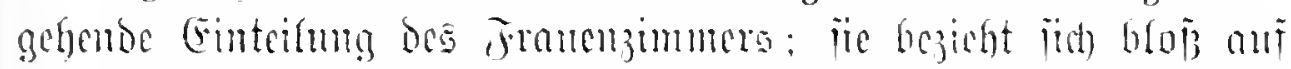

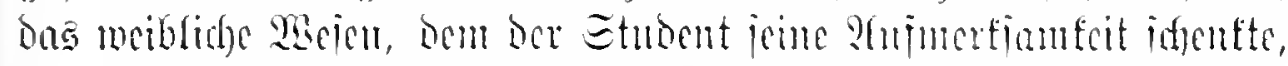
nul bie Charmante. Sener alte Bierfonment - Bas goipitium

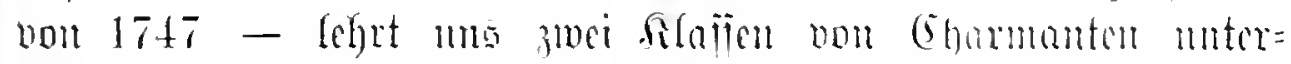

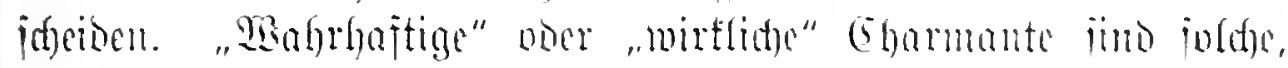

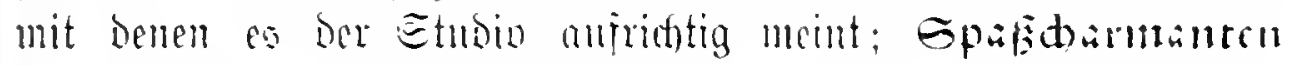
aber jüb zum Brituertreif anserforen mo bon ibuen gilt bie

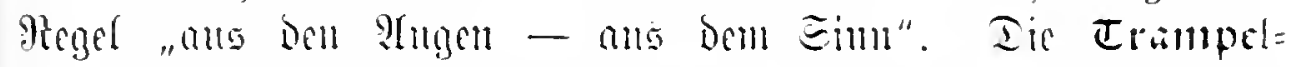

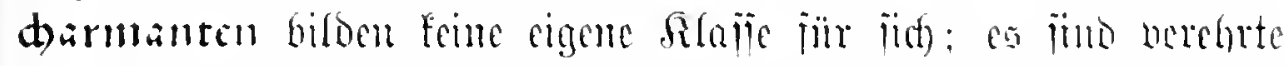
Mäbden, benen ber buridje - trampeno - isme Fenter=

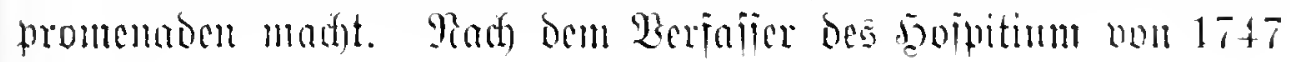


merden charmante mo 21 mante lunteridgicostos gebrandst, ob=

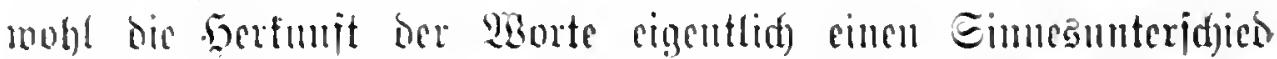

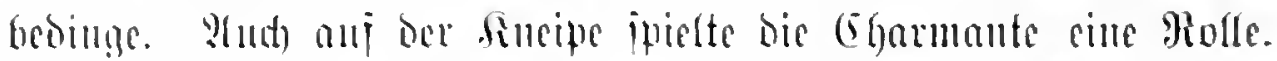

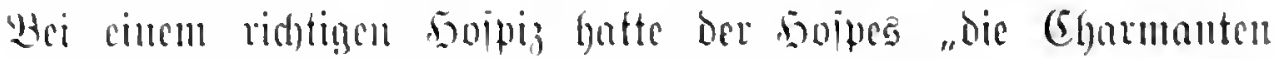

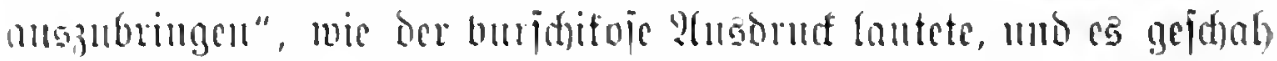
in orci Simben - jür bic Eharmunten in loco, in patria mo

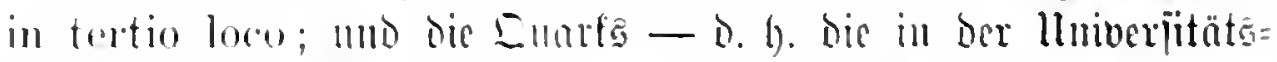

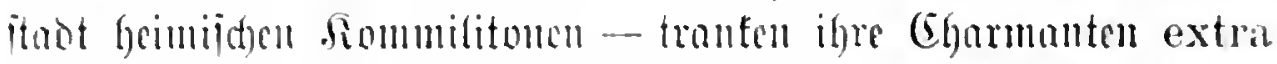
patriam, mo bic ïbrigne igre (Egamanten in patria tranten.

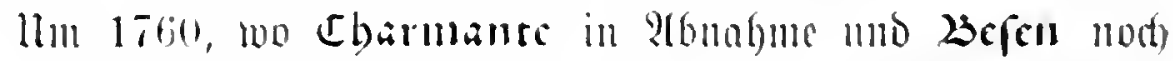

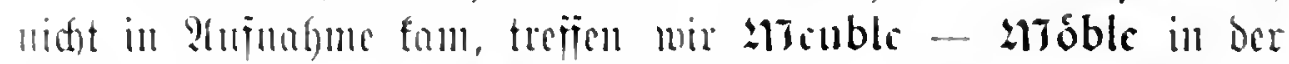

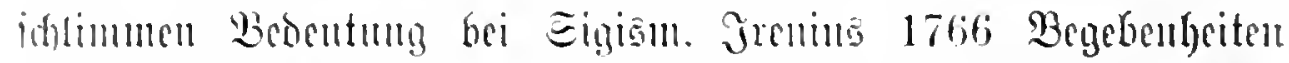

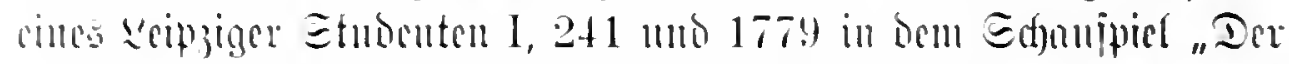

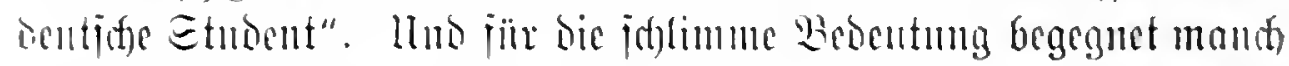

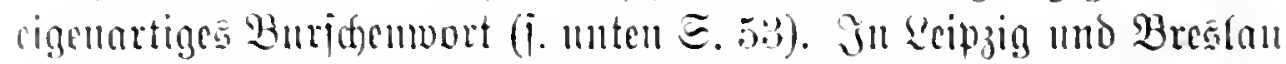

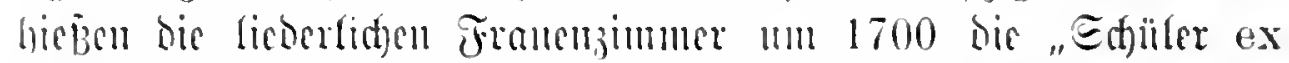

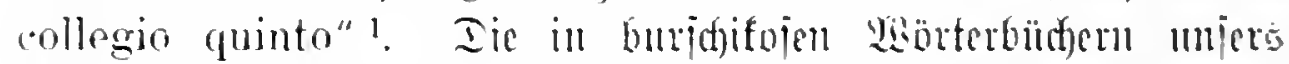

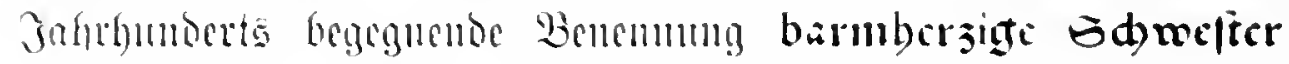

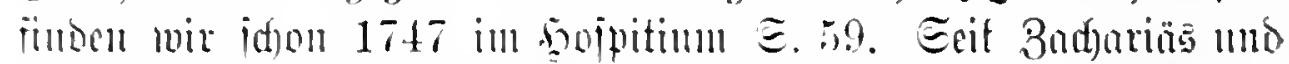

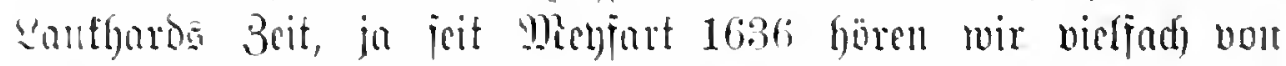
:aympben, hejontors non "Gras=, finall=, Etallubmphen". Ilno Inu 1500 begeguet saclend in gleidfer Bebentung.

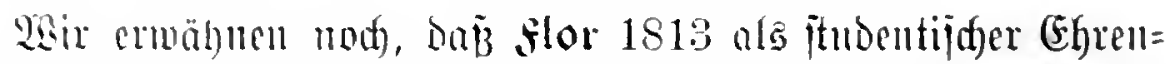

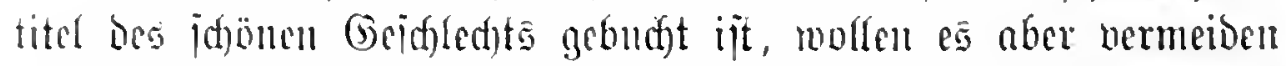

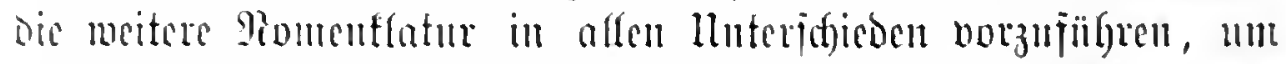

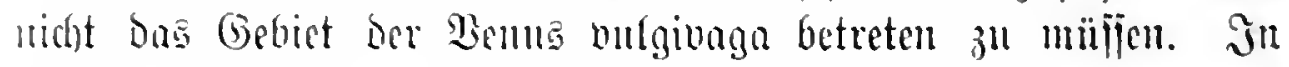

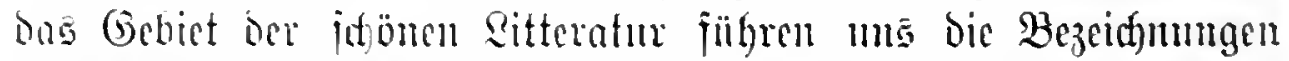

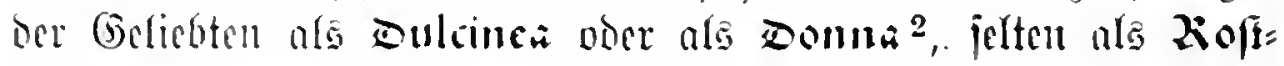
nante ${ }^{3}$.

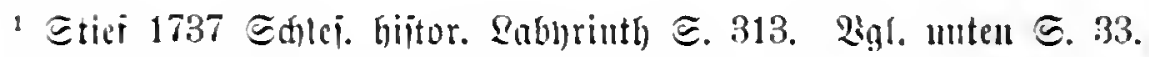

2 Bricic über Erlangen 1792 ङ. 107.

3 J. (5. 5emu 1794 Sertranti 2ricic S. 20. 


\section{Trunfenlitanei.}

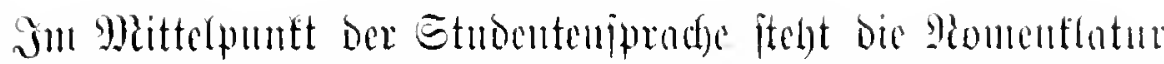

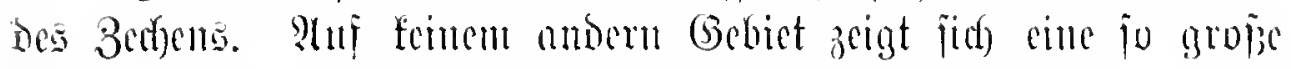

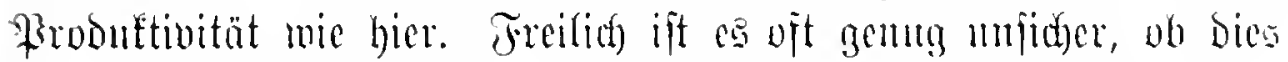

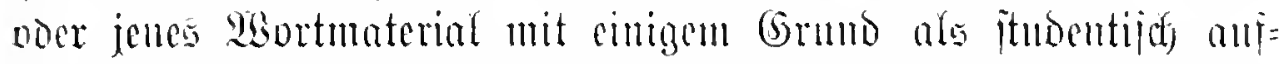

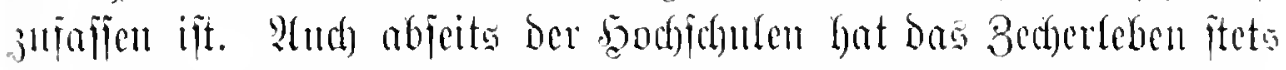

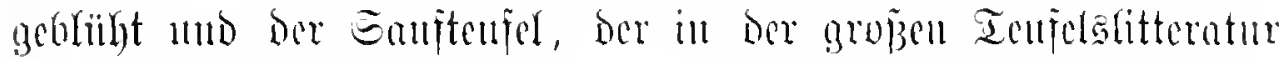

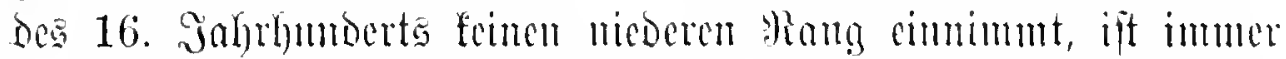

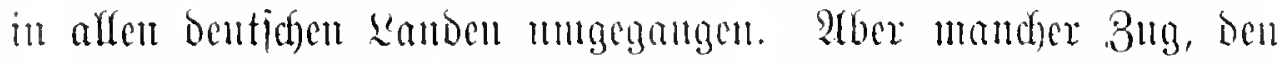
wir in Fifdarts Iruntenlitanei finden, zeigt jidh in fpäteren Beiten auf Den Stnoentenfueipen. Bielfeidgt in feinem anderen $\mathfrak{B}_{\text {sult }}$

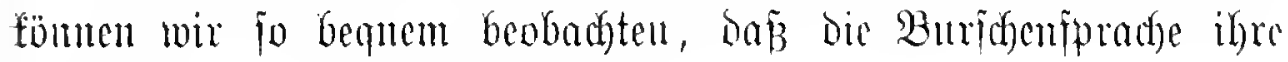
Mgurzelu im 16. Jahrohumbert hat.

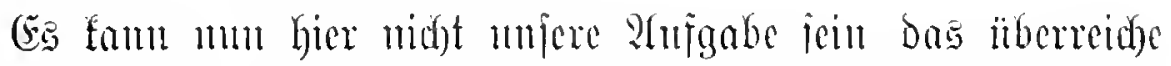

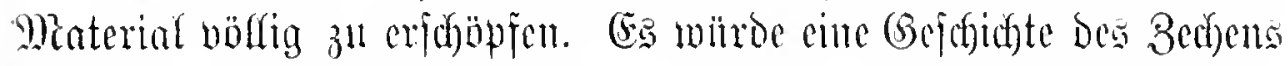

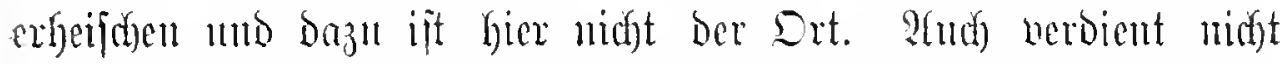

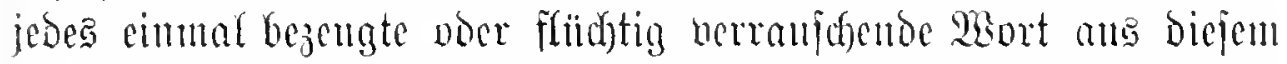

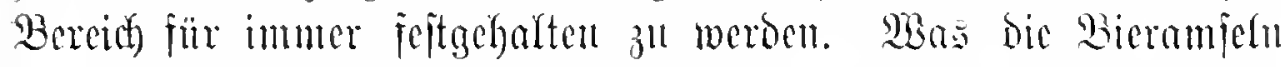
und Bierfinfen, bie Biermöroer und J̧opfenbriider bes 16. Jaht=

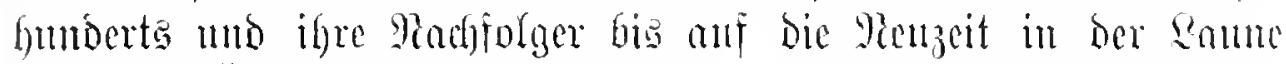

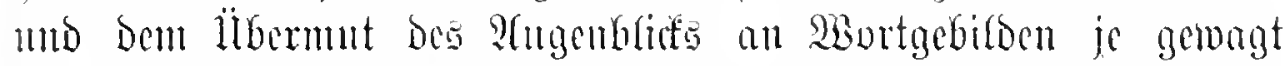

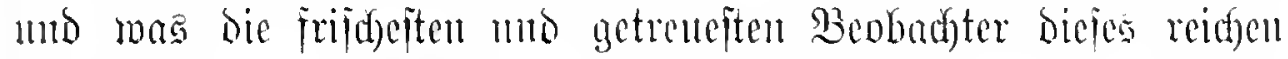

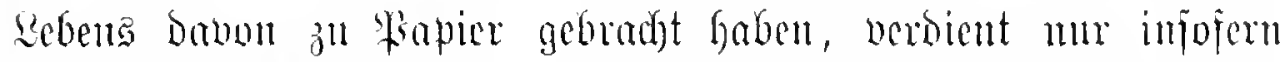

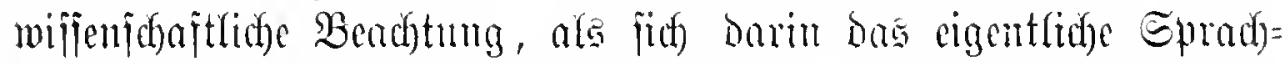

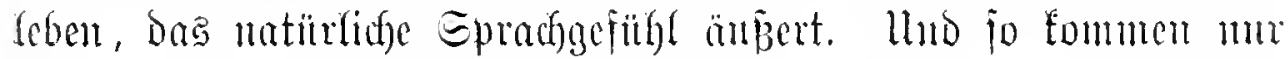

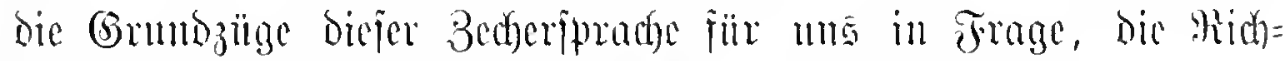
tuntgen, in benen fid biejer ipezictle Emandgeift bewegt.

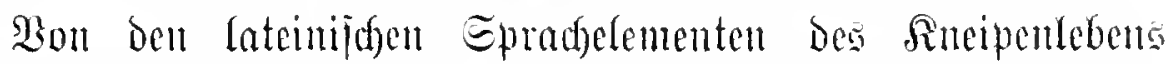
jehen wir zumäajt $a b$, weif jie mo in anderem Bujammenthange

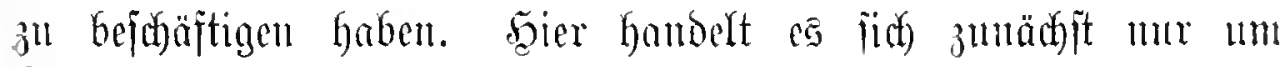
(5rmppierutug Des Mortmaterials.

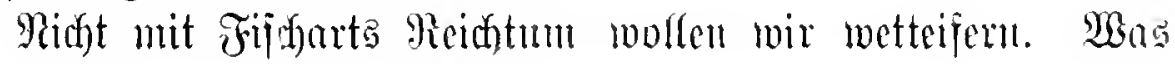




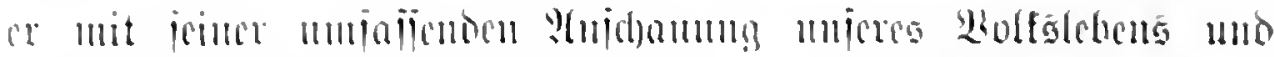

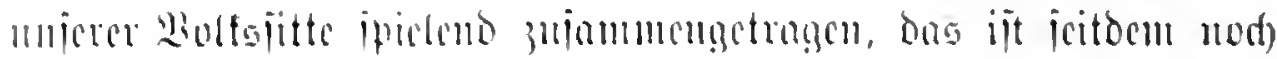

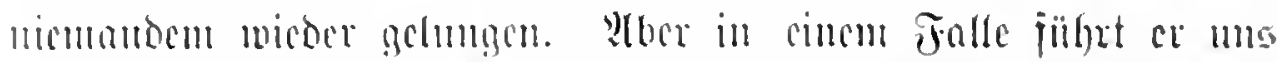

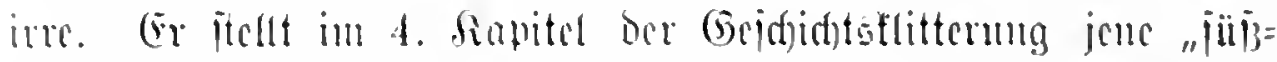

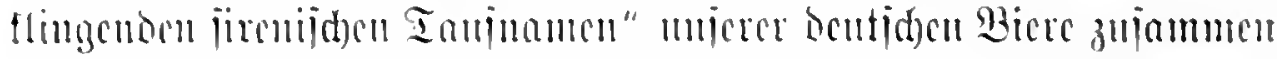

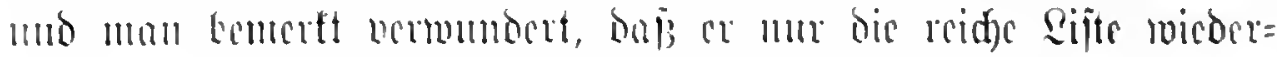

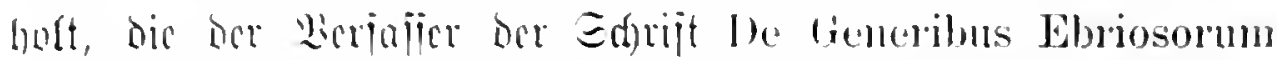
in cincu bejustu dinpitel be diversis cerevisiae nominibus mijicut. Ed)on Dicier ucunt bic Bicrmancu hirolatronibus adeo amahilial. adeo anditu jucunda. It puoties talium quippiam nominaretur melas sirenas sese andire putent et jam tum ad ipsats voees sitiant. Weir begiuncu mine Materialion mit

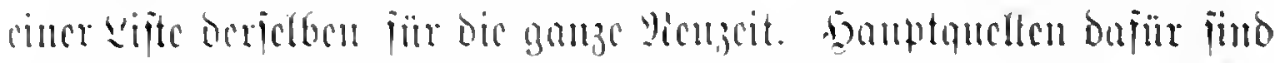

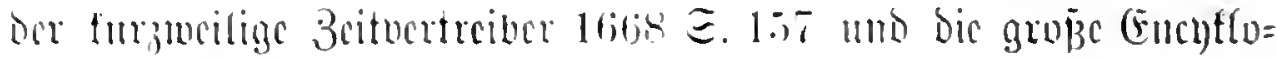

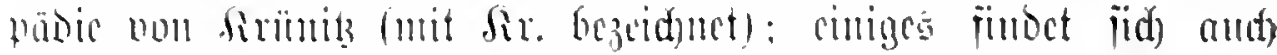

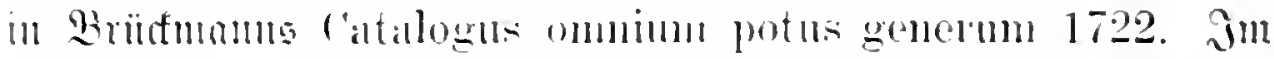

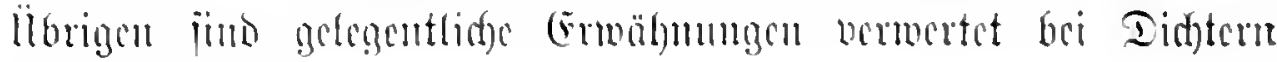
mon y'roinitern.

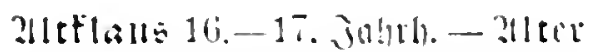

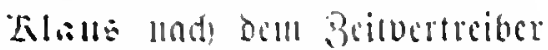

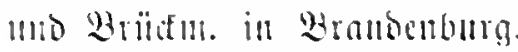

?ugenblemsia 166 s 3cilnertrei=

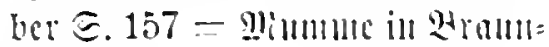
idulueig.

לattats 16. Jahnl).

B.a5mann 16. Sabth.; Had) bemt

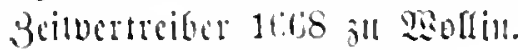

betiscrman 16. Johth.

benitten 17202 Stumeburt.

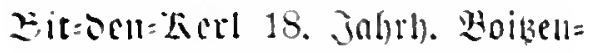
burg; Safiur somsenterl 3eit= vertreiber.

Jilladel 29ollin Beibertr. 1668. Slad 17.-18. Silnth. Golberg.

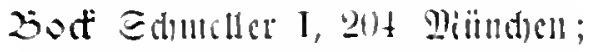

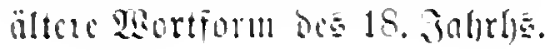

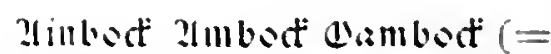
(5intecter Mtier). Belen fïr bort 5. Dorrin! $18: 31$ jauit in bie= wanbe ber 3cit e. 68.

botthiallate 17.-15. 3h. Wgollin.

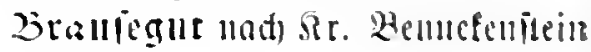
$\left(5 \times r_{3}\right)$.

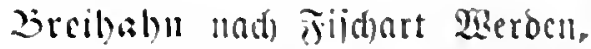
mad) 3coler briebabu Jalber= itnot, Hach bem 3eibertreiber

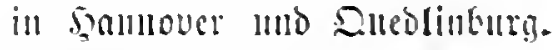
Errllenbier bei grijd) $17+1$. broripos!

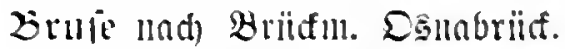
לuர̃, Puர̃ nad) Etiele:1691 \$alle (aıtc) Arätoriıs 1663 Saturnalia ङ. 16); idfoll ill 16. Infrh). bellitebor. 
binfel 16.-18. Sabu. Frant= furt a. D. $\mathfrak{D}$.

Suje $=2$ inuje.

Sacobulle Eafiabella 18. Gabrif. (5acuniöroc.

Slabs (alter) i. 211tflaus.

Dosenfopf fedute in gietit. 17. Jathrt).

Dorftenfol mad) Brtidmam 172: Prmmerbad bei Sent. Edyon in ocr Salinde $174 t$ and Fander 1729 Foet. Rfeinigfeiten 5.45. Drretitein 18. Sabrh. תänig rutter; als Dubltein hei (5hr.

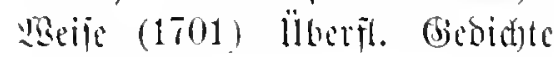
ङ. 344 .

Dummer Tenfol hidarift. mu 1700 Siernar boi Micifter.

售佂e?

ferjer 16. Jahrly.

fioclis 16. Sabrh.

fil 16 . Jahrh. : nad) Stieler 1691

แ110 18. Jahrh. Whagberura.

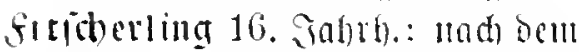

Brifuertreifa S. 128 (- D286.)

Miagdecurig.

Garlei 18. Sh. Gardeleben; Weta (5hr. 2asije (1701) Ilberfl. Ge= bidite $5.3+4$.

Gandium Eeipsiger nno Torganter Bicr in Scipzigy nad) (5br. spegen= opref 1526 Lincomium Ebrietatis aa VIII.

(Fante mach) Gen. Ebrios. mo 18. 3h). in (boslar; als Fole boi

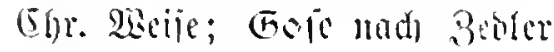
Dincolimburg.

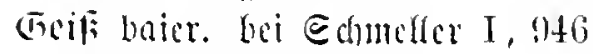
ibinmer als boct).

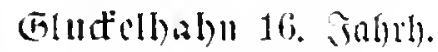

Fole = Faale.
Frouling Edultert I, 1011 mbs. y̆riuz̧ine.

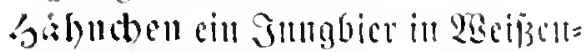
icta 17. Saburit.

Sorlemside 17, Jahrh. Sefme jitäo:

Galtenacte 16. Jobrh.; nadh bem Beituertreiber Silbed.

beiseter mad Sir. Werjeburg.

beiliathater=eid! Gd)miller ], 62 Miind)en.

2lume beinte nob. ein geringes Sier I286. 11, 891.

beinzel, beinzlein baicr.obers. iffon im 16. Jafhrh. (6mijti 1562 Grammat. E. 237) - cin geringes sicr.

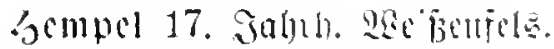

Sorlemstiot 16. Solntr.

bojing 17.-18. Jalmb. gevolgait.

Gotenbach 16. Sathet.; ium 17. Sahth. Sectutitat.

bumo 18. Sabrb. Ialient (Bram= j(c)weig) mis (Corvei.

The weils nitht wie 18. Solth. Butermoe.

Tirael 17,-18. Soluhl. Lübet.

Ttax 16. Sahrh). (anth) Giargantuta Penor. S. 145); Silbet mad iem Beitvertr. Triak.

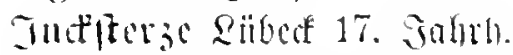

Tunfer nad) Sr. Magochura (17-20 Sugac Venalen 170).

kalbergagel 16. iahth.

Lapar obero. bei Edtutfer: ge= ringer als beinjel.

Eater 18. Sabrh. Etabe.

Gatlerine (it)nelle) 17. Sabr\%. Manmbura in ben Samanipiefen ber engt. Somöbianten (5reisi= Inth) ङ. 273. 
Scute = Koite.

Kenterlung 18. Th. IBettin; Kay: terling im Beifvertreiber $15 \mathrm{~s}$

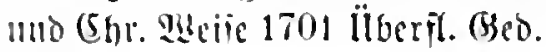
340 .

Eisegern 16. Jolirh.

Clapit, Kalspit 17,-18. Jahloh. indmitrot.

Klatith ein Dïmubier in der Plt: marf Evi Ianucil ङ. 103.

Slatit), Elatide in Beituertr. 1 lifis unb bei Eticler 1691 Jema; nad) Entinde ic ijdes Elabtbier. 2(ud) ill Peibzity.

Elebebier bei Mrenter; oniür bei Tilid)art fleleriges Bier. Bal. I2BL.

alotmilth $17-18$. 3h. 2anken. Clume Dieflenburg mad) Brïdu. Enilenatt 18. Sh. Giititrow. Erroll bei gijanart in D约.

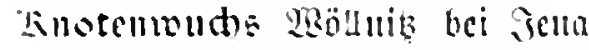
mad S(t)mabel Iluin. = Suhreu 1835 ङ. 94.

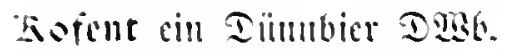

Covite, Kollte 'verevisia hataviea' M!urmelins 1513; 15 Sh. Inïniter. Eolleter afobem. Ronvittbier in

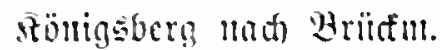

Koppreilier 17. Sabrh. Dhrie: burg.

Eorfinf, Lillrint 16. Juhrh.

Eorjgele an ser Wans hidritl. แIII 17010 Mlericturg;

Erabbel an Ber wallo 18. Sh. (Sisteben; ehenio 1781 in jiilder fromiider 2 mridinde $\lesssim .46$.

Grellen 16. Sahrh

Elltutt nad) Ştieler 1691, Juriid 1741 แ. ล. SBittenberg (aนd) (5ht. Bïuther ell. Litzmann
E.43); bei Бohbern แIb (5olerus: allgcmciu 17.-18. Jahloh.; $\mathfrak{b g l}$. ba: T吸!

Guhidwall 16. Jahrh.; 18. Sh). Telitid) in Böhmen. Heleg: (Shr. sisije 1701 Überil. Gedoid)te 344. Euyt bei $三$ dottel $1664=$ Eoite. Lammelbier idlci. 18. Jahrh.

gangiel gangwel Rangweil jalej. heii. (i. ₹286.): nad Stieler 1691 cerevisia secundaria.

gaute 17.--18. Sahrh). Mlölfen in Qanenburn.

Rsuginer balle nad) 2 bel 1701 Seilumcoicus ङ. 191.

Lord 16. Snhrh.; Sorde 18. Sh. Zieflans.

Lotenale 16. Safhrh.

Lumpenbier Merningerobe 17. -18. Iafurf.

Duntid und Sticler 1691 Erfurt. Miratglust Had) Ir. Tejdell.

:lisulelel 18. Jahrh. Jena.

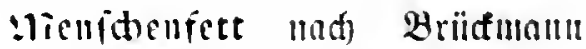
1722 Srojpeda bei Selta.

Mioll 18. Suhrh. gimmenen.

"Nioro uns Ioorblag nad Etielcr 1691 und 18. Sh. Styris; nad) bem 3eituertr. utro hidjitl. um 1700. Miortpotner Miorbosner 16. \$b. :liutteujenf 16. Jahth.

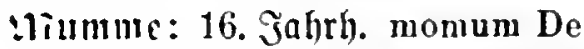
Generib. Ebr.; idon im 15. Jahth. Eezeugt fïr Braunidı. ; $2 l \delta e l u n g$ gibt es alud fïr $23 i$ mar alt. Gel Moito of nadh bem 3eitbertr. (1) wie! Rimbad) mad) Brüđtm.

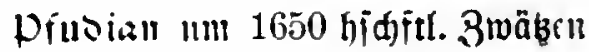
bei Jena.

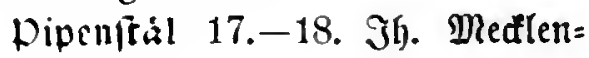
burg. 
Dreuling 18. Sh. Tanzig; nad tem Beituertr. Fethte in sgeil= ialen.

Prifanbier bei Fildart.

Duti i. Buif.

Mllactelocis math Sir. (5aterliforbe.

Muitfart 16. Jahrh.

4horolewits $11 \mathrm{~m} 1700$ Rönigs= itein (bidfftl.).

Siamenad) 18. Sahrh. (biülitat (5oliteiul).

Jismua 18. Jathrh. Serioto.

Tiafemalln 16. Sahrh).

Siaftrum Seipsig: in Stastred)= umugen jeit 14St; im 18, ms 19. Sahrh. als Ziajter eill seip= jiger Dimmbier. P(potrnphe Ien= tumg leitete $a s$ ab von eintem eijermen gedhen (mit einem (Bfaje. als Wirtidajtazciden.

Ticiletopf 16. Jalnh.

Siolingsbier 16. Snhrh.

Siommelseja, Ziummeloeur mad, itr. cin Püfefer Bier in Innzị uno in 17. - 18. Jahnh. cin Rier in Rabeburg; vgl. I㫮.

Silltetop 16. Salfrli.

Sabl=oen=Eerl 18. in. im sande Dodellt.

Ealst 16. Jahth.

Scerp=ber hei filifart; in ser Genma Gemmarum 1503 ats Sharber.

Stbenper Gemmi Gemmarum 1503.

Shlaghratt mad st. Fistebent.

Edlipithliap 16. Sahrh.

Sd)

De Gener. Jibrios.: bağll der Vagister Schlunz in Universi- tate Erfurdiensi in ben Epist. Obscur. Vir.?

Ships mad Etieler 1691 und Had) $\mathrm{Kr}$. Brestum.

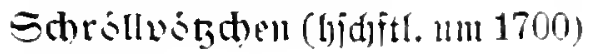
3eib.

Sd) intetiappe 18. Jahr. Mitter Ijamen (\&ramid).).

Sdrmellvóghen 17. Jahrh. Mler= ieburg.

scormornm und) Murmeling 1513

Pappa D I Iïuiter.

Spcrpipe 16.-17. Snlyrf.

Etaffeling 16. Sabri. Trantfurt a. D. 5 .

Stampr $=$ mI = Die = 2lithen $16 .-17$. Jahrh.

Stiar Edtejich is Jabrb.

Stirtenterl 16.-18. Ifr. Ieren= burg.

Strectperjel 16.-17. Johrh. etiirjchanzel.

Strobetugen 16. Jahrh. (3) Seinede).

Srurgebarsel nad Stieler 1691 Nerjeburg.

Dummer Teufel (hid)itl. um 1700 ; Werga bei 界eizen.

Tibr Soli mat) Brïål. Pram= id)mein in Sirusfloiter.

Witte 18. Jahrh. Siel.

woittenticl 18. Gahth. Sabüningen (Bramita)w.).

toller Mrangel 17,-18. Jahrh. 幽restall.

wúrge 18. Jafru. Berbit.

jals mad) ntr. in (silentourg.

Sigenllle nad) Brindm. in Man mo nud) itr. in ber Mittefmart. 


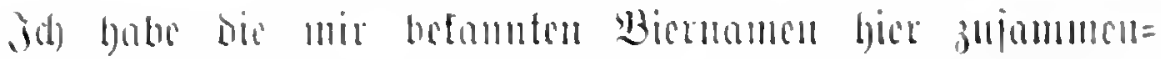

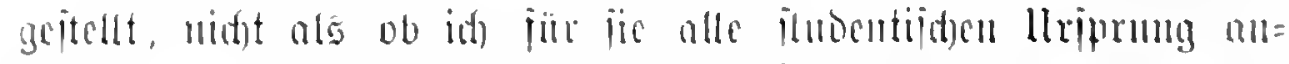

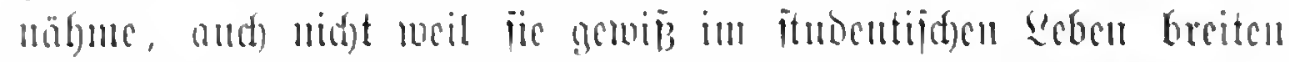

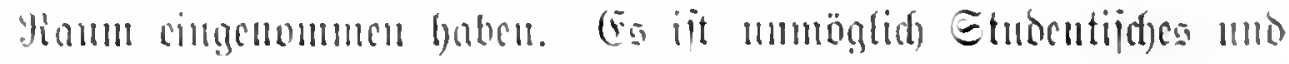

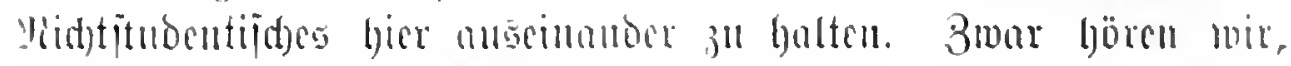

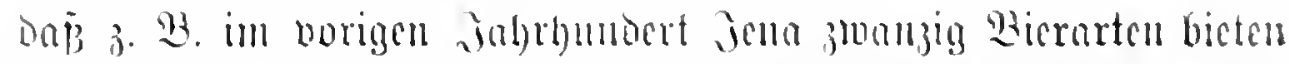

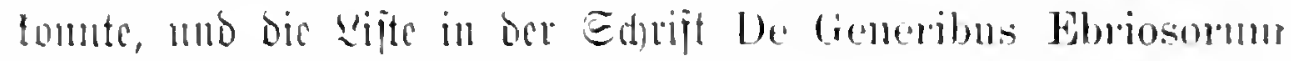
zrigt allein jür Frantiunt a. D. D. etwa 30 Biernamen. Wher bod mur menige tragen itubentijase Epuren. Ing Scipjiger liastrum

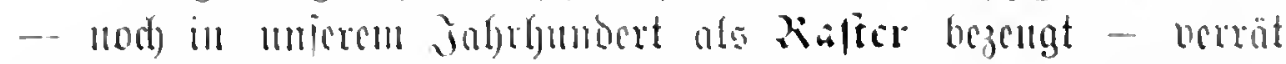

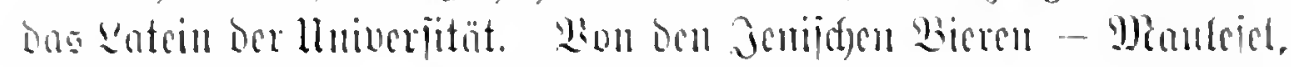

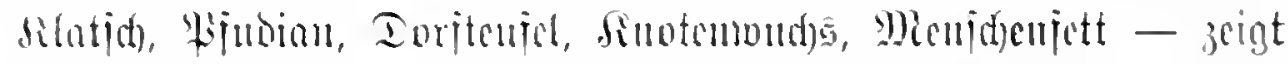

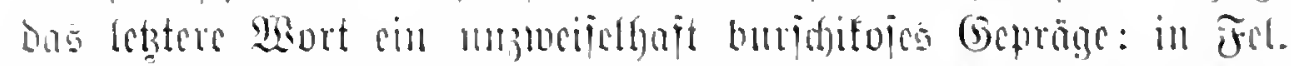

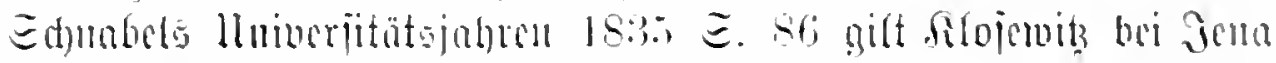

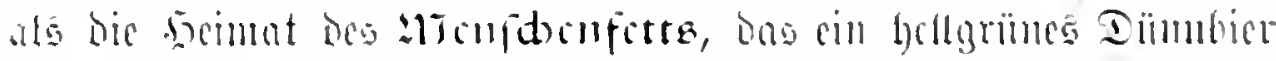

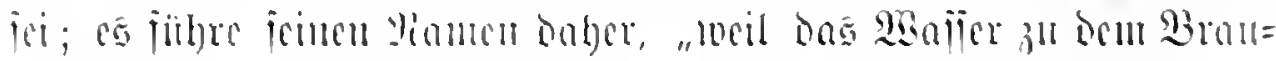

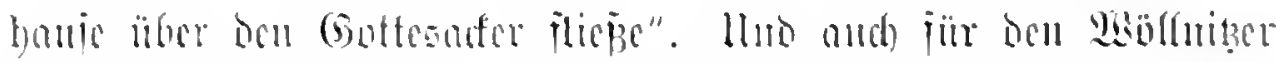

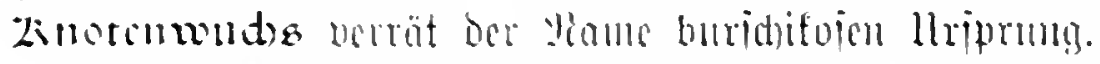

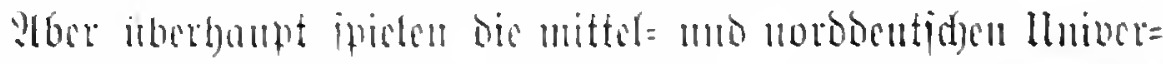

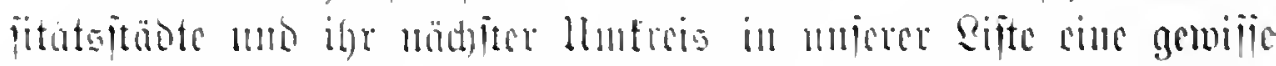

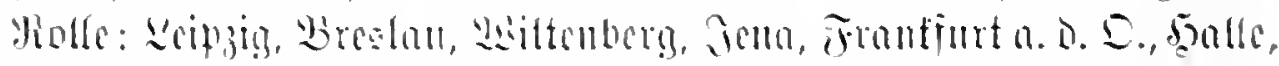

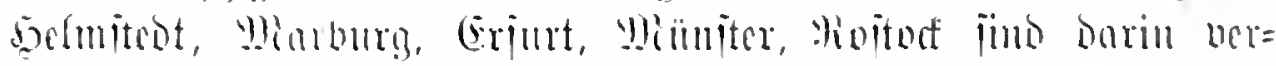

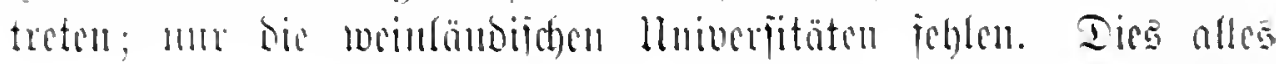

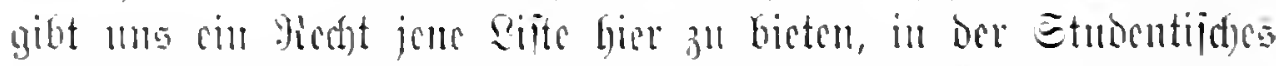

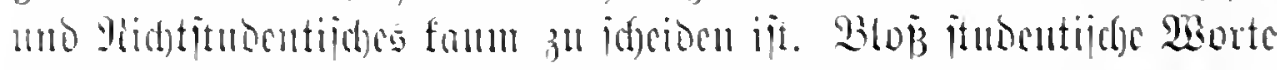

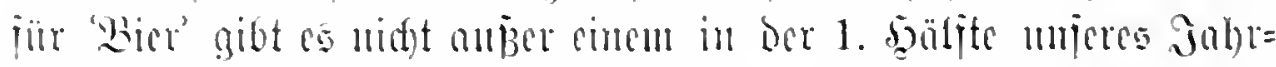

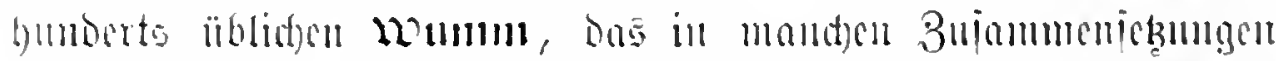

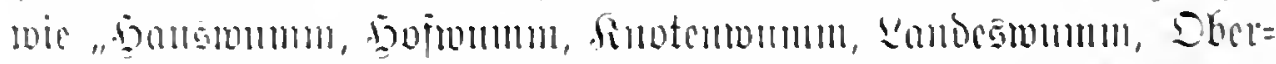

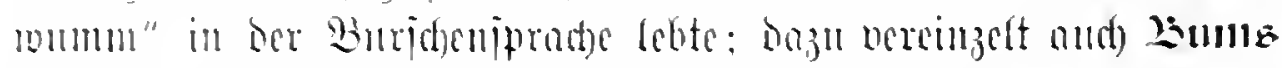

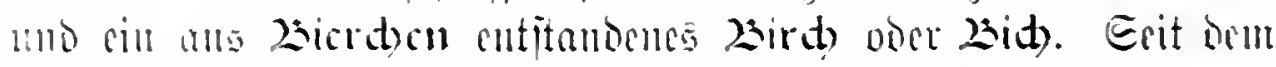
worigen inbrbumbert bat cin allgemeines Gtoff jidh cingejtellt.

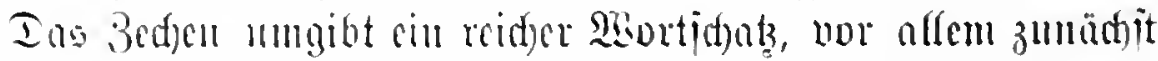
gemeinjanes Bedyen mit Wevbadytung jeiter Formen und bem

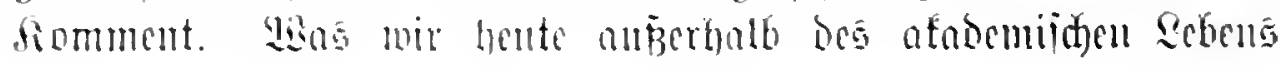




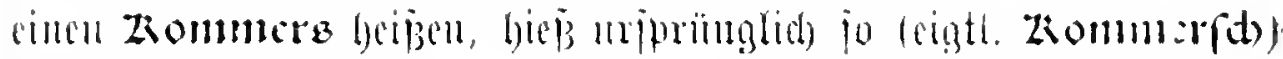
um in ocr Etmontembelt. Jm 1s. Jabrfundert gilt jïr Dos

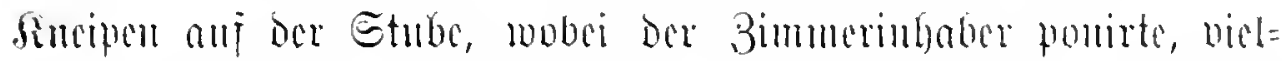

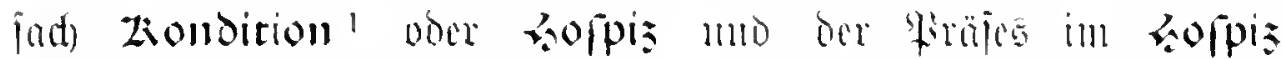

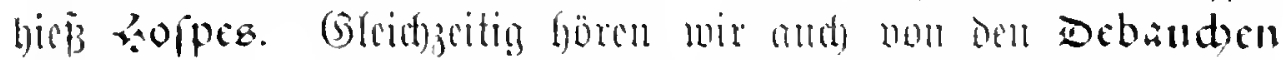

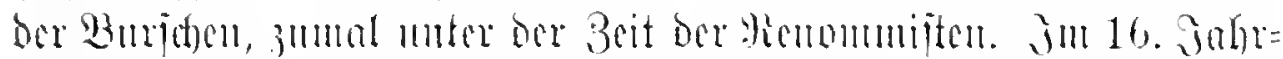

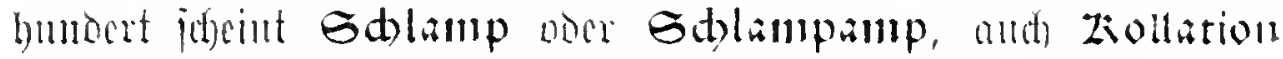

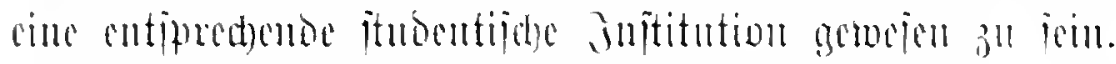

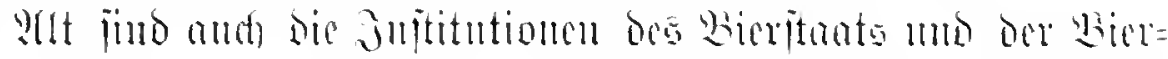

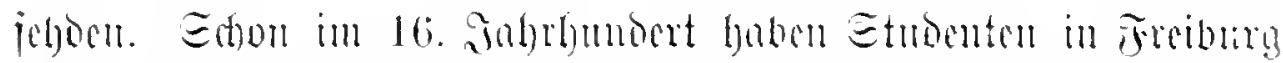

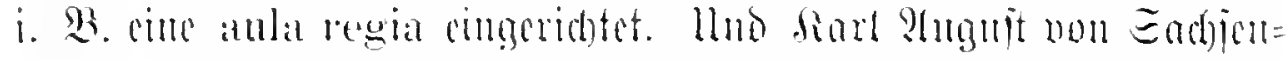

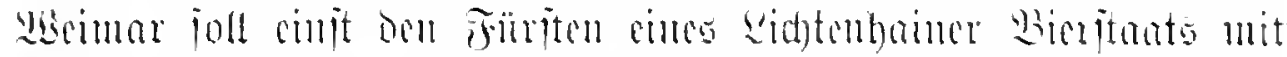

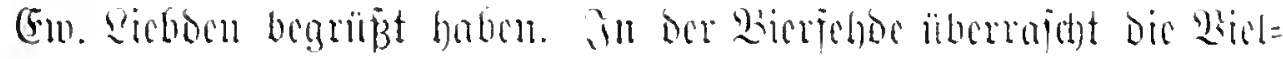

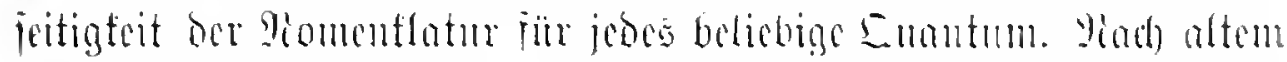

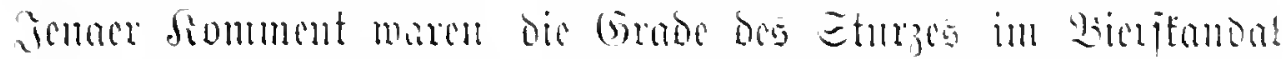

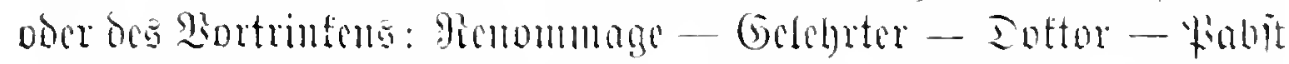

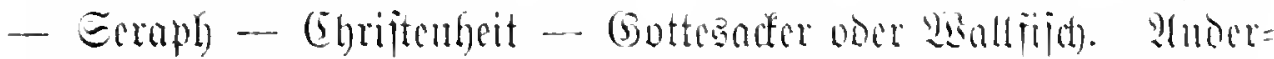

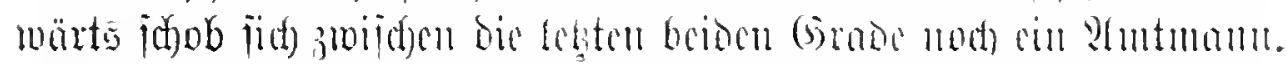

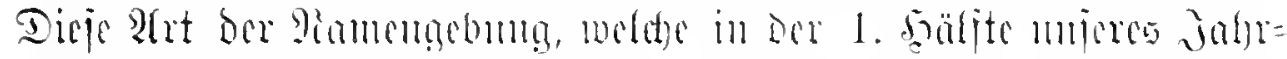

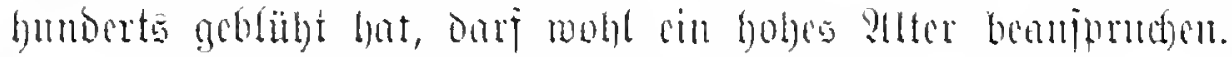

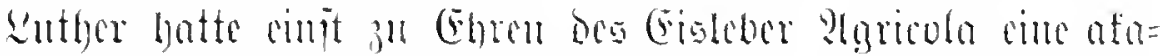

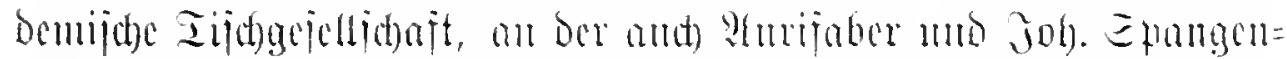

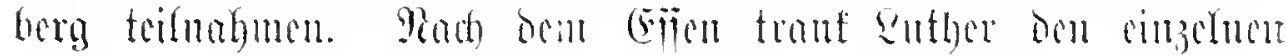

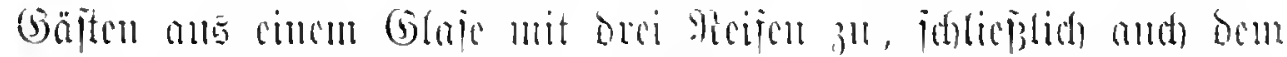

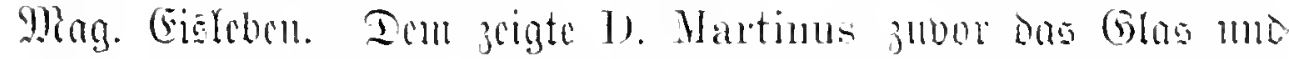

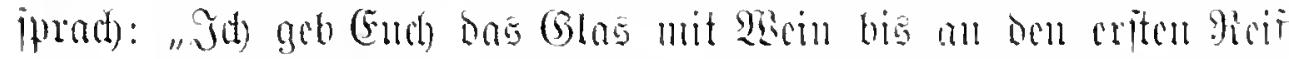

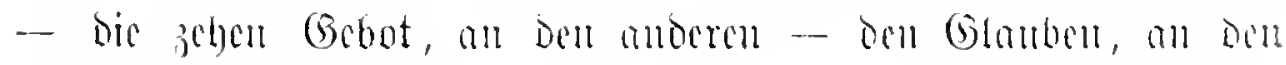

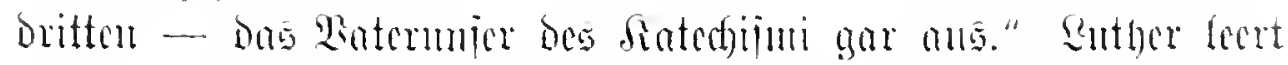

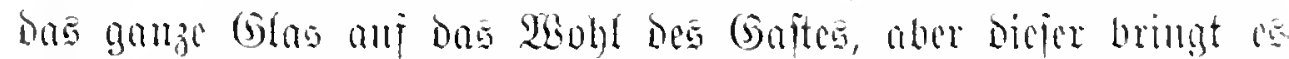

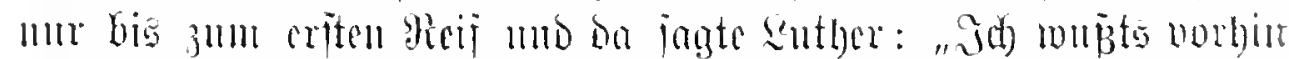

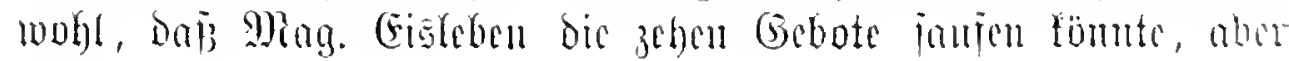

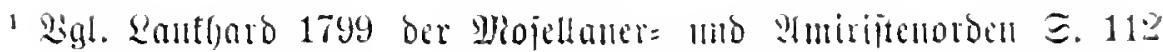

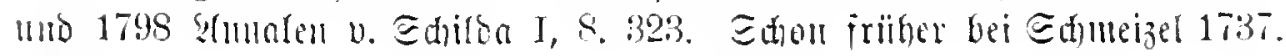




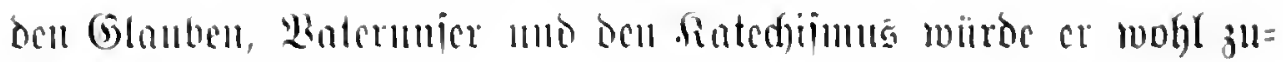
iricberl lafien".

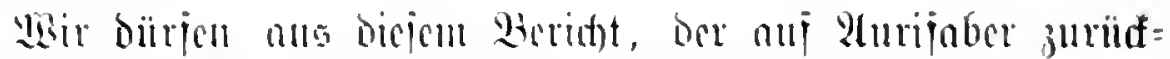

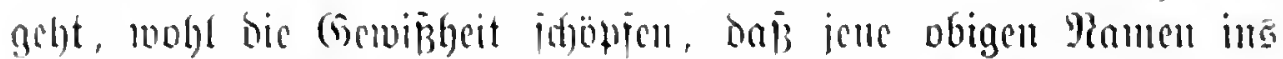

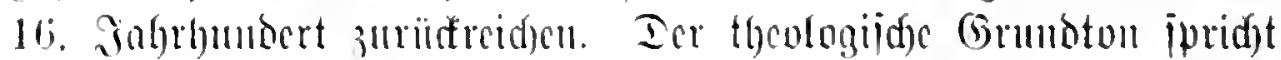

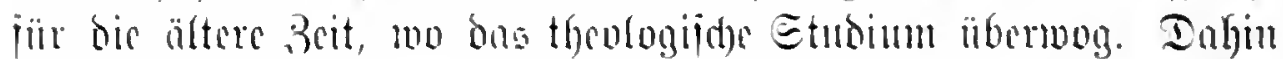

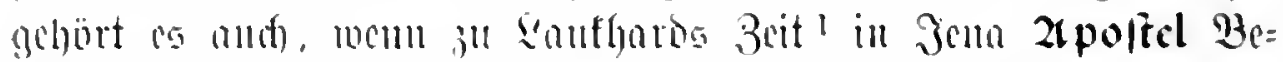

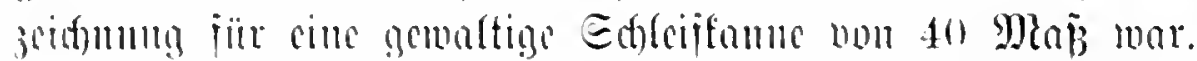

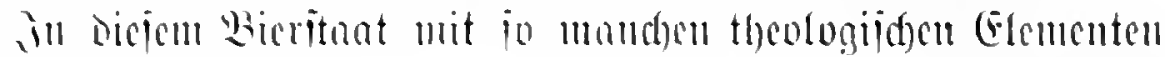
- man bentr nud) an dic Emijucije - batte bcjonders bas

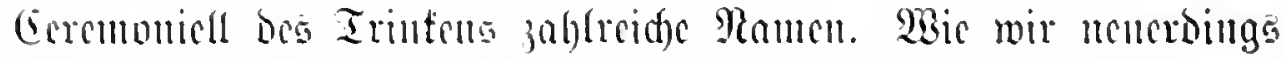
icit etwa 5) Jahren bon Ealamanocr, io hatte jedes Beitalter feinc offijicllen Formen mo Jurmeln. E(t)mollis mo fiouzit eridoll im devipiz ju Rautharos uno Simblebens Beiten grade vie hente.

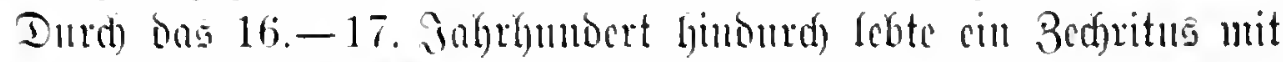

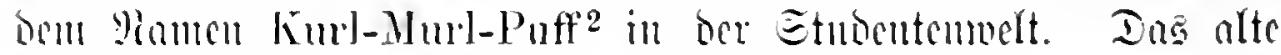

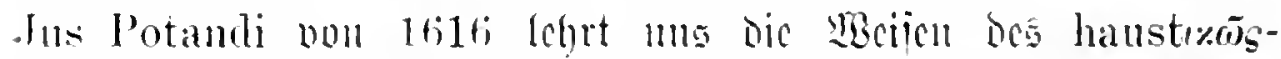

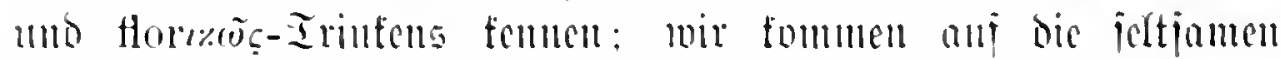

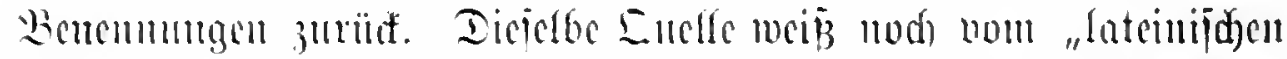

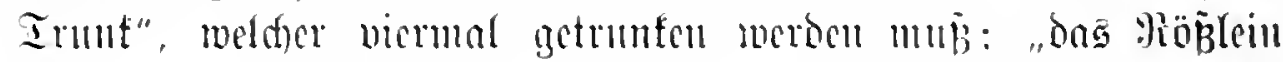

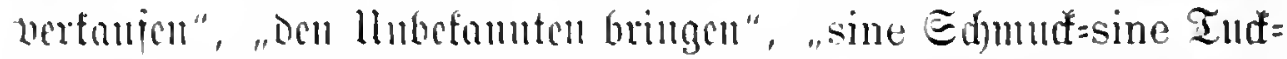

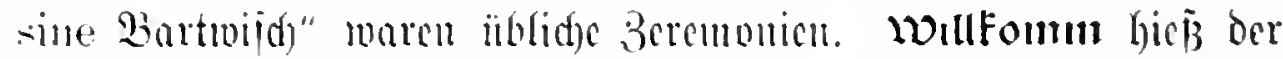
Ermut, ber bcul Gajt gebrad)t murbe, mon jugleich bas poculum

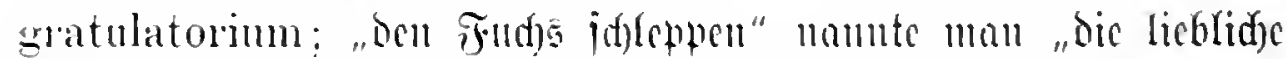

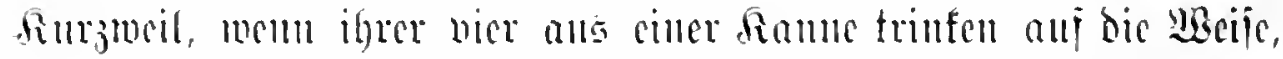

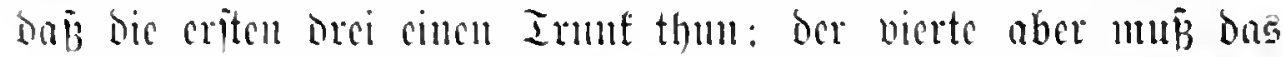
anocr allos, was nod) biuteritellig, exsiceiren mo anstrocfucu" (.Ju: Potandi $\$ 331$.

1 Miojellaner: nub SImiciltenorben 1799 હ. 51. 53.

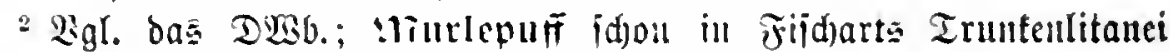

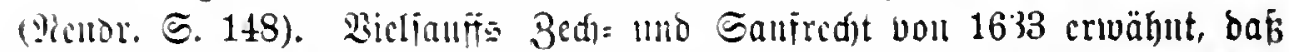
et viel jeltiame $\Xi$ djuaten und Boijen in jeinen llingang veruriaht. Seturl=

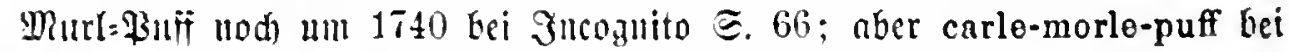

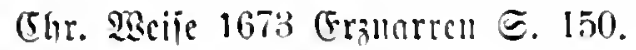


Gine bejondere Beffart war bie kimon. Viad) alter nieder=

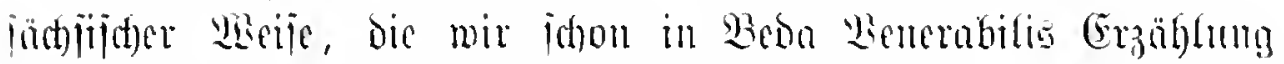

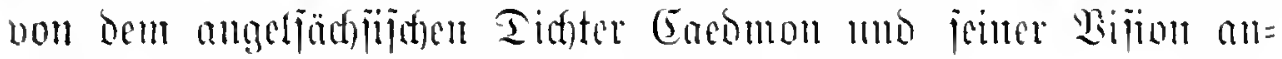

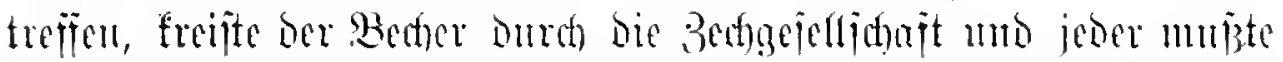

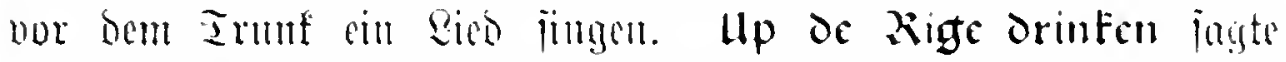
man im 16. Sabrhmonert platt bajiux: mo ba morhte moht eill bed ut, beel ut, Etalbroer ben Irinfenton animieren - viefleitit

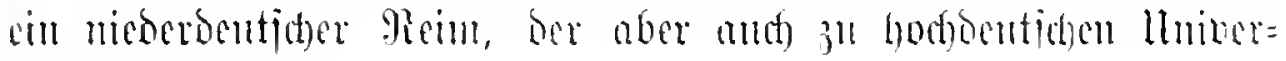
jitäten gelangts.

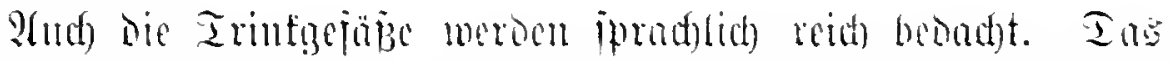

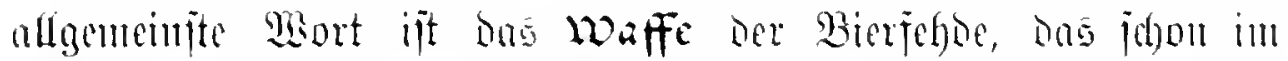
nlten. Jus Potandi non 1616 mo jeitom dit besulgt ijt. Eo

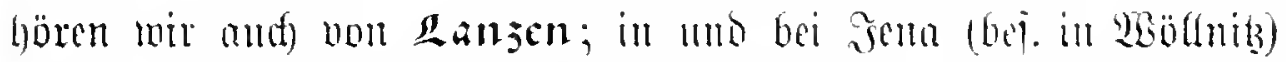
b)

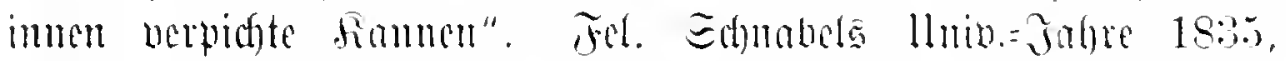

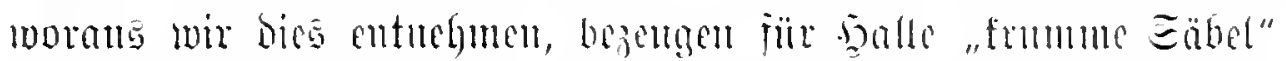

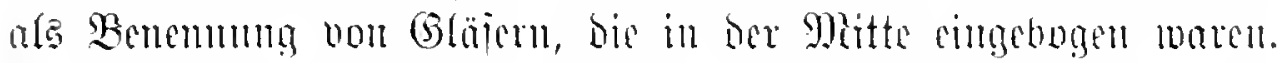

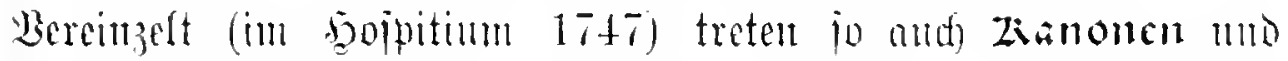

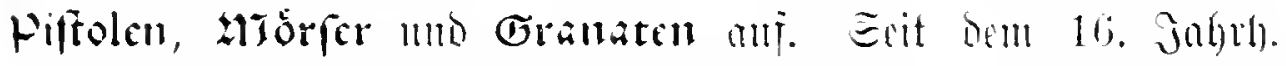

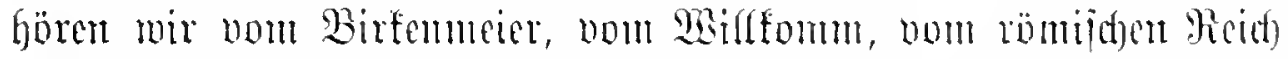

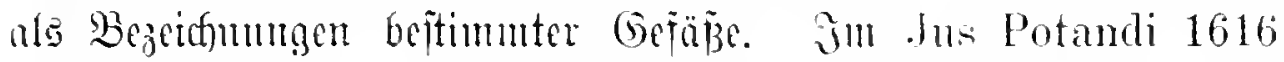
wiro ein jeltames Sabafafe gleidfuertig mit waffe gebramt.

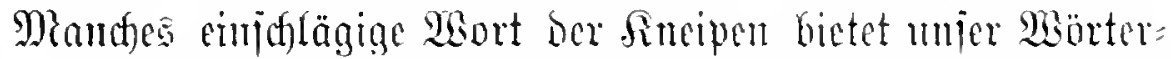

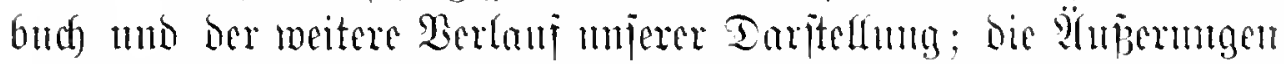

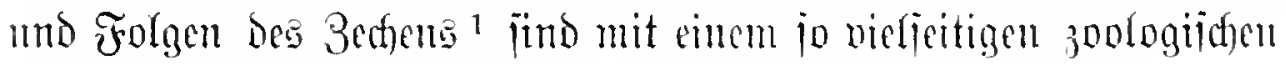

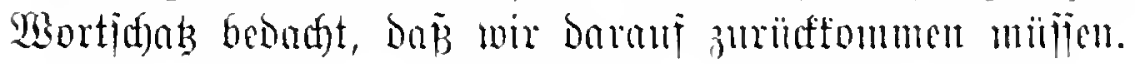

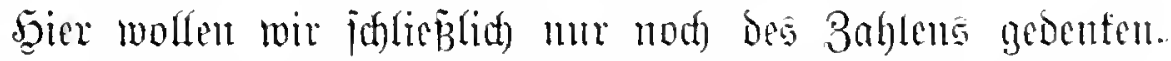

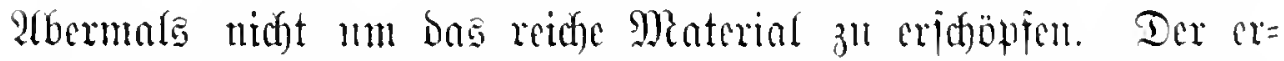

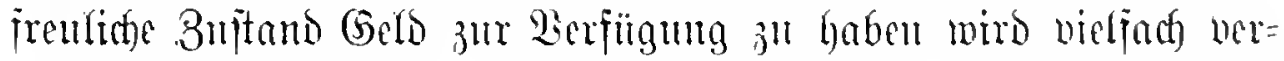
ichleiert. sitoneten, nunquam-retrorsum. Zinopfe, Epicke,

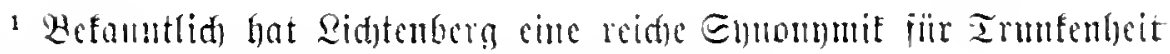

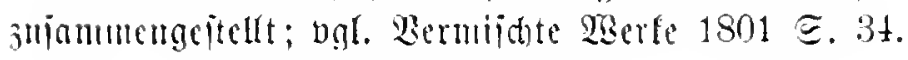




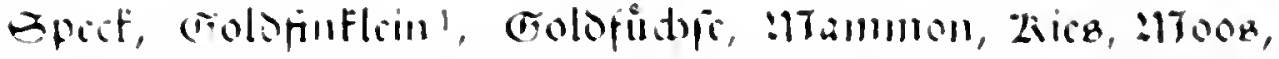

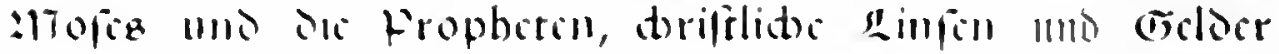

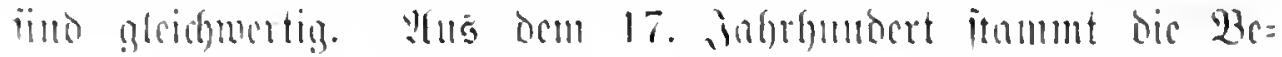

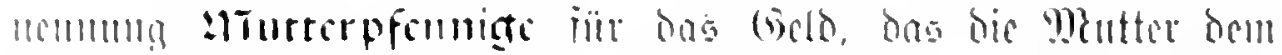

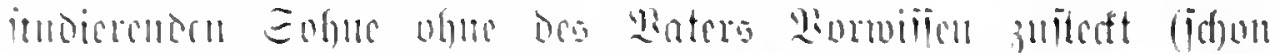

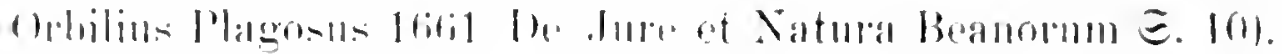

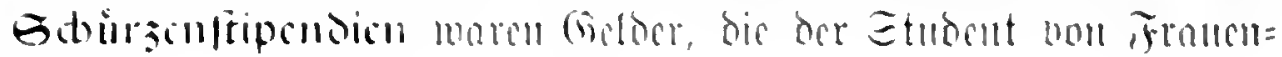
simmerie nerehret betam.

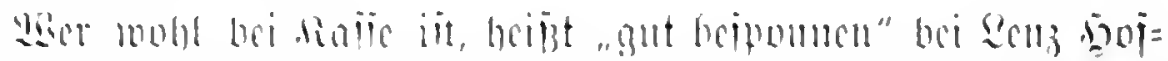

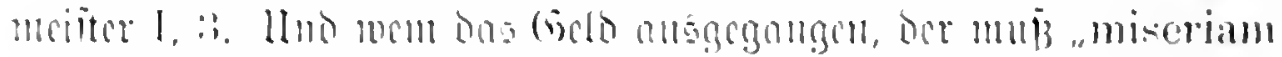

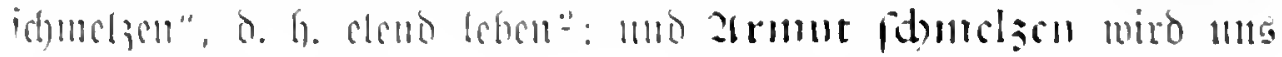

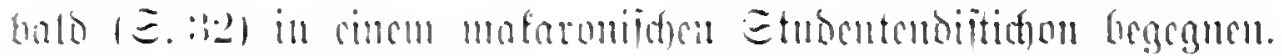

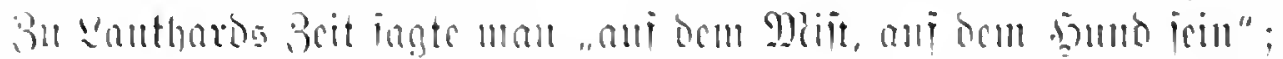

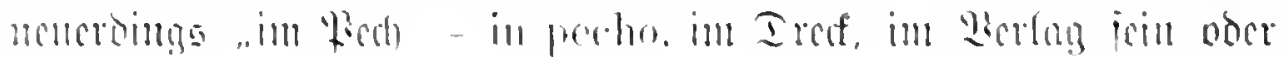
itretin".

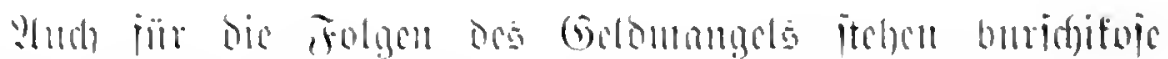

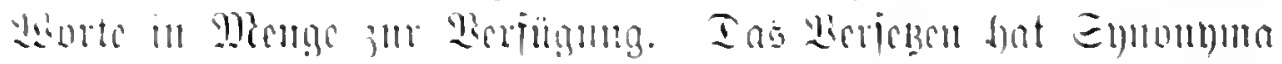

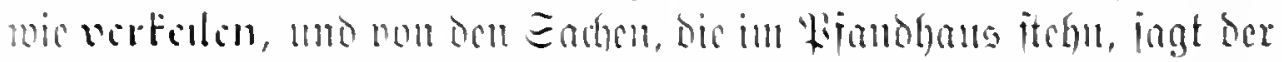

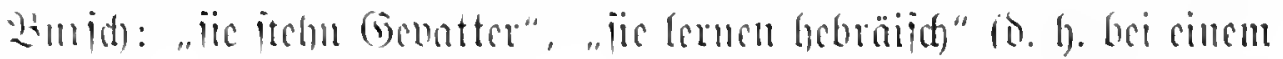

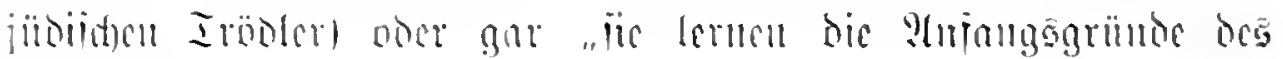
Emriid)en".

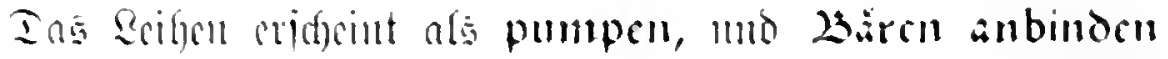

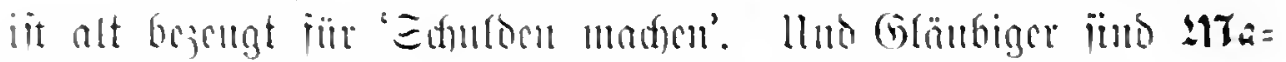

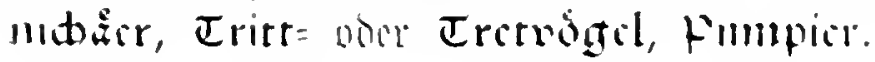

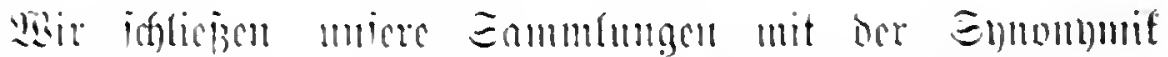

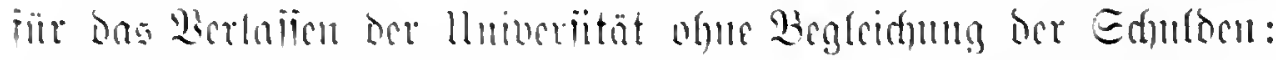

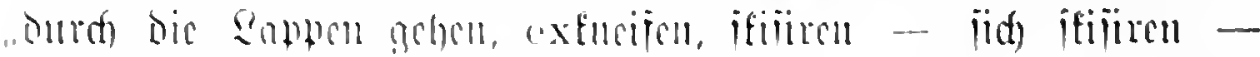

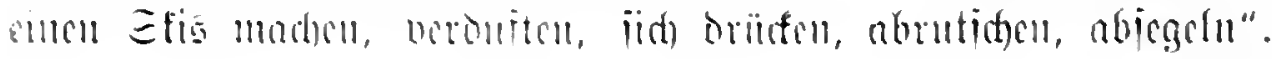

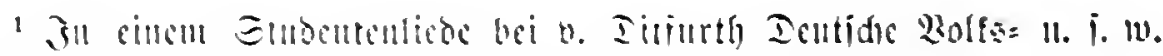

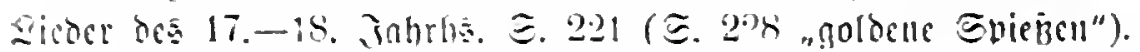

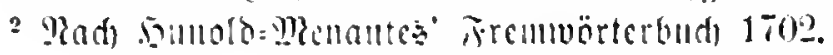




\section{2lutife (5) femente.}

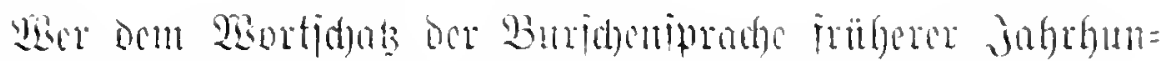

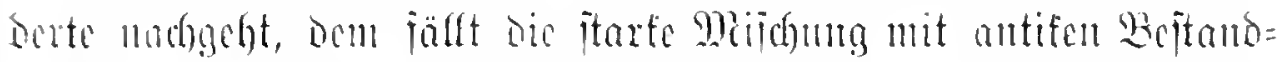

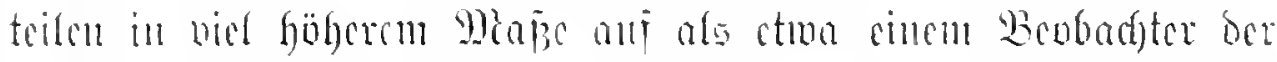
hentigur Etuocnteniprache. Ens Satcin hat ncit über bie Bcit

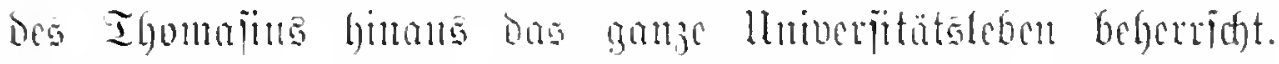

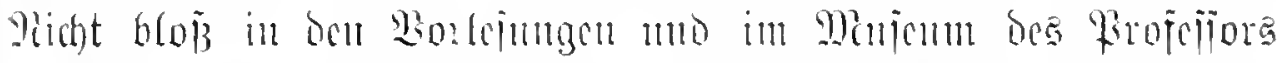

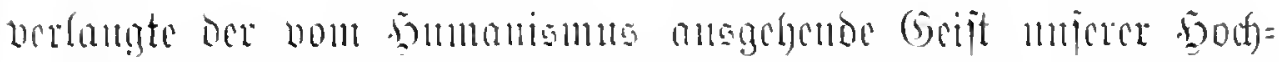

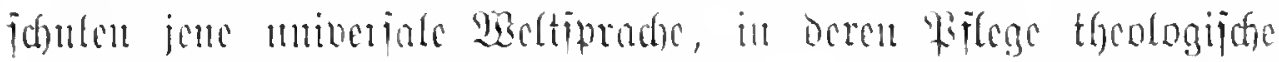

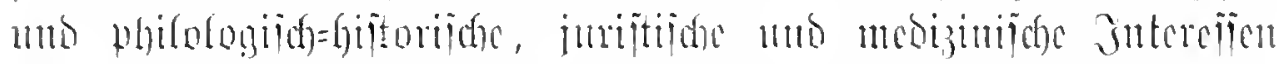

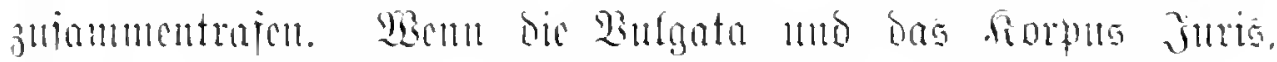

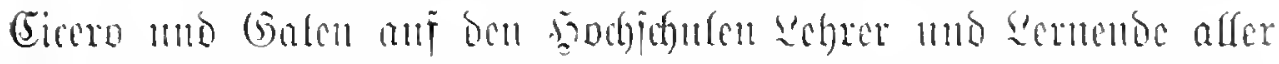

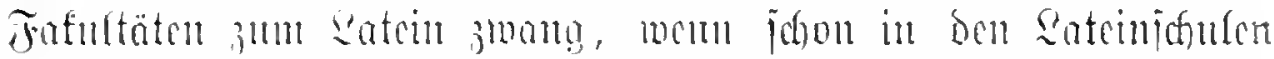

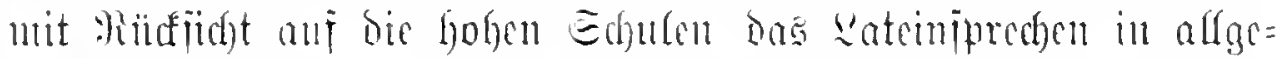
meiner lifung jtans, jo birren mir crwarten, baj bas satcin

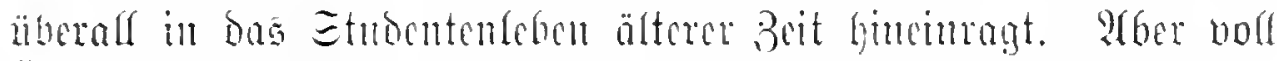

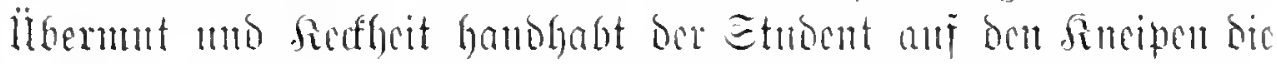

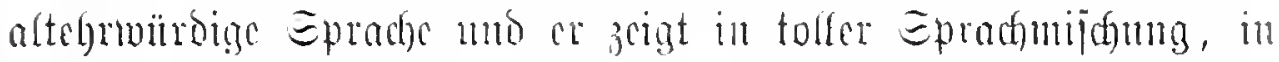

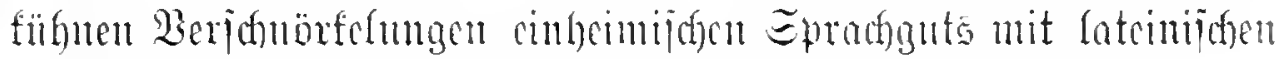

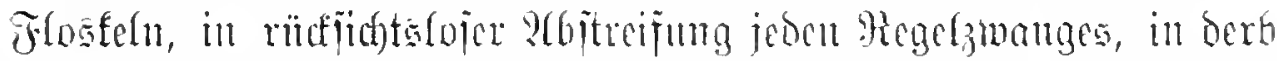

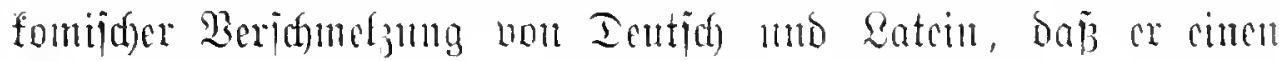
läjtigen 3wang mit summor anch beciur Bier erträgt. In der Igat mus bas Ratein im Jus Potandi, int Bierfoument mo in ben fincipen bes 16. mo 17. Jafrbumberts cinen brciten Raun cin= genommen babent. Into cin alter Eprnth, ben uns Fijdart riber= licipert, bejagt:

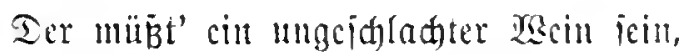

Der cim nidst giç̄̈t Satein cirt.

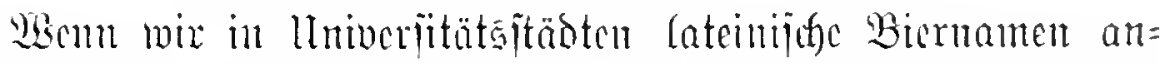
treffen wie das rastrum und bas gandium in Reipzig, jo bürjen wix mohs die Etndentenfreije bajür nerantroortlidy madyen. Into

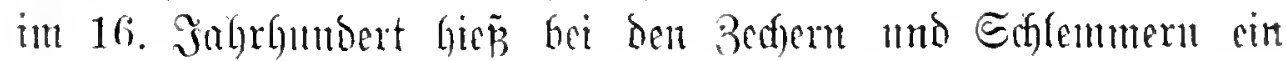




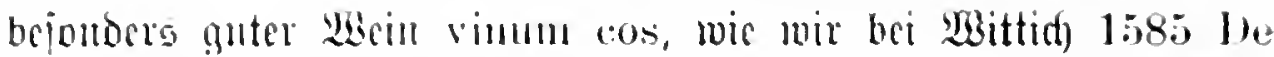
conservanda raletuline $\Xi$. 5i crjobren, und bicje burjefifoic

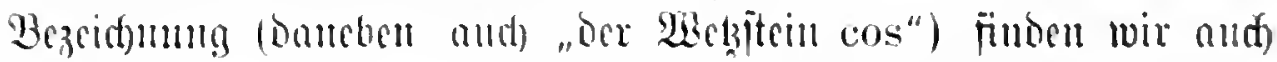

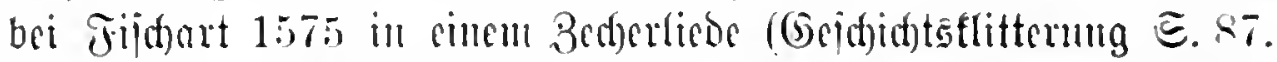

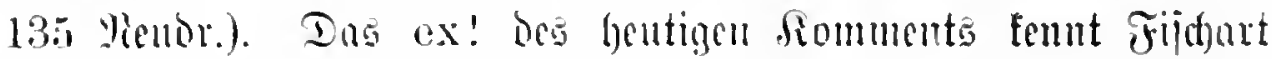
and) im zicoe: "Irints gur atrs! totum ex!" llub bns alte Jus

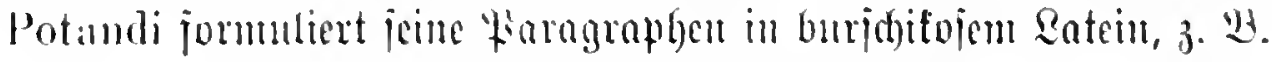

Qui bibit ex neigis, ex frischibus incipit idem.

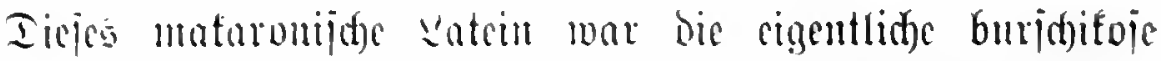

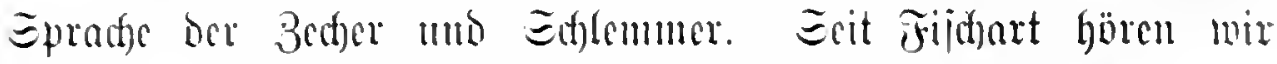

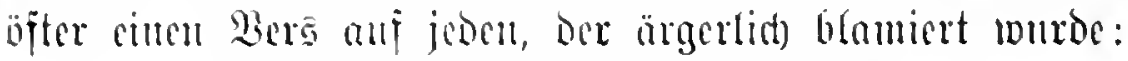

sic jacet in irecko qui modo reuter erat.

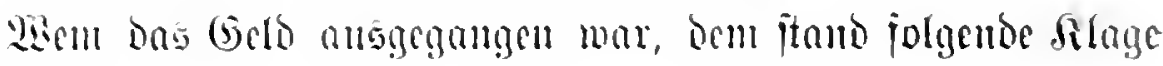
311 Grbote :

Tos miseri sumu: nee Brod nec Butter habemus

Edomeljimus armuthium, leibimus onne malum.

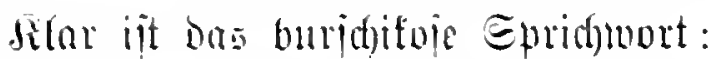

Qui multum scheret multos, scherehitur ipse!

Uno in biejem mafaronij(t)en Satein bewegt jids bie ältere buridji= foje Sitterntur, dhe es beutidfe Burjefinden, Jobjinden, Budfelinden, Backeliaben nij. gegeben lyat.

Diejes Satcin:- Gat in oer Burjugniprange bie tiefïten Epuren Ginterlajjen, wto cine Fülle übermütiger M(ije)biloungen, in Denen Dentide Terte lat. Enomigen ammehmen, charnfteripiert die Bur= idjeripradje.

Eidf ielbjt bejeidneten Die Etuoenten mit einem lat. Worte.

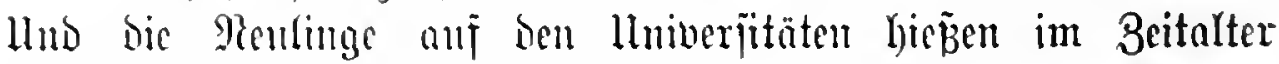
Des Remuliômu Quasimodogeniti, Neovisti, Innocentes, Imper-

'Rgl. Meimbalos Ifabemien = mo Etubentenipiegel 1720 ङ. 238. 244.528.

21627 eridien eine balb belieft getoorbene Studentica, 1689 sa Certamen studiosorum cum rigilibus nocturnis. 


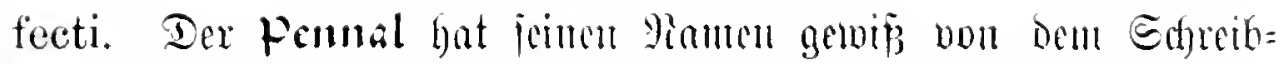

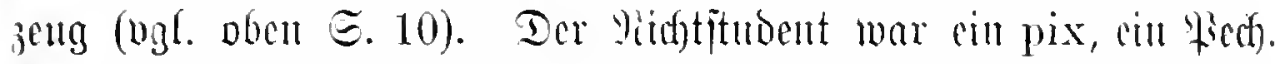

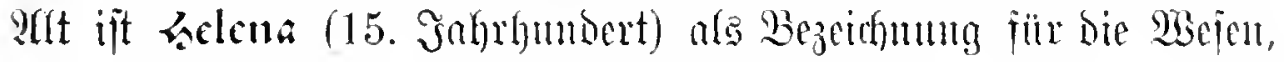

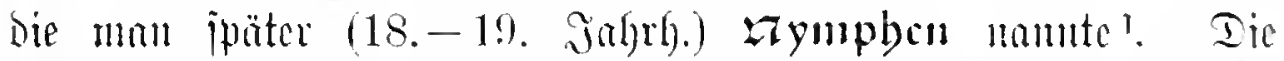

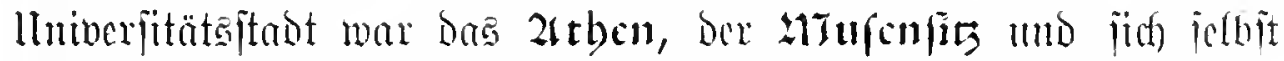

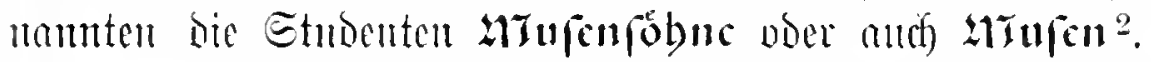

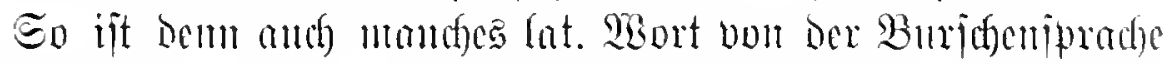

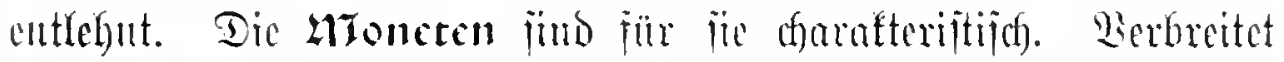

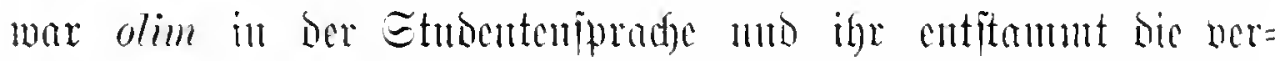

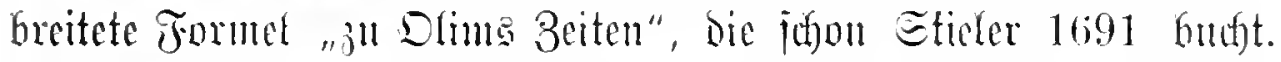
"Semper luptig - munquam traurig" tor in 17.-18. Jafyr= bumbert -... Jumal in Den Etammbütheru - éne befiebte Buridgen= Devife ${ }^{3}$, bie mohl cinem alten fionumershiede cutftammt. "Sine Edyund - sine Tud - sine Bartwija" war eine Formed Des Jus Potandi. Eareander 1710 ?Imnr auf lluiberijtäten E. 119 zeigt

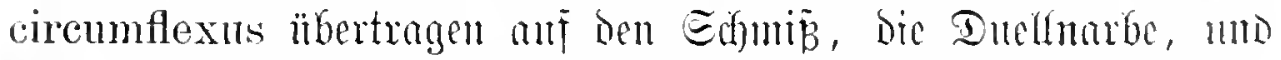

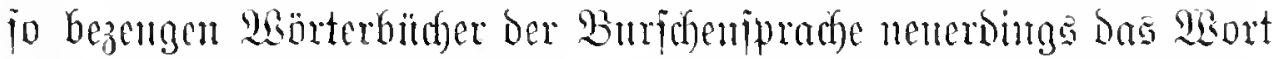
Circumfler. 2Beit berbreifet - in neneren finmmersfiebe mic

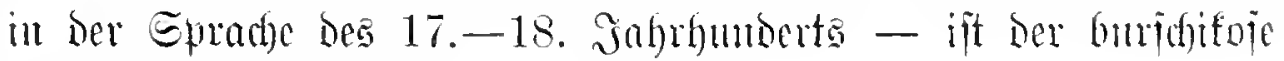
(Bebrautch von capitolium fïr 'Sinpl'”

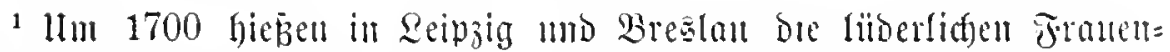
jimmer "Shülter ex collegio quinto" (Etiej 1737 Sdhlej. hijtor. Raburinth) ङ. 313). "Töinfte Frafultät" swar Damale gleid lupanar.

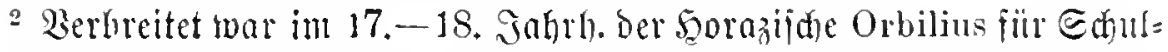
meiter 3. B. bei હtoppe I, 141; II, 106, 115. Bgl. Die Quaestio Status de jure et natura Beanorum quam praesidente Orbilio Plagoso adseret et tutabitur Tyro de Afflictis 1661. Die Rewlic) vermel)rte ßemal= mo

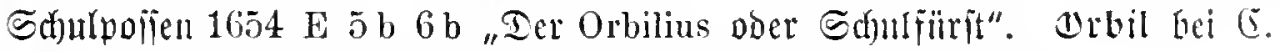

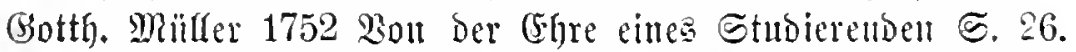

3 Taubmanniana 1737 S. 89; Saufhard 1799 miojeflater 5.60.

4 2igl. dag D2Bb. unter Capitolum uno Eapitolimm und Jus

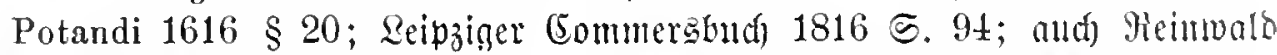

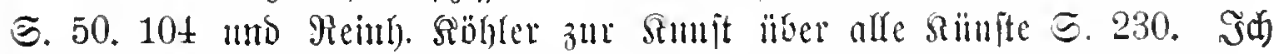

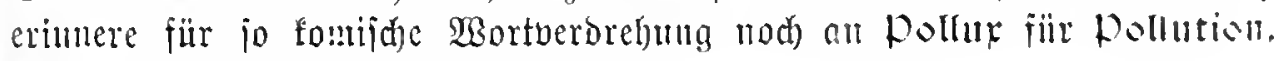
Triptolemiog (für Treo) bei Boflumant.

Rluge, Deutide etucenteniprad): 


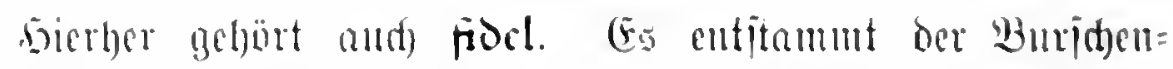

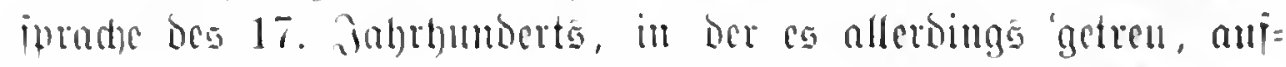

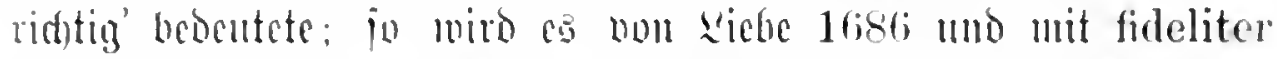

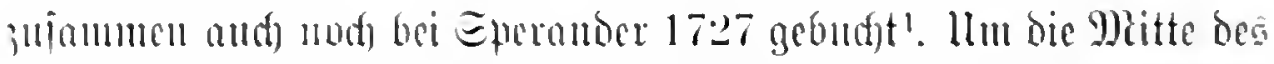

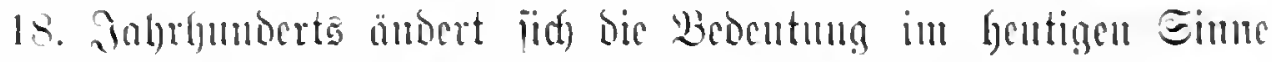
und bie Penije bes dirnubantbuliften 1745, toujours fidèle et

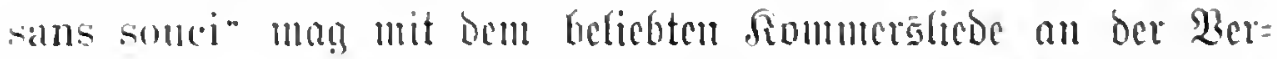

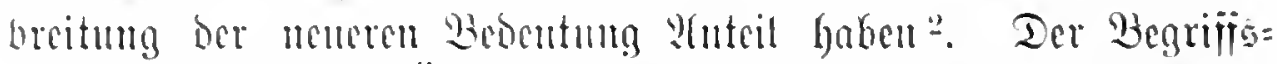

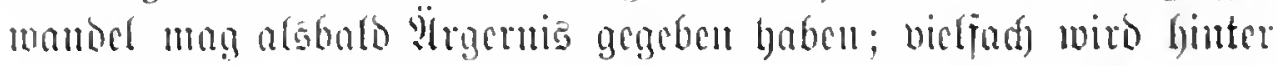

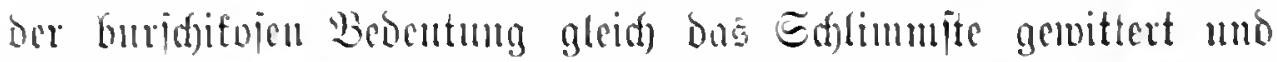

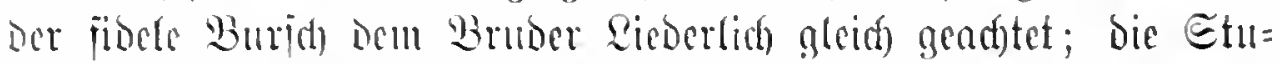

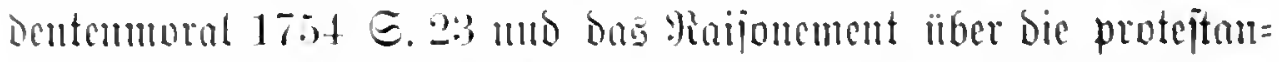

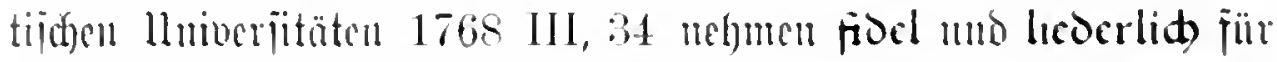

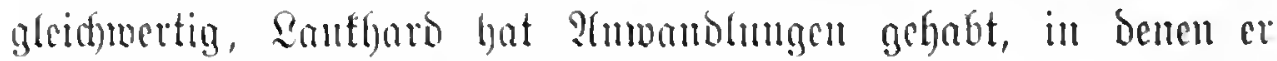

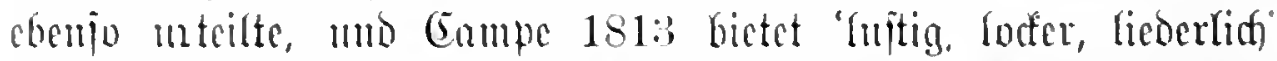
als bie Besentungen des itudentijenen Mortes. Die harmlojere

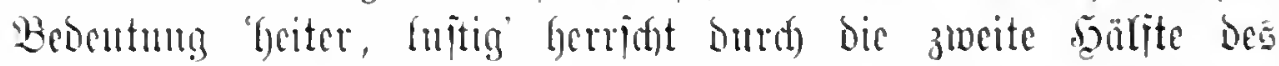
18. Jahrbunderts; (Socthe gebrantyt jo Das 2 Bort fiflon int

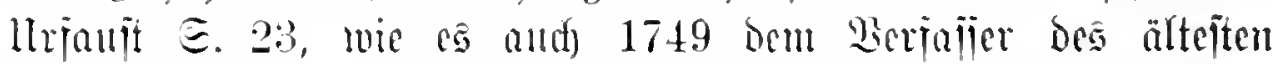

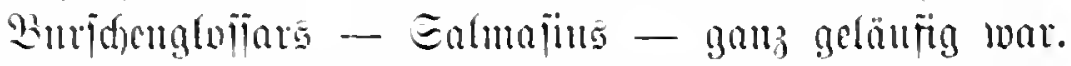

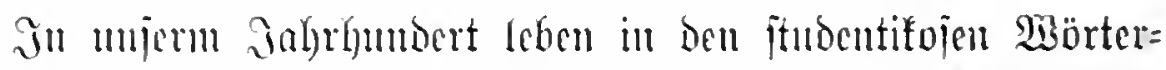

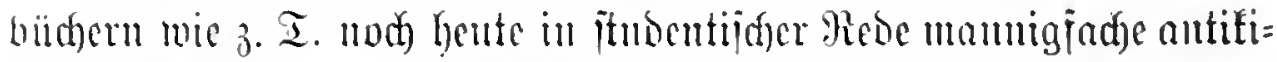

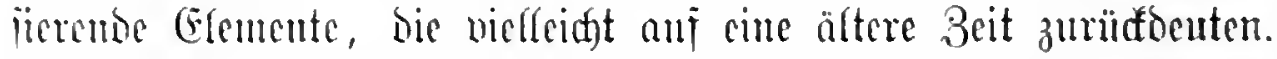

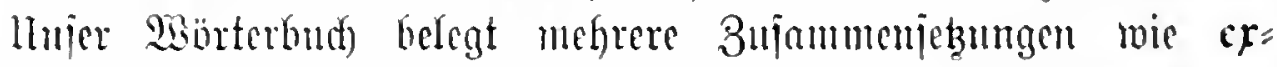

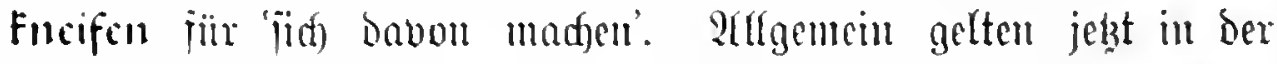
Buriffeniprad) Bilsungen wir Exfncipe voer Zonfucipant. "こid) bene thun", "ad patres reijen" - joldje Menbungen hält

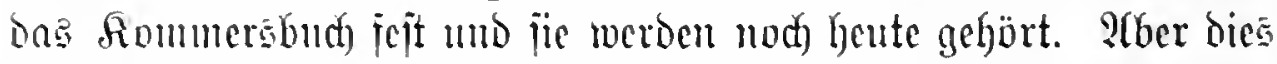
Satcin miro jonberän befantoclt, bie lat. Edfulgrammatif bleibt

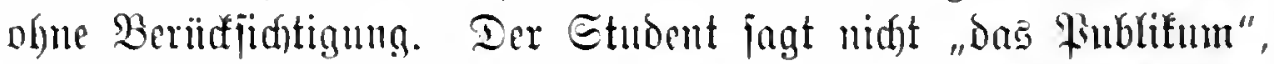

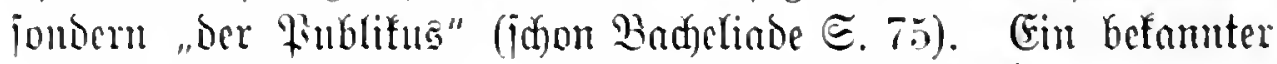

1 fideliter communiciren bei 2Beije 1673 Cranarren Mreubr. Є. 132.

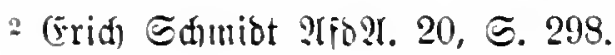


Erkmeipe. Hififfifus. Ed)wadmutifus. Sonfucipant.

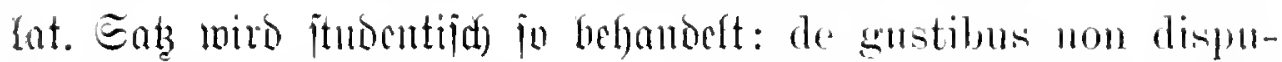

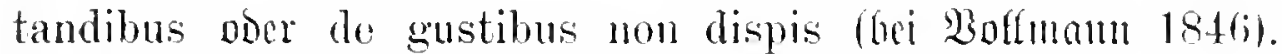

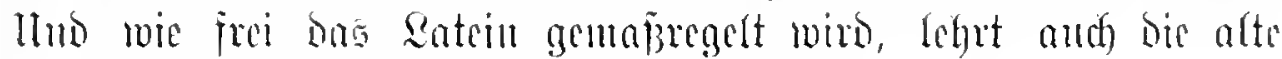
Regel: deficiente jecu- deficit omne -nia.

"(5) jino nicht alfe Enteinct, bic Gabclus=sintug tömmen".

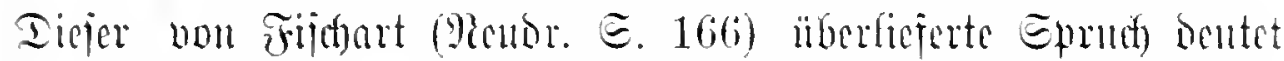
barant bin, wie bic (Enbung -ns ab dem Satein cigentümfich ant=

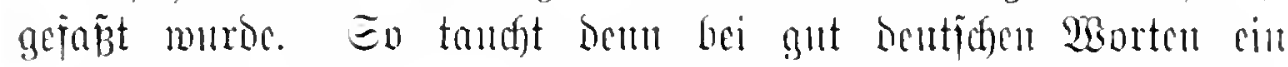

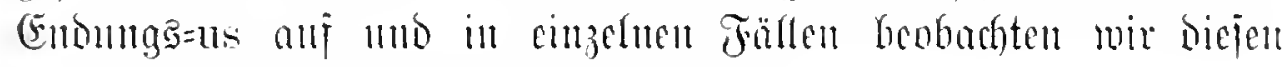
Iypus mirflich in ber Burjomentracte.

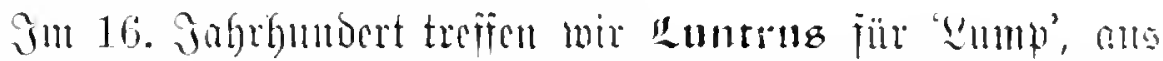

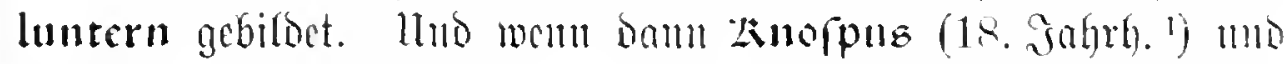
Znofpes (16. Sahrh.) neben einamber als Echimpinmete boftehen,

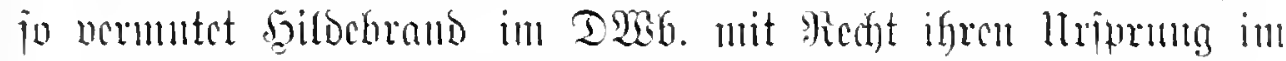

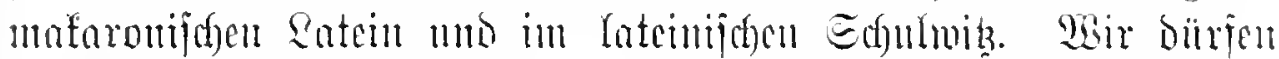

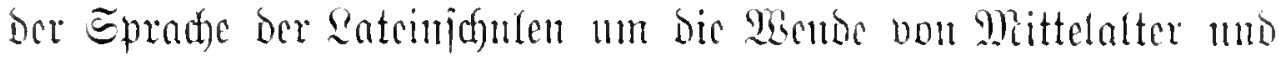

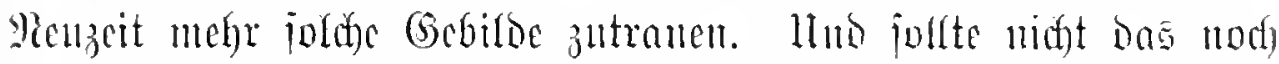

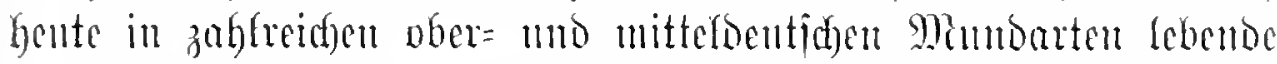

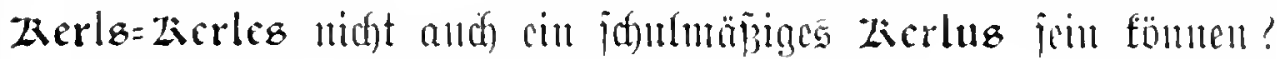

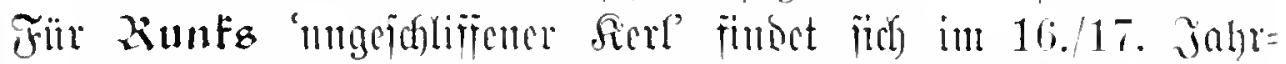
Gumbert wirflich latinipiertes Runfus. Sn Sanfhards Entigranten=

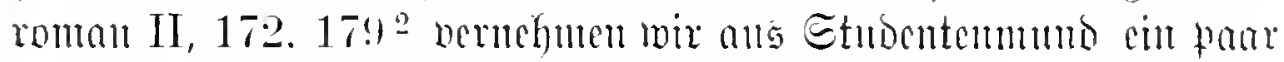

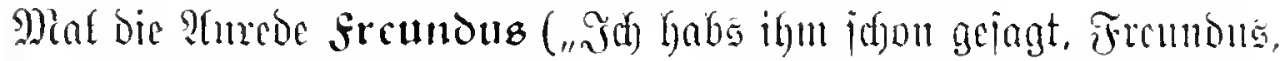
baj iff nidht alfe (5jel femm" - "Meint ifre bem, Frembus,

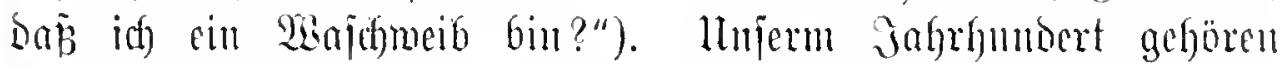

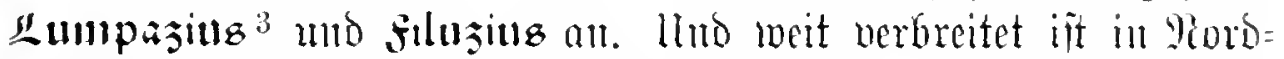

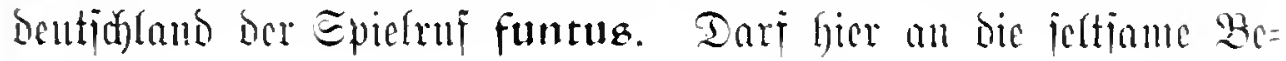

13. 2. bei Goedefe III, 267.

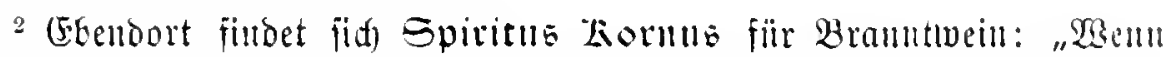

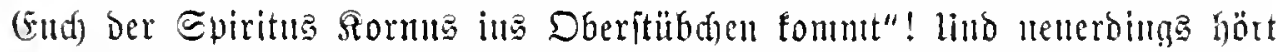

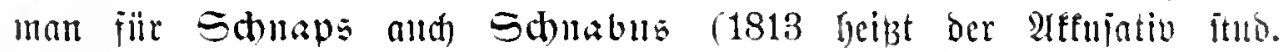
Eduabem), wie and eill biffer Menid) oft cin Dittus gentant wirb.

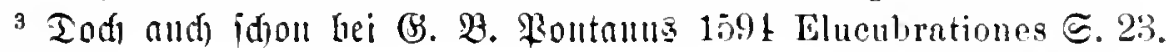




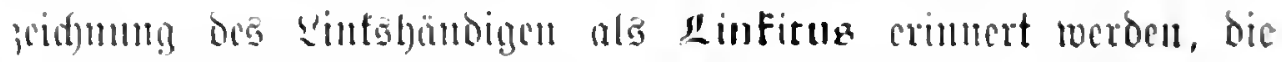

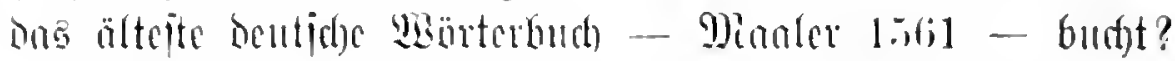

Dem gleichen Rrinzip entitammen moderne Bilsungen, mie

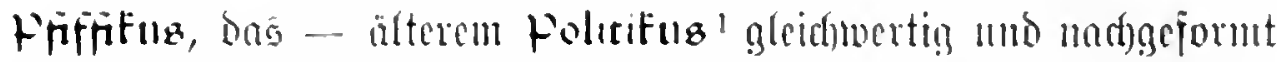

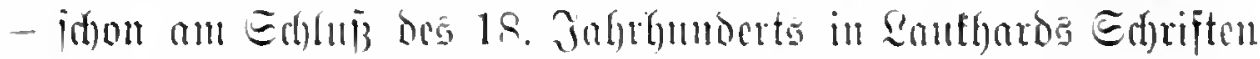

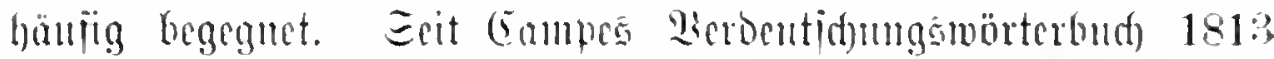

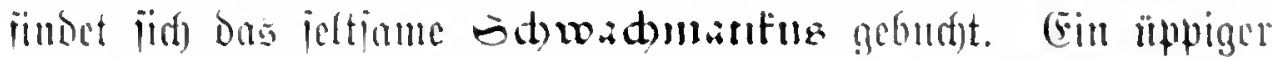
Etmoent beibt nath Q

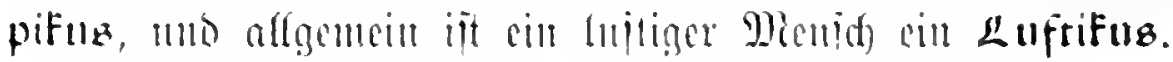

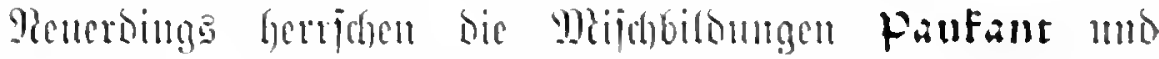

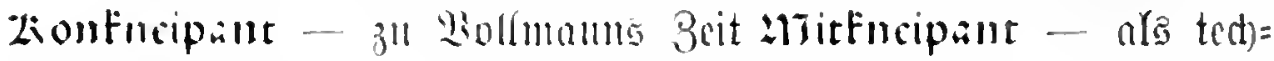

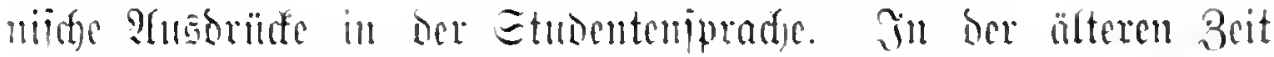

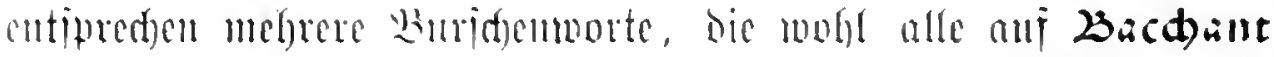

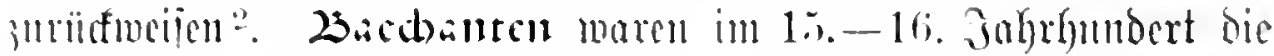

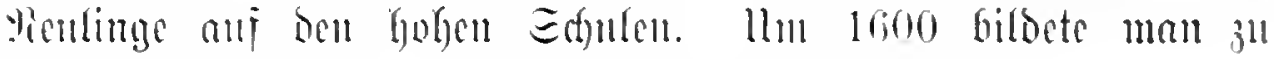

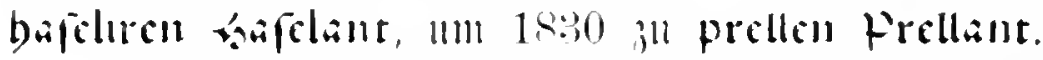

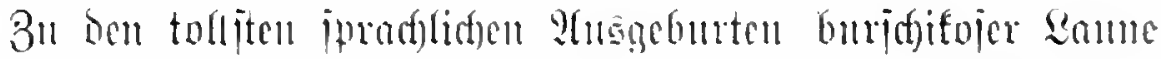

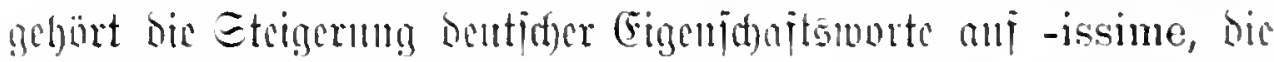

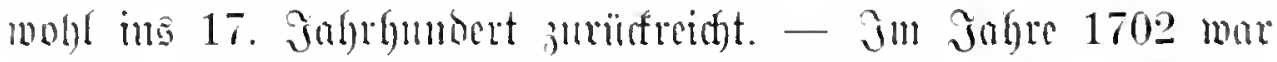

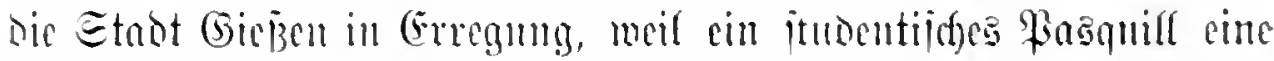

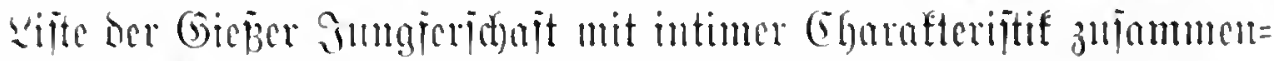

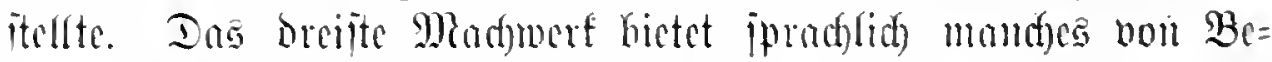
lang. Iis cogaratteriftif ber (Eimzelfeiten bewegt jide zenjurbait in Ponertien bes tolliten Senteins: optissime?', non vixissime; die Fiibe cincs Piädfens erfalten bas Bannis plumbissime und

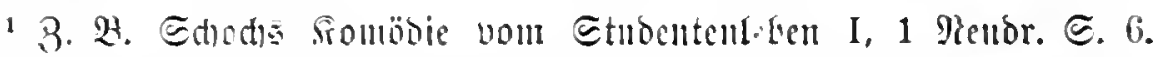

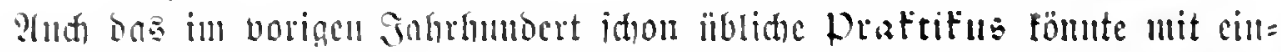

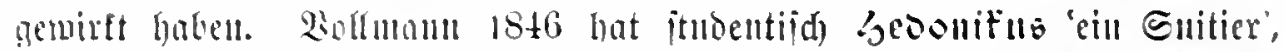

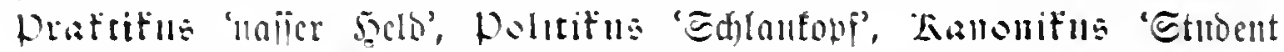

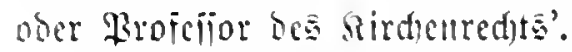

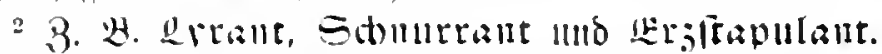

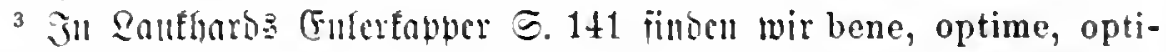
missime! in bur beitätigenden 2luttwort. Taz̆l bene benissime Sokitabe II ㄴ. $2+32$. 
Bifififus. Rontueinant. Sdtledtissime. Plnuberunent.

Der Bujen eines anbern fdeledtissime; nub "olim gut", "olim

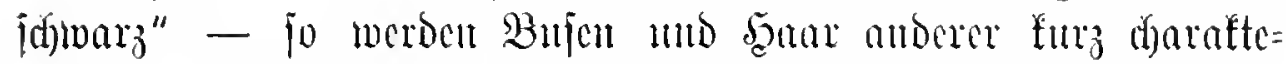
rifiert. Ed)led)rissime f(t)eint im Mnjang bes vorigen Jahre hunderts berbreitet geweien all jein; wir treffen cs 1714 bei bent Jejuiten Calenberg, Der an burjoffojen Wortgebrlon iiberhant jeine Fratioe bat 1 .

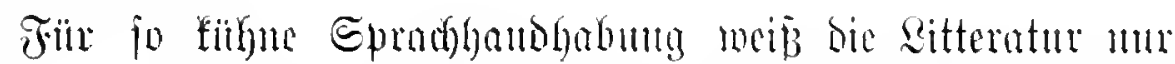
geringes Belegunterial anjubringen. Sie lebt rent eigentlid)

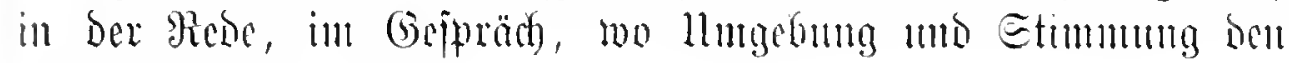
Mint bagn gibt.

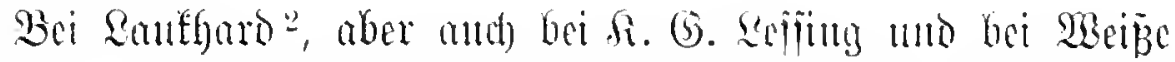
begegnen wir cincm Gurfobitojen fpendabel 'ireigebig', zu Finfen wird kussibilis gebildet vou $\%$. Eeverims 1670 Der ver=

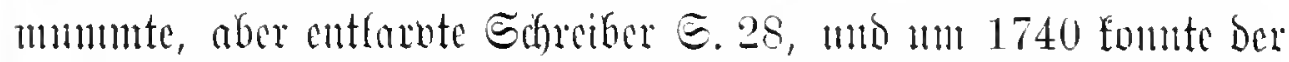

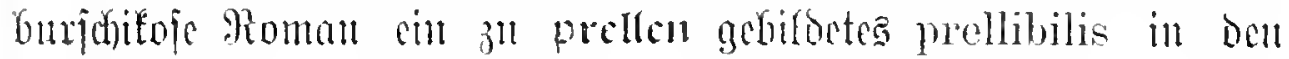

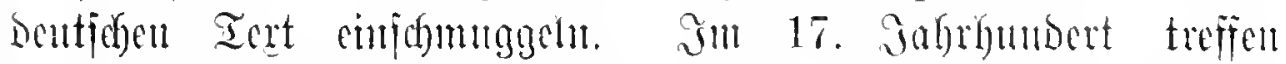
wir cin Gubrides Plandernent ${ }^{3}$, Das mmatourtfids in Baicru

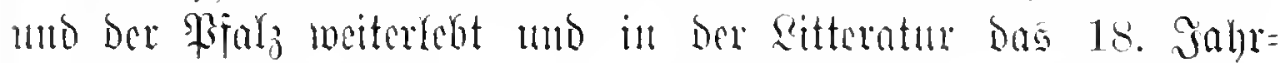

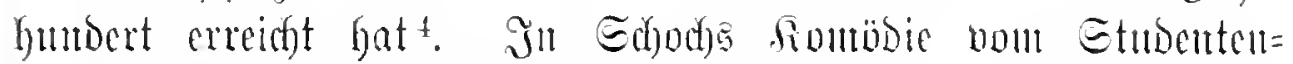

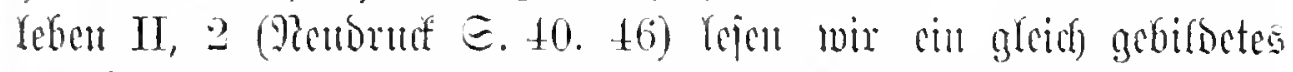

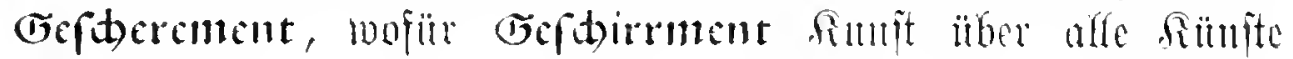
厄. $236^{4}$.

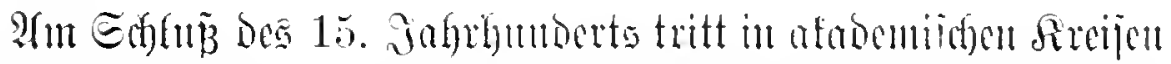

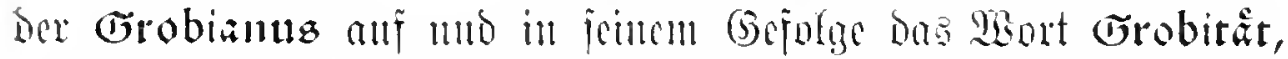

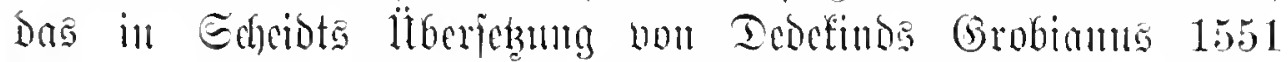

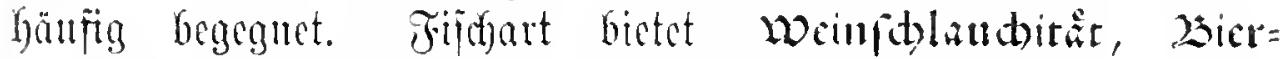

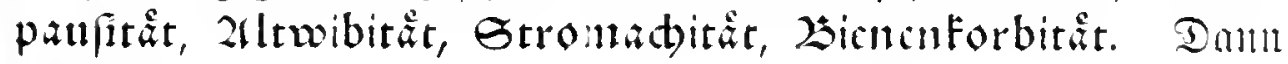

1 Quasi ๔. 48; Wurmatia ๔. 18.

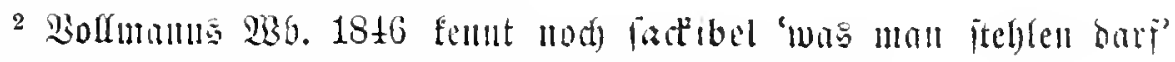
jonie fnallibel - riggibel - búritibel - bủririabel.

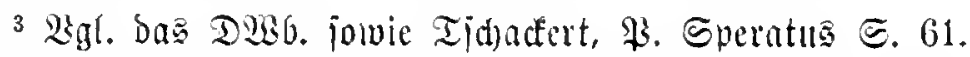

- Renerbing Poufeneur = Doulfage mitel im $\mathfrak{x b}$. 


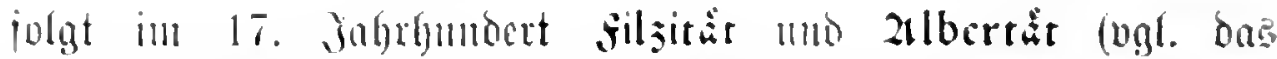

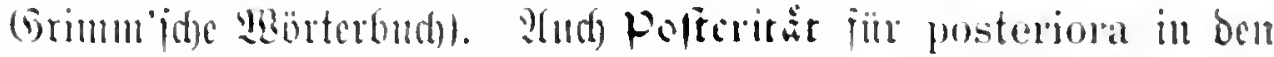
Pambmammiana 17:37 $三$. 16:1 mo bas gleidabedentende Epon=

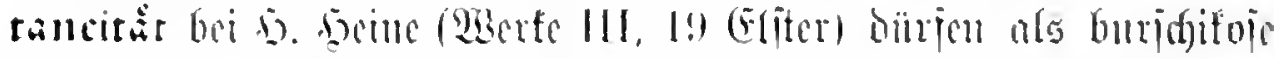

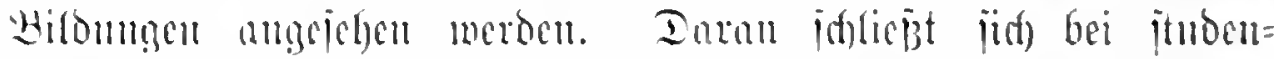

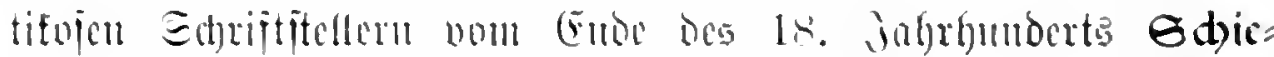

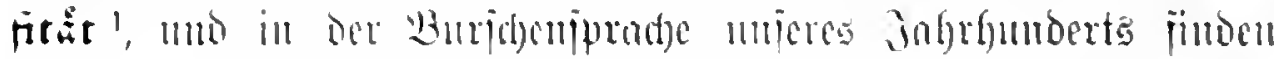

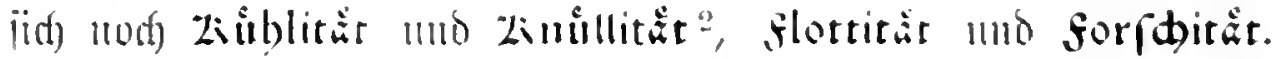

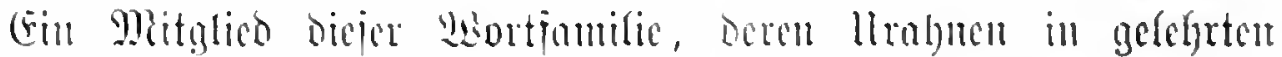

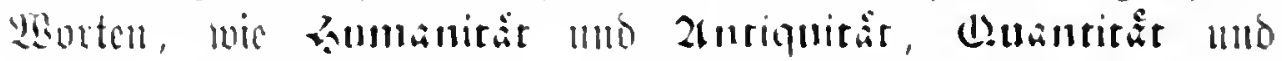

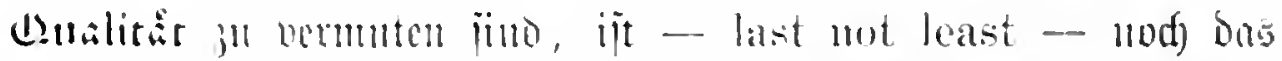
meit nerbreitete Bd)wulitur, bas jid) icit fimblebens Etmbenten=

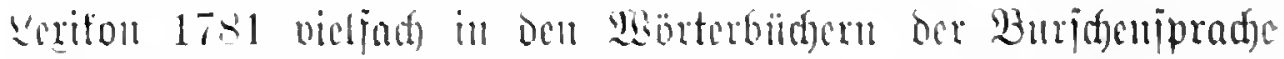

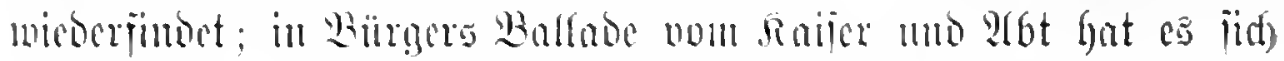

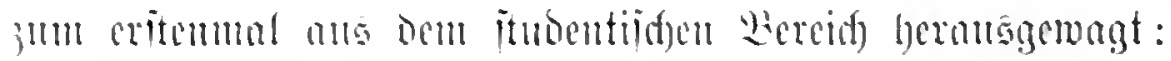

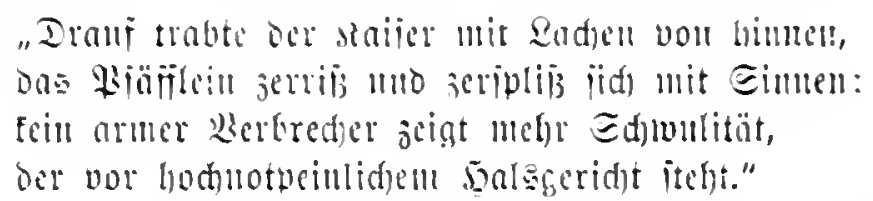

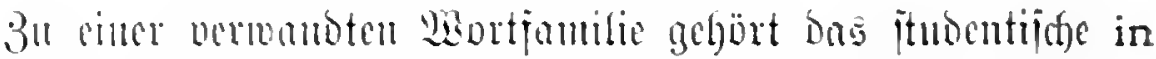

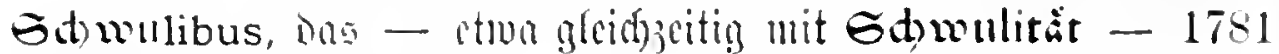

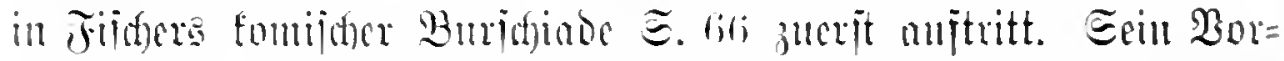
lämjer ijt sin älteres in floribus, bos burjofifos jofon 1729 in Etoppes (jedidgten II $\Xi$. 108 bejengt iit, mo mix etimern an dis iobiinde I, 黑. Sost:

"כo gut als der bejte Reademifus lelt er täglity in floribus."

Iicics "Deben in floribus", bom eir "Peben in splendombus" $171+$ in Calenberga Wurmatia $\Xi .22$ entppriat, hat fix

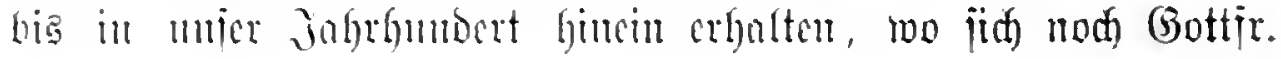

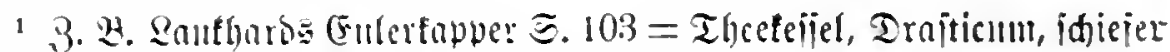
Serl; aud Mroicllaner હ. 10, 2Utobiogr. I, 158.

" "2ritiber in knïllitate" bei Bollmam 5.174. 


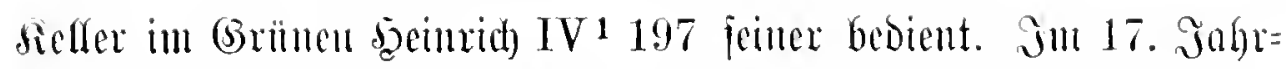
fumbert verfond ber 3ecter mit biejom in floribus nore cinen

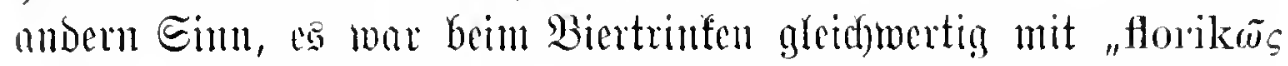
trinfen" mo flores war was wir bie Bhlume nement. Jn biejer

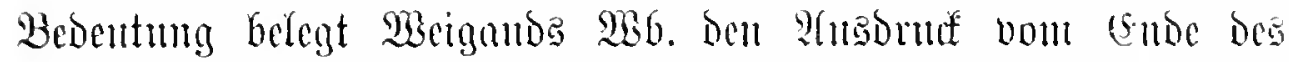

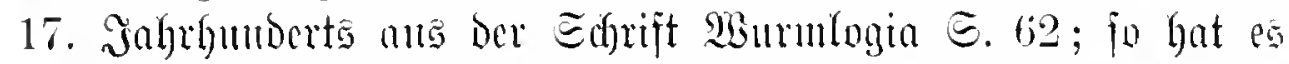

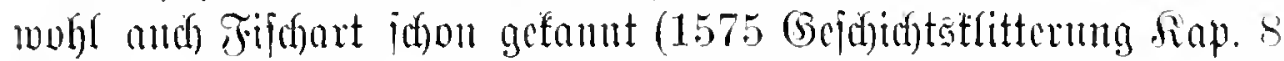
Mentor. E. 149). Itno barans hat fid vereinzelt cin sloribus

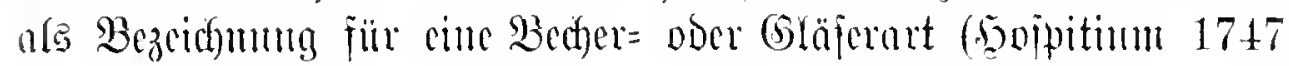
\$ 36) abgezweigt.

3 bicjer (Bruppe in thoribus und in splendoribus bat fitif am

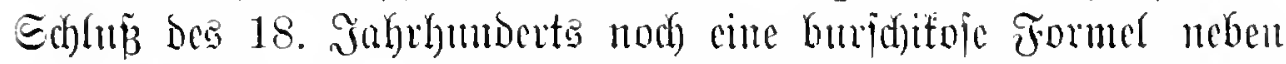
in schwulibus gejeflt. Seime Banrid)aft nemt ber Etnoent ber

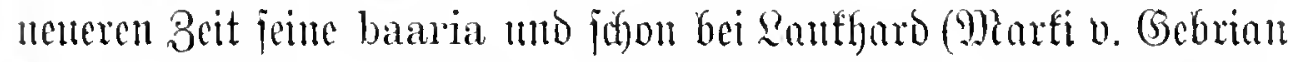
I, 35) leper wir: "6000 Livres in Baribus"; tunb biejcs in baribus beiregt Eumbers autrit) ans b̧oltei. ${ }^{2}$

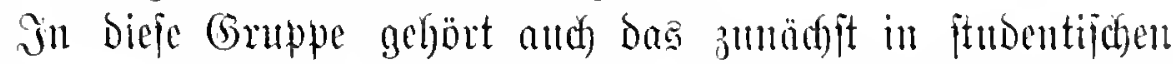

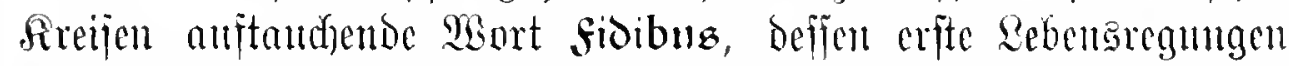

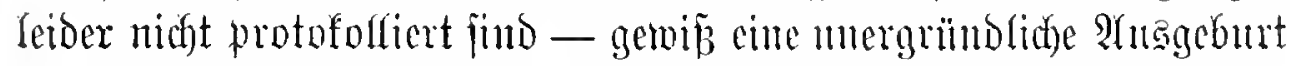

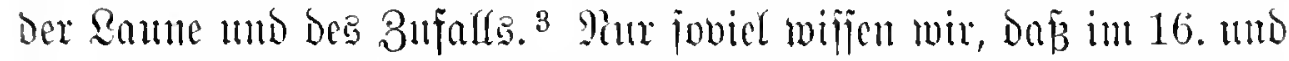

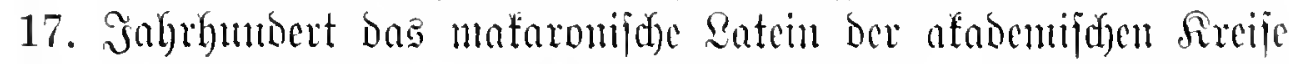

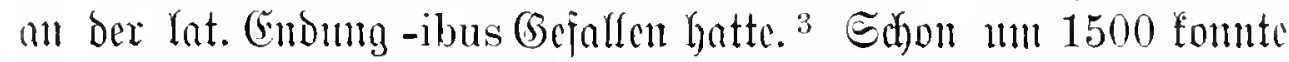

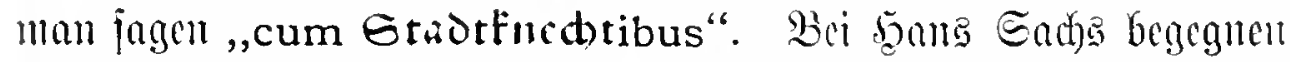
einige Banberiormeln mit Sijenbolatein, in bem -ibus cine gewifie Bolle fpielt, j. $\mathfrak{b}$.

1 Meitere Belege ans dem 18. Safrhmoert i. im DQsib. unter flor; and) Speranders Sento=2exifon 1727 unter flot.

2 Ӭlnuliche Formeln bei Bolfmann 1846 finto in pecho, in manschettis, in stifelis.

${ }^{3}$ (5: ift nicht ber Mïhe wert, bie verichicoenen ofit geiftreichen Meimnngen

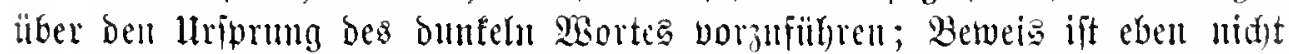

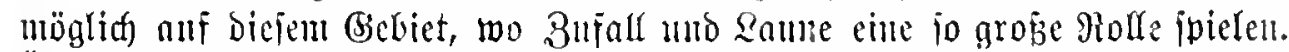

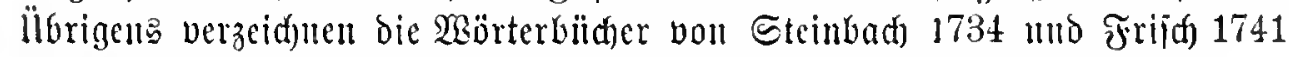

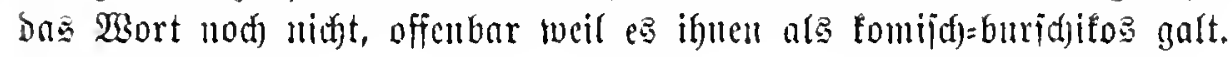


nvenite ilır unlunldibus

bringt bengel her uns stultibus

die semper mit uns spentibus

sub capite et lendibus."

Mir jeben, -ibus hat jür cin untrïglictes fenmzeid)en bes

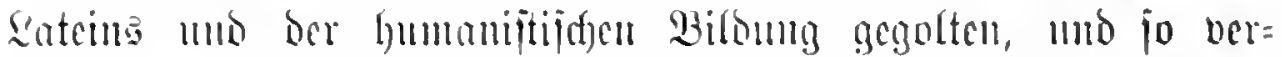
munern un jene bajtarbartigen in schwulibus, in splendoribus mo in baaribus nidft, anth) ber fioibus mo ber floribus nidjt.

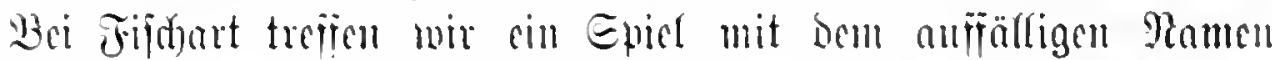
23.rbedoribus, unb bei bemidben jolgt rin buntes "Spectormm fialbertanzen" im Satcinmuterridyt mij ben Eab: "Ireib Den Eohn

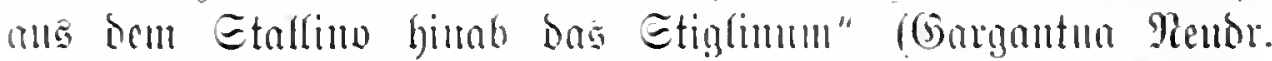

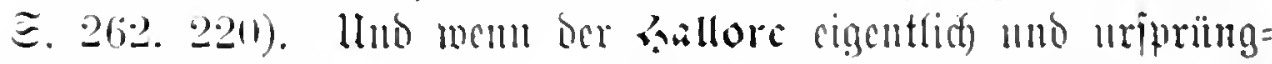

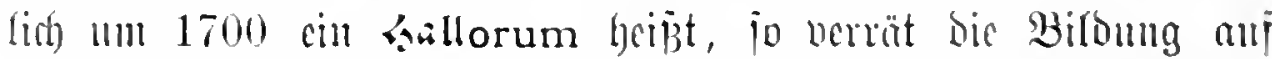

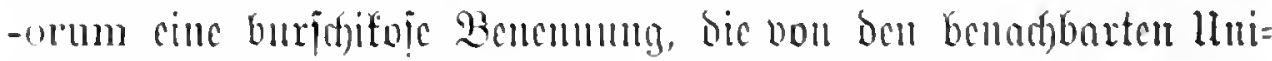

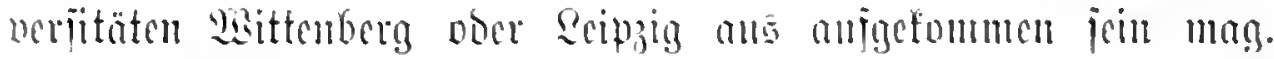

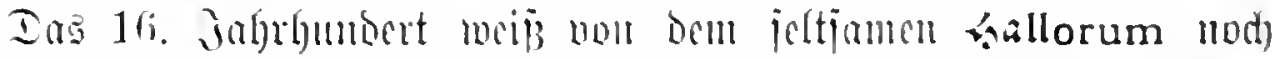

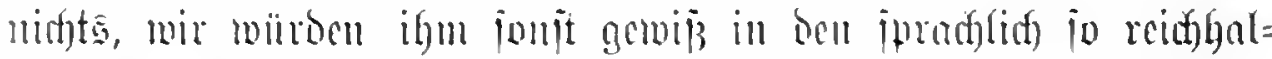

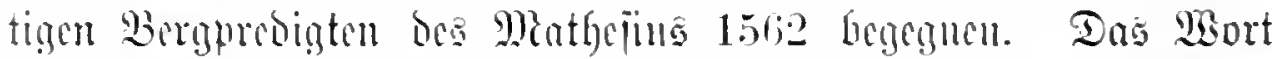

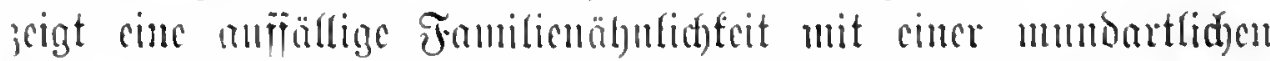

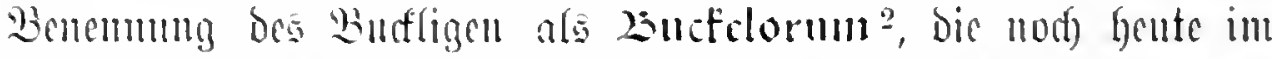

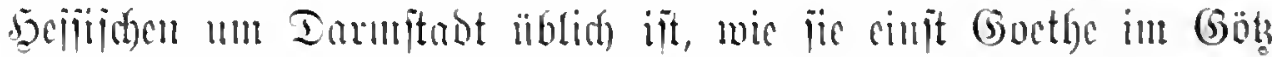
gebrancht hat. Luriffifos ijt ber 3njammenbang, in meldem ein ieltiames piftilormu bei saufgard auftritt: "Ei jo joll ja ber

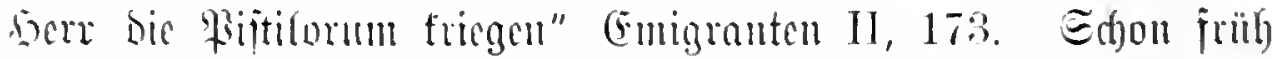
- In 1500 - cridfunen dic posteriora nts ber posteriorm bei Elcarins De Fide concubinarum erga Sacerdotes.

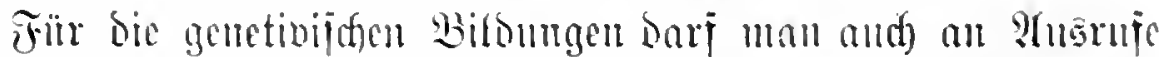
mic o jermm ober an lirmm-larmm crimern. Dic Frenbe an

1 Esciumar. Juthrbuth II, 427 ï.

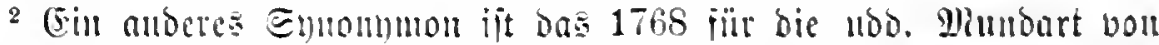
Plemen Gezengte Pukkelhomo-Pukkelhomini uno "Brnoer Bufellomini"

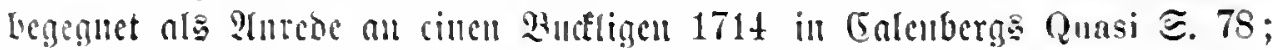

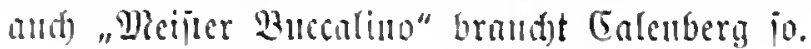




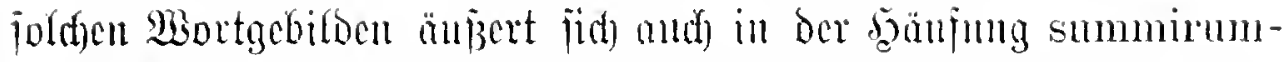
summarum bei $\mathfrak{B e i j e} 1673$ Erznarren Mendr. $\Xi .91$ mo ber

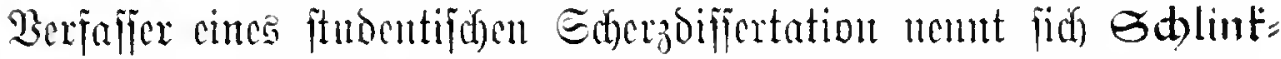

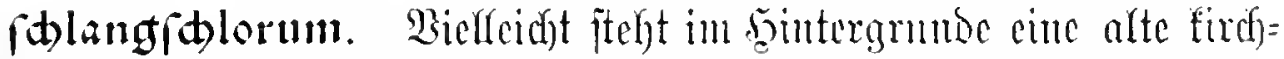

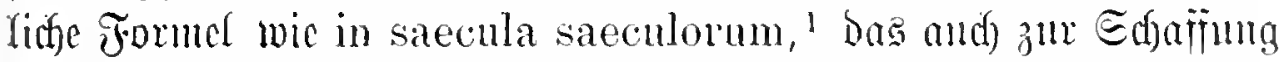

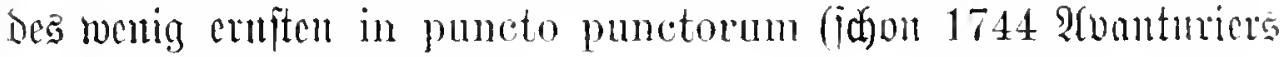
E. 191) Borbiro mar.

Sm 16. Jahrhumbert bören wir bon armen Exhiltern, bic

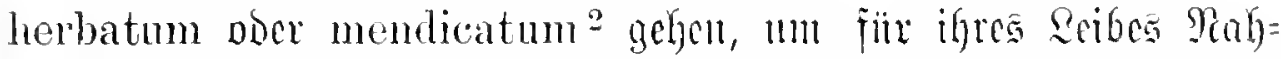

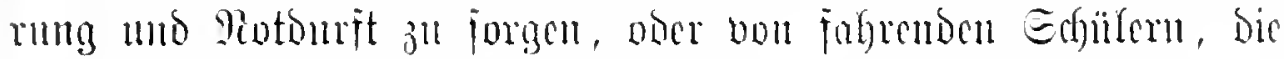

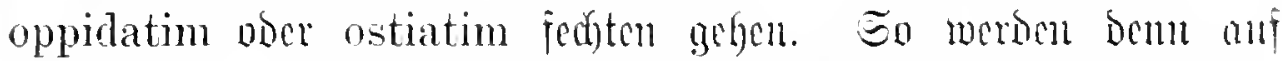

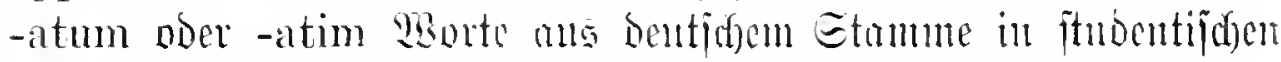

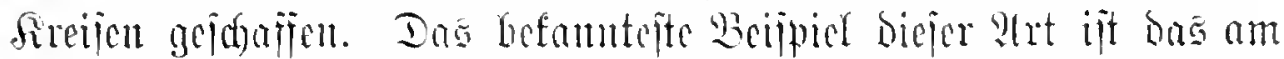

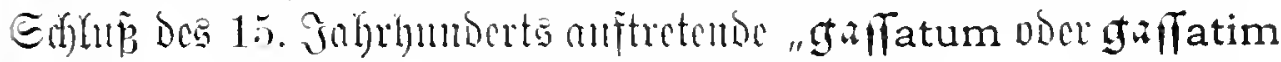

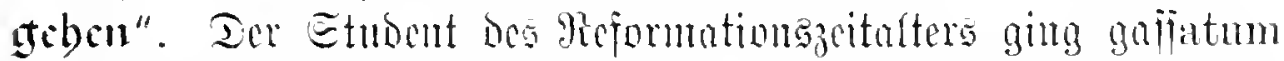

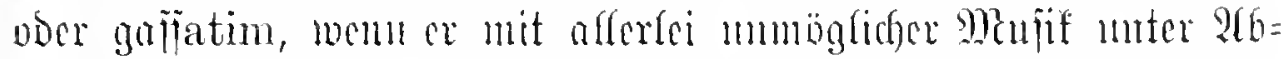

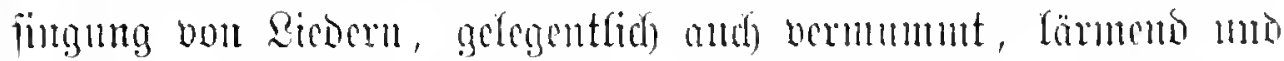

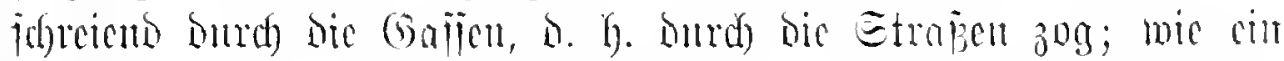

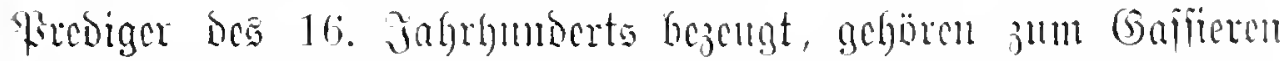

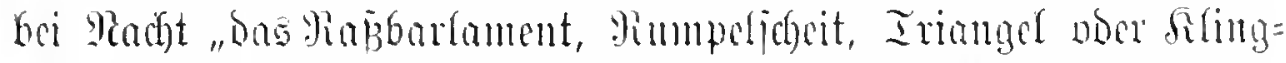

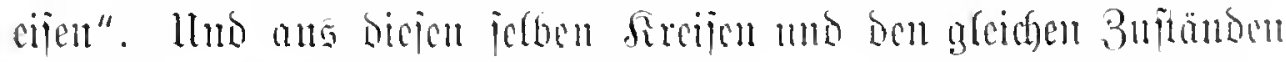

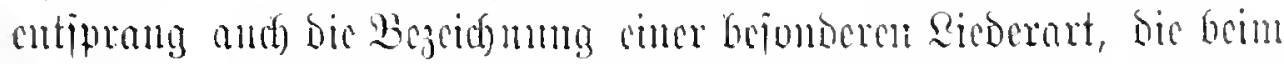

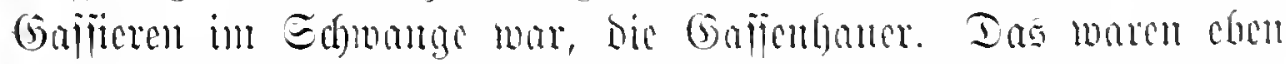

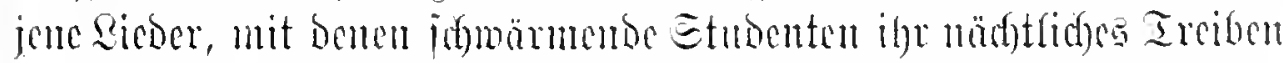

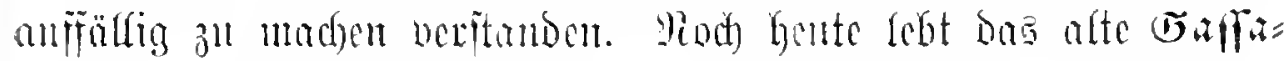

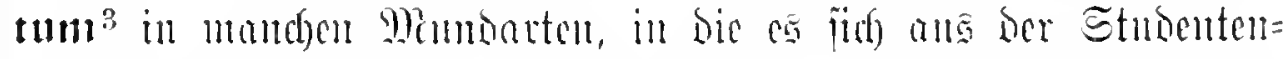

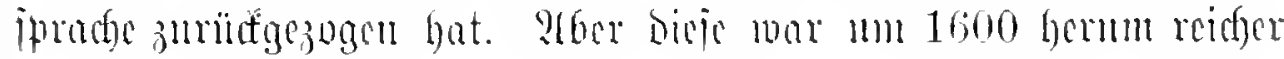

1 Bgl. bajll die ?adfbilomig per omuia klingklangorum bei Zucas be Leuna De Jure et natura Pemalium 1611.

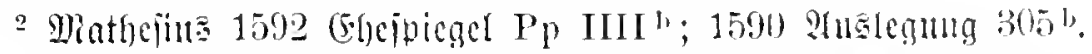

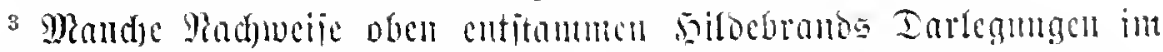

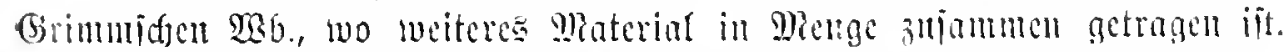

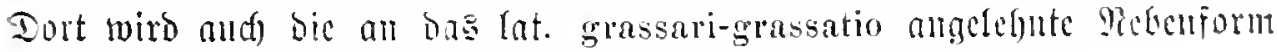
graflaten erörtert, bic ans bem ?ob. in- Däm. gebrumgen ift. 


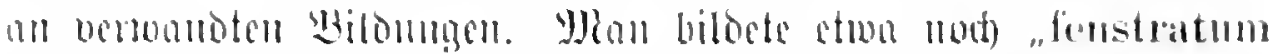

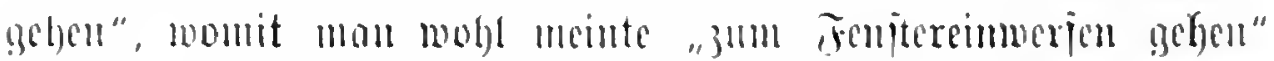

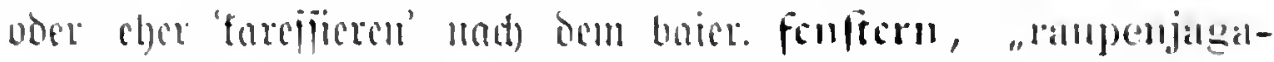

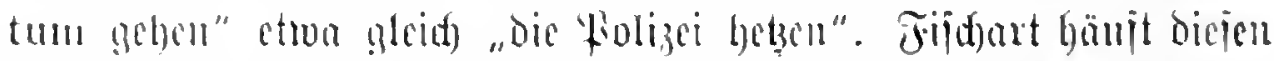

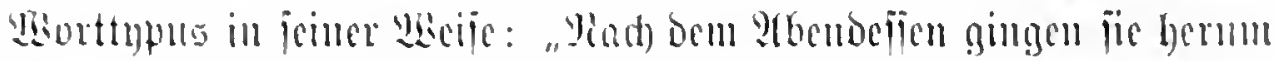

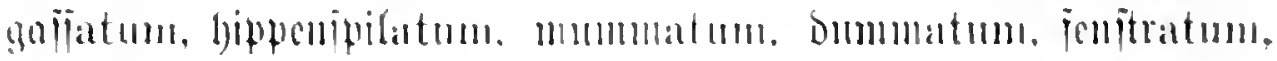
rmpenjogatum"!

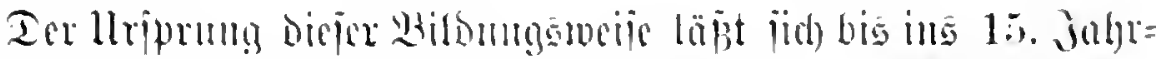

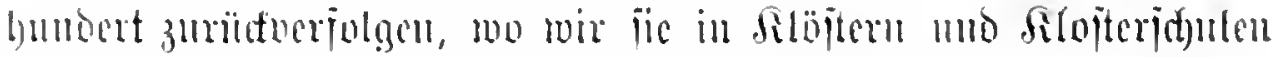

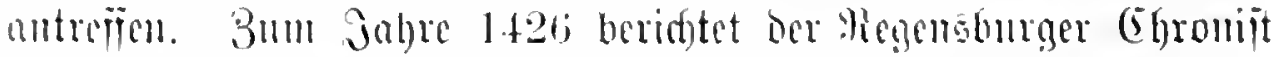

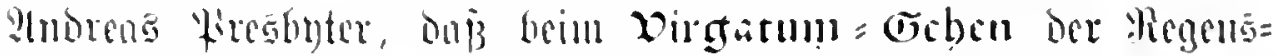

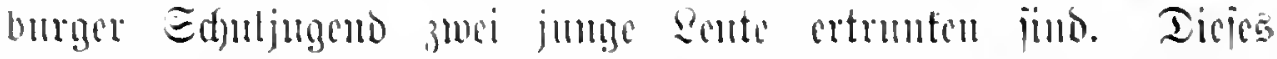

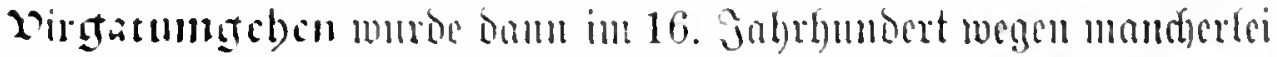
Injugs, dex bei oen jugembipielen nortam, Duret) Batsveroro=

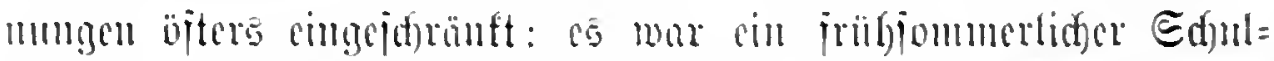

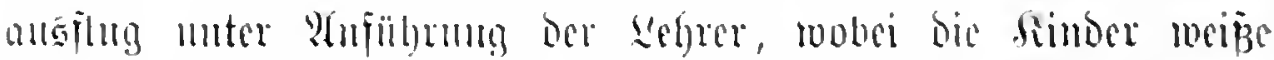

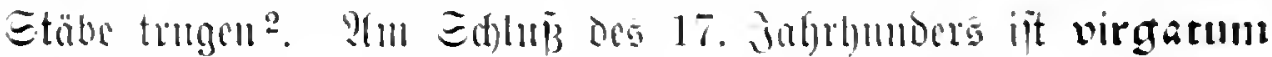

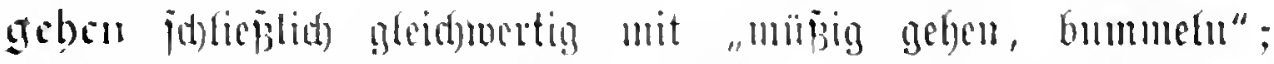

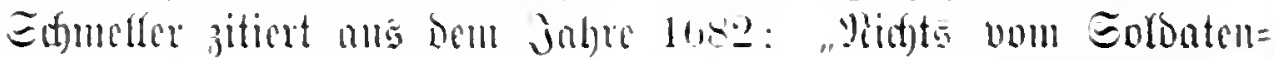

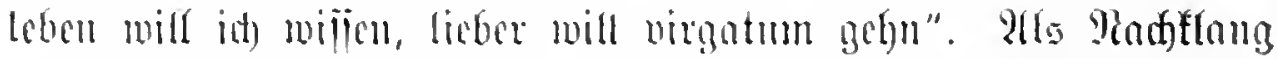

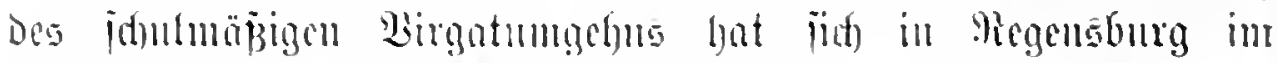

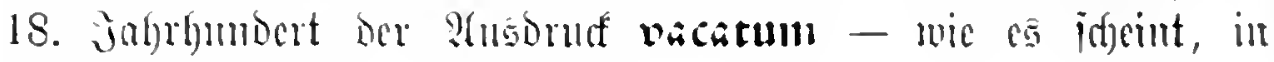
gateider Bebentmin - crbaltrn.

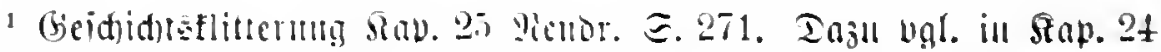
Viust. E. 25. cine 3echerrigel mit buriditojer Eprnd $=$ mo Micimbants= habung: "giad Bircu gib potum - nad potum cile encotum". Daz Ratcin ber Imnfelmämnetbricic femt cill spaciatum ire 'ipazicren gehn': ivit in ecclesiam rel ad forum vel spaciatum in rubetum. Imb Eim. Mothg

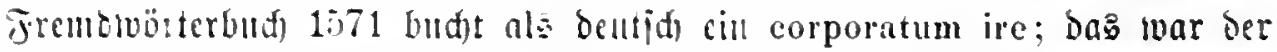

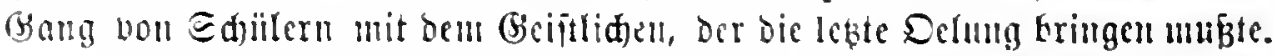

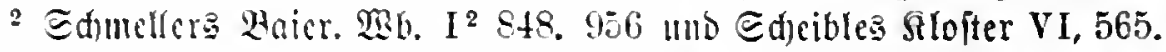
Eimon Mothe Fremblvörterbu(d) $15 i 1$ bud) "virgindemium ober virgatim" wno veriteht Sarmiter bas Ruthenunden ober Meifigholen der 2 Hben fïr Sel)rer nol (Beijulide. 
Biemlief) gleiffyeitig mit biejem "virgatum" begeguet in

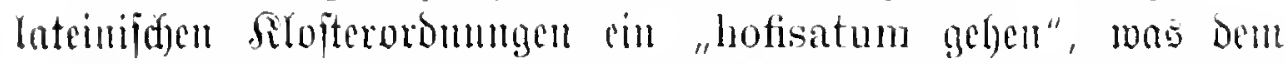

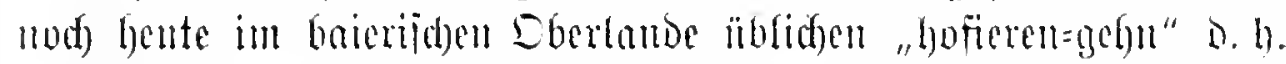

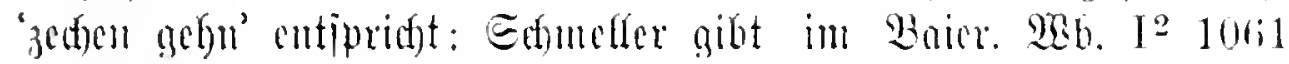

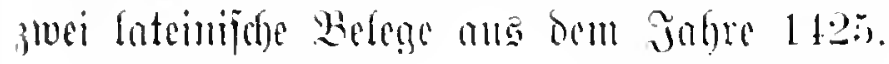

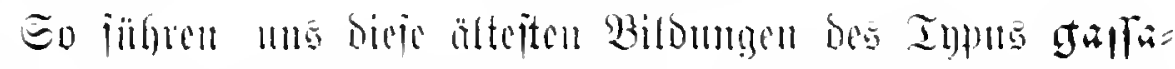

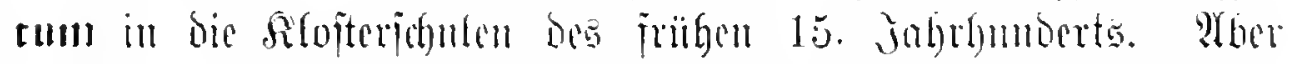

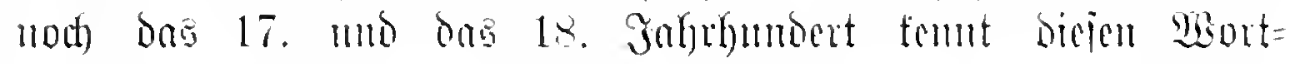

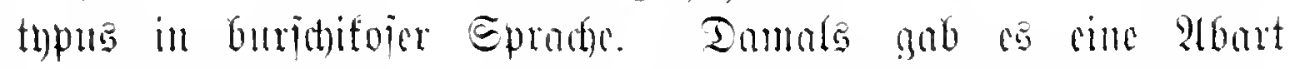
Des (Bajpatengebns, bic man "stellatim ober stellatum-gefun"

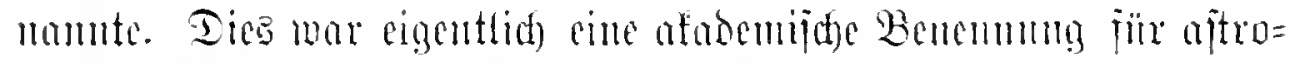

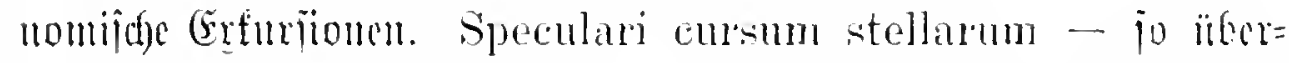
ickt Weismann Lexicon Latinogermanicum $17+1$ bus Dentighe

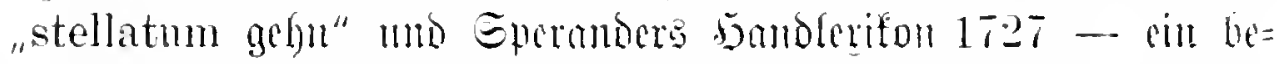

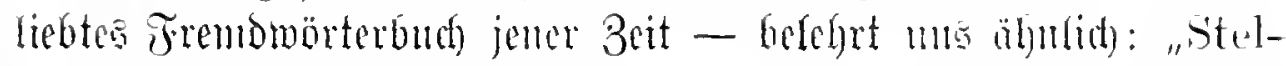

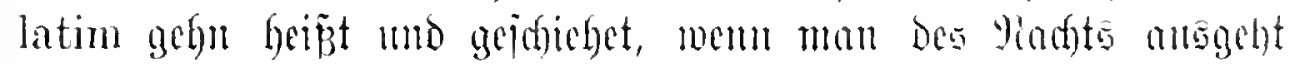

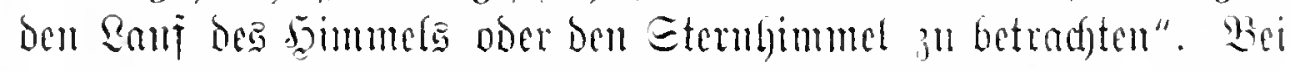

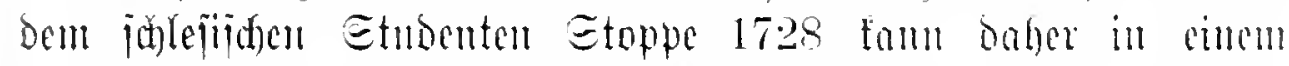

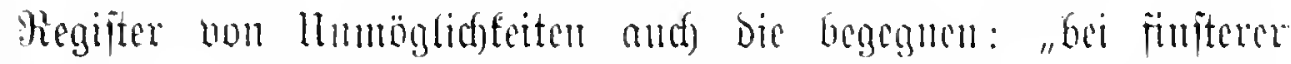

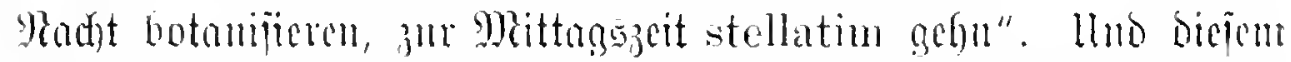

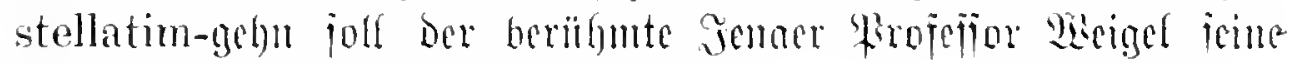

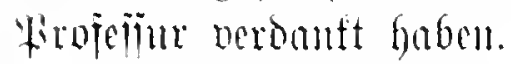

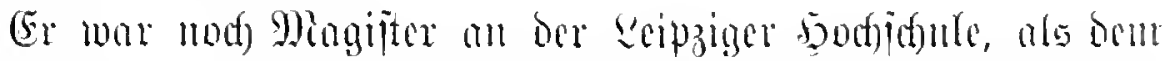

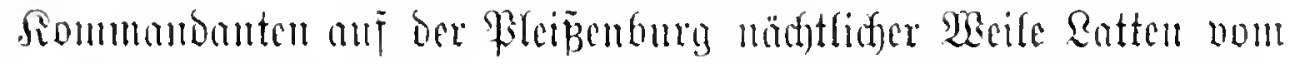

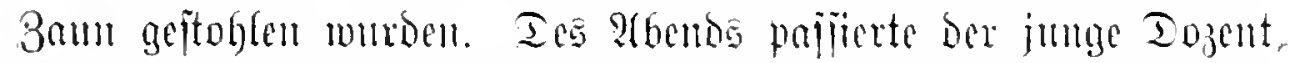
Der aftronomififge Beobudstungen mathen wollte, mit cinigen

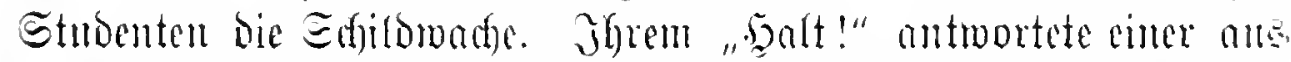

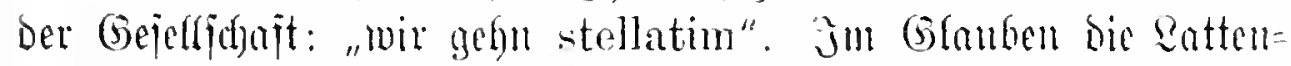

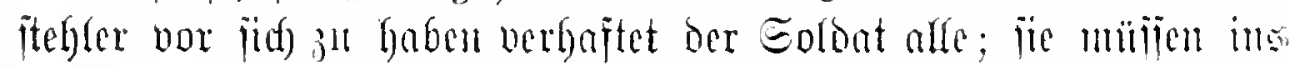

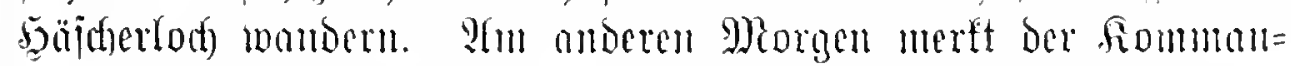
Dant bas Miēperîtänonis mo refommanbiert ben ifjur befannter

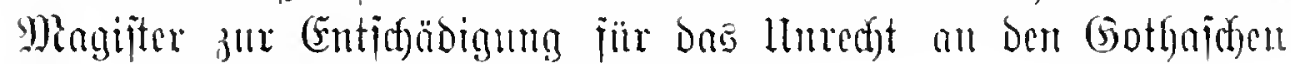

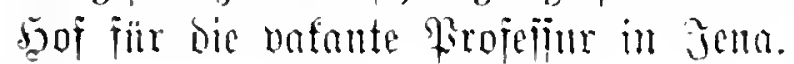


Qlace bicjes stellatim- oocr stellatum-gebn ijt nidgt immer

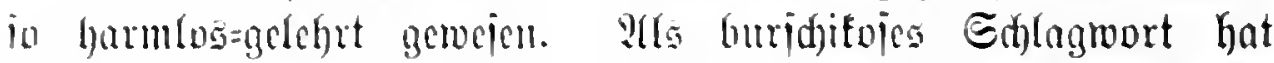

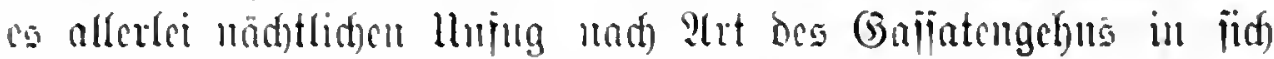

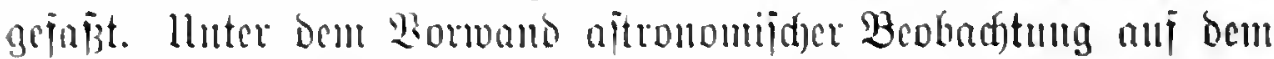

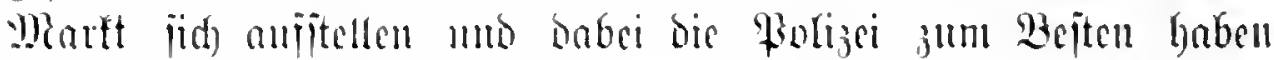

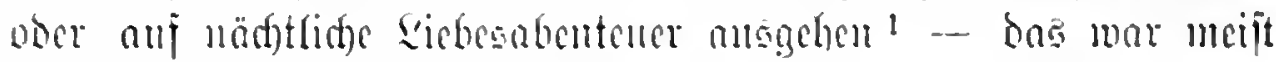
Der Bmed bes Etellatumgerms.

$$
\begin{aligned}
& \text { Stellatim piflegt man ghad): ;il gehn, } \\
& \text { wo idoüne sesifer woluncu }
\end{aligned}
$$

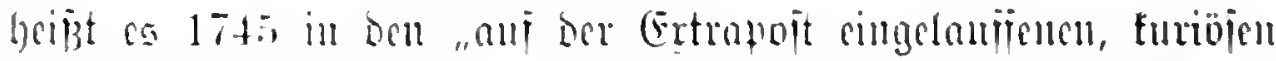

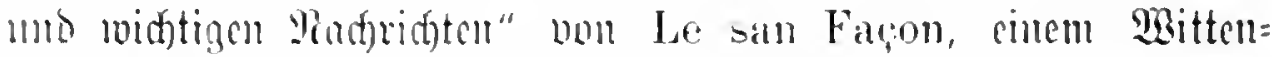
Serger (Elaborat nou bedentlid)en (Charafter.

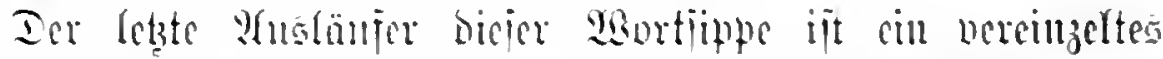

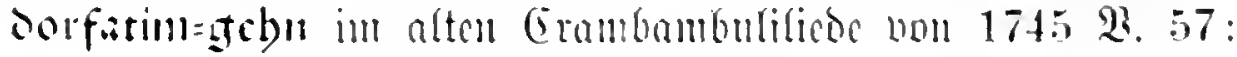

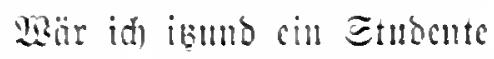

3̈ Dem berilluten Eatathen,

此

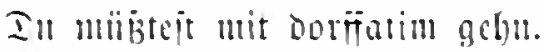

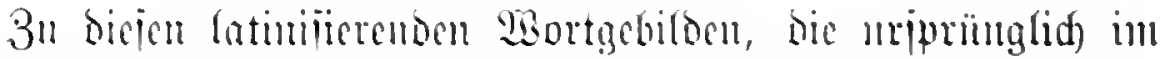

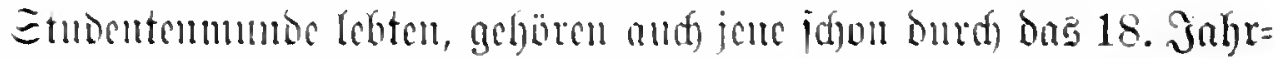

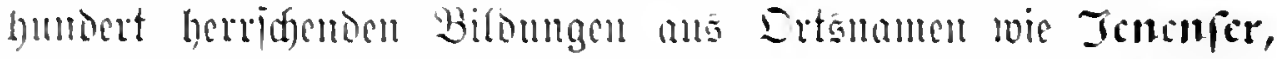

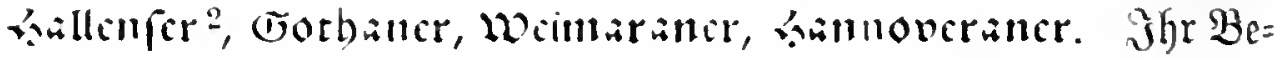

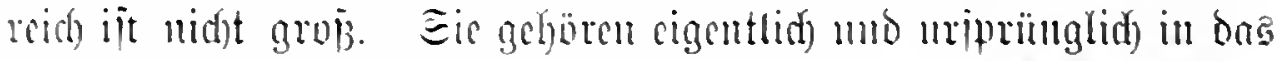
Satein ber Meatrifeln mo ber Dotturbiplome, Der Difjertationen

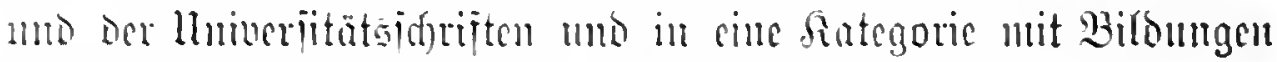

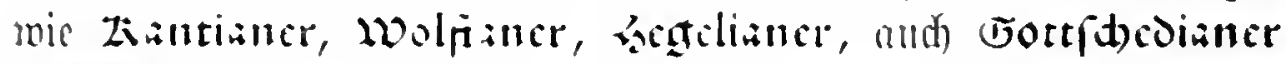

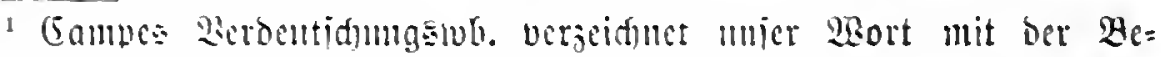

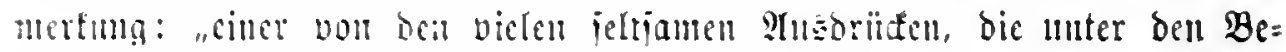

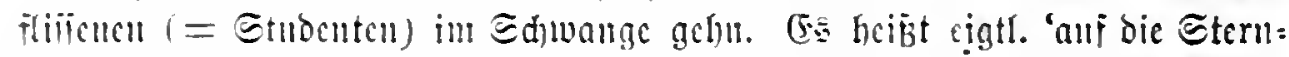
idfane ans

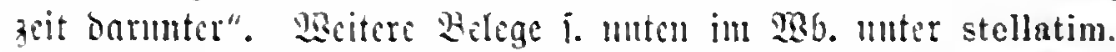

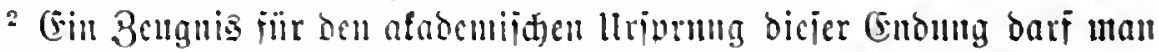

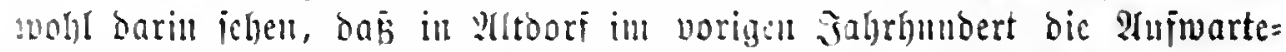

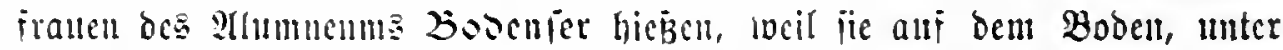

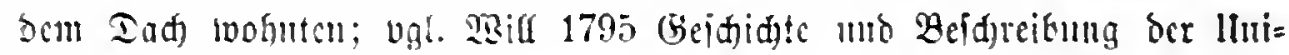
weriität $\mathfrak{9}$ ltbori ङ. 193. 
¡̈telfatum. Dorfatim. Jenenjer. Beimaraner. Bränz̧inner.

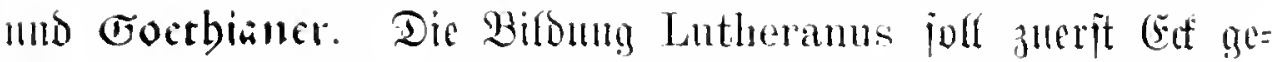

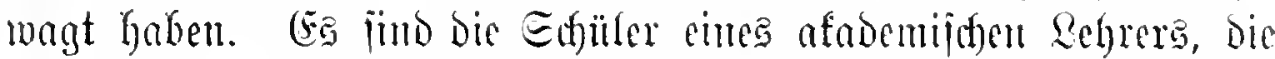

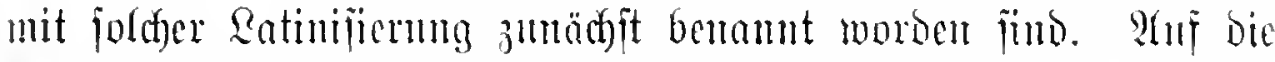
Lutberance folgten bie fläianter ober Illyrifuncr, bie 21 me $=$ Dorfinner. Der Tupus itammt Deutlidg ans Den theologifden

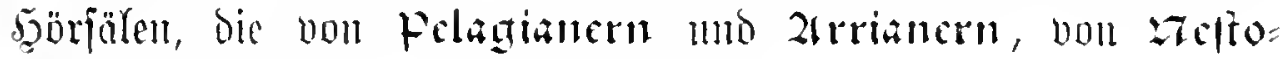
rinnern und Celiftinnern mieserhallten.

Ifber jefon jrith it bicie Bifoungsweije ans ifren nutilt=

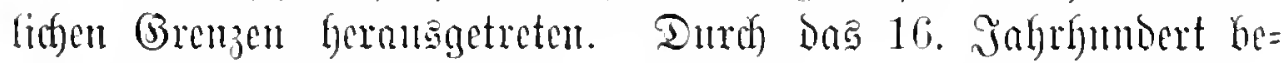
gegnen wir alf Eefritt unt Iritt dem Grobiantlo ober Grobiancr. Gifeidge Biflong zeigt rin peltiames ziariancr,

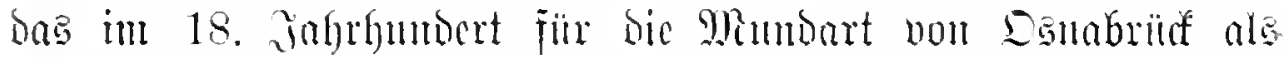

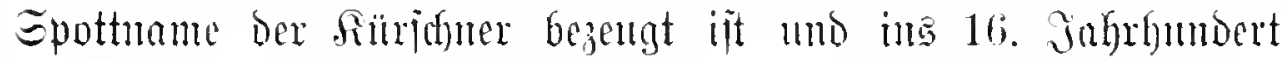

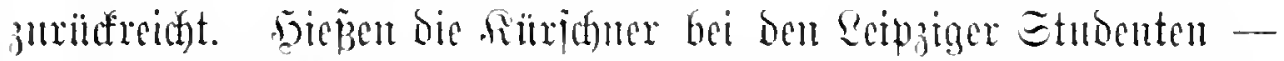
nach Sindeners fontipori 1558 - Ziagenfitinder, jo waren jic in

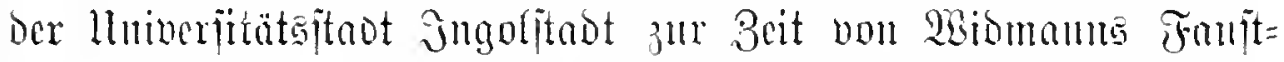

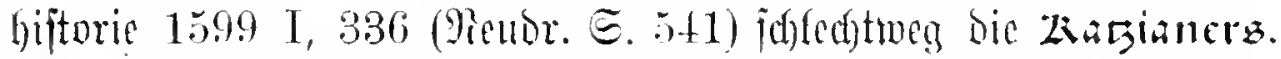

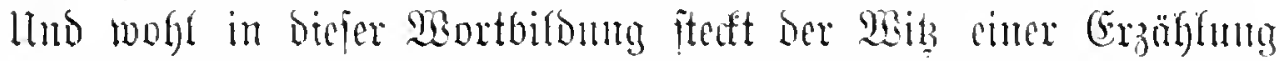

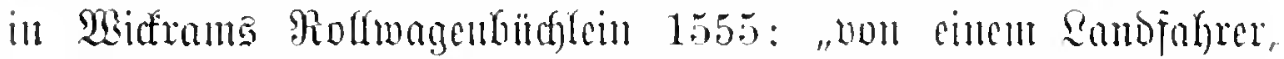

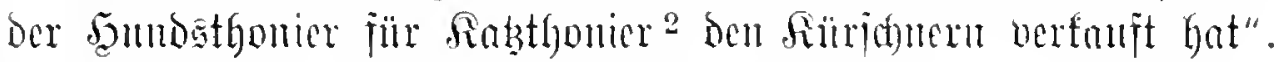
(5rimmelafganjen bezeichnet mit cipriance cillell, ber bas Bipper= lein hat. Stiefer 1691 buffit Pfäfianer jül Hfäfic. Donn

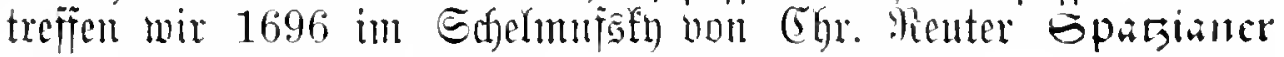

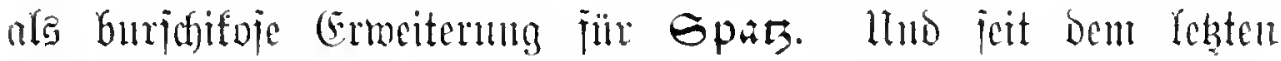

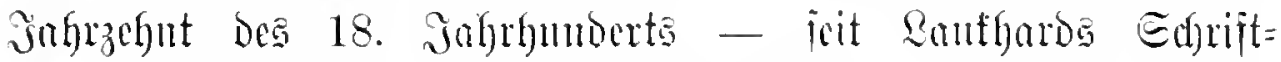

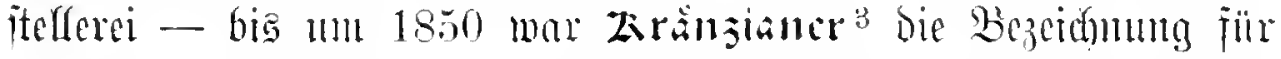

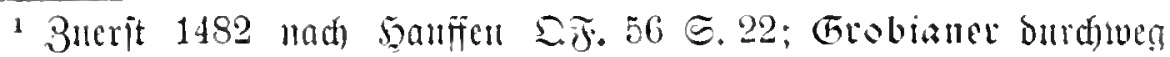

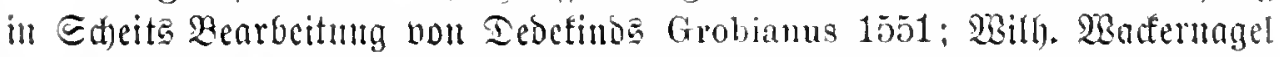

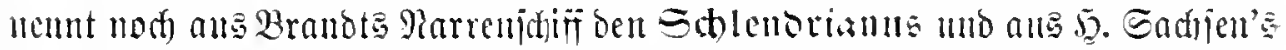
Fajtuad)täpielen ben Sanft Stolprianus, Der mit bem Eanft Brobiants

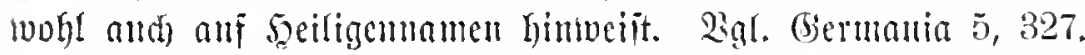

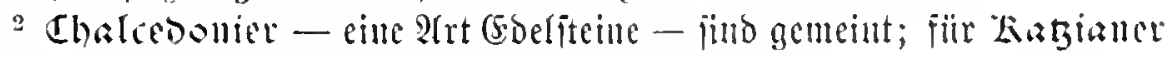
barf eime Mekenform Kargioner voranegejest werben.

${ }^{3}$ (Fine verwanbte Bildung zeint bas alte Sworift (neben Schor=

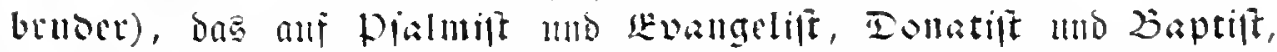

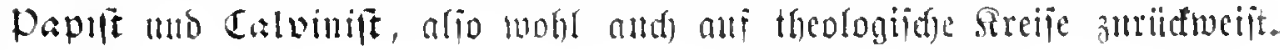




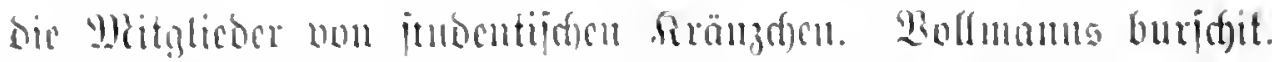

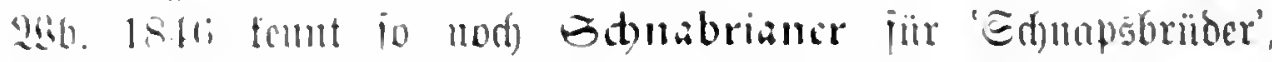

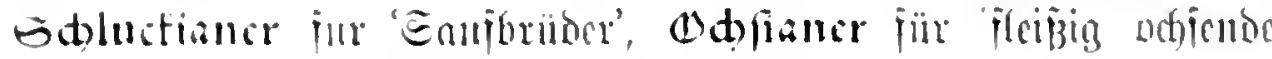

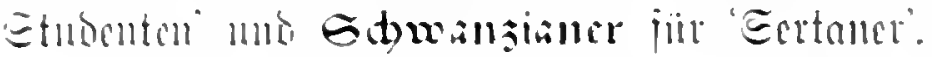

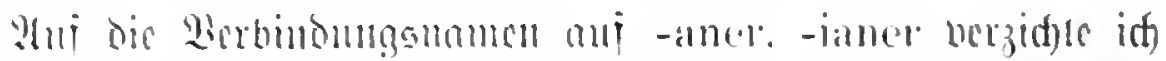

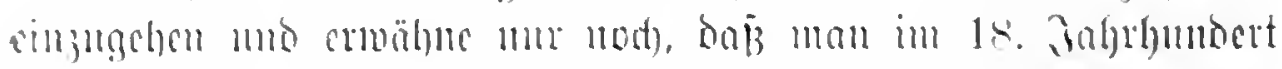

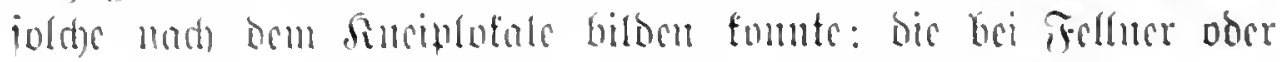

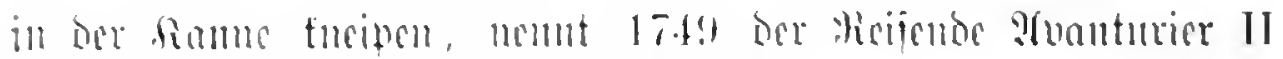
包. fin fillnerinther unt Zinnnerianter.

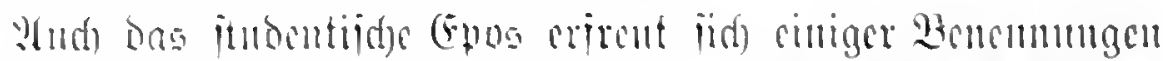

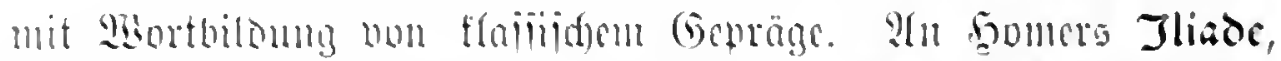

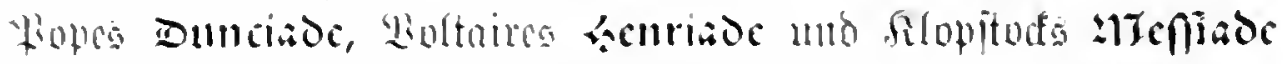

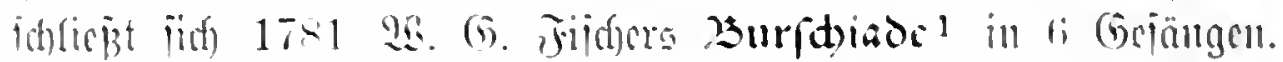

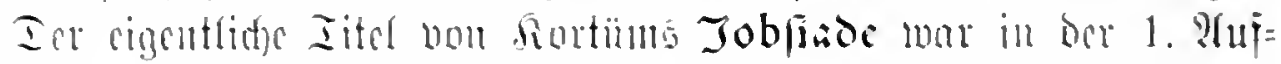

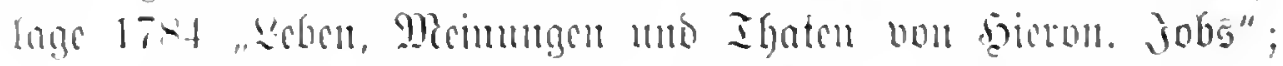

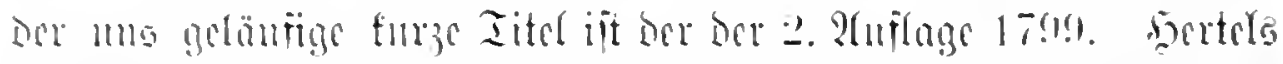

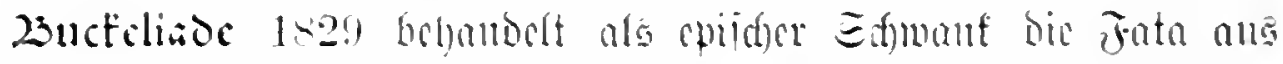

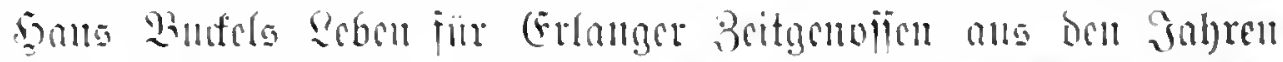

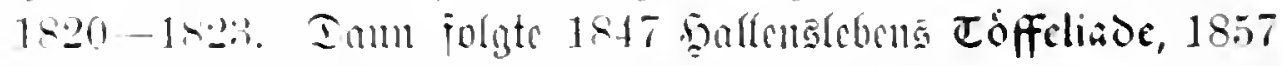

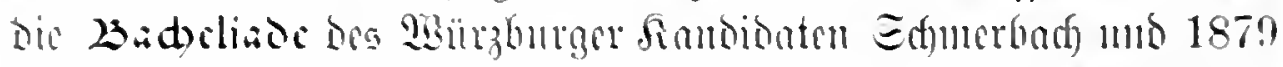
sime Piftoriabe. Ther bicie Bitbungeneije ijt nidgt auj Etubenten=

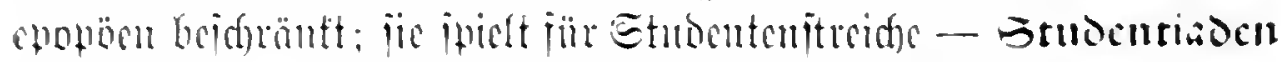

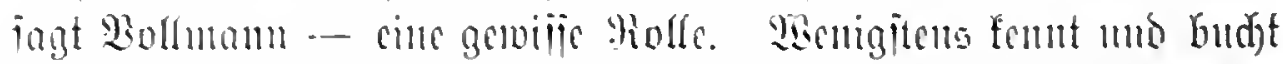

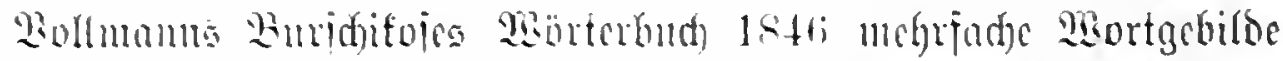
ali -iade: Zinotiade jür 'soljeri', Sandogmanniade jür 'Alleiperei

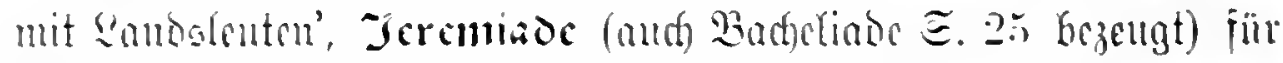

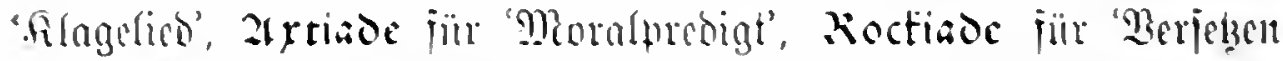

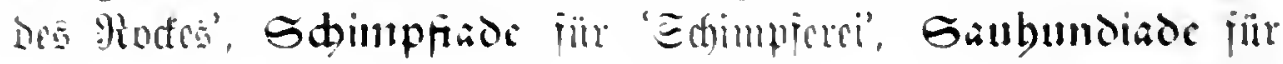

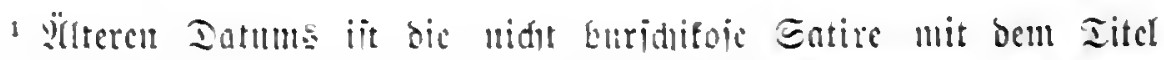

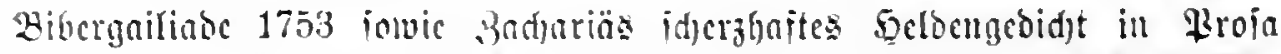

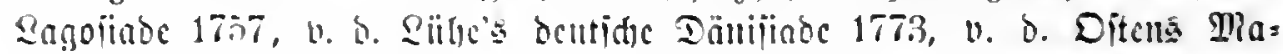

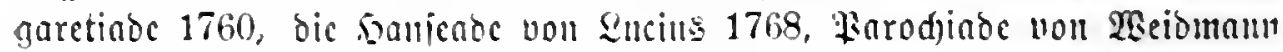
1781, Eclaboniabe 1779 - alle bci Bbocede IV. 


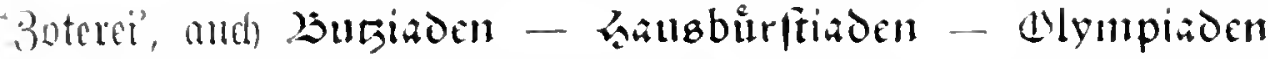

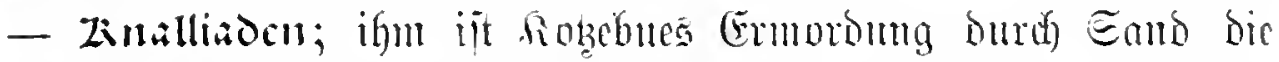

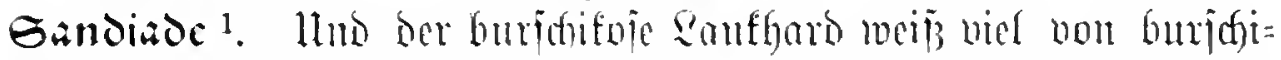

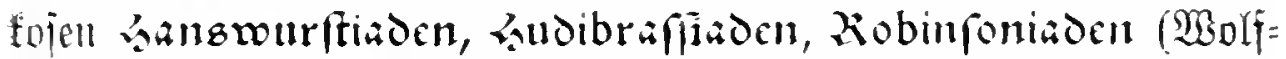
fteill II, 314: fint Magnuts 3i. 181). Non barlefiniaden

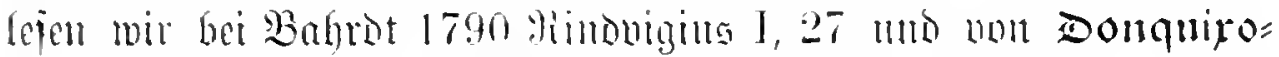

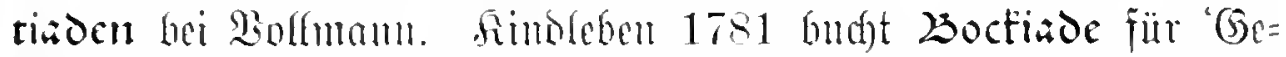

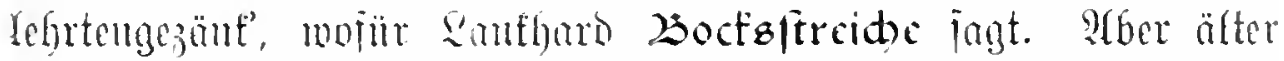

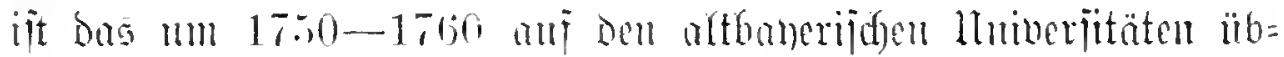

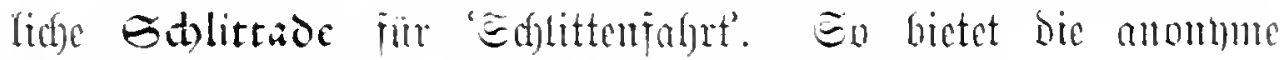

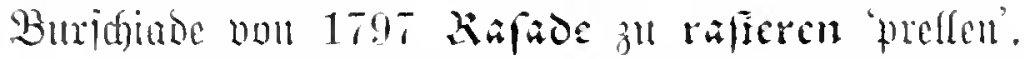

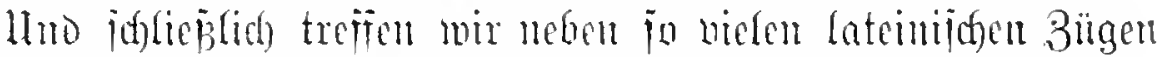

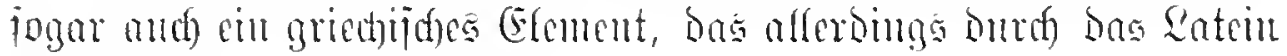
bimourdh gegangen ijt.

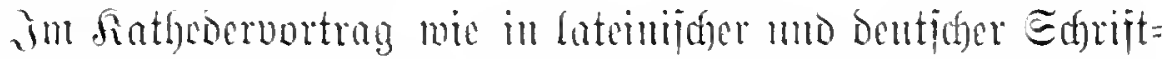

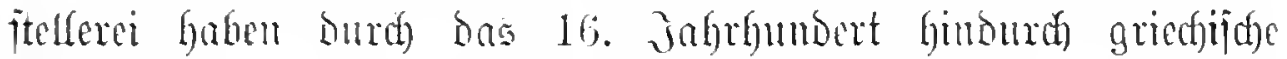

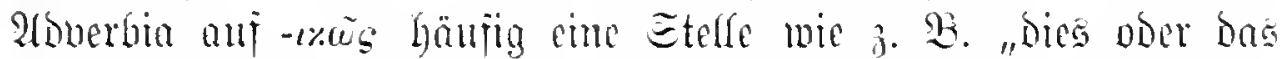

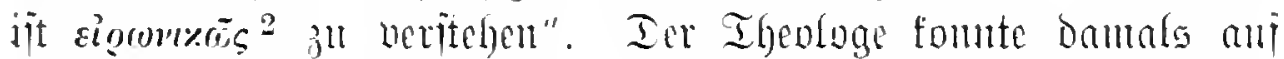

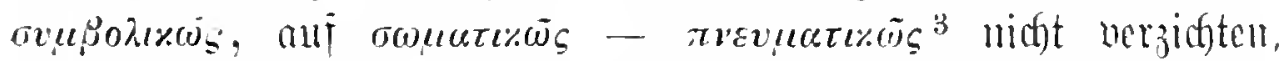
madjoem jie jidf ans Den Pranlinijgen Briejen non jerbjt ergaben.

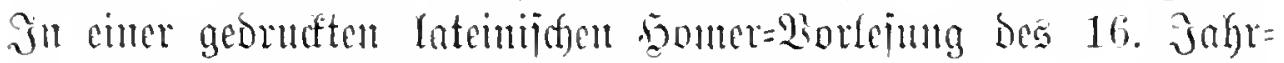

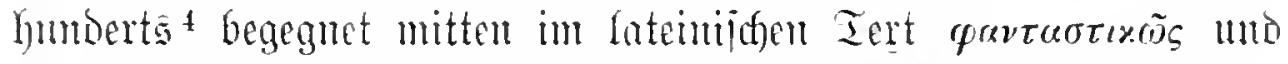

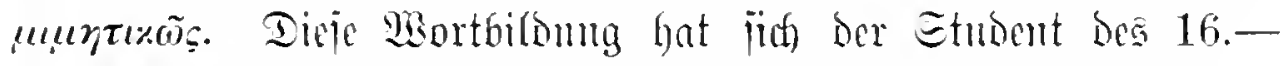

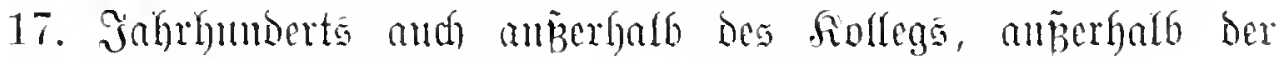

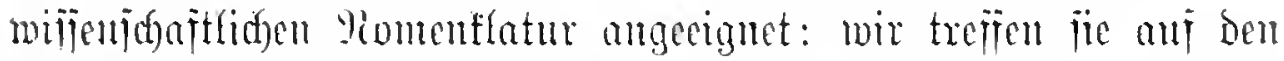

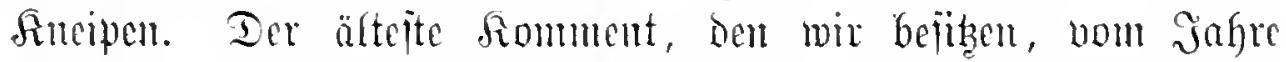

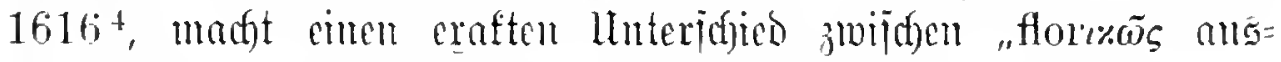
trinfen" uno "haustures anstrinten": "florixoss, wem man bic

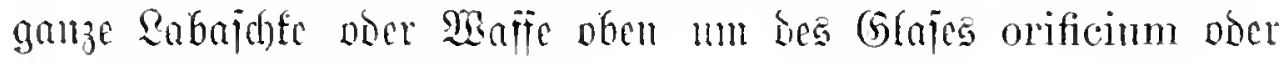

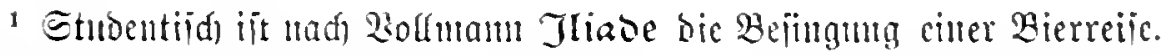

2 Matheium 1590 MhElegung $240 \mathrm{~b} 72^{\mathrm{b}}$. - Oratio Sebast. Theod. Winshemii $1553 \mathrm{D} \mathrm{IV}$.

3 Mart. Crusius, Orationum liber unus $48^{\mathrm{b}}$.

4 Nultibibus 1616 Jus Potandi $\$ 9-12$. 


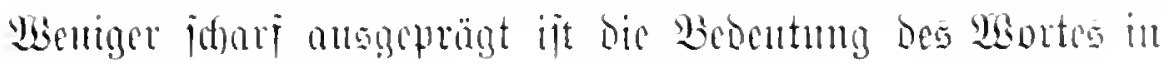

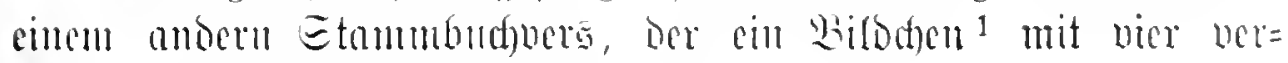

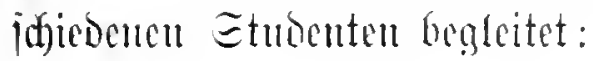

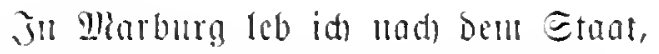

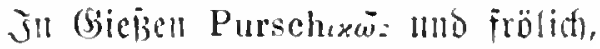

Snl sicrbon wie cill Sanbibat,

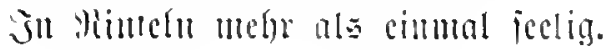

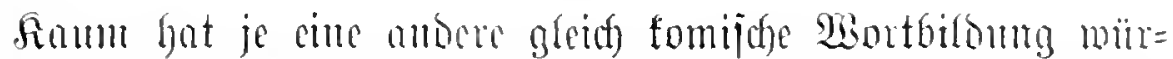

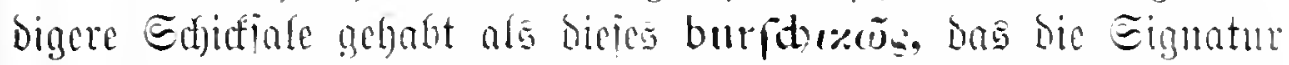
jencr Bcit war, Die wir an Baffartäs lienommiten fennen. Sien

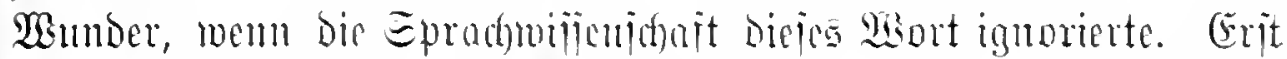

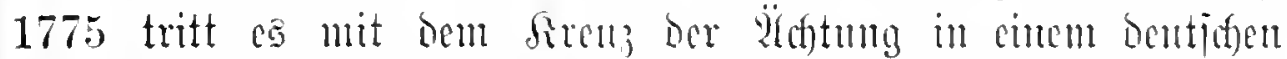

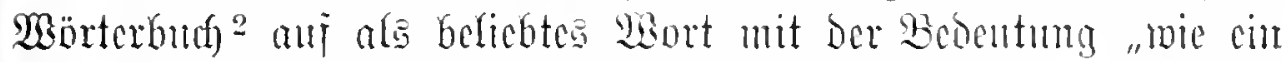

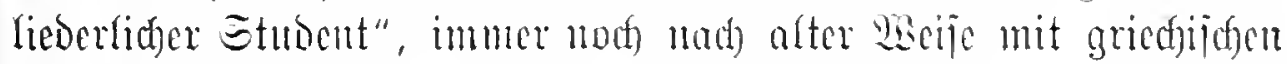
Settern in ber Endung groudut. Ju ber 2. Däljte bes 18. Jahr=

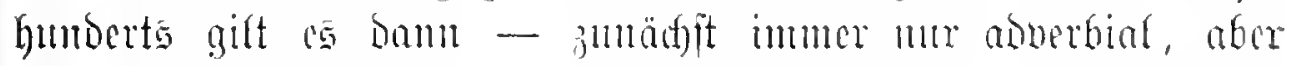

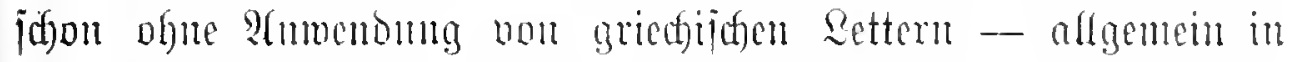
itnoentipger Ritteratur: "Ẽ lebt man buriffifos" 1764 Ier tugeno= uno lajterhajte Etudent C Ia;

Jo6s lebte inmer feill buridiftos, ieill brob erhaltener 9 itum war gros.

Jokitnde (1799) I 2.. 903 ,

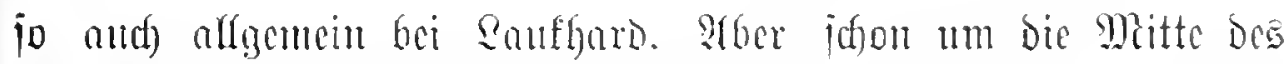
18. Jahrfunorts lejen wir bour "bursicosen 2ictuatien" in ben

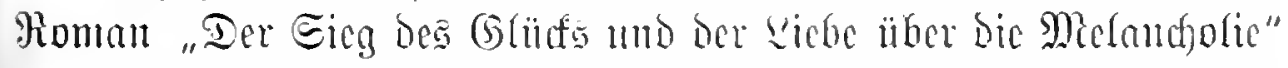
(1748 હ. 198) unb um 1780 gilt allgemein "cin Guridgifoles, cint

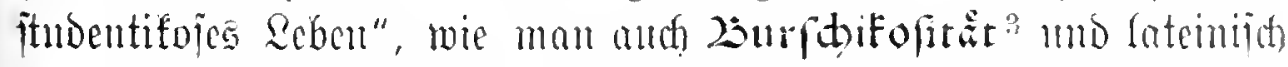
res burschicosae bifoetc. Ilno bam wirb as icfliejplich litteratur=

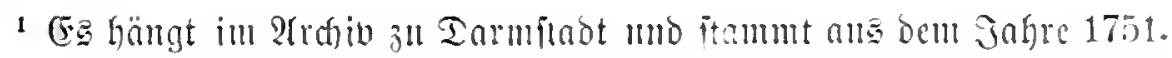

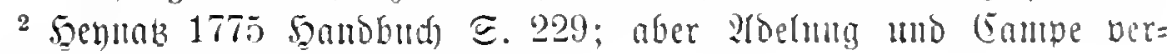
zeidutell 63 nod) nidit.

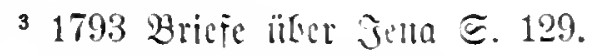




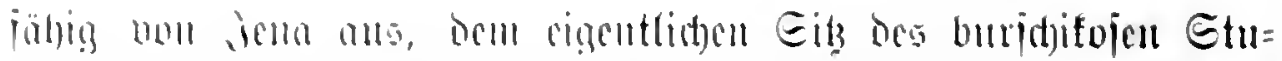

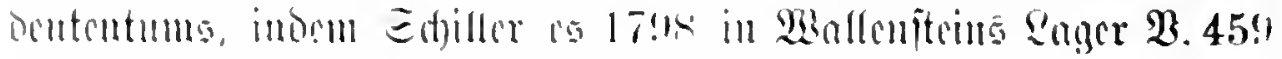

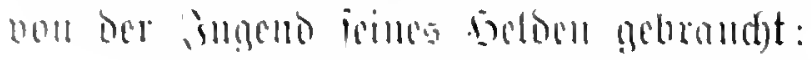

"3u vilroori in Embententragen

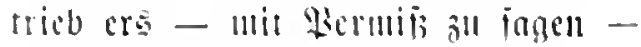
cin menig locfer mo buridjifos."

\section{Burridjitoje Soologie.}

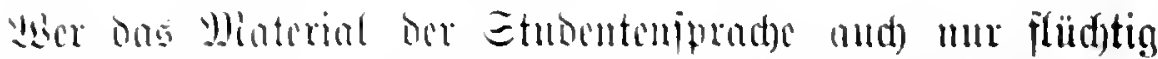

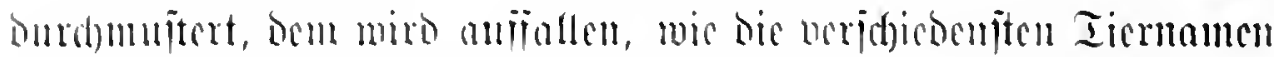

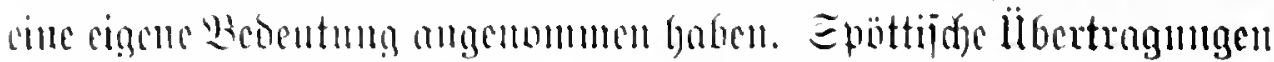

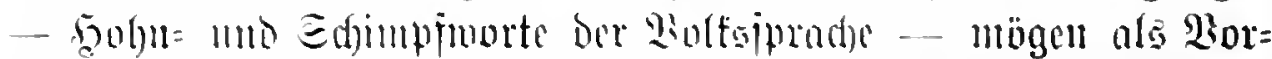

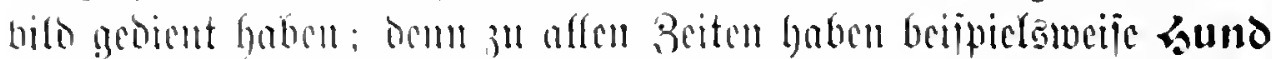

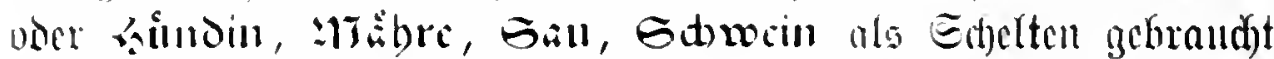

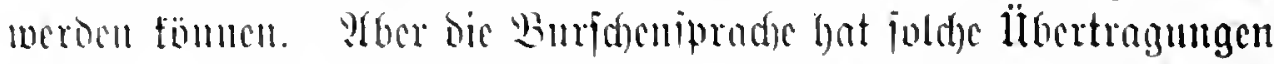

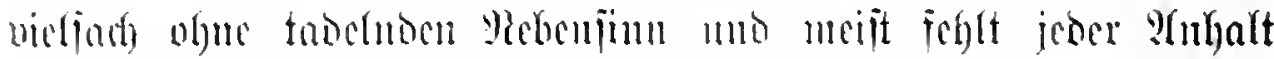

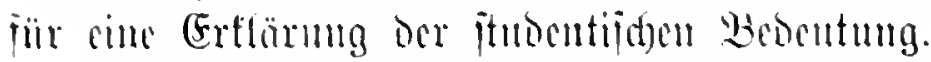

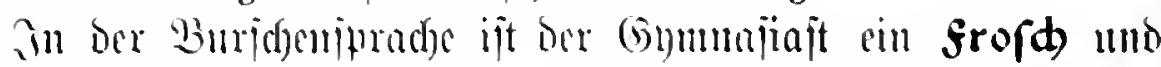

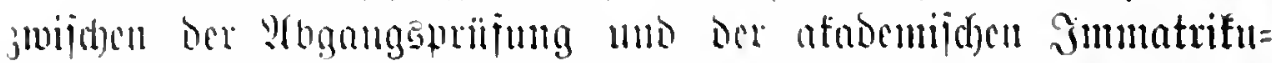

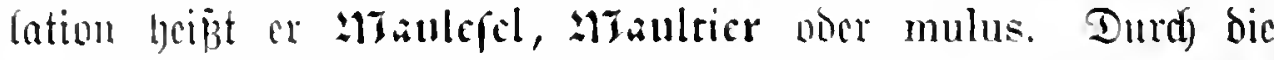

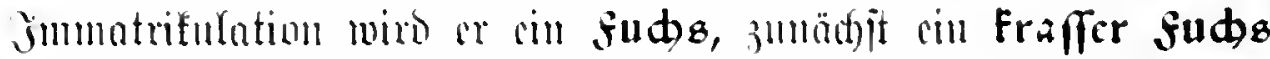

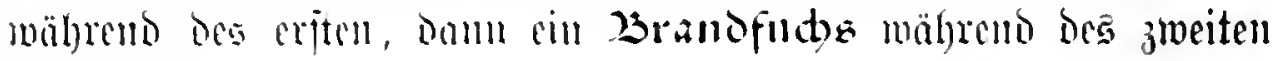
Ecmeiters.

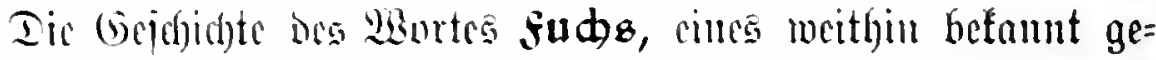

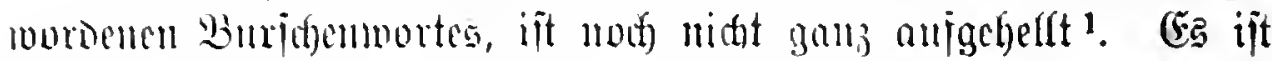
jajt 200 Jabre lang in beutigen Eime bezengt und in unfern

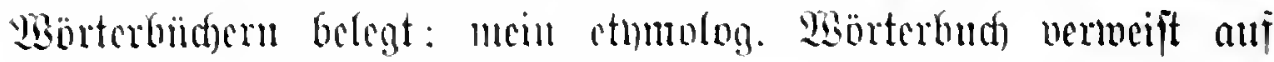
Etople 1728 Gedidite 1, 133

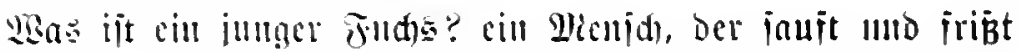
uns von ber Sateritadt brei Soffr berwiejen iit.

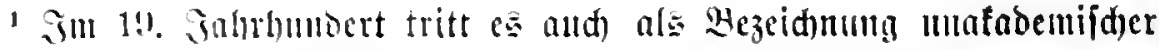

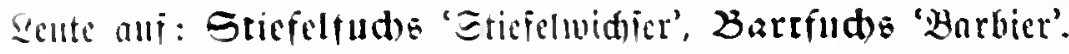


(5e war cigentfich $\sigma$ d)ulfud) als 'juvenis qui ex schola in academiam defertur', ijters ijt

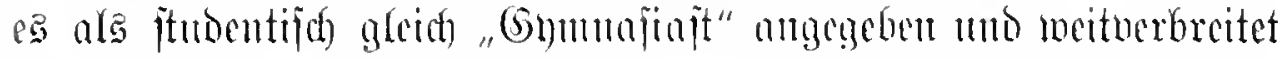

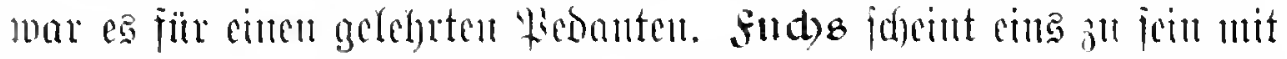

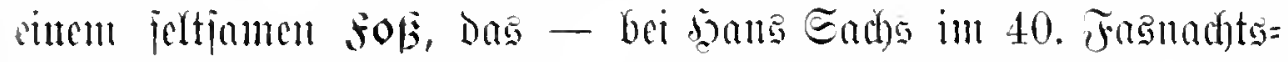
ipiel Der Partefenfar 23. 348. 366. 369. 382 belent - nou

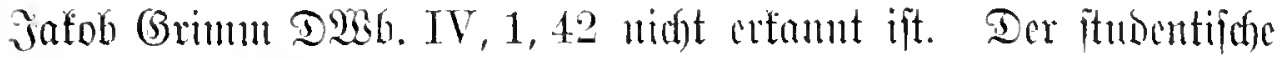

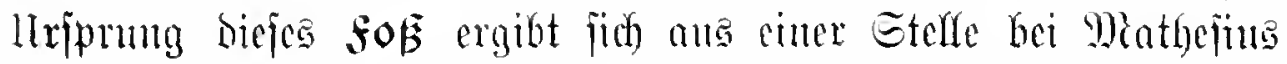

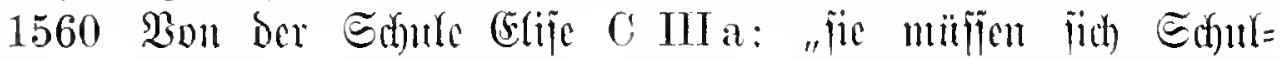

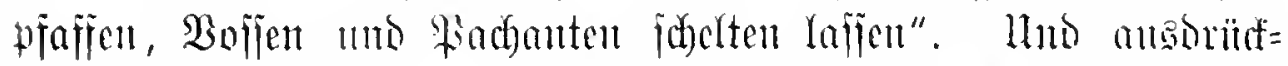

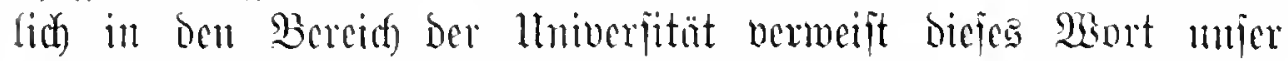
äteftes Fremomörterbud, Eimnn Rothe Dictionarium 1571.'

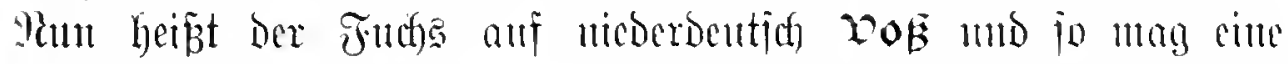
nieberdentiche Mniverjität - ctwo kojtorf, viclfeicht and $23 i t t e n=$

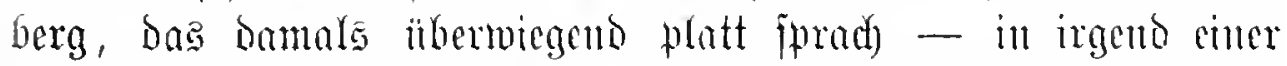

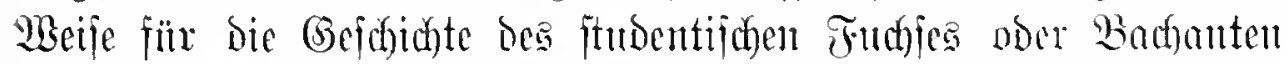

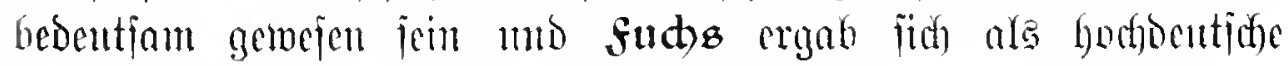

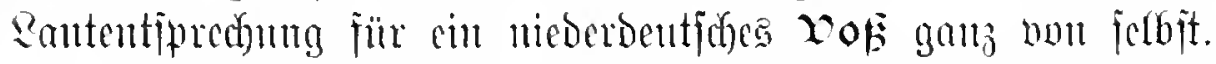

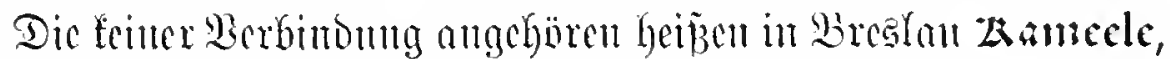
in Jena finten, in Bern Lären. Sint 18. Jahrh. ift ber Stubengenoife Grubenfaball, im 19. Bubenfancel. Pomaden:

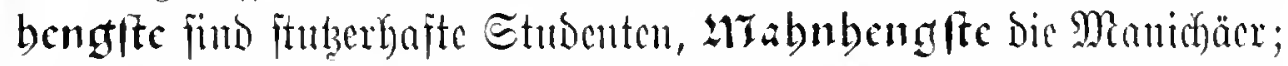

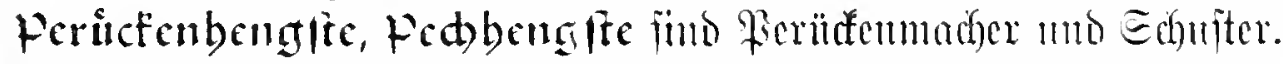
Jim 16. Jahreumbert heipen bie 3eder 23icramfeln voer Bicr=

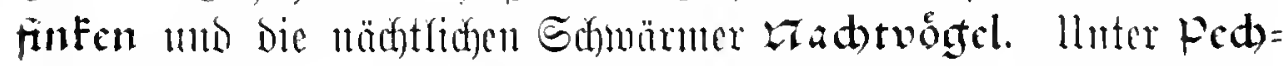
vogel werftegt bic neuere Burideniptude ben, ber immer Pred hat.

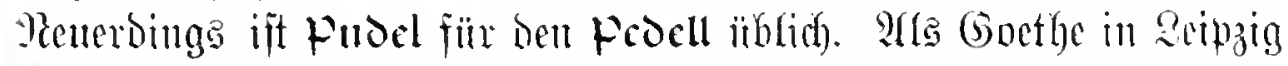
itnbierte, hatten bie Etabtjolbaten bort ben Epottnumen meeron -

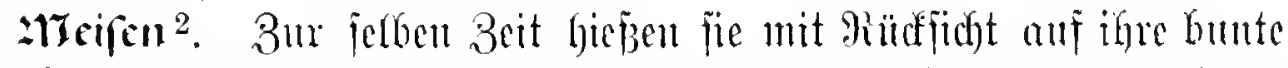

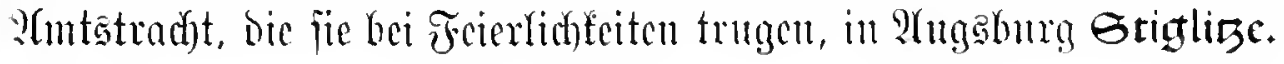

1 Moth jagt: „Phos eill Epottwort Der groben llıgelehrtel, bamit jie meinen bie Beldhrten und ponit Entoiden zu verleben."

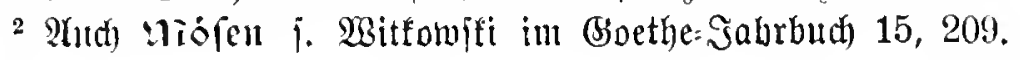


IItber bie Etubentenipradje.

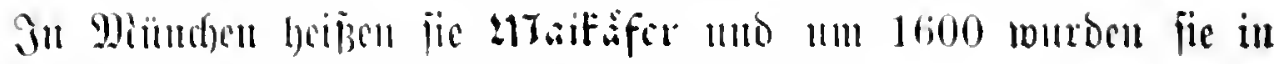
Dherbentichlanto allegemein Biupen gerujen.

Iic (s)lünbiger ljeiben Eritt= ober Eresroggel. Hno and) jür

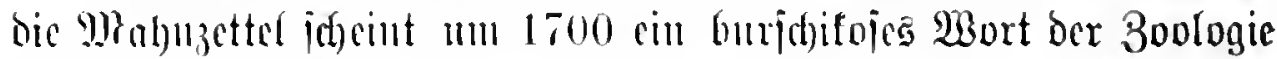
gegolteu 3" haben: bun (Enlenbern in :U(manad) S. 146 hören

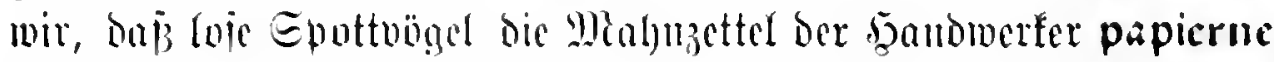

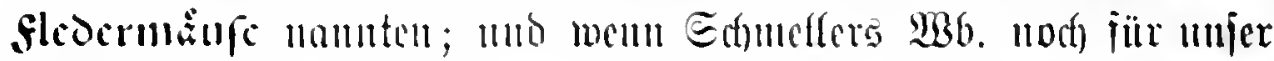

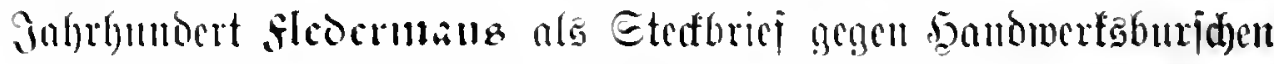

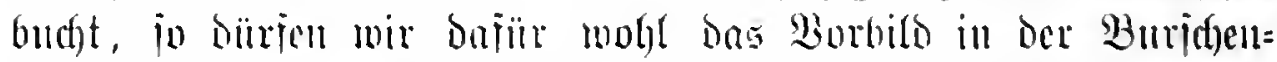

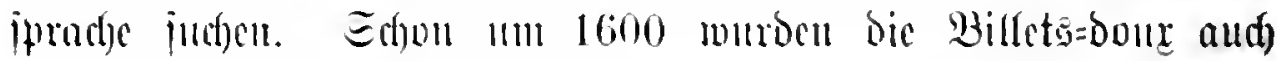
matse fleocrmanto gentumit.

Injere Irmufenlitanci $\Subset$. 2.2 bictet weiteres Minterial jür

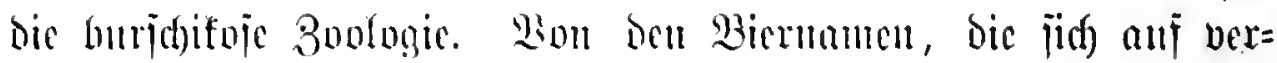

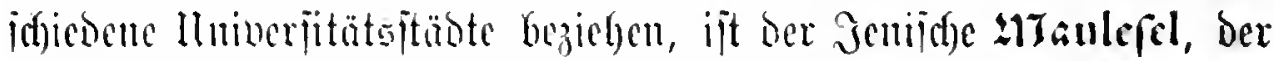

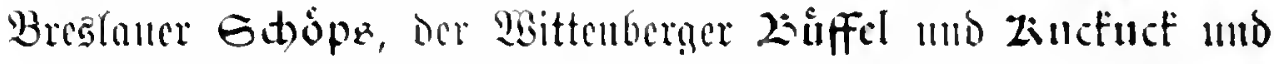
Der N) sulno, Zinter, Grair, Geis unt Enti, Gluctelbabn, jomie

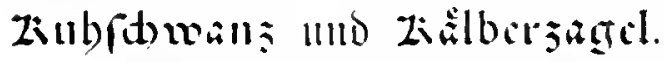

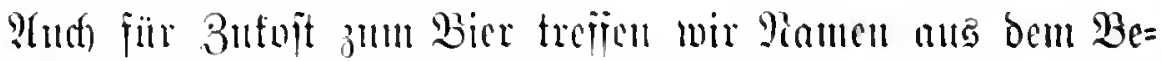

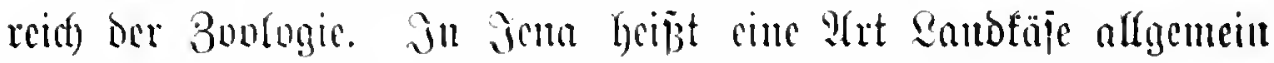
Erutbubn. Ilno int 16. Jofgrbundert namuten lujtige 3edjer ein

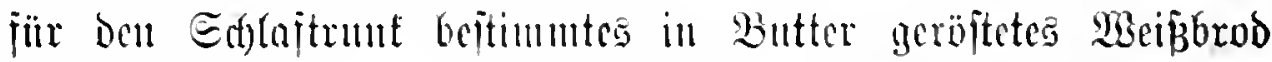
Zirammernoggel - aber auf Eatein scala vini nad) doieron.

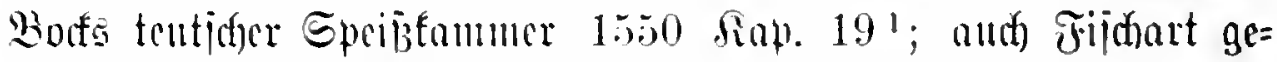

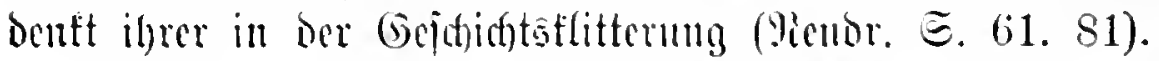

Ins Jus Potandi, Der moderne fiomment bat it cinem

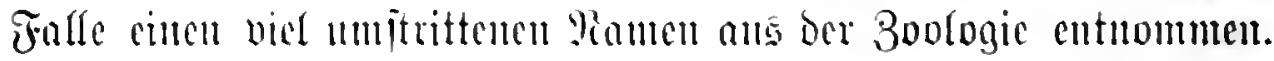

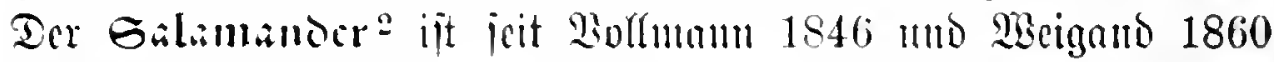

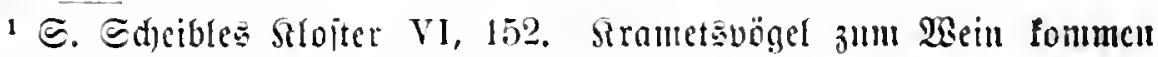

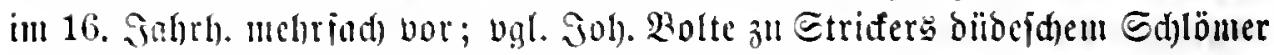

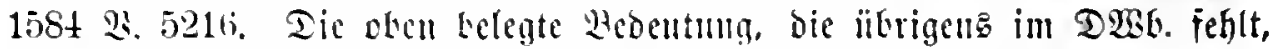
diirfte fïr mandie der betreffenden Eteflen Das Midjtige trefien.

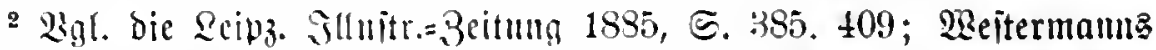

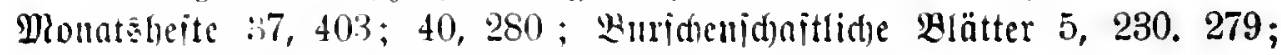

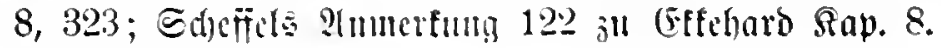




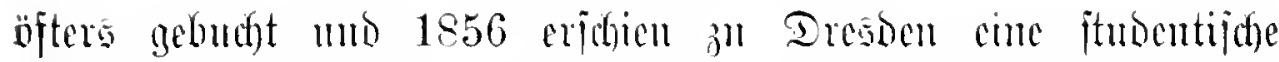

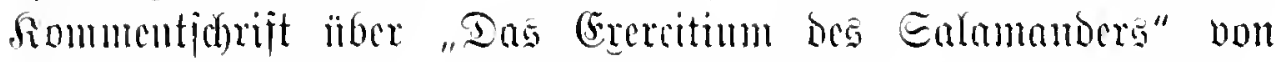

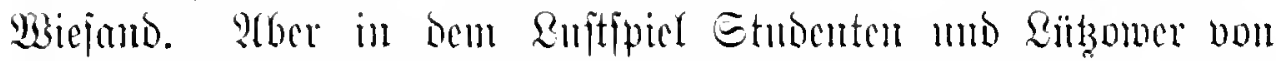

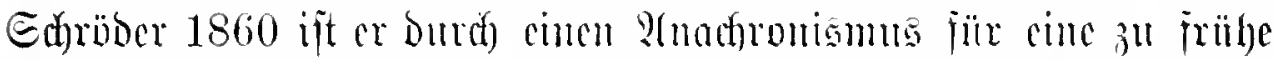
3eit angejebt. IIm 1830-18t0 war \$art mo Eacje in Bom

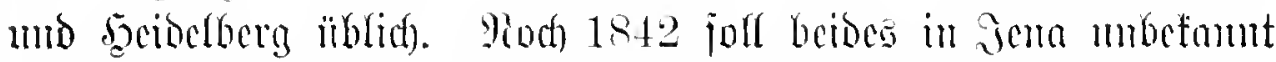
gemejon jein unb wir bürfon cilue guten Trabition wul) glaubeu, baßj in Jena zun crften Male im Eommer 1843 anf bem Burg=

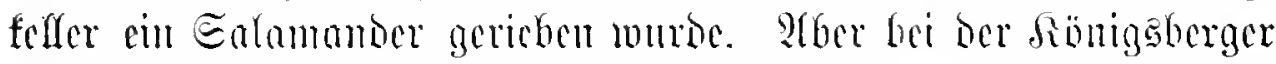
Jubeffecer 1844 fammte man ben Ritus noch nidft (i. Burjeten=

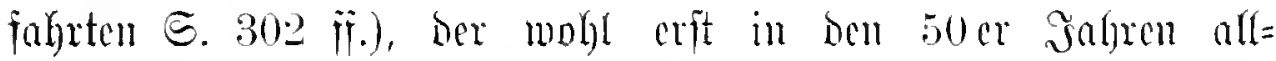

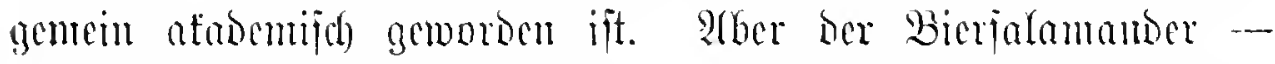

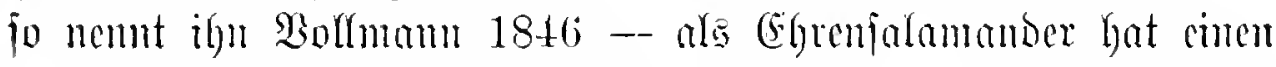

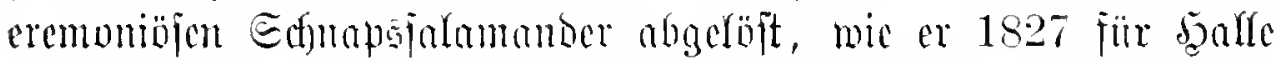

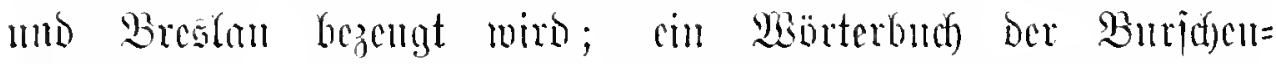

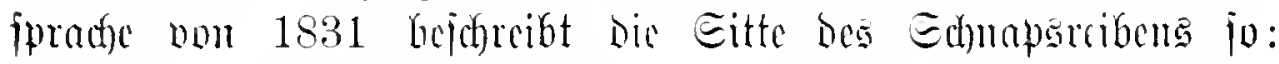

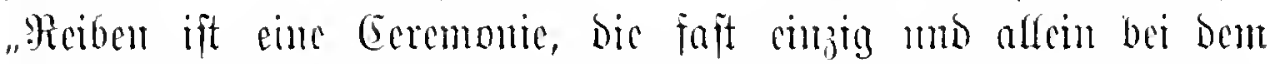
Echnapstrinten Eitte ift. Jal ber Regel tommandiert jemand ans

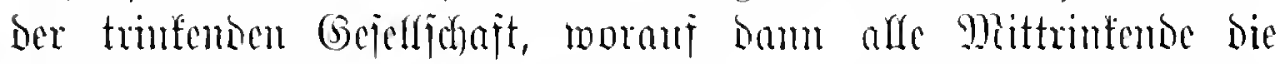

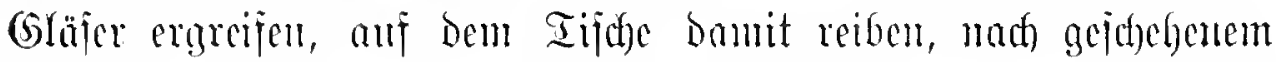

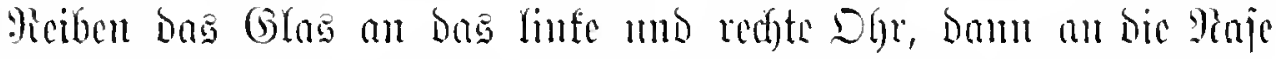

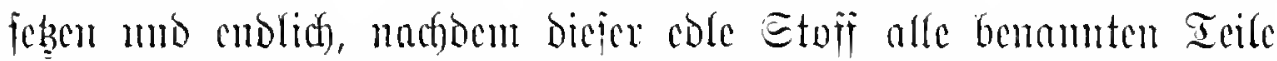
wenigitens mit jeinem (Scruche crirent hat, fom ber Irinfento

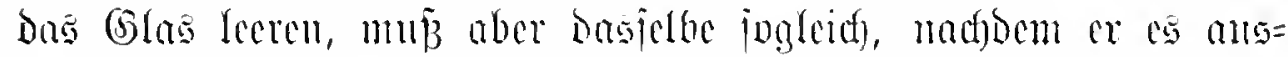

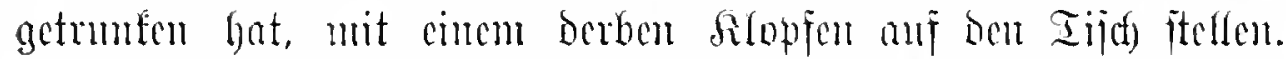

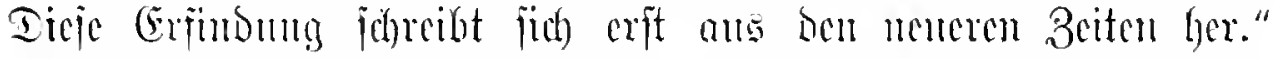

Dicfer Beridet, in bem mir allerbings dos Mort Bula:

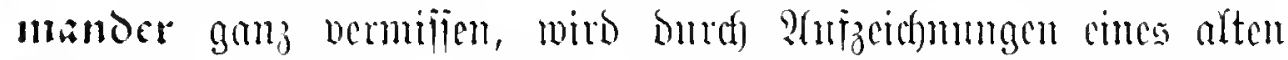

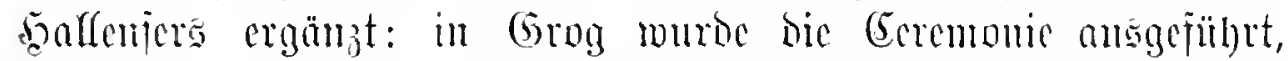
während des Reibens murmelten bir Enlamandriften Drcimal

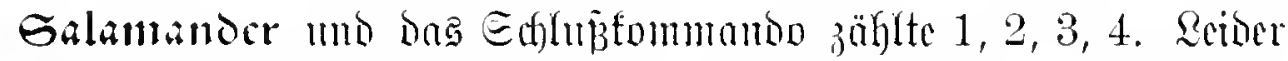

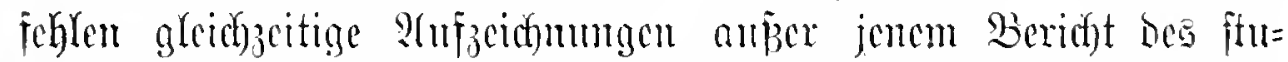

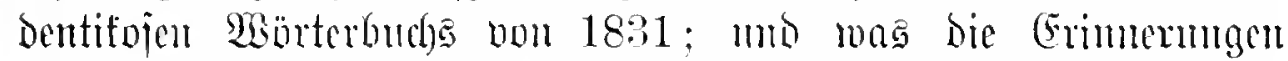

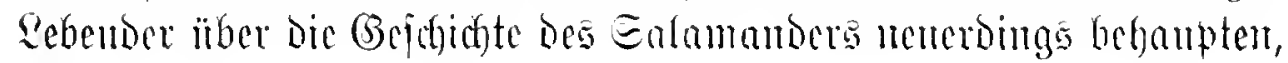




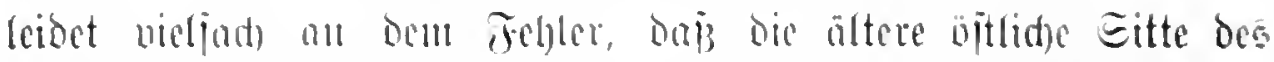

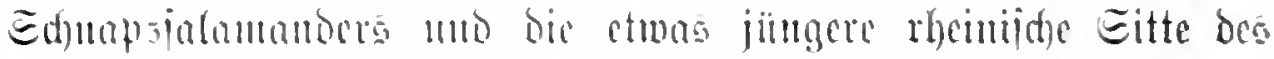

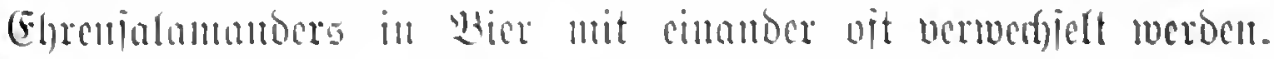

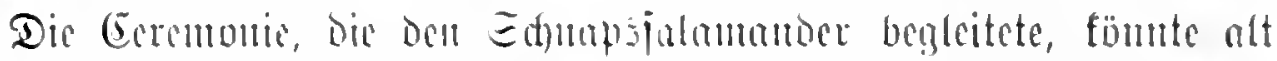

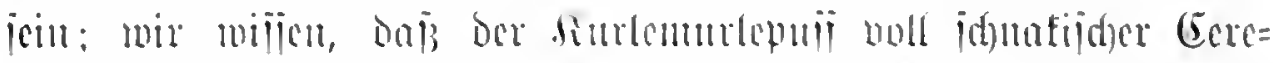

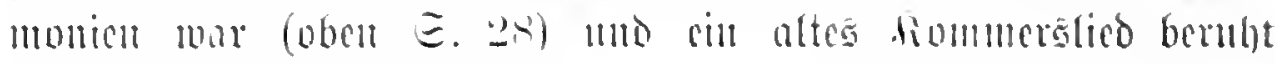
ani jonter Eorentunic:

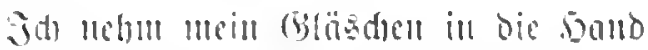 \\ llno incir bantit ing Iluterlano. \\ id) hot bor (bitasdent wieber berwor

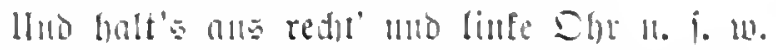

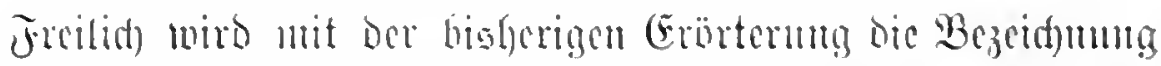

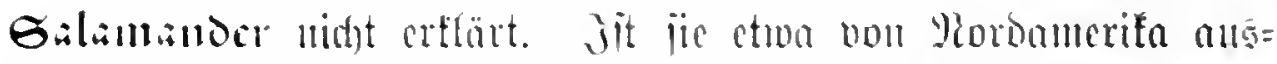
gegantgen, wo man in bur 22. bälite bes 18. Jahrefunderts einen

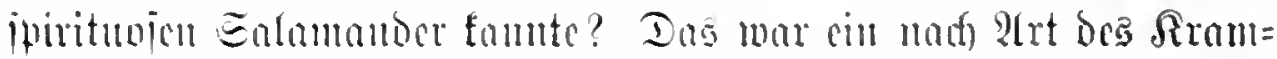
Gambuti angezintueter seiqueur, ber brement getrunten wurbe.

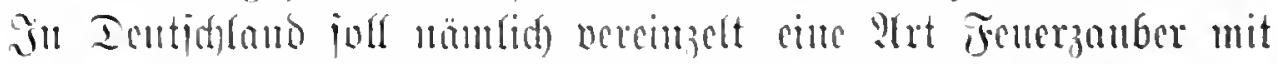

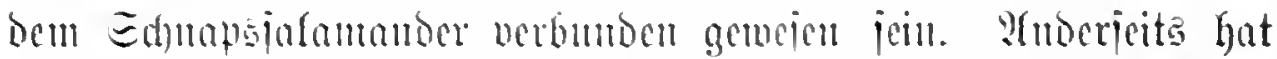

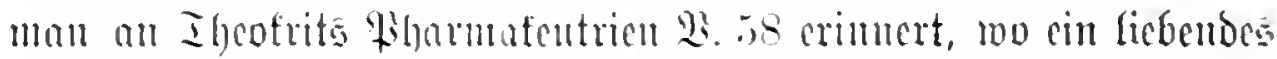

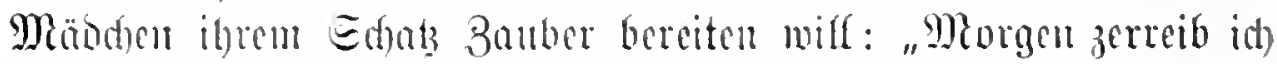

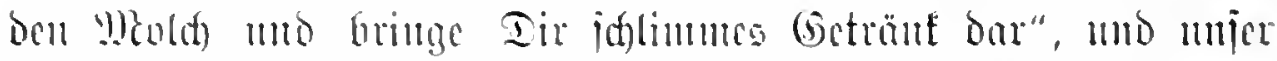

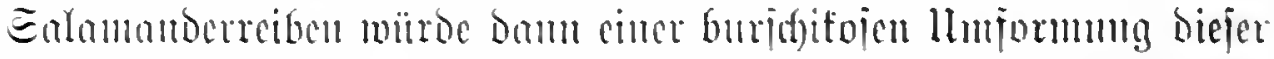
Etelle Den Pannen banten. Eo möglid) manche in ber reiffen

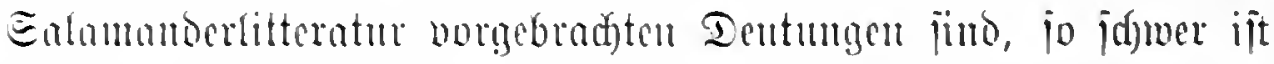

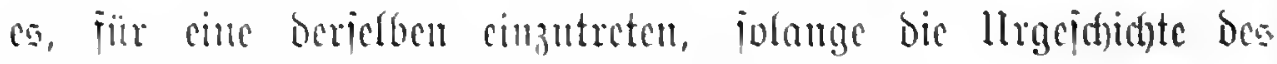

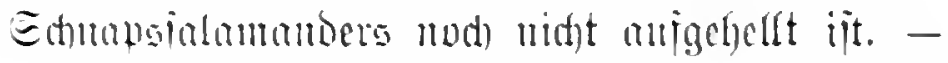

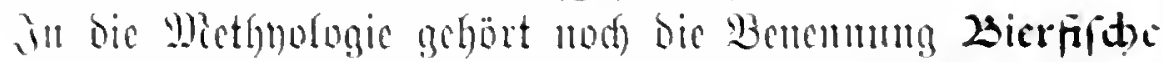

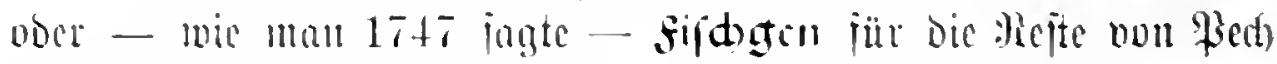

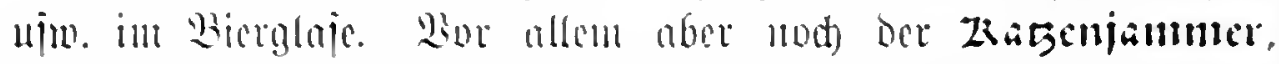

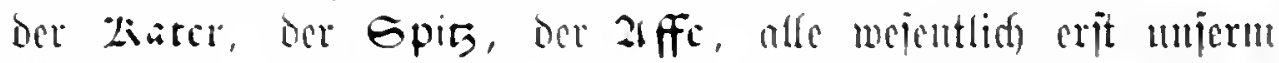

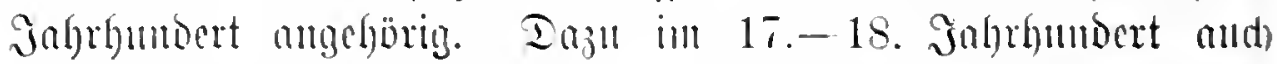

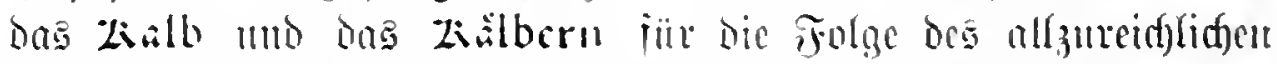
Biergemrifes, Die mun im 16.-17. Jahthumbert mad) Dem hlg.

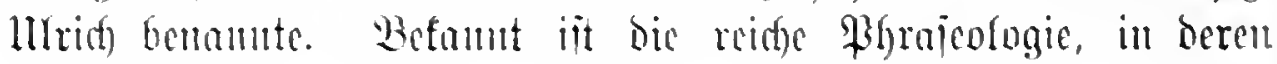




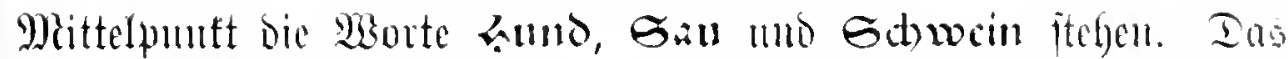

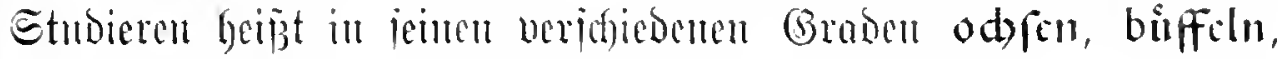

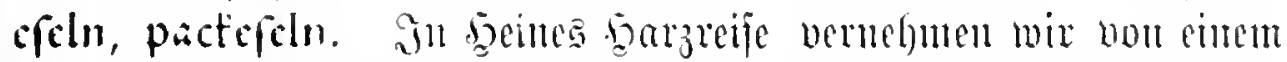

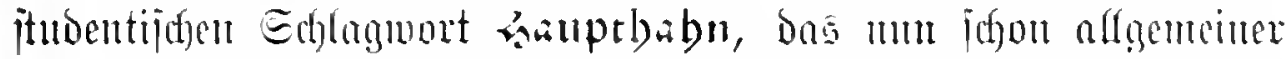

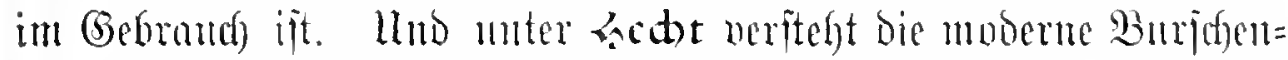
ipradye cinten langmeiligen Eonderling, bantenen and bie iffmere Rueipenulut nolf bun beizendem Iabafrandf).

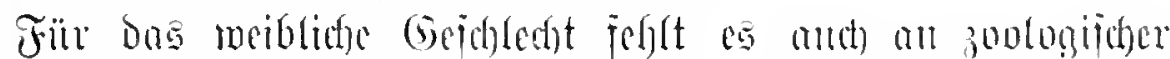

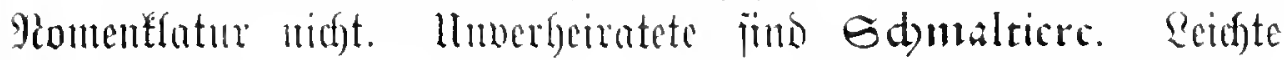

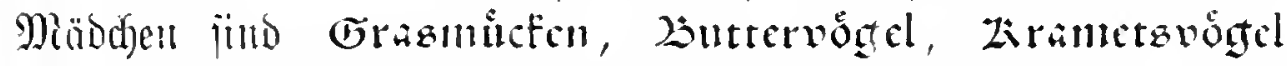

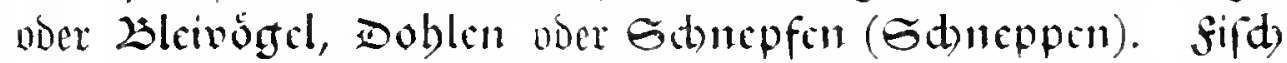

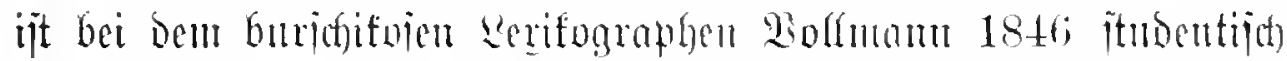

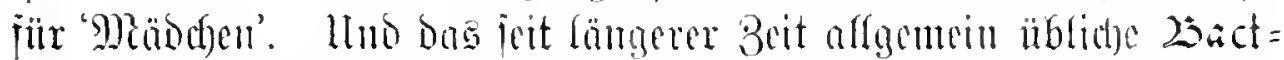

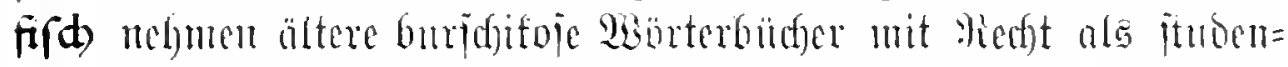

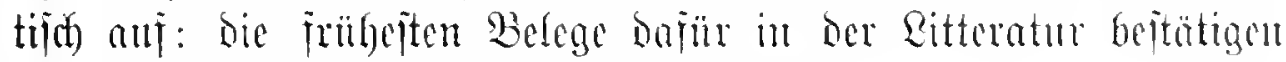
burjujifojen urjprung mit noller Eidfergeit.

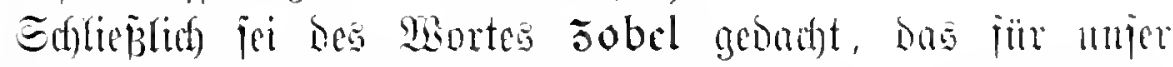

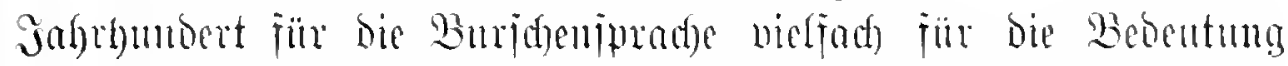

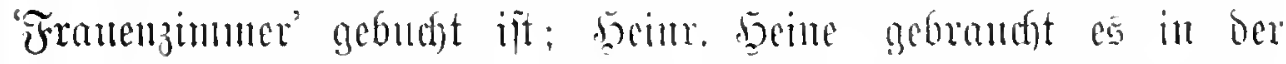

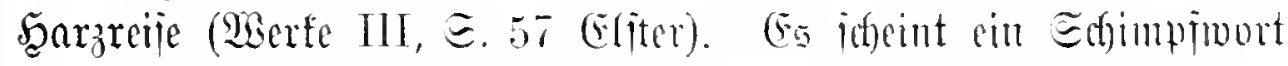

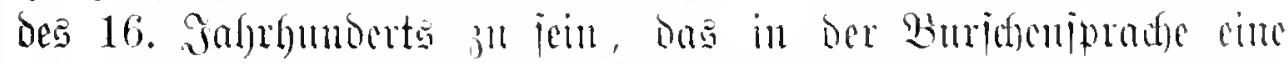

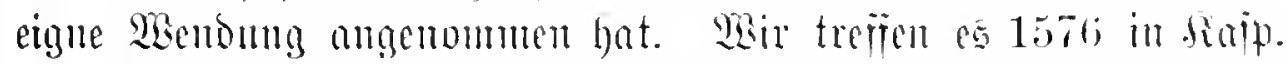

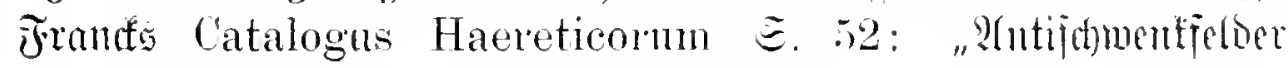
jeind joldfe Joblen, weldfe all ifre Ead) anj bas Estrein ligen"

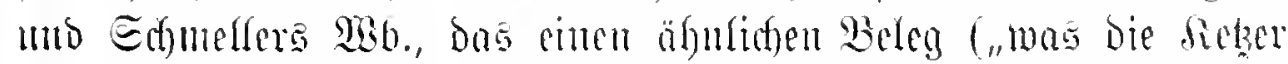
iür jeine Zobehn ien") ans bem Jahre 1581 beibringt, belegt

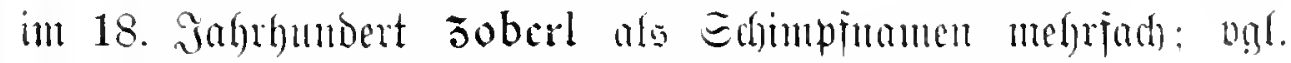

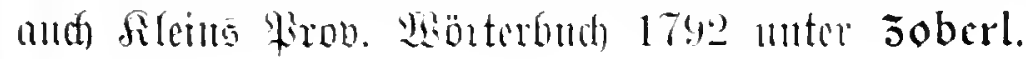

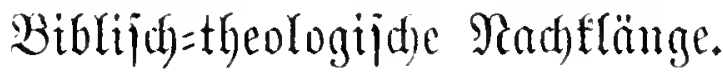

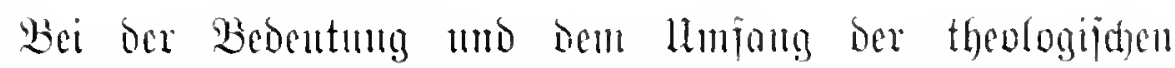
Studien in 16.-17. Jahrlumbert ueritehen mir es, menu ftuben= 
ïber bie Stubcutenipradie.

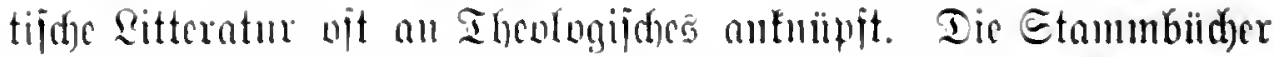

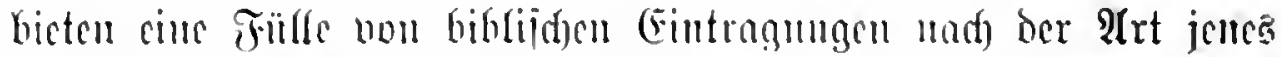

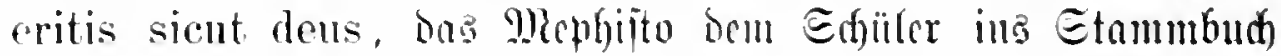

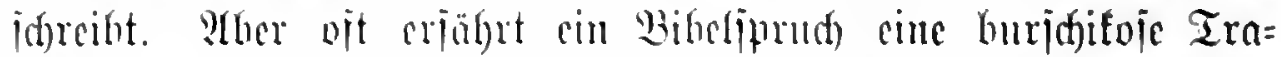

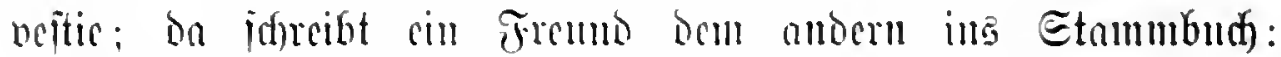

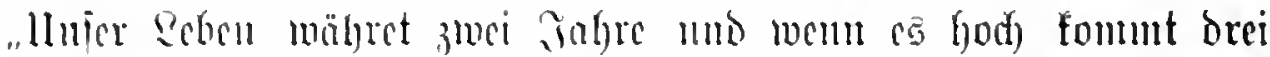

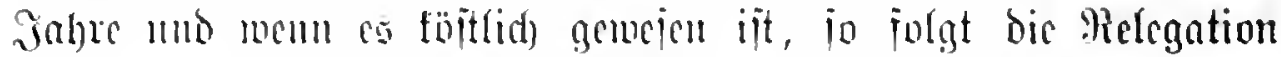

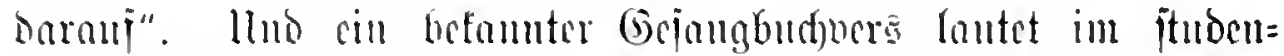

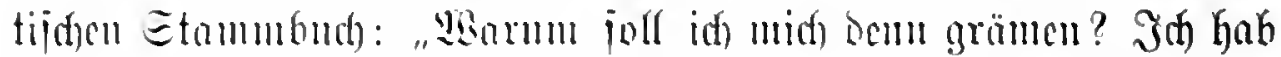

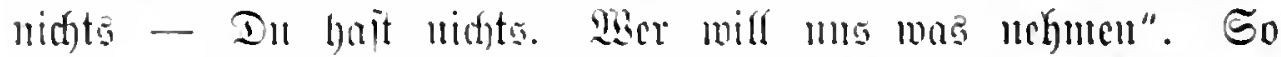

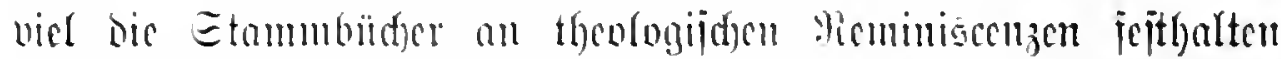

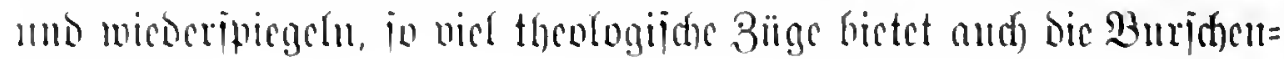
imracts.

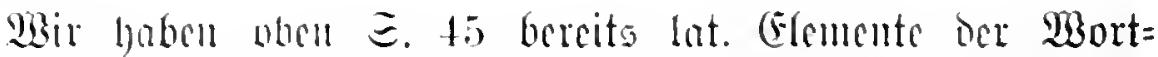

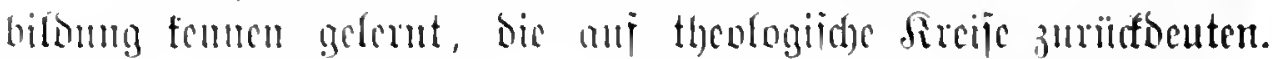

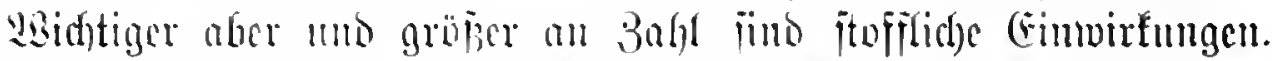

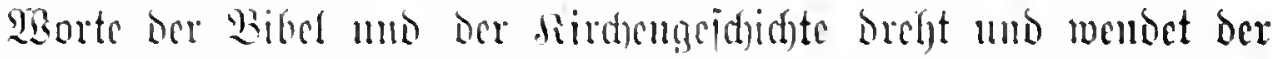

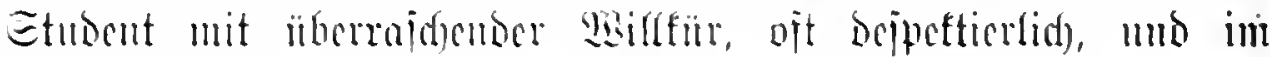

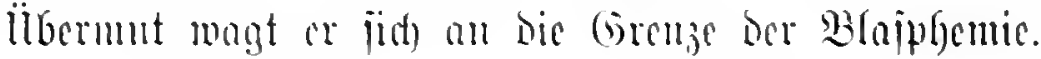

Jun 17. Jabrbundert war bo: Fudfa cin Quasimodogenitus

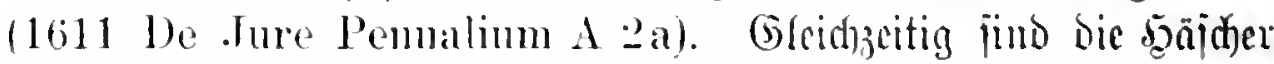

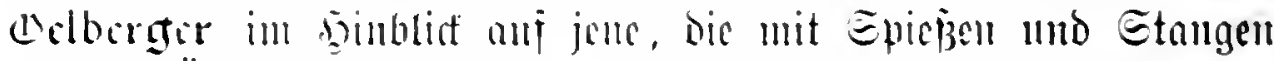

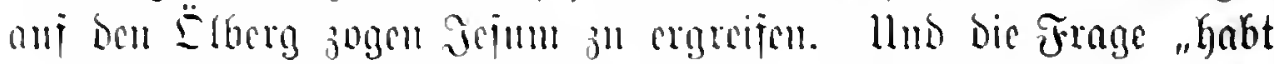
ifre nidft :15ofon und die fropberon" (Eufas 16,30) hat ber

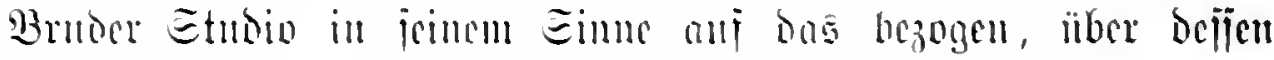

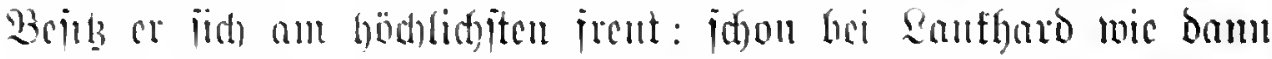

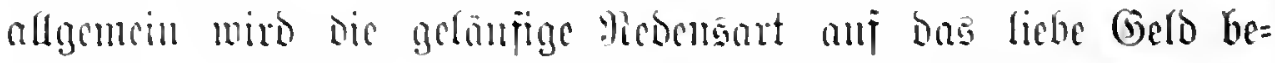
jogerl. Iir Eafte ber \$1Tunid)

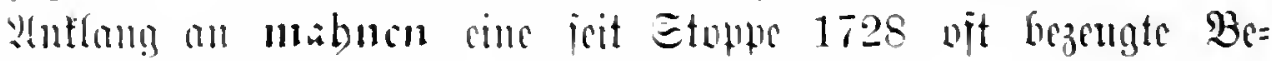

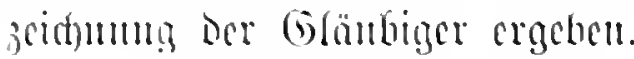

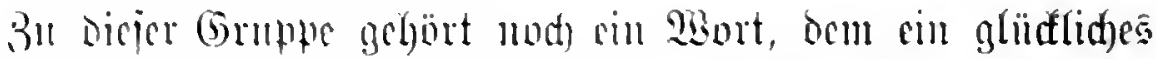

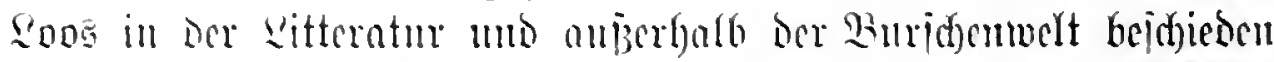

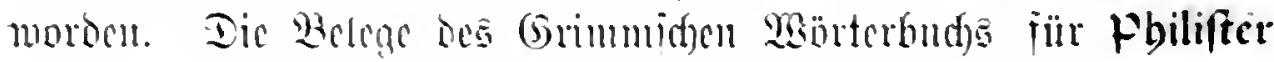




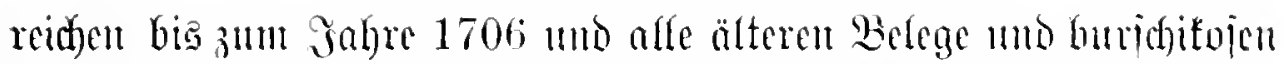

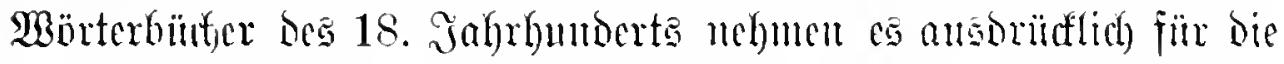

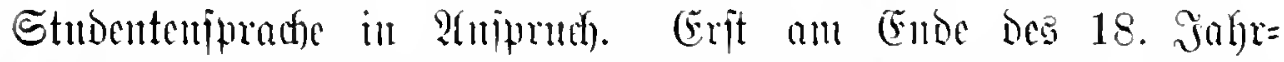

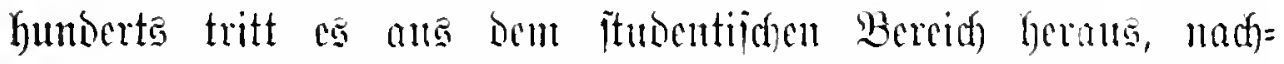

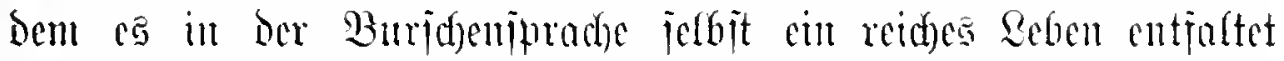

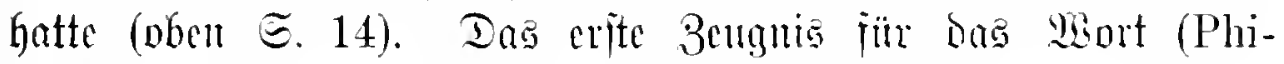
listrus quidam, vos aliique Philistri) finde idf idfoul 1697

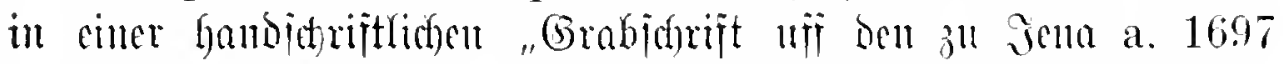

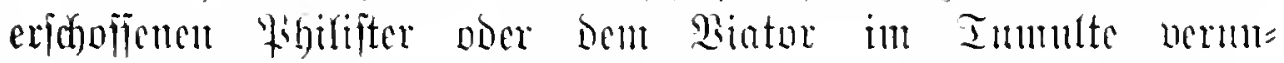

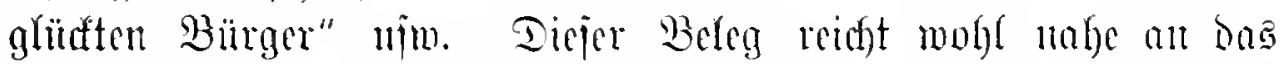

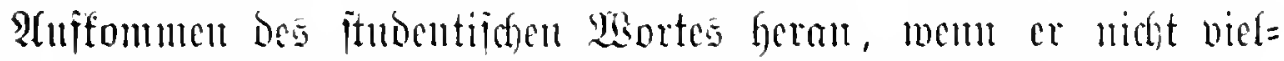

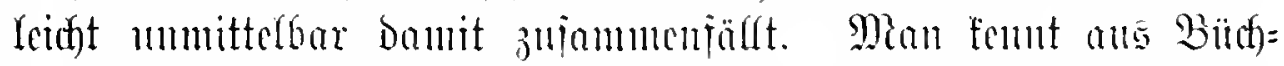

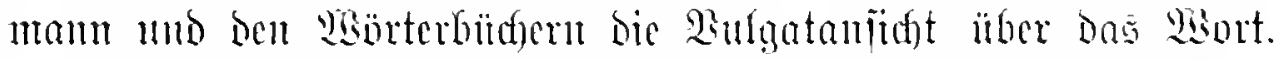

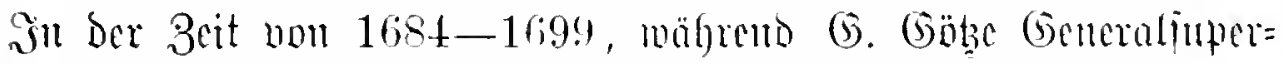

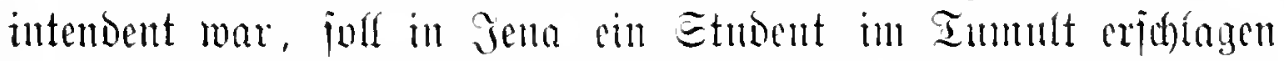

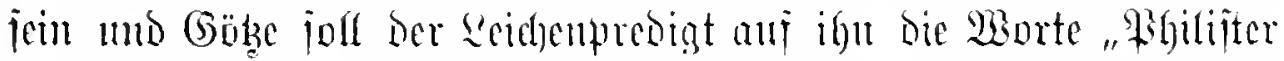

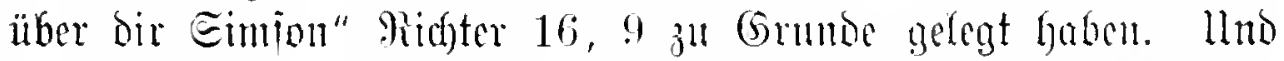
bieje Fresigt mird allgemein als bie Suelle bes itudentijgen

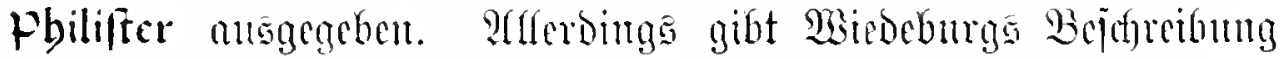

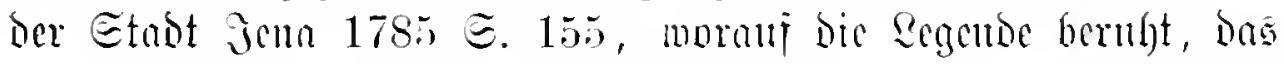

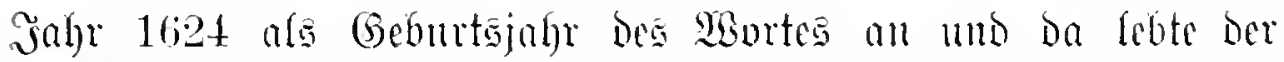

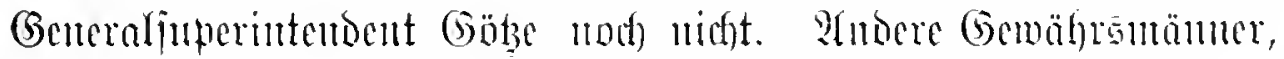

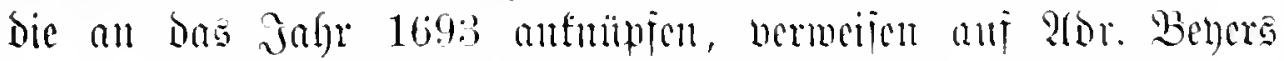

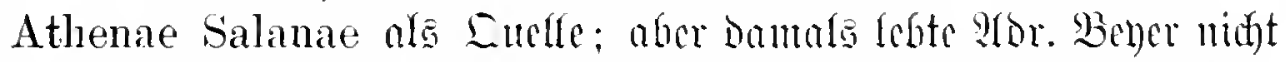

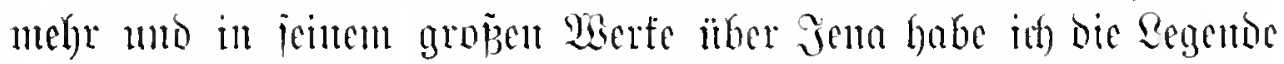

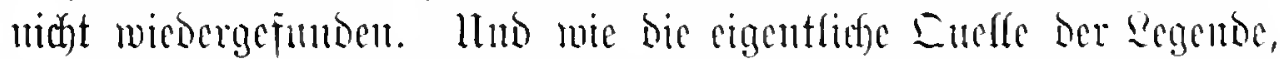

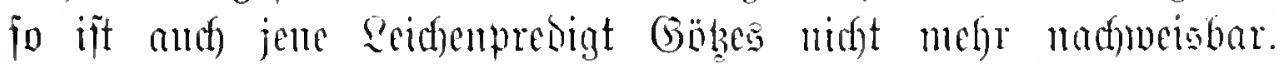

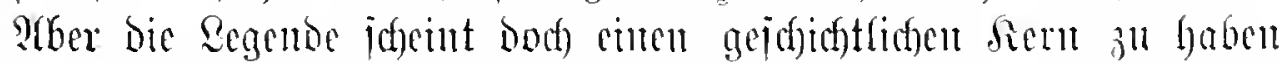

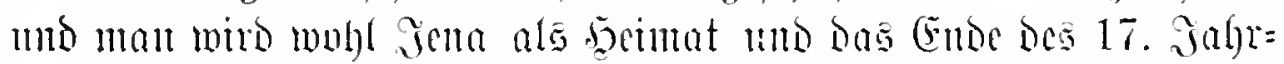

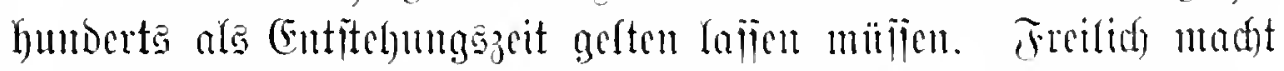

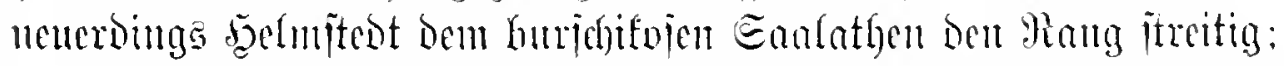

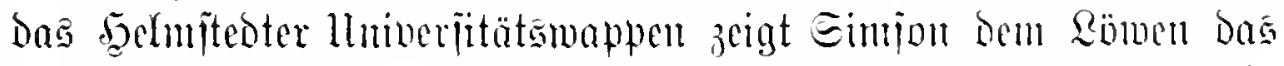

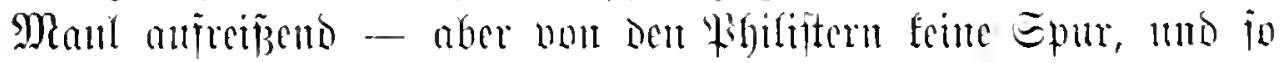




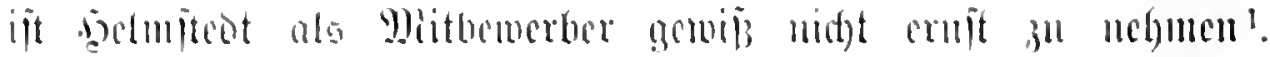

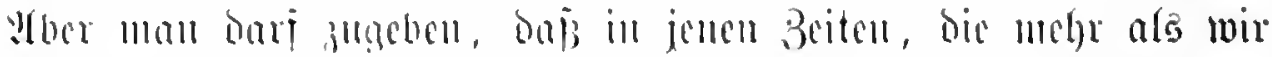

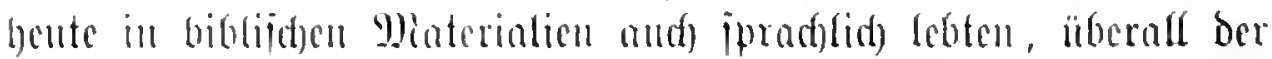

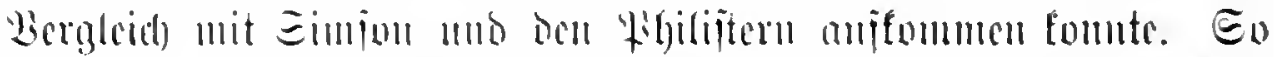

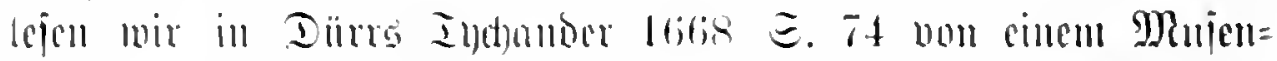

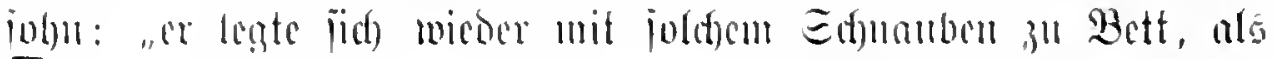

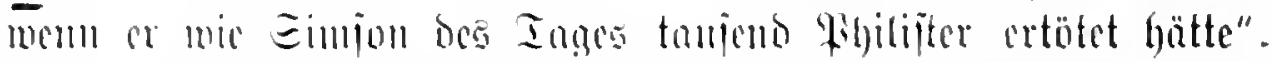

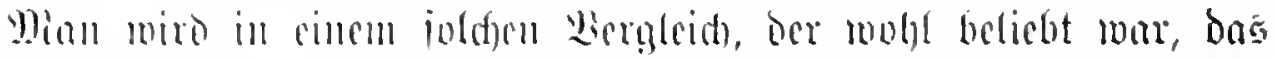

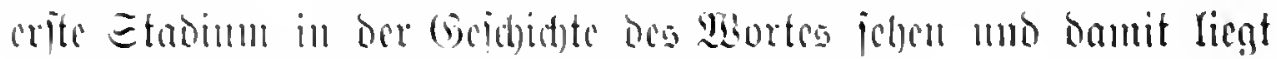

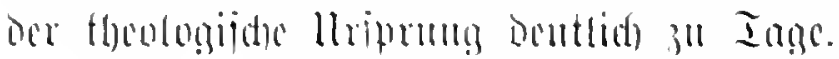

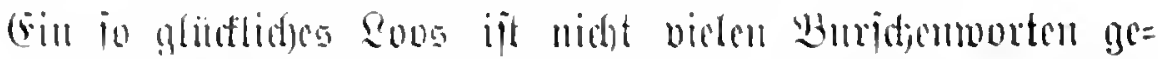

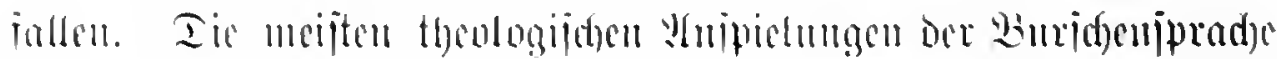

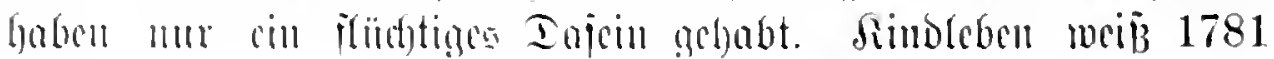

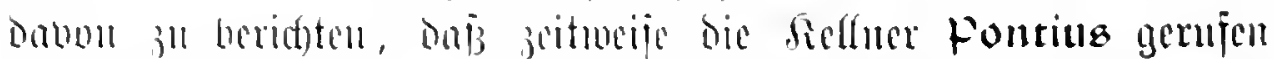

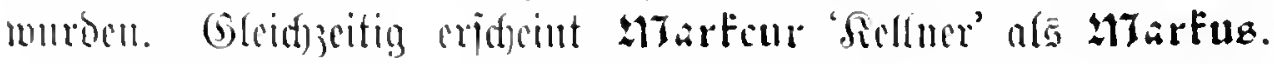

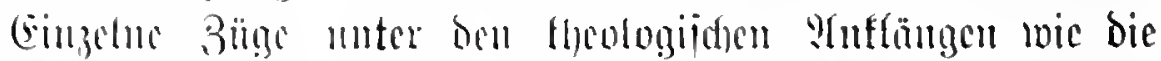

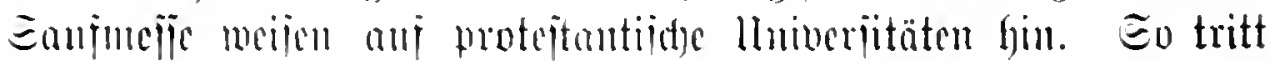

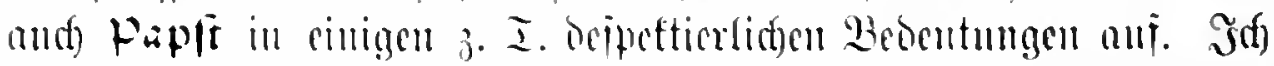

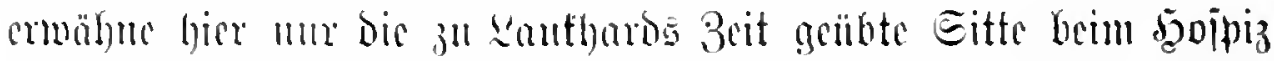

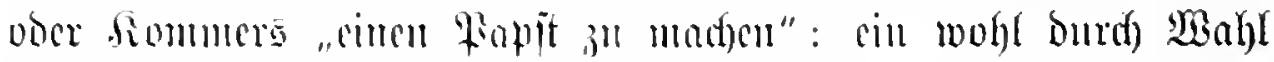
Gejtimmter Etmoin jebte jids anj rimen Etubl, ore am bem Tija)

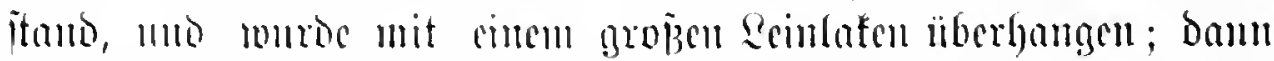

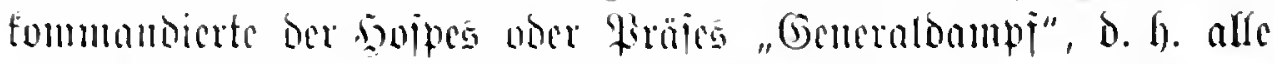
mmïten Den Eabatsiand unter bicies Safen bfaien mo mu

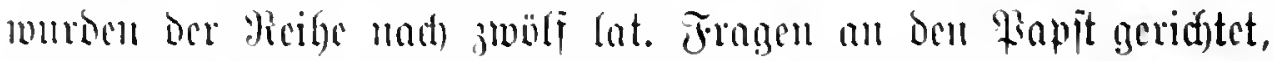
Der jie lateinijid Geantworten muibte, jebesmal cin bolles Glas

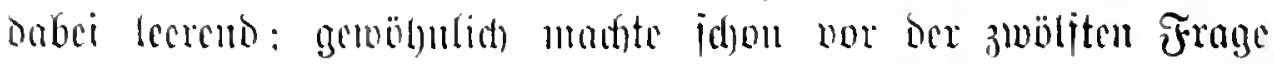

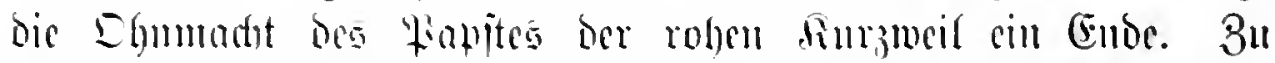

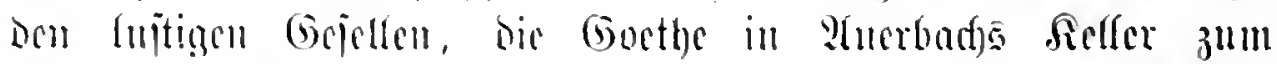

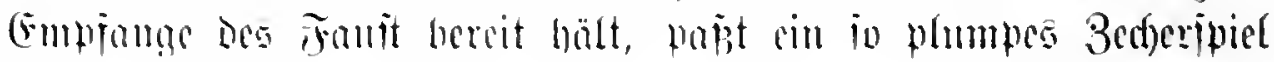

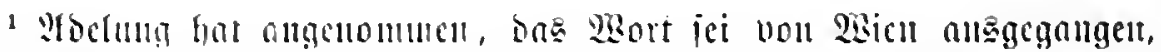
wo die Etabrioloasen Darmuter veritandon gelueien ieicu. Llub ber Initige

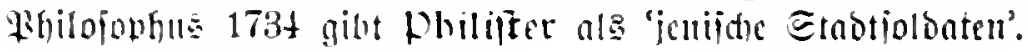




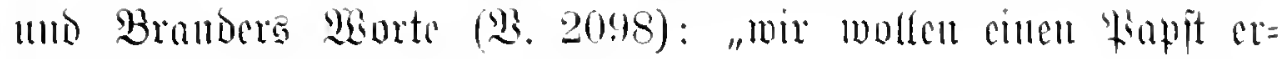
wähleu" faum woh)l barali jielut. ${ }^{1}$

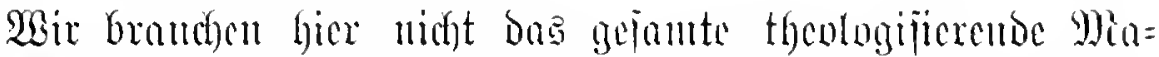

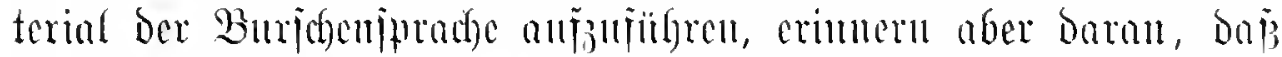
Das Stubentencpos in Eramensicuen fomijerse 2Birfungen mit

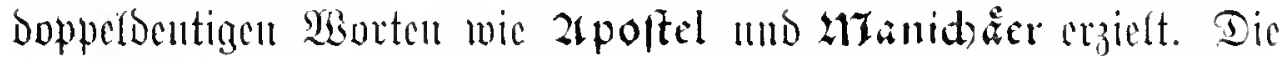
Ranbibatemprïjung Jobjens idseitert baran, baj ibm bie fituben= tifd)en Begriffe näber ficyen ats bic theologijgen.

\section{Şm Bam bes Protwelja.}

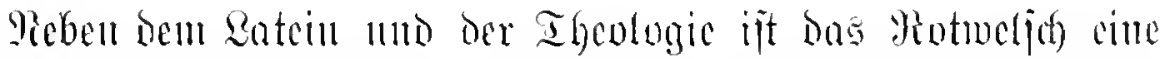

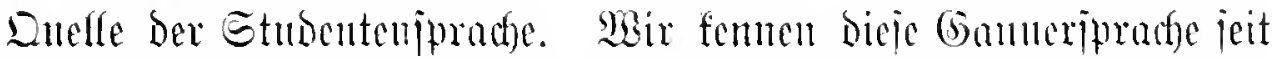
ben 14.-15. Jabrhumbert ans reidhartigeren und vieljeitigeren

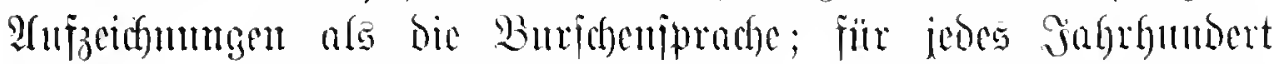

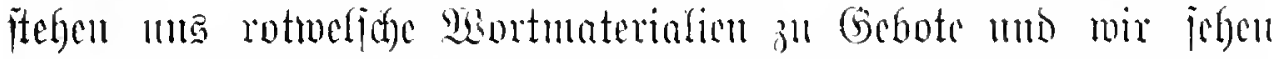

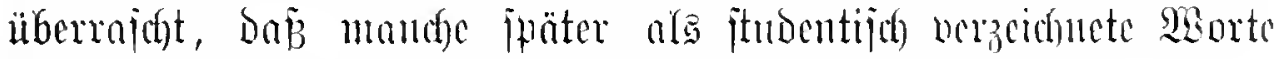
juerjt (Sannerworte bes Rotwedich gemejen jüb.

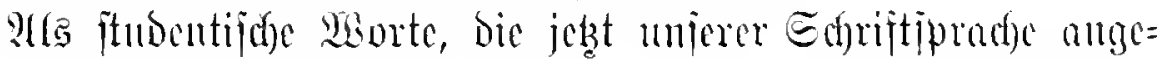

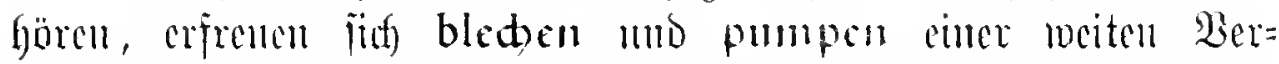
breiturg, aber jie cutitammen bem Rotmelich. Lled) wiro fïr

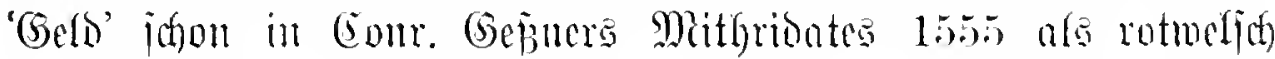

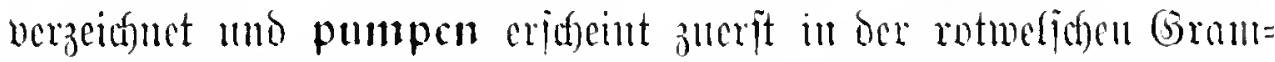
matif non 1755, bum erit 1781 mo 1795 in fubentifojen

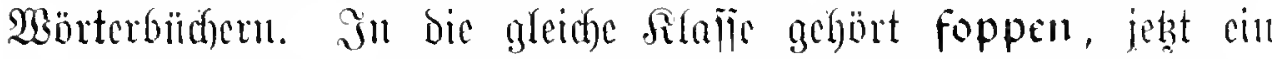

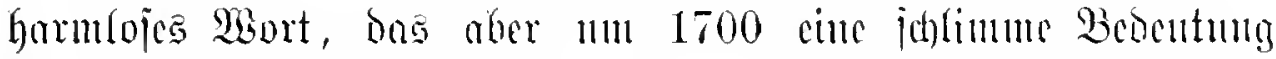
Gatte: Etiders Worterbud) oun 1691 bejeidfuet os als rocabulum plebeium et sordidum. SGm Gaftete böfes Dormetel an injolge

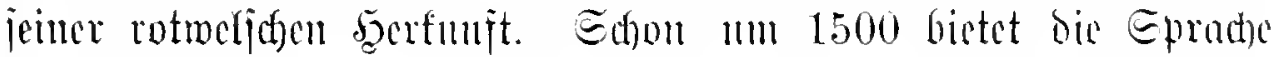

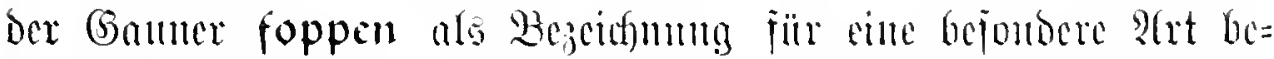

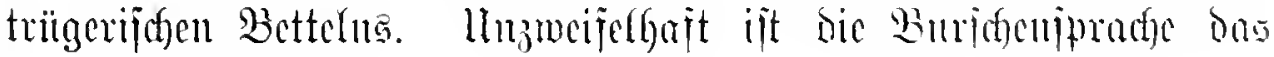

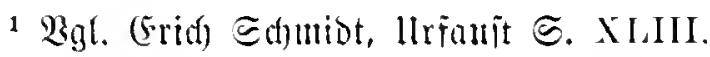




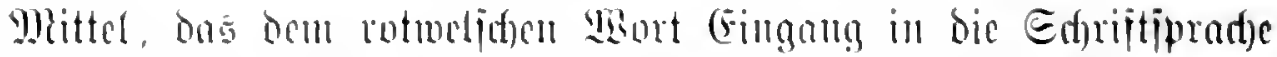
veridguift hat. Edjon im 17. iabrbundert begeguet foppen in

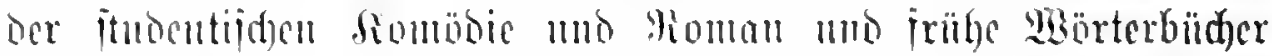

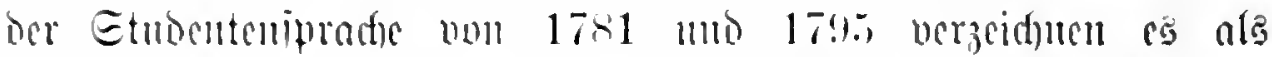

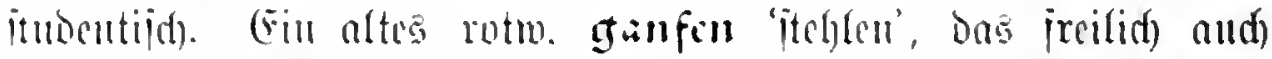

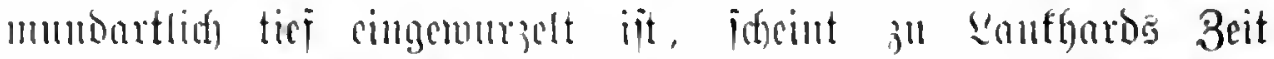

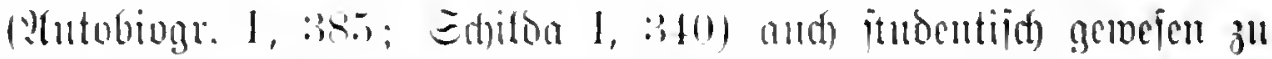

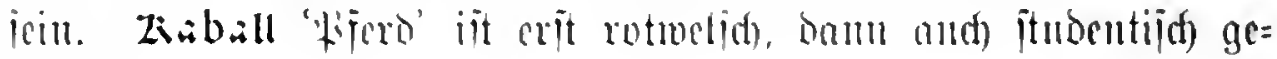
mist.

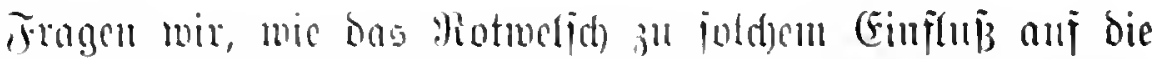

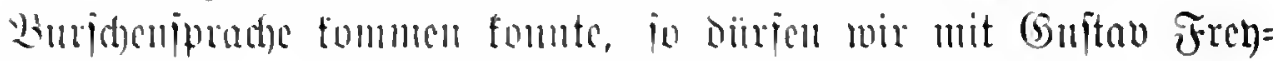

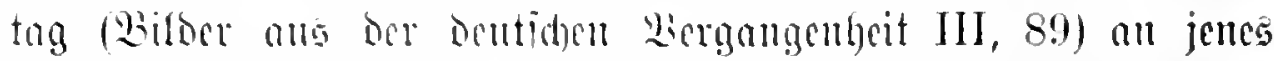

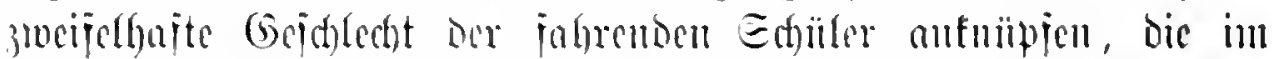

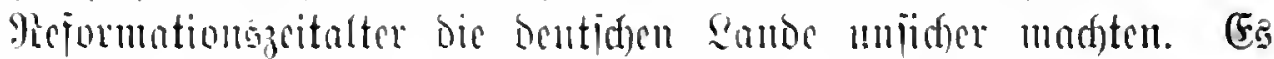

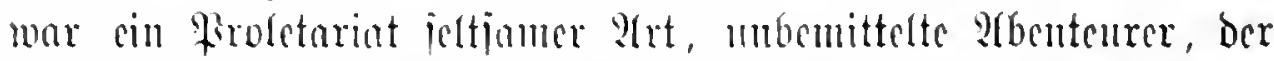

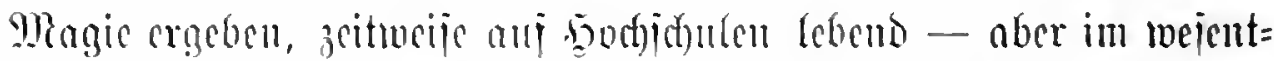

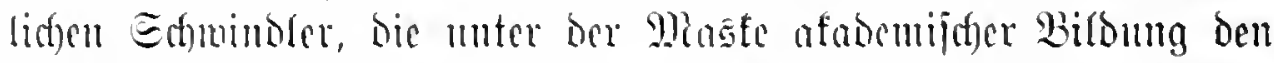

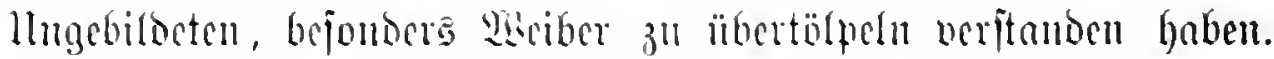
lluter zagtreict)en ?iament meroen jie in ber Damaligen Ritteratur ermäbut; jic beijen Eombjabrer ober Eanoitreicher, Etridflinge,

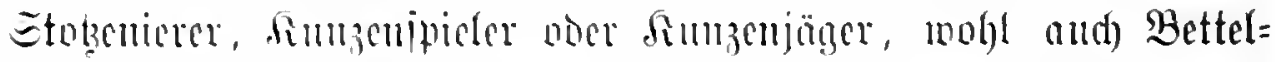
itubenten und jumeitit dben fabremise Estüler. Bejonders anf alemanniictem Boben bahen fie ilgr llumcien getrieben. Trithemins hat jie uns mit Mierfmalen gejejiloent, bic er and an jeinem Zeit=

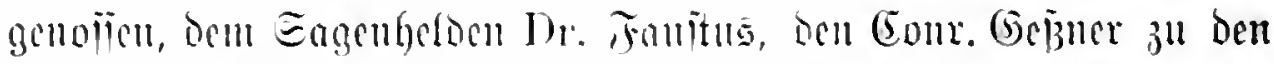

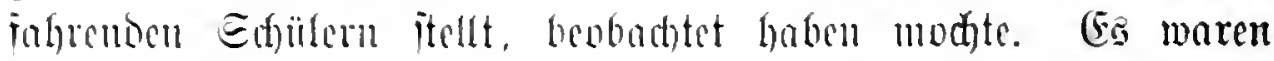

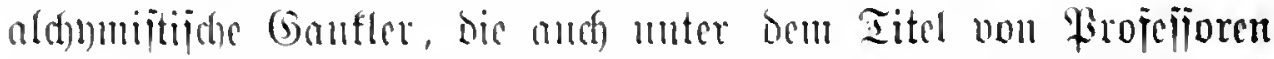
Der Yefrommutie gemaltigns nerjurecten mo antünoigen. Sie

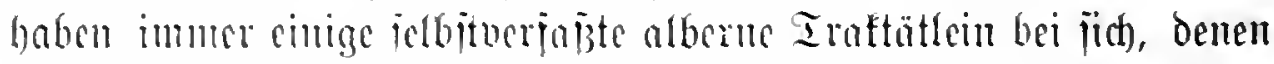

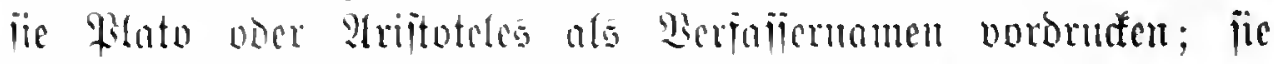
hanticren mit alten Banberjormedn, wie iir im 15. uto 16. Jahbr=

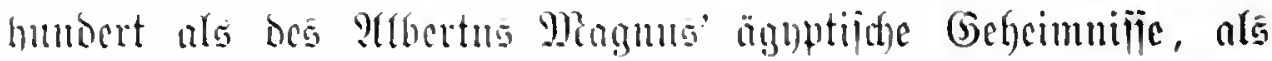

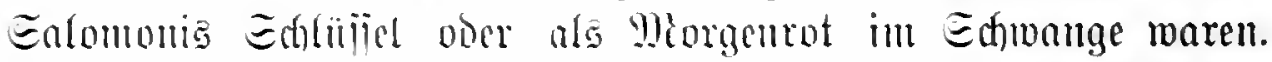

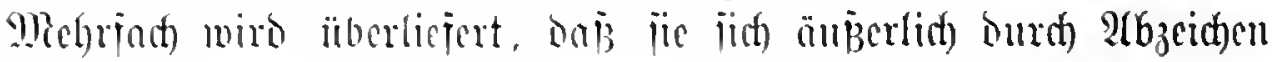




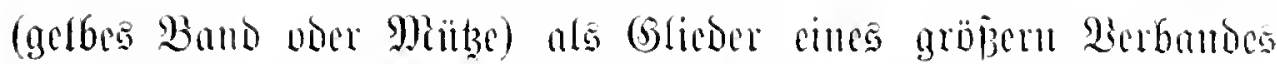

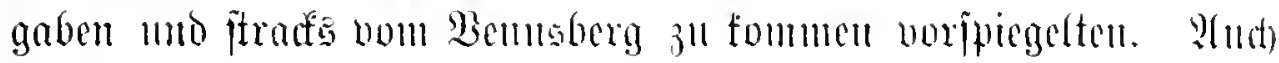

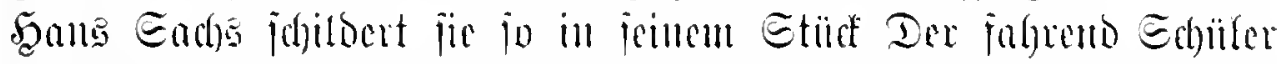
im Farndies; mo in rinem andern Etün - Der fahremb Esjüter mit bem Ienjelşbanten - legt er cinem Bertreter biejer Menjoche

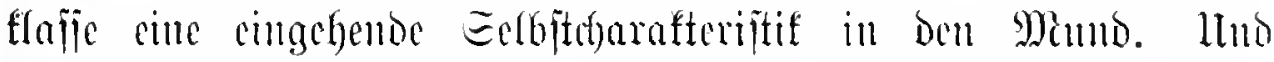

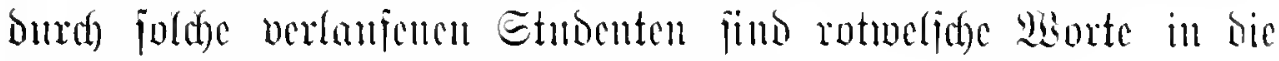
Sincipen Der llniberïtätsitäote geraten.

Fiir nenere Beitn liegt as and) nabe, munittedfor an bos moderne Jubendentich anzufuipfen. Ev gehört dos verbreitete

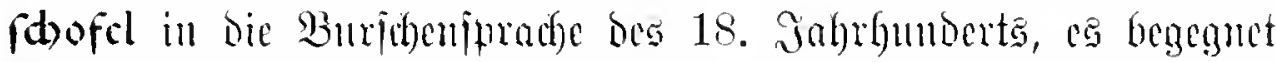

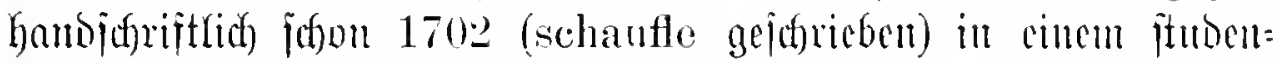

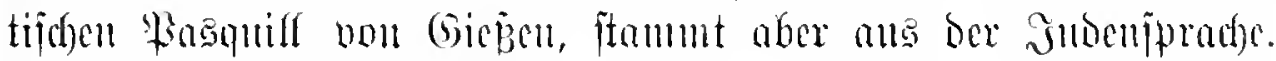

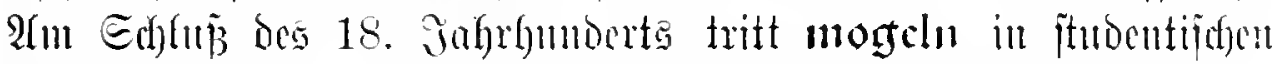

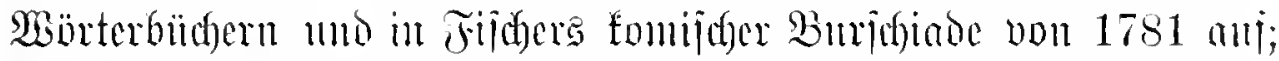

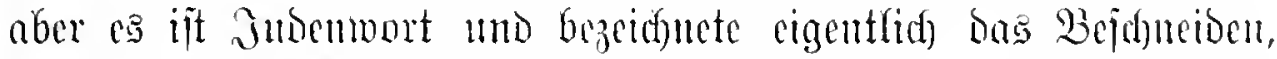

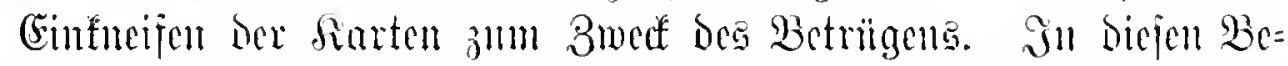

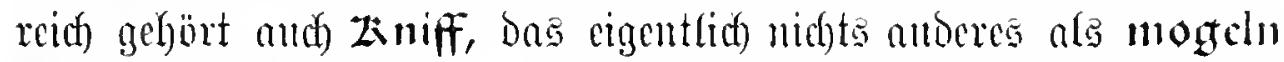

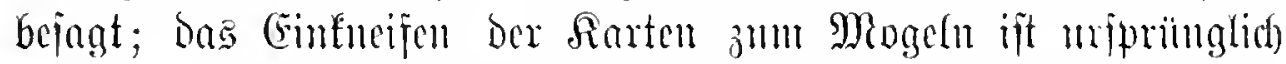

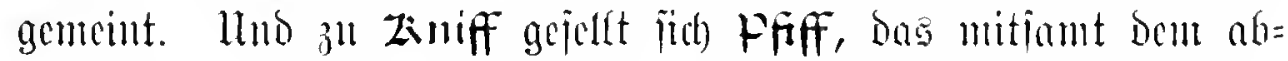

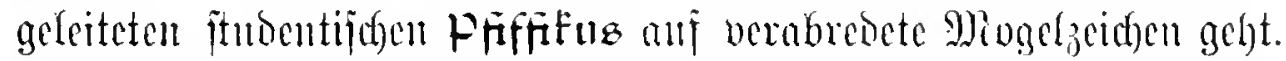

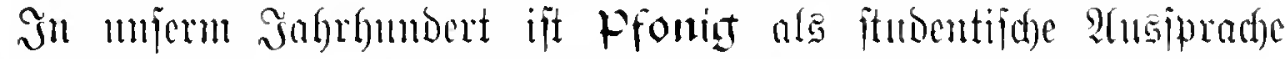

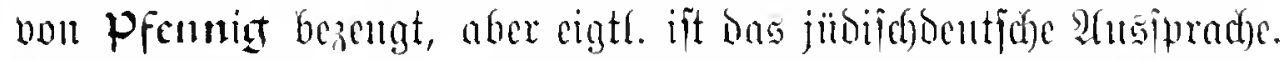

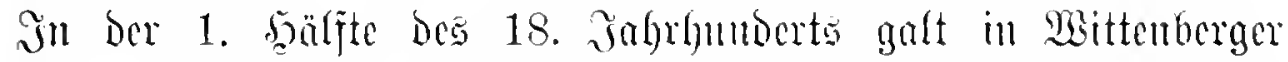

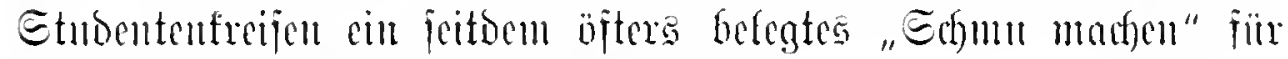
Das hebr:=jübiedye schmu "gedede".

Mit bem Rotme(id) hat bie Buridgeniphende nody andere Büge

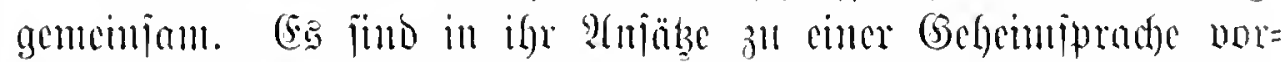

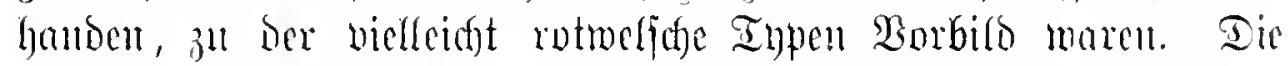

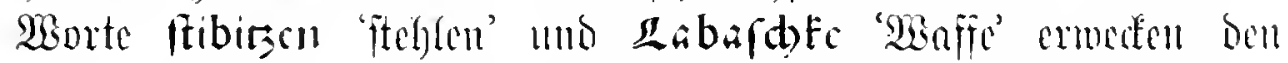
Berbadyt, als ob jie ju ber jog. pi-Epradhe gehören fümuten, bic

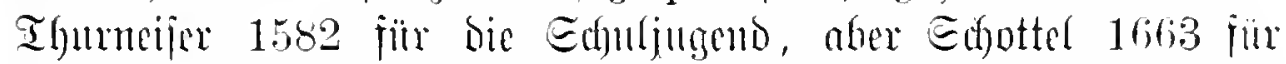

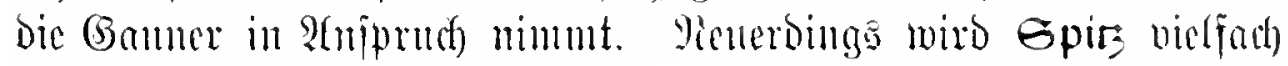

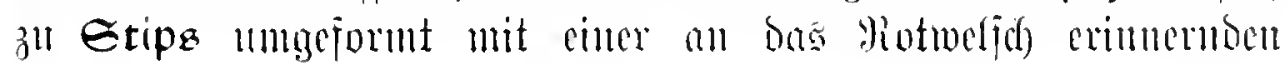




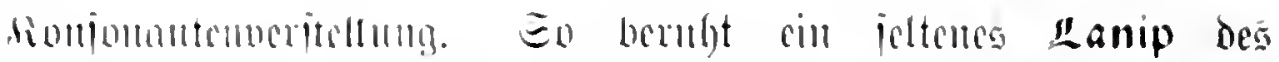

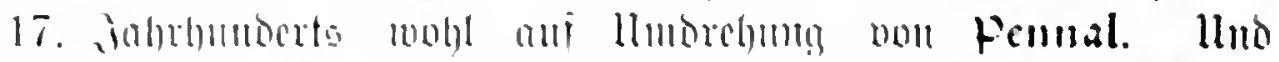

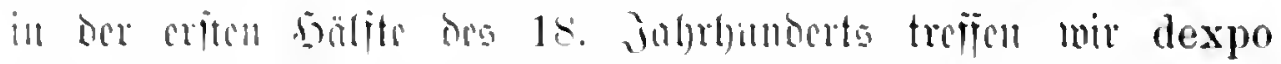

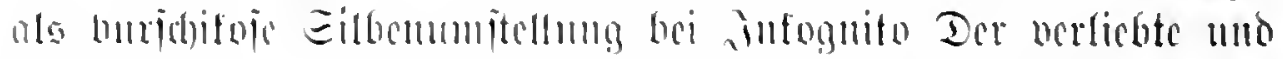

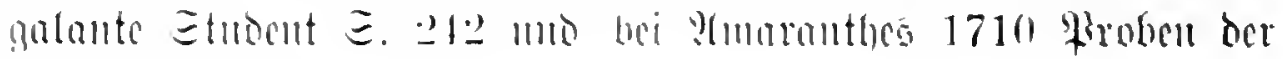
Honciir e. tent.

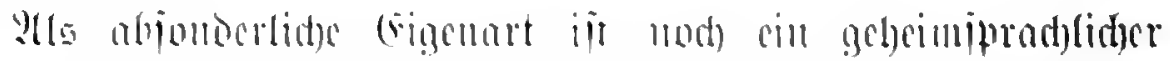

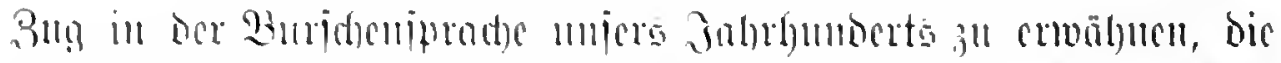

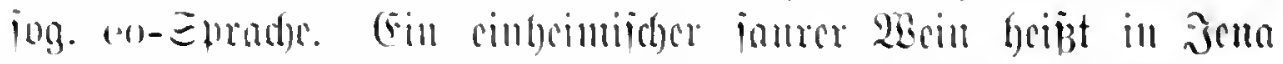

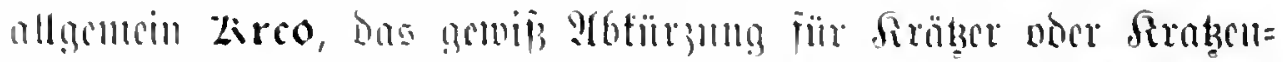

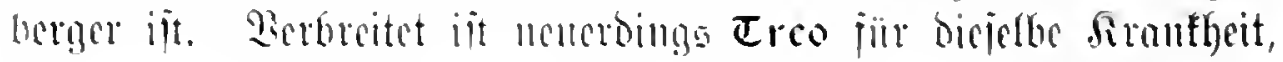

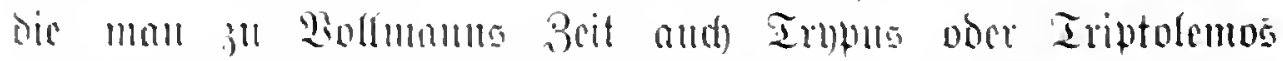

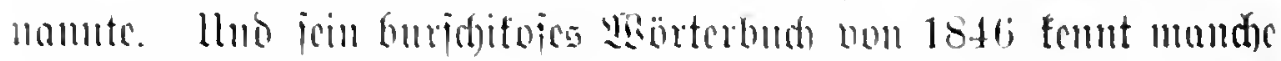

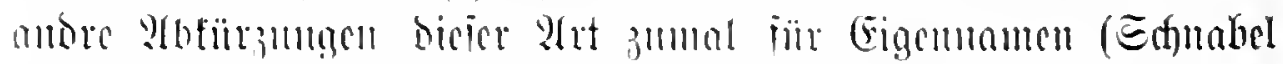

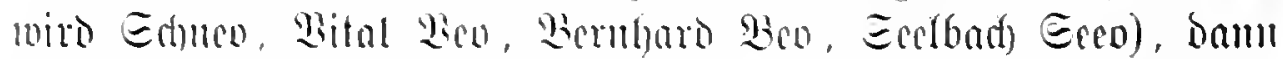

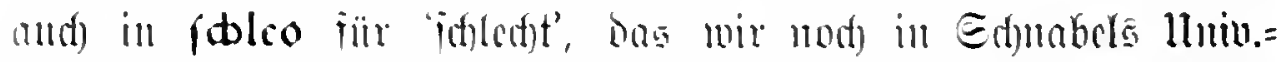

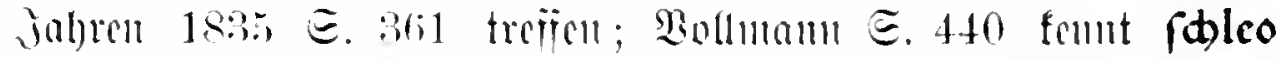

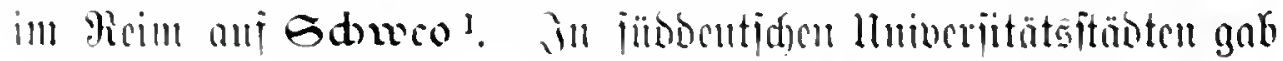

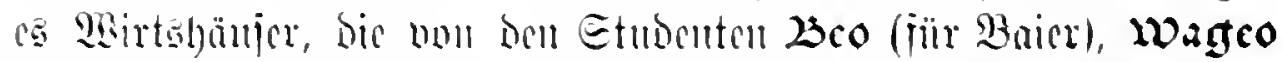

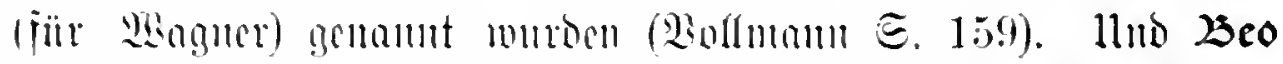

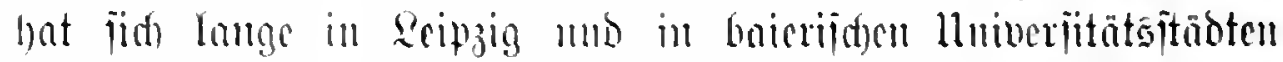

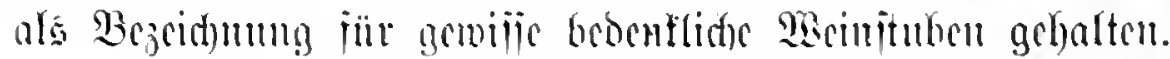

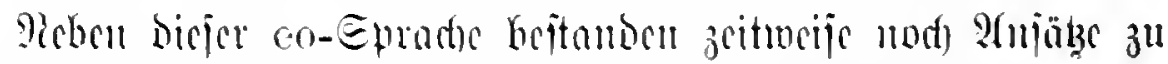

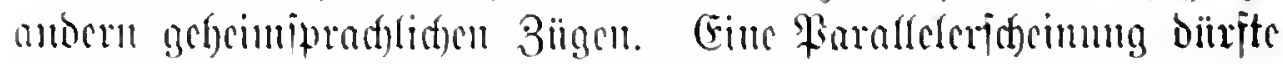

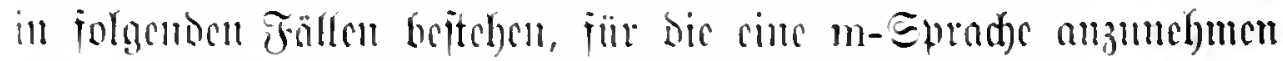

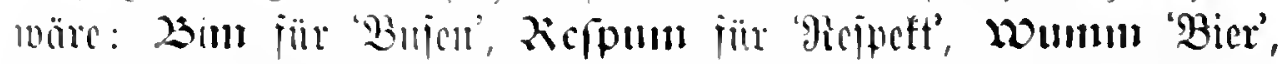

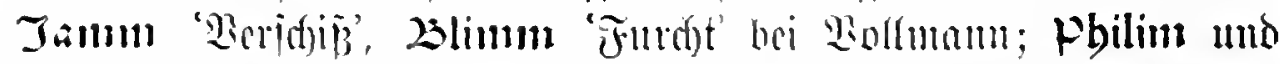

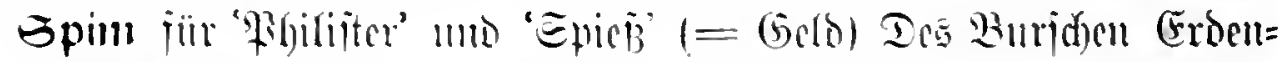

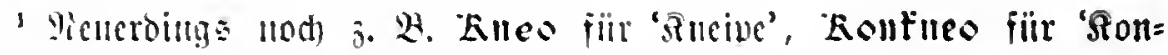

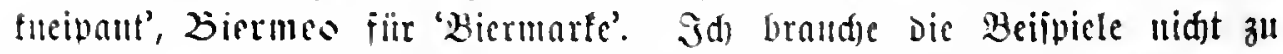

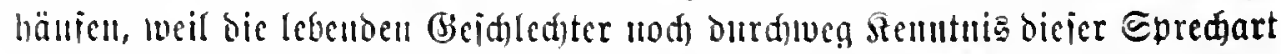

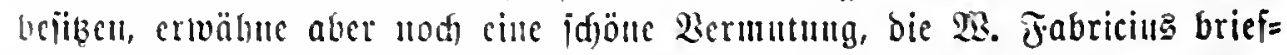

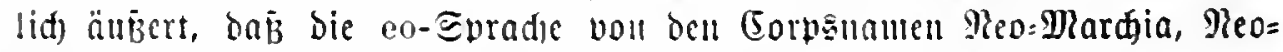

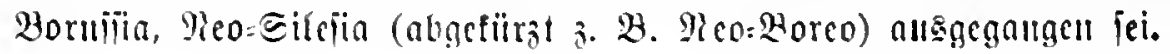




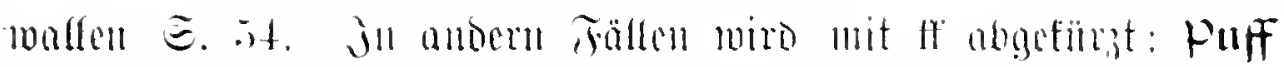

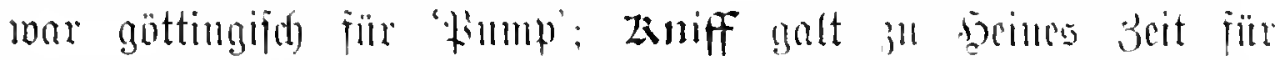

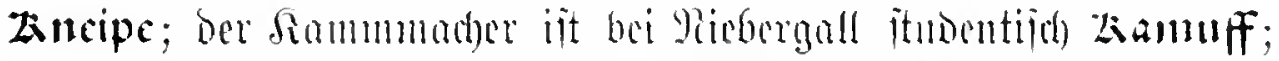

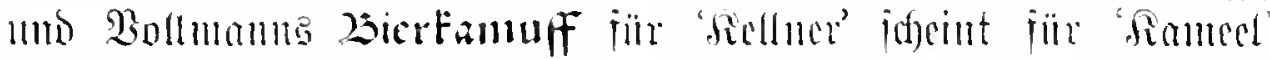

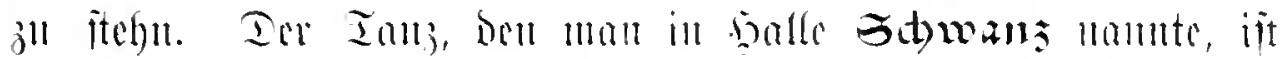
allgelleill $\mathbf{E d}$ woof.

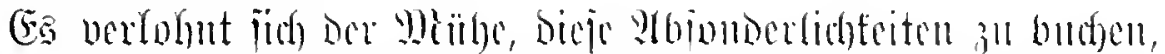

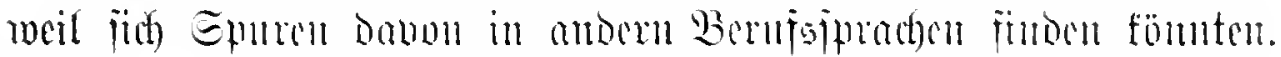
Bon Der eo-Eprade, die an allen Dentichen bod)idylen in

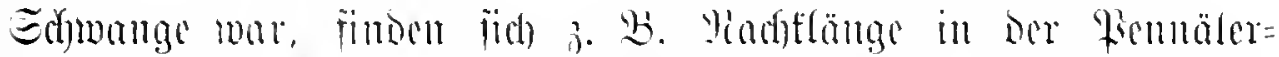

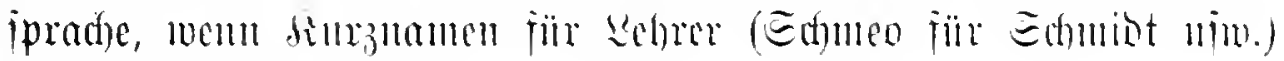
damit gebilont mersen.

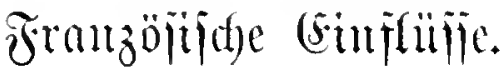

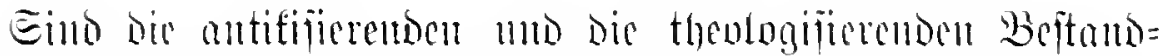

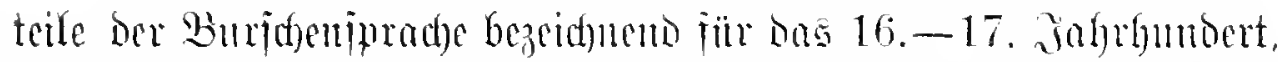

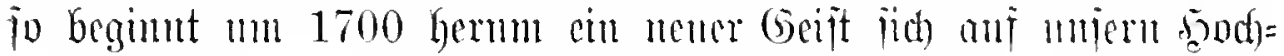

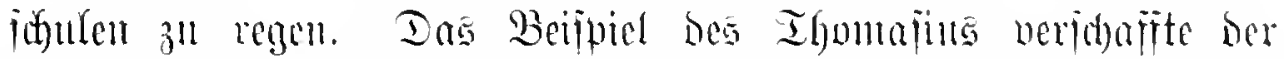

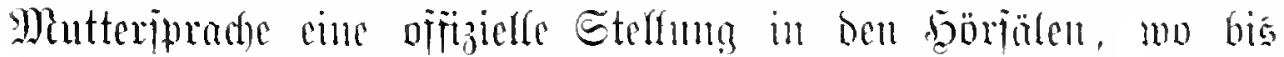

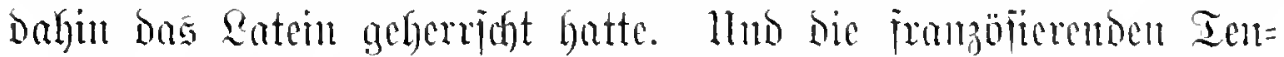
Denzen, die bei uns ben Beitalter smbunigs XIV. Folgten, Gradsten

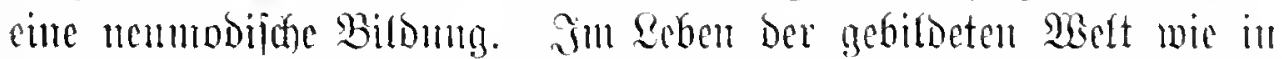

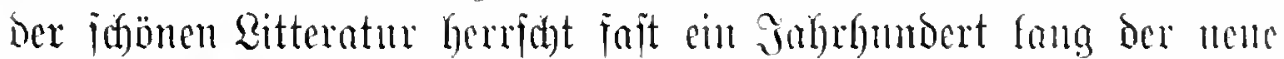

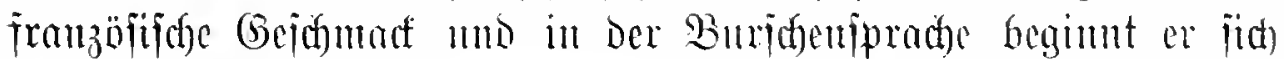

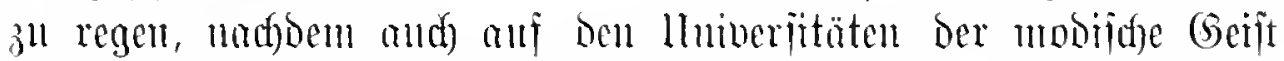

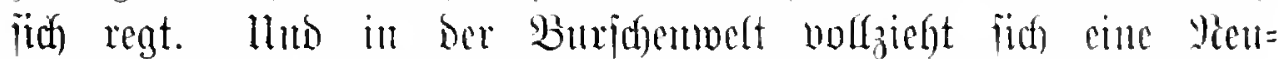

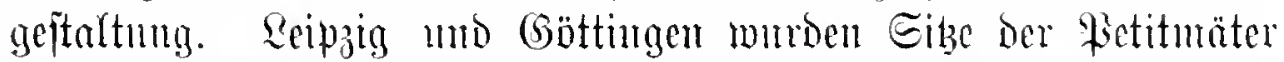
und ber Bierbenger mb mit Entjeken faben bie senommijten

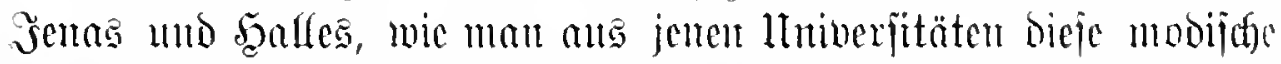
Gerferei "bei gauzen Bentuen herbolte" mb bamit bie afte Burr=

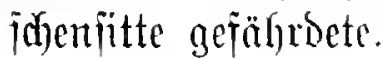




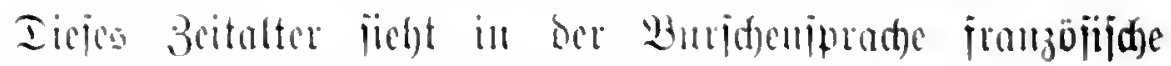

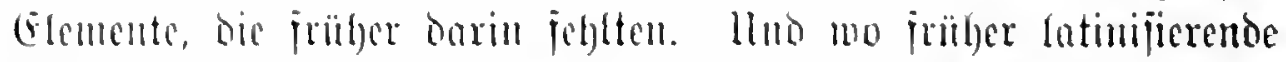

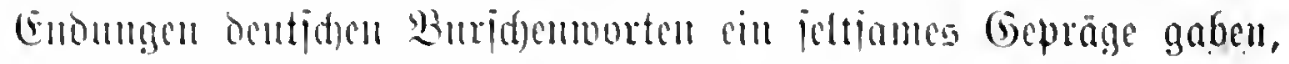

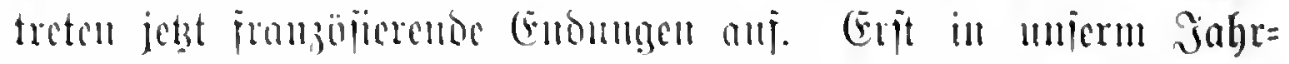

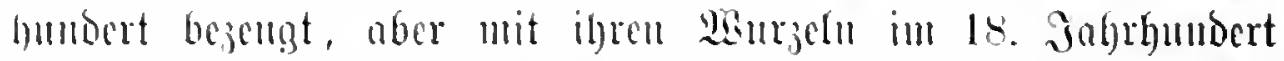

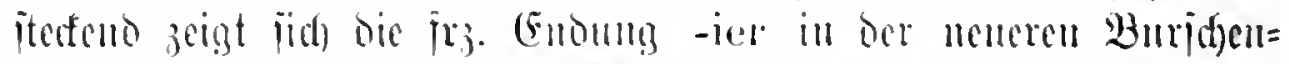

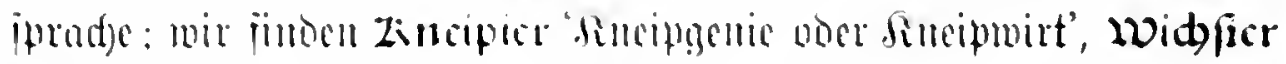

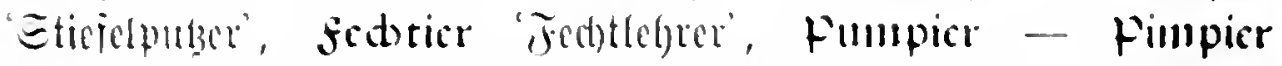

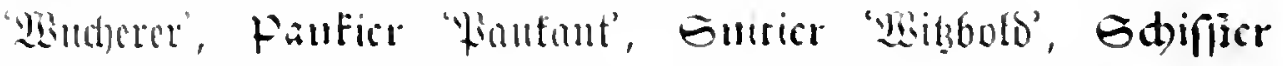

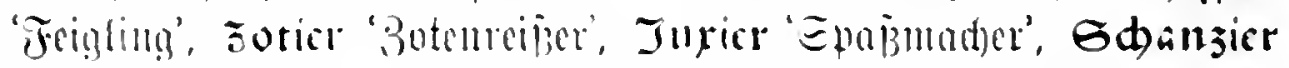

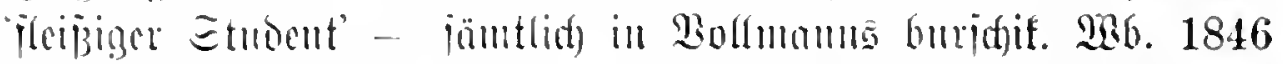

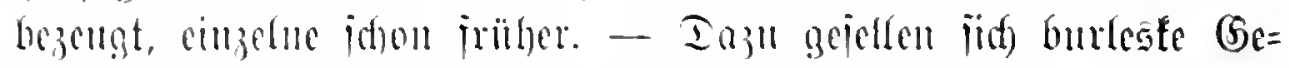

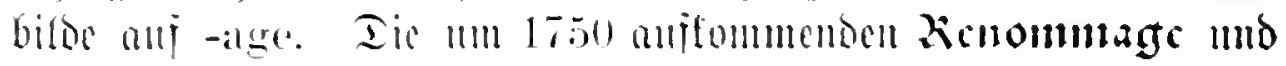

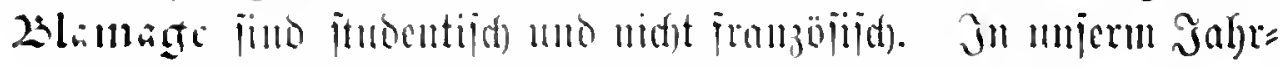

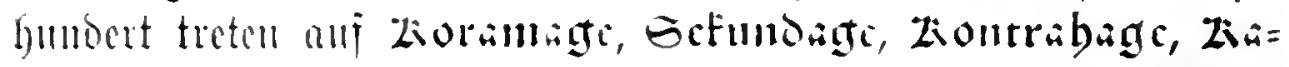

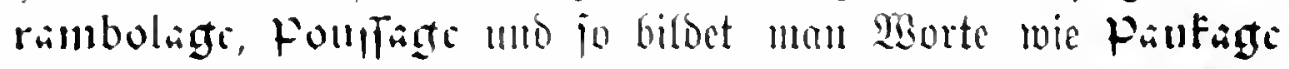

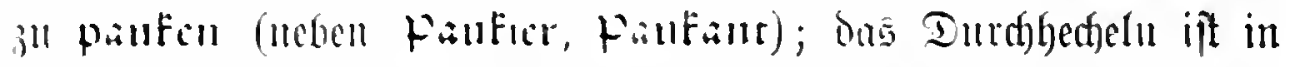
Den Etnoentemnizzen 18:3! E. il bie rectuchage. Man hört

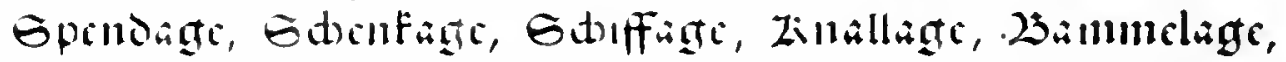

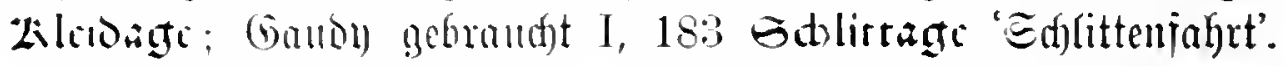

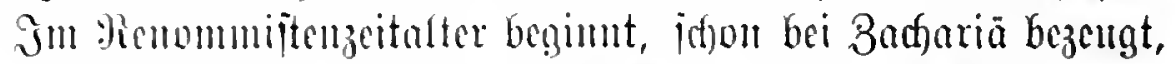

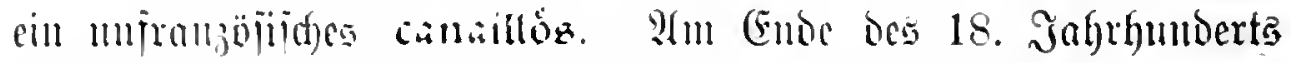
trejien mir bei Sauffard ajters luberoz. Penerbings gelten málitiog mo ped)óg - pedión allgemein; philiftróg tritt anf

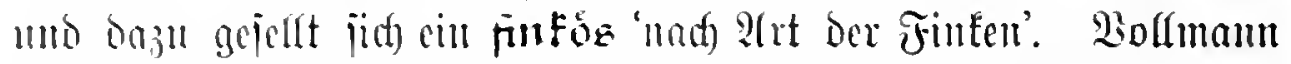

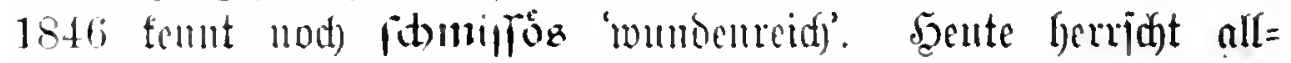

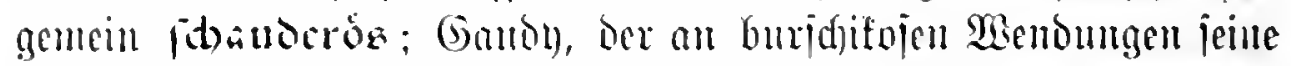

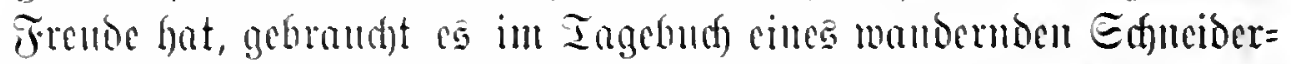

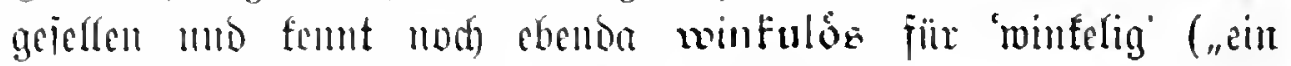
minfuliojes Veje” Merfe I, 179. 199 Meïller).

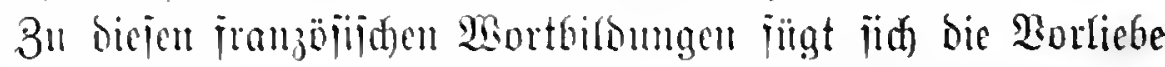

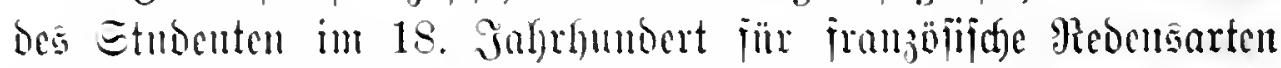
von interjeftiontelfm Eharafter: enfin. sans doute oder votre

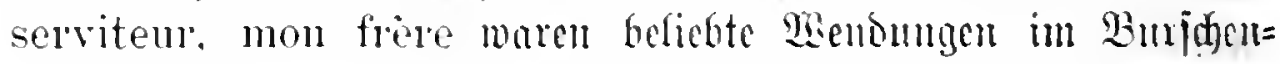




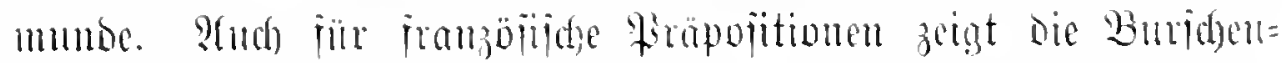

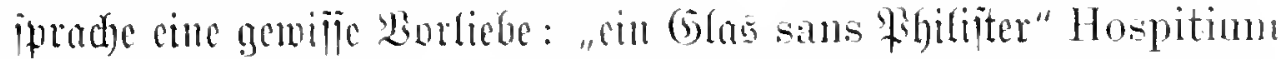

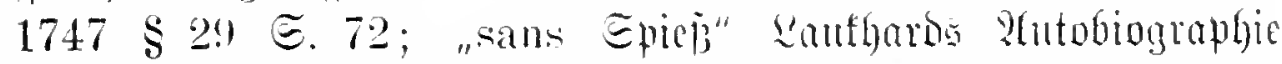

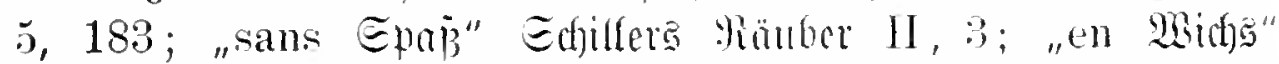

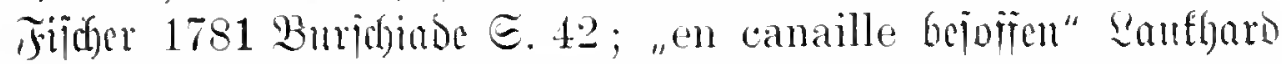

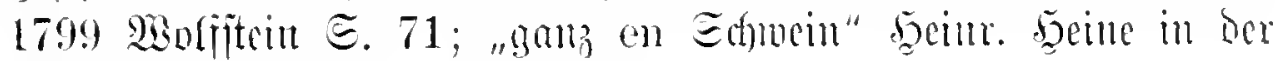
Dararaije (Worfe HI, 65) (Eliter).

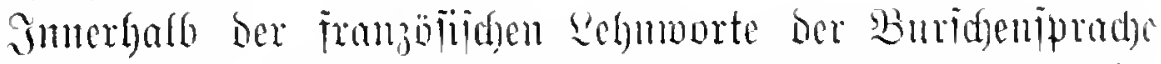

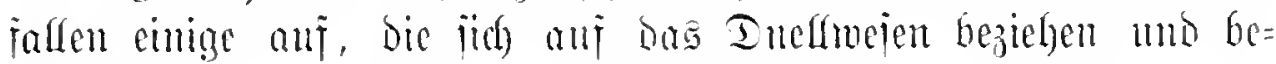

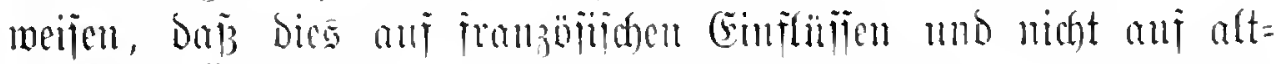
Dentiffer lïberfiejerung bernlyt, wie 2 vantagge nur Tondhe:

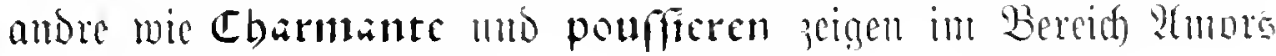
jramzöijiche Mione wirfiam.

\section{(Siammatijhe Sigentart.}

Wer ben Begrifï ber Epradfe ben hente herridemben 2 in=

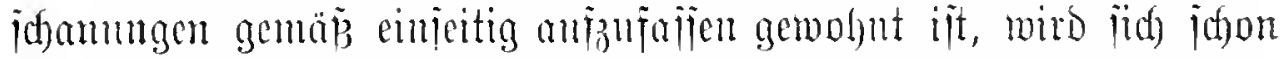

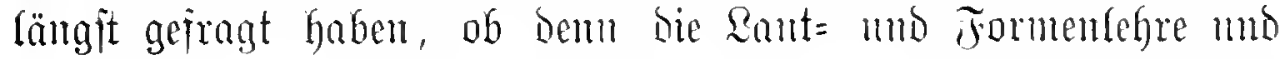
die $\ddot{b}$ brigen grommatijgen hategorien ju Bemerfungen feinen Wulaj geben. Sn ber That jeflen ber Buridenipracte grom=

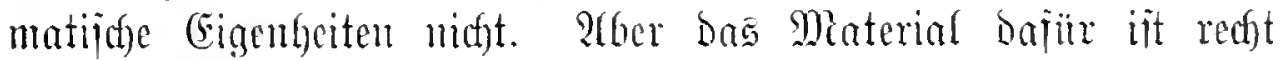
bürftig. (sibt cs bod) eigentlich feine jujammenfängenden Terte

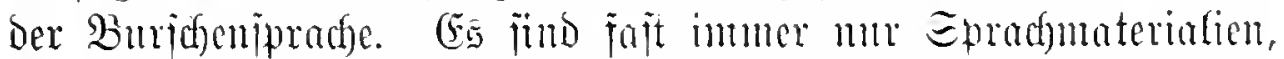

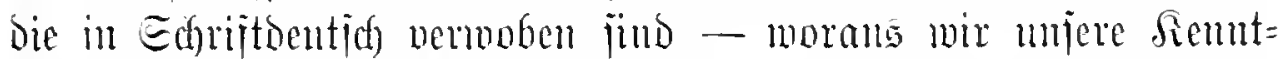

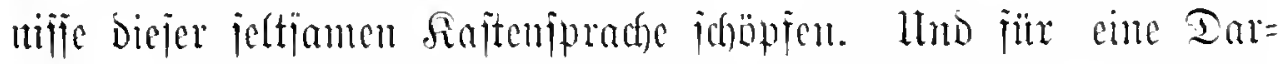
iteffurg ber (Srammatif mil̈̈te jie in alfer Reintheit mo in $z^{2}=$ jammenhängenoer Dariteffung juberfiejert jein.

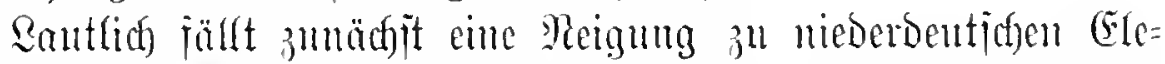
menten anj: verfloppen neben verflopfen, Sdueppe neben Gdunepfe; Gnote iit mos. Fïr '(5endije'; flott jtammt ans bem

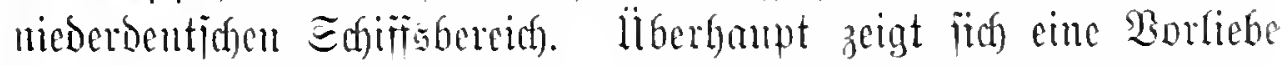
für bie mumbartfidge Santjorm anjtelfe ber jdfrifippradfichen. Bid) 


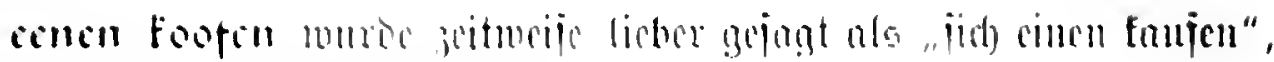
senteon licber alo stemein.

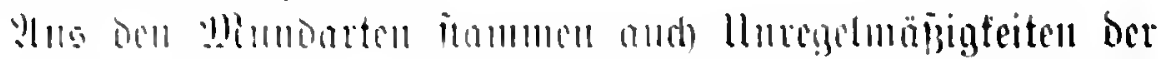

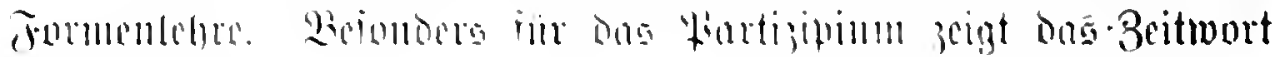

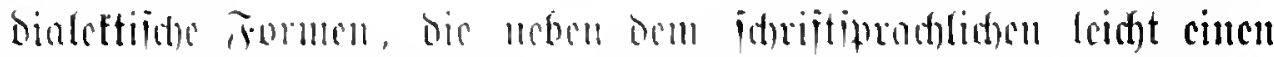

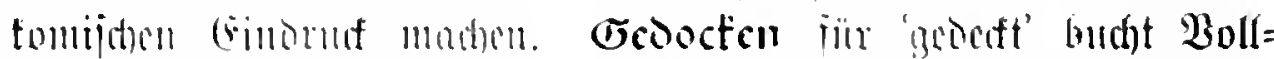

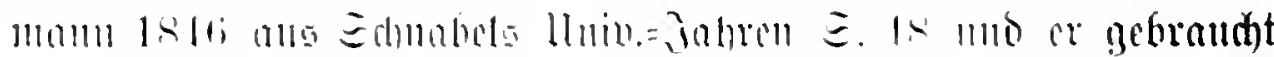

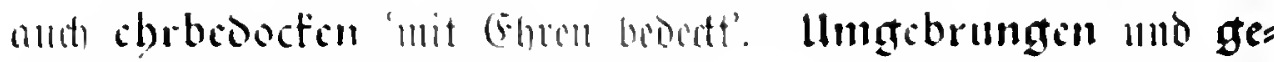

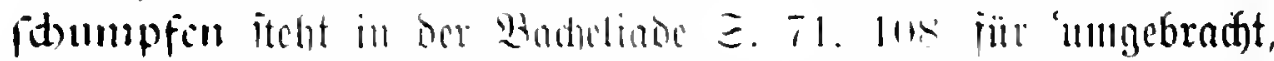

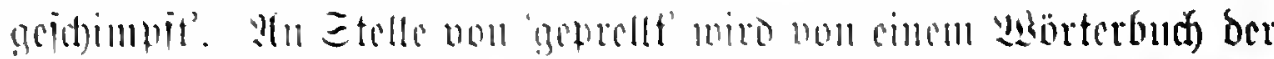

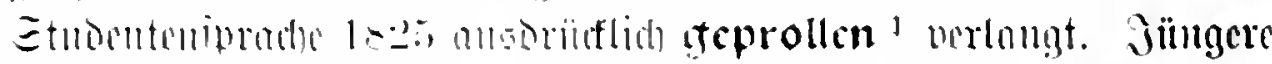

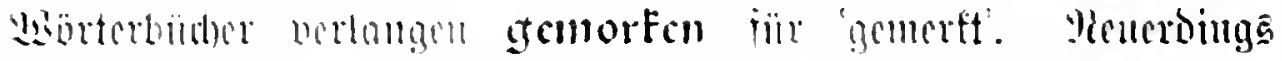

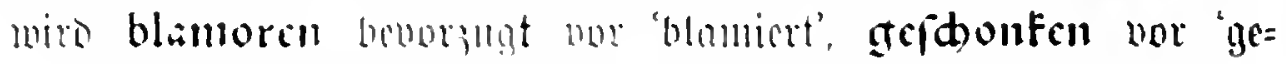

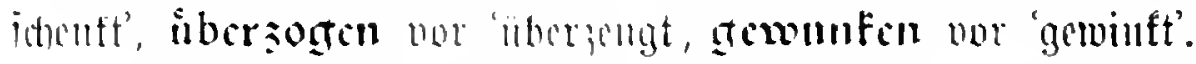

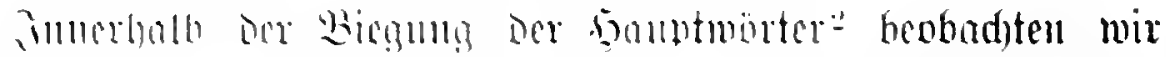

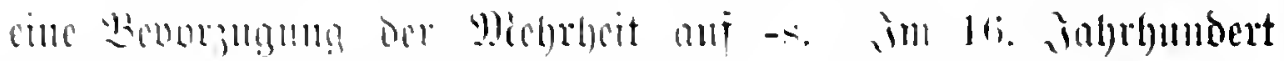

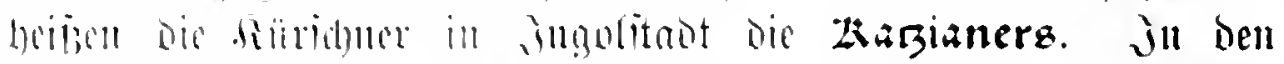

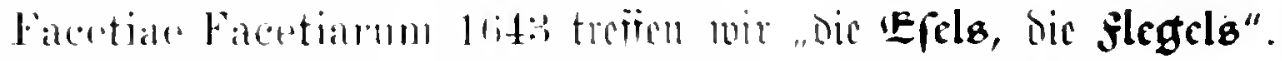

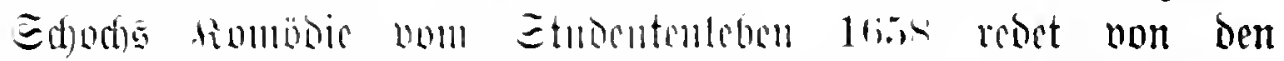

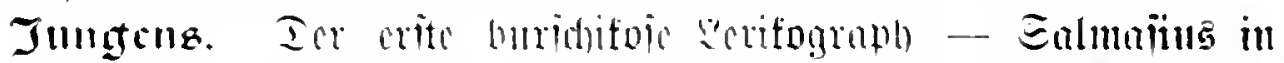

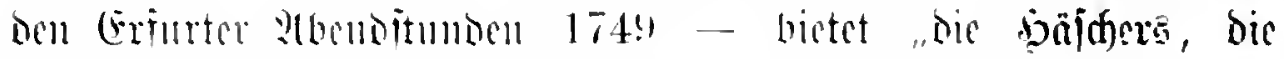

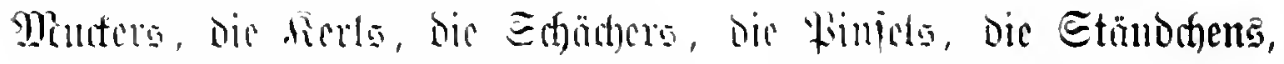

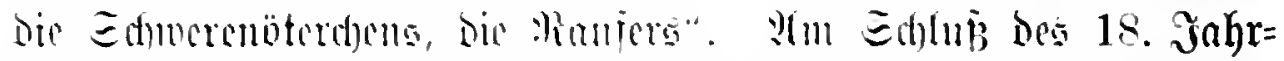

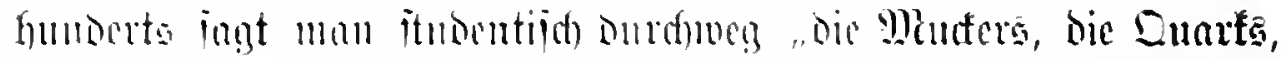

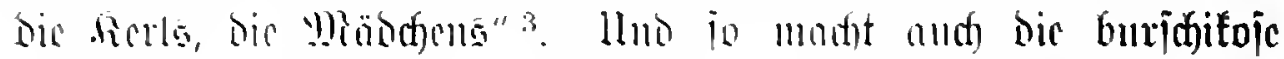

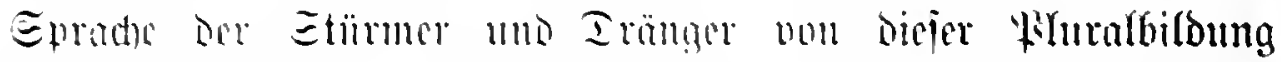

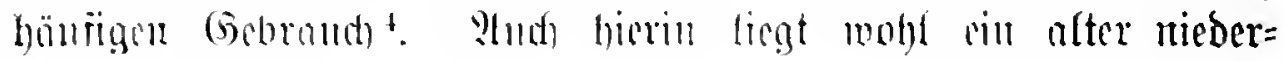
Dentider (Ëmfln nor.

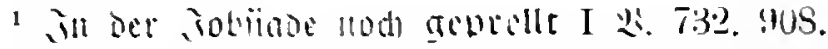

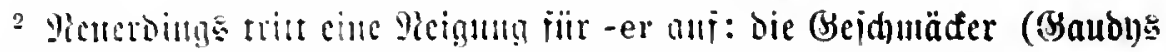

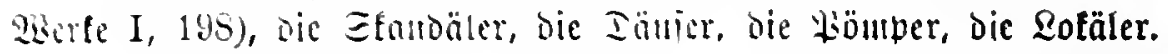

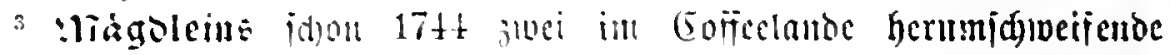
Shanturicu $\approx .99$.

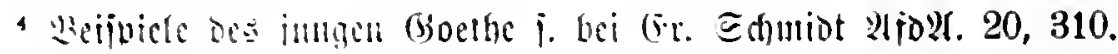




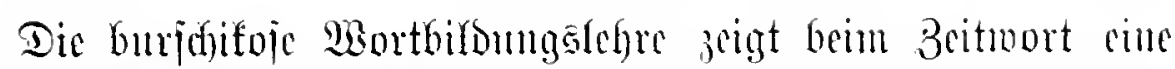

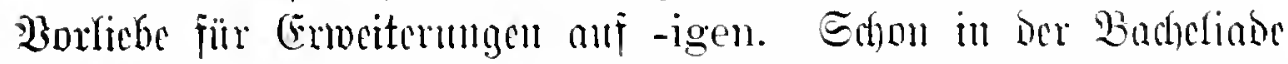

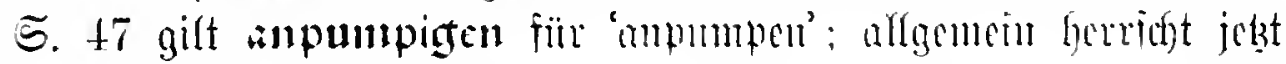
berappigten fiir 'bcrappen'.

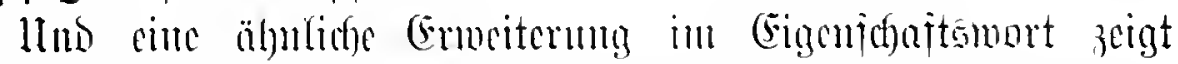

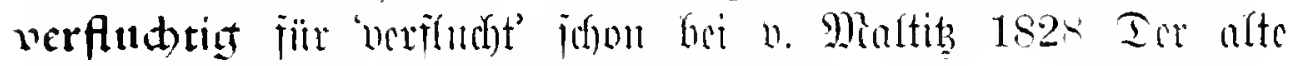
Stulent II, 2; II 4 : "nerffudftiger firrl".

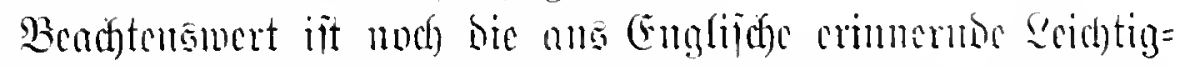

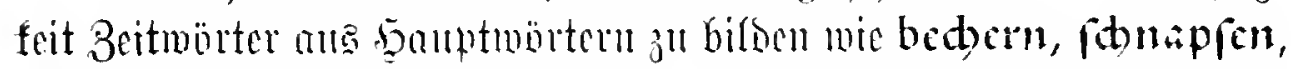
bieren, grocten, d)anpagtnern 1 mo weinen. Eo mirt ats

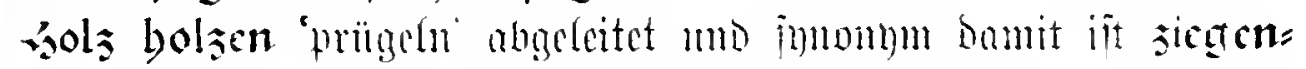

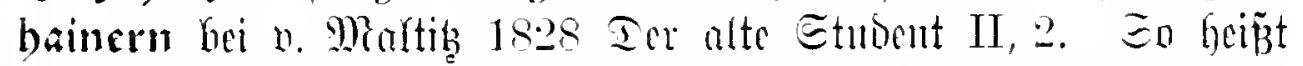
tabafen 'Tahat ranchen', rappieren 'irdh mit Dem Bappier itben', odyen und büffeln 'arbeiten mic cin Echje, cin Büfjel'.

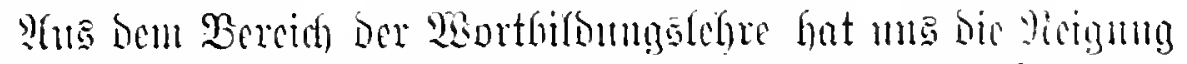

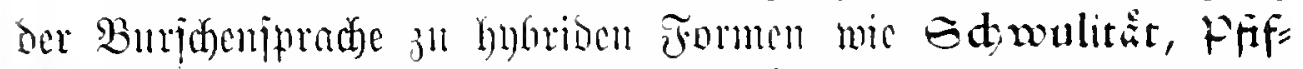

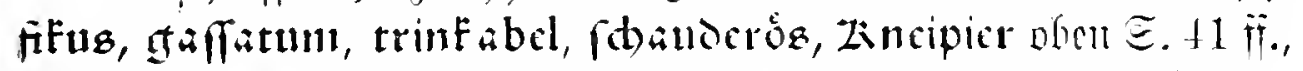

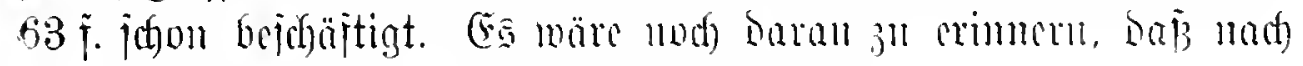
Goloatesta cill Zinotesfia grichaffent murbe.

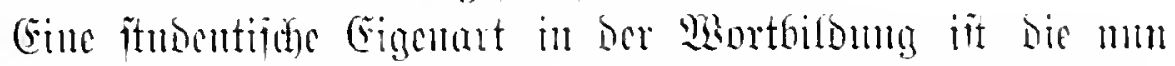

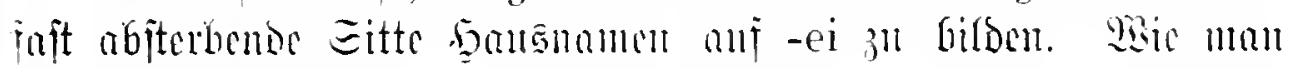

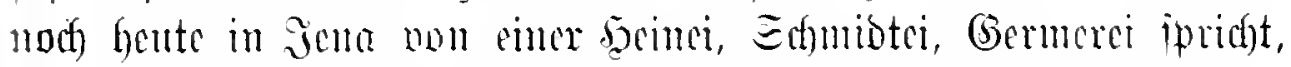

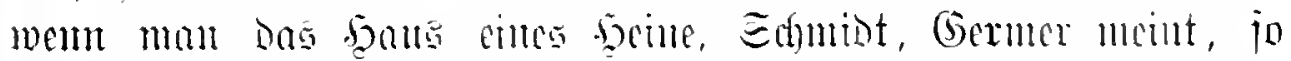

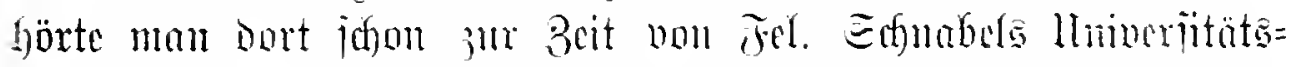

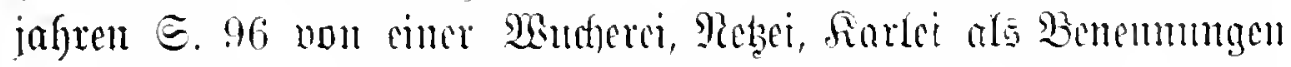

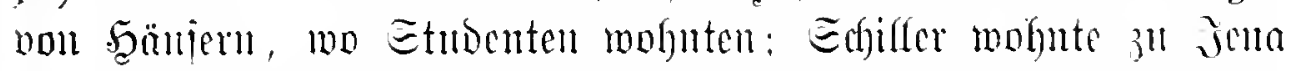
in eincr Ed)

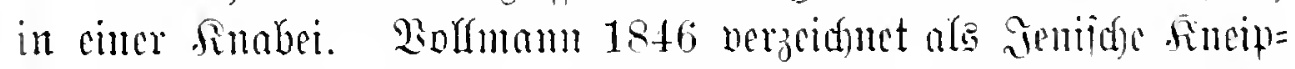

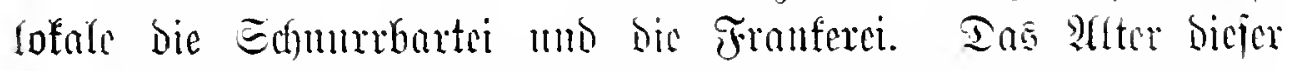

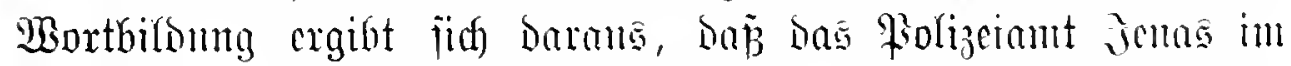

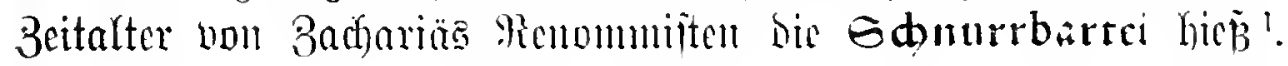

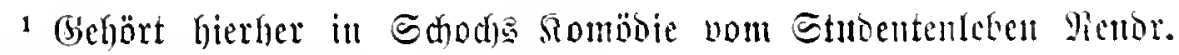
ธ. 30 bag Balanci=\$̧an fïr bie Falanci? 


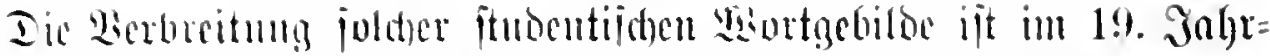

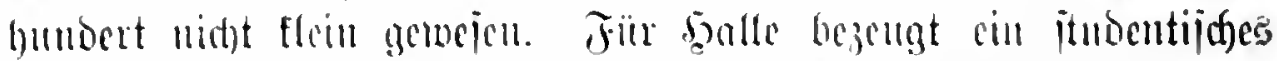

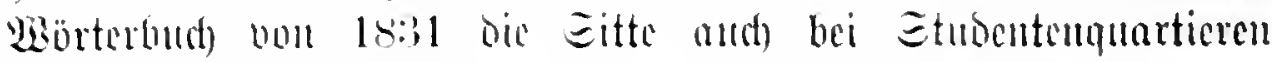

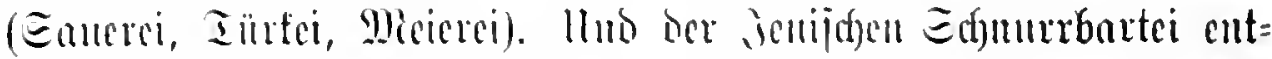

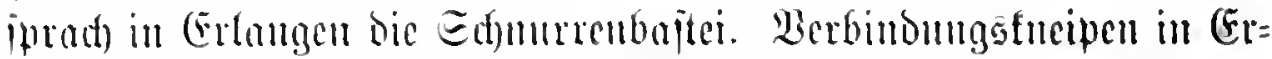
Langen fomuten un 1820 Etahlei, (Erichei, Berfei, Fricoridfei, Dppelei

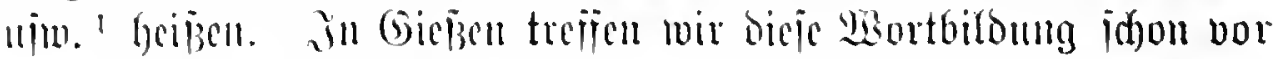

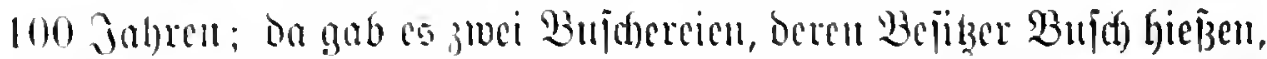

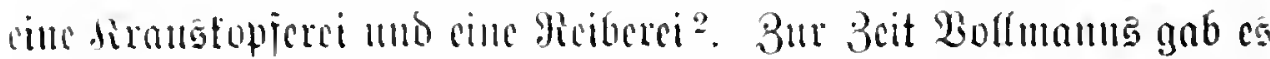

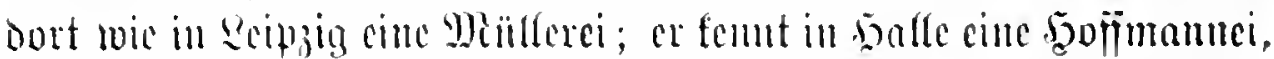

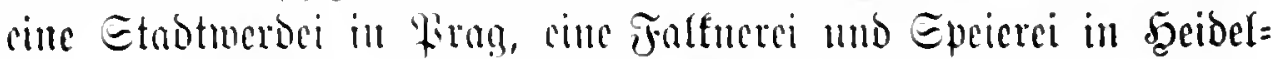
berg, wo Sioble usu 1818 cime Goutumanre unb jpäter Fet. Edyuabel eine Enttlermiillerei traj. Fïr Tïbingen hören wir

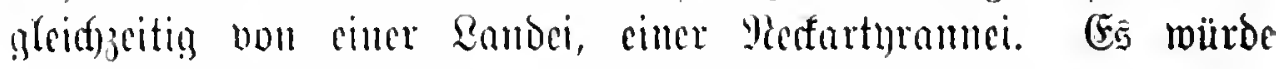

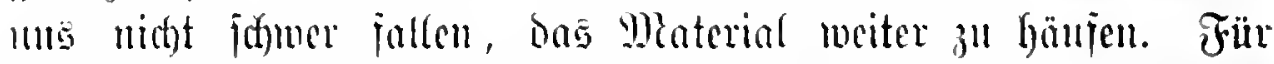

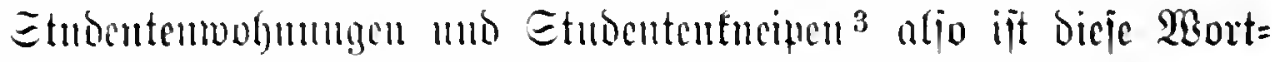

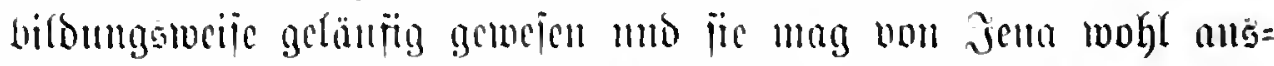

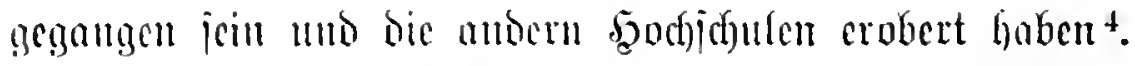

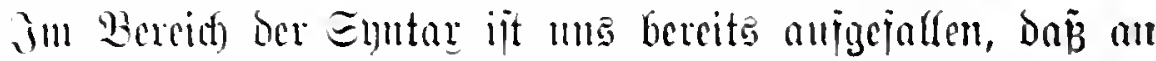

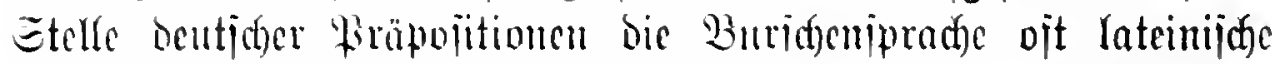

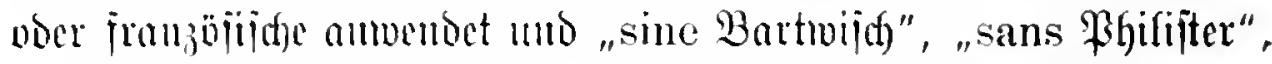

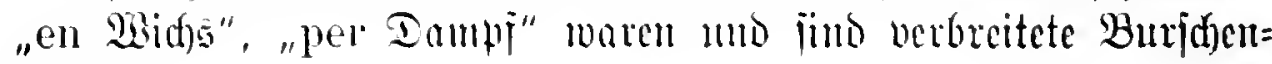
phrojent.

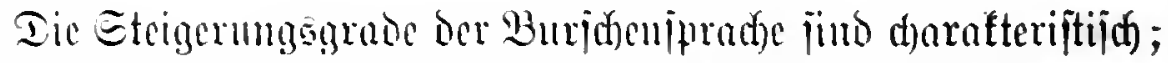
die Fillle un Begrifisiteigerungen Durde (Elativabverbien verbient

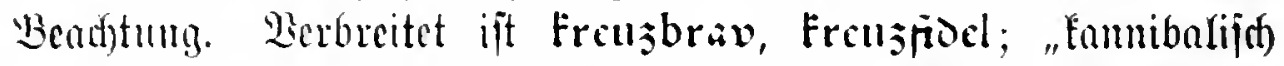
mobl" büren mir ans Etunentemmunde bei Govethe im Fouft;

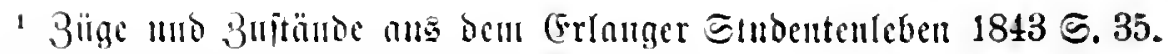
111. Tie Bufeliade 1829 bejengt bie Eppelei.

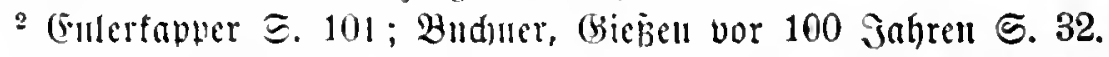

3 Sn ber 2olfsipradie ift Piarrei, Brobitci, Ted)anci zu vergleiden.

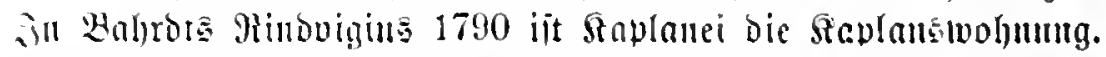

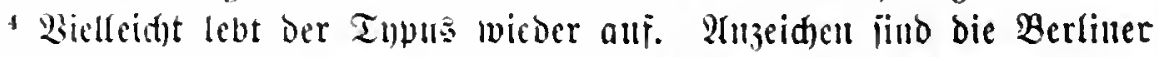

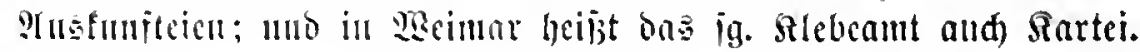




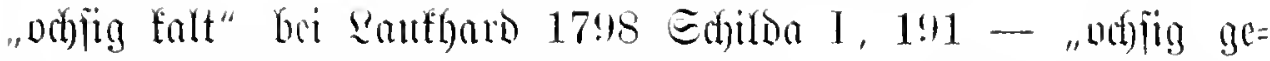

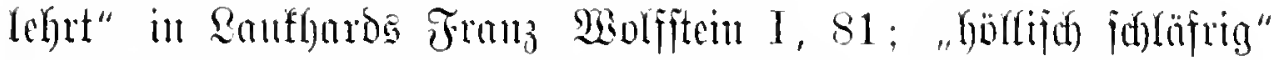

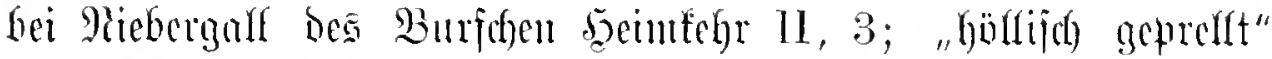

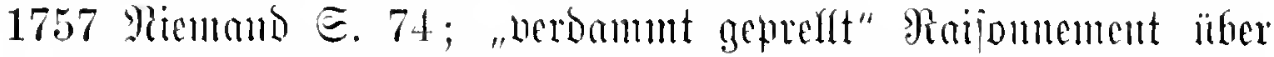

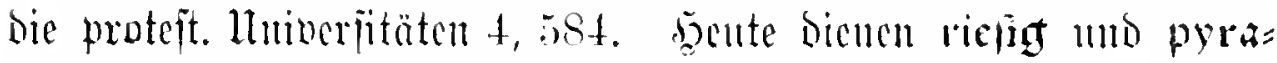
midal bem Bebürinis des Etmosnten, fräjtige Farben iür ịcitue Epractie zul bermenten.

\section{Uriprung ano Sierbreitung.}

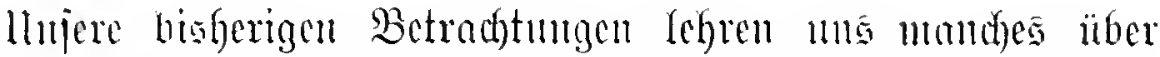

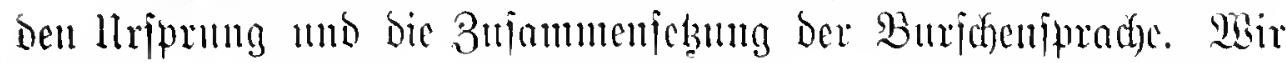
Gaben un bas 3abr 1700 cincul llmicthmung fennen gelernt, $100=$

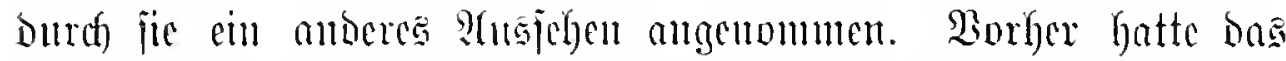

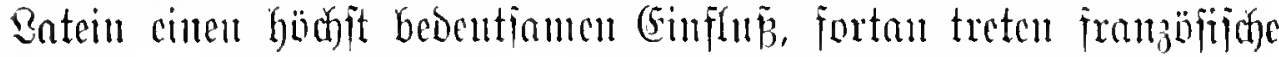
Elemente ftärfer herbor. Das Ratcin bat zahlreiche Sefmutateriafien

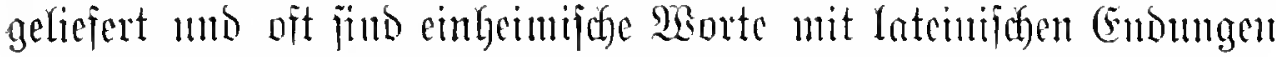

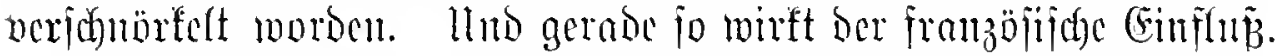

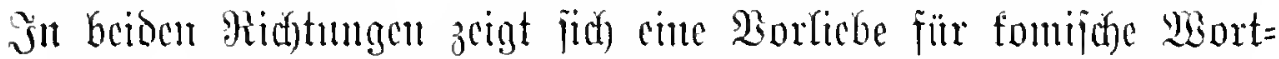

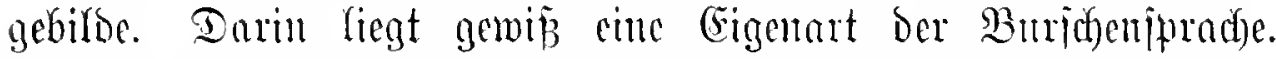

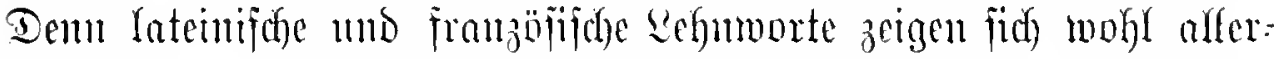

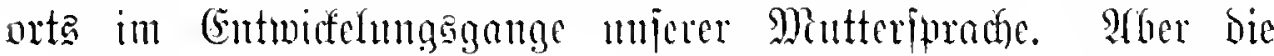

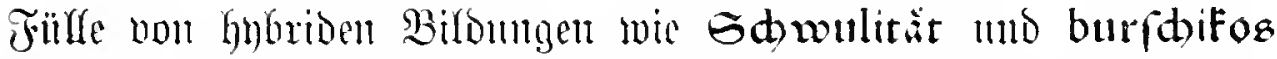

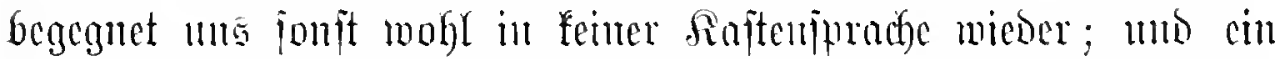

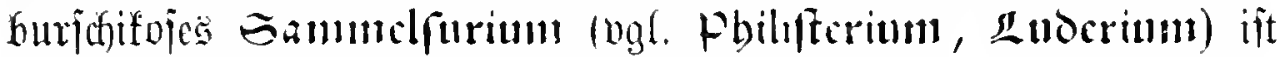

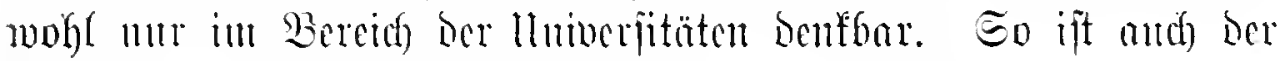

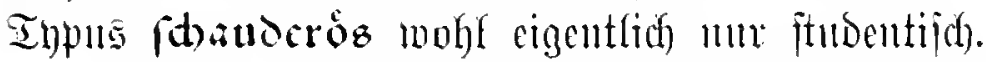

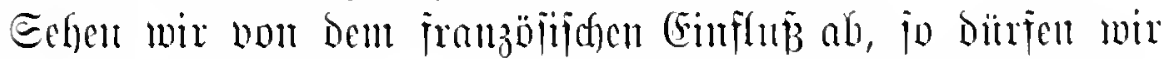

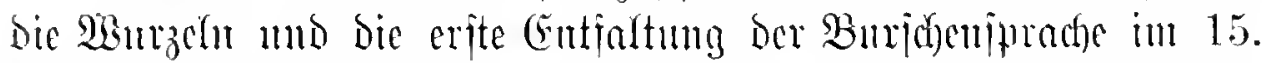
16. Jahihundert judden. Der Morttypus gaffatum muzelt in

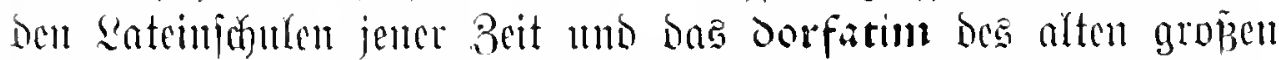
firambanbulifiebs non 1745 gebt jurïf anj ben Inpmes oppi-

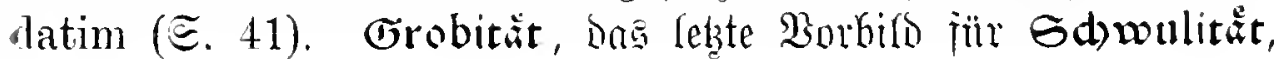




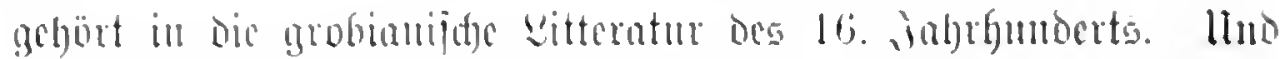

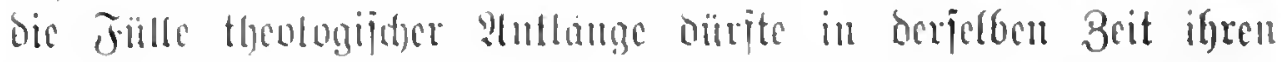

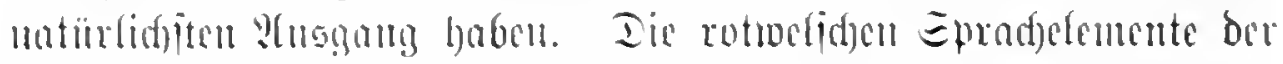

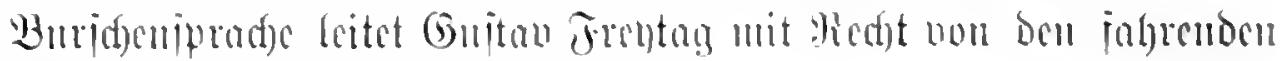

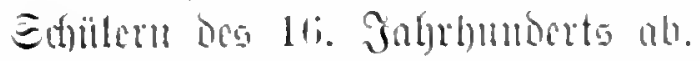

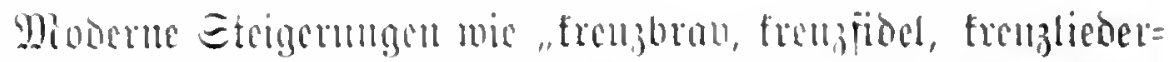

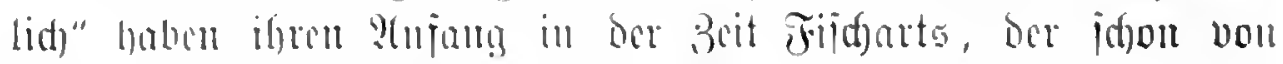

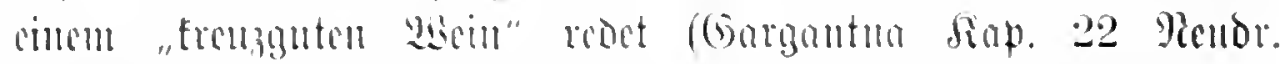

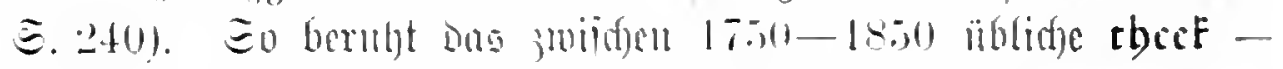

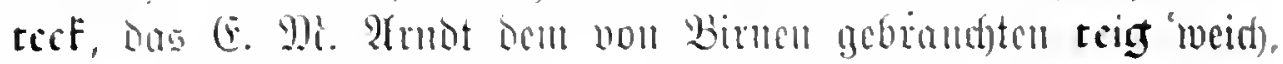

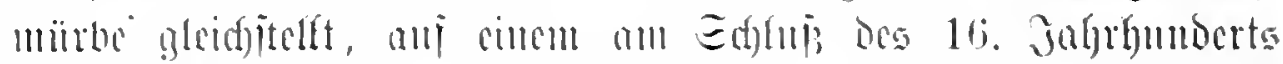

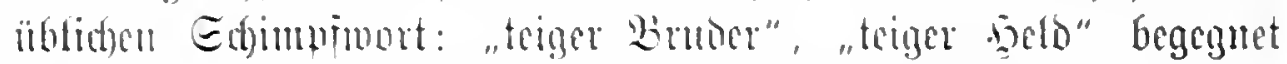

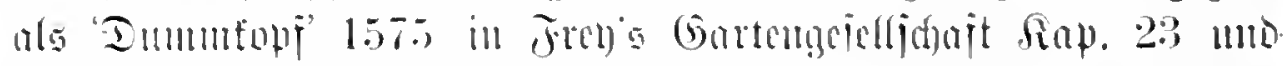

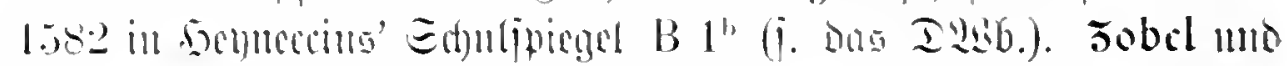

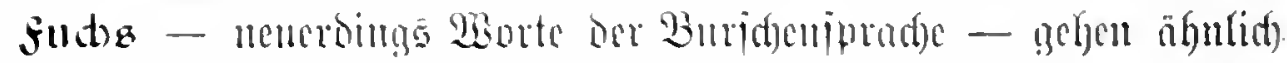

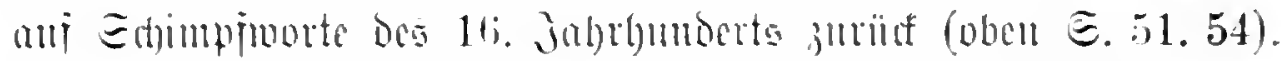

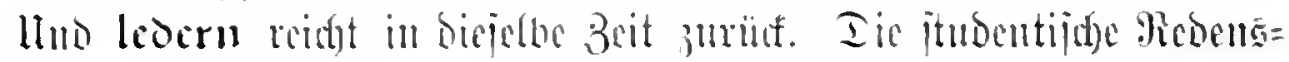

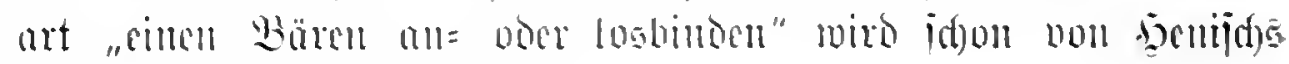

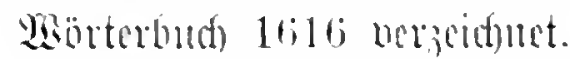

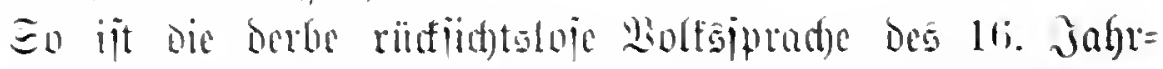

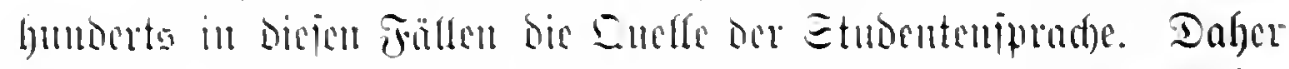

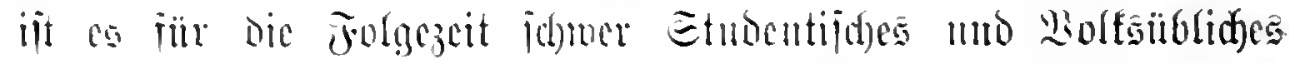

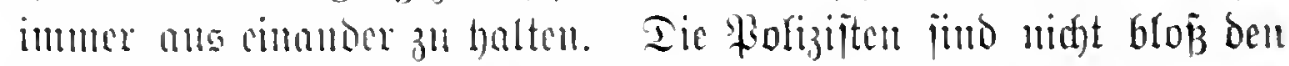

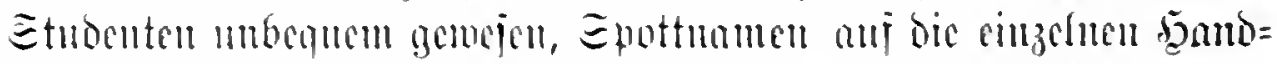

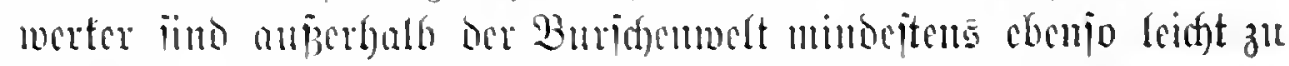

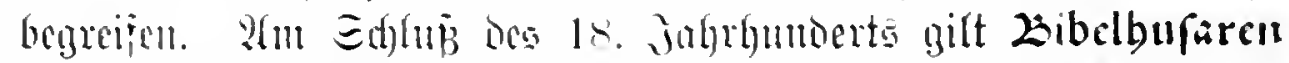

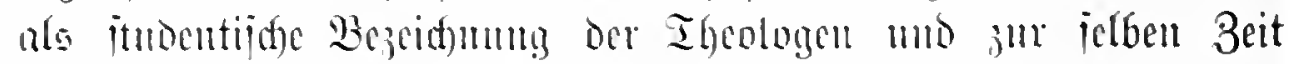

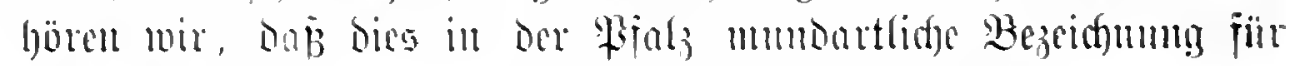

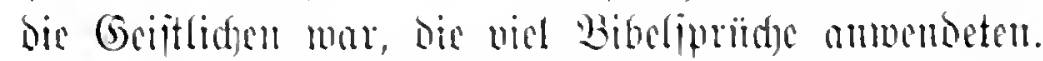

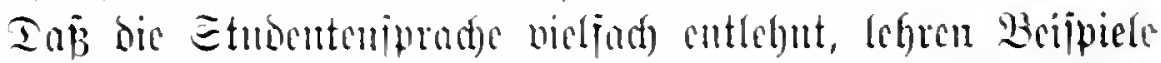

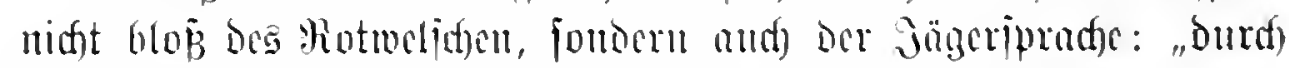

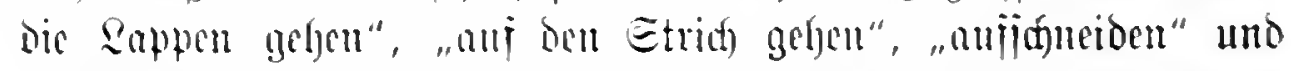

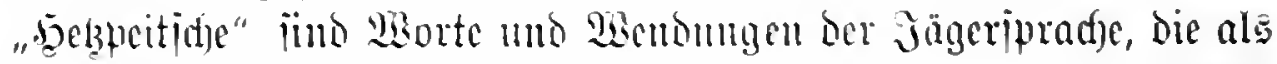

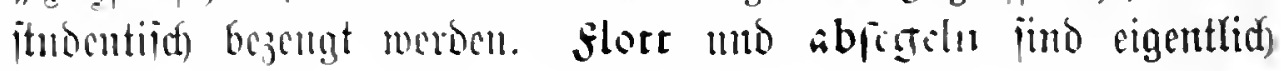




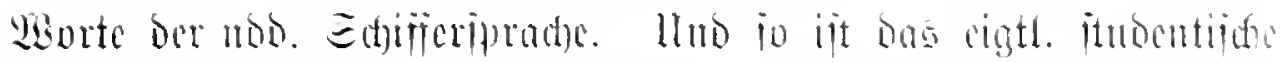

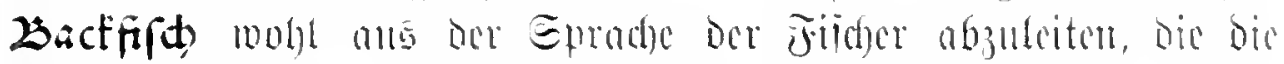

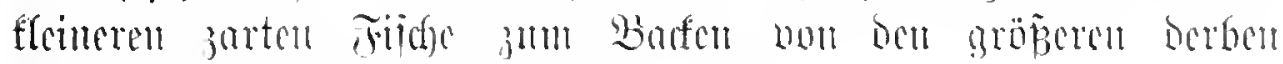

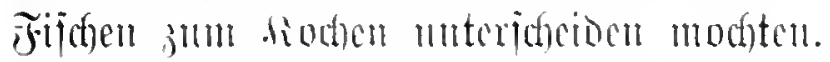

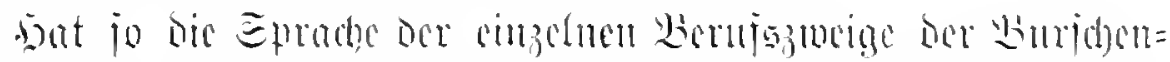

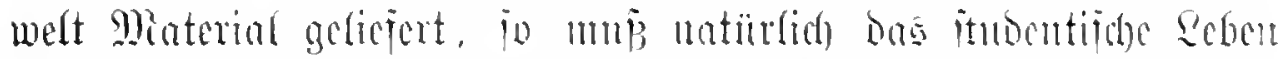

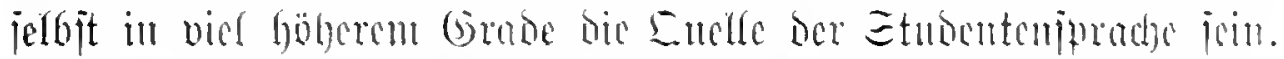
Mit ben 2Borten ungebobelt und ungerdyiffen itelen wir im

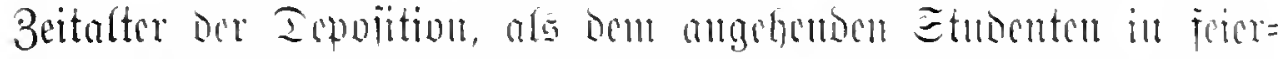

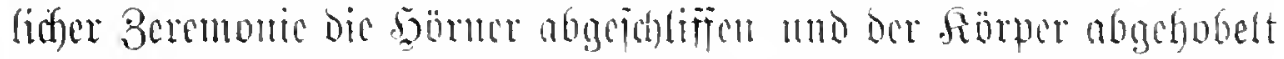

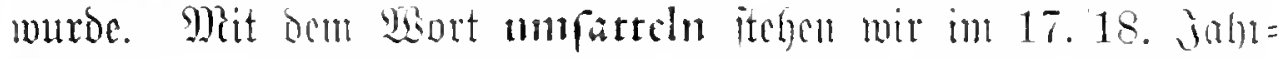

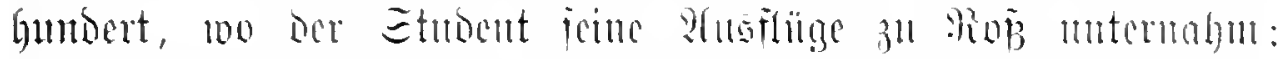

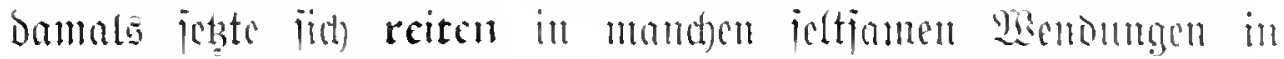

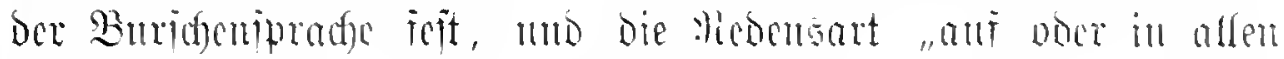

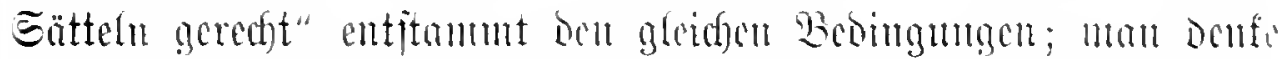

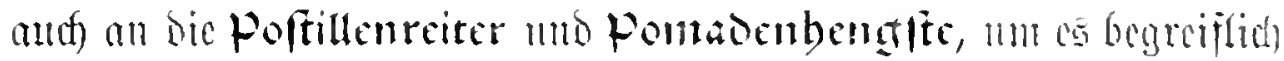

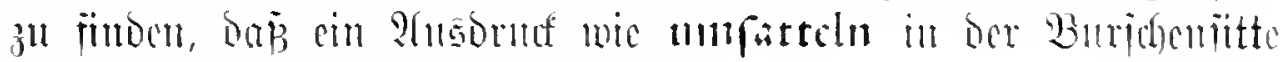
fritherer Gahroumberte murzeln farm.

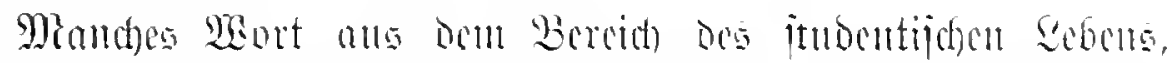

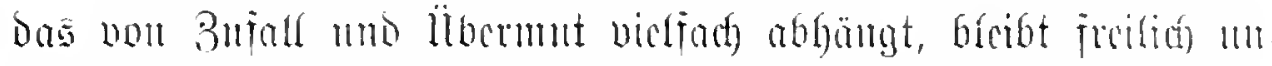

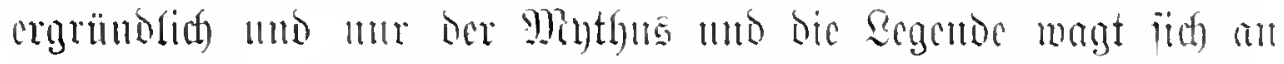
Dentungen. Ier Galumander miro nadh cintem Batuter llni=

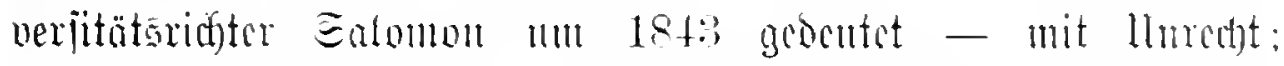

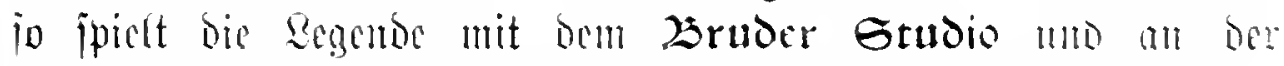

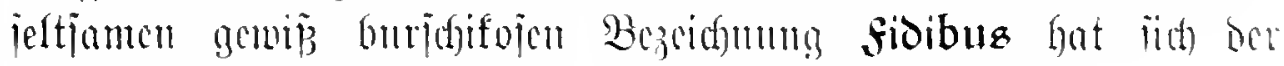

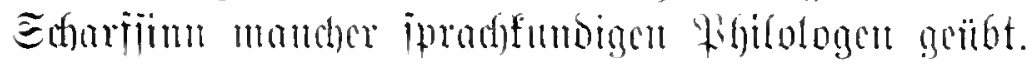

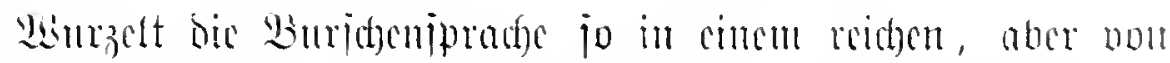

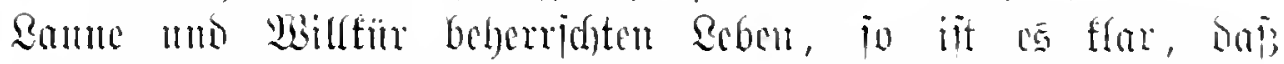
mantfes Dariu ans rein lofalen Bebingmigen hervorgegantgen icin

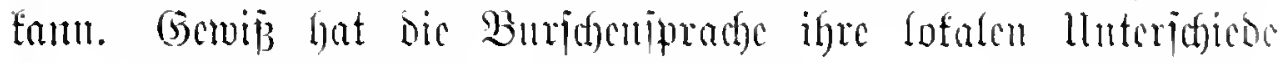
gefabt jeit alten 3citen. Ias kaftrum war unb blich natur=

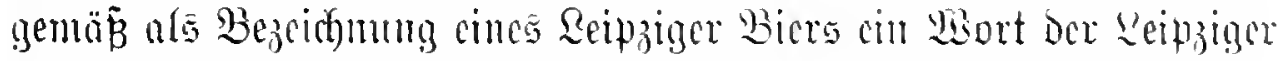

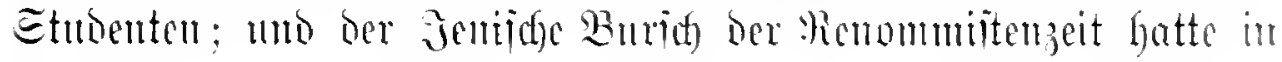


jeinem Epradjidfon einige jontit unthefunts Biernamen wie :Haul=

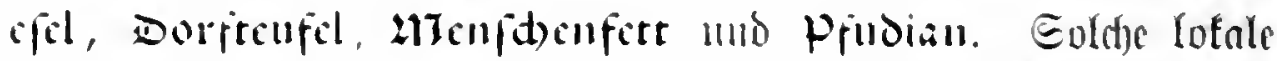

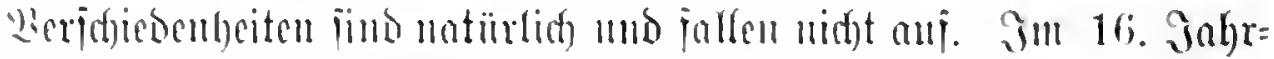

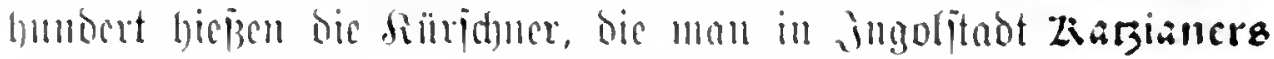
namte, bei bon Scipziger Etmoenten ziargenfdindor. Für bie

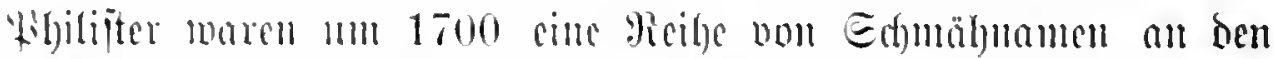

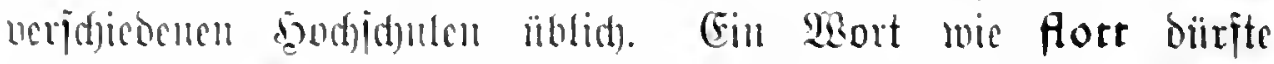
rigentlict) einer maritimen llninerfitätsitndt angebören. Haber

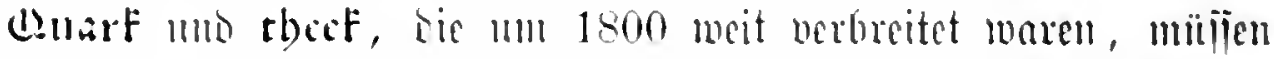

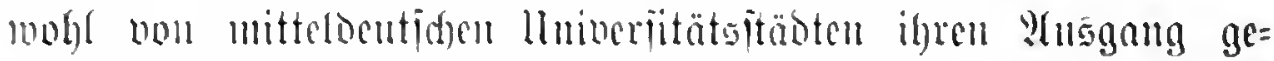

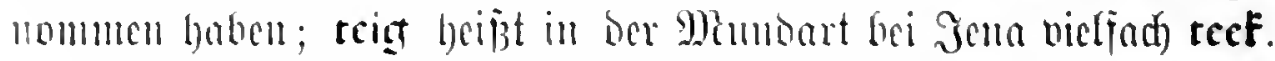

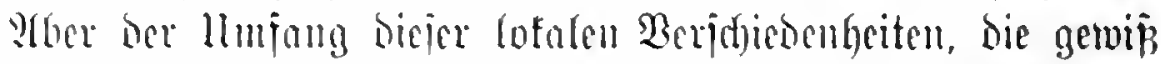

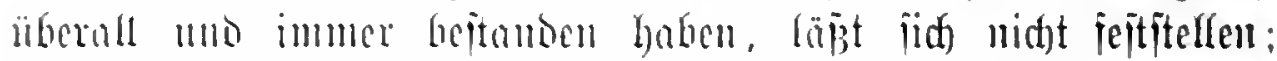

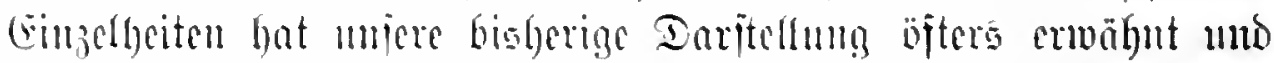

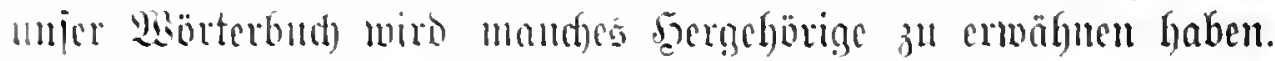

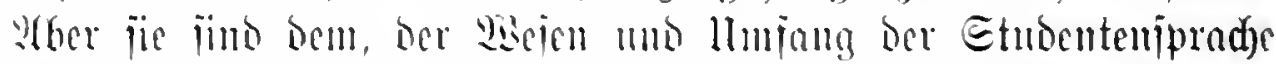

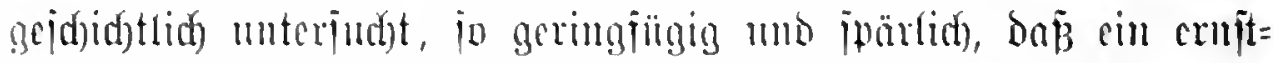

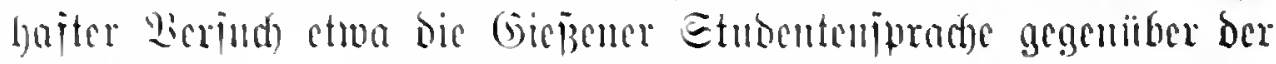

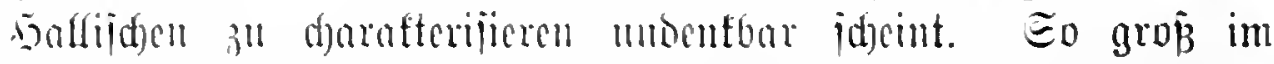

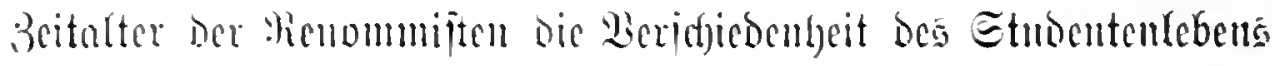

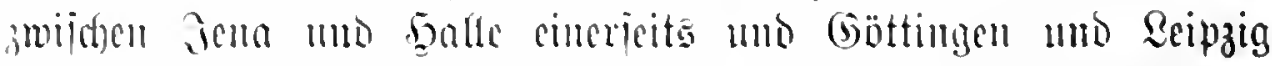

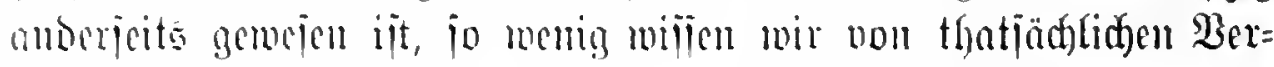

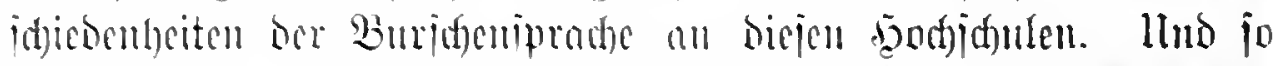

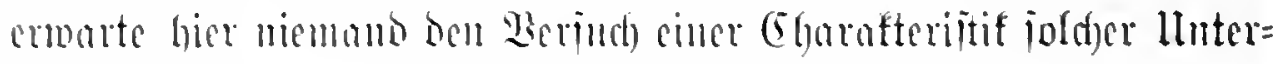
idjicos.

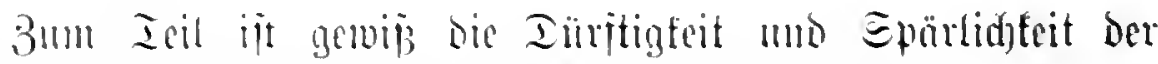

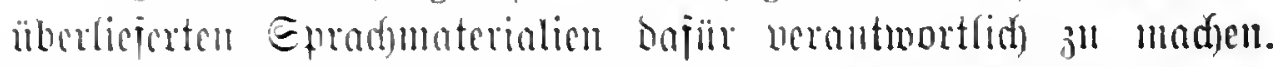

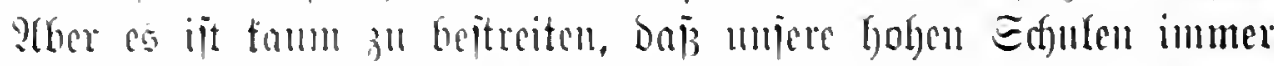

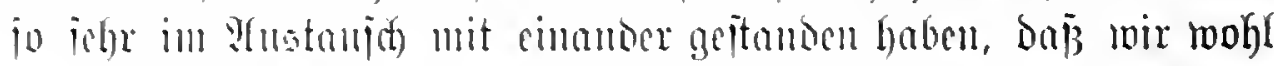
nu ciner cinbeitfidgen Emradje jür alle reben muijien. Jedes

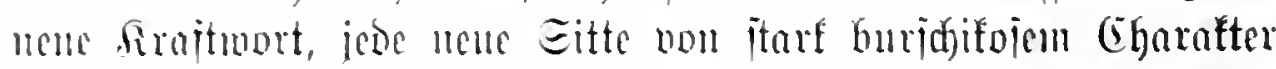

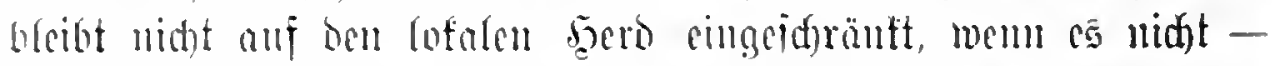
wie jenc Bientamen - mit lotalen Elementen nervadjen war.

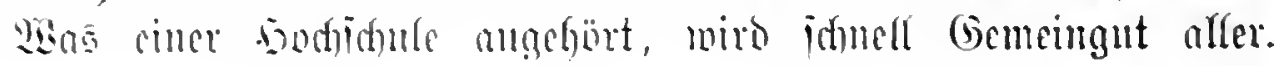




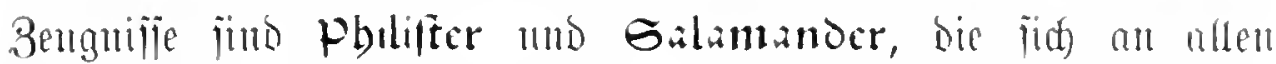
llniverjitäten cingebügert haben, aber cigentlich mur lofale 2 Borto getpejerr fillo.

Dies aber gibt ber Burifienturatige igren sert und ifre

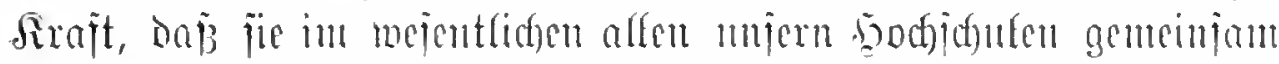
ijt. Das macht ïe itorf mo mächtig genng, um unter alfen

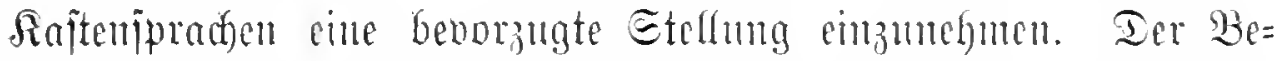

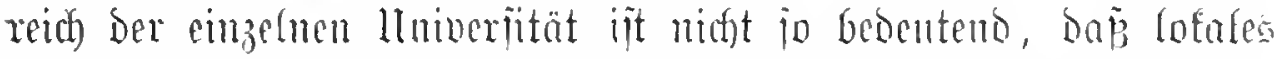

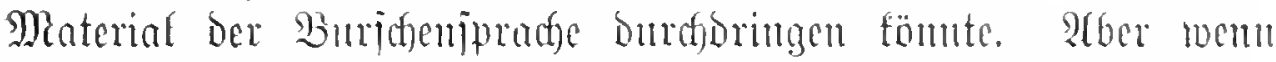

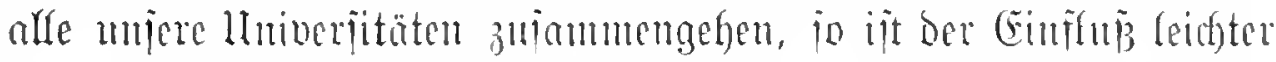
und nahbaftiger. 3u alfen Beiten hat baber nujere Etmoenten=

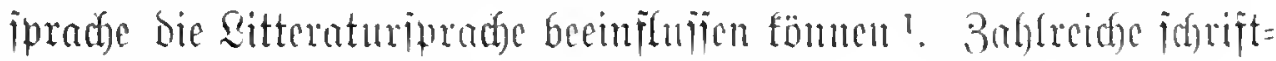
beuticte Sorte entitammen igr.

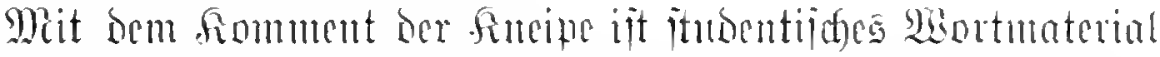
in bie meiteften Areije gedrungen - jo bie Morte Zincipe nmo

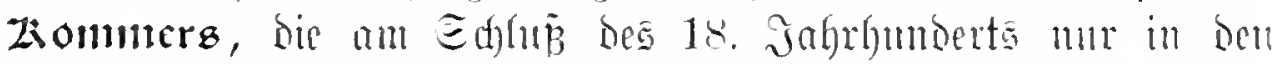

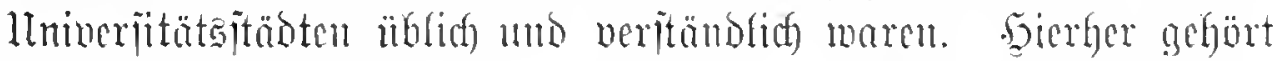
Das Bort sumpen, das im 16. mb 17. Jabrhmbert mur ber

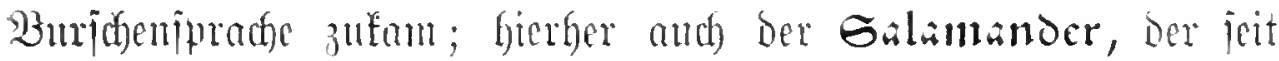
ben $60 \mathrm{er}$ Jahren meitere fireije gemomen hat, nadbem er zunor

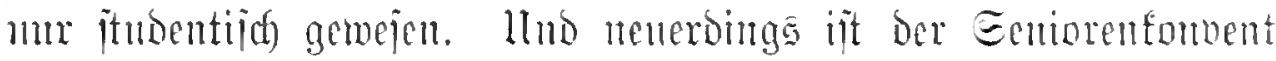

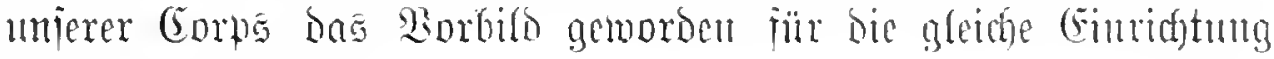
unjers Reichatags.

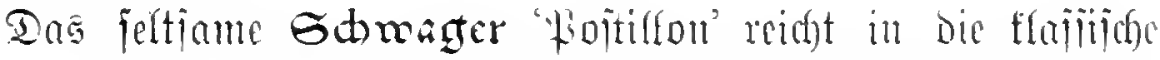
Sitteratur finein, gebout aber eigentlicf dor Burichenipracte der

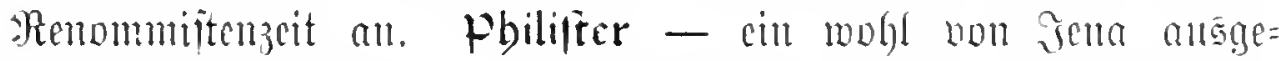
gangenes Burjocnwort - nimmt bei mijern in burjefifojen Eturm und Irang heranreifenten Alajifferm cinte cigene Basen=

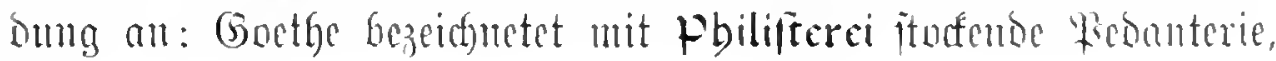

1 Ten 3ujammenfang Der Etmoenteniprad)e mit unierer Silteratur=

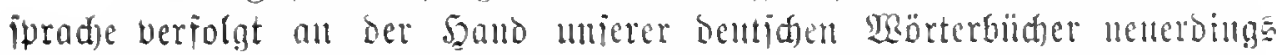

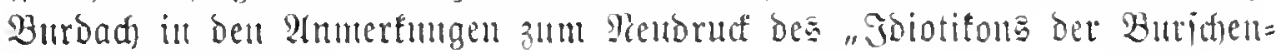
ipradee" voll 1795 . 


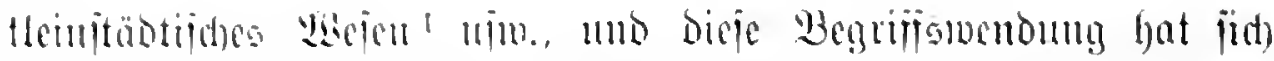

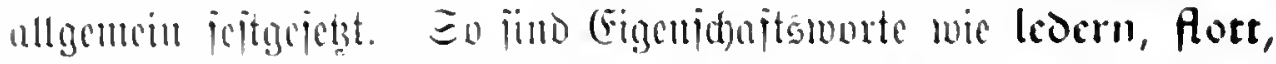

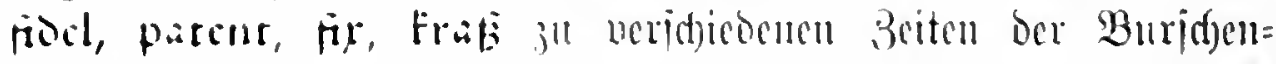

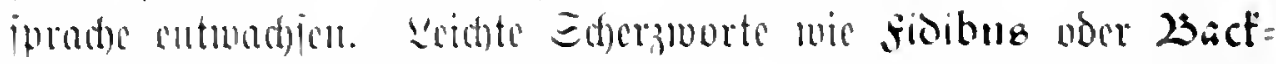

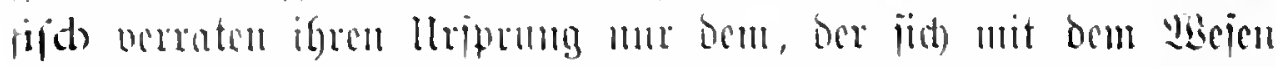
mijeres Etmoentenipradxe betaunt gemadft bat. 3eitworte wic

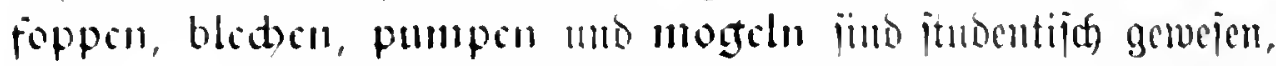

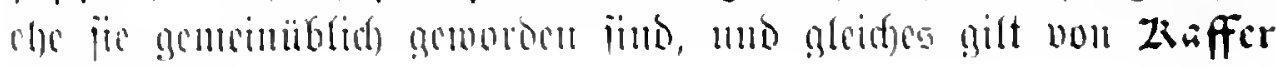
IIII) fit) oficl.

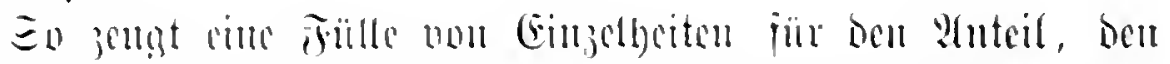

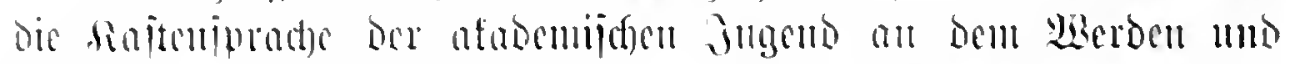

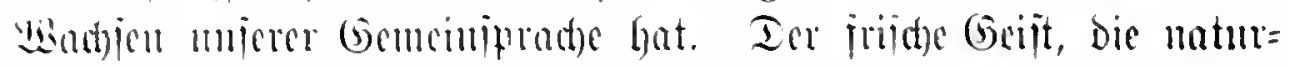

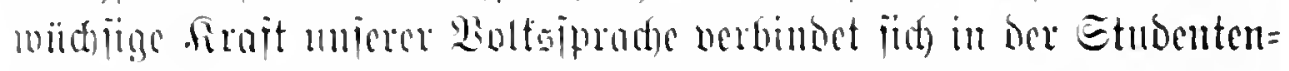

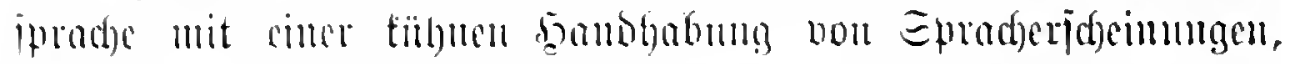

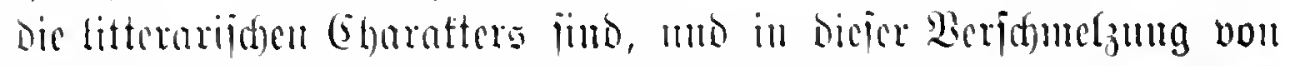

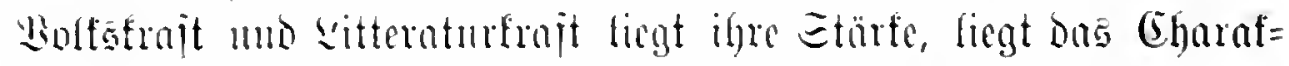

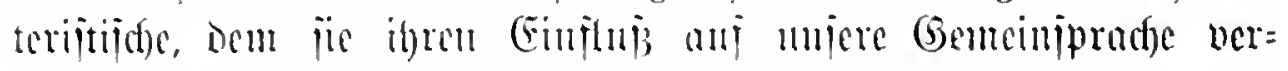

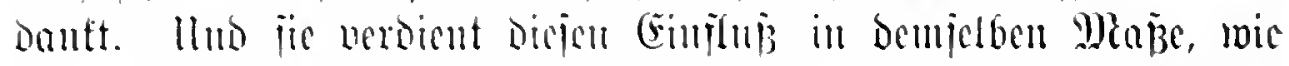

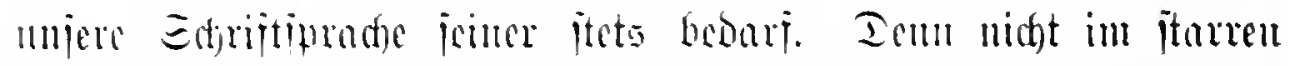

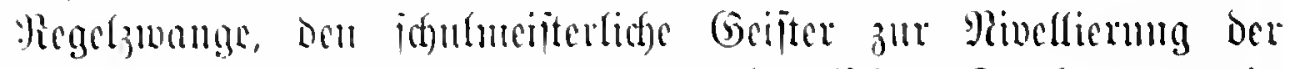

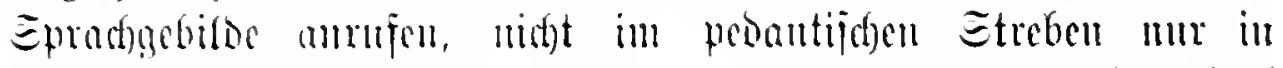
ang afgegrengtem llujang Vientrungen zul Dullow, liegt bas dzeil

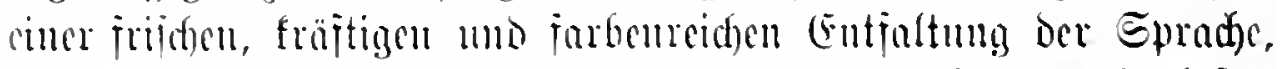

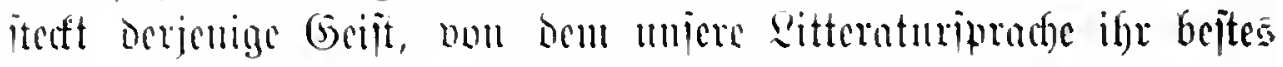

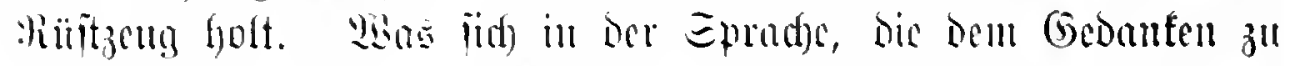

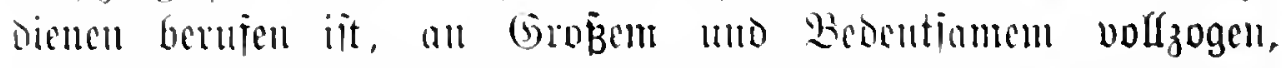

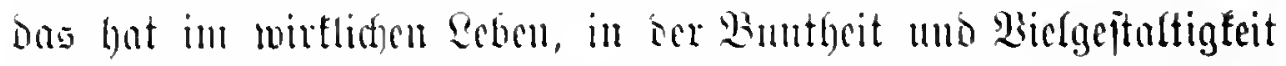

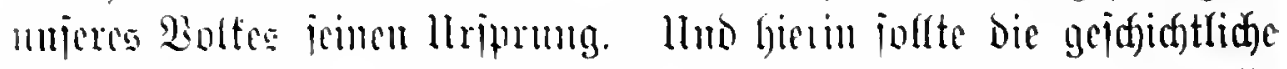

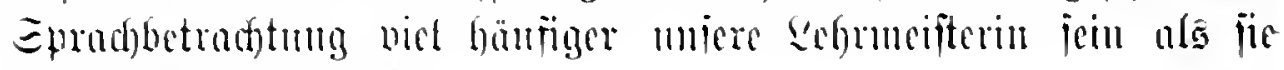
is in wistidffeit iit.

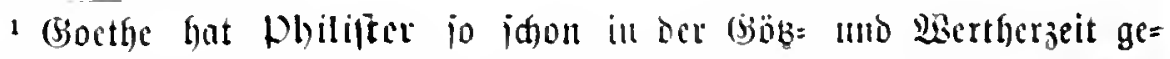
uraudt; vgl. Brieje II, 10. 11; Smtrger biotthe III, 245 und Mollen,

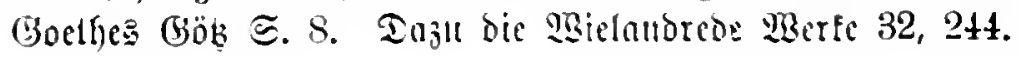




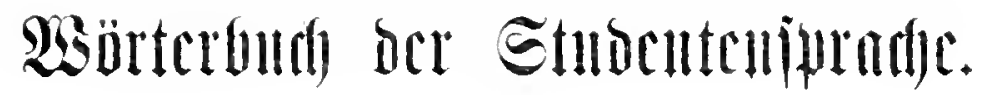

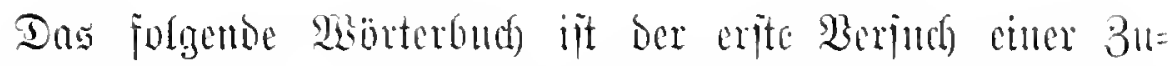

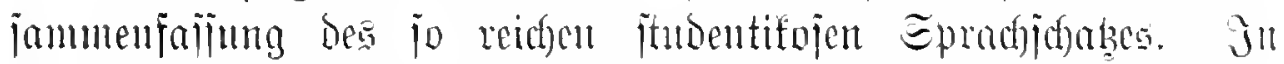

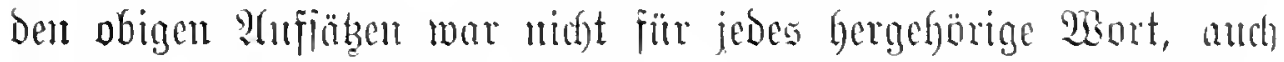

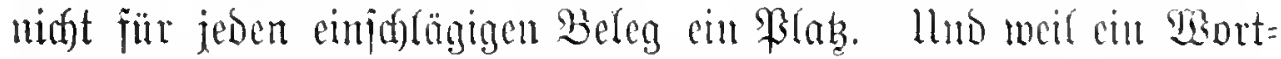

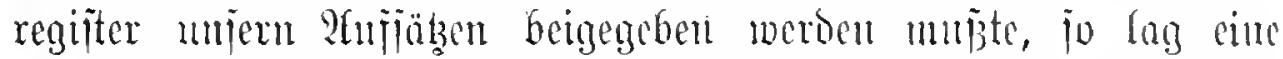

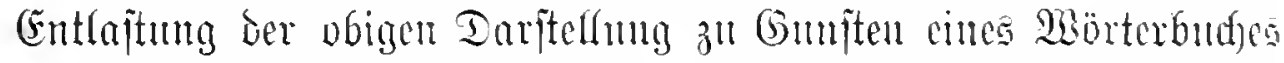

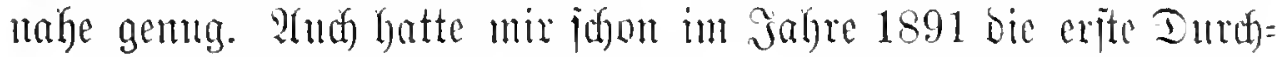

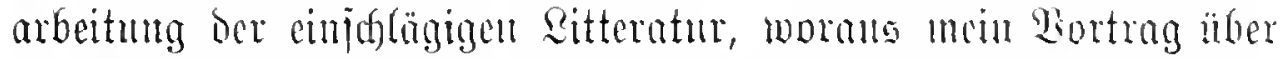

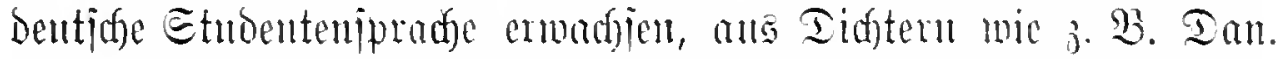
Stoppe voer ms jonjt mubefammter itudentifojer Sitteratur wie

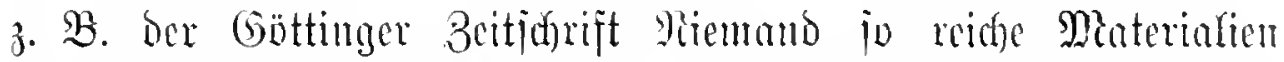

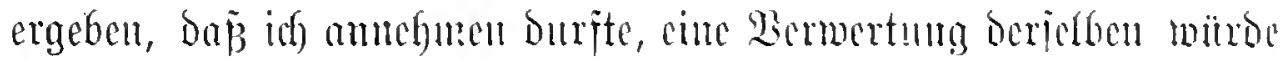
Dex bentichen Rexifographic jul Gute fommen. IIm jo cher Dumpte

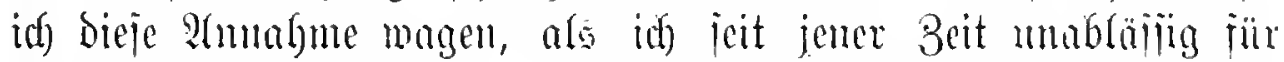

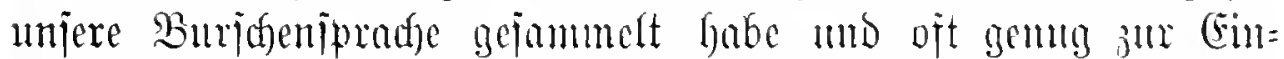
jitht gefommen bin, ba $\tilde{B}$ die Epradis mijerer Sitteratır, jogar

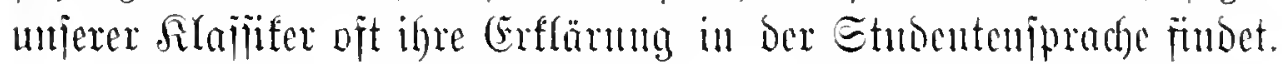

Das jolgende wärterbuch bietet bie Epradgmateriatien in

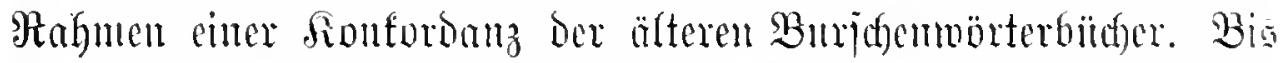

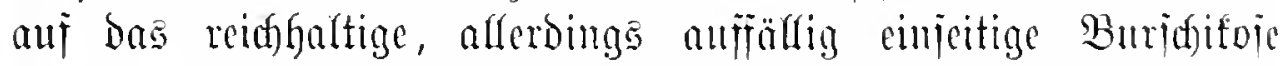

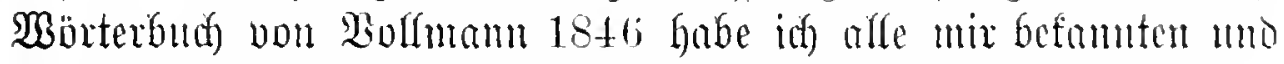

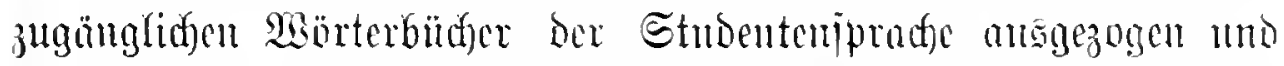
verarbeitet, jo baj bas ältere lexifalijge Matarial jebst bequen

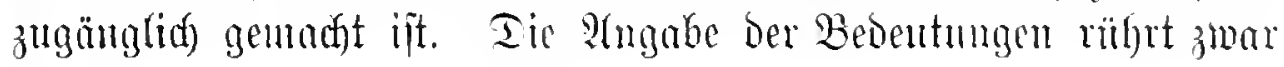




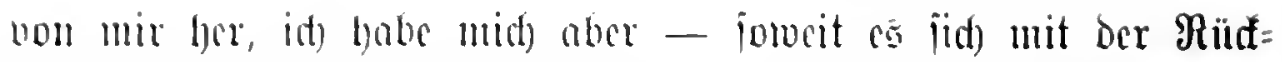

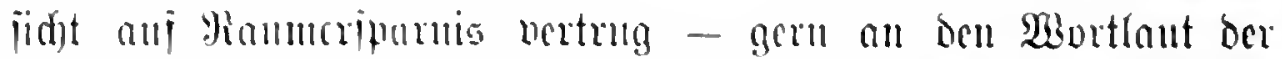

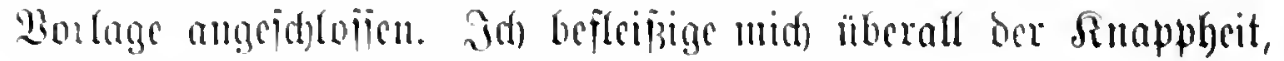

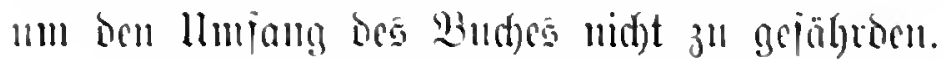

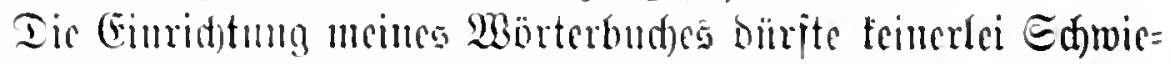

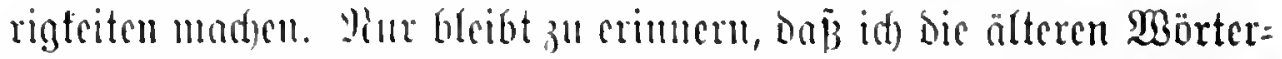
bïd)er als Euellen nur furz mo bünoig mit ber Jabreszabl ifres

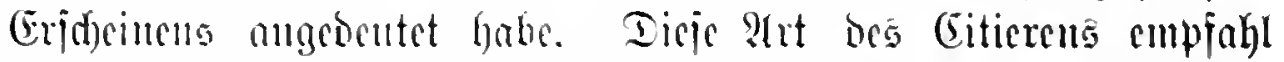

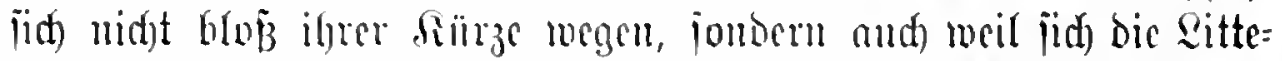
raturbelege um jo bentlidfer non ben leritalijaten Belegen abheben. llijere sitteratmebelege jind wobl an jid) Dentlid), fïr die lexi= falijd)en (Eitate aber gelten jolgente (Bleid)mngen:

$1749=$ Sergniigte $\mathfrak{M b e n d i t u n o e n , ~ E r f o r t ~ 1 7 4 9 , ~ 1 I , ~ 6 9 . ~} 353$.

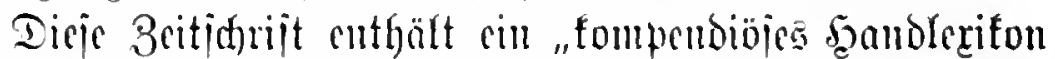

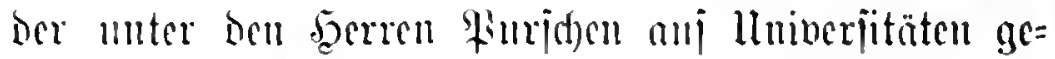
Gräud)(id)jen fimnjtwörter" non Ealmajus mit शlad)= trägen vou lirotax.

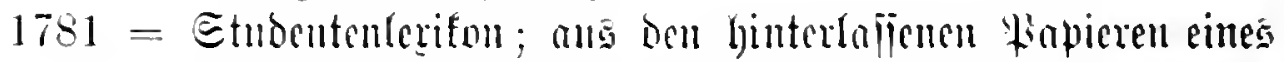

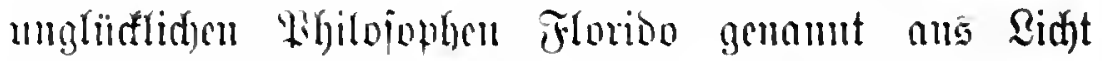

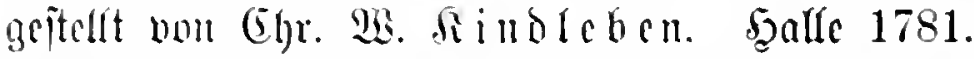

1795 = Demertumgen cincs Utabemifers Ḧber Salle und bejien Berovhner in Sriejen, nebjt cimem anbange enthalteno

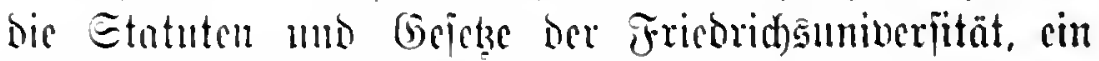
Sointifon der Buridenjprade unb ben ig. Buriden= fomment. Bermanion 1795.

Einen ?irubur Des Jointifons ber Burjuenjprade Darans mo Bemertmingen ïber Chr. Fr. Bernh. ?ugujtin alo Dierjajier des anoummen Budes betet Burbad) in Dem Budfe "Etudentenipradic und Etu=

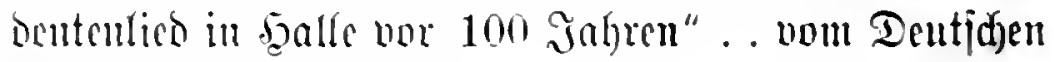
Y(benio in balle 1894.

$1813=$ Ier Büttinger Etmbent vorr Bemerfungen, Ratjoläge InL Belchrmmgen ïber Gïttingen und sas Etndenten= leben anj ber Gororgia Auguita. Göttingen 1813. 
$1822=$ Das Seben anj lluinerpitäten oder Daritellung aller Sitten uno Gebränthe 1. j. w. nebjt einem Berzectefnis

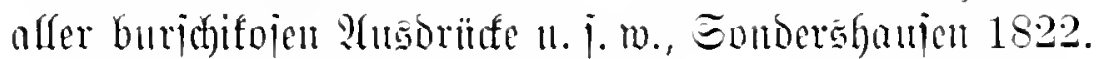

$1825=$ Etndentifojes Eomeriationslexifon oder Sehen, Eitten,

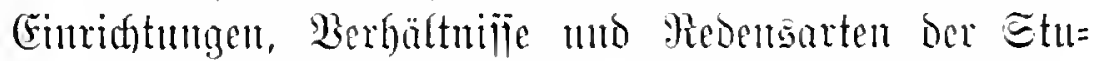

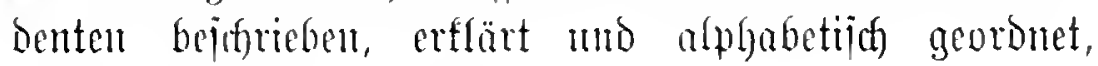
Reipzig 1825.

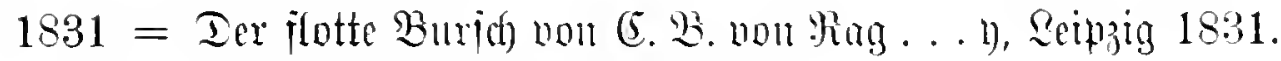
$1841=$ Etudentifoies Jobiotifon, Jenta $1 \varepsilon+1$.

Die ipäteren (shloijare ïno abjicttlich soentig zugezogen anf jie - wie überganpt anj bie Epradhe ber lebenton (bejeflecteter -

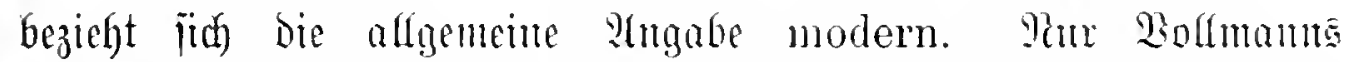

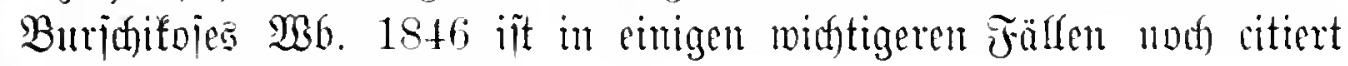
mit Der Jahreşahl 1846.

Iastnobeu $\left.{ }^{1}\right)$ alte idumbige $T a=$ batspieife 1781. 1795. 1811.

2) Sđhimpin. 1795 .

sbbanen weggehn 1791. $18+1$. abbrennen mit sinterlajint bon Squnloen burchgehn 1831. - $\mathfrak{a} \mathfrak{b}=$ gebranut ofne Beld 1846.

abowmmeru abjiehen, abgehu 1831. aboructell iterben 1781, 1795̃. -- „fi( abor." meggefur 1841.

abfabren ${ }^{\text {1) }}$ weggeldn ${ }^{2}$ ) iterben 1781. 1795. ${ }^{3}$ ) at(aufen 18+1. ${ }^{4}$ ) beim 19 ïr $=$ felu bie erforderliden $\mathscr{U}$ ugen nicht wer fen 1813. ${ }^{5}$ ) ịcinen 3wecf nidist er= reiden 18t6; ald Laukhard 1800 Erzälungen u. Novellen I, :85. abfallen ertappen (bej. beim Inell; aud) "bie $23 a$ fifen -“") 1831.

abfingern (,Iu faumit Tir bas (ceid)t -“) erfläten 1813. abfübrell 1) jent. mit 2ierweig meg=

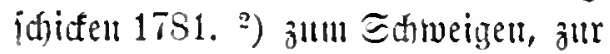
Ruhe bringen ( $j$. B. ,denl Serl habe id) gut abgejiihnrt") $1831:$, ,utit (5ilauz abgeïint" glänzenb beiiegt 1846 . abgel)n bie llniverịtät verlajien (mit Bejriedigung ber (3länbiger; (5g). jtijice 1i) 1781. 1795. 1841.

abteilen abfanjen 1831. abflaviren $=a b j i$ ger 1813. abtileifen jent. etw. abloffen 1841. ablallen jent. Dutch jein Betragent zum llnwillen reizen (= an (anien) 1795. 1841 ; ,er loat einen ablaujen, abfahreu lafien" $18+1$.

ablegen ${ }^{1}$ ) nidht beittehen (,er legt

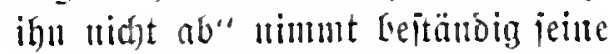
Bartie) $1781 ;{ }^{2}$ ) gebäreı (hei. am Geim(it)en Drt) 1781. 1795̃. 18+1. 
Rörterbud) der Etmbenteriprad)e.

abludion = 14dicu 18t1. :bomutten aburiigeln 1813. abrapiten ') iid orïfell, weygebn 1751. 1795. 1831. 18t1. 2) iterten 1795. 1841.

abronsmmeren lem. Sil iibertreffon juden Ciaudy. Werke III, 16.

sbrutidon idfuell bie lluineriitüt verlaijen miiiien 1831.

?lbithicosuchicl beiu ?thgang bou ber llıiverjität ăแ Edulocutilgma 1813 (unter $\mathfrak{B}$ ed) jel).

abjarammen milder als abbrell= uc $1 \mathrm{~S} 31$.

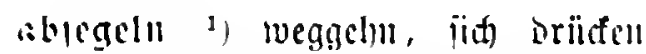
1781. 1795. 1831. 1811. 2) iterhen 1795 .

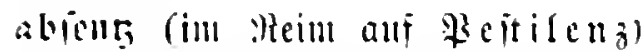
abicite Laukhard Schilda I, 190. abjocten fortgehn 18t6. Laukhard Schilda I, 3reg.

"bionser" in ber Mebentart „it

Jin abgrionbert" id) habe mein Gelo Leripielt mo ivicle midst melor mit $1795.18+1$.

abstemin. entfaltian 1769) (beid).

Gött. હtIlo. III, 294. :bituten abjahrell 1846.

abifolicn (Bscld) jum Eergnitgen anberer opiern 1813.

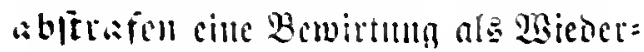
vergelemgerandugen (man itrait jem.

ab, bell mall juvor traftirt (hat) 1813. abtreten lafjen jem. von der Meniur vecijen 1831. abtrollen weggehn 1781. 1795. 1841. abjichen jid) bavon madell 1831. 1846 .

sicenticren bas vorgetrunfelte Duan= tum Leim . fommers 1831 .
IIticr|tuseut Ëlonom 1781; $\mathfrak{B e r =}$ walter, Julpeftor 1831. - Anbers bei Ericler 1691.

sianurieren i) ill Epiel getwimnen 2) iiberh. ctw. crlaugen (and burd) सorgell ober Eteblell); aud „eine :Icaniiition mad)en" 1795. Laukhard. Emigranten II, 224.

Ilema Hicifenfovf Fischer 1781 Burschiade 6.5 .

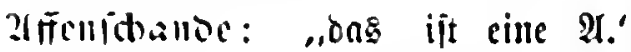
modern.

?llunug:, teinc - " Hcill modern. Iltumith Stubent nad) bem 3. Se= meiter 1822.

?llter 2ater 1825. 1846.

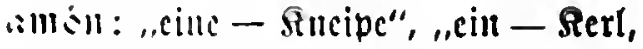
Mäod)en" 1831. Gaudy I, 187. (illsuldell: ,ilid) eill Duartier viuc Bube mieten Hippel 1779 (Lebenslänfe II, 122 Göschen). allumsen: ,mit jem. -". jid mit jell. in cincu @treit einlaffen 1749. 1781. 1831. - "fltrz angebunben" leidt anfgebradit 1781. Bgl, $\mathfrak{B}$ är mitio at alb.

"ubremen: ,nid)t gern - laffen" alles gern mitmad)en 1813 (unter flot 0 ).

allosumern:" jem. cine Bumbe im Tuell beiluringen 1831.

?moiente cinc bejt. Duellwumbe 1831; 1845 Burschenfahrten 5.102. wroblen anjduauken 1795 (unter (a $\|$ j (f) $u$ ard e e l).

suribren betrïgen; "angefïbrt toer= sen" Şadon erleiden 1795. 2Iniulyer wer einell feierliden 3ug anfiil)rt 1795 (unter $\mathfrak{B} e\{$ d l ieß̧er). ?ugitrible Cnlimberhut modern. 
anbángen: „jem. eine Partie Billaro anly." abgewimen 1749 .

2(unbieb bor 1. 5rieb ill eitem slenims= gange modern.

anboien 1), ifich anf." jich bie sojen anąichen 1781. iiferhomt jid) an= fleiden 1795. 18t1. ${ }^{2}$ ) зит Mcuim anbanbagiren modern.

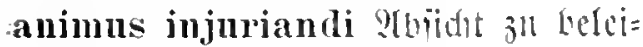
bigen 1825. 1846.

sutenlen amidatien 1795. 1813 (,Gero ante" (Gelo bejdatiten 1781. 1795. 1831. 1841). - jem. fïr eime 2 ere binbung ju getwinnen ind en 1822. 1825. 1831. 1841 2c. - 3miammen= trommteln Fischer 1781 burschiade ธ. 19.

anlaufen jem. ourdj jein Betragen unvillig matfen 1795. "jem. aul. Iaijen" Salinde $17 \pm \pm$ ๔. 89.

supergen Demuziren 1781. 179ŏ. 1841. arpimpen $1813=$ a npแmpen.

astpumpen (trani. Geto van jem. borgen 1813. 1825. 183126. suquslen (B̈̈̈her, siteiber) anthafien 1781.

suracen etro. mit Mithe herbeildaifen 1781.

sureifien: angerijicn angetrmfent 1816.

anfacilien 1) betriigen, aniiifrem (bej. mit sidntbezalylung ber Edmlden) 1781. 1795.1831. 18t1 2c. $-{ }^{2}$ ) im Drell eine beit. 2Bunbe beibringen 1749. 1781. 1795. 1822. 1825. 1831. 1841 (angejuijic un twerben eine

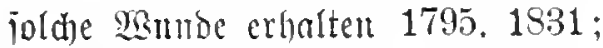
Laukhard, Emigranten II, 42)). 3) Eei Bierfehden jein (stas znerit antrinfen 1825 zc. ${ }^{4}$ ) inphilitija ant =

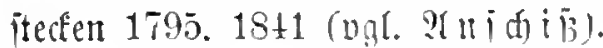

Znithif 1) eine beit. 然mbe in Duell 1781. 1795. 1822 2.; ; alld Hauff, Nemoiren d. Sat. Rał. 8. (Dazu

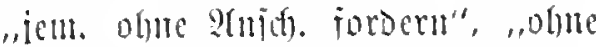
2(mid). losgehn" 1831.) ") eine vene= riide Sultedung 1781. 1795. 1531 . $18+1$.

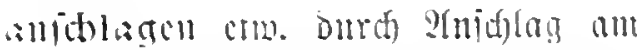

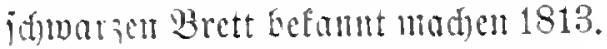

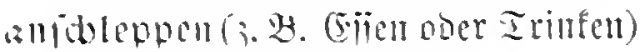
berbeibolen 1813. 1831.

(nllithmicren jen. beim Serfanfen iiker= vortcilen oser Eetrïgen 1781. 1795. 1stl. - ,iem. chw. anjim." ami= oringen Fischer 1781 Burschiade כ. วัวิ.

amithuallen: ,iidh etw. anidumallen" anióaffen 1781. 1795. 1831; j. 迟。

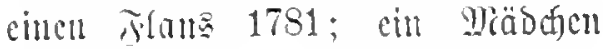

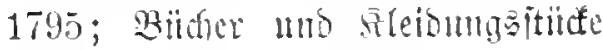
1831 .

: 1781, 1795. 1St1.

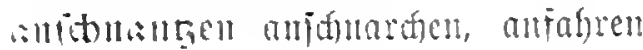
1781.

:mithullen cinem cins zurrinten à la ronde 1781.

arbuntren anjabren Laukhard 1798 Schilda I, 137.

amithoten anid)affen, herbeifhaffen 1822. 1831. 1811.

2lufalt: "Ëniglifye 2fnit." lupanar 1795

?minano vornebme Gejellidat, bej. Damengeiellifaft 1831.

aniramoig: "cin anit-er Whilijter" ber gern ptmtut 1831 .

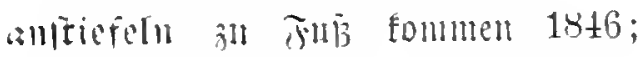
"angerticielt fommen" modern.

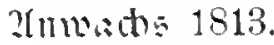


amushlew antoumen (, ,id) werbe halb anw.") 1813. - ") lherbei= bringen 1822. 1841; "antu. Iajien", (3. 24. Tabat) holen lajien 1813. amvithen ctu. anidarifen 1781. alsapfen anpumpen 1831. :mjtchen" biebe andenten obme lie ju id)lagen 2), „oen Plilititer anz."

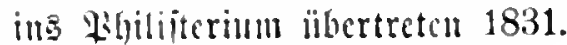

appellieren romere $1749.18+1$. "llad) Darmz rejp. Eveicr app." vomere 1795 (unter ipeicu).

?trminthier leidhte Duellumbe am Irm 1781. 1795. 15t1; aud) Mart. Schluck 16.

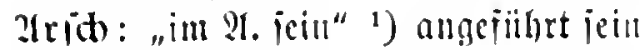
1525. 1831. ${ }^{2}$ ) im Dref, in Ped jein 1846. - „iem. alli bell $\mathfrak{H}$. jeken" jem. iatwer vermumben 1795. Zrfitpander Eraicher 1825. 1831. 1846. - arichereinlid mahridjeist= (iti) 1846.

Slithfuthen Topisuden 1795.

2lthen llniverịtät产tabt in ङaal:

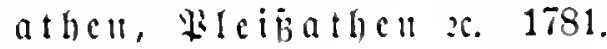
1795 und jonit.

IItrention Mui Des Pointeurs beim 5ajarbipicl 1795.

attilibe :Tadht fibele Yiadit 1829) Buckeliate હ. 65; 1846 (aud) $\mathfrak{I} t$ tica).

auf sic "lisulur! Rut im Begim

bes ভlandals 1831. 1St6.

"ufbinsen, aurhangen, aufheften jem. jalides glauben mad)en 1781. : 11 brummen (a. B. "eiten bummen Suntgen") cime provojicrente He= leidiguta äuß̉em 1831. 1841. O. L.

B. Wolff Naturgeseh d. d. Stud. "zufsomern: "iił aufo." (ïd) aut=

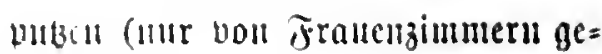
jagt) 1531.

suftlaviercll: "iid) alfel." fid auf= puben (৩. Ilämแerı) 1831. anjtorgen besahlen, traftieren 1749. :uffratien (ïd) busen 1781. 1795. 1531. 1841. - auigetrabt ver= guiignt 1781. 1831. - "auffraken Ialien" autiiden lailen 183 . " madhen ober aud jür Minjitanten, Pluibürter w. i. w. Gelo zujammen= brimgen 1795.

a minugen tleine jebler jem. über= treiteno vormerien 1781. 1795. :arpartill jid fortmachell Laukhard 1798 Carl Magnus S. 4 . :ufpalien aujuertian jein 1781. 1795. auriobren animuben 1781, 1795. surlacten auf́abcn, aujbüroen 1781. 1795; jem. eime Süge als $\mathfrak{B a h a b h e i t}$ aurbindell $18+1$.

aufithuciocu mit llebertreibungen er= zäle 1795.

allifbiniln Gitien in vorzitglider glenge herbeijdarfen 1795. 1841. anfiteden verraten (in Deitr. Baiern) Kleins Prov.-Wb. 1792. anfianen lebhajt iverben 1846

Schnabels U.-Jahre 1835 ऽ. 236.

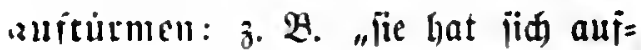
getiirmt" von Tamen mit hoher Jrijur 1781. 1795. $18+1$.

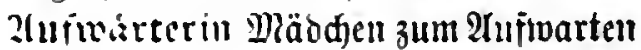
1781. 1795.

alfwidbien, allwiren ') Epeife.und Iraul ieinen Bäjiten aujtragen \affen, vorjeken 1781. 1795. 1831. 1841. and) Mart.Schluck 1798v.Burschenkomment $\subseteq .25$; Laukhard, Eulerkapper ङ. 105 - v. Gebrian II, 
62. 2) Stuat madyen (j. 9 i i (f) 1795. 18t1: "iid) anim." iid, iehr elegant fleiben 1831. 3) (bas (5igrent= wort) gebell 1841.

anfieben ") jem. narren, neften 1781. 1795. ') aufiduciben 179.5. $18 \pm 1$. Daber lufjug cine gar zul hod ge= ipannte uno ansgedelute (5rzälung 1795.

21 urjug feierlider Êtudentenzug 1795. 1813. 1846 (i. a lifjiefen).

2lugen: ,in bie - ited)en" gejallen 1795.

allsfallen ${ }^{1}$ ) Geld herangrïfen Laukhard 1800 Erzälungen und $\mathrm{N}_{0}$ vellen I, 251. 2) beim 厄̧fagent und auf der Meniur 1831; Daz̆l ber II UE a a II 1831.

allsfedten in ciller छtreitiad)e bie Sberhand behalten 1781 unter fecten.

ansflitten prïgeln Mart. Schluck, v. Burschenkomment $\$ 13$.

a 11 śrill)teu itibiken 1831.

anshanen keim Duell rejp. Duellgang 孔lerit hauen 1813. 1825. 1831. Daวu Zushieb eriter siteb 1795. 1813. 1825. 1841.

"arbolen "jem. bie Sïmite abfragen" 1781.

austlingeln ") jem. heruntermadien

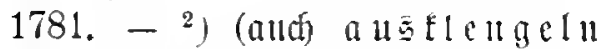
1795. 1841) im Eviel bas (belo ab. แehmen 1781. 1795. 1841.

ansfueifen heimlia burdgedn 1831.

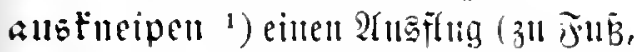
殀Ferb oder Magen) madjen 1813. 2) allg̨ghn 1822; ipaziren gelu,

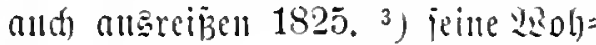
แung veränbern, auşzieben 1831. 4) cinen Tallz, Bier= ober Meitl= Rluвe, Deutide Etutentenjprade. tuifi bejuten 1831; die Aneipe be: jud)ell $18+1$.

allsforgen romere; aud) "bas ijt 3u! 2f แ立." 1831.

sllatramen vorbringen 1795 (unter e i lt ba fe e ll). 1St1; erzählell Fischer 1781 Burschiade S.41: Laukhard 1800 Marki v. Gebrian I, 39. anstrabell entrliehen 1825. 1846. Inslage cime beit. Fechteritellung;

baju fint anslegen 1813, 1822. 1825. 1831.

allslcoen Durdiprïgeln 1841. "asmadou: "cimen Efandal ausin." iif) Duelliren 1822. 1825. 1831 2c.; and eine Bieriegde 1831.

aแsmifren jem. im Epiel Gseld abge= twinuen 1795. 1841.

allspreifeu ') bent atab. Eefrer in ber

Rorlejung jebes Mipipjallen burd)

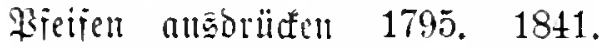

3) Willfommen Der Fithje von ben älteren Ettroenten 1781. 21 sputger fleiner vermeis 1781. ausciumelt gebn Laukhard 1802

Anecdotenbuch I, 231. atureitien entfliehen 1795 . alloridten ${ }^{1}$ ) angehn; ${ }^{2}$ ) bie lluiv. per consilium verfajien miijien 1831.

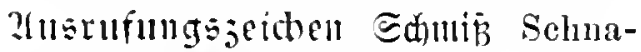
bels Unir.-Jahre 厄. 315 . allsibarren zum Beidjen des Mi jallets im Solleg idarren 1813. allsidmieten ') abidreiben 1781. $\left.1795 .{ }^{2}\right)$ burdprügetn, idflagen 1781. 1795. 1813. 1841. 3) Eeim Spiel (bej. Billarb) viel (Geld abgemimen 1795. 1813. 1841.

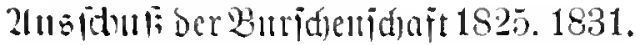
ausipeien bezahfen, traftiren 1749. allofechen (trani.) Sen Pang abge= 
wimlu (bej. in ber (B)mit ber Wlä̀: d)el1) $1781.1795 .18+1$.

: deutsehe Student S. 11.

allstrommel" (mit beu Stöden) 1) die Fïnje als gentiuge im solleg

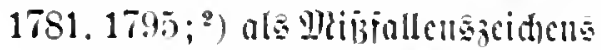
fiir ben Rrojelïor ill stolleg (mit den Etöden alli ben Tilliboden oder mit Fäniten anj bic Iijd) id)langen 1795. 1831. 1841 .

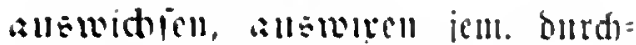
prïgchl 17s1. 1795. 18:31; im Tuell itarl id)lagen 18t1. - ansig =

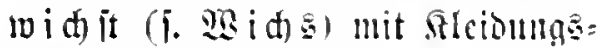
itüctell verichen 1831.

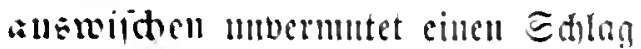
beibringell 1795. 1841.

allsjichen (intr.) 1) bie lluiv.= Etadt heimlits werlailen ohne Iilgum ber ङかulden 1757 Niemand હ. 159; and) 1749. 18+1. ${ }^{2}$ ) jeine solonuma veränbern, and bie Flludt ergreifen 1795. ${ }^{3}$ ) (trani.) in Evicl (Gicld ab= nel) men 1813.

susjiihen 1781. 1795. 1841.

21 valltage: "in Q. jein" I) heim Epiel 1795. 2) beim Incll 1795. 1813 :c.; andh Laukhard schilda II, 200. "iid) in 21. jeben" 1825. 1831. avec: "mit a." gnt, mit (Érjolg 1846. - "ben a. Ioglhahen" Montine haben 1835. Schuabels Un.-Jalıre ङ. 182.

Baaria, Baaribus Baariataj $18 t 6$; baria 1813 mo 1835 Schuabels Unir.-Jahre $\Xi .221$. - ïber in baaribus obell 5.39.

battifich cill nod) nidt namubares Pläbđen 1813.1831. (vgl. oben હ.19.) balbiercn jur lleberlait jein (v. läiti= gem Beju(h) Blaustrumpf $17 \notin 6$ vier possierl. Gedichte S. 4. 2Gal. barbicren.

ל̇albut, Hatbier 1822. 1841. - 23gl.

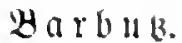

bans Drochazeidell der Rerbindungen 1N25. 18331.

Bansage 1831. 1846. Gaudy, I, 216. ḃant: "ritredit in Ränfen" \$ump vollani 1846 ; Schuabels Un. Jaltre ธ. 3339 .

1. Żat (in Bern) Mtidtwerbindmg itthent 184ti.

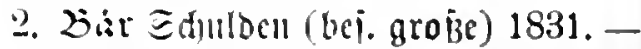
"cinen $\mathcal{H}$. anbinden" Sdullben maden 1781 (unter a n bi in o e n). 1825. 1836. - "cincu B. Ioşbinben" Edulden hezal)lell 1825. - "Die \&äreı rciben lid) Ios" id) mur abbrentuent 1 s31.

barbicren täniden 1795. 1841. barbur, $18+1=\mathcal{H} \cap\lfloor\mathfrak{b} u \mathfrak{b}$.

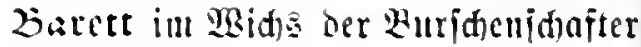
1525.1831.

Batrinth: Hatbier 1831. battuen die Atlunge des Beguers her= unterbrïden hojditl. 1820; den (Beguer entwafinen 1846.

bessmmert wer jidh idfuer jured)t finbet 1825; bormirt 1846 .

bosoutsus arielulid 1813.

betbingen: "Dicbe - " beim Fedten 1813. 1831; "bas $\mathfrak{B}$;mpen jem. beibr." 1831.

betneipen beinden 1813. 1831. bci. nom Beiud) ber Stndenten einer Iniveritütsitudt bei denen einer alloem gehrand) 1831.

betnullen („iid) befn. wie cinc @tanb= jüule"); befüult eriter (Srad ber

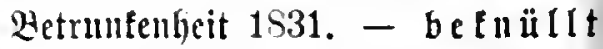
aud) Harring 1831 Faust $\Xi 71$. 
bet'sten (iid) romere 1751.

befonoitionmen traftieren Lankhard, Eulerk. હ. 105.

betotgen (iid) romere 1781 ; iid) bie Stleiber dabei Feindeln 1831.

belegen 1) int böriant eimen Plabs burdh PHiflegen eines Papiers anf ben Iijh ober bie Banf ketegen 1749 - einen Bifaz im sörianal belegell 1825. 1831. ") Das Rolleg= gets bezahlen 1813. " "I) "Ias bitt" ich zll belegen" bas iit etmas bej. Isidhtiges 1825; dos iit jehr un= wahriateinlia 1831.

beriebte mo entping Pechmming 1785 Beytr. z. Statistik r. Göttingen @. 106 ; auต i

belofieln übertölweln, ïberlijten 1795. $18+1$.

beludien Indjen 18t1. $18+6$.

bemogeln betrïgen 1S13. 1846.

bemosit ergraut, erfahren; bei salfe 1846. - bemooles baupt heifit Der 巨tmbent im 5. ङemeiter 1813 - im 6. Eemeiter 1822. 1825. 1841 - im lebten @emciter $1831 .={ }^{2} \mathrm{~B}=$ mooiter Saerr" 1825; Goethes Faust II, 2. 6638 mo 1822 I.eben auf Unir. こ. 50. 51 .

bene: "ïid) b. thum" jïr) giitlid) thun 1831; and) Bahrdt 1789 Religionsedict, Vorwort.

benebelt leidnt bejedt $18+6$; "iid be= nebeln" Laukhard, Fr. Wolfstein I, 50; Bahrdt, Rind vigins I, 71.113. beneventiren ims. Plnfmit beglït= wimichen Menantes 1726; Musander 1709 Studentenregeln 5. 7.

beritten 3. B. in ber Mlediju = mndig 18t6. Jobsiade. belaufen un in bell Redenarten "jid) en canaille, en canone tiej." 1831. berichlelier wer beim arujug ben Bug ber Reiter bejaliejt $1895.1813 .18+1$. befonmmelu betrïgen Marianus, Scenen aus d. akad. Welt S. 160. bejehen: "Gelder, einen Mmidib bej." 1831. "Den beiebe id" id bin ifm iiterlegen 1831.

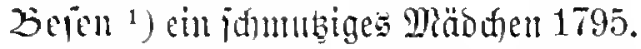

2) jedes Dienjumäbden 1822. 1825.

3) jedes meibliche Weien ("Nolor=,

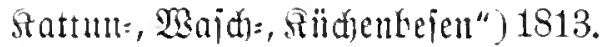
1825. 1841 :c. $-F$ lo $r=$ mid $\subseteq$ t $a \| b=$ beicn werden miteridieden 1831.

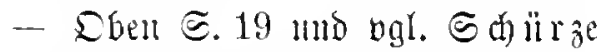
mo Sefribejen. Bei Fischart erideint 5ausbäjem (i. das DWb.) als edimpimort.

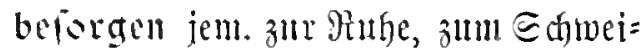
gen fringen 1831.

beiponten: "gnt bejp." bei Salje Lenz, Hofmeister I, 3 .

Seltie: "fleme $18 . "$ altes sans 179 $18 \pm 1$.

bethumigeln (iid) jids jo jtarf be= trinfen, da $\bar{B}$ mant twie angenagelt

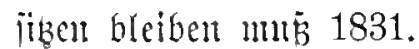

betribens ïa) alli Roiten jeme. be= reidjern; $\mathfrak{j}$. B. "id babe ing im 2Bbit um einen Thaler betritbt" ihm abgemonnen 1831.

betruven ärgern 1846. - betrmut traurig, veritimm 1825. 1831. 1846. bejeden (iid) fïh betrinfen 1781. bejichen anï̈thren, betrügen 1831.

bibelhularen Theologicitmbirente 1795 (unter reiten). 1841; aud) Studentenwitze 1839 ङ. 65.

bich Bier 1825. 1831. 1846. - Dału bich en itarf trinfen 1825. 1831. 
bieren Stier triuten Kohbe $1840 \mathrm{Wp}$ innermugen $11,6 \pi$.

Bierfilde modern = Fijdge 1 . -

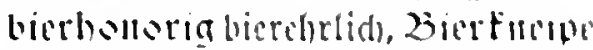

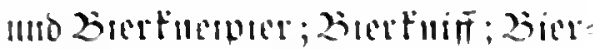
fomment bein somucto; $25 \mathrm{iel}$

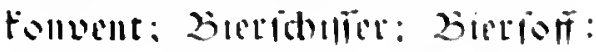
"cill 出. lyaltell" obue sommets fucipen; bierwis fleiner Summers; bierfitof 1831. - Eirtounent, bierprocal mo Bterverith 1841. - 9lubre zaldreid)e 3uiammen= jebungen mic $=b a j,=b o r i,=e h r=$ lid, =geridtt, =orben, = recije, = ialamanbcr, =waljor nifw. 1846.

billet sour Ziebcobriejden $17+9$.

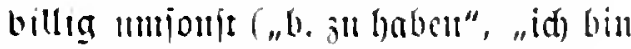
6. Daran gcfommcn") 1813.

bim M. Bujeu 1825. 1831. $18+6$.

binse ber Raufanten auj ocr Dienjur 1831.

binsen: "die Alingen beim Fedten b." 1813.

binsrasifiren ängitliç jein, flein bei= getell 1795.1841 ; bajür binsfasiren Laukhard 1798 Schilda I, 301.

bird Bicr 1846.

bilchof in ber Pierjehde 2 (bläier 1846.

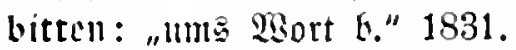

Slamage, blamieren 1781. 1831. 1846. Dng $3 \mathrm{tw}$. anth Fischer 1781 Burschiade $\odot .9 .-B \mathfrak{B}$ a $m$ a $\mathfrak{g} \mathfrak{e}$ aud) Lauklıard, Eulerk. ङ. 113 - Autobiogr. I, 97.

blak: "nidat bie blaije Soee, Probe" 1813. - "Dch Blaifen triegen" it Etauncu geraten 1813. - "ben Blaijen vorue weg haken" Laukhard, schilda I, 303 - Corilla Donatini S. 104.
Wha॥ itärfer betrumfen 1831

Bled Eadic von geringem Diert $1795.14+1$.

Hedtu bezablen, aแszahlen 1781. 1795. 1831. 18t1; aud) Laukhards Leben II, S7 utto Bahrdt 1790 Gesch. d. Prinzen Yliakanpol $\Xi$. 106. 必gl. ohen 厄. 59.

bledtusc meretrix 1822. 1841.

Sler: „int Wl." bei Staile 1846 - ill

(belingen II. Mors, Erinnerungen ธ. 34.

Slentifte die Etangen bor Edfanr=

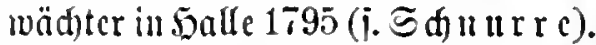
1841; fiir Ģöttingen bezengt 1846 t11t 1835 Schuabels Univ.-Jahre Є. 316; jïr 5alle nod) 1798 Briefe über Halle $\Xi .31$.

bleivogel lieberlides Franenzimmer 1781. 1795. 1841.

bluten Bier veriduitten bei ber Bier= ieljoc 1831. - Gluten mitilen Geld l)crnıšrinden miiijen 1781. 1795. 18t1; Bahrdt, Gesch. d. Prinzen Yhakanpol ङ. 303.

bosocu (iriiher in Falle) ungeididt gearbeitcte Fanomen 1831.

bosen J゙e(t)thoden 1825. 1831.

bonis: „ill b." bci Ralife 1846; Laukhard, Eulerk. S. 169.

bons mots ("bag Vifero madet b. m." - "mad)e mir nid)t lange b. m.") "„Eprïnge, $\mathfrak{A u b c t t e n , ~}$ Biehern, E(j)ltwörter ऑ. dgl." 1749.

brampi @dunas Niebergall $1837 \mathrm{~d}$. Burschen Heimkehr II, 7.

ḃrallo Manid 1846; "im Br." be= trunful Harring 1831 Faust $\Im$. 71. Biransbrief 1) amtlide Rejacinigung

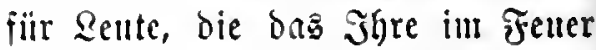
verlorell haben um Mitleio ăll er= 
Legen ; ${ }^{2}$ ) Bettelbrief 1781. - biano= geloer eingejammelte bisloer fïr einch, bem bas Eeine verbramt ijt 1745 Auf der Extrapost eingelaufene etc. Nachrichten 5. 18. ḃranber - 2̇rambiubs Gtubent iut

2. Semeiter 1781. 1813. 1822. 1825. 1831. = 2jramofuchs, 2jomutr 1795. Dafüt 28 a a ber redgt lijtig ansijebt mo jiet unter bie Eraben Burjac gar nidft finden fam" " 1749.

brechen: "bas (5h)renwort bx." 1831. breiter Steill bie Witte voer bas

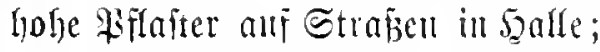
"auf beu br. Єt. jtefu" (in bafle) gleid) "Gicuatter itely" 1781. 1795.

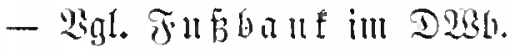

bremer 1795. 1831. 1841; Fischer 1781 Burschiade ङ. 51. $19=$ Bエณ

bruber Stubio Etmbent 1822; আ Writoer studeo jobll 1744 Zwey im Coffee-Lande herumsebweiffende Avanturiers. brummen im 9 rrejt jiben $18 \pm 6$. bilfell arbeiten 1822, 1825. 1831. 1841 ; Gaudy, Werke I, 198 ; Schna-

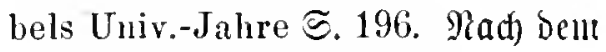

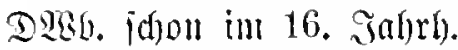

brigeln futuere 1745 Auf der Extrapost eingelaufene Nachrichten ङ. 41 ; and $18+6$.

bulnumelu 1857 Baclieliade 5. 5. 44. Sirger: "afndemijther B." 1831. Bumith ${ }^{1}$ ) Stubent mad Dell 1. Stu= Dicujahr 1795. 1813. 1831. 2) Stll= Dent, Milemiln 1781. 1813. 1831. - Daz̆ burichentomment 1781. 1795. 1831. - 23mrit)enwid.

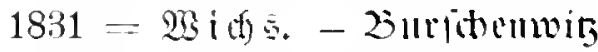
1) itehende Pedensarten ver Stu= Denten. 2) Sutperei 1831.

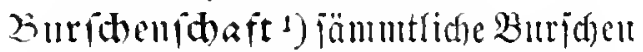
Laukhard, Sehilda I, 176 - Autobiogr. I, 131; ; ${ }^{2}$ jeit 1815 wie hente. buriditos (ohen $\sigma$. 48) buriduen:

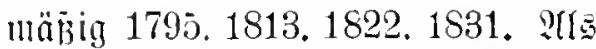

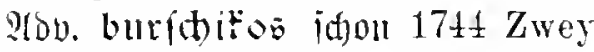
im Coffee-Lande herumsch weifende

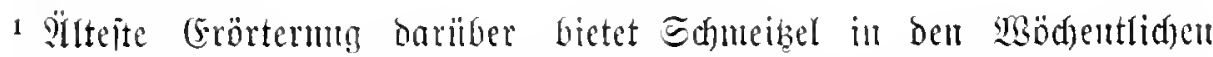

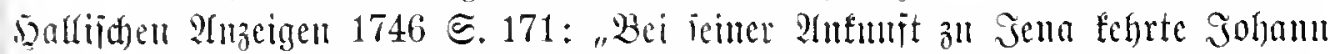

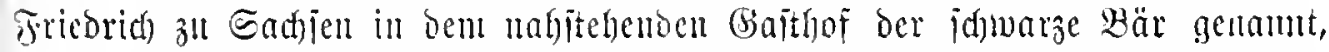

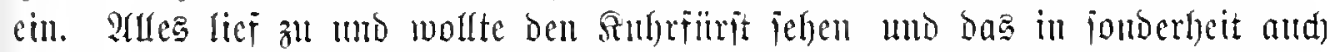
bie jumge Stubenten. Ia aber bieje gar zu häufig, and) wohl gewöhmlidger

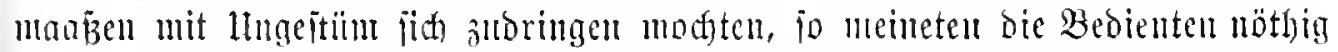

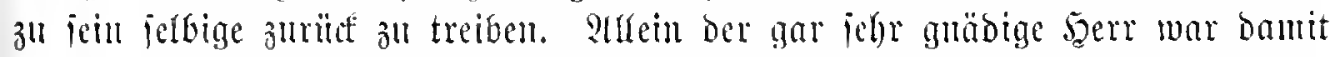

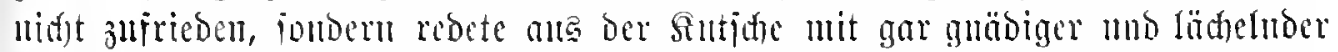

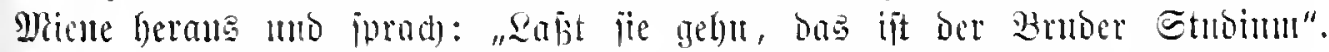

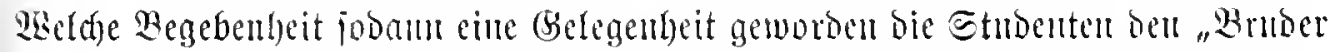

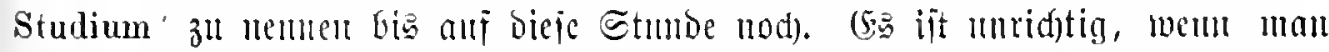
Bruber Studio redet uno jafreibet. Sigl. Joh. Rosini, Vita Johannis Electoris; Wolfg. Heideri Orati. I, IV; Casp. Sagittarius, Hist. Johamnis Friederizi $\$ 25$. 
A ranturiers S. 3; Lankhard, Eulerkapper Є. 151. - Burrititolia Sammlung vou Ead)en H. i. Iu., bic ben Embenten als jold)en intereitiren 1813. - Eburithitolitat 1831. 194ti. butiten 1) ansidelten 1781; itraien 1795. $1841^{2}$ ) futuce 1781.1846. butteruoral meretrix 1781.

buren itibizen 1781. 1795. 1841; and buriten 1781.

Serevis I) Bhier ("eill gutes (5.") 152i). Marimus, Scenen ans d. nkad. Welt $\subseteq$. s(i7. ${ }^{2}$ ) beim Irinfull bas (5hrutuort "bei meinem (Eerevis", "ia gebe Dir meill (5." 1525. 1831; "alif (5." 1846 jowir Schmabels Univ.-Jahre $\widetilde{\sigma} .262$ und Nicbergall 1537 Des Bursehen Heimkehr III, 4; IV, 1. - Dic heutige Hedeutung des Disortes jehlt bis 1850, mur erit (5erevisuilibe, = fappe 'Bierfapuc' listi.

Campagneru Selmabels Univ.-Jahre ธ. 308 .

Shapeau shouneur bei Plujignen

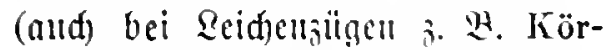
ner 1812 Nachtwächter IV) eine (5hrendarge reip. ber Epreder 1795. 1813. 1\$31. 1846.

Shapcau Bouleur öber (Gicielle 1795.

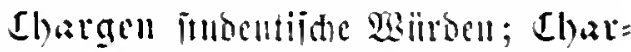
gentriager bei ben Drbensbriberu 1795. - Sbargierter1831.

Sharmante 1749 ; vit in Hospitium 1747 ङ. 33.45 ர். mo in Zachariae's Renommist. 2igl. olien ङ. 89. Sbor 1831. 1841 ; i. Sorps. Shriftulheit bei ber wierfehde 6 oder 3 Ğläı 1831. $18 \pm 6$.

Sircumiter Sdjmarte in (beijust 1749 $=$ sin Estmib bou einer beit. Form
1825. 1846; and Leler. Blanstrumpl 1746 vier possierl. Gedichte S. 5.

cittrell vors roncil ober and mugnificun beitellell 1825. 1831.

Ionleur Blaie 1831.

Dach ("cittem zu D. itcigen") prïgelı 1781; and Harring 1831 Faust כ. 72; coramiren 1831.

Dactrlus penis $1846=$ "Tröiter "mbo dactylus" 1633 \%ech- und Saufrecht $\$+7$; aud) ill Stammbüchern. Damenpusel Jungiernfued)t, $\mathfrak{B e i b e r}=$ lued)t E. M. Arndt, über den Studentenstaat 1815 .

Sismmern id)lendern, bummelu 1813. 1831; and) Schnabels Univ.-Jahre ङ. 50. $\mathfrak{D a z u} \mathfrak{D a ̈ m m e l e r , ~} \mathfrak{D a} m=$ uerïur it Bummler 1813; Däm= merfïrit eill bequemer und babei träger Mlenid 1822. 1841.

Dampi: "mit D." mit Srait, fdnell (z. B. "mit D. abziehen") 1831. Salmpfen itarf Tabaf rallden 1781. 1795; Hospitium 1747 5. 49. 52. Siampren jem. Durd $\mathfrak{B o r t}$ oder burd bie Allinge fleill madyen 1831.

จálïg einjültig, umbejouncn 1781.

Sallern bemitleidensmert jein 1795 . 1841.

Deballbe Sdivelgerei Speranders Handlexikon 1727; Callenbach 1714 Alnanach 5 . 60 interid)eidet "Mecill=, Pier:, Tabat=, Spiel= uno (5arejiendebaud)en".

sebaubiren (beboudiren) idinel = geriiđ) Ieben; and Behmeno 1715 Poet. Cabinet S. 121. secten trani. Freihalten, traftiren 1781; (i id bea en beim Fedten und wo etwas all riafiren ober zu bezalen 


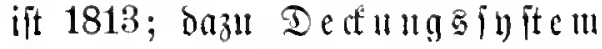
1813; zudeffen trañ. wit Saflä= gell iiberlaben 1781.

Deute nithe Saran (anth) "id)"): Mna

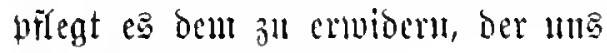
IIII etrag bittet 1813.

Dentjettel Pritgel 1781.

Seponiren 1) "iid) als student ans "thuten Iaffer" 1795. 1781. 1841: onzu Depolitiongiulu 1781 . 1795. 1841. $-{ }^{2}$ ) futuere 1781 (jo j(hon 1744 Avanturiers 5. 42; hänfïg 18. Jahrh. in Stanmbüdhern "virginem deponere licet"). $-{ }^{3}$ )

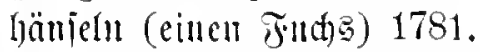

8epreciren 2rbbitte thu 1813. 1822. 1825. 1831. 1841; aud Harring 1831 Faust S. 69.

Desavantage Madteil beim Spiel und beim DHell 1781. 1795. 1813. 18+1; Laukhard, Schilda II, 200. Dexpo Poder oben S. 62.

oitt (oicte) ${ }^{1}$ ) alferbiutge, ja (20nt= wort 1781. 1795. 1841. ${ }^{2}$ ) iteigerno 3. B3. in "bict bejofien", ,er zrwingt "se bif" hat viel Geld 1781, 1795.

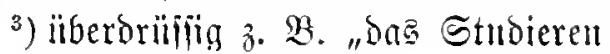
fobe ich fïr bente biff" $1813 .{ }^{4}$ ) "Did thum" renommiren 1846 ; jpröbe jein Stammb. 1759?

Dientiteren 1846 ; i. Be ien.

Dimenfionen: "nad) allen D. Eeilen" Köhler $18 \pm 3$ Academ. Welt I, 38. Difcippel Dijciplinargeridgt Niebergall 1837 d. Burschen Heimkehr III, 10.

Dottor bei der Sierichoe 3 reip. 2 (Biäjer 1825. 1831.

Doma Belictuc 1792 lsriefe über Erlangen I, 107; Schnabels Univ.Jahre 5.49.
Dommerbejen grop̉e Periife 1795. Dorfatill oben 5.44.

Dorntuippel bicfer Stof der Re= nommifter mo Ranfer 1781.

Draftitum [Gieß̈en] = IGeeteifer, Binjel Laukhard 1799 Fr. Wolfstein I, 138 - Eulerkapper S. 103 - Autobiogr. I, 97. 116. 180. Dazul ora it ifich Eulerk. S. 107; Drajt ic ität Eulerk. @. 125.

Dred": "im Dr." auf Deul Şunb 1846. Eulerk. S. 110.

orillew - trillen futuere Stammb. แIII 1752 .

(iich Orinten 1) jid hemnlid lweg= begeben 1795. 1831.1841. ${ }^{2}$ ) weggeh川 1813. 1825; and Schilda III, 71. I, 303; v. Maltitz 1828 Der alte Student II, 2; Schnabels Univ.Jahre S. 157.

Duell, dafür ftud. Słanbal 1831. Suften ausbiuniten 1781.

Dubmen (5ุ a

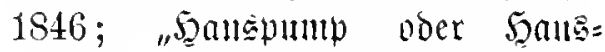
Dulfmen" Schmabels Un.-Jahre S. 96. Dn:Eomment 1831.

Dilljinea Geliebte 1831. Bahrdt, Rindvigius I, 67. 69. 104; and Dulcinc 1779 Der deutsche Student S.11.27. Dumun: ,jem. o. fommen" Laukhard, Fr. Wolfstein I, 128.

Dummer erg. In g g Tujhuort 1831. Du: Kamment Dukberfältuig 1831; nad) Schnabels Univ.Jahre 1835

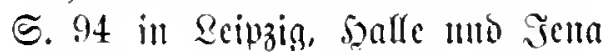
iitblid).

Surdbobren provozirend firirell 1831. surdbremen dic lluiveriität lyeimlid) verlafitell 1841. 1846.

Durdballen ungefdictes vorbringen (3. B. bermuglinfte $\mathfrak{s i b e}$; and) "ben 
Imitiall befommen" 1795.1841. Das (5xamen nid)t bejtchen 18t1. „\$Benu cin Standidat bei eincr Predigt nid)t gemählt rvirb, jo jagt man and, er ieidurdugciallen ober habe dell Durd)iall befommen" 1795. 1841. - Durthrall Durd) Examen 1846; ugl. and Haulf, Mann in Mond ङ. 65 (Hempel).

surdgerben prïgeln Laukhard. schilda IIl, 100.

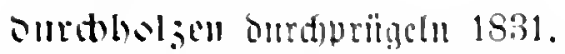

surdthemen ans ciner sume in bie andere jichen; untd cine Etnot paiiiren 1831.

surd)lauthe 18t1, surdblauthtag 1795. 1St1 löd)erig, boll @öd)er, jer: riiien (vou Âleibumgsitiiten) 1795; ourdjiit)tig Jobsiade II, 2.. 1736;

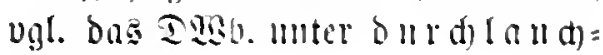
tig 3 .

Surdpitältern durdjinden Ineognito,

Der verliebte u. gralante Student こ. 77. - Burd)lejen 1776 Briefe dreyer akadem. Freunde I, 68. surtwidien frore Hritgeln 1795 (unter a $\cong$ wid) jen).

Intbormser 1781. 1795; Laukhard, Marki v. Gebrian II, 7t; 1764 Über die protest. Unir. Є. 23.

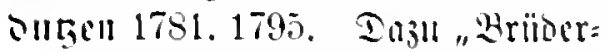
idaft ober anf ocu Ink rinfen" $16+5$ Facet. Facet, ङ. 66. 告ber: "iid) wie eill (5. hallen" 1831.

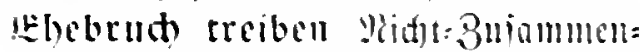
gehöriges anjommen gicbrand) (5ijen unb Tabafrand)en ober Taboe

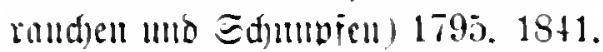
(E)benburith Egargierter bei jeier: lidfeiten; 歺brengang, =geritht, =mutgltes, =ițmalls, = mort 1831.
Hhrengand Duell mit itumbieu Mappieren bei verid)lofienen Thüren $18+1$.

[igen Tujd)uort 1831.

IEimer: "nuf dell (5. briugell oder fommen" all (Ğumbe geh) 1831. IEimsarm Teigling Niebergall 1837

d. Bursehen Ileimkehr III, 11. emfrallen beim Fedten. - "es jällt mir nidt cin" idf benfe nid)t barou 1813.

emateben jem. etw. mahe legen 1831. ringebn (beim Dnell) einb:ingen 18.5. eintleisen in bie serbinbung .mi nehmen molern. inturipen einfelyren 1822. 1841. eimpacten múlfen im Meden, Dij= putieren nid)t weiter tömen 1781; iī) vergeksidge $\mathfrak{P}$ ü̈he und llmitämbe maden ("Damit fannit bu mur cin= pacfe" v. nidtigen (Grïnden) 1795. 18t1. - pa cf cill bör allf 1831. - "Der tam mur cimpacfeu" fid an: Bem @taube maden 1831. cimpanten cinlernen, cinjubiren 1813.

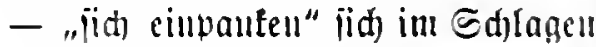
cimübell 1831 (= cinfd) (agcn). - ,jid) Durd Trinfen ill cintell Sieritaat eimpaufen Schnabels Univ.Jahre @. 30.

emprügeln (ïid) jeinen Tamrn cill= iduciocu (in Tijd)e, Stöđe niw.) 1813.

'mrücten cingąehen 1831. cinjhlagen: bie fïndje twerben vou alten \&urid)en eingeid)lagen 1825. cinjpringen (beim Tuell) jwijden ivringen 1813. 1825. 1831.

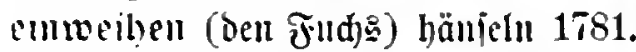
IEllenleutmant Rabendiener in cinem Giejdäft, wo mit ber (Elle gemeijen 


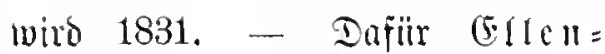
r(eiter) bei Braseh, Gesch. der Univ. Leipz. ङ. 15.

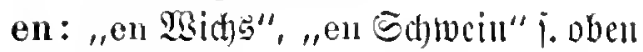
ธ. 65 .

cutanonieren ( $j i d$ ) ïh betrinten

Laukhard, Schilda I, 189.

entricten: „eilen @fandal, PHum entr." 1831.

IErontiflol Stlloent 1781.

cotflectlich: "erkl. jid) amilijuen", "cr=

fleatlidyes $\mathfrak{X e d} " 1813$.

erid) willgen erwerben, geminnen ("man

faum nicht vicl babei criftw.") 1813. erjbrav, erjifod 1757 Niemand ธ. 83.106.

cr;ielen anjăffen (3. B. "täuftlid), wohlẹeil er3.") 1813.

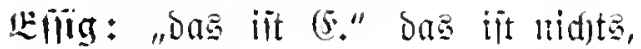
idjledyt 1831.

占trage: "in ber 3. (5." ontudgejallen 1795.1841.

Etcetera ${ }^{1)}$ Taugenidgt Salin de $17 t 4$

๔. 67 ; Jobsiade II, 2.76 . -

2) meretrix Jobsiade III, 2.1 1234. 佔var Todtet meretrix 1831.

ex z॥ Enoe, jertig 1831. 1846. - exgerbell vomere 1846 . - exgreifen 1846. - extueifen jid bou ber llniwerituät heimlid cutfernen 1813. 1831 ; vou bautu gefm 1822. 1811.

- Extneipe 1846. - extrieiper " "aus jeiner ftreipe rïtof un etw. onl initifiren" 1831 ; all bic (5x= fucipe gehn modern. - eptraben entffiehen 1825. - 佔piprige 2113: flung modern. - exifreifen jdunifu 1846.

eptlubirren: "ans einer Lierbindung extl." 1795. 1831. 1841. - Iałll

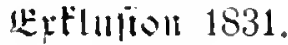

Epperimentalphylit (Worlejung ba=

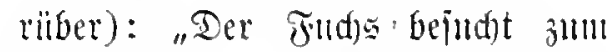
Beitucrtreib bic 2orlejungen madjt mit Der (5rb. Den Pufang" 1765 Der Freymüthige oder der engl. Greis von Young 5 . 60. Rigl. bei Hunold-Menantes 1710 Satyr. Roman der gal. Welt ङ. 37 "einct Fran cill collegium physicum experimentieren". "(5. hören (bei cilter Frau)" Bretzner 1790 Lebon eines Lïderlichon II, 11.

extra gebn Taubmanniana $1703 \Subset$. 84. 115.

Eptration im ballijden SBaijenfans cill geringer Freitild 1795. $18+1$ (and) 1781 s. 96).

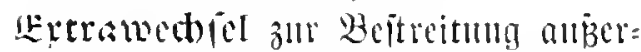
gemöbuliche: QYugaben ("Der Rater mui cinen (5. lostituen") 1813; Fischer 1781 Burschiade ङ. 69. fisteljug 1831. $18 \pm 6$.

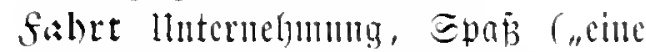
Fibele, betrnote F.") 1525. 1831. falll us (ältcr famso) forifh, flott $18+6$ : alth) Gauty, Werke I, 16t, 168.

framulus armer Etmbent, Der hei

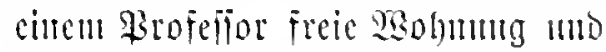
ande Borteile hat, iu ben söbräleı

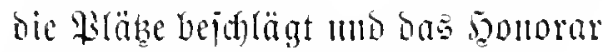
fïr bie Sollegia eintreibt 1781.

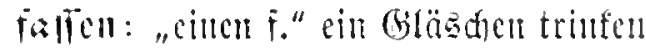
1831.

ied)ten (fechtmeifter) 1781; bafïr

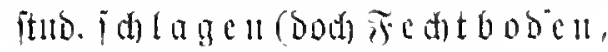
= jaal, =lyaujiduha 1831.

feserfudier Ectretär 1831.

focru p:ellen, rupfen 1839 Studentenwitze 5. 32.

Seoern: jid sul jeiten 尔. verjanmeln" 3 3ett gehu1793 Pliil. Dulder: 
feger tildtiger, bratuer Burid 1831. fentilotạ an anitündig, an jein 1831. ferlengels gebon (and) "dic Terjen seigen", $17 !$ ó.

fereng minetrmuten 1s:31.

ferser Degen DWb; and Behemo 1715 Poet. Cabinet ङ. 121.

fensiren (gSittenberg?) itehlen Reinwald 1720 dealemienspiegel $\Xi .155$.

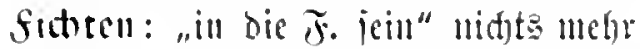
gelten Laukhard, Eulerk. ङ. 104.

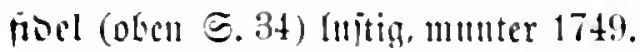
1795. 181326 - Sn 5alle: "hijt bu iibel"? wie gehts?"; "fibeler frerlfibeles Suber" 1795. - „fibeles \&ehen,

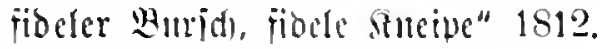
fischtist um 1765 ill Jen. Stammbüchern; Laukhart 1799 schilda I, 180, 181. III, 204; = Fidelité 1813; Schluck 179s Bursehenkomment ङ. 25 .

fiseliter fiofel Gaudy, Werke I, 176. fisibus i. bas DWb. mo oben ङ. 39. rioul 1831; Bacholiade $\Subset .49=$ ñod.

- fisum Sertranen 1822. $1841=$ fioll; 18t6. - fisujt Flutwort alif $\subseteq$ d m oll i 1813.1825 .1841$.

fin Fein 1831 .

pusen unienit ober fïr eimen geringen Hreis erhalten 1795. 1S41. - "finte er jid)": jo jagen brave Buriden bei

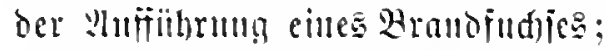
beliebtę 巨án̈̈herwort 1749.

Suten ${ }^{1}$ ) (im lluteridied von 2 is i d e fleibige Etmbenten ohne stomment:

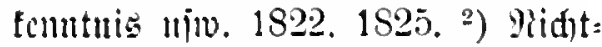
verbindungsindenten (in salle uns Jena) 1831. 1841. 1846; Schuabels Univ.-Jahre 厄. 20.91. 312.
Stute 1 beim Fedtell 1781. 1795. 1813.1841.1831. - ${ }^{2}$ ) (Shifane'1795; s) "Tinten reiBen" 1795. 1841. silitgen (oben S. 54) 2ieriilde 1747 Hospitium $\Xi$. 73 .

frital in solle Fanulns=Stubent

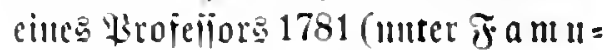
(11 Avanturier II, 477. 510; oft bei Laukhard 3. 23. Schilda I, $133-$ Eulerkapper ङ. 183.

fitrich loderer Menid Niebergall

1837 Bursch.-Heimkehr IV, 8. ir. 1) idntell Schoch 1657 Kom. v. Studentenleben DIIbGb. - jibel, lie= benswiiroig 1831 ; "cine fire Streipe" Hauff, Mem. d. Satans Stap. 6.

flaichen tüđ)tig losgel)川 1822. 1841. flanich 1781. 1795. 1813 - flalle 1813 Rod, bei. Winterrod.

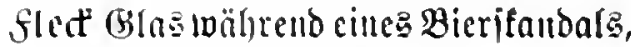
worans alle trinten biurien 1747 Hospitium ङ. 77 .

Hecten vom grled bringen 1813. fleormianie oben 5.52 . - fleoern itart jdilagen (anj ber Meniur) 1831.

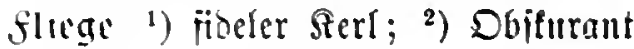
1831.

flor (Eh)rentitel für bas jübune (B)e= id)led)t, Damenwelt (,ill ber (Se= iellidiat war wertig F." ) 1813.1825. 1831 ; vornebme Damen 1822. 1841. (i. and) salbilor). - florbefen Mläbden vormehmer Eltern 1825. 1831. 1841; Hauff, Mem. d. Satans Stap. 6.

flores ${ }^{1)}$ Sduanm, H(ume des Bieres iIII Bínje Jus Potandi 1616; 2) in floribus oder floribus eine Trint= cercmonic ebenda; ${ }^{3}$ ) "in floribus lebren", jeften "in flore leben" i. Dn 
DWb. unter F(or. ${ }^{4}$ Floribus cinte (3) äjerart 1747 Hospitium $§ 11$ (vgl. oben 5. 38).

Prorieren bei Raffe ieill 1781. 1795. 1841; wolgl allf jein 1831.

Horixäs obell 5 . 47.

flóte Mlago 1846. - Etouf Schnabels Univ.-Jahre ङ. 363.

Hott gut, fdön, berrliff) (flott trinfen föuten") 1781; (njtig, muntter 1822. 1831. - „fl. leken, ein flottes miäo= ()en" 1795. 1841; cinl fl. Ferl, ein $\mathfrak{f l}$. Miäbdhen, fl. MSirtịhaft 1813 2c.; ein fl. Fract Fischer 1781 Burschiade 5. 18. - Frottität $18+6$.

foppen necten, narren 1781. 1795; jafon 1633 Zech- und Saufrecht

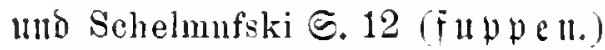
Foroern zแut 3weifampi 1781. 1795. 1825. 1831. 1841.

Forsern: "iid) f." eillen Seybold 1816 Der umgekehrte Faust $\odot .6$.

formalifiren: "fid f." iid iiber etw. aufhalten Laukliard 1797 Schilda II, 168; III, 113 - Erzälungen 11. Novellen I, 220.

rorid) (nod. mu forsch fidjou iul 16. Saldrh.): "eill forid er serl", cr madte fid forid)" 1813. 1846; Schnabels Univ.-Jalire ङ. 90. Foridität 1846.

forftswe Stunent der Foritufabcmie

Wolff, Naturgesch. d. d. Stud. ${ }^{3}$ 64; Dafïr For itpola of 1846.

frattur: "予. mit jem. id)wäken" prügeln Niebergall $1837 \mathrm{~d}$. Burschen Heimkehr III, 11.

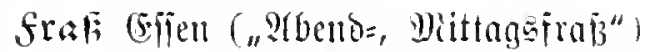

1813. 1831. 1846; and) Bahrdt, Rindvigius II, 30 IIIID Studenten-

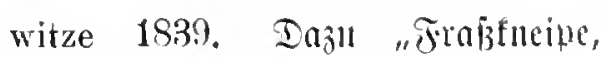
- fncipier, - fuiri, - munt" 1831.

frequentiten bie Sdjule bejuchen Taubmanniana 1703 ङ. 182; Musander 1709 Studentenregeln હ. 2; Salinde ๔. 65; Reinwald, Academienspiegel S. 258.

Fritaificen zeridumettern ("Der Eatau folf ifn fr.") Lankhard, Emigranten II, 172. 244.

Fundbringense (Gejellidaft Ditfler= ejel 1795. 1841.

futhe (ohen $\widetilde{5} 50$ ) junger Burich, ber erît ganz nen anf llniveritüten foum 1749. - Stubent in 1. Se: meiter 1781. 1795. 1813. 1831 26.

fubicifun ${ }^{5}$ ) Sporen 1815 Burschenfalirten હ. 101. ${ }^{2}$ ) Futhegeit 1846.

1. fuchen 1) beim Billaro sälle mod)en, an bie man uidnt geoadit hat 1795 . 1841; cill joldher Ball heifst man

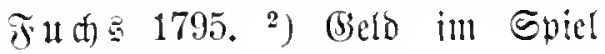
zujaumenj)lagen 1795.

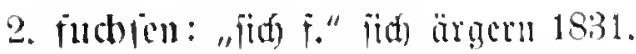
fubstollegiun 20 rlejung ïber Sogit 1781. 1795. 1831 (and) (smentlo= päbie). $18+1$.

fuchetommer 1831.

fudjer guter Salüger 1831.

fudbumappe nagelnetc Rollegmappe 1795 (unter mappc). 18t1.

fubsullajohe meretrix Jen. Stammbuch 1759 .

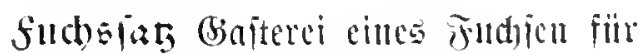
jeine Freutube uin. 181 ?

riibren beitien (3. 2. guten Iabat, Bütuer) 1813.

funjel alte Rampe 1831.

furit voll Iborn i. miter Thorn. 
fur; in llugemitter: "cr iteld une cin - " ar prablt bon jiunen Inten mo ift ber erite, ber flicht 1795.

futtietell: "ii(t) $i . "$ iid) Ë̈unuern Sehilda I, : :(1.2); i. ons I) Wb.

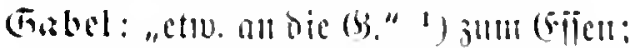
2) cit Fronensinuer 1846. - "mii Der (5) jd)(agen" bein Fedtell 1s:31.

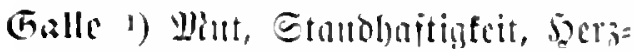

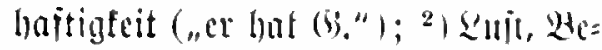
lieben, Eierlangen ("bait bu (6). ba

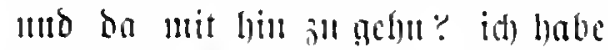
jebt feine (35.") 174!). - "Balle jcigen" 1763 keils Stammb. G. 272. galfen itellen roben 厄. 60 ) Laukhard 1802 Anekdotenb. I, 235; Niebergall 1837 Des Bursehen Heimkehr II, 10.

Fallg beim Dutell 17!5. 1813. 1825. 1831. 1841.

gassatim obon $\Xi$. 41. (5)alfonballet 1795. 18t1.

gaudinm 18t6. - (iia) ) ga Jobsiade II, 1393 Laukhard 1802 Anekdotenb. I, 228 Eulerk. S. 102 - Enigranten II, 225.

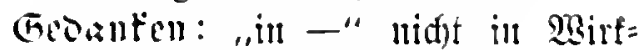
(id)Eeit, nein 1795. 1841.

Exefălle: "tin gutes (5). haben" viel anj ciumal hcruntertrinfen fümtı 1831.

Fichali uasutulus Sticler 1691, vgl.

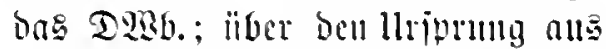

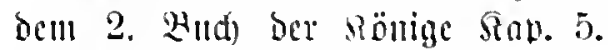
ugl. Wolfg. Heiderus, De vulpeculis Seholasticis હ. 12.

Feige: (cineแt Mläbd) añ ihre (5). itreid)en futueres Incognito, Der verliebte u. galaute Student S.210.

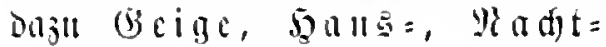

geige meretrix 1846. - geigen future strumbuch v. 1735. Fincor vie! Gielo 1831. Felstili in (belo allagezablter Frei= tiij) 1781 .

(5)lleter bei ber Sierfebde I Gla 1 1525. 1831.

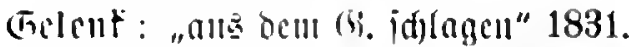
nemlin: in balle "cill gentecner Sterl"

$17 ! 5$; "cin gentodner Sterl" 1813. Finctislompi allgentines Handell

IIospitium 1747 ङ. 76. - General. frallung minctio omnium 1781. 1795. 1841.

grtbell vomere 1846 ; SchuabelsUniv.Jahre S. 35)!

giruril: "jid) g." jid) betragen 1825. Fophafte mact)en in Epiel gemintmelt 1795.

acritliffen: "mit gejdsl. 2aafren" bei bur Bierieboc $=$ mit bis jum Raube vollent Gíäicm 1831.

geidallen fin verliebt jeill 1795; Lankhard, Erzählungen und Novellen I, 257.

gejuns tlug; tapier; bei Salje 1831. Gevatter frelsu int Ljandbans, verieşt jeirl 1781. 1813. 1825.

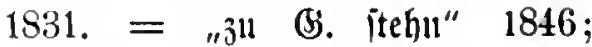
und) ciujad) "itel)n" 1825. - Mod "ju Bicuatterı itehn" Lenz, Hofmeister I, 3. Mlber "Biev. iteh" Hospitium 1747 5.45 ull Salinde 1744 ङ. 167.

Fuevatterbube [5alle] Dbitbube 1831. (5evatturin Dbithänderin [5ुate]; in

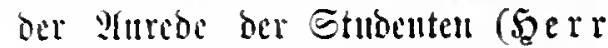
(Gevatter werden jie mieder ange= redet) 1781. 1795. 1841; ältejter Welea fïr Salle 1749 Der reisende Avanturier II, 492. 483. 
gemaichen: "iid) - haten" vorjintids icin Lankhard, Eulerk. 125. giefien raja trinten 1831.

Gimpel reider mgeriloster bounirter Sert 1831.

(Elans: "fith in ocn Gil. toerfen" [Senta] fid bolltommen in Bier be= nebeln Laukhard 1799 Mosellaner ๔. 54. - "mit Gir." jambs 1846.

Glas: ,bie (s)äjer bh. Jentiter cill= merien" 1791 Letztes Wort übel Göttingen 5.14.

Gleoitden Blaustrumpf 1746 Gedichte $6.5=\mathfrak{A l a n b i t a n d .}$

gleid) : "bie $\mathfrak{Z}$ affen gl. madon" beim Bicrifandal 1831.

glimmern fareifiren, Yiebherzen 1781; Laukhard, Schilda I, 199.

GHot'u 1795. 1822. $1841=$ fit $\mathrm{D}$ te

1. Folorilch Golditita Laukhard, Emigranten II, 215.

2. Goloruds Futhes im 2. Eemeiter auj ber 2. Lluiveritüt 1825. -- હtı= bent inr 4. Jahr 1831; aber @in: dent im 1. @emeiter Mart. Schluck, v. Burschenkomment $\$ 19$.

Golomenja amöne Mäbden 1831. Gollenredt in (Söttingen 1846; Beytr.

z. Stat. v. Göttgn. $178 \tilde{5}$ ऽ. 164: Schnabel $\subseteq .314$.

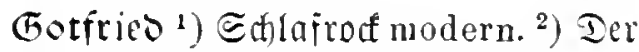
alte Flaus, Stlltagînof $1846 ;{ }^{3}$ ) Sommer 1831. Hauff, Mem. d. Satans 6.

Gottescter bei ber Bierfehde 8 Griajer 1846; Schnabels Univ.-Jahre ๔.50. Grasmulde meretrix 1795.

Grobballs ein 5̧ajardipicl 1795 ; Laukhard, Wolfstein I, 355. grodten Broct trinfell Kobbe 1840 Erinnerungen II, 67.

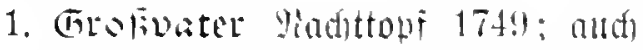
1747 IIspitium 3 . 22. 7t; 174t

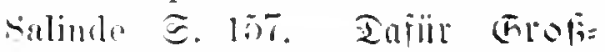
prapa Jen. Stammb. 1762.

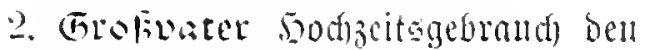
getabenen Stnocnten die Tünzerimen ju ïbertajicn Reinwald 1720 Academienspiegel E. 23:3; Tanjcu bei ber 50 djocit mad) aujgehobener Mat= zeit Salinde 1744 5. 116. 117.

Grimling Itureifer v. Maltitz 1828 Der alte Student I, 1.

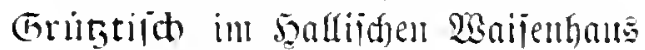
$=$ (5xtratij (d) 1781. 1795 .

Gucts $1781=\Im \Perp x$.

gut was doul ङtndenten gejäll 1795; gute Norgen gute Groiden 1813. - G ut en Merge Etmben= tengrub in Porbdentichlano, der ben ganzen Tag gebrandt wirb 1846. barbentel Malid) 1781. 1795. 1841; Laukhard, Eulerk. ङ. 96.

babemus Pranid Gaudy, Werke I, 194; nad Sanbers and boi Holtei. habes: "hat jidf was!" ja, ridjtig! I. Köhler 1843 Akad. Welt I, 45. -- ".Sie bante ihm. "sat iid

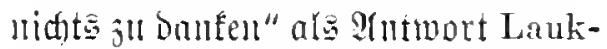
hard, Emigranten II, 223.

battiaten 3otell reiben 1795. 1841.

- Dajur battion 1) 3otenreizer 1795. 1841. 2) Bote modern.

Gabll: "cin tiidtiger F." $^{\text {flotter }}$ Stubent 1831; and Schnabels Unjvers.-Jahre Є. $315.327 ;$ i. 57 a $\mu \mathrm{t}=$ hahn, samafhahu.

balbtlor Tädter mo Frranen bou Sanfifuten mo 2 camten 1S22. 1841. haltell : ", "halt cr fidt"! ${ }^{2}$, "halt" Mü ber ङefmbanten auf der Miniur 1831. 


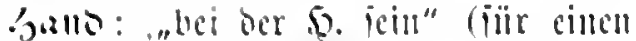
Tumult ) $17+$ ?

h.illae" ") "mit jem. h." in Efambal mit jent. itel)u 1831. "2) "büngt" id) nehme an Kohbe 1840 Krinnerungen I, 176.

brilleln de jild jie 1781.

biatling harter Thater Schmabels Univ.-Jahre ङ. 82.

bali, galelant, halelicten 16. -18.

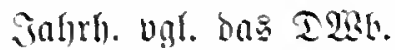

baleupanter: "Dos 5. ergreifen" 1795; and Salinde $\Xi .325 ;$ Avanturier: G. 65.

Ganserer Solmfutidar 1846.

ballell anj ben bieb fedten 1795. 1813. - "ïbers Dhr h." betriigen 1781. 1795. 1841. - ball omment 1822. 1841.

baller Iegen 1765 Der Freymüthige ธ. 62 .

Grapt j. bcm o o it. - . bauptbabll ",iit ber gröbte (Ehrentitel, ctwa als criter Burid alterfamut" IS 31 ; and bei Heine, Werke III, 16 mI Kobbe, Erimnerungen I, 64. bauptferl jait $=-$ goupthahn 1831. balls, altes b. Fremojodattidye $21 n=$ reoe 1795; "fideles 5." 1825;

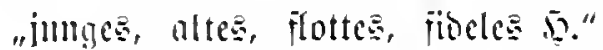
1831. - 6allsbeicn Iienitmabden im sanic 1831; i. ofen muter Beicu - ballsburid Etmbent im gleid)en Sanje wohnent 1813. 1831. - ballotuedt Meit in ber Pifeife Bacheliale હ. St. - ball: fuedt - ballstiodell Sans: id) liiliel modern. - baustnoden und $=$ sansururid modern. bullstommerich fleiner Stommer im Salic 1S31. - Gauspliliter
1831. 1841. (Schnabels Unir.-Jahre כ. 361); balloblilutuelfe 1831 .

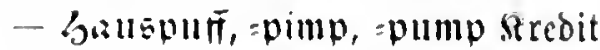

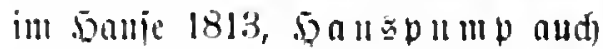
1831. 18t1 (i. and $\mathfrak{D} \| \mathfrak{h} \mathfrak{m} \in \mathbb{1 1}$ ). balliell lebell (j. B. in ber Stueipe) 1831.

b)aแtixis: pben E. 47 .

heben trinfen; "ber hat gut gehoben" iit benchelt 1831 ; Marianus, Scenen aus 1. akad. Welt S. 162.

bebrifith lernen in Bjanbhang jeill 1825. 1\$31. 1846.

Gedt $\left.{ }^{1}\right)$ Ilnwiiliender Fischer 1781

Burschiade 5 . 18; als höhnende

Inrebe Kobbe 1840 Erinnerungen (.. 17. ${ }^{2}$ ) biffe Smeipenluit mad bemt Iabafrandien modern.

berte jum Madjidreikell ber $20 r=$ lejungen 1795. 1813.

beise: "wic cin 5." janfen; and) heisemunisig (ianien, paufen) 1831. beimgrigen wegididen Niebergall 1837 Burschen Heimkehr II, 7. beimleudbten Irenius 1766 Begebenheiten eines Leipz. Stud. I, 175. berallsbeilien: "ben xer Stubenten $\mathfrak{h}$." Jer lluiverïtät $X$. (5hre maden Wolti, Naturgesch. d. d. Sfud. 128; "ïit) h." gut wegfommen 1846.

betallstomunen: "babei ijt uiduts

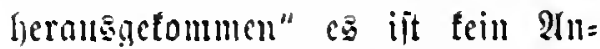
id)ißis uargejallen; ${ }^{2}$ ) verraten werden 1825.

heraแspanden: "ïd ans bem 2 er: (đ)í) h." 1831.

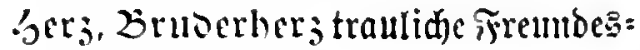
aurede 1831.

berjog im Bieritnat 1831.

1. begen jell. z̆m Duell gegen jent. antrviegelı 1831. 
2. beรe'n cin mantänoiges Sple beim bojpiz (bas mähere iijt micht an= gegeten) 1747 Ilospitium ङ. 36. baz̆l $\mathfrak{B}$ ärentebe, bas mad) 2 s. Fabricius ein nidit jelten obicönte Wergnitgen ber Reinziger Stubenten anf den Törfern un 1813 gelvejen iit.

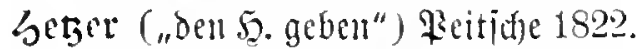
1825. 1841. Jet5peitiche ${ }^{1}$ ) beim Meiten, 厄わlittenfahren 1795. 1831. 2) jum Bejdimpien be (begners 1781. 1795; Laukhard, Eulerkapper ङ. 206. - Emigranten II, 220. 221

- Fr. Wolfstein I, 128.

benpferd bormirteg Gubjett 1831.

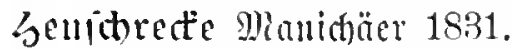

sieb 1) beim Fedeten 1795 ("einen

5. geben, beibringen"; "Der 5. Jizt" 1831). - ${ }^{2}$ ) ein fleiner $\mathfrak{I}_{\text {rumf }}$ Brantewein 1795. 1841 (, cinen 5. nel)nten" Brantewein trinfen 1831). $-{ }^{3}$ ) "einten 5. haben" betrunten jein 1825 .

Sieber Degen 1781, 1795. 1813. 1831. 1841; and 1786 Beitr. z. Statist. v. Göttingen $\subseteq$. $179=$ 'Yeid̆ter ๔äbel'; "grȫer 5." Bahrdt, Rindvigius I, 124.

bieb fomment 1813. - biebffallool Duell ani Sulläger, Begen ober frumme 厄äbel 1831.

bimmelreich lop̈djiter Єtură im Bier= ifandar (32 (s)läier) 1831.

himpeigen, huljeben wohin gehn 1813.

birid Sidatuerbinoungâtutoent 1846 ; Schnabels Univ.-Jahre ङ. 312. bobeln polieren, zuituben 1831 . bodbeinig ") (3. 28. "f). Beiten" Laukhard, Schilda II, 146, 151) büritig, geldarm 1795, 1841. - ${ }^{2}$ ) „er geht h. wie der Jroid im Mlonden= ichein" iit betrunten 1795 cunter fla all m). 18t1. - 5odbe illige Stude ut en reide, wohthabente Lankhard, Schilda III, 253.

bollanoer Tabafaide an ber innern खsand ber Bifife 1795 (nuter 将于eife). 1841; juon 1747 Hospitium $\Xi .76$.

1. Sols: "Ia i it viel $5 . " 1)$ telter, galant, idjön, gelehrt z. B. pon einem id)önen Fleio, einer guten Brediat 1749. - ${ }^{2}$ ) viel von einer ङadje 1813.

2. bolj Sdhläge ("willit Dul 5.?" "Da hat es tïdtig 50. gejebt") 1813 ; "es hat 5rolje geietst" 1825. 52013 - Solze Etoffpritgel 1822.

boljen idjlagen, prïgeln (and) $a b$. holzell, burdholzen) 1813. 1825. 1831 2c. Etoctprïgel ans= teilen 1822. 9luch Hauff, Mem. d. Satans Sap.6. Iaju goljere 1813. 1846.

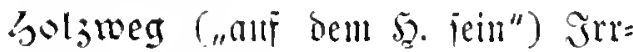
tum 1813.

bonett: "ein h. Burift", "ein h. Dejen" 1825.

bonorith angejehen 1795 ; bonsrig ehrenhajt 1822 ; jofon Balurdt, Rindvigius I, 90; Fischer 1781 Burschiade S. 17.

bopfenfeimben Bieriijde (Meit von "'Pech oder Shleim, Sefen ober Tabaf im (GIajc"") 1747 Hospipitium $\subseteq .72$.

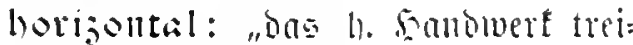
bell" coire 1831.

Golpes ") Hräies im goipia 18. Jahrh. 2) Geitwirt; Ganowirt 1846 . bopits: Gaftwirtin; Sallâtwirtin 1846. 
Golpitant (biat ') int Bolleg 181:3;

2) Deill Rommer 1831. Dasul hoputicren (bajt fein'; im sollen 1813. 1s31; $\left.{ }^{2}\right)$ beim stoumers 1831 .

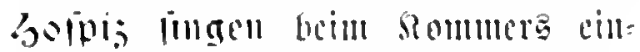
jelste Derie jolo hermu jinṭen, wo= bei ber Mejrain allgemein geiungen wird 1825. 1831. 1846.

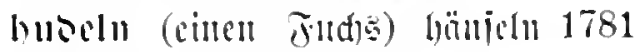

(unter bänie

bujen itifitacn 1749.

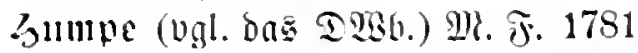

- bumper 18t6. - bumpen bedern 1846.

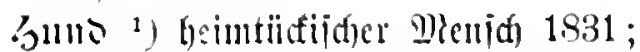

2) Eadje voll geringem 2 ert 1795 (unter sta zend recéc); "bag Miäb= dhen iit fein 5." iieldt nid)t iibed aus 1795; Lankhard, Eulerkapper ธ. 147 - "Ias ijt unterm 5." minter aller Srritif 1825. 1831. 3) Starzer 1831. Fuher "anf ben G. fommen, bringen" in traurige llmịn̈ıอ 1825. 1831.

buแลśatt Thidmort 1781. 1831. 1846.

bumbolase whegen ben Sater burdi) trinfen vertreiben D.Wb.

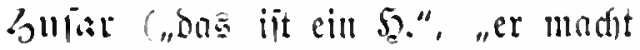

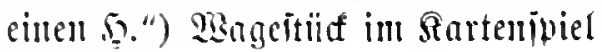
1795.

butiden nad) bem Edmoflistrinten ceremoniös die Steider gegenieitig tanid)en mit allem, was in ben Tajuen niw. iit. Der bution iol= (h)es Steibertamiden 1749.

but = uns blingenarrete ober, 5 gut = und mitristitium" Stommentêregel, monlad niemanb sut ober Mrike beiun Slomuers abuebmen bar 1747 Hospitium 5. 35.
Tamm Nerrui 1846;1835) Schnabels Univ-Jalure G. 104.

Tanirjicharenmult bie veneriiden Strantbeiten 1813 (bajïr $\mathbb{P}_{\text {njif }}$ ober tiirfijde Wh ni if 1846).

Taupe, Tope iblechter Rod $1795=$ Sllpe 1781.

Jikger Heine's Werke III, $57=$ ll niveritätajäger Sclnabels Univ.-Jahre 5 . 316: in Söttingen

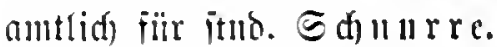

leuen (oit $i$ d) eifen geiprodsen) ipielen 1813. 1831; vgl. bas $\mathfrak{A}$ ob bej (f) eif afs Sarteripiel bei Kobbe 1840 Erinnerungen I, 53.

ighvriren 1831.

illuminiert beiofien 1846. impertinent Tniditwort 1846.

in dulci jubilo leben 1831. 1846.

in silentiis trinfen on pro-poenaTrinfen cines alten

Tuventarium alter Stubent 1795 (unter $\mathfrak{K} a \| \delta \in \mathbb{l}$ ).

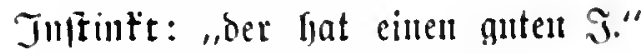
einen guten Riecher 1831.

jotlbaft, Jot - Iofub, jotas 1831 ; jul $\mathfrak{S}$ 나.

Jrrjal Srrtum 1781. 1813; "in cinem gelintoen 3. id)weben" 1813.

Tubal Frenteleben 1781.1795; Spá̉ 1831. - jubelı jid) ein Rergnïgan maten 1781. 1795. - jubilieren jibel jein 1831.

Juben " "I. haben" Frurdht labbell 1846. Sclmabels Unir.-Jahre $ఠ$.

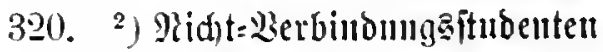
(iir Bürid) 1846.

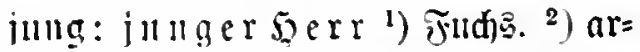
tiger lieber fremblidfer $\mathfrak{g}_{2} \mathrm{err}$ mit ber $\mathfrak{B e j t c} 1749$. 
Jungburith Etmbent in 3. Gemeiter 1822.

Junge: "blamirier S." Tulldwort Laukhard, Eulerkapper S. 106 "D un meer S." Tuidumort 1795. 1813; Eulerkapper હ. 109 (,ijem.

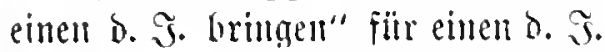
ertlären - "eitten D. S. idhident", ihu durd) cintell ambern bafiir er= Elärell laffen 1795).

Jungfer twer bie Inniveriität verläßst ohne gepauft, gefejiel tmb losgclegt zu haken 1825 - obue gepauft ån habe» 1831 und OLB. Wolff, Naturgesch. d. d. Stud. S. 131.

Jinge Saffer, bounierter Serl 1831. Jorg Tujhwort 1846.

Jur (G) ॥ cf 1795 2c.; Laukhard, Eulerk. S. 108.

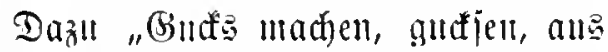

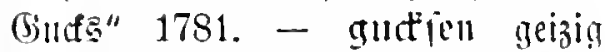
icin, Brofit madhen; Futtomactor Batderer 1781. - Turier Mibsbols 1846.

Eaaball ') Bjerb Salinde 1744 ๔.75. 196; eime rotwelidae Bezeidymurg. $\left.{ }^{2}\right)$ bgl. Stubell=, Tifallabalt.

Kadaver Förper 1831; Schuabels Univ -Jahre 304.

Eaomusbruber Ranfbold? Joh. Chr. König 1781 Akad. Studium S. 161. Eaffer Baucr, bäurif̧der M(enid 1831; TIIf)iwort $18 \pm 6$.

Kalaber Sert, Bater (bei. als 24 h rcoe) Laukhard, Eulerkapper $\widetilde{S}$. 189. 203 - Sehilda III, 71 Emigranten II, 177. 216 - Fr. Wolfstein @. 89 - Steins Abentheuer II, 21.

St u ge, Deutidie Elubentenipradie.
Kalb: "eill At. aubiuben" romere 1781. $1795.1825=$ "ein $\Re$. machen" 1795, fílbern 1795. 1825.

Kaloaunenit) ucter arme Studenten, bie mit faledter Rojt vorlieb nebmen miilien 1781.

Lameel 1) gidat=Serbindmagitndent Niebergall 1837 Heimkehr III, 10. Schnabel 312 (in Şeidelberg uno Freifnrg 1846). - 2) wer teill Inriduifofes Reben fiibrt; bazu $\mathbb{t} a=$ melifixen 1831.

Eampilabll Schnabels Univ.-Jahre 259. 2igl. 5a a pthahn.

fampuen ${ }^{1}$ ) bie ganze Madht liber anf unb (nitig jein. - 2) bei einem an= dern ïbernadten. $-{ }^{3}$ ) auj den Dörfern liegen 1749. - Bedeutung ${ }^{3}$ ) and 1749 Der reisende Avanturier II, 57 mb Michaelis 1768 Raisomnement über die protest. Univ. IV, 171.

Kanmuர Rammunader Niebergall 1837

d. Burschen Heimkehr IV, 1.Diertamuf Rellncr 1846.

Gallaille: "feine blaile $5 \mathfrak{y e l e r} 1813$ inter bla $\mathfrak{b}$.

fallailló oft jeit Zaehariä, Renommist I, \$2. 100. 196.

Eambel - Saubeel Sandidat, Student nad bem 4. Semeiter 1795. 1831 (aud) Mord f a mbeel).

fanmibalif(b) 1846. - Goethe, Faust I, 2. 2293.

Banone: ${ }^{1}$, ,bie bölfige M."Betrunten= heit Laukhard 1810 Steins Abentheuer I, 192. - 2) grofise Bicrflaidne Laukhard, Emigranten II, 260; 1747 Hospitium ๑. 66. 
Ranonen Meiteriticiel von gebramutcm Eeser 1813. 1831; iteiic Sticjel bi an bie sälite ber Dherid)enfel $1 \times 25$.

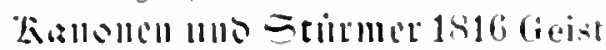
des Studentenlebens hes. in Jenn 厄. 29.

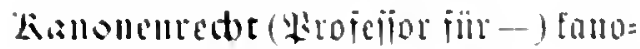
niides Red)! 1sto; "strmonch=?t."

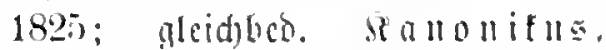
stanouenplotial $18+6$.

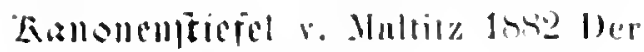
alte Student I, 1.

E.apitolum sopi ohen E. 33.

tapsers: "f. geth" Jist6.

Eariamboligg holsehfth. mut 1820.

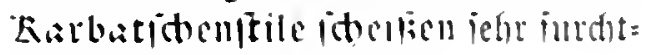
jum iein 1795.

Farlanten fanlenjen 1741.

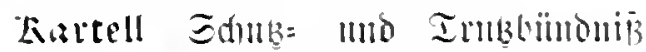
( "in Startell mit icm. ithen") 179\%. 182.2. 1825. 15:31; Sareandel 1711 Amor anf Unir. $\Xi .41$.

Eistelleriager 1831.

Earjer: Carcer 1749. 1831. Barjeritiz garzerwäter 18:31.

Eajten stovi, Simu ("etw. im $\Re$. hatien") $1>46$.

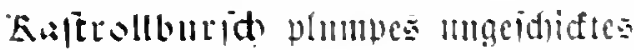

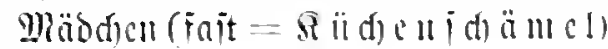
1831.

Enalus 1846; "ods it moin casus" io meine iff es, bas iit red)t iiir mid, Sie baten es getroffen 1749. Lalus Goethe, Faust I, 堊. 1324; Gaudy, Werke I, 165?

Aater Babcniammer: vor 1850 mn= belegt. Injn in Laukhards Autobiogr. I, 240 ,beiotien wic ein $\mathfrak{s} . "$ ?

Eattumbcien Dientmago 1846 (i. seient.
Garg balten jeflu bleiben Salinde $17+t$ E. 102.

tarab! (iandy, Werke I, 202.

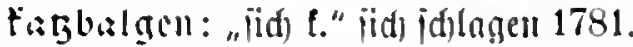

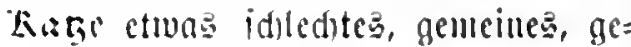
wöl)ulid)es ("Don iit féine $\mathfrak{S t} . "$ ) 1749. - "Iunter ber ภt. jeilı" itärter als "muterm Sumbe jein" Is25; = muter allem Enoce 1 sit6.

Casbunored" Geringwerthiges: "mein (Gelo ijt doda tein st." 1795.

kingillammer: "cinen furditbaren 3. Gaben" 1813. 1831; älteiter Weleg Wichmann 1768 Antikritikus Z.60) (6) (6. gicbt cine Strantheit bes seites, die juneilen migliidlide Wlenid)u mit ben Staken gemein

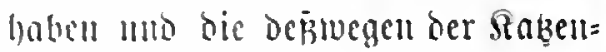
janmer genamut wiro uiv."); Brenrano 181j Griundung Prags (5.439 mad)t jiber bas gat die ?Hmerfung "ein Mame, mit weld)en bie vollen Wriider bic Madwehen ber Trunten= heit bcacid)ucu"; 1I. Heines Werke I, 132. 411. Bgl. Mloralijater. Gater ift in biciem Eimne bis 1850 nod) Hidt nad)getuiejent.

Cargenmulit 1st6; bujür im 18. Jahrh. EHottmuif Sehreiber, Gesch. d. Unir. Freiburg; Polter= mnif 1749 Der reisende avanIurier 1T, 489.

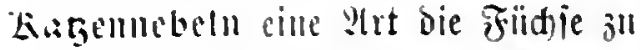
quälen Leo 1836 (Akad. Monatssehr. $(\mathrm{r}, 286)$.

Eaginjamber, Eabianer i. oben ङ. 16.45 .

faujen: "id) babe mir einen gefauft" (ober eeuen gefooft) ïber ben Durit getrunfen 1831.

fiulflitb erjielen funjen 1813. 
faut vorïd)tig, beridnitżt ("ein fauter Serl") 1831.

Ravaltade Burjuenautang zu Brerde 1831.

Gebrbejen Dientmätodyen Gaudy, Werke I, 219. 2igl. Bejen.

fehrell: "der felirt, wo er himfommt"

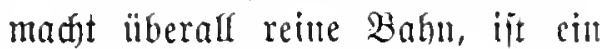
geï̈hrliđer Begner 1831.

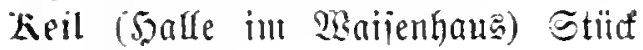
Brod 1795.

Eeile bereben Sabläge befoumen (meitit "tiildtige, fïrd)terlidhe $\mathfrak{\mathcal { H }} . "$ ) 1831.

teilen f́ăen; ỉhlagen 1831.

feilenl 1) faujert. 2) im Epiel mefrr bieten. ${ }^{3}$ ) prïgeln $1795,{ }^{4}$ ) erhajd)en, crlangen (oft per nefas) 1813. ${ }^{5}$ ) 'anfommende Stubenten abjangen' iit nenerer Mortgebrand nad) 1843 Züge u. Zustände aus dem Erlanger Studentenleben @. 51; L. Köhler 1843 Akadenı. Welt I, 38. Eatlbaur Reihhan 1795. 1813.

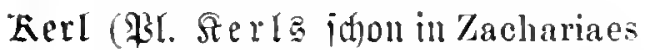
Renommist) inl guten "uto böjel Einn: "ein lieber, oummer, idblechter Serl" 1795.

1. Kelïel da Salliige Waijenhan: ふe ijel a uer ieine Bemolnter 1795.

2. Eellel Mluder Mart. Schluck, v. Burschencomment $\S 12$.

Regerbiftorie Rirchengeidichte 1825. 1831.

太立é Gelo, Gelder 1831.

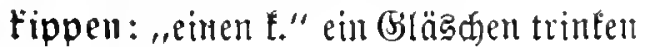
1831.

flat̃en von 28 unden 1813. 1831.

frlamm lein feill Gield haben 1781. - $l \mathfrak{l}$ amm döritig (.flamme Bei=

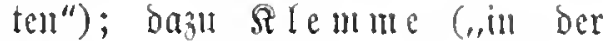
KI. jein") 1795 .

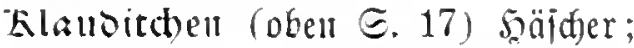
vgl. das DWb. Inter (s laubit= (4) en und oben unter (bied it dien. colepper itartes Mrietpierd 1831.

flemmen itchlen (bon crlüubtem 2hus= fiilhreu) 1795. 1825; genaner "Sadjen

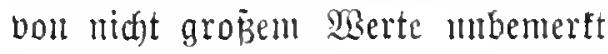
mituehmen" 1831.

Lilinge: "eine (gute, id)lectite, jeine)

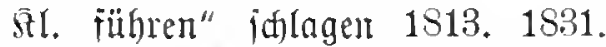
"eine gute fil. jameipen" gut jula= gen; "eituen vor bie Altinge triegen" 1825.

Flifiren preflest (unt eine Buttelje Bein, un ein (5ijen, แn eine 巨(d)littenfahrt) 1749.

Alober lange Bifeife 1846; Niebergall 1837 Des Burschen Heimkehr II, 2.

floben mieis, itarf orauj loghanen beim Duelf 1813. 1831; bazu eill= $\mathfrak{f} \mathfrak{l o b e} \|=$ "flobig einbauen" 1813.

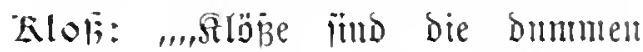
Ferrela, die inmer in die follegia Iaujen, jith ben fopf jerbredyen,

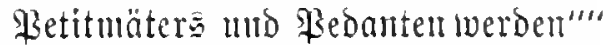
in Gigi. zll bell braven Burianen

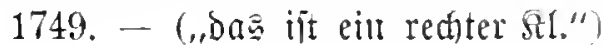
onmmer, einjärtiger Mnenich 1781. "alfes twas nicjt Buriche ift" 1757 Niemand E. 57. 112. 133. 153.

florgen bezallen 1781, viel zahlen 1831 (anc) Laukhard 1800 Erzälungen u. Novellen I, 245); vou alteu 巨(d) gelderu 1795.

tlogig grob, umbüflids 1781; jtarf, vicl, gropi $17 \%$ ). 


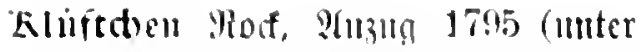
Sove); idfon 179:3 Philipp Dulder I, 87 mo Laukhard, Sehilda I,

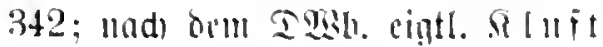

$=$ iiì. Khâlîf 'Stceio'.

celuntern biselocer 1749.

filallen futuere 1781. 1795. 1846; bran enallende Gruloitonen Laukhard1-COAneedotenbuch I.8S. Linallhiste lupanar Lankhard 17os Schilda III, 63; ce iit nads Stlem Prow.=296. 1792 piälitid.

Ellare (- Ellart: Bahrdt 1790

Leben re. des Pastor Rindvigius I, 212.

Killagter 1) guter Manditnbaf 1781. 1795. ${ }^{2}$ ) altes Butd) 1795.

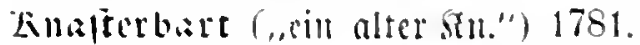
Ellanl: „beiofien tvie ein . Rn." 1S31;

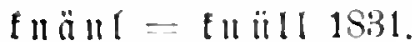

Limanlet Geizhale 1795.

fuciren trani. (and) , ctm. jem. ab: Elleifu") jem. omst Bureten babiu bringen, daj er belo oder (beloces: wert hergibt 179.).

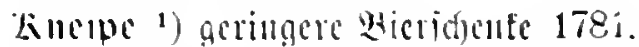
1795. - 2) 9girtabats 1813. 1822. 1831 (bei. Lierbi lloung afue ipe).

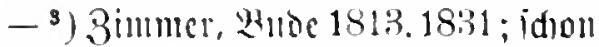
1793 Phil. Dulder I, so (iiir: Misttenberg): ,jell. mí die sin. rïfen" jem. beildhen 1813. 2lud) Hauff, Yem. d. Satans Mał. b. - Tïl Hedentmig ${ }^{2}$ ) in (Erlangen viclmeht If ommershans 18:55 Schuabels Univ--Jahre $\Xi .234$.

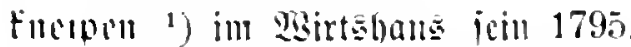
1813. 1822 26. 2) herbergen, logiren Schnabels Univ.-Jahre §. 80 . -

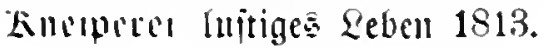

Elleipgeme twer gern fneipt 1813; cherio Linewpler 1813. 1831.

Ementir Bainvirt 1822. 1831. 1846. Emerumart als iron. Bezeid)mun Schnabels Univ.-Jahre $\Xi$. 36.

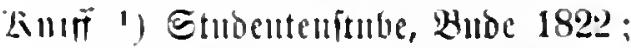
2) Feincre Sureipe 1831; and Heine, Werke 11,58. 3) Streuzer 184t; hdschftl. un 1s:30; Schuabels Univ.Jahre @. 362 .

Limitrbeologle 1831 (ङ. 115) wohl verbruct fiir $\mathfrak{R}$ nifieologie (ohen

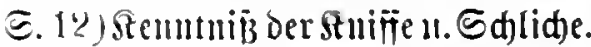

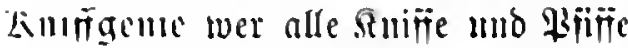

lennt 1793 Philipp Dulder II, 118.

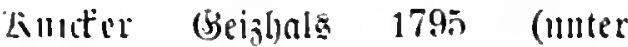
at un uifr).

Emill Fleijd, Braten $18+6$.

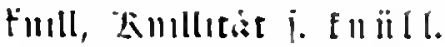

Limtteltomment 1822 = s แ to $\|1 \mathrm{me}\| \mathrm{t}$.

knobd miriel - tu obel 1813.

fnödchn in salle witrjeln 1795 (unter a b $a j$ d) $e \| 1)$; and in (5öltingen 1781 Beitr. z. Statistik v. Göttgn. ङ. 205; Laukhard, Fr. Wolfstein I, 134 .

Lllodicn 1) gemeines Mä̆dd)en (i.

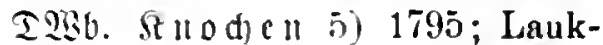
hard, Emigr. II, 167 ale 2 Inrede an cine Ed)enfwirtin. $-{ }^{2}$ ) STerl (,ein Fineler Suod)en") Studentenwitze

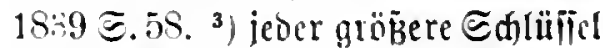
(3. 旦. Dan= mo Etubenfunden) modern.

frollig grob, mugejittet 1781. 1795 knspre: ") "Sur. haben" (beld haten 1825. 1831. 1846. "\$n. ipringen laijen" (bels veridjlagen 1846. - 
2) "Sil. Htathen" bic (5om mad)en Lankhard 1795 Schilda I, 21S.

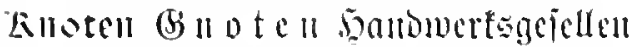
1781. 1795. 1831 (antd) = mans:

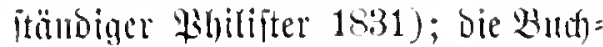
stufergeiclles fint ansgenommen Iach) Laukhard, Schilda I, 80 Eulerk. 69). - H 11 otig Den Stu= Denten midnt jienculo; "Enotiges Beld" viel Beld 1795. - genteill, orbintizr 1831. - Se utentud plumper Wis 1781 .

Enoteata Menge Stuoten (z. B. „es

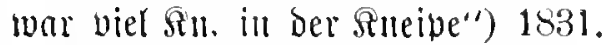
funffig tïntig, gehörig 1839 Studentenwitze હ. 31. 93.

fuill, Enullitist 1825. 1831 (and) "ein Eniller Rerl" cin originelles Sibjeft). Stulill it ät Schmabels Univ.-Jahre 164.

Emúppel Sandwerfägcjellen, Sehr= intugen 1781 miter $B$ ur ithe.

Emippelasjutant die miterite (5harge Fischer 1781 Burschiade 57.

Emippelfomment \&rïgelei 1813 (untergich omment) =suittel= c o ut ul e ut t (Gebrand jich mit Stö́fen żl prïgelı 1822. 1831.

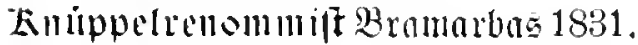

Eober Rebbaber; "ein naffer $\AA$." eill Riebhaber phne belo 1831.

Lodbloffel: "jem. Hifrer ben $\mathfrak{R}$. bal= bicren" als jtıb. Lankliard, Schilda I, $12 S$ - Fr. Wollstein I, 74; aud bei Vollmann 1846.

Eormi() saninmann modern.

Eobl Ilufim 18t6; jonoll Bahrdt 1790 Autobiogr. I, 25); Lankhard, Fr. Wolfstein 1, 89.

foblen "biel ourd)einander fured en, obme 2thïht mb 3mianmenhang

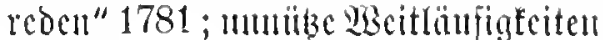
im Bortrag mad)en (and) "cr mad)t viclen $\left.\Re_{0 h} l^{\prime \prime}\right) 1795$.

Lolibri fleiner Selluer Heine Werke III, 1!).

Eollegic Sorlejungen 1781. 1795; j d $\mathfrak{x}$ ä $\|$ ze $\|$.

Eollé: bic intuere Geitc all Dor Bande beim Billard; "ber Ball jeht Rollée" 1795. - "Rollée f(c)Leppen".

1) dell Ball mahe an dic Bambc jebell 1795. ${ }^{2}$ ) alf Dell Siarzer bringen (v. o. Şäjuern) 1749. 1831.

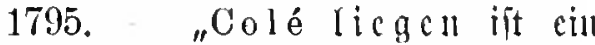
Wittenberger terminus: wenu eiucr fein Beld lyat, jo fagt man, er licge colé; heibt audi) joviel alo trumm liegen" 1745 Auf der Extrapost eingelaufene etc. Nacbrichten ङ. 70 .

Roller ("in K. mb Rannnen") dic gelben Rederhofen des Stlloentent en Wid) 1846.

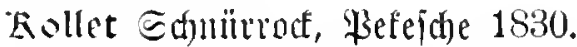

Gollet folien $1781=a$ af it o $\bar{B}$ e It 1795 beim Jed)ten jent. in dic 5nge treiben. - 3 u S ollette it eigen icul. Siormilife manen 1795. - "anl bas $\Re$. follumen" ôll Dad) iteigen 1831.

Esolloquinm beim semmers 1825. 1831.

fomiich Illitwwort 1813 Göttgr. Student S. 107.

komitat beleit fï eiten abgebenden Mitburijd) reip. Loititation 1813. 1831; Laukhard, Eulerk. @. 127. - $\mathfrak{t}$ om i = tiren bas Geleit geben 1813. 1831. fommanoire'l bei Dnellen mo kei Bierfebden 1831. 
Fommen: "cincul etw. f." vortrinfen modern; vgl. DWH. V, 1640.

Komment (bejeke be wurjdenlebens 1795. 1s13. 1831 ibei. Intll=, Discrtonuent); "cin tranriger, nafier Somment" cinte mantänbige \&ehens: urt, jolectre (Gewofntheiten, häblid)es Metragen; "co ifr cin nafier Romment unter ifhen", "er bat eimen naijen Bomment" bojen llugang mb Ber= fiht 1795. - Eturenteubraud) 1522. - "Den Tromment wiijen ober ver= itefu" iid) ju Hehuen wifien Campe 1813. - Kommentruterei Sehnabels Unir.-Jahre Є. 104.

Konmerith Stommers 1781. 1795. 1813. 1831; dału foutucrid iren 1781. 1818. 1831. Stomerid)= brnder wer an sommerien jein cinjigce mo grö̈teg Rergnitgen hat 1795. - ( 5 o m merce, (5 o m merce= is ruber Campe 1813.

Kommeraienrätl)in bic mit imngen Mäbden cin fommerzinm mad)t 1795.

Gommilitomen joun oit 17tt Salinde

ङ. 15̃1; Reinwald 1720 Academientpiegel ङ. 117 .

fommun ("eine fommme Birtid)aft") gemein, niederträdtig 1813. 1846. toncesiren bei ber Bieriehoe 1831.

collcilium consilium bas afabemijate (Berid)t 1813. 1831 (ill Seipzig Ânjill.

Eonsition nafferzelellidaft (and) 5ouslehuritelfe) 1795 ; oit bei Laukhard (j. oben 5.27 ).

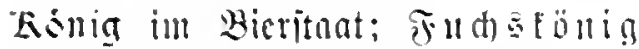
THofmajor 1831.

Somilium 巨trajerfenntuiz, Etabt mo Stabtgebiat anj 2 Wieilen in ber
Mumbe in 24 Ctmbent 3ll rämmen; conilliren ba stoufilum gebeu; "Das (Somilinum muteridrciben" cille milisere Form der Etraje 1813.

Eompitution (scicke cinter Berbindung 1831.

Falltablatlen beim Tedenten (Bgaj. "Eeftion hanen") 1795. Foutra: rufell, Fontrabetgll bei einer Mempclei cinem ber ruit ober webat (bej. Itod) in Mintelu) 1749.

fontrabiren mit icull. 1831. 1846; bajll Routrabage 1846.

Lomvent 3miammentumf um gemein: uiißzige Begenitänbe abzuthandeln mit jll be timmen 1813. 1831.

Konvift in Reipjig 1831.

Esuvivimm Stmeiperei $18+1$.

Kopilballger Miuter.

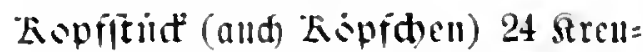
jer $1 \$ 35$ Schnabels Univ.-Jahre อ. $100 ;$ i. $\delta a: ~ D W b$.

forwu nebmen jur Mebe itellell 1781 = foram triegen 1813 (and) Bahrdts Autobiogr. 1780 厄. 79 $=$ toramiren 1781. 1795. 1825 . 18312 . (alld) Laukhard, Schilda I, 156; Jobsiade II, 15); herall: jorbern 1781. Dazn Koramage Tadjirage durd) einen britten ob ctw. habe beleibigen jollen 1822. 1825 (unter it ï $\mathrm{r}$ jeli). 1831 (unter (urios).

Estb Etubentenitube 1846; modern. - "jen. ben $\mathfrak{H}$. aupipielen" $\mathfrak{A a b e n =}$ milif maden 17. Jahrh. (in Schreibers Gesch. d. Univ. Freiburg II, 453).

Kotnclití Sater 16/17. Jalurh. ugl. Reinh. Köhler, Zaehers Zs. I,452. 
Corpe (ancrit als cloor feoc land: utantid)aftlide Sirbindmug 1831) ala Bonner Wort in Bouncr Ber: bindungêtatuten 1826-1829 àtrit unitreteud. Iazn Shorburid 1831 $=(5 o r p s b u r i d) c 1846$. Ob eigtl. iscutija mit $S_{\text {tubentend }}$ Stnbentenifi in ber אirdje Reinwald 1720 Academienspiegel 5. 417?

Eoltphilifer Speijewirt 1846. Schna-

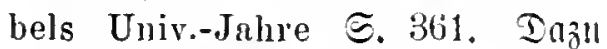
Soithrilitrejie 1846.

Kotge vomitus, fotgen vomere 1831. Ester Stubententund Pistoriade.

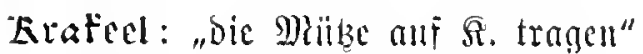
idhief, anf dem red)ten Slor 1846. Arafeeler wer ami Drelle ans= geht 1831.

Gramtivogel meretrix Hunold-Menantes 1710 satir. Roman d. gal. Welt ङ. 57. - Cine andere Bedentung im 16. Jahrhmbert $;$. obelt 5.52.

Lräljaben itud. Perbindungen an Stelle Der Drben 1785 Beytr. z. Statist. v. Göttgn. ऽ. 207.

Aranjianer Rerbindungatubenten, Mitglieber eines firündhens, einer (Beiellidhait oder Burichenjafjaft 1822. 1825. 1846. - Dit bei Laukhard; vgl. pben ธ. 11.

fral; umpolirt, raulf Fischer 1781 Burschiade ङ. 19 ; einfältig, hölzacrn ("ein fr. Bentehnen," "eine fr. Soee")

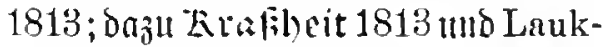
hard, Eulerk. S. 111. - krajier Fud 1846. - fiaijer 占hilifer Th. Köner 181ㄹ Nachtwächter 4.
Elavatte: "einlent unter bie Rr. jeben" cin (G)äst)en trinfen $1 \times 31$ (unter pífife it).

Kirawall 1846.

freisu cille Bed)e anjarciben lischer 1781 Burschiade 5.7.

frupieren fterben; "ill bell Subnben tr." ohne Einwernehnen mit ben (Släıbigeru weggely 1795.

trenjfioel jefr berguight 1846.

Ereujphilifer Sdjimpfimort bei Laukhard 1798 schilda I, 142.

Lrippe in Breslan ber Egl. Freitif(t) 1831.

Eritif: "miter aller Sir." 1846.

Eroten: (5roten Philifter, Bürger

Reinwald 1720 Akademienspiegel ธ. 153 ; i. oben Є. 13.

Krummer (, anf Grumme erg. Säbel losgeh" frumm liegen obne Beld jein 1745 An der Extrapost eingelaufene Nachrichten 5. 70; Studentenwitze 1839 ธ. 88.

fuerviren (Beld) dnrdybringen Musander 1709 Studenten-Regeln 5.27. ¿udbenprofellor §uchenbädfer, Der zllaleid Siquenrs und Ronfitment verfanft 1781. 1795; Sindhenbäcter $182518312 \mathrm{c}$.

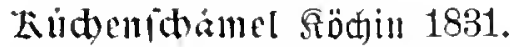

Eub) fbwoof niebere? Tambergnïgen 1831. 1846 (älter in 5olle $\mathfrak{R} \mathfrak{H}=$ ¡đ đf a Univ.-Jahre ङ. 139. 257.

Kunmel Rebcllsmittel 1822. 1841. 太ưmmelturte Etudent and der gä̆he von 5alle gebürtig 1781. 1795. Gtndent defien Seimat nidis über 2 Meilen entfent iit 1813. 1822. 1831. Th. v. Kobbe 1840 Humor. Er- 
innerungen II, 171 gicht an, R. 시 ill siel, wer bort abjolvire olune cille andere llniberiität beind)t ill habel. In̈rfe mirt nenerbing uls crepitus ventris in ber Penüler:

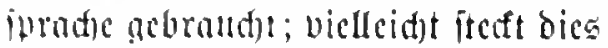
eigtl. in ocr 3ujanumenicbung.

fribu "alles was jid ctwas iber bas (Bowölunlid)e crbebt (cine fiibule joce)" 1813.

Rubpapa Ddje Bahrdt 1790 Rindvigius ङ. 31.

furallin, Niebergall 1780 Des Bursehen IIeimkehr III, II; and DWb. furiแson but(t)prigelu 1781. furen, coureu (bei ben Brofelioren) QIniwarmmg mad)en in (5öttingen Zs. d. Harzvereins 22, 423; Heun 1792 Vertraute Briefe II, 80 .

furiss cill Injdwort 1831.

curlemurlepuri oben $\Xi$. $2 \%$.

Eutri=?linrr (cill 200rt ans ocu Mlopsorben?) jubcilen = baar (bicld 1749 .

Fillen: "cin Dunttier anm t." eine icine Bude Hippel 1779 Lebensläufe II, 122.

kussibilis obell ฮ. : 37.

Eutithe: "couche - recouche contrecouche" als Beleibignungen bci Mart. Schluck $\$ 12$.

cuticher: "er hat sarten mie $\Subset d a n=$

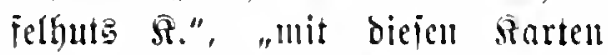

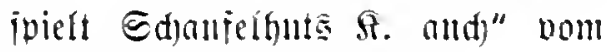
Bsliiaf im startenipiel 1831.

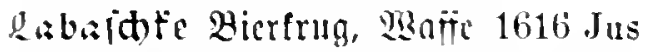
Potandi $\$ 9.41$.

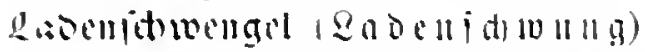
1831; Grudy, Werke 1, 21s. Ta= fïr 2 abcuid wanj $18: 39$ Studentenwitze $\Subset .7$. labm: "bies ift cinc I. Sade" iie hat cincu Safen 1831.

Sallocevater 1779 Der deutsche Stu-

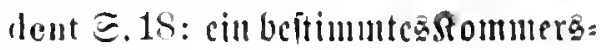

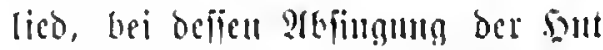
mit ciucm $\mathfrak{T}$ cgen burditoden wirb 1781. 1795. 1813. 1831. 2igl. Zachariaes Renummist I, 23. 305. Lansomianniकait Rerbindung aus Stubcutent beg gleiden Baterlnubeg 1781. 1795. 1831 ions Mhitglico ift cin $2 a \| b a m a n j$ idaftcr).

1. Lallje: "eine gute \&ayzc, zum Mingelremen nidgt bequem" obse. 1745 2Ali ber (5xtrapoit cingelanfeuc Miw. Taduridten $\Xi .26$. "S. breden" illt Welllesritt futuere 1749 der Meijende 2lvanturier II, 59.

2. Ianjen beit. Giläicr 1825. loöl= zerner Bofal aus Sena 1831. "eitue 2. bredten" alo Bieritandal 1831. Lappen: "Dutrd) bie \&. gehn" Fliehen, a॥ - Niebergall 1837 Des Burschen Heimkehr II, 10.

lisppiic) ung̣creimut, cinfältig 1781. 1795.

giztijol Enite, Etreiane (in Mänden) modern.

lanern jebnlid) crwarten; beim Fechten iid) bectell mo eille bequeme $\mathbb{G}_{e}=$ legenl)eit zum 5ieb oder Stó $a b=$ warten 1795.

Laus: "teine S." ntein, gar nid)t modern.

Laus-Deo Hedumung 1795 ; allgemein 17. - 18. Jahrb, $\mathfrak{j}$. 2A. Laukhard, Erzählgn. 11. Novellen I, 22:3.

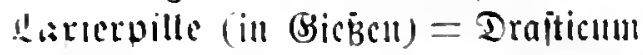
hei Laukhard 3. B. Autobiogr. I, 180. 
berer: "bou ?. jiehen" blant żiehen 1781. - "311 \&. itcigen" 1795. 1831

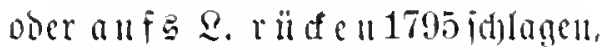
haut zuleben. - aufs \&. jaujen fitalf zutrinten; ansledern ourd)= priigeln 1795.

l'oern ná), theef, tranrig, erbärmlid), emuntyat 1831; Fiseher 1781 Kom. Burschiade 5. 16 ; "leterner Eal= buder" nach Büehmann idion 1591 ill Hans Clauerts Historien 92.59 . Lebrgelo gebell beim Spiel, da mant no(f) nidgt recht veriteht 1795 . Leib: "anf bell Reibe haben" ") be= mittelt jein, $\left.{ }^{2}\right)$ seera haben, Seritand bejikels 1749.

deibburithe $18+6$.

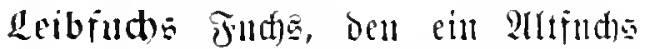
einjdlägt 1825. Fondis, der bem alten $\mathfrak{S a n}$ fleme (5hrendientite be= jorgt 1831.

Meicbnam Difizier, Mlilitär 1846.

leidthimnig luitig; and wer mbe= bactijam (5redit gibt 1831.

veiotragender Freund mo siebling

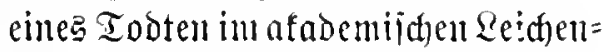
zug (bon 2 chapeaux d'honneur ge= führt) 1795.

Leimpieser giddtwerbindungattndent; leimitedernodien 1846.

Sotrionballen beim Feduten gervifife Soiebe in jeitgeiekster Sromung uah cinander hantell 1795.

Ler Reftion Lenz, Hofmeister I, $t$, Licbt " "er ijt allögegangin wie tin

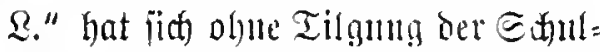
Den bavon gentad)t; ${ }^{2}$ ) "R. weg" 消年 1749, - "wie ein 8 . neridninoen"

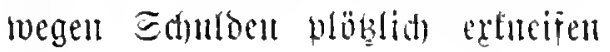
1831.

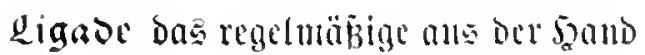

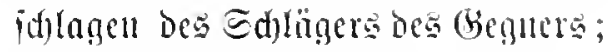
Dajll ligite ul 182?.

ginfen: "d)rittlidbe 2." (belo; "mit "thiden d)r. ?. helfen" Bacheliade ๑. 24; Studentenwitze 18392 ङ. 11. 54.

liftı einfältị, ja)wad) ( Serl") 1749.

Qitteratm Etmoent ber PGgilojophie?

bei Laukhard, Emigr. II, 167, 295.

loca: ad loca beim fommers 1831. Loch: "Söher in ben Reib fragen" viel jragen 1813; "wie cint Sod) ianfen 1831.

loffel Tölpel, onumer einfältiger Wienid) 1795. - „über dell S. bal. biren" 1795. Bgl. Sodflöfel.

Liffll1: "iich l." O. L. B. Wolff ${ }^{3} 56$. los Srommantoo beim Tull 1831.

lorgeben jid) idflagen; ,losgelyen auf ङäbel Єđ̆läger" 1831.

log haben (z. B. viel, bie Wandeften) wiflen; los truegen lemen; los

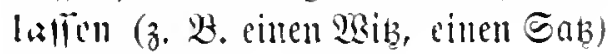
3แu beiten geben 1813 .

loslegen futuere 1825.

losmacben einen Broeifampf beiteln 1822 .

Losichictien anjangen v. Maltitz 1828

Der alte Student II, 2; Wilh.

Sehröder, Studenten u. Lützower I, 4 .

Writhlagun vertanfen 1770 Begeben-

heit mit einem Gött. Stud. auf Plesse I, 182.

Lo foin Wicujur haben Köhler 1843

Akadam. Welt I, $7 \tau$. Lovis Sonito or 1813.

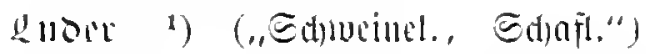
Fleijd); ${ }^{2}$ Fifero; ${ }^{3}$ ) meretrix Laukhard 1798 Schild I, 192. - 
"iu ะ. icin" inupicu stoppe II, 17!) "ill $\because$. licgen" innien 16 .

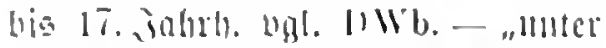

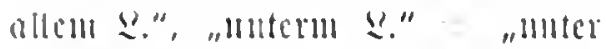
Der Mabe" 1htt - "Illter bell :"."

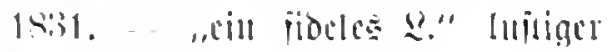
Merl 17\%).

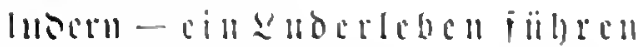
licocrlid) lewen 1795.

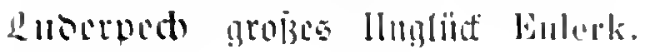
Е. 120 .

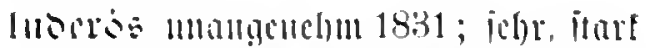

Lankland. 17!'s Schilda I, 333. 192. lutures gendunen 1795.

Immpen: "iide llid)t 1. Iajīen" Giaudy,

Werke I, 174.

"mmpcubums nid)täürobiger Ser! 1831.

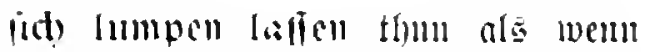
man arm wäre mu cinc bicldam= ansgabe àl vermeioen 1795; filziç= forg icill, wems ant (5hre an= commit 1781. - Gaudy, Werke I, $17 t$.

Lumpig idfled)t 1831; Laukhard Schilda I, 302.

gutbarimm Eopha? Krinderling 179i Reinigkeit d. d. Spr. G. 195.

2ungenb!c 1) itarfer Bich 1795. ") Bernucis, Eatire 1781.

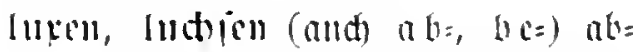
id)!unbell, abitibiken 1781. 1795.-

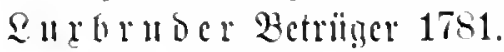

Sc. Macedonianus: „ber bartherjige Lapa boitcht (beim Ed)ulbemmad)en be Eohus anfm Sct. Macedoniano" 1793 Phil. Dulder હ. 93. Siacessmice = Jube, Dhabencifer?

Magniticus Iitel oce lieftore ober Broreftor 1831 .

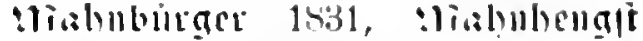
1九ti. - עl

Siralice Heine Wosteit (, Mh. ani jem. Iraben"1; ,iid) nit ber y․ oriiden" iad)tc, geränid)los weggehn; III a! $i$.

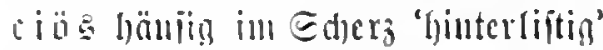
1s13. - "mit Malice treten" 1831.

malitiso gerieben, durdytricben 1831; Injthtwort 1846; Eulerkapper $\widetilde{S}$. $1 \leqslant 7$.

Mialitisier cine Etcigermug dea Mappicrumuen 1825; F. and) Stul p. :lianidbá (Ğlüulviger 1749. 1781. 1795. 15.22; id)on bei Stoppe 1728; mahmen ijt itub. ma॥idäeru $1631=$ ma uid)ircu 1795.

:lianllidbaft: „junge Ml." dic nen= angefommenen Etubenten 1795. (muter $3 \Perp \mathrm{m}$ a d) 8 ).

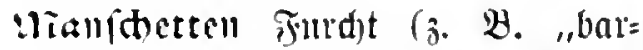
bariiđfe M.") 1S13. 1846; Laukhard, Schilda I, $302-$ Eulerkapper 5. 147. „Die $\mathfrak{D}$. zittern if)m" el iit eridurocfen Fischer 1781 Burschiade G. 16; in manschettis in Plugit 1846. - $\mathfrak{M}$ ani duetär Feigling modern; Ml a uidet tier Illidjwort 1846. "lianfकetten= junter Dnanijt 1781. 1795.

:Tiappe Sollegmappe 1795. 1813.

:Tiartus Marför, Selfuer in Mein= ober Staficehäujerı 17S1. 1795; altd) Harling 1831 Frust 5.76 .96 ; Koch u. Köhler 1843 JubilarAlbum d. Univ. Erlangen 5.5. :liartlubrioer Bäaergejellen 1846. (id) Marticun ïđ) a॥şzeidnc॥ 1813. 1822. 1825. 1831.

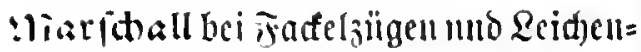
begümgnijien 1831 . 
mafine plump, derb Salinde $17 t 4$ ธ. 157. Nicbergall $1 \$ 37$ III, 11. :lianlefel Etmoent nor der Summatri= fullation 1822. $1831=$ Wa antita Kobbe 1840 Erinnemugen I, 2.

mallifbellitell öfter fei Laukhard i. 2 . Schilda I, 142. 147 - Antobiugr. I, 105.

"Micifen (Miceja, Etadtmeju, Whäju) Stadtioloaten in seiping 1831; when ङ. 51 .

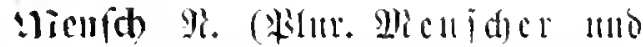

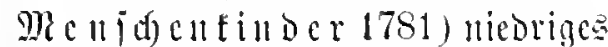
Trratcutziututer 1781. 1795. 1831.

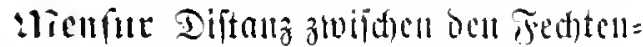
De॥ (,D?. แchmen") 1S13; anf Der D?. Ftehn11 1822. 1825. 1831.

merfen: "lní Dir nithte mertélu" iei ruthig ober Du hajt Iluted 1795.

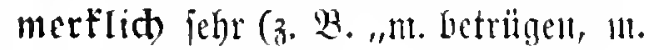
größ̄el“") 1813.

merfwairdig Illid)lwort 1813 Göttgr. Stud. ๔. 107.

Mieịen, Etadmejen in Reipzig bie Stadtiofoaten 1831 ; i. Mi e i i en.

mielle = S anf me ije 1831.

lieuble, menble meretrix Irenius 1766 Begebenheiten eines Leipziger Studenten 5. 241; 1779 Der deutsche Student ein Trauerspiel 厄. 5, 12, 19. - Laget = lionble meretrix 1749 Das Luft-Schloss

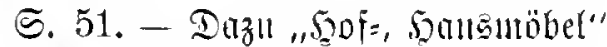
1846 unto "cr foat cin fehr gefälliges Amenbentut" $18+6 ;$ Diöbcl ob itinates najemeijc Mäsd)en 1831.

Liíe z meretrix 1781. 1795.

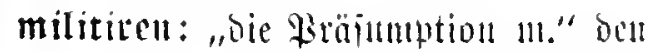
Sdinein erweden jollen 1793 Phil. Dulder ऽ. 117.

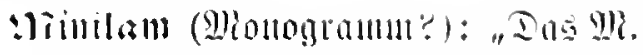
Der Pifeife ift juön" Harring $18: 31$ Faust $\widetilde{S} .77$.

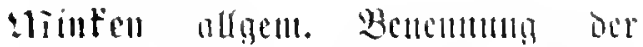
Bürgermäbd) 1822; Nariunus 1832 Konn. Scenen 5. 152.

mijonen ipicten (bei. najard) 1831. miseriam fomeljell elcus leben Ienantes: obcu ธ. 30.

wiift Werfegentheit, trantige Sitnation 1781. 1795. - th 11 f be in Gilswerlegenthet jein $17 \mathrm{~s} 1$ (nnd) Laukhard 1798 Schilda I, 5 Eulerk, 147), - ex i jit a It d d II e ijt ans mit ilym - cr tollt $111 \mathrm{t}$

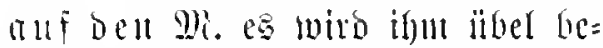
foumten; a lif oc m Mr. flepiten clendiglid) itcrben 1795. - "Ml. ansiührcu" Dummbeiten id)wabecn 1846.

"riaffinten Bantu Jobsiarle II, 2679.

thilfifer 2 gronom OLB. Wolff Naturgesch. d. d. Stud. ${ }^{3}$ ङ. 64.

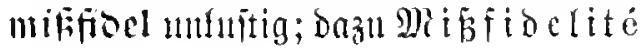
1813.

mitgrbeu beifieu iteylen 1825.

Miitfueipant 1846; Sclmabels Univ,Jalure S. 37.

mitleisige Sbwelter = barmlycräigc G(f)iw. 1769 Gesch. d. Götting. Studenten II, 202. $\subseteq$. $\subseteq$ di $\mathfrak{w} \in \mathrm{c}$ it $\mathfrak{c} \mathrm{l}$. lisibel = me uble.

Miobejourmal, Midodella a de als beleibigende 241 reden v. Maltitz 1828 Der alte Student II, 2.

mogeln bein Spid betritgen 1795. 1813. ("cr mogelt jidd etwoe zu= jammen" 1795) 2c.; alld Fischers Burschiade 1781 ङ. $34 .-2 i \mathrm{D}=$ ge I c i Betriigerci; "Ml. zieft" feillel 
Wetrm gilt 1s13. - Mto o e le Hotrïger int Extel 1795.

1. Sishren in jena arme sunben.

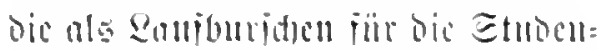

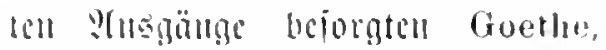
Thur- und Jalureshefte 1804. in ienijucu Etrmmmbind)rn (Kóril,

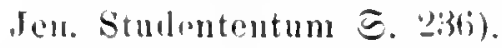

2. : lishren haben fidt) pïrdten (and)

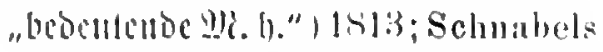
Unir.-Jahre 5. 110. 176, 304. 3:20. Yiad Sander hebr. mora Furdt. mollia 18 isi.

molum: „er ijt m." bejoffen 1 is 25. 1831. 1846. - Laukhard, Fr. Wolfstein 5. 50.

:Tiomptrs Bled), lluïm (.,mad) Dod) nidut jold)en 2$)$ :") modern.

STinkathen Gelocr 1846.

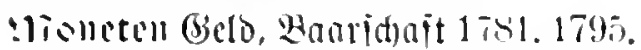
1813. 1831. 26. 1, cr hat flotte wl?." 1795 ; “ci hat odjige !!l!." 18133 . Belege fiir Wloneten Laukhard 1798 schilda 1, 5 - Eulerk. ๑. 16. :1isos (biclo 1846. OLB. Wolff Naturgesch. Є. 53.

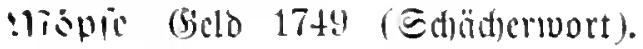
1846.

Distalıper trig. Mabenjummer 1831.

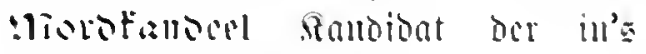
(5ramen geb)t 18:31.

biorstarl Sgmutferl 1831.

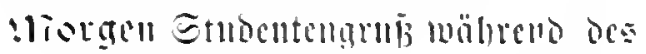
ganjen Tauce $184 t$.

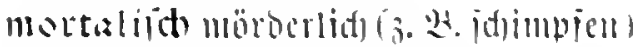

Eulerkapper 109. 231.

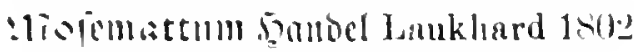

Anckdotenb. 1, ti. 50.

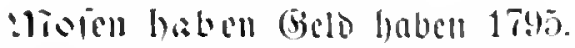

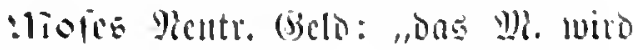

Feble1" Laukhard 1799 Fr. Wolfstein I, 135.

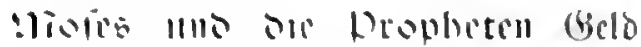
$17 \%$; Lankhard 1810 Erzïlungen 11. Novellen 1, 273-Eulerkapper 5. 139. Schilda 1, 380.

mutt'il priigelu, mores lehren 1813. Sillder nad) (Bombert id)on bei (Bïntber $(† 172: 3)$ und 9ientird) $17: 27$ bezengt, jollen und) (35. Trant, (beid). ๖. proteit. Tlleologic II, 148 zuerit ill sena dic Bulsörer des pietiftifall Brofejior Bubdele $(1705-1729)$ nenamut worocn jein. - Stloßj; Pietijt 1749. - Stubenichwiker, philijtöier: Stlldent 1781. 179; ; , cill 5galliid)és Qcibwort" Leber. Blanstrumpf 1746 Vier possirl. Ged. S. 4. Beleye fïr 'Wictijt' 1745 lirzählungen zweyer Musensöhne Є. $60 ; 1776$ Briefwechsel dreyer akad. Freunde I, (69) (aud) Keils Stammb. 1739. 1745 巨. 206. - Mlu if er gerviditiger Burith, ber burd jein ?hiftreten und 3urcben andere bej(t)widtigt 1831.-

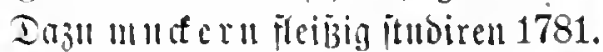
Du :limteren 2 Büriel; bie $\mathbb{M}$.

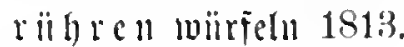

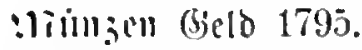

!rimlinuñ oben E. 28.

Milli ') Etıbent 1822; Laukharil 1798 Schilda I, 192 ; zucrít blidftl. iu Dolle 1727 Hallischer Universität angeschlngene Pasquinische $\mathrm{Be}$ grebenheiten; Hospitium $1747 \subseteq$. 16. 41. 2) in salle Miethppero 1781. 17!); Laukhard, Schilda I, $19 \%$

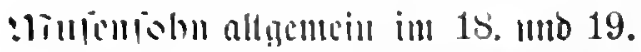

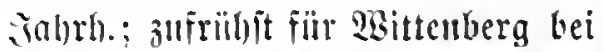
Megrlofilus 167s Herzentsprung- 
enes Freudenecho. so denen edlen vier Musensöhnen Fürst Apollinis 2c. (ands ङ. 10): Musander 1709 Norwendige Studentemregeh $\Xi$. 132. (jebudht 1691 bei Stieler.

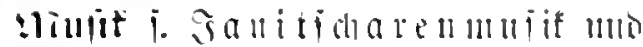
Sa a be mu mit.

Miuterfaliden sier i. ons DWb. unb ,eine 5 cräitärtung ans beut 每." Bretzner 1790 Das Leben eines Lüderlichen $\widetilde{\Xi}$. 363.

:linterpiemigo ${ }^{1}$ ) ,alle auberorbent: lidten :

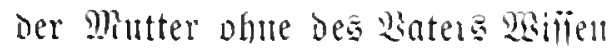
1749. 1831. Salinde 1744 ङ. 51. Hacthauell ben bieb des Gieguers er= wibern 1813 ; bazn 9 a d h h 1813.

maditerlen beim (Grobhans (5ajarb) (Seld nadabieten unb bie andern $d a=$ ourdh zrwingen, entweder zuzujesen

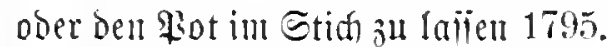
nachochlen fleibin mad)reitei 1831. nachpfeifen: "Die Manid)äer fännen iezst nadppeifen" àtiehen, wie ite ihr Geld hefommen 1831.

mad)reiten (speite, Siolleglyeite) nad)= holen 1813. 1822. 1831; and Heines

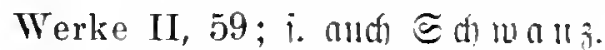
nacbriuten nad)fommen (,id) rïcfe Dir fald jur sueipe mad") 1831. Had) jaufen im Irinten nad)tommen 1831.

nad)perg̣en im Trinken nadbfommen 1831.

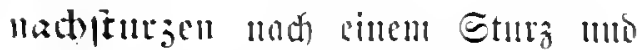
Iuell aberutals itüràtu 1825. 1831. :Tact)thatt (biesante: "mir ijt ein ?h. anfigegangin" Niebergall 1837 D. Burschen Heimkehr III, 10. 2injl. ant ceifeniteder.
:Tadtmmit (E)mentändast, iberl. Etäusocu Sichluck Bur-chenkomment 厄. 50.

Madtrinten 1813.

:Ta(b) (iil Tinlningen) 1st(i; Schnabels Univ.-Jahre Є. 366 .

:Tactusogel meretrices 1is1.

"rat)itehc" im Irinfen nad)fomme.1 1831.

Hagdn, allmagitn coire 1781. 1stti. :ingelprobe 1831.

"lishen: "Der iit genäht" hat im Duell einen 2 njidis erlualten 1831.

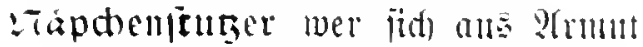
ober buffahrt nim. anf ieiner Etube ineijen lä̉t Reinwald 1700 Academienspiegel $\Subset$. 296 .

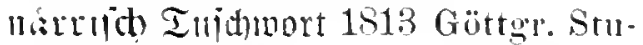
dent ๔. 107 .

Liolemtruber Mart. Schluek, v. d. Burschen-Comment $\$ 13$.

na: ichlecht, mangenthu, manjtïunig ("ein malier sterl") 1795; i. nud fomment. najier Prim Lankhard 1799 Fr. Wolfstein @. 73 - Eulerk. 124.

:Tatur: als QIntwort fïr "Mutiulid, ja" Laukhard Emigranten II, 17 s (5r ijt weggereift? "glatur it $\mathrm{el}$ ueggereijt") - Schilda I, t. ,92. fneipen" - "9l. jimpel" modern.

:Taturallit wer duellirt bhute die Feditmut crlerut zu haben 1795 : and) Beytr. z. Statistik v. Göttgn. 171; Danu uat ur a lijice n 1795. :iebel: „im $\Re . “$ beranitht 1846.

:iogoce giscdjelgeidärt; ,ein 97. reibeu" (Gelo alli 1795. 1813; and Burschiade 1797. 
nehuntu: ,einen u." ein bilibstren trinfen 1s:31.

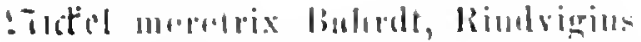

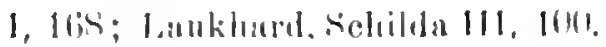
mesertradeta non allem man=

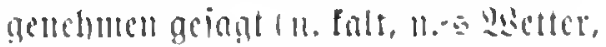
11. Imugerigh 1761. 17!5. - , id) B̈n" lankhued 17!5 sobila I, 192.

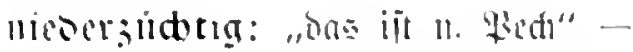

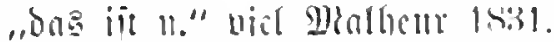

:Tiquiter Etmont ber iid) idfmar, malt mu merkannt an deu Ermben ill icill 1750 Beytr. zo Statistik r. (iiittern. 134.

:ille 1) ugl. Worreiter. 2) meretrix Stoppe 172- Gediehte I, 135: l.eb. Blaustrumpf $17+6$ Vier possirliehe Ged. E. T reambillen ibid. 20); Salinde a 5 ").

nobel idiön (.,oer shod itcht hödit nobel, ou hait cine noble Mianue") 1813.

Siminam - retrorsmu Golditiof

Bertr. z. Stat. $\because$ Grittgn. 1755 ๔. 65 .

:iurbe mburidjifojer Emoent 1831. :inf(be idfuädflidjer, flingenjoenter Etmbent 1831.

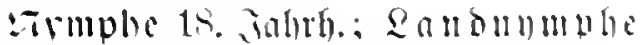
Salinde 1744 厄. 110. $: 266$.

erberlans (beim Brommers) Bie ohere Tiidhlyälfte $18+6$.

Obermelifer voritglider Spieler 179\%. obifur unitudocutiod 1825. 1831. Shon Weise 1673 Erzmarren Seudr. $\subseteq .122$ "ein obienrer \&eren= henter". "in obscuro leben, ob= ifuriren" 1 s 31.

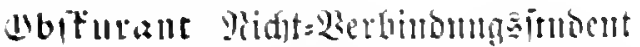

Sehnabels Univ.-Jahre $\subseteq .312$.
ज wob!: gewöhnlider (5imunri hei idmer sul glanbenten bebamptumgen 1.itis.

shlen (,böllid) od)jen") arbeiten, imbiren 1s13. 1s:31; and Niebergall 16:37 Des Burschen Ieinkehr 111, 10: schmabels Univ.-Jalire E. $113.106 \%$.

sdpig viel, grois 1781. 1795. 1813.

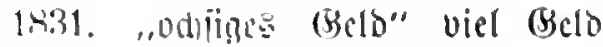
17il: ,odhipa bemogeln, odjig noll" 1413; oit bei Laukhard z. $\mathcal{H}$. "od)iig lalt", .rid odfig irenten" schilda I, 192, 302. shre: ,oic Eade, bng iit nidht ohme" iit gut 1831. - $\Xi$ dhon im 16. Sahrl).

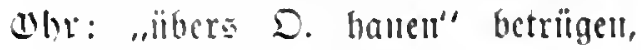
lintergeben 17s1. 1795.

spiecu fezablen, Giclo bringen 1781. sppsuiten bei der Promotion 1831. 山roe"l gemilie Etmbatenverbinbungen

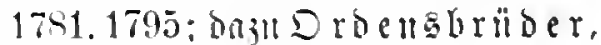
Droenszeiden, Drónu= rege $\{11$.

orgelu, and) "ani ber Drgel ipielen" futuere 1731. Dit in Etammbiiderm. Hablt ") "cimen $\mathfrak{P}$. wähten" Goethes Faust I, 2: $2098=$ "einen $\mathfrak{P}$. mad)en" bei Laukhard 3. 2. Sehilda I, 183 Emigranten II, 227. - Eulerk. 203. 206. 209. Dann „סas grobje Habitipiel" $17 \pm 9$ Abendstunden II, 75. - $\left.{ }^{2}\right)$ im Bieritandal 3 (släjer 1813 - 4 Bläjer 1831.

parfen raudell 17S1; idnell randen 1795.

pactell: „iid) p." abjiehen Laukhard, Emigr. II, 43 . pastejell tharf arbeiten modern. Psilsct tenwurm Surijt 1831 (ఠ, 120. 
122) = Pandettenritter 1795 (unter reitell).

Danier Wappen Der Sandomann= idhaften 1831.

Darabel Feriicte 1795 (nnter Ber iif a c).

Dalade 2hrolage beim jednten 1813. Darbujlow lupanar Leber. Blaustrumpf 1746 Possierl. Gedichte ङ. 6 min Laukhard 1798 Schilda III, 63.

Darlewn Franjüïn ober Mademoijelle für bie Stinder 1749; and) 1757 Niemand 厄. 109.

parteiifber jeuge beim Inell 1831. paridell i. a bpa id ent.

patent galant unb żwar in jeidenen

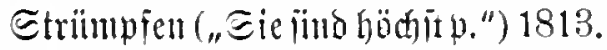
alles was dem Buriđa) gejüllt 1831. Sehnabels Unir. - Jahre ङ. 49; Gaudy, Werke I, 178; and Niebergall 1837 d. Burschen Heimkehr II, 7. Daju Patentringer $\therefore$. Maltitz, der alte Student II, 2.

Patenthengit 1846, aud) Schnabels Univ.-Jahre ङ. $257=$ Datent ibeilier 1846.

patres: "ad p. gehn" heim reiicn 1825.

patria: "lro p. losgehu" im Mamen einer ganzen Berbindung oder gegen cine ganze Berbindnng losgehn 1831. Dautage Tnell Kobbe 1831. Humor. Skizzen ङ. 42.

Pautaut OLB. Wolff, Naturgesch. d. d. Stud. 3 @ 66.

Dautarjt beim Duell 1831. Paut binse 1831.

Paute Predigt bei Holtei und bem DWb. -- bazn

1. pautell presigen 1781 ; ald Lauk- hard 1798 Schilda I, 14 - Autobiogr. I, 2366.

‥ panten prigeln 1781, 1795;

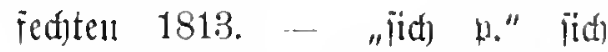
Duellieren 1795. 1813. 1831. Taßn Danterei Duell 1813. 1822. 1825. 1831; Pautbut, =bole 1813.1831; "Bantfomment, \&aukfabit, Baut: jaal, Raufitube, Panduidus" 1831. - Dallfwir allh Hauff, Mem. d. Sat. $\mathfrak{R} a \mathfrak{b}$. S. - $\mathfrak{B}$ anfhahn Schmabels Univ.-Jahre @. 219. 243.

Panten (ein paar 名.) eine veneriind Arrankeit 1781. 1795; ङ人meller be= legt 秘alle 'Benle' alle bem 17 . Jal)rh.

Palltier guter Edaläger 1846 .

De(d) llnglïa 1795. 1813. 1831 ("h)e $=$

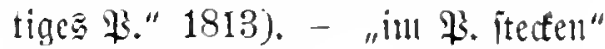
Niebergall 1837 d. Burschen Heimkehr III, 10 - "\$3. kaufen" ebenda I, 10. - "\$3. geben" anseizen $18+6$ (and) NWh. all Holtei).

pedos ("eg gefjt mir p.") 1813 -. pedhiss modern - pechenle 1831 - pedells Schnabels Unir.-Jalire 厄. $86 ; 1846$.

Pedbengit 厄dulter Sehnabels Univ.Jahre ๔. 140.

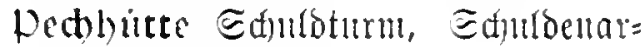
reit 1846 .

Pechuogel als itndent. bei schmeller II 2522.

Pedant fleibiger Etudent, Der nidft burichifos, jondern nad) ben Gejeben lebt 1749 .

Dennal Gymmaïait 1813. 1822 ("ein üppiges P." 1813).

1. per: „p. Iampijaifi, p. Droinke" Gaudy, Werke I, 219. 222; "p. Dampi, p. Tidanto" Bacheliade 
こ. 110.135. 15i3. ,1. Edmuans"i. muer E d) a a ₹. - .p. Evals" be lent Sontoer ans brimmelshanicu.

?. per burd, port, we?; per ach iit per madten ourdobremen: Perganger Rollution 1846 .

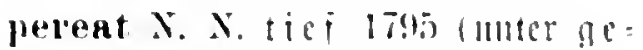
reiteul: preresent dic Whiliter

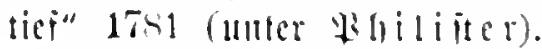

pereat, aber weul ber wurid el: grimum iit, pereat tie i 1831.* perstren cill pereat bringen $17 s$. $1795=$ perman 1749.

Deruito eine Fignt bein Dajaroipicl 1795.

Petmastre allgemeill Wobenhengit;

?) buriff. Pedaut $174 \%$.

pethiren jukem 1795 .

Petitogell (= Ethkertegen) 17si lutter $\mathcal{D}$ igen.

petum Iabat 1846.

perge'l flagen, flaghar werben 1795 ; beben verraten, anzeigen (eilt Etı beut bell Y(noern) 1525. OLB. Woltl Naturgesch. d. d. Stud. ${ }^{3}$ ) 67. S. antebent - Bscerei Eulerk. 111.

Peuts haben Turdt haben 1813. 1846; Schnabels Univ.-Jahre ङ. 110. 176 .

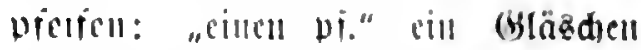
trinten 1s:31.

Primuginder Geizhals: ivarjamer Etmoent 1795.

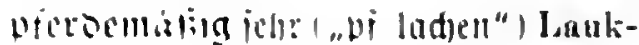

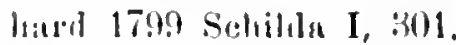

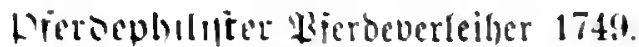
1781 (muter abilifter). 1825. Dit ill ber ?. sälite de 18. Jahrhas. i. 出. I7tit Jalirb. d. Brockens v. 1753 -1790 I E. 81; Lankhard, Schilda I, 66. 221.

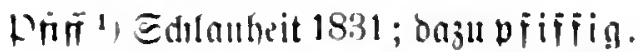
Bi iififus.- - 2) Iabalabicife 19:2, 1831. ... 3) halbes Duantum Hicr oner sien 1846.

Pripitlis 1846; oit bei Laukhard, zb. Schilda I, 432. III, 12. Dajür Pritropi ibid. I, 141.

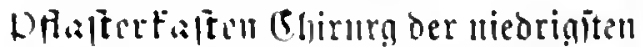
Slajie 1831.

Pliafterteter 1) imbicrendes Stadt: fillo 1795.1813 ?c. ") armer fleibiger Etubeut Lankhard, Schilda I, 204. 3) Betitmaitre ebenda. ") Bummler $1 \$ 31$.

Proulg Pieming 1813; cigtl. juben= bentid) $\checkmark$. Hundt-Radowsky 1820) Truthähnchen 5.116.

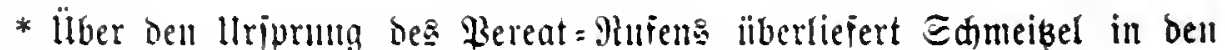

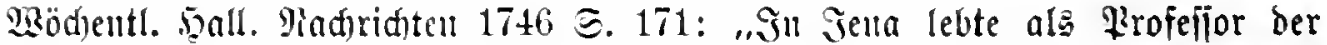
icel. Burc. Gotth. Etrune, cill Maum von vieler Heblidłteit mo Brideibenheit. 1712 war er juerit Proreftor gewejen. (5r modite es einigent von benten Stubentent nid)t red)t gemadt haben; bahero ale er am 4. Alugujt bas Plegiment niederlegte, jo entitund zu Plad)t sin gräulidher Muflani, bei weldhem u. a. nud) on: pereat, Struve, he! pereat! dic ¿njt als eill juäblider mid id)änd=

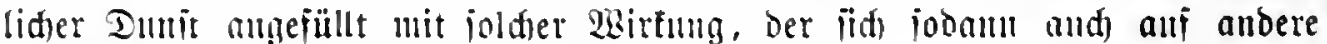
Iluiverijitüten befaunterutuajen ansgebreitet hat." 
Pfote Syand ; vgl. Pig il i jerpiote v. Maltitz, der alte Student II, 2. pfropien: "alli den $\widetilde{F}$, rz gepfronft uerben" jid) in serlegentheit befinden 1795.

Dfundipornell Sporen all bell Sa= none" 1831.

1. Pbilifter*) Bürger Der lluiverii=

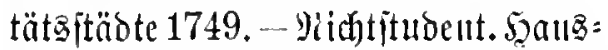
uvirt bez Stubenten; Student nad) beut Rerlailien der llniverjität 1781. IHes Männlidue, was nidht Stubent ijt; Stndent, ber ieit Prbgantgajentunis bat; ßferbevermicter 1795. - Geder
Der niaft Etuleut Imb faton jein

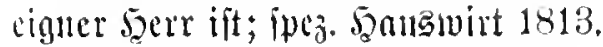
- Bereinzelt wirs im 18. Jahrh. Das ftubent. Philititer ipeziell auf bie Stadtioldaten bezogen z. B. Sleing Prov.=28b. 1792. Bereinzelt (z. : Zachariae, Renommist I, 2. 104; VI, 2. 40; 1779 Der deutsche Student S. $10 ; 1798$ Vertraute Briefe über Halle 5 . 26) = Bferdephiliter.

2. Dlyilifter Mietpierd 1795; and 1756 Jahrbiicher d. Brockens 1753 -1790 I $\subseteq .26$; Schnabels Univ.-

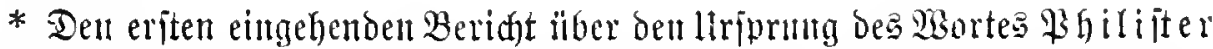

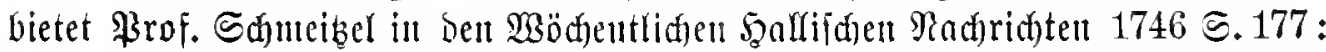

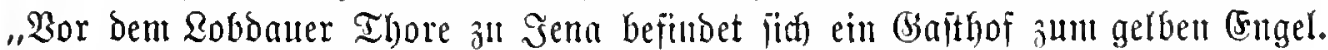

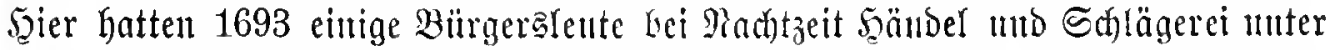

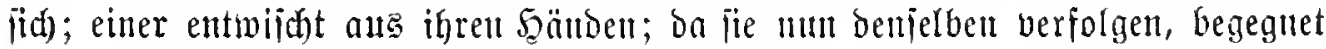
ifnen ein unifhulbiger Stubent bor bejagtem Thor. Diejen halten fie vor ihren

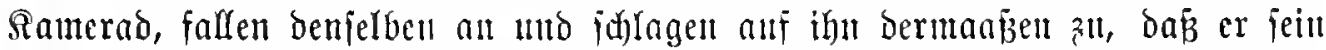
Reben verlierent miiffen. Deti Somntag branf itrafet ber Superintendent bieje mïrberijhe That mit biefan 2 Gorten: Da jei es hergegangent wie Dort gefaricben

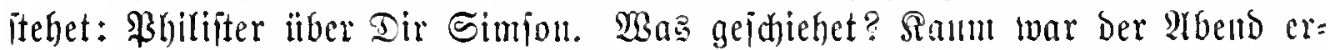

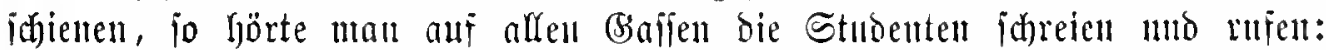
Bhrilijter, Phbilijter itber Dir Simjon. Mithin war dieje Begebenheit zugleid)

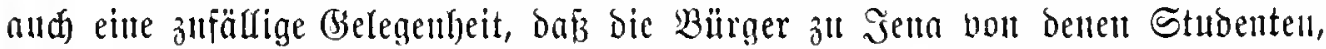
jobann ordentlidjerweije mur Philifter genemet worden, weldye (Sewohnheit nod

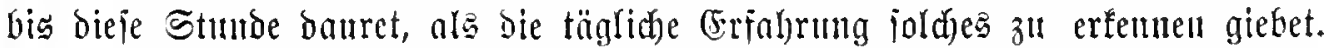
- Sn geitehe ez germe, baj̉ mir in öffentlid)en Sdriften von biejer Begeben=

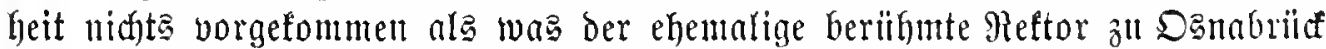

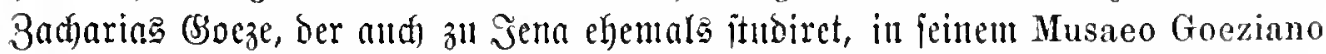

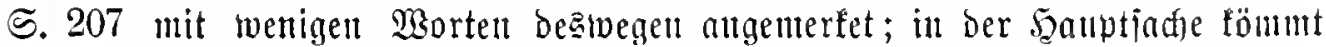

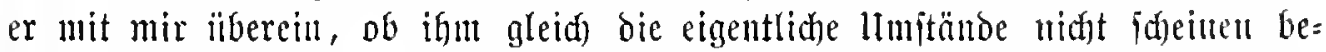

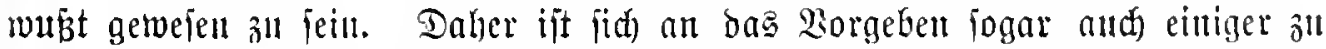
Sena nidit zu fehren, bie hierbei ganz anders rebeu. Wer auf einigen andern

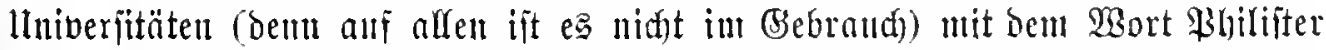
heut zul Tage beleget werbe, taul id) ïberboben jein z̆l melben, weil ca gar waz befanteg ijt." Pađjtrag ăll obell $\subseteq .57$ 
Jahrb. E. 191. 192. 2igl. Bh $\mathrm{i}=$ li jerpierb.

3. Phtllter Deicureite im Bierglaic 1747 Hospitim $\widetilde{\Xi}$. 72 - Meit Tabal in ber Picie 1795. 1825: ll̈berbleibiel in oer Pirife, vic es

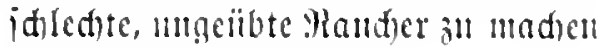
pflegen 1781.

Dhulliergaul Mlictujerd 1831.

Phullecprets gemietetes $\mathfrak{B i e r d} 17 \pi$ Briefwediel brener atad. Fremide J, 68.

Philifterimm PGilititerleben (Ivent ber Burid ansitmoint hat, jicht $\mathrm{cr}$ ins Philiferium; ar wiro bor bem Dit jeiner Ecitimuma Philiter") 1813. Pl hiliterium bas bürger= lide Treiben unto Thum, bas clter= lid) Sgans, in Das ber SHrid Mad) Der Embienzeit eimzient 1S22.1831. Philifertiorren :

Nemoirell d. Satans Kap. 6.

Phuliftans Etmoent im Ietsten (ti.) Eemeiter 181:3.

Philittelle 1) Rïrgerin, QÄ̈rgerepran 1546. 2) Fansiran, 5ams= mo soit= wirthin ( "Datshl)., Stoithh." 1831. 1846.

phtifruten ') cin philifterleten fïhren

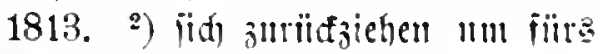
Eranten sul odjien 1831.

philitrs: midt huridifos. $-p h i=$ li it re uie 1831.

Dhrifiter $=\mathfrak{s a t z e n i a m m e r} 1831$. $18+6$.

Did $=$ Ped 1831 .

piden itart zedien (bei. nom Bier= trintent ) 1781. 1795. J831; bi d e 1825 (i. 2 i d ).

pictien modern.

Pietift itammt und Epener 1692 ans ber Reinjiger Smbenteminrade (ogl. bas D 136 b.).

Difejide Etmbentenrod mad) polniider 2I rt 1781 (muter RIe ider).

Dulen geben cinen Perweis geben; beim Indf von Sugelu gejagt 1781.

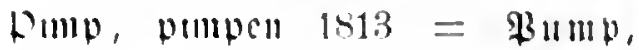
bumpen. Pimpregiter Red)= แmแ ami Credit 1813.

Rompur $=411$ mpier 1825 .

Pinlel 1) Atos. 2) Beizhala 1749; inmmer ungeidicfter Mienid 1781. 1795; Stoppe 17:28 Gedielite I, 156; II, 126. 197. (E i If a lt $3=$ pinicl idon Lessings Jung. Gelelurter III, 1s; aud) Schilda III, 115. Wigl, on I I

Panlelei Lauklard, Eulerkapper 125. Hinlin ") Elagen, antlagen 1781. -

2) jid) wie cin Liniel betragen, äugit= (id) mo zaghait jeill 1795.

Pintolen iteife Sticiel bis ans Snie 1825. Meititiciel bis turj ïbers Suiv 1531.

Pijtolentiansal Wiitolenonell 1831. Platte: . die PI. nuben" jids jort= mad)en Niebergall 1837 d. Bursehen Heimkelır IV, 1.

Platter Bürger, Whilifter Reinwald 1720 Academienspiegel S. 13.

Hempe 2Bafie Schelmufski 5.120 ; Ble แ $\downarrow$ e J. S. Semler 1781 Autobiographie I, 91.

plumpen durfis Exumen fallen 1831. Dlunser = Diöpie 1749 . poden int sajarbipiel 1795.

poena: "pro p. trinfen" beimt soun= mers 1831.

Poladt Reit in ber Tabafappieife 1781 - int Gfajc 1770 Der verführte u. wieder verbesserte Student $\Xi .40$. 
Polife, Police Polizitit 1S25. 1\$46: (autch 'Aledell' 18+b).

Dollur Lollution $18+6$.

Domaoc ${ }^{1}$ ) Slcinigteit 1795 ; 3. 岁. "Das iit mir P." 1781. 1795. 1825. ${ }^{2}$ ) (5) mäd)lid)łeit ("mit ber gröbten $\mathfrak{B}$. geht man in einer Stımbe borthin", "id liebe jehr die \$.") 1813. 1825. 1531. Daz̆l pomasig kequem, whlegmatiid) ("wir wollen es ung p. maden") 1813. 1822.1831.- poma: Dificrell jidfs bequem madten, ge= miitlylid) leben 1822.

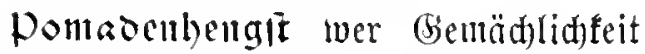
und Bequemlidafeit liebt 1813.

Domaocumulble (lupanar? $1753 \mathrm{Jahr}$ bücher des Brockens I, 5 (bajïr $1846 \mathfrak{B}$ el żühlel $)$.

pomadenjamanli Leipziger Stud.Geographie bei Brasch, Gesch. d. Univ. Leipz. S. 15.

pommer Brlït ("rajentés Bonmer habeu") 1813 ; nod in ter Reipziger $\mathfrak{M a}$.

DónaltarzerStubentengeiünguia 1781 unter pro poena.

poniten traftizen 1831. 1846.

Pontins "vor einigen Jahren = Mar $\mathrm{r}=$ f us gebrand) t" 1781 unter $M$ a r 411 . Doftillenreıter Hönn 1721 Betrugslexicon 5. 165. Kleins Provinzial-

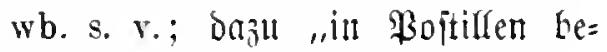
ritten" Jobsiade I, 1638.

Dounaoc 1822. 1831, = Doniliment Riebjuaft 1813. - Boujienr wer Delt ફ口of madit 1813. 1831. -pouifireu bie (5our maden 1822. 1831.

prae: "bas p. habell" 1831. Prafes Boriänger beim Rommers 1813; präibiren den 2orfit beim mers hatell 1813. 1831.

Prellall Iver prefft 1839 Studentenwitze ${ }^{2} \subseteq .11 .32$; vgl. oben $\subseteq .36$. 1. prellen 1 , bic giid bje bei ihrer $2 n=$ fumft ungebeten bejud)en mb jich vou ihnen traftiren lajien; jem. um etw. bringen $\left.1749 .{ }^{2}\right)$ betrïgen, hinter= gehen 1781, 1795 ic.; (bie Fiidjie) prellett 1781. Litterarische $\mathrm{Be}$ lege sind älter z. B. „jem. mm fein Bield prellen" $17 \pm 9$ Der reisende Avanturier II, 63. - $\mathfrak{B a r t i z}$ ge $=$ prollen, nidit gevrellt 1825.

2. prellen (Mäbd)en) verfïhren 1765 Der Freymüthige oder der engl. Greis ron Young $\Xi$. 64 .

Prellerei Betrug 1795; Eulerk. 140 Der Şausinirt prellt ben Stndenten; Eulerk. 140; and Hichaelis 1768 Raisonnement 4, 568; Beytr. z. Statistik von Göttgn. ङ. 226.

Preller crepitus rentris 1747 Hospitium ভ. 75 .

prellibilis twer fid preflen läp̆t Incognito (1740?) Der verliebte und galante Student @. 227 ; vgl. obell ङ. 37 und triufabel.

Prollo Rrellerei 178s Das Leben eines Lüderlichen ๔. 106; แm 1830 Leipziger Stud.-Geographie bei Brasch, Gesch. d, Unir. Leipzig ङ. 15 .

Drime beim fodedten 1795 unter $\mathfrak{T}$ er $z$. Prinz: "eill naijer $\mathfrak{B} . "$ ein mint: itehlicher Dienich 1795; theefer Br. Schilda I, 129.

Prife: "eine munderlidje $\mathfrak{P} . " 1781$ -

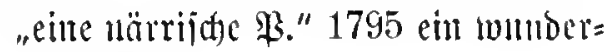
lidjer Serl. 
Drivatfollegimm, Prirntivaimum 1813.

Privatsojentill meretrix 1831 ; and Suiten 1825 S. 23 mIb แad) En॥= bers bei Doltei, eigti. Werlinija. privatijirell ') zuritafgezogen lehen 1 m. 3 arbcitell: ${ }^{2}$ ) meretrix iein 1831. Privatitume nebmen: "D\| mußit nod) odriige \$3. nefment" Dul ver= itelyit bas nidst; "id luill bir Ririvat= itmtiden geben" (d) veritehe es beiler als Dll 1795.

Privatverguigen 1813.

Profaller wer in feiner Q Berbindm!n iit 1825.1831 .1846$.

Nrofar \$rofelior 1846 ; in (3öttingen Brorettor Heine III, 16 (aud) OLB. Wolff Naturg. d. d. Stud. ${ }^{3} 66$ ).

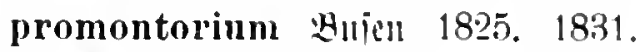
1846.

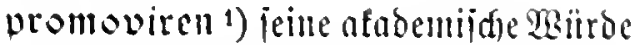
erlangen 1781. 1795, $-{ }^{2}$ ) itellen (won erlanbtem Diebitohs z. $\mathfrak{B}$. „er hat mir meine Sacfe promovirt) 1781. 1795; 1633 Zech- und Saufrecht $\S 43$; Musander 1709 StudentenRegeln S. 30 ; Speranders Handlexikon 1727. Remerbings nod fïr den Reipziger Sonvift bezengt; i. Sdillten.

pro-patria-Stalloal Duefl von $2 \mathrm{ker}=$ bindung gegen $\mathfrak{B e r b i n d m i n g} 1822$. 1831.

pro poena trinten, faupen cin Straj= quantum trinfen 1781, 1795. 1831; 1747 Hospitium S. 25.

proquellis: "in p. leben" = in floribus leben 16. - 17 Jahth.; ’.. $\mathfrak{B}$. Waldis 1527 Der verlorene Sohn 2. 523; Gumpelzhaimer1652 Exere.
Acatem. S. $115 ; 1654$ Pennal- ete. Possen I: II ${ }^{\mathrm{b}}$.

Profit, Droft! Brubiormel 1781. 1795; Kindleben 1781 gibt \& 1 roit als "jeit cinigen Sahren anj einer gewiifen llniveriität Dhode getworben". - Mad) Kindlehen 1781 aud beim Irinfes: baber proiten trinten 1781.

proftitmiren blamiren Sobsiade I, 这. 2137.

Provin; "ill bie 18 r. reijen") Seeinnt 1825

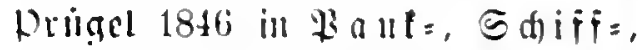
Sdie is=, Sefllubirpriigel.

Publicum öfientlide 20 orleiung 1813. Pusel Bedull 1822. 1825. 1831. 18t1 etc.; and Heine, Werke III, $16 ; 1839$ Studentenwitze G. 27; Schnabels Univ.-Jahre S. 58. 363.

1. Duif ") hallijdes Etabtbier 1781. 1795. (bazu Buifteller ein Bier= feller unter bem Hathanie) 1781. 2) in salle jeser tleine Bieridant 1795. ') lupanar 1795. 1831, alld Laukhard 1798 Schilda III, 63.

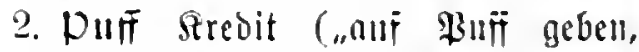
nefmen"); pufien zur Borge geben mib nehmen 1813; ala nbd. Stuben= temworte Kleins Provinz. Wb. a z. Statistik von Göttgn. ङ. 238, wo auch "iem. pufien" iem. janl= big bleibert.

Dump Sredit ( "allf $\mathfrak{B} . "$ ) 1781. 1795. 1813. 1831. - "חuf $\mathfrak{B} . "$ alf $20 \mathrm{r}=$ id) IIIt Fiseher 1781 Burschiade S.9; Laukliards Leben I, 94. 95; bei. 5ुaug̃ ump Sredit be马 Stu= denten bei jeinem Sausivirt 1781. 
1795; "einem झ. rijtiren" Gelo aufuehmen 1825. - p u m pen borgen 1781. 1813. 1831. etc.; Mart. Schluck 1798 Burschenkomment ङ. 18; 1798 Vertraute Briefe über Halle Є. 26; Fischer 1781 Burschiade ङ. 65. 70; Heun 1792 Vertraute Briefe I, 61. (5)eine Z3erfe II, 67 mit ber urjprgl. $\bar{y} m \bar{B}=$ note "buriditolet 2ussornd fiir borgen").

Pumpier Beloreiher 1822. 1825. 1831. Pumperei Wump 1831 ("ill Der Ftreipe

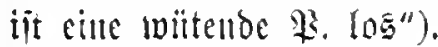

Dumpregifter 1829 Buckeliade ङ. 11 ; (5rebitbut), Sircibenbrett 1846.

Purzelwalfer Brantwein 1781. IInalm Maud $1846-q u a[m e \|$ (Tabat) rundent Laukhard, Fr. Wolfstein I, 3à - Anekdotenbuch I, 88; Gaudy, Werke I, 202. ellarts 将ur. Stmbenten aus den PRađbarbörjern ber llniveriitütsitaot gebüntig 1795. 1822. Sing. Dnark Mart. Schluck, v. Burschenkomment $\$ 12$.

quasi als ob, gleidjian 18t6; Jobsiade II, 816.

Quasimodogeniti Fittuje 17. Jabrk.; 1744 Salinde ङ. 120.

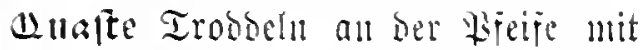
bell Jarben ber Serbindung 1831. quatji öbe, onlmun Laukhard, Fr. Wolfstein I, 81 - Eulerk. 125. Wuelve Manidäer? jäjđer? 1764 Der Tugend- unr Lasterhafte Studente XII.

aletichert: "iid) an." ourdagehn 1831; anioiple Franluzimmerthen 1781. "eine alte D." ๔and)e von geringitent getl Cons gilt mir jobiel wie eine alte Quioipie") 1795; wol mit dem verbreiteten dial. $\mathfrak{Q}$ uintipie 'cunnus' identisch.

Tiano Mand („halt bent M.“) 1831, and) Niebergall 1837 IV, 11.

Rialloal, rambalieren 1831. 1846.

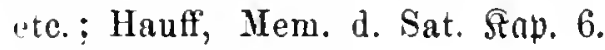
- Tiansaleur 1831.

rappeltispfig verriidt 1781, 1816.

Sisppier Feưttlinge 1795. 1831. rappieren ïd im Fedften ilbell 1795. 1831 ; nad Sanders aud bei Goethe. - Viappierjunge (oner = $\mathfrak{A} \mathfrak{n} \mathfrak{b} \mathfrak{e}$ ) Illdwwort; Dazu "einen Mappier= jungen ftïrzell, aus̄mad)en" Menijur alif Rappiere 1813, 1825.1831 etc. rajerel ân 2 ejten l)aben Laukhard, Fr. Wolfstein I, 85; prellen 1846; Siafose \$relferei Burschiade 1797; Siafeur $\mathfrak{B}$ uderer $18 \pm 6$.

raufen los̄gehแ, 5ändol juffen 1831.

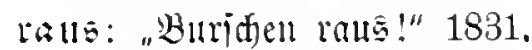

re $\left.{ }^{1}\right)$ gleidfalle $\left.1846 .-{ }^{2}\right)$ Antwort oder G5egengrus auj Brojit 1825. 1831. 18t6. - regeiofien veriofien 18t6; aแd Schnabels Univ.-Jahre ङ. 218. - regetommen berbummelt Schnabels Univ.-Jahre @. 253. - refritzen eine $\mathfrak{B e l e i d i g u n g ~ m i t ~ e i n e r ~}$ alloeru ertwibern; Der Reftur 1846. - Zieprofit $=$ re ${ }^{2} 1846$. reconche Metounfuticte Mart. Schluck 1793 v. Burschenkomment ङ. 38 .

Tiealavantage 1822.

rebus: ,nicht in worten, jondern in rebus" Reinwald 1720 Acad.Spiegel ङ. 477 ; aber "jein r. gut madien" sc. in coitu Incognito (um 17t0) Der verliebte und galante 
Student (5. 85; aud Bahrdt 1790 Rind vimins.

recipieren in cine Rerbindung anj= nehmen 1822. 1825. 1831. Daßı Sicception 1831.

regalieren traftieren 1831.

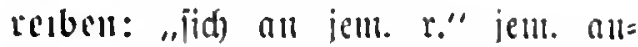
haItend zum (sicgenitand jeiner Mad)e maden 1781. - reiben eille Irint= jeremonie, ,bic jait cinjig uno allein mur bei bem Squapstrinten Sitte ijt" 1831 ; i. oben ङ. 53.

reilien in Epiel getvinuen; etro. unter= nel)men (J̈inten, Eniten, Zoten, cine Hegoce) 1795. - "cincu wib r." - "x hat gute Belder gerifien" erhalten 1831.

ceiten trani. ') ( 3.8 . Die Pandetten, bie 5efte, eit Bud, bie Rogit) itl= biren 1795. 1\$13. - ${ }^{2}$ ) futuere 1795. - ${ }^{3}$ ) "er reitet auj bem 5olzwege" er jührt anj bieje $\mathfrak{E e i j e}$ bie Sadje midt burd 1831.

Reittsllet Pefeid) 1831.

refommanoiren anj Plurage beim Sommers = Präles moniren, z̆ er= innern haben 1813. 1831.

Sictitor Der Ilniberiität 1781.

Relegat Melegierter 1831.

Sielegation Berweijung von Dor llni= veriität 1813; daan relegiren 1825. 1831.

tempclı 1825. 1831.

Sicrommage renommititide Beleioi= gung, 2Trrogallz 1822: "cine traile oder eine blaife $M . "$ jade Bramar= dajieren 1831. - sienomme ') $\mathfrak{2}$ ijs

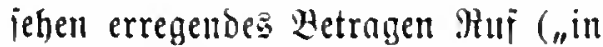
id)led)tell $\Re$. itehn") 1825; "viel $\Re$. habelt" allf Der !lniverïtät jehr be= tant jein 1831. - Sienonmierenen

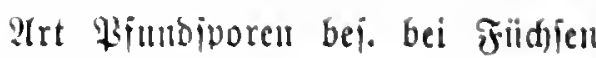
1831. - renommieren jid ala Denid autiiilyren, ber weber gött= lide nodn menidilide Medite aner.

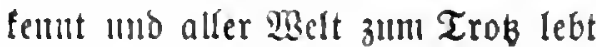
1813; grois thun, prahlen 1822; bramarkajicrell 1831; "an jem. r." jell. mit Mrenommage zn beleibigen verindhen 1825. - Tienommift wer vor allbern bas prae judjet mib einell grö̉en état von Balgen 11. Edjlägerei madjet Speranders Handlexikon 1727. - ver iïr Geld oder cinelt Edt)mans bis Eadie eines antorn ansîfid)t; baun and wer ingrimmig und gefährlid) ausiieht 1749 . Maulfold, ber and dem Ganjen er= gebert ijt mo nidt itudiert 1781. 1795. "Jekst benemut man jo einen Boltroll, ber viel idreit und wenig leijtet, thut alo ob er alle ver: id)lingen wolle unt babei exa jurd)tiam iit" 1795. Dazu Renom = mifterei, najies Renomie= rell 1795 .

Siensuce ') eine Itrt Sronfneipant, wer iid) zu ciner 2 erbindun bält ohne Ylitglieb zu jein 1822. 1831. ") well man uid)t alı̄itehen taun 1825. renoncieren als Renduce" leben 1831.

respum Mejpett 1846.

iictourfutide ("R. zieht nidat" iit ıแzuläifiig) 1813. 1825. 1831. Dafïir Sietourchaile 1846; aud "er fährt retour" 1831.

revoricren miderrufen, eineBeleidignng zurïđürehme! 1822. 1825. 1831. rieden: "id fallu bell Berl uidht r." alsistehu, leiben 1831. 1846.

rippel Sumpen (in IIIinden) Mors, 
Erinuerungen $\cong .74$; Kobbe, Elinnerungen I, ङ. 149 als seibel= bergilid.

riftiren hei. beim Eviel 1795 ; „eillen Pump r." 1831.

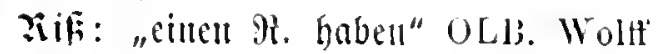
Naturgesch. d. d. Stud. ${ }^{3}$ ङ. 68.

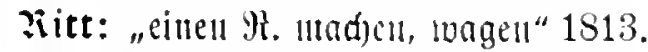
Siojenbaner jäadher? Stadtiolout? (iil 5alle: oder Franfiurt a. D. D.?) 1749 Der reisende Avanturier II, 57. Riofuante ") Bierd Fischer 1781 Burschiade $5.19 ;{ }^{2}$ ) meretrix $18 t 6$; bei Heun 1794 vertraute Briefo

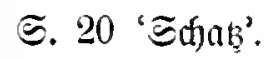

ruitten: "iem. alli die sillipe r." zul jent. auj die Stutbe gehn mut ihn zu foramieren 1831. ruberu itarf trimfen 1831.

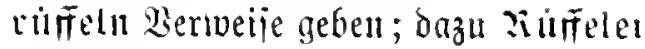
1813.

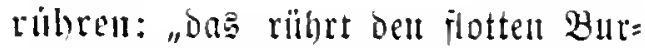
idjell uidft" 1813; aud 1781 unter id) $\mathfrak{x}$ i $B$ e 11 .

Silunogefedt cille bej. Frt Menjur 1822. 1846. - Tulnogerang 1831. $18+6$.

Tilipel Ed)lingel, Siinmel 1813.

Siabelitalloal Säbeloutll 1831.

Sabell mabell rem habere cum puella $1795.18+1$.

listen Delt Eptelgeminn einitedent 1795. 1841. - ¡̈tibibell 1846.

Salamanber (oben S. 52) * "Der afa= Demijale (Ehrentoait" (aud) "Bier=

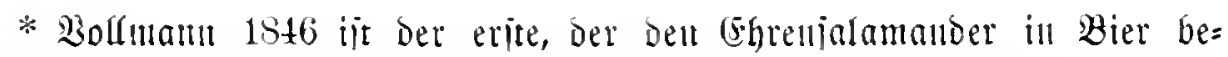

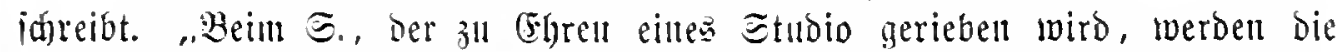
Burjaen an bell Tafelu in Sränze getheilt uto biejen Plufieher ober Exercier= meijter vorgeiebt, hiernu bie (Bläier geiiullt und jobaun anj bem Tiih) unter 2lusipredung Der Worte 'Salamander Ealamanber' gerieben, bis vom Eenior

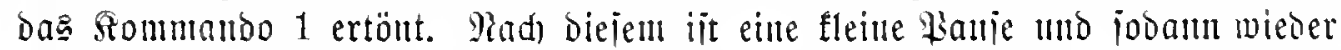

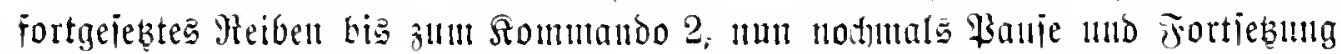

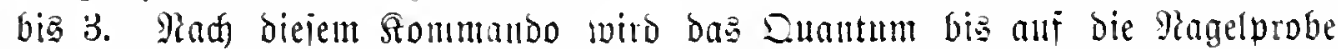
geleert, bie Bläier aber erit mit bent Sommanbo $t$ auj bell Tijd) gejebt.

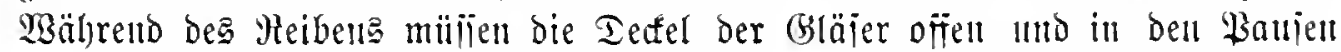
bei Straje gejuloijen jein; wer jid bagegen verieflt ober ău jpät trinft, mü

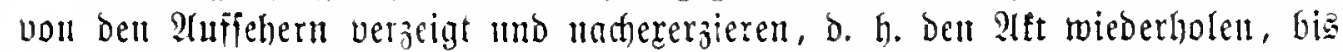
er vour Eellior fïr legal erflärt ijt." lluter Bierjalamander giebt Boll= mant 1846 jolgende (Erflärmtg: „Bierjalantander, ein Bierjpiel in 3 Tempos, bei sueldem bie ganze (Bejellifala bie Gläjer reibt, anf bas Rommando 1 und 2

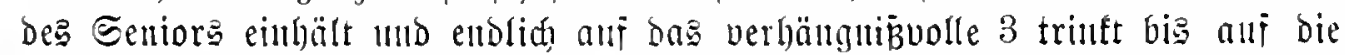

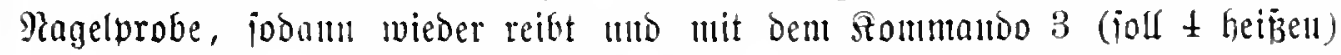

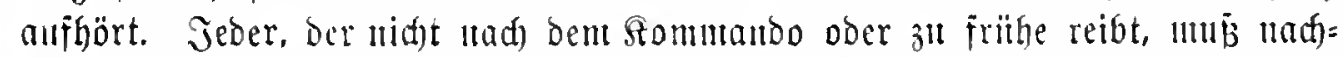
exerzieren utb zur Etraje das Tupluni reiten. Der Ealamınber wirb unr zu

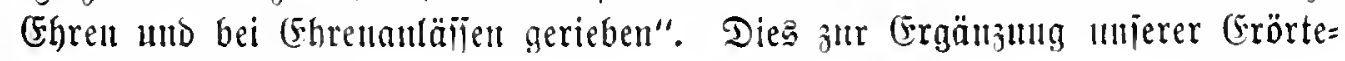

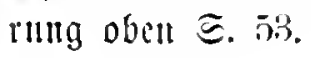


jalmmander", "einen S. reiken” ober jala

sans obell G. 65; vgl. sine.

Gapienjtina|ter (in joibelbern) bic armen theolog. Etubenten, die auj ber jog. Eapienz wobntell miīīan Laukhard, Mein Leben I, 294.

Satisfattion Bentgthum 1825. 1831; 174t Salinde 5.68. - Tazu iatisiattiongfäbig 1831.

Sar, Baterei, Edumanierei 1813. 1831; "einen S. gchen (Thee=, Stafiee=, 2(bendjabs)" traftiren 1813. Sall ") alles was idjled tut mo man: gench m ijt; "eine $\Xi$. im Reibe haben" mverniujtig hambuln 1794. 18t1. 2) BSlïđ ("eine mubändige ङ. an Reibe baben" ichl glïf(ia) jeill) 1813. 1831; Hauff, Nemoirell d. Satans kap. 6.

lallen (= jud)jen) in Epiel viel gesvimlell 1795. $18+1$.

jaufen itart trinten 1831. - Daju Saufloumint 1831. - Saui= meije 1831; iffoll 1747 Hospitium ธ. 53.

Saubreb unregedmänitger sọeb 1795. $1525.1831 .18+1$.

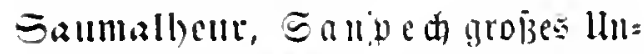
glüd 1795. 1841. - Salwe d) alld Eulerkapper ङ. 120.

Shaarmadter in salle bie jä̈jer 1781.

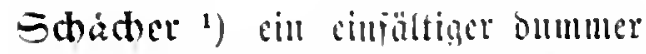

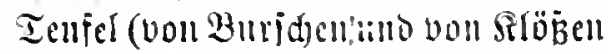
gebrumdt) $1749 ; \quad$ ) ("eiu armer Ed.") cill elenber Stïmper, der feime Elementarteuturifie, hat 1781. 1795. 1841; Iaakhard, Schilda II, 88. - 2igl. da D D

Sbaftaic ill Reipzig baccalaureus ministerii nad) Spencr 1692 (vgl. dng D $\mathfrak{W b}$.) und bei Paullini, geistu. weltliche Merkwürdigkeiten ; Schmeitzel 1746 Wöchentl. Hall. Nachrichten S. 180.

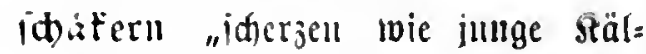
ber" Leber. Blaustrumpf 1746 Vier possierl. Ged. S. 21.

idansbar iđändidh, mangenehun (3. B. vom 2 Better) 1813.

Sdalloe treiben Qärm maden 1813. S(balljc Studentenitube; bazu iक all= zen arbeiten; S d a lizier fleiżiger Student $18+6$.

亏かalljer ill Etrab̄burg Snformator Laukhard 1792 Mein Leben II, 37;

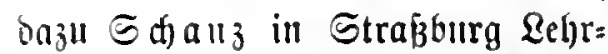
itumbe ber 52 ansinjormatoren Kleins Provinz.-Wb.

S(barinibterbieb eill Fed)terbieb $179 \overline{\text { m miter }} \mathfrak{T} \mathfrak{e} \mathfrak{\jmath} .1841$.

liharren mit ben Fï̈Ben als Beiden ber llı̧̧uriebenhe it im Solleg 1813.

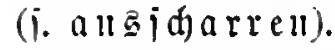

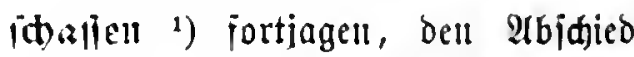
gebell 1781; Fischer 1781 Burschiade 5. 65; Laukhard, Fr. Wolfstein I, 139 - Schilda I, 174 - Eulerkapper S.117. ${ }^{2}$ ) im Duell 3ૈuriidtreiben 1795. 1813. 182\%. 1825. 1831. 1841. 's) ¡đłwäızen 1825.

ihauscros Gaudy, Werke I, 179 ; obell §. 64 .

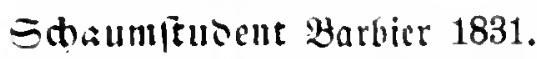

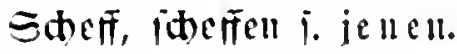

id)cinen yejallell ("es joll dir wohl

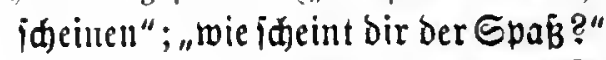
"mir idfeint ar odjigg") 1813; 1825 Suiten ङ. 114. 
fbeiken angit แnd bange jein 1795̃. i hei isidel ichl lujtig mo aujge=

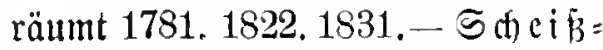
ma $\mathfrak{l} \mathfrak{h} \not ̈ \mathfrak{r}$ Laukhard, Schilda I, 131 . juenten jem. Der Mrïhe iiberheben

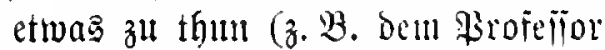

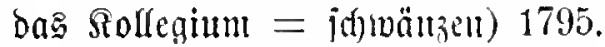
1841. - „Dell Rejt der (Erzäl)lung ¡đ." = erlaijen 1745 Auf der Extrapost eingelaufene etc. Nachrichten ธ. 28 .

fcheren (bie Fithjie) veriren 17. Jah - "fid ifd.“ abzichen Laukhard, Emigranten II, 43.

iđbicten $\left.{ }^{1}\right)$ volt der ltniberijtät weijen, releqiren 1813. 1831. $18+1$ (and) Niebergall 1837 Heimkehr III, 10). $\left.-{ }^{2}\right)$ (Seld int Gpiel verlierm 1795. 1841.

1. latheben itigizen, atsiühren Mart. Schluck, v. Burschenkomment $\$ 4$. 2. idsieben Fortyehen 1795 ; aud 1790 Die verkümmelte Hochzeit 5.29 uוb Laukhard, Schilda I, 302, - gehell 1831. 18t1. 1846; i i a juleben (and jith iortidjiebent) jortgel) 1813; gehn 1831. 1841.

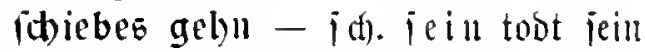
Schilda I, 302 - verloren, flöt $\mathfrak{1}$ gehn Laukhard 1804 Corilla Donatini S. 104.

(b)iefer Kerl Laukhard, Eulerk. 103;

- Brinz Mosellaner ङ. 77. (5omment Eulerk. 125.

Sd)iefitist Binjel Schluck 1798 v. Burschenkomment IV; Laukhard, Mosellaner S. 10 - Enlerkapper 103.

1. jobielian itibiken (o. erlunbtem Stehleti) 1795. 1813. 1831. Schnabel 198; jteflen, dus menu nidyt
Dabei ertappt wird 1781; ftehlen 1825; aud Salinde 1744 ङ. 115 , 122; Laukhard z. B. Schilda I, 174.

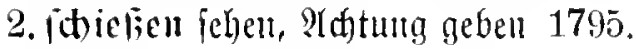
1841.

libielibar ireflbar ill Göttittgen "lwą unter cinem $\mathfrak{I}$ lyaler wert ijt" 1835 Schnabels Unir.-Jahre S. 198.

S(bielipringel Flinte $18 \pm 6 ;$ O. L. B.; Wolff Naturgesclı, ${ }^{3} 69$. S. Brïgel. Sdifif (bei. Beldidifi) Briej 1825; 2) Maditgeichir 1795.

jhiffen vesicam evacuare $1781,1795$.

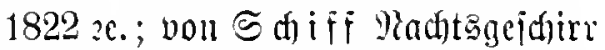

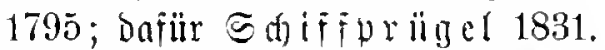

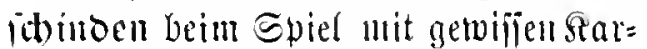
tell jo lange marten, bie man ba= bur(f) größere Sorteile erlangen fam 1795; benad)teiligen, betrïgent 1846.

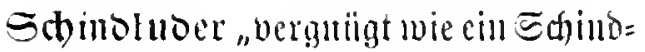
luber" anjerorbentlich lnitig 1781. "Sch. treiben" jent. zun kejten haben $1846=$ "Sd). ipielen" Laukhard, Eulerk. S. 20s.

Sointen ") "alte Sd." alte 2 iildher Chr. Giunther. ") fleine Boggen= hrode in Seipziger Stonvift ("Sd). promoviren" bas bröbden bei Tija weguehmin) modern.

ld)ippen ${ }^{1}$ ) itoben: ${ }^{2}$ ) (aud) "Die Sdiphe friegen") relegiren 1825.

Schil; 1) Sdfulden ("Sd). haben") 17\$1. 1795. 18t1; und Fischer 1781 Burschiade $5.9 .-{ }^{2}$ ) P(ngit 1825. 1831. 1841; Ed). ljabet bej. vor bem sosgefelt 1831. $-{ }^{3}$ ) ", all bell

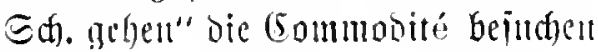
1831.

Sdiller 1) Spottname fiir fleibinge, ordentlib) Stubenten (Begeni. an 
Renommijt) 1781. 2) Jeigling, der alle weleibigmucu anj jid jiben 【äвt 1795. 1825. 1931, 1841: Eulerkapper 111 (Eraidiijer): (Mehen= form Edfifiter 1846). 3) wer in 㤁errui erflärt iit 1822. 1831.

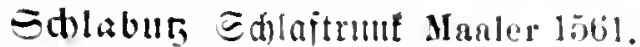
lifh ithlagen ") fedten 1831. ") jït) Dnellieren; oajn $\Subset$ d) (äge re i Duell 1813. Cithagen ') ben Fechtboden

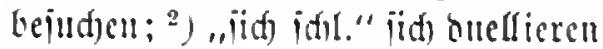
1825. 1831.

Shliager 1) \&antwarie. 2) wer gut id)lägt 1822. 1825. 1831, 1841 .

id)lall (gern if a $l$ gejuroden) 11110

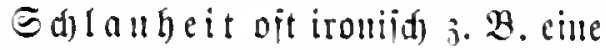
id)lane Soce 1813.

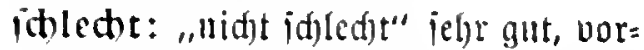
trefilid); iid) idfledt madell iid) blamiren 1795.

ड̈blemm cin Epielterm. ted). 1813 (unter fle de ll); alle Etidue 1816. (b) leppen ${ }^{1}$ ) auja ftarzer bringen 1831. 1825. 1841. Bahrdt 1790 Prinz Yhakanpol ङ. 30t; Laukhard, Eulerk. 111; Dafür collè jđl. 1781. - Sollee ið)l. 1795. 1831.2) bie Füudie miilien bic Marfen anj den łaufplá idjl. 1831.

Ethleppgels Geld dajïr an bie Seäidher 1781.

Shleppruds juthe, der die Sieber

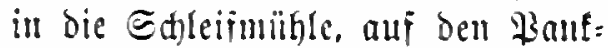
plaze tragell muí 1831.

ithles juledt $18+6$; obeu S. 62 .

Sdliagerflinge 1765 Der Freimüthige $\Xi .62$.

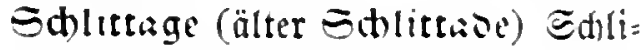
tenjalirt i. oben $\Xi .47 .64$.

Sdluntí Serl Eulerkapper $\Xi .45$. idmust)ten nad) jem. "cin Etuber= ausornd $=$ iid) uad lem. iehmen" 1781.

Sd)maltire Inverheiratetes Frauen= simmer modern.

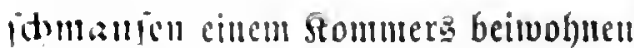

1795.1841 . ittmel;en i. miseria.

it)merig: "ben Edmierigen ipielen" artig joill Niebergall 1837 Des Burschen Ileimkehr 1I, 3.

三かmil; 28momalen 1846.

Sd)miter (,,der alte Sd)m.") eill alteg idjled)tes Budj modern; bafür irvifger $\subseteq$ d) öd)er 1781; and Laukhard z. B. Fr. Wolfstein I, 60 - Schilda I, 289 - Eulerk. 5. 215. idomollen grollen 1781 .

St)mollis Mui beim Butrinfeu auj Briideridajt 1781.17952c.; Frübeiter Belcg 1749 Reisender Avanturier II, ङ. 57: „Benn ธđmollez oder Brandtwein getrunfen wirb, joll jid) feimer weigern 30 Gläier augzz"=

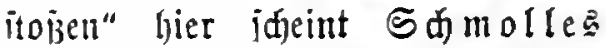
ein Betränt jein? das Bengnia be= jielyt iid) anf salle? ober Frant= furt? In Stammbüchern bei Keil 5. 260. 270 aus den Jahren 1754 . 1764; in einem Gicssener Stammbuch 1752 ,气dmolles, ifr Brüber".

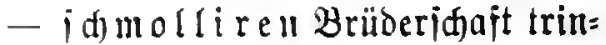
fen; $\widetilde{G}$ dmolligbruder Duk= bruber 1795 ; idmollixen and 1822. $18+1$.

Sdmul madbe ${ }^{1}$ ) eincul uner (aubten Wiropit haben 1781; 1749 Reis. Aventurier II, 374. 385. - ${ }^{2}$ ) ,iit ein פBittenberger terminus ulb heibst jo vid als "d)armiren" 1745 Auf der Extrapost etc. eingelaufene ङ. 63. 


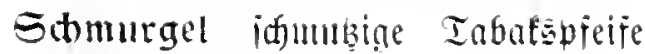
1795. 1841.

Sd)mut, Bürger, 米们ilifer Sehüttgen 1747 Historie des Pennalismus ธ. $19 ;$ um 1600 and Iat, sehmutzo.

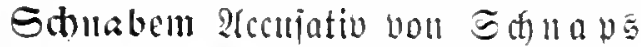

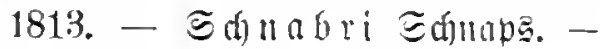
Sthutrianer Sthna 1846.

(d) natiid) poijitlidy 1781; Laukhard. Eulerk. 178 - Schilda II, 1599, IIr. 117.

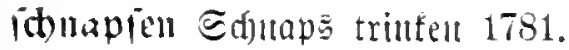

Sd)uart)er Manferds 1781 unter a $11=$

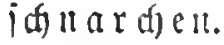

Sthnefrer Exfuneider 1831.

Sdueio: "Śfun. jat etw. habelt" Niebergall 183i Des Burschen Heimkehr IV, 7 .

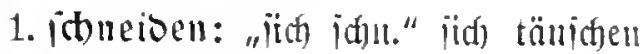
1825. 1831.

2. fonteioen prellen Salinde $174 t$ ธ. 153 .

ichnellen betriigen 1781; Lauklaard. IIosellaner ङ. $56 ; 1749$ Der reisende Avanturier ऽ. 375 .

ichnengen ${ }^{2}$ (puellam) futuere Incognito (un 1740) Der verliebte und galante Student ङ. $\left.108-{ }^{3}\right)$ um B̧eld preflen Emmerich 5, 135.

Schuiepel 1) eleganter Sabenijamengel 1825. 1831 (Sd)uippel 1846). (5le= gant Gaudy, Werke I, 193; Schnabel 257. $\left.{ }^{2}\right)$ Leibrout 1831. - i in nie pelu Fein angefletibet jein 1831.

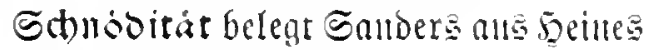
Berfell 19, 302.

Sd)upftud anitergium Lulerk. 123. (d)unren (ums Beld) prellen 1764 Der Tugend- u. Lasterl. Studente
A4; Schöttgen 1747 Pennalwesen ธ. 118: Oleander 1721 Gedanken v. d. academ. Leben S. St.

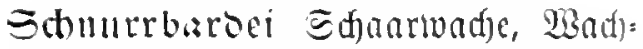
Lofal ber ভstiuntröate 1749; Zachariaes Renommist 174t; 1757 Xiemand 79 .

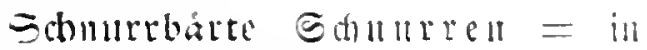

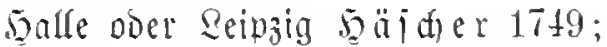
Schnurrbärte Incognito (1740?) Der verliebte u. galante Student 194. 225 und Salinde 1744 5. 76. 5ో)urrbst in Hallischer Universität ete. Begebenheiten (hidjift.) 1727: and bei Ineognito (Iㅔ 1740) Der verliebte und galante Student 47. 52 b "nit 300 @timmen twurbe ein Tutti gerufen: $\widehat{\widehat{y}}$ djuntrbart, Edjelmbart, Macferbart."

Sdulurbarte hallell all bell jed)t=

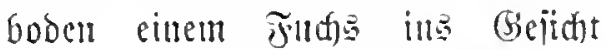
Ganten Leo 1836 (Akad. Monatsschr. 4, 287).

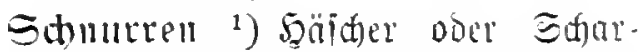
röadter 1781. 1795. 1841 (jontberlid) in Sent 1781); ?aantwändter modern - Etrotioldatell Der Itniw.:itad

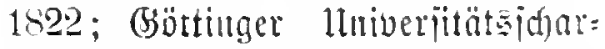

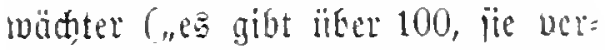
tretell fait bie Stelle bes Militärs") 1831. Heine III, 16. 486. ') \$olifen, tomiinde Einfälle 1781. ichurren Eetteln 1781.

ithunrig Laukhard 1802 Limigranten II, 42. 171. 257; Bürgers Ballade rom Kaiser u. Abt.

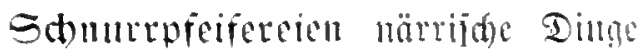
1781.

i(t)ury: "Das bleikt ï(d) idu." iit cinterlei 1831. ïthoiel verädtlich, idjled)t 1781.1795. 
1831. 1841; Jobsiade II, ㄱ. 1676; Laukhard, Schilda II, 11s Eulerk. 204.

Shorlit alter Stmbent (\$n lकeren) 17. Salbrh.

schorus Edmuna; Gdorbrnber 3ed)brnder 1616 Jus Potandi 5 . їbranben anjichen, neđfen 1795. 1811; aud) Goethes Faust I, 23. 2180 .

Shrectenberger \$opanj, Sdrect= geitalt 1795 ; Finte beim Spiel $18+1$. idreiben: "id) tann nidst unter= Inifen an Did) z" ja)reiben": "lviro bei allen möglid)en belegentheiten gebraudet" 1749.

idosipfen futuere 1745 Auf der Extrapost eingelanfene etc. Nachrichten ङ. 53.

亏drumpelbeutel itub. હdjimpinort 1769 Gesch. d. Gütt. Stud. II, 166. S(bul): "in ben @d)ulyen frepicen" ohue dic Edulden ju bezablen alt: reizell Mart. Schluck $\$ 4$.

Shule: "Sd). jd)lagen" beim Fedteu 1831.

SЪulfub) = Mqaultier 1822. 1S41;

(Snmunajiajt 1841 ; bojdfftc. ImI 1820 Sd)üler mif ber ङdule, vou Mtanl= tier unterid)iebcu; Bemunl 1831. id) 1781. 1795; and 1800 Der Weltbürger 5.219 . - eillcu von ber Eeite remen, rempeln 1822. 1841.

St)irze Fran 1826 Bruchstücke aus K. Bertholds Tagebuch $\cong .13 ; \delta a=$ zu Shurientallsiont Rallbiont Der Frauell ibid. S. 18. 19.

Shirjeuftipensiun (belogejdente von Franenjum!tern 1781. 1825. 1831. 18t1. 18t6; im 18. Jalyh. aud) Beiber= oder Frauenjimuer= it i pe 1 b i $11 \|$ bei Incognito un 1740 I116 Avanturiers 1744 5. 80. 85.

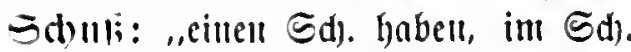
jein" s"l viel getruufen lyaben 1831.

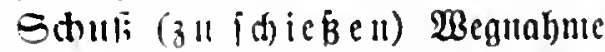
geringiügiger $\Xi_{a d) e n}$ ("einen $\subseteq$ d). unagen"; "eill herrlitber Sd).") 1813. cinte Sकuा baben uidet redt flug jeill 1781.

St)wadullatitus 1846. S. oben ธ. 36 .

Sthwaoronenlieb cine 9rt irregu= lärer J̧iele 1831.

(क)wasroniren viel Borte mad)en

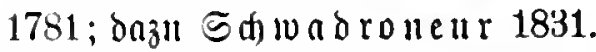
亏かwager ${ }^{1)}$ Poittillon 1781. 1795. 2) Snallore 1795. $-{ }^{3}$ ) Dorfumilfanten Salinde 1744 S. 173.174 (bie Stabt=

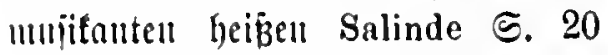
baug(e\|te); vgl. aud Better mito Bevatter und obell 5.15.

Sकmant Saujmann (iiehe $\subseteq$ d) wung mb $2 a b e n=$

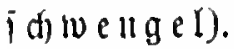

Sd)walj Ball 18t6; Kulli(b)alj แad) 1831 in şalle für neueres Ş () $10 \vee 0 \%$.

ธか) lejtung 1813; Rüđe in Stollegheit 1795; Fischers Burschiade 1781 ๔. 38 แ1ठ 1795. 1822. 1831. 1841.

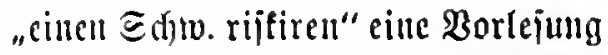
veriäunte; „einen Sd)w. nad)reiten“ eime Borlejung mad) bem \$eft eines anbern nadhyolell 1813 (and) $H$. Heine II, 59). - "per Sdit. hören" (eine Borlejung) ¡đjinden 1813; aud hei H. Heine (Sallbers)?

亏(t)wanjontatcn Sdürzenftipendia Incognito ( $\mathrm{ltm} 1740$ ) Der verliebte und galante Student 5.195. 
1. f(b)wizen (eine Bortejung) ver: fäumeıt 1749. 1781, 1795. 1825. 1831. 1841; ald vom Brofeijor ge= jogt = einte Bortejung antejeken 1781 .

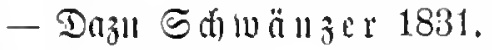

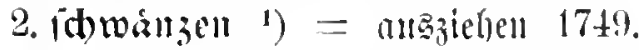
$\left.{ }^{2}\right)$ "Inm etio. ianm." Hrélen 1749; Stoppe 1729 Gediehte II, 181; bei. Iell Wirt, Traitem nicht bezahlcu L. Blaustrump f 1746 Gedichte S. 4 ; 'mit ๔(fulden Durdigelnu' 1764 Der tugend- 1. lasterhafte Studente B5̃

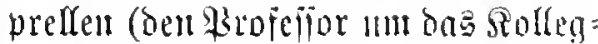
gerdo); jhon Steinbach 1734 'be= triigen, hintergehn'. ${ }^{4}$ ) futuere in Stammbiichern $11 m 1750$.

(d) warjes biett 1813; jofon Im 1700 bejeligt.

Sdwarjmantel Ifyeuloge 17. Jahrh.

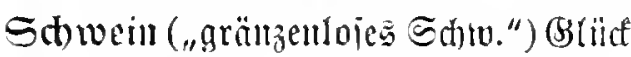
1813. 1844; Niebergall 18337 D. Burschen Heimkehr I, 700. - „ein Sd)w. iu Reibe haben" unverniinfitig Gandelı 1795.

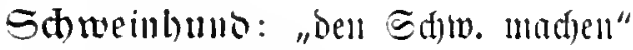
ansighelten 1846.

(đ)weinigelu unflätig reden 1781. (d) wefeln ${ }^{1}$ ) juitifitren; ${ }^{2}$ ) viel trinfen 1831.

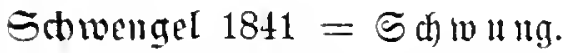

1. Shwerenóther $1795-\varsigma \mathfrak{d}$ were $=$ แöthrige\| 1781 fleiner rumber

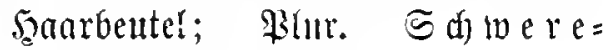
nöterd)en 1749.

2. Sbwerensther Durdhtriebelier Sert 1798 Fritz Reinfeld II, 52.

Sabefter: "barmherzige ऽatw." meretrix Stoppe 1728 Gedichte I, 58 ; oft bei Laukhard; anch Hospitium 1747 S. 59. Dafür $\mathscr{R}$ a no i d we fter
1747 Salinde a $5^{b}:$ i. and $m i t=$ le ibig.

Stbwimel i) wer lidits thut Imb feime Borlefungen bejudgt. 2) wer vou ciner Sueipe in bie andore ziegt. 3) wer Dronmitg uno Droentlichfeit uicht beachtet; Dazu iff wimeln 1831.

fat)windeln närrijdge Streidhe mathen;

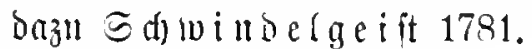
Shwiten $1795=$ Sultu.

(申)wizen ${ }^{1}$ ) eming arbeiten 1781. 1795. 1831. 18t1. 2) in Serlegenheit fein 1781.

Shnof Saffermball, niebriges Ianz= bergniigen 1846 ( $\mathfrak{x} \| \mathfrak{l}\{$ d) wo o f 1831).

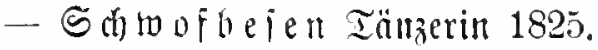
ibwofen tanzen 1825; wiitend tanzen 1831.

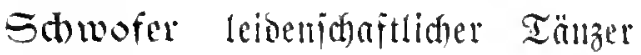
1831.

schwnlibus 1841. 1846; "in schw. jein" 1831. 1846.

Shourititen 17S1.1795. 1831.1841; 1831 - $\subseteq$ d) full lite $\|$ 1795. $18+1$ Berlegenheiten. 2igl. $\widehat{s}$ d) üb it ät uno oben 5.38 .

Sかwung Sadendienter 1831. 1841. 1846.

scribax Sefretär 1831; Jobsiade II, 2. 324.

Seelcutleiter in Reipaiger Ronbift (B)rïbbrei 1825. 1831.

Sefumdant I) beim Duell 1795. $\left.1831 .{ }^{2}\right)$ bei Bieriniten 1825.

Setunoc ein Fechterhieb 1795 (unter Terł hanent).

Seiscul(pinner?) Leipziger Stud.Geographie bei Brasch, Gesch. d. Univ. Leipzig S. 15.

Seifenfieoer: "ba gilly ifm cin 


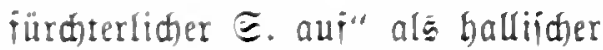

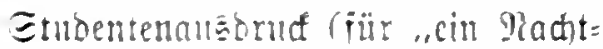

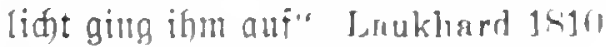
Streins A bentheuer I, 5 t.

ietunoiren beim Iuell 1795. 1731. 1811; Ectun bierpriige! ieinere Art Rappiere, mit benen ietumbirt wirb 1813.

Semeiter (Eommeri., Winteri.) 182.5. 1831.

Sentor ') bei Eroell und Rerbin= dungen 1781. 179526. 2) bei Trei= tiidhen 1795.

sequens: vivat \& ruit ber Bräiés beim Solpiz 1831.

iegen traktiren (bazu 厄ab) 1813. 1825. 1831 (bei. ofue (5inlaburg). einen Sieb ieken, Euarten, Ierzen jezen 1813. - ieben la jien ins Srazzer 1825.

Setgt an Ruf beim Bieritanbal 1831. Sellijerbret Guitarre modern.

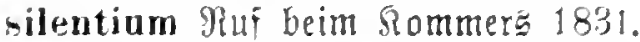
sine 5dmudt sine Tudt sine bart. roild Incognito (แm 1710 ) Der verliebte und galante student ङ. 66\%. 厄. oben ङ. 28 .

litgen ${ }^{1}$ ) bon bieben = treffell $($ "ber Sieb ïnt" hat getrofien) 17\%5. 181\%. 1831. 18t1. - ") im Starjer itectell 1815. - ") C C fibt id habe da Duantum, bas id) bor = ober thadt= conimen muibte, gelcert 1431.

ifalliren (ïber et tw.) fidh liber etw. moquiren Jaukluard 1802 Anekdotenbuch J, 95 (nach Sanders auch bej Gorthe "alli jem. it." ant jem. idimpieny.

Stanoal (łhur. Efanbäler 1822

Loben auf Lnis. E. 30) ij Etreitio= keit 1795. 1841; ¿erbrü, ¿ärm, lingelegentheit 1781. - "Ef. mahten" lärmen - "Et. haben reip. iuchen" Etreit 1795). - Etreitigfeit, die ein Intl zur jolge hat 1813; ,Et. unit jem. haben" eill Duell mit jem. vorbabell 1813, cin beboritehendes Tuell 1822; "einen G. falfen" ton= trabiren 1846. - ifanda Iö an an= itösig, ehrenrührig 1781. 1795;

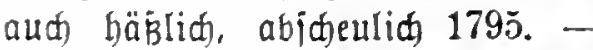
„fid) moriiber ifanbalifiten“ 2MnitoB woran nehmeli 1781. 2) Biennig (in Gię̧en) Eulerkapper S. 106. 238.

Ifansiren mingere 1781.

Sfat das helannte Sartenipiel 1846. Sti, @f h eine Sarte im Tarol 1781. 1795. (val. H. Schraders BilderBchmuck d. d. Spr. ${ }^{2}$ 408). ifiiirell ") bieie Starte bor jidt hinlegen 1751. 2) ,iidh if." ofne (5inbernehmen mit ben Bjläubigen bie liniverität verlailen 1781. 1795 $=$ iid) jibiren 1781 (unter $a b=$ gehen) 1831 (auch Mart. Schluck \$4 i id iquijirent).

Stis: "eillen Equis madbelt" $=j i$ d itijiren Mart. Schluck $\$ 4$.

ifitgen ftehlen, entwenden 1795. 1841. Smolliz 1813 - Edmolliz. ionberbar Tuichmort 1813 Göttgr. Stud. E. 1117.

Sorten: "fid alli bie E. madben" leije und jullell babon gehen 1795 . - 3rm. i o de en gehn, meggehn 1846. Soff ${ }^{1}$ ) ununäß̈̈iger Trunf 1781. ${ }^{2}$ ) (Se= tränk 1813. $1831 \quad$ (z. B. "Das i it eill idjlechter, belifater Soff").

Sobll: "einen jungen 5 . befommen" mit einem ßedfel erirent werben 1749. 
Spobu Beld Körner 1812 Vache wächter IV.

pownen nad) bem Bedjen now eine Fjlaige mit einent andern juiannten trinten 1781.

Spartam et Martham Bifure uno Euarre Stammbücher: $19 \pm 6$; Salinde $17+t$ a 5 .

Spargeliteder is raf́ 1946; Wolff. Naturgesch. d. d. Stud. $\Xi$. 60.

Gparitarmante oben E. 19.

ipajjig: "ip. gefru" 1749 Abendstunden $\Subset .362 \%$

spe-fubs arbiturient in Breslau 1831.

ipectell beionser (,ib. Peeth, in. Ma= lice"); "aup einem Evegielfen treipen" in serlegentheit jein 1313.

Epedt ("Ep. in ber Iatide haten") Beld schelmutiki ङ. 110.

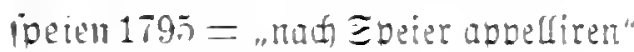
$1781=$ "nat Eveier mo morms abpelliren" 179.5 fï erbrefier.

Spei-multum: „eit כp.-n. begeht" romere 1616 Jus Potandi $\leqslant$ t5.

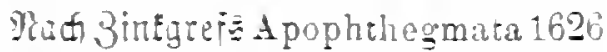

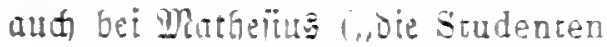
maden igre Eqfubreden bei mein mo Bier it Ferison und spei multum"1.

Eper Epejiesthuler 1795. 1922. 1841:

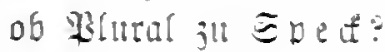

pidfen: "Den Belobeutel io." 1itt Avanturiers @. $t 2 ; i$. Eved.

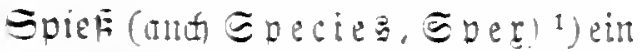

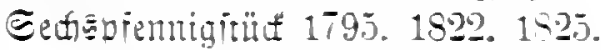
2) Beringmertiges (,if gebe mith einer Eviés ourum") 1795. Epiébe Gelber 1795. 1513. 1822. Laukhard. Schilda I, 201 - Euler-

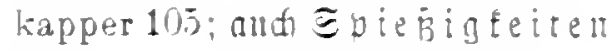
1825.

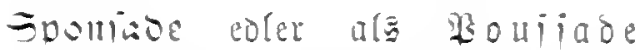
1S.31; aud Gaudy. Werke I, 194; Daglt હoonjenr 1831 mo ibou= iirend die (5our maten 1831.

Eptrg Mauid 1st6; Ftudentenwitze 1839 ङ. 68: Schnabels Univ.-Jahre

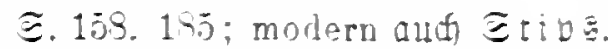
Eprigbinthe Mäodien 1791. 1946. iortgen reiten ober fuhren; Eoris.

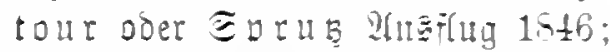

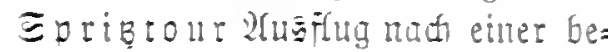
Itatibarten Etuot O I. B. Wolff. Naturgesch. d. d. Stud. ${ }^{3} \cong .6 t$.

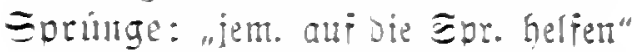
fem. Den Seitrober an bie sand

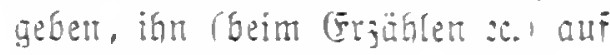
Den reffert 浔eg bringen 1795.

Eouj Evejiestuler 1813.

Etabcurrelt 1795:- Etad meeien i. Meeien.

Stallbeier Itemirmänden $14 \pm 1$.

frallell mingere 1731. 1795. 15+1.

Grommbut oes Erubentelt 1731.

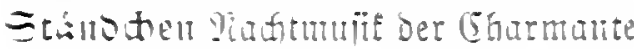
gebradit $17 \pm 9$.

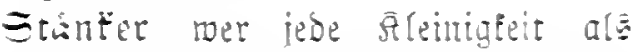

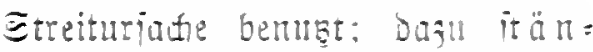
Eern, Etäulereicn 1795.19t1.

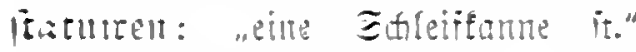
boniren $17+9$.

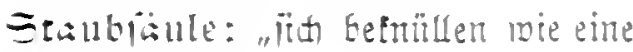
Et." 1831 .

|Fe⿻上丨: 1) "einem erm. is." heintion

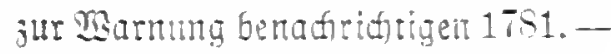
") "Wtit Ravbirent it." 15.31. " I gitt trinferl 1531.

Erewer trambortable Tintenfäilier aus Forn 1s13; 19t5 Burschenfahrten $\widetilde{E} .71$. 
Iteife Gesanten molliiftige Gedonten 17's1. 1795.

itrigen "gehn; "in Dorje it." $17 \mathrm{~s} 1$. 179\%. 1531. 1st1: 1749) Der reisende Avanturier II, 50. 66: , 311 Bicre it." 1795. - "2) vortrinten 1846 ; "id iteige bir eins vor" 1831. stellatim atben cohen E. 43 ); "(B): banfen ben bem st.-a." cin analreont. (Sedid)t Abendstunden 1750, 335; Stoppe 172s Gedichte I, 43. 114. 151. II, t5; Servius. Mïgdetrïster ङ. 147; Schnabel 1750 der aus dem Mond gefallene Prinz.

frellen ausfiihren, burdiezen ("er tam nidut viel it.") 1813.

iid) frellen jid) einfinden (zแm Duedr) 1795. 1825. 1841; "iidh nidt itellen", eitue Forbermun nidit aumehmen 1795.

fribitsen liitig itehlen 1781. 1795. 1841.

Stit) Summent 1813.

stifelis : "in st." jul THi 1546.

Stiftler "toie ber cuang. Theologic beflifiencu (veraltet)" 1841.

Stratmidier 1781.1795; Sticiel. w 1 (⿻) $18: 31.1845$; nuth Bursehenfahrten అ. 93; ভticieliud) modern (and) Bacheliade S. 102).

Stori jede Enantität non Bsetränfen 1831.

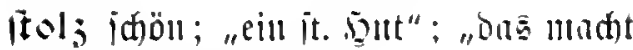
iiá) itoly" 1813.

Stoppelhopier Defonom modern.

Stiplet Serl 1793 Phil. Dulder I, St.

Stordbeill als Beleidinung v. Maltitz 1828 Der alte student II, 2; ob jul Stordicr?

Storder Goethe im Urfaust nael AfdA 20,304 jull Stordbeill? cher (wie Di a r ft id reier 2 . 2179) $=$ Storger 'Randjahrer' (Stór. (her C'ulmbach 1714 Quasi vero כ. 033 .

Stolien (nid)t in 5ralle) $=$ (Srobhan 1795.

Ifolicn mit Etoprappieren fechtell 1813. 1831; ito B́t an Rui int Bier: ifandal 1831.

it riajlidb grob, jefr; "itr. Rangetweile", "fict jträllid) cunnuiren" 1813.

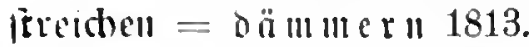

Etruif ("cinell Etr. madhen") $\mathfrak{F} \| \bar{b}=$ tour, Bummel 1757 Niemand 5.84. Strid) : "alli ben Str. gehen", "cin munterer Str." zul itreiden 1813 ; aud Laukhard, Autobiogr. I, 345 "ani ben Str. gehn แแ⿰ Mäo= dell wie Eerden jangen".

Etritbrogel i. 3 ugügel.

Stritt Sterl Eulerk. 187.

Strobrensmmift Bramtartras 18:31. Strobm Gtrold Eulerk. 187.

Etromer $=\Xi$ d) wimel (bei. in Seinsig) 1431. Stromer Bummler Sehnabels Unir. Jahre 5. 254.

Strumpf: "ali bein Str. jein" IHarring 1831 Faust 5.97 - "auf ben Etriïnjen jeill" Eulerk. 187. Stritmpie: „jid allf bie Str. madten" idnell wohin gehn unt eille Sache zll bejorgen 1795.

Itrumpien all bem Strumpie jein 1846.

Stubenarteit 1781. 1795. Stubenburith 1795. 1×13. 1831 Stubeuburjare 1781. Salinde $174+5.64 .65$ und 1747 Hospitium ङ. 23. 24. 68 Stubengenojie. Tajïr Stubeufaball 1781; alld Salinde 1744 ङ. 83 ; Stubell= 
burid) nod) Körner 1812 Náchtwäcliter III.

Stubentaneel modern $=$ Stnben= fam i jol 1831; vgl. Sya jol şallgnenolie O. L. B. Wolff, Naturgesch, d. d. Stud. 66,

Stubenfowiger = Mn ater 1781; dafiir 1795, 1841 Stubenjizer. Etubeut: bafïr immer Stubio 1831.

findentitos itubentemuäßig 1781; 1737 Taubmanniana 厄. 150 it $\mathrm{s}=$ be $11 \mathrm{t} \%(\overline{11} \varsigma$ - Reinwald 1720 Academienspiegel ङ.9 studenticòs. Stubirent balber $\mathrm{um}$ itmbirell ăll jollen 1846.

Stubl: "శึ̆ St. fommen" zur Muhe tommen 1813.

1. Stulp, Stur pe gropier Fechthand= i(f)uh 1 s31.

2. Stulp Sturz ani 12 reip. ¿t Bänge; malitiöier Et. Strm ali 24 reip. 48 Gänge 1831.

funden: "(bie Sollegiengelder in meliorem fortunam) it. (a)ijell 1846 ; and) Schnabels Univ.-Jahre $\widetilde{S} .17$.

Stummel Bipeife Heun 1792 Vertraute Briefe I, S. 16.

Sturn: "im St. jein" beranjet jein 1831.

Sturnter 24t Miikze 1816 Über den Geist des Studentenlebens in Jena

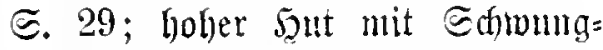
federu 1831. (5igtl. Sturuly

Sturz jede fommentmäßige toudyirende Beleibigung 1831.

ftúrzen: "Beleioigungen it." 1813. 1831. - bei Betränf́en itïrz̧t man eiuten (Belebrten, einen Doftor, einen Bapit, indem leide Teile je 1 (5ikis reip. 2 oder 3 Bstajer trinten 1513. Sluge, Deutide Etubentenipradie.
1825.; "eiten bumutu Smugen it." jo iffimpfell 1822.

Stus Mï̈ke, Sout 1816.

Stuger "thigndeifen joviel wie ein Benommit, bismeilen wie ein \$eti= mäter; Dan uer ben einen Shoob upu der Weite auf bou 2 trme trägt unb jith ärger in bie Banit wirit als ein Bettelinde" 1749; (bigi. ว̆l Menomutif 1781 (and) Mart Schluck \& 16). - ङ(hon im 17. 11 . 18. Jahrh. 3. B. 1611 Stutzerus 二 Tutho De Jure et Natura Pennalium A $3 \mathrm{~b}$; Reinwald 1720 Academienspiegel $\subseteq .453 ; 165+$ Pennaletc. Possen B II a III $^{b} \mathrm{~V}^{\mathrm{b}}$.

Subjertior 1831.

Suite $\left.{ }^{1}\right)$ Dueff. ${ }^{2}$ ) Sommer. $\left.{ }^{3}\right)$ Eiebes= ahenteuer. ${ }^{*}$ ) jebe anberordentl. Fahrt

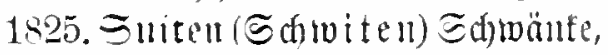
Streidye 1795. 1813. 1831. 1811; "S. reißen" (nitig und iibermiithigiein 1795. 1813 (Goethe, Dichtg. U. Wahrh., 7. Buch); "eille freuzitiole Eutte"; Suitier wer alle Etreidhe mitmadt; initijiren Burjuen= itreidhe verridten 1813. 1822, 1831. 1841. - Sulte nba dultiger Rerl 1822. 1841.

Sulphurifr 1) Rugeter, Demuniant 1825. - ${ }^{2}$ ) wer fid gegen die Ramb: mannjoffell mit anbern verhumbu

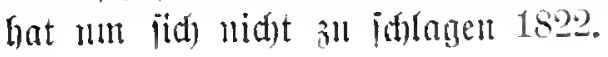
$18+1$.

summum bonum biffe mo jecife 3öpie 1781.

lumprell modern (verinutien Wilh. Grimm, K1. Schriften 1,51s). Suvi $1846=\Xi$ d) $10 \circ$ T.

juperbe („i. 2Better", "i. Rereat", "e 
priert iimerbe") in Plumbe tor Edjäđder and lietimüter 1749. Suppenaller batituirt 1721.

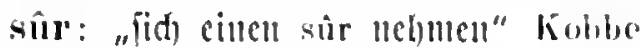

1810 Erinnerungen I, 12.

iiili Fillen rodicem lambere $1 \mathrm{~s} 13$. Sritem Iabafopicife O. I. B. Wolff, Naturgeseh. E. 126.

Tabulas eine Plit von "nstaraser fiir Sie braben Buridhen, die ibre (5ollegia nid) bezalen toollen" "1749. - Dit in Stammbiichern $14 m$ 1750 "2ior Sarjer mo vor Iabulat behit uns Syerre jriih mo juat." Tabulat= d) en Sarzer in Senta bei Leber. Blaustrumpf, possierl. Gedichte 1749 ; Salinde 1744 ङ. 127.156.

Tad: „InIm iit es T."? Gdüdheran= Druct 1749.

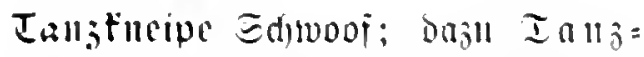
tueipier sirt cines Tanlotals 1831.

Tanjturi anitionsinger Extwovo 1831. tapiren mituchuren, itehlen 17\$1.1795. 1841.

tect $=\mathrm{t}$ beet.

Ter; ballen beim Fedten 1795.

Ther: "anf ben Thee fommen" jibel anlanjen, miglitefids metsen 1795. $18+1$.

thect (oben $\subseteq .70$ ): julerît "eill teefer ङduurr" 1766 in einem Jenisehen Stammbueh zu Weimar. - Dann elent, idfled)t, gering, fade 1 ,ein th. Serl") 1781. 1795. 1822. 1941; "eill

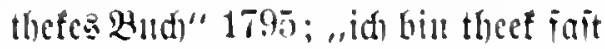
ielyr" id) bin bettelarm 1781. tcef 'meid, mürbe' "cill edjter Buridenantsond" F. M. . I rudt, ïber den Studentenstaat. 1815.

Ibectellul ein einfültiger Mienja, ber lid)t gern mitmad)t 1781. 1795. 1831 ; aud lanukhard 1799 Sehilda I, 16i - Eulerk. G. 103; Lenz 1774 Hofmeister IV, 6. - Thee: fejieltite nornelnue Damentelt 1846 .

Ibierchell: "mein Th." zärtlid)e 2(11= rese wie alte 5 and 1795.1841. Thorll: "Tintit von Th." 1831: o I. B. Wolff, Naturgeseh. d. d.

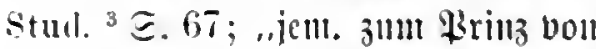
Th. madhen" eime cigene 2 rt fid iingeno 3̆l betrintell Laukhard, Eulerkapper G. 229.

Thonpringel weije Thonpieife 1813; i. If riigel.

thun: "סas fann er wol th)." altorntef 1749 .

ticf: "pereat tiej" 1781; baher "tiej perciren" gropese Gleno an: wiinid)en 1795. - "t. in 巨dul= Den iteden" 1795 ; "t. in Der Blamage itefell" menlig (5hre habell 1795.

Tiegel: ,in I." inl llnglïa 1846.

Tiidburid Tijdgenolie 1703 Taubmanninna $\odot .97 ; 1744$ salinde ङ. 24 ; dafïr Tijofauall ill Kejls stanmb. Є. 171 aug (5r. Langen 1749 .

Tobid Tahat 1822. 1841 (Tobiạ 1831); hojditt. idjon 1692.

toll bejofiell Laukhard, Sehilda II, 125.

Tólpel; "jell. iiber dell $\mathfrak{T}$. werfen" beïiegen 1747 Hospitium 5.56.

Tortel bilïa modern

Touthe Beleidigung 1822. 1825. 1841; Campe 1813; Heines Werke II, 74; als Ionid) 1831. - toll= d) iten beleidigen 1821. 1831. 1841; alld) 1747 Hospitium $\subseteq .36$ (tou = 
idiren 1822. 1831); fđdult 1744 Salinde 5.71 .76 (I D $\|$ d) e ule $u t$ 5. 72).

Touph Miliif zum Rivatrūen 1831. Trampelabarmante i. obell S. 19 ; daju Trampler wer Fenlterproule= maden madt 1747 Hospitium S. 46. Tramermantel, =vogel mumegitedidider Menij) 1795.1941.

tranrig mangenefin, elend 1795,1541 . Treffer mterwartetes Britid (eigtl. im Bärrelipiel, wo trefrell "ge= wilnten' bedentet); "er hat viel $\mathfrak{I} r . "$ 蹋 glïat ibul allig 1795. 1841; Bahrdt, Rindvigius I, 107; "er hat eitten guten $\mathfrak{I} r$, all Reibe" er mirft iul Bürfelipiel jelgr glïdflid) 1813; „einen Treffer poer Ped haben" Laukhard $1799 \mathrm{Fr}$. Wolfstein I, 135.

treten trani. ") heftig allfeţell, woju verniögen 1781. 1795, 1841 (ว. $\mathfrak{B}$. ,jem. um (Beld treten" 1795); and) ipeaj. ${ }^{2}$ ) jem. bereden, eine (Siejellid)ait mit Rafiee 2c. an regaliren 1795. 3) utahtuen 1813. 1825. 1831. 1841; ipez. a1t Duell 1822. 1831; Dazı

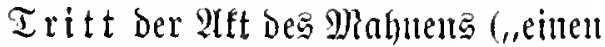
gegelinden Tritt erhalten, geben", "Trittbrief") 1813. 1831. - ${ }^{4}$ ), ,ill Tl. jein" einen fleinen Mamj habeu 1831. - "treten laijen" cinen Serans= geforberten an Das Rosutacten eines Duelf erimtern Iaijen 1822. 1841. - Irittoogel $1846=$ Ireter 1831.

Tretbrief Młahubrief Niebergall 1837 Bursch.-Heimkehr III, 10.

trintabel Gaudy, Werke 1, 182 แnь ogl. oben S. 67.

trift trantig; Ulubehagen azengento
1795. 1825. 1831 ("eill triftes รูañ").

Tritt f. tretell.

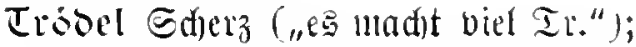
tröbelhaft jherzhaft 1813; "cittell Ir. amitelfen" Studentenwitze 1839 ธิ. 89.

trollen: "jidh tr." weggehn 1781.1841.

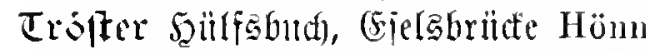

1721 Betrugslexicon $5.165 ; 1747$ Hospitium 5. 18.

Irotticell $\mathfrak{T}$ rott reitell 1781.

trumpfen einen Lerweis geben, Lidr= witrfe madyell 1781. 1795; "einen Trumb worali jeken" etw. itark befräftigen, man werde etw. jicther jo ausfüthrell als man zul thum Bailleng ijt, went das Bsegentheil gejdehen ober nidjt gejdhehen jollte 1795.

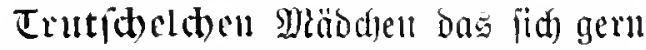
lieblojen läst, bej. Tirolerimnen, bie Init Balantericwaaren ljandeln 1781. Tumult 1749.

lllf, Spás, Tumult 1831; IIIt treiben= nltin 1846.

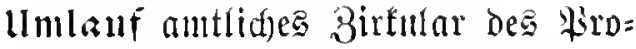
reftore oder bes Detalle 1781.

IImiatteln Motschmann 1729 Erfordia Litterata I in Megiifter: Joh. Jak. Lehmann, De Mutatione Studiorum (2oul Inijatteli) 1715; auct Niebergall 1837 D. Burschen Heimkehr IV, 8. Tazu lt miattelung (1720) Faustbuch des Christl. Meynenden ङ. $2 / \%$.

IImptopen allein oder in Bejellidaft iell. anf die Stube fontment mo er=

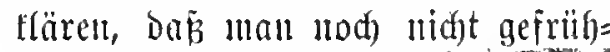
ftüatt loat 1785 Beytr. zur Statistik von Göttgn. S. 130 . 


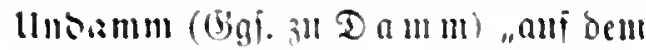
II. iein" modern.

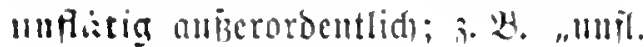
buritig" 1795. 1841.

Inageriblifene !'bute Hacdianten bor ber Iepopition Reinwall $1720 \mathrm{Aca}$ demienspiegel હ. $\$ 2$.

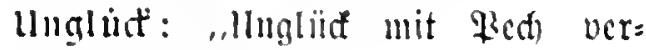
miide" 1795 .

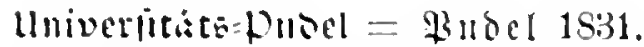
Uniform ber Eanozmanuidaiten 1831. mupartciipher jenge 1831.

lluinn afs 2tczcid)um fïr allez mas bem surjaen nid)t geiällt; bazı "ui inuig: "ein mi. Sans" cin prigineller, toller Serl 1831.

Unterbans (beim Srommers) dic $111=$ tere Tiidhälite 1846.

unterlanjen cine llurcgelmäßzigfeit frim Ducll 1525.

Inwernimftig aniserorbentlid 3.3. "muvern. Flcibig" 1781. 1795. 1841. "muern. heifi", "muvern. ja)lajen"; "ber sert hat cime muem. Ean" viel Ǵlïđđ 1831.

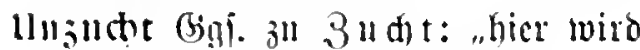
llng. getrieben" hier geht es wild burdf und ilter cimanber 1813.

lippig: "ïd - machen" OLBWolt Taturgesch. d. d. Stud. 3 厄. 128. Jeppitus (oten $\Xi .36$ ) llebermittiger 1 s.6.

Vascmecum Tiener kei Sdmeibel 1797 (cinte red)tidnatienen Stndenten Slugheit all leben).

Lerbinsumg 1831 .

verbủiten (Gicls) burdfbringen 1 แ! 1600 Fei Ant. Banmaister. Fin schon neues Fastnachtsspiel $A 6^{\circ}$. versominuen (Gels) verthum, ver= iubeln Reinwald 1720 Aeademien-
Spiegel S. 453; Hönn 1721 Berrugslexikon G. 408; idon 3ind: grefo Thophthegmata 1626.

wersonnern (zu cinem Efrafanantmm beim Sommerā) 1831.

Lergnugen: "eill miduldiges Ber= guiigen" Enanic 1795. 1841.

fid) verbauen eimen faliden bicb thun 1795. 1813. 1841.

verjubeln im Subel ourd)bringen 1813. verteilen i) vericken 1781. 1795. 1813. 2 verfanfen 1795. 1813 . 1822. 1825. 18:31. $1841 ;^{3}$ ) Niebergall 1837 Des Burschen Heimkehr III, 10. - ") "iing berf." fid ber= licbell Niebergall 1837 Des Burschen Heimkehr III, 12.

vertigell icin (seld im Spiel ver= lieren 1831.

vertleifern Denumeiren Bahrdt, Rindvigins I, 101.

vertloppen verłanien 1831.

vertueipen in \$Birthähäujern ourd)= bringen 1813. 1831.

vortisidben berfanfen 1831.

verfiumeln verfaujen 1822. 1831.

1841 ; all $v$. Maltitz 1828 Der alte Student II, 2.

Verlag Rerlegentheit 1846.

verlusern (Geld und Zeit idjedft ver= thm 1831.

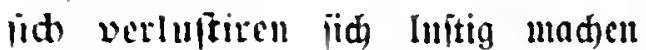
1781.

verms seln verfaniell 1831.

verpaitalen (bas (Seld) verjubeln 7schr. d. Thür. Gesch.-Vereins XI, 6 .

verplempern iid) platoniig verlieben 1825. 1831.

verpsinen (j. B. cine stneipe) in 2 er $=$ ї)is thut 1831. 
verpumpen berborgen 1831. verauálen bertanfen 1813. verquetionen verfanten 1 s.3. verrintt: "lvie verr." wie toll $1 \times 31$.

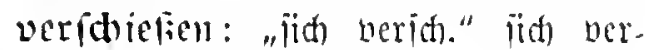
licben 1825. 18:31; verido ojien verliebt 1341.

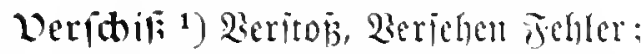
"in 23. jein" gegen dic Megeln be Suridentomment fehlen 1781. 1795;

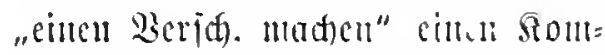
mentiegler begefnit 1595 - "in den Qerja. thun" fïr chrtos crflärem 1841. Laukhard, Eulerk. 116 Schilda I, 9 Mosellaner ভ. 97 ; "an1

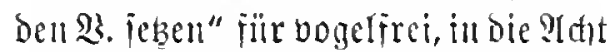
crffären 1795. -- ${ }^{2}$ ) (Ebrlo 1822 ("temporärer Serjđ)., perpett=

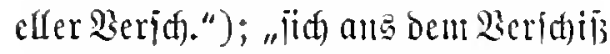
id)Lagen" 1825; 2errui, (shrlojigteit 1822. 1831; daju eill 란 $\mathfrak{r}=$ idifiener 1822. 1841.

veriblemmieren verprafien Jobsiade I, $\mathfrak{2 .} 886$.

veriduallen berfanfen 1781. 1831. veridnappert: "ïid verjint." Naltitz 1S28. Der alte Student II,2. veribwimeln (Beit uno Beld) ver: thun 1831.

veriđwitifiren jein (Seld buriđifos burdbbringen $1831 ; i$. Suite. verianisen verlernen 1831. weriatwrofen (Beld) vertanjen 1831. verieten Gelo anj ein Bafano borgen 1795.

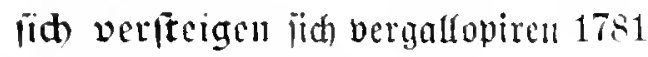
(unter it eigent).

verwitbien buthluingen 182.

verwithien 1) fït do seripreden geben etwas nid)t ant thut 1831. 2) Durabringeu $1 \$ 41$.
Detter, frall Detterin Pfurede an bcu 2Birt mo die geirtin auf ocm Jentaer Butgfeller 1831 Der Student u. die Steeknadel I, 104; Kobbe $1 \mathrm{~s} 40$ Erimerungen I, $15 \%$.

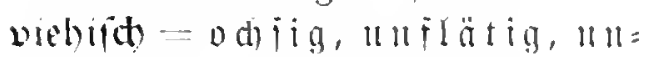
berllïlftig 1795. 1841.

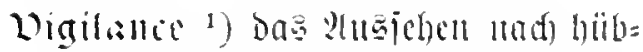

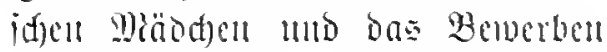
mu ifre (sinut 1781. 2) Das iduöne (Bcichledut bei. ber höheren Stämde 1795. 1841.

vigriliten ${ }^{1}$ ) jübuen Pläod)en nad)= idjaten 1795. 18t1. - Bigilauz "Mltgenueide am jraneminner" Fischer 1781 Bursehiade $\subseteq .46 .-$ 2) beint sajarbjpicl bie Sarten lang= jan Durdziénen 1781. 1795.

virgatim oben 5.42.

Dotativus ein burdhtricbener fict Eulerkapper ๔. 10.

volticen Fedterterminls: 1813 .

vor: "eins vor" $=$ iø trinfe Dir citt (Gias vor 1831.

vorfabren herbeibringen, herbolen, vorfiithren ("Iás mir cincu tïdtigen

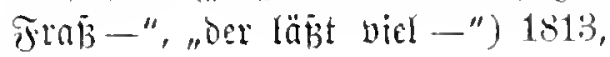
1 1.831.

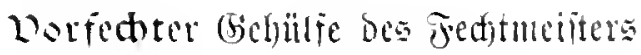
1831.

Dorgebirge $=$ promontoria 1825.

vortosen (nad) boutriufell ge= biloet) 1.31 .

vorreiten ${ }^{1}$ ) prïjentiten (obic vien= linge werben vou ihten ?andstenten Den andern Etubenten borgeritten"? 1s13. 2) portrinfen $17 \pm 4$ Salinde ङ. 65 .

Dorreiter bei Galittufahrten 1831. Dorreiter eime (5harge bei Prujäigen Edy Yittenfabrten 1795. 1E31; "cint 
ante Worreiternille haleu" ba Bars reiten gut veritehn 179\%).

Dorrosmmerel vortrinfell (iff)

Eir eint vor") 18.31.

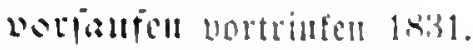

Dorfteher Eentor bei ien 2 mujden: iffaiten 1-331.

Doridomsen bortrinfen 1739 keils

Stammb. E. 206; Ilospitium ङ.5t. bortengen vortrinten 1:31.

Dortrag promontorium 1;25. 14:31. Dortrution 151:3. 15:31.

warmalden gut anjitiden 1831.

sarjithen uortrinten 1831.

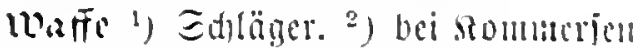
Das Gin 1825. 18:31.

Wallad : "non possum iit cin 然。” id) taum mid)! 1831.

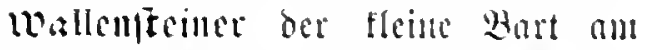
Simu 1431. OLblWolf Yaturgesch. S. 134.

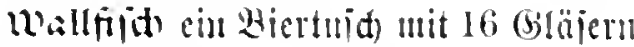
1331.

Wedfel des Entuduten in Der hentigen

Bedentum 1795. 1813. 1831. 1ist1 ete. (id)u Behmeno 1715 Poet.

Cabinet ङ. $121 ; 1745$ Aut der

Extrapost eingelaufene Nachrich-

ten $\subseteq$. 70); ïberhanpt (bels, Ber= mïgen 1825 .

regtruegen lemen (3. W. Das Lisein= trinten) Laukhard $1799 \mathrm{Fr}$. Wolfstein 5. S2.

mearutideu nfgehn (von der Edfule)

Schilda III, 269.

vegltibityen = itibisen 17 .

whlit) ") vou Pierden ?c. mntig.

2) itmo. itbermiting 1781 .

werslidh red)t iehr ("einen w. Mus=

bunzen" beruntermad)en) 1781.

weilen iid) anigalten 1781.
Weintueipe =tuepier, =tmin 1831. welt: "alle in ber $\$ 2 .$, mur bies nidft" abjd)länlid)e 2utwoort 1749.

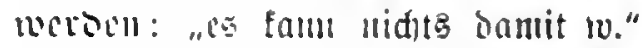
iit uid)t gut uto wito uid)t gut 1749 .

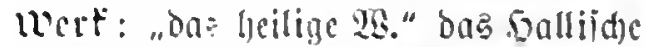
isaijerbans 1795. $18+1$.

wrgo" in die Eteine banen 1749; mit bom Ingen ani ben Etein ober bem Etcinvilaiter, io bais Junten

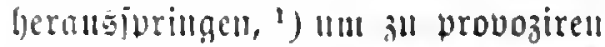
1781. $\left.{ }^{2}\right)$ a (s. Beiden bes 2luiruhrs $1795,1+1$.

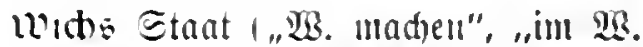
jei1" 1795. 1831. 1844. - "en WBir" ist (bolla, jehr gevubet 17s1. - jïh in 照. Iverion Laukhard 1798 Schilda I, 191. - Widtitleis 1778 Zs. d. Harzrereins 22, 423 - wirtlis Etuatäfleio Fischer 1781 Burschiade E. 1 s.

tridhicu prïgeln 1781 (and) Nart. Schluck \$ 13); id)lagen 1795. 1831. 1841.

Widjiar Etiejelwidier 1841. 1846; aud) L. Köhler 1843 Akad. Welt I, 40; 1I, 33.

wilser wer iid nid)t ju iemen Lands: leuten hält, sver teiner Ranbsmann= juaft angeljört 1813. 1831. 1841; (aud) nidjt ala Menonce) 1822. $1 \varsigma 31$; Schnubels Univ.-Jahre 312. wils l'bombre jajarojpiel, bej. \&harao 1813.

Wintelqualt keim jod)ten 1795 unter Terjha ue ll.

Wijcts: "ein Derber $\mathfrak{2}$., eimen $\mathfrak{W}$. ciniteden" Laukhard, Emigranten II, 1 ss.

Wit, andh von Soanblungen ("guter,

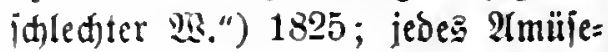


ment; i. Biertuik, Buriuen= $\mathfrak{w}$ i 1831 .

witgfaat Abart bes Bieritatis OLB.

Wolff Naturgesch. d. d. Stud. ${ }^{3}$ ธ. 176 .

wolle: "in ber $\mathbb{2}^{2}$ jiben" in guten Berhälmitien, bei Geld iein 1781. $1831=$,ill ber פs. icin" 1795. 1841.

worti Bud modern.

Wortflauber wer fid tei Sleming= feiten anffält und in Der Sagh obe: (Erforidung ber worte pebantipa) unl ängittid) iit 1781.

$\mathfrak{w}_{\text {ud }}$, Stiefelmude 巨tieielmidjer 1822. 1 \$ 41.

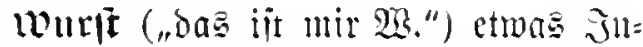
Differente $1822.1825,1931,1841$ etc.; ctros (bleidhgittiges (aud) wurstum est 1846). Schnabels Univ.Jahre ๔. 160 .

touritig: "Das i it cine w. Cadje", "Das iit mir muritia" eine Blemigkeit 1831. $18+1$.

Wurjel Minfur 1841; Nart. Schluck, v. Burschenkomment $\$ 12$.

wuitrid 1831.

$x$-illal modern.

jatt: "ïid) 3. madiest" fjïbid, itatt=

(id) ansiehen ("ei! Das madit ïd) jart") 1813.

jelle Stube, Bube 1846.

jeng alle Slleibungsitütue; "aū bem Benge jein" iid) munter uno wold Eefinden - "jem. etw. an Dem Benge flifen" jem. Edaden thm oder ihm etrv. allbaben 1795. 1841; "was bas

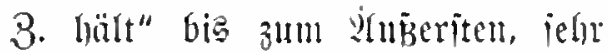
Laukhard, Emigranten II, 169. 171 .
Seuge (parteiijocr, mtparteiijater) 1831.

jengmart eine (Sharge (wer die seafien, Fahuen, Binsen uin. Der 2kuriden= id)aft unter iid hat) 1825.

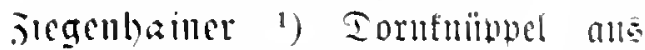
Biegenhain bei Jutra 1795. 1531. 1841 (and) Keils Stammbücher ङ. 283). $\left.{ }^{2}\right)$ gewöghtider Єtudenten= itod (merfwitroig Dafei die Eitte, bas biffe (5nde untell ju tragen wie bei einer Seule) 1S13. 1831. 1841. - "3. geben" mit einem 3. Durd)= nritgeln 1813 - jiegentainern ohen $\Subset .67$.

jiegenlyolz Biegentainer 1831. 1sts Burschenfahrten $\Xi 71$.

jiebell ${ }^{1}$ itart pro poena trinten („зich, 厄dimmel, jieh") 1781. 1795.

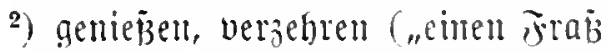
mit פisein j.", "einen Ednatem j." 1813. 3) gelten, erlaubt iein ("Mie= tourfutide zieht nidut") 1813. 1831. 1841. 4) (5ine (5ntidulsigung, 造e

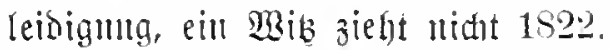
1825. 5) in 2lupuahme jein ("eime IIniverīität j̇ieht", ,Minteln $\mathfrak{z} 0 \mathbb{a}$ midut") $\left.1813 .{ }^{6}\right)$, mm Geld jem. $\hat{z}^{.}$" prellen Salinde 1744 A 4 ".

jinten im Gefide ?aje Niebergall 1837 Des Burschen Heimkehr III, 11 .

\section{jirtumfler (5irfumitex 1749.}

jobel Franenzimmer in Iflgentuen 1822. 1841. 1846; आnch Heine 1824 Harzreise (Werke III, 57 Elster); Mäbden ber niederen Etände, bei. Dientmäbden 1831. Canber be= legt 3 obel de n meretrix ans Grimmelshausen. Bigl, oben $\mathfrak{\subseteq} 55$. 
SNSol morbentlides büblides Trmen. simuer ma den niederen Etünden 1 1s:31.

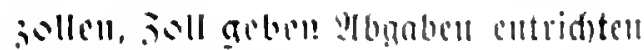
1781.

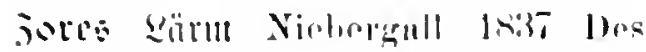
Burschen Ilrimkehir II, 10.

Sotologic Lauklund, Fimigr. 1I, 4.3

- Marki v. Cebriun II, 121. jusetten bein Tedten bell Grequer tiid)tig zuinumentranten 1s13; jem.

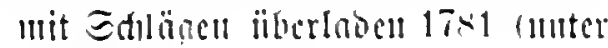
be efell).

suruther (im Startuiniel) gliuflid) sufnuisn 1795 comter indient. $1+41$.

juguogel meretrix 1831. "Bllg= ober

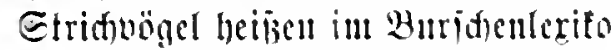
Mädden, die inm Bapientreidn bie Bromenase regelmäbig bejuden" 1785 Beytr. z. Stat. v. Giittgn. 294. jureten anlermen, belebren (bag bie:

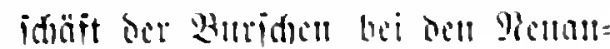
acfommentin) 1813.
Surütuchmen cine (iibercilte, mbe= abiidntigte) Welcibigunn: iic jo gut nla nid)t neidjehen extlinten 1795. $1825.18+1$.

surnitt liul lluredt babou, bic Gade nidst neritehn ("bu biit nod) weit 3uriid" on irrit onrin iche) $179 \%$. $12+1$.

slliammenidhlagen hict im Epiele aelvimuen 1795. 1841.

illi(hallicll beridaafien Laukhard 1798 Schilda I, 3-1.

juroat), junger juwact)e die nell= angefommenen Shbirenden 1795. 1841.

swingen in autem 3uitande, bei Beld icin 1795. 1k41; , „er zwingt es bife" hat viel Geld 1781.1795; "cr famm nidit jwingen" loat feill (Gelo daz" 17-1; Laukhard, Erzälungen u. Novellen 1, 413. - ..jid 3w." fid Giewalt anthun, etw. itherwinben (id) miïite mid) gıviugen) 1795. 1813. 1441. - Eulerkapper 5. 107. 


\section{VERLAG VON KARI, T. TRUÜBNER IN STKASSBURG, I

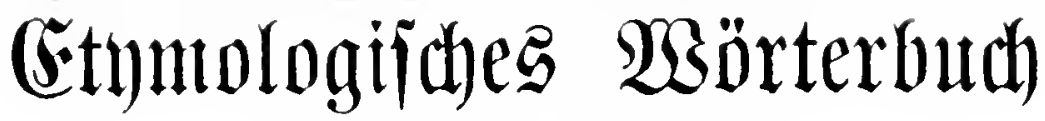 ber beutidyen Epradje}

Doll

Frricorid Slltge,

ort. Arofefior ter teutiden Eprade an ter iniberfitát greiburg i. Br. Tiinfle verbefferte uno ftark vermelprte alufinge.

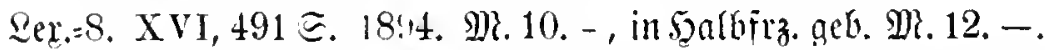

Der Verfasser des vorlicgenden Werkes hat es unternommen, auf Grund ler zcrstreutu Einzelforschungen und seines eigenen mehrjährigen studien ein etymologisehes Wörterbuch des deutsehell Sp rachsehat zes auszuarbeiton, las dem gegenwärtigen Staude der Wissensehaft entspricht. Er hat es sich 7ur Aufgabe gemacht, Form und Bedeutung jedes Wortes his zu seiner quelle zu verfolgen, die Brziehungen zu den klassischen Surachon in sleichen Masse betonend, wie das Verwandtschafisverläiltuis zu den ubrigen germanischen und len romanischen Spracher. Selbst lio vergleichung mit den entfernteren orientalischen (8anskrit und Zend'. Jen keltischen und slavischen Sprachen ist in allen Fälen herangezogen. Wo die Forschung eine Verwandischaft festzustellen vermag und wo diene Verwandtsehaft zugleich Idicht auf die Urzeit des germanischrn Lebens wirft.

Eine al]gemeine Einlcitung behandelt die Geschichto der deutschen Sprache in ihren Umrissen.

Di e Verbesserungen der ne uch A uflage bestehen i ll der velwertung lier ueuesten Forschungsorgebnisse a uf lem Gebiete ler gexmanischen Etymologie; die Vermehrungen in der $\Lambda$ uf nahmeeiner grositen $A$ nzahlsolcher Fremdwöter, welche seit dem 16. Jahr h. aufgekommen sind, und seitden einen unzweifelhaften Bestaudteil der deutseben sprache ansmaehen. In den meisten l"ällen ist es dem Verfasserge]ungen, genau fest\% ustellen, wann und auf welchem Wege das eiuzelne liendwort in unseresprache eingedrungen und damit Nachweisungenzu bieten, welehe a userdem sprach lichen, ein hohes rulturgestehtliehes Interesse be:anspruehen.

(Ankïndigungr des Verlegers.

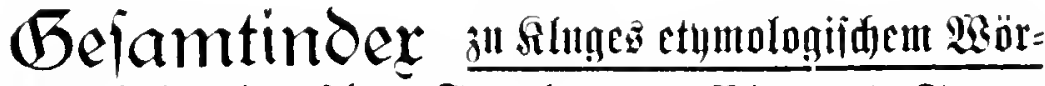
terbud ber deutiden Sprade non Bincent Frans Janijen. 2er:=8". 284 Є. 1890. M. 7.-.

„Dieser Gesamtindex ist eine sehr wertvolle Ergänzung zu dem ausgezeicbueten Werke von Kluge, das so schnell zu einen unentbehrlicben Ratgeber für alle Sprachgelehrien geworden ist. Erst mit Hülfe dieses Index, der offenbar mit voller Jiebe und Jingebung und nicht auf blosse liestellung gearbeitet ist, kann der grosse Schatz wohluberlegter Aufstellungen und Kombinationen, dic in Kluges Werke enthalteu sind, voll gehohen werden. Man sieht, welch cin Reichtum uns in diesem Gesantindex geboten wird, und es larf sich tenselben Xiemand entgehen lassen, ler der Kluge'schen Arbeit eine wirklich wissenschaftliche Benutzung zuzuwendon in Standre ist. Der Preis ist tür das hier Gebotene sebr billig."

(Lit. Centralblatt 1590. 23.) 


\title{
Bon Suther bis Rejiuntg.
}

\author{
Epla (t) gle \\ bor
}

Fricirid Pluye

Profejior an cer lluiberfitat ìrelbutg i. Br.

imute, surdageicheme ?lutage.

inhalt: Stitd)enjurade und Loltäptad)e. - Mharimilian mub ieille Stanjlei. - Euther undo die beutide Eurad)e. - Edjrift=

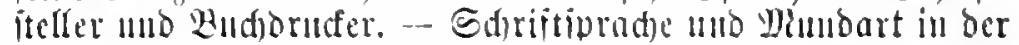
Edwei3. - Dberdentider mo mitteldentidjer Mortidjab. -

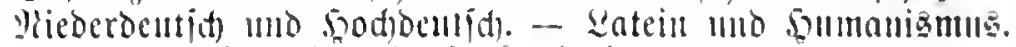
- Dberdeutidinno und bie Sutholifen.

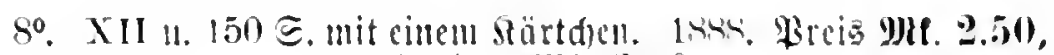
getultben 9 Mle. 3.50.

rSchon del (iegenstand an sich, den hier ein auch velteren Krelsen bereits dureh sein trettliches Etymolugisches wörerbuch ler deutschen Sprache" betanute Ge'ehrtel nicht blos mit der Zurerliissigkeit des Fachmannes, sondern auch mit den Geschich und feschmack eines gerandten Schriftstellers behundelt hat, sollte wohl darnach ungethan sein, dem Büchlein unter den nicht gelehrin Freunden der deutschen siprache lieb. haber und - Küufer zu erwerben. Denn dass die liragen, deren Beantwortung den Inhalt dieser schrift ausmacht, in den liereich des Intcresses ler höher Gelihdeten fallen, braucht lem nicht erst bewiosen $a$ werden, der weiss, wie treu gerale die fieschichto unserer sprache, mehr wohl uls irgend etwas anderes, den kampf und den Sieg unseres Volkstums widerspiegelt. . In Welebem Geiste der Verfasser seine Aufgabe erfasst lat, bezeichnet er selber, welln er in Vurworte sagt, dass auch sein [Büchlein \%engnis lavon ablegen solle, . was len Ewrwicklungagang unsrer Iation gehemmt, was ihn beschleunist und gefördert hube: es will zeigen. warum Jakob Grimm unsre Schriftsprache einon protestantischen Dialekt genamit hat, warum erot seit 1580 luthers surache eine autoritative Stellung alangen kounte, warum der liegensitz von schrifispraehen uul

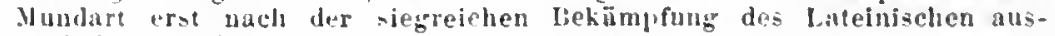
greglichen worden ist.

Nicht mit dem Anspruche, ane wollstïulize Geschichte der deutschen sprache $\%$ bieten, tritt $\mathrm{K} J$ uge aul, er will in einer keihe unverbunJener A ufsitze- nur -zusammenfassen, was Fachleute vor und sejt Jakob Arimu uber eiu paar sprachwissenschuftliche Probleme ermittelt haben". biese Aufsütze aber fiigrn sich von selber zu einem innerlich zusammenhängenden Fanzen, sodass wir hier in der 'That eine höchst aazlehende Darstellung der Lebensgeschichte unseres Veuhochdeutsch von selnen An. tängen um die Wende des füafzehuten und sechzehnten Jahrhunderís b!s zur Begründung seiner Alleinhersschaft un die Mltte des achtzehnten Jabrbunderts vor uns haben.....

(Die Grenzboten 1350. Nr. 19.) 
Soeben erschien:

\section{MINERVA}

Jahrbuch der gelehrten Welt herausgegeben von

Dr. K. KUKUf,A und K. TRÜBNER.

1V. Jahrgang $1894 / 95$.

Mit dem Bildnis Lord Kélvin's, radiert von Hubert Herkomer. $16^{\circ}$. XVI, 930 S. Preis brosch. M. 7.-, geb. M. 8.-.

Früher erschien :

Minerva, Jahrbuch der Universitäten der Welt. Herausgegeben ron Dr. R. Kukula und K. Trübner. I. Jahrgang. 1892/93. 160. VI, 359 S. 1892. In Perganent gebunden M. 4.-.

- Jahrbuch der gelehrten Welt. 11. Jahrg. 189:2/4:3. mit dem Bildnis Theodor Mommsens, radiert von W. Krauskopf. $16^{\circ}$. VI, 827 S. 1893 . In Halbpergament gebunden M. 7.-.

-- - III. Jahrgang. i893/94, mit dem Bildnis L. Pasteurs, radiert von $\mathrm{H}$. Minesse. $16^{0}$. XVI, $861 \mathrm{~S}, 1894$. In Halbpergament gebunden M. 7.- .

„Dis ausserordentlich nützliche und treftlich geleitete Unternetmen bedarf kaum einer Empfehlung mehr. In gleicher Weise, wie in vorigen Bande sind auch diesmal die Herausgeber bemüht gewesen, es auf seiner Höhe zu halten, und nicht unwesentlich zu verbessern. . . Besonders praktisch ist die im Eingange gegebene, nach Ländern geordnete "Geographische Vebersicht der behandelten gelehrten Institute", durch die man in den Stand gesetzt ist, von den in jedem einzelnen Staate vorhandenen wissenschaftlichen Anstalten mit leichter Mühe ein liild zu gewimnen, ein Vorgang, der für Schwenlie's Adressbuch der deutschen Bibliotheken sehr zul empfehlen wäre. Katum ist es nötig, auf die elegante Ausstattung in dem bekannten bequemen Formate noch besonders hinzuweisen, die die Berutzung 7.4 einem gewissen Vergnügen macht."

Literarisches Centralblatt 1894. Nr. 22.

Aus dem II. Jahrgange einzeln :

Professur Mommsen's Porträt. (Rarlierung auf grossem Papier). II. 3.-. 


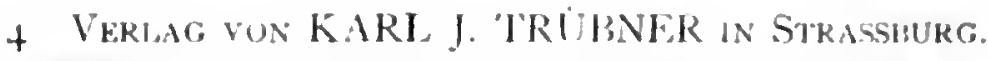

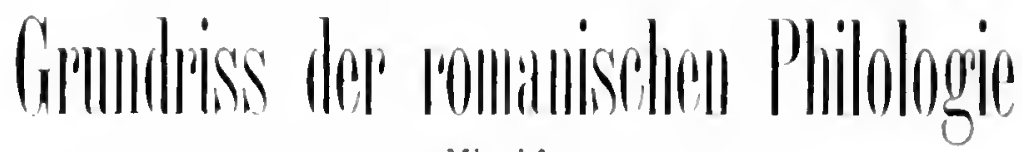

muter Mitwirkung von

G. Baist, Th. Braga, H Bresslau, T. Casini, J. Cornu, C. Decurtins, W. Deecke, Th. Gartner, M. Gaster, G. Gerland, G. Jacobsthal, F.ikluge, Gust. Meyer, W. Meyer, C. Michaẻlis de Vasconcellos, A. Morel-Fatio, Fr. d'Ovidio, M. Philippson, A. Schultz, W. Schum, Ch. Seybold. E. Stengel, A. Stimming. H. Suchier, H. Tiktin, A. Tobler, W. Windelband, E. Windisch

$$
\text { licrausgegeben von }
$$

\section{G U S T A V GRÖB E R,}

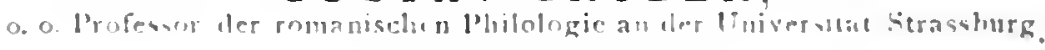

\section{Plan des Werkes:}

l'opineutischur "la il:

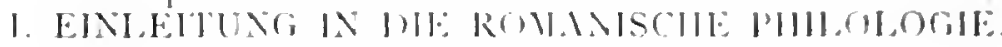

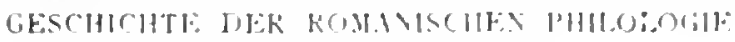

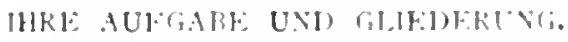

Metho discher "le th

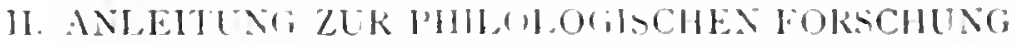

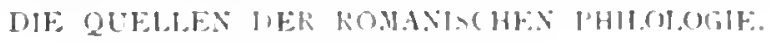

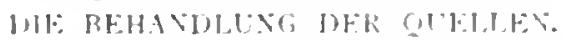

$$
\text { Realer Tril: }
$$

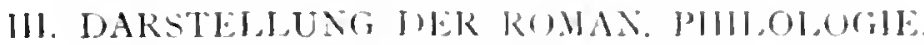
ROMANISCHE SIRACHIORSCHLNG.

a. Die vorromanischen lolkisprachen der romanischen Lauder.

- b. Die romanichen spraclien.

METRIK DER ROMANISCHES SIRACHEX.

I1. 13d, I LTTERATURGESCHCHTE DLIR ROMANISCHLN VÖLKER.

1.Abb. Die latein. Litteratur. - 1)ie französ. Licteratur.

II. Bd.

2.A ht. I

Die provençalische Litteratur, - Die catalanische Litteratur. Nie portugiesisclie litteratur - Die spanisclie Litteratur.

Die italienische Litteratur. - Nie rumänsche Litteratur. - Die rätoromanische Litteratur.

IV. GRISNZWISSENSCHAFIKN.

GESCIICHTE, DER ROMANISCHEN VÖI.KFR.

CULTLRGESCHICHTE DER ROMANISCHEN VÖLKER.

KUNSTGESCHICHTF, JIER ROMANISCHEI VÖLKER.

DHF: WISSENSCHAlTEN IN DEN RONAX. LANDERN.

Bis jetzt sind erschienen:

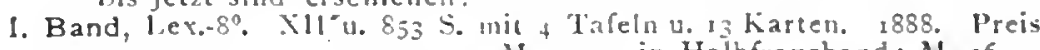

M. r..-, in Halbframzband: M. 16... zu haben.

Auch noch in einzelnen bicferungen 24 M. 4. - . M. 4. - und M. 6.-

11. Band, I. Abteilung, 1. Lieferung. 16 Bogen. 1893. II. 4.-.

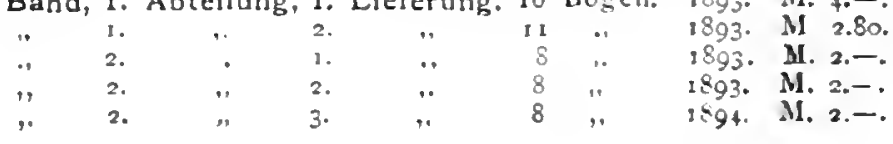


Verlag von KARL J. TRÜBNER in Strassburg. 5

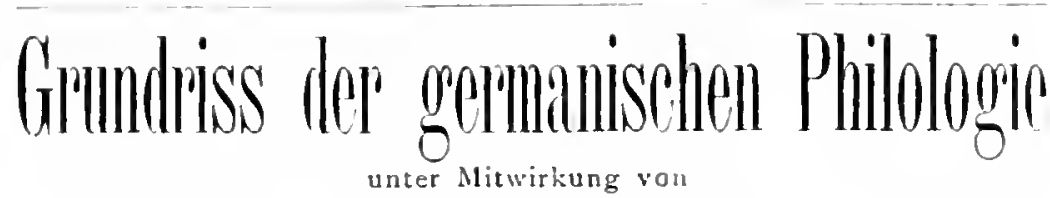

K. von Amira,jW. Arndt, O. Behaghel; A. Brandl, Bernh. ten Brink, H. Jellinghaus, K. Th. von Inama-Sternegg, Kr. Kalund, Fr. Kauffmann, F. Kluge, R. Kögel, R. v. Liliencron, K. Luick, J. A. Lundell, J. Meier, E. Mogk, A. Noreen, J. Schipper, H. Schück, A. Schultz, Th. Siebs, E. Sievers, B. Symons, F. Vogt, Ph. Wegener, J. te Winkel, J. Wright

herausgegeben

voln

HERMA N N PAU L

ord. Professor der reutschen Sprache und Literatur an der Universitit München.

I. Band. XVIII u. 1138 S. I8gr. Mit einer Runentafel und 2 Karten. Brosch. M. I8.- ; in Halbsaffian geb. M 2r.-.

I1. Band I. Abteilung. ' $X$ und 1072 S. 1893. Brosch. M. 16.- ; in Halbsaffian geb. M. 18.50 .

II. Band 2 Abteilung. VI und ${ }_{4} 84$ S. 1893 . Brosch. M. 8. - ; in Halbsaffian geb. M. 10.50 .

Die einaelnen Lieferungen acr usspriinglichen Lieferungsausgabe werden, soweit der Vorrat reicht, auch jetzt noch einzeln abgegeben.

\section{N HA L T :}

1. ABSCHN.: BEGRIEF UND ALFGABE DER GERMANISCHEN PHILOLUGIE.

11. ABSCHN. : GESCHICHTE DER GERMANISCHEN PHLOLOGIE.

1. Lid.

III \BऽCH.: IIETHODENLEIIRE.

IV. IBSCHN. : SCHKIFIKUNDE.

V. ABSCHN. SPRACHGESCHICHTE.

(Nit Anhang: Die Behandlung der lebemlen Mundarten.)

11. ABsCHN : IIYTHOLOGIE.

VII ABSCHN: HELDENSAGL.

1III. ABSCHN: LITERATURGESCIICHTL.

11. Bd. (Nit Anhang: Übersicht über die aus mündlicher Über I. A bt. lieferung geschöpften Sammlungen de! Volkspoesie.)

1X. ABSCHN. MEIRIK.

X. ABSCHN.: WIRTSCHAFT.

X1. ABSCHN: : RECHT.

XII. ABSCHN: KRIEGSWESIX

11. Bd. XII. ABSCHA.; SITTE.

2.Abt. (Mit Anhang: Die Behandhug der volkstünlichen

XIV. IBSCHN: KUNST. Sitte der Gegenwart.

(XNMEX. SACH- UND WORTVERZEICHNIS. 


\section{Deutsche Grammatik}

\section{(io.isch. Alt-, Mittel- und Neuhochdeutseh von}

\section{W. Wilmanns}

- Professor der destschen Sprache u. Litteratur a. A. Universitat Bonn.

Erste Abteilung: Lautlehre. gr. $8^{\prime \prime} . \mathrm{XIX}, 332 \mathrm{~S}$. I 893 . M. 6.50. In Halbfran\% gebunden M. 8.50.

Das Werk wirl in vier Abteilungen enscheincn, deren jede furchschinittich 20-25 Druckhogen umfassen wird: Latuthehre. Worthildung, Filexion, Syntax. Eine fünfte, die Geschichte der deutschen spuache. wird sich vielleicht anschliesser.

\section{Die zweite Abteilung (Wortbildung) ist in Vorbereitung.}

.. Es ist selur ertreulich, dass wir mun ein Buch haben werken. welches wir mit guten Gewissen demjenigen empfelslen hömnen. der sich in das Studium der deutschen Fiprachgeschichte ainatrheiten will, ohne die llöglichkeit zu hahen, pine gute Vorlesung äber deutsche (irammatik zu hören: an Wilmanns wirl "r hierm einen zuverlässgen. auf der Höhe der jetrigen Forschung stehenden liührer finden. Aher anch dem Studierenden. der schon deutsche Grammatili gehört hat, wird das Buch gute Dienste leisten zur Wielerholung und zur Frgänzung der etwa in der Vorlesung an kurz gekommenen Partien. Jedoch anch

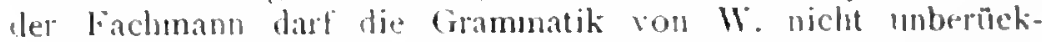
sichtigt bassen. Denn alle in Betracht kommenden Fragen simb lier mit selbständigem Urteil und unte' roller Beherrschung der

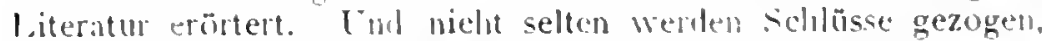
die von der gewoihulichen lutiassung abweichen und \%um Mindesten zur eingehenden liangang anfiordern. so dass niemand ohne vielfache Imegung diese Iantlehe ans der Hand legen wird Besonders redeh an neuen Dullassungen jst uns die Jehre ron der honsonanten arschienen. Sher ancls dio übrigen leile, unter denen die bisher weniger oft in (irammatilien dargestellte behre vom Wortaccent hervorzheben wire, verdienen Beichtung...."

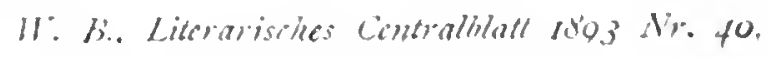




\section{Geschichte}

\section{Deutschen Litteratur}

\section{bis zum Ausgange des Mittelalters \\ von}

\section{Rudolf Koegel,}

ord. Prof. für deutsche Sprache und litteratur a. d. Universität Hasel.

Erstel Band: Bis zur Mitte des elften Jahrhunderts. lister lej l: Die stabreimende Dichtung und die gotische Prosa. so. XXIII u. 343 S. 1804. Preis MI. 10...

"... Kögel hat eine Arbeit unternommen, die sehon wegen ihres grossen Zieles danklyar begrüsst werden muss. Denn es kann die Forschung auf dem Gebicte der alt dentsrhen Litteraturgeschichte nur wirksamst unterstützen, weum jemand den gauen vorhandenen l'estand von Thatsachen und Ansichten genau durchprüt und verzeichner, dann aber auch an allen sehwierigen Punkten mit eigener Untersuchung einsetzt. Beides

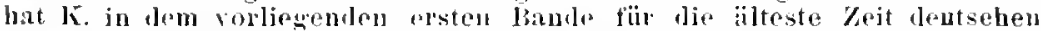
Geisteslebons gethan. El beherrscht dats bekitunte Material vollständig. er hat niehts aulgonommen oder lortgelascen, ohme siel dariber sorgftiltig Rechemschaft zu geben. Jiein titein auf dou Wege ist von ihm unumgewender verblieber. K. hat aher aurh den Stoll relmeht, einmal indem er selbständig alle Ililfsguellen $(\%$. B. die Simmlungen der Capitularien, Concilbeschlïsse: u. s. w.) durchgearleptet, nene Zaugnisse den alten keigefïgt, dis alten beriohtigt h..t, Terner dadurch, dass el aus dem Bereieho ler iilurigen germanishen litteraturen herangezogen hat, was irgend $\lambda$ us-

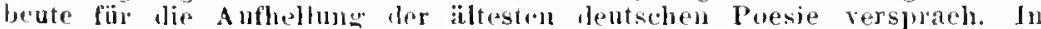
allen diesen Dingen solnoitrot or aul don platen Karl Mïlenholl's, dessen lirösse kein anderes buch als elued las seine bessey würligen bejut...."

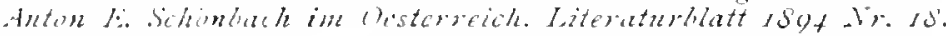

"Koegel bitut Mejsteru wie Jüngern ler Gormanistik eine reiche, willkommene Gahr mit seinen Werke: vor allem abel sci es lel Aufmerksamkeit del Lehrer des leutschen an höheren siohulen enpfohlen. fiil die es ein unenthohrliches lliilfsmittel werden wird durch seinen eigenen Inhalt, lurch die wohlausgewäblten hibliowraphisehen Fingerzeige und

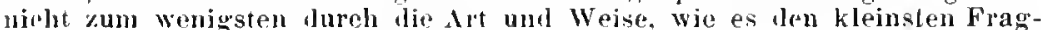
menten ein vielseitiges ntersse abzumwimnen und sie in grossen greschiehtlichel Yusammenling \%u stollen vorstelt Wie es mit warmer Theilnahme für len riegenstand enopheitot int, wirl us gewiss auch, wic der Verlasser wünseht, Frende an dir untionalen Wissensehatit werken und mittelbau auch zur Belebung des dentsolnen litoraturuntorichts in wisvenschaftlichnationalem sinne beitrawan,"

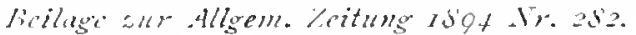

Das Werk wird aus 2 Bänden bestehen, die in je 2 Teilen ausgegeben werden.

Soeken crochien: Ergänzungsheft zu Band I

\section{Die altsächsische Genesis.}

lin lieilna zur liusehichte der althentechen Inichtung u. Verstumst von

Rudolf Koegel

"rrl. Profensor an ler Umuersität bissel.

80. X. 71 S. 1805. M. 1.81. 


\section{$(\mathfrak{b e}$ ddjidjte}

\section{ber \\ (5)aglifidell Ritteratur ten}

Germljarit ten Grink.

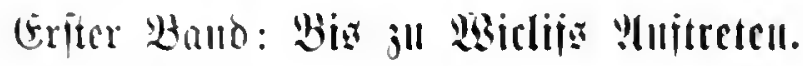

8. VIII, 470) 5. 1877. M2. s.-, geb. Ml. 10.-.

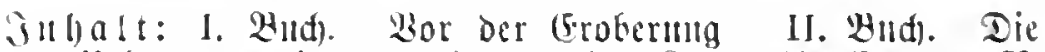

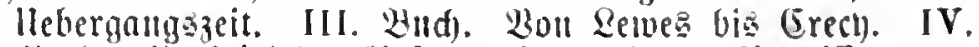
Hud). Loripiel ber Mejormation und ber Menaillance.

\section{Bmeiter Bano: Bis jur Jicjormatiou.}

berangegeben volt Mlois girnilol.

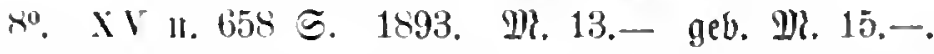

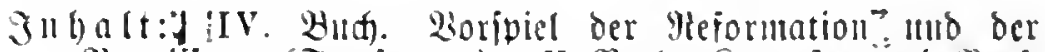

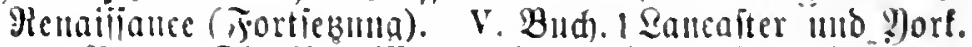

Il Bud). Dic Menailiance bis ă Surren's Tod."

Daran cinzeln: Die in Februar 1 s93 cridtientene 2. Şälfte.

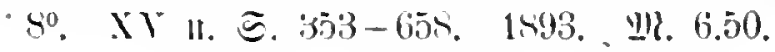

"Wie Fortsetzung zeigt alle die glänzenden kigenseluaften des ersten Bandes nach meiner Ansieht noeh in erlühten Masse; grïndlieho Gelehrsamkeit, weiten Jlick, eindringenden Seharfsinn, feines ästhetisches (iefühl und gesehmackvolle Darstellung."

Berlin. Fulius Zugitza, Densehe Litteraturzeitung 1889. Mr. 19.

Bernhard te lt I riuk's Litteraturyeschichte ist ohue Zweifel das grussartigste Wurk, das je einem englischen l'hilologen gelungen ist. Mehr noeh: es ist eine su meisterhafte l,eistung. dass es jedem Litteratur. listoriker zum Iuster dienen kann Und dies Urtheil hat seine volle Kraft trotz der unrollendeten tiestalt des Werkes. Wäre es dem Verfasser vergünnt gewesen, es in derselben Weise zu Ende zu bringen. 80 würde es leicht die hervorragendste unter allen Gesammllitteraturgeschichten geworden sein.... Museum, I893, Nr. 7 .

Die Bearbeitung der zwei weiteren Bände hat Herr Professor Dr. Alois Brandl übernommen. 


\section{(5efdjidjte}

\section{rer \\ Stalienifiden Riteratur \\ voll \\ Toulf Gnspary.}

(5riter Bano: Die italienifac Qiteratur im Mittelalter.

so. 550 ธ. 1885. M2. 9.-, geb. M2. 11.-.

Sulfalt: (Finleitmug. - Die Sicilianiiche Dichteridnulc. Frortiebung Der Inrijaen Diattung in Mittelitalient. - BMiov Sninicelli von Bologna. - Die franzöf. Bitterbithtung in Dberitelient. - Meligiöie unb moralifae Poefic in Dberitalien. - Die religiöic snrif in llmbrien. - Die Broja im 13. Jahrh. - Tie allegorifd)=bibattifite Diatung mo bic philojowh. Surif der nenen florentinifinen Sdule. - Tante. - Die Comöbie. - Iag 14. Jahrhubert. - Betrarca. - Betrarca's (5amzoniere. - VInhang bisliographifither mo fritifder Bemerfungen. - Hegi iter.

Bweiter Band: Dic italienijase Eiteratur der Menaifinancezeit.

80. 704 ङ. 1888, Mr. 12,-, get. Mi. 14.- .

Jul alt: Boccaceio. - Die Epigonen ber groben Florentimes.

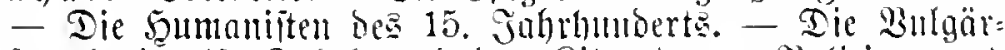
ipradje im 15. Jahrir. uns ilure Riteratur. - Poliziano mo Borenzo dé Miedici. - Tie Mitterbichtma. - Putci umb Ro= jardo. - Rcapel. - Pontano und Eamnazaro. - Miaction welli unb Giniccialdini. - Bembo. - 2trioïto. - Eajtiglione.

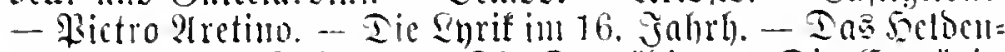
geoidht im 16. Jahrh. - Die Iragöbic. - Tic (50möbic.

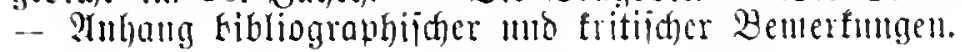

"Jeder der sich forcan mit der hier behandelten Periode der italienischen Litteratur beschäftigen will, wird Gaspary's Arbeit zu seinen Ausgangspunkte zu machen haben. Das Weik ist abel nicht nur ein streng wissenschaftliches für Fachleute bestimntes, sondern yewährt nebenbei durch seine anziehende Darstellungsweise aueh einen ästhetisehen Genuss: es wird daher auch in weiteren lireisen Verbreitung finden."

Deutsche Literaturatitung.

Die Fortsetzung dieses Werkes hat Dr. Wendriner (Breslau) übernommen; ihm sind Gattin des verstorbenen Verfassers die Vorarbeite sich solche im Nachlasse vorfanden, ausgehändig 


\title{
Neuhochdeutsche Metrik.
}

\author{
Lin Handbuch
}

vin

\author{
Dr. J. Minor, \\ 11. is. l'rofessor an Alor Universität Wies.
}

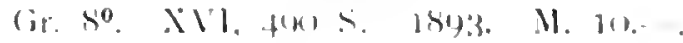

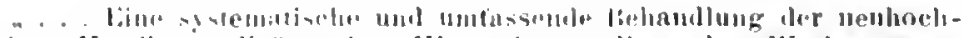
leutschon Metrik iu lieleru hat Mims in volliegenden Werke unternomm

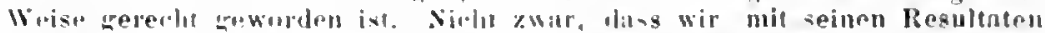

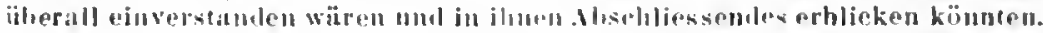

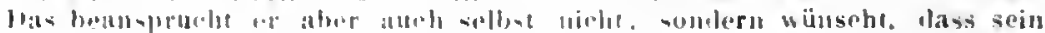

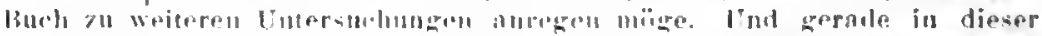

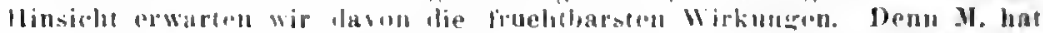

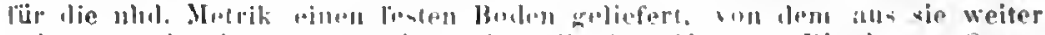

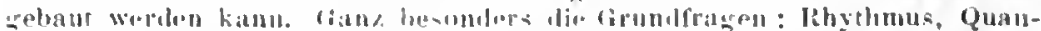

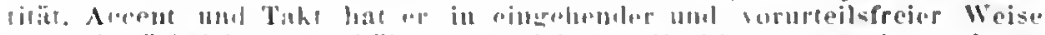

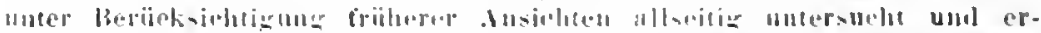

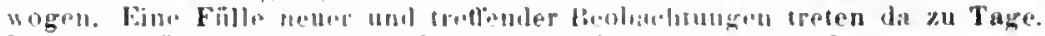

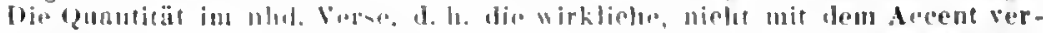

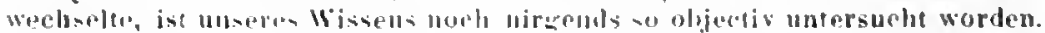

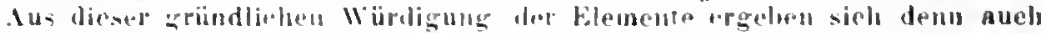

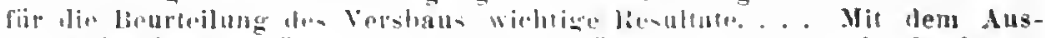

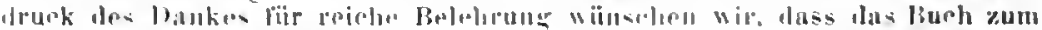

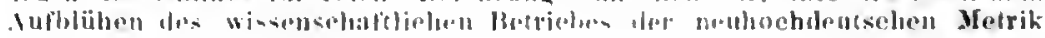
Verilniasollte grabell mïgre."

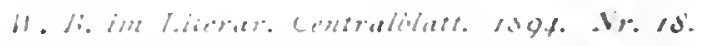

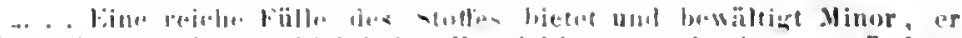

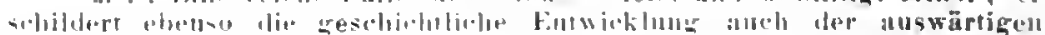

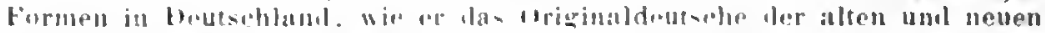

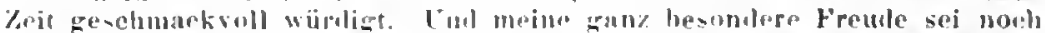

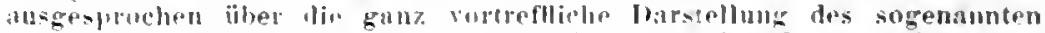

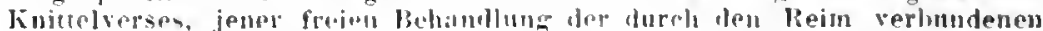

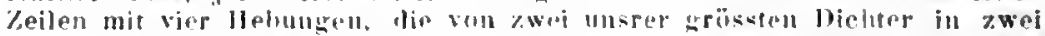

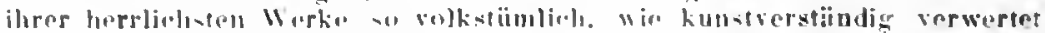

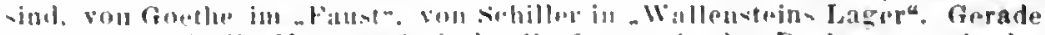

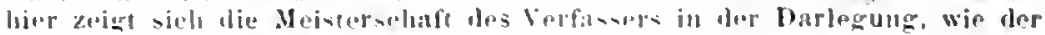

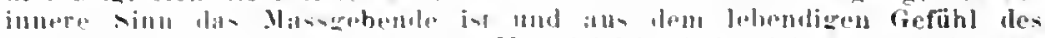

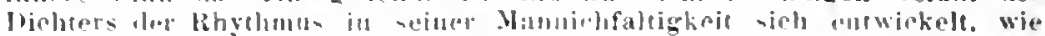

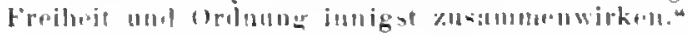

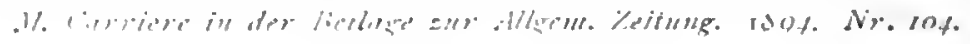




\section{A b ris s \\ urgermanischen Lautlehre}

mit besonderer Rücksicht auf die

nordischen Sprachen

zum

Gebrauch bei akademischen Vorlesungen

von

Adolf Noreen.

Vom Verfasser sellist besorgte Bearbeitung nach lem schwedischen Original.

s. XI1, 2-8 $\therefore$. 184t. 11. …

"Schon die schwedische Ausgahe, die ror medreten Jahren elschienen ist, hat in diesem Blatte warme Anerkenmmg gefunden. In noch höherem Masse verdient die dentsche Bearbeitung das jener gespendete Lob. Sie ist eine überrasclend reichlaltige, übersichtlich angeordnete und fast durchwer zuverłässige Darstellung eines der wichtirsten Kapitel der germanischen Grammatik. Die umlangreichen und sorgfäligen Literaturangaben sind besonders dankenswest; man wird kamm eine Stelle von einigrer Bodentung vermissen. Ausfühliche Wortregister erhöhen die Brauchbatieit. Schon die altislindische Grammatik in Bratlie's Sammlung und die Geschichte der altnordischen sprache in l'aul's Grmndriss, heides Musterleistungen, haben das grosse Tilent Noreen's lür dir Pewältigung sprörler Stoffmassen gereigt. Dieselhe Begabung bewährt sich anch in dem neuen Werke Ls zerfillt in zwei urosse Abschnitte, dic conanten und Konsonanten überschrichen simb Jeden diesen Teile geht ein kurzed Überblick ïher den idg. Jautstand roraus, der mit Hülfe des Indischen, des Griechischen und des Janteinischen erschlossen wird. ham folgen die urgermanischen Latutgeset\%. Den beschluss micht jedesmoll ein umfingliden

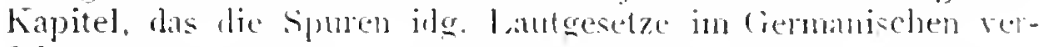
folgt.

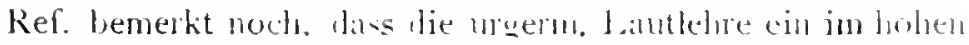
Grade empfehlonswertes Buch ist. dem ein roller Erfolg im Interesse der germanischen Grammatik lebhaft gewönscht werden muss. - - - - - - " 


\title{
Dic \\ deutschen Runendenkmäler
}

\author{
heratusgegeberl von \\ Rudolf Henning. \\ Mil, lafeln und an lloleschuilles.
}

Mit Unterstüzung der kgl. preuss Akademie der Wissenschaften.

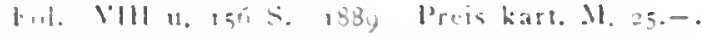

111.111: I. Dic Speerspitre rom liuwel. - Il. Die Speerspilse vin Mäncleberg. - II a. Die Spererspitze von "lorcello. - 111. Der Goldring von P'ietruassa. -- IV. Die Sponge von chamay. - 1 . Wie spange von Osthofen.

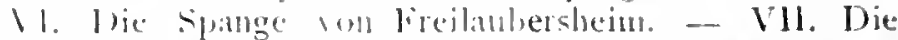

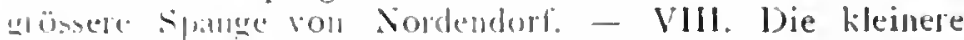
Spange un Nondendont. - 1X. Wie Emser Spange. -

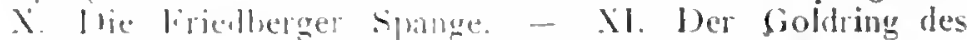
lerline Musenus. - Xll. lled Bracteat von Wapno. -

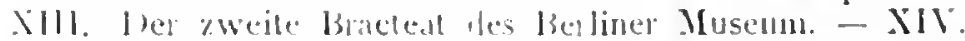
Hie D.mmenberger Bracteaten. - XV. Der Bracteat ans lleile. - XVI. Das Thonköpfelien des Berliner Museums. - Jigebrisse. - Mnhang und Register.

-... Der Verfusser, der in den Fragen prälisloriseher Kultur, zuglopli aber auch in ter Jeutschen Sprachgeschichle wohl zu Hause ist, liring ron jedem Denkmal einen genauen Fundbericlı und giebt eine Lnatu Besuhreibung, an die sich lann spine beutungstersuche anschliessen. Jan muss die sorgfalt rühnen, nil ler alles in Jetrach Kommende erwogen ist, und in einer lieihe von füllen ist ihm auch gewiss geglüekl, das Richlige zu lindeu... Es muss uns hier genügen, unsere Genuglluung übor das kirscheinen les Buches und das Resultat unserer Nachjrüfun dahin aususjrechell, dass dor Verf. gewiss len auf ihn gesetzten Frwartungen in Wesentlichen eutsprochen hat. IVir weltere Forschung iber unsere deusschen Runen wirl auf der Grundiage dieses Werkes zu arbeiten haben. Wenn wir sehen, in wie rerbältnismäsigkurzer Zeit sieh das Material zusammengefunden hat, das uns hier vorgelegt wird, so ist liv Hoffun gewiss bereehtigh, dass die Funde sich nuch ferner vermebren und diss dann auch nene Momente für die Eirklärung sich ergeben werden. 1 Literar. Cemtrablatt sqo. Nr, 20.)

Vun demselhen Verfasser erschienen frïlier:

Henning. Rud. (P'rof. an der Universitit Strasslurg). D a s leatsche Ilat in seincl historischen Entwicklung. Mit 64 Holzschnitlen. so $^{0}$ 183 S. 1882.

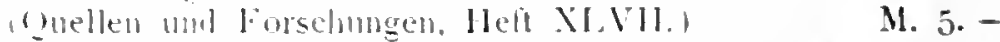

- - Jic Ieutschen II a ustyen. Nachtrigliche Be-

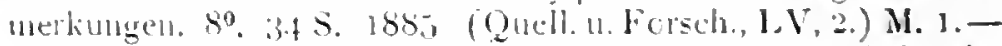
- Ueber lie Sanct-irallischen sprachdentmäler his zum Jolle Karls des Grossen. $8^{0}$. Xlll u. 159.S. 1875. QQuellen und Forschungen, H1.) M. 4.-. Nibelungensiudien. "so. Xill und 329 S. 1883. (quellen uml fiorsclungen, XXXI.) M. 6.- 


\title{
Gxitroliagent \\ neuhucholentlehen dinutlyltens.
}

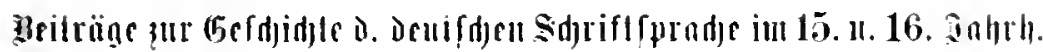

voll

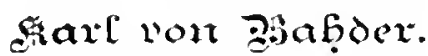 \\ 80. VII, 28t 巨. 1890. ㅍ․ 6.-.
}

Juhalt: Finleitung: Iis Sanjleipmaden. - Tie taijer= lidge Panjleipradje. - Das gemeine Deutjh. - Dic Druct= ipradelt. - Die bairifatu Drufe. - Die Plugghurger Drudf=

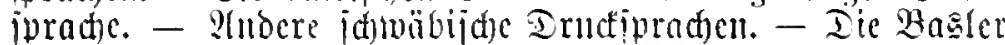

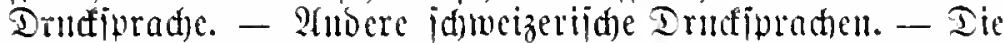
Strabburger Drmfiprade. - Jijhart. - Die Bïrnberger Druffiprad)e. - 5ante Sadj. - Dic Mailzer Druđjprache. - Dic Meichabidjede. - Vlmore mittelrheinithe Irtat=

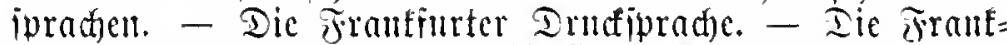

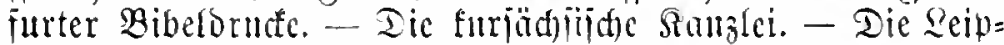
ziger Druafipraduc. - Die (Entwifelung von Dnthers Eviadhe. - Dic @pradje Der Bibel von 1545. - Dic von Suther abbängige mitteldentidhe Riteratur. - 2 hweid)ungen Der mo. Bitteraturipradie vou Euthers Epradie. - Silteite Stho=

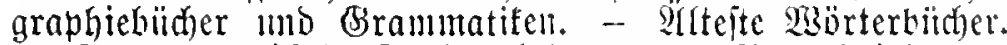

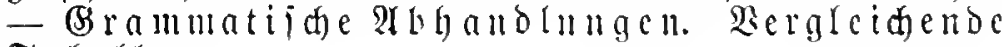
$\mathfrak{I} \mathfrak{a b} \mathfrak{e l l} \mathfrak{e}$.

\section{Geschichte der \\ Schwäbischen Mundart im Mittelalter und in der Neuzeit.}

Mit Textproben und einer Geschichte der Schriftsprache in Schwaben dargestellt von

\author{
Dr. Friedrich Kauffmann \\ Professor an der Universität Halle a. S.
}

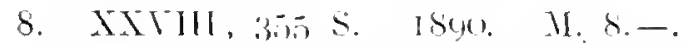

"Auf dem Gebiete rer deutschen Dialectforschung dürfe seit lang"ep beit kejn Werk von ämulioher Iodeursankeit "rschienen sein, wie das gegenwärtige. Wenn mau weisn. Was es heisst, die Geschichte einer hestimmteu Mundart zu selneiluen, wic vielerlei da \%u beobachten ist. wio. heikel die Benutzung älterer spratehquellen ist, so wiml man von rornherein keine geringe Forstellung von der Arleitsleistung eines Werkehaben, das sich einen solehen Titel gibt. Diese Erwartung wird abel nicht getäuscht; es ist hier eine grosse Arbeit gethan worden, wic sie in dieser Art noeh für keine deutsche Mundart hesteht, und sie ist mit Fleiss, Lmsicht und Sachkenntniss gethan worden." 


\title{
Geschichte
}

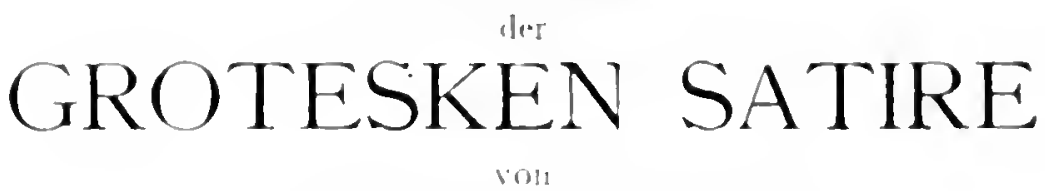

\section{Dr. Heinrich Schneegans,}

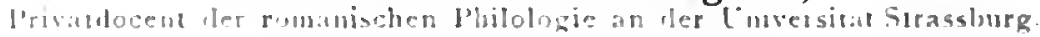
llit 24 11, lillungen.

$$
\text { ar. so. X1. }
$$

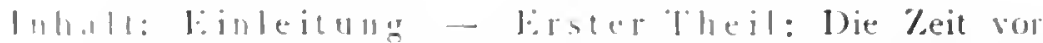
katelai, Kin. I: lie lieine les grotesten Satire in Minclulter. Kipr. 11: l)ie italirnisclue Ritterdichtung. Kap.

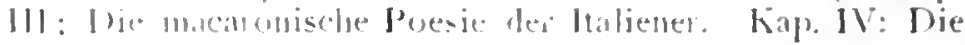

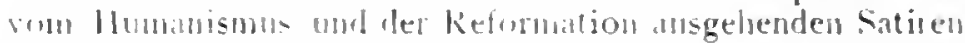
leculachlumls. - \% weiled "lheil: Rathelais. hap.J: Die

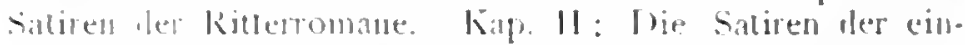

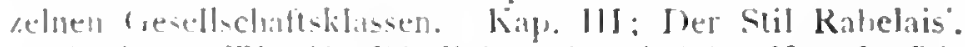

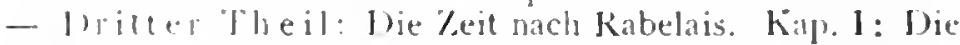
iiuseren Sachahne! Kabelais' und die von ihon heeinllusste Kunsl. Kill. 11: Die französische Satire in Geiste Rabelais:. Kill. III: Das Groteslie bei Fisclart. Kap. IV: Die Ausläuft der groteshen Salire und les grotesken Stils. - Schluss.

$$
\text { Die Bísa-Saga }
$$

\section{nebst Proben âus den Bósa-Rímur}

\author{
heratrogereben
}

\author{
$\because n n$
}

\section{Otto Luitpold Jiriczek.}

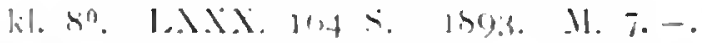

Die lbeina-suga ist seit den Jaltre 1530. Wo sie mit willkürlivben A uslansuggen in len Furnaldarsügur ersehien. nieht mehr gedruekt worden: - ic crshein lier in vollständiger kritiseher Ausgabe, zusamnen uit einer bi-ber gathe unbekannten jüngeren Fassung aus dem 1\%. Jabrh.; neu sind r.benfalls die im Aulange nitgeteille apokryphe Huslubien und proben der lissarinur. Dite usführliehte Fiuleitung behudelt nebst ler Texikritik lice liebieliungen beiter Fassungen unl der Pinur untereinander und zu anderen lyginügur und biete in ilnell Untersuchumgen über die innere

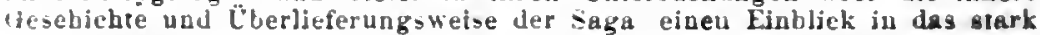
vernachlüsslgte Geblet der älteren islündirehen märchenhaften Isglsaga. 


\section{(5ijal) ulld Studien}

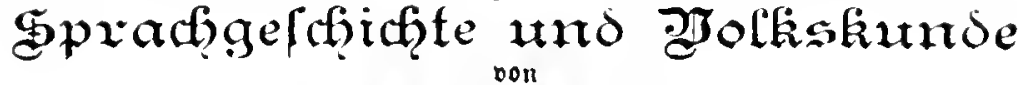

Gufftu Me Mer,

Brofeffor an bet univerfität braz.

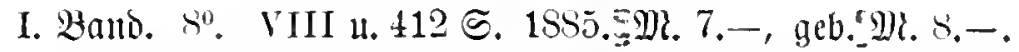

Sulart: Bur Eprangeiduidte. I. Das indogermaniide Itruolf. II. Die etrugtithe Evradfrage. III. lleber Sprade

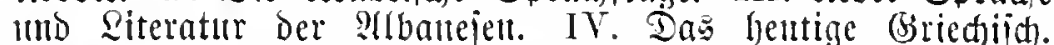
V. (5omitantin Sathas nnd die Slavenfrage in (5iried)enlant.

Bur vergleidgenden Miärdjenfunde. I. Jolttore. II. Mlärdben=

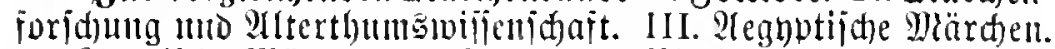

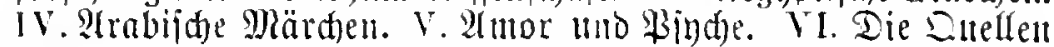
des Decamerone. VII. Siidilavija) Dlärdhen. VIIt. Ter Hatten= fänger von Syameln. IX. Ter Bathe des Todes. X. Mip van Wintle.

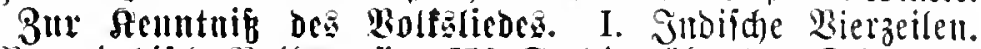

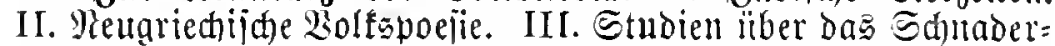
hïpfel. 1. Bur Siteratur Der Ed)naderhïpfel. 2. Bierzeile uno mehritrophif hes sico. 3. lleber den Matureingang bes Ed)nader=

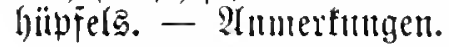

II. Band. So. VI แ. 380 ङ. 1S93. Ml. 6.-, geb. M7. 7.-.

Snhalt: 1. Franz Bopp. - II. Beorg Eurting. III. Beltipradhe und Beltipradyen. - IV. (strustijues ans

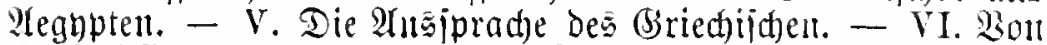
Der id)leiijan Mrundart. - VII. Bur (Sharafteriftit Der indijath Biteratur. 1. 2Ullgemeine (5rrumblagen. 2. Der Weda. 3. Sâtli= Dâia. - VIII. Bigeunerphilologie. - IX. Soltslieder alts

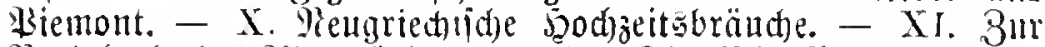

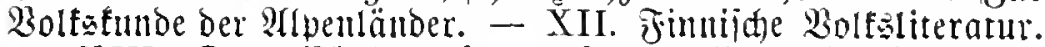
- XIII. Das Mänbermejen ami der Balfanbalbimiel. -

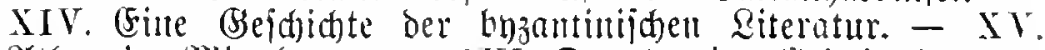
Ithen in Mittelalter. - XVI. Das hentige (Briedhenland. XVII. (Briediid)e Meijemomente. 1. Sont Seriu nad) Mthen. 2. Ithen. 3. Sul Rattoe der ßelopiden. - XVIII. Zante. XIX. 21pulijhe Meijetage. 1. 2out Brindiji nad) Recce. 2. Recee. 3. Sialimera. 4. Tarent. - XX. Bei Dent :Hbanejen Staliens. - XXI. Das Jubiläum der Uniberijtät in bologna. - Mlı= merfungett.

Der wissenschaftliche Werth eines Werkes von Gustav Meyer ist stets über allem $Z$ weifel erhaben; das vorliegende ist aber vermöge seiner glänzenden Darstellung von A n ton Schönbach für würdig befunden worden, in seinem Buche, Über Lesen und Bildung, 4. Auflage" unter den Werken aufgeführt $z$ u werden, die einen Ehren. platz in dem geistigen Haushalt jedes Gebildeten verdienen. 


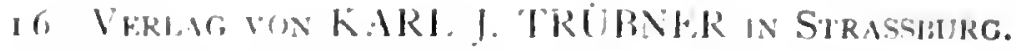

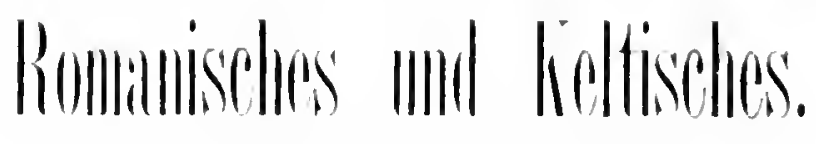

\title{
Gesammelte Aufsätze
}

\author{
Hugo Schuchardt, \\ lerofewour an der Universitut Gran.
}

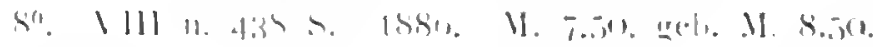

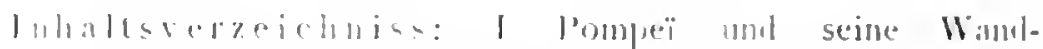

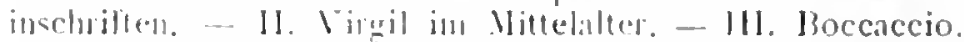

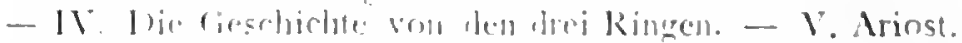

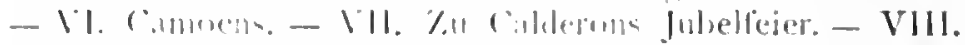

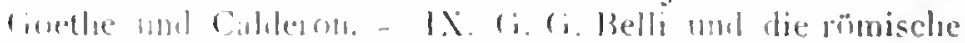

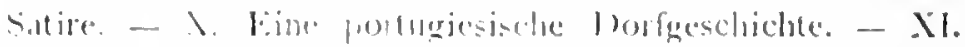

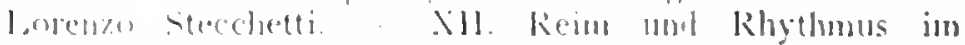
Dentschen und kimanisolen. - XIII. Lielesmetaphern.

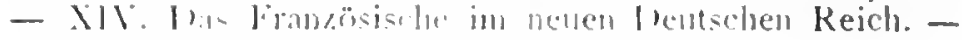

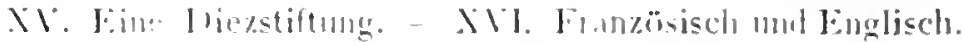

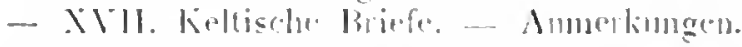

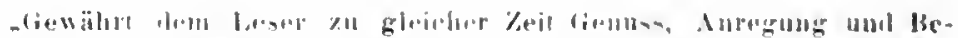

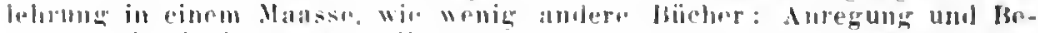

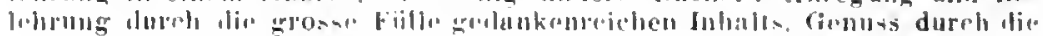

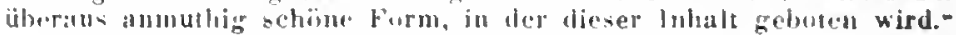

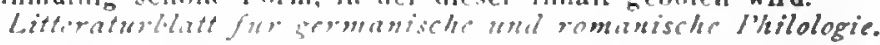

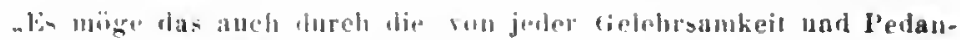

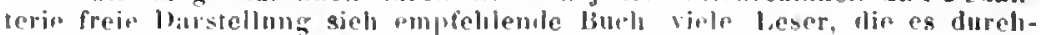

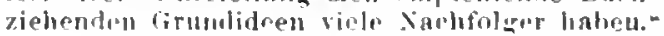

Deutsche filteraturzeitung.

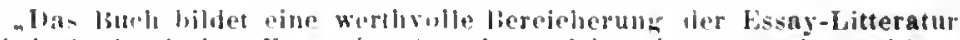
in wahrhafi elassischel Form der sprathe und luereuter, sprachgewaltiger litriollung Hochenscherft fir klassische Philologic.

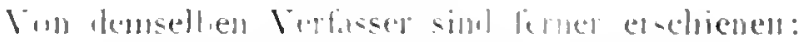

\section{Auf Anlass des Volapüks.}

so. Is 2 . 1888 . 11. 1. .

\section{„Weltsprache u. Weltsprachen".}

\section{An Gustav Meyer.}

\author{
$8^{0}$. 5.t 5 1894. M. 1.40 .
}




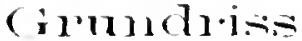

der

\section{vergleichenclen Grammatik \\ d $1+1$}

\section{indogermanischen Sprachen.}

Kurmefasste loustellung der Geschichte iles Altiodischen. Altiranischen (Avestischen und Altpersischen), Altarmenischen, Altgriechischen, Liteinischen, Tmbrisch-Samnitischen, Altirischen. Gotichen. Althochientschen, I.itanischen u. Althirchenslarischen

(*)

Karl Brugmant1

or. lyofesol. der undugerm

Sprachwiscuschaft in leeiprig

\section{Berthold Delbrück}

uru. Pofessor des Sanskrit unul (l. r verel. Sprachtiund. in Jena.

1. BH. EINLEITUNG TND L \U'T,EHKE von KarI Brugmann. gr. $\delta^{0}$. XVlll u, 568 s. Ik86. (Vergriffen.)

11. Mil.: WOXTBILDUNGSLlEHRli (Stammbilungs- und Flexionslehre) ron Karl Brugmann. 1. Hälfte. Lorbemerkungen. Nominalcompositar Redulicierte Nominalbildungen. Nomina mit stambildenden Suflixen. Wurzelnomina. gr. so. XIV und $462 \mathrm{~s}$. I888. M. 12.-.

- - 2. llafte, 1. I.ief: Zahl wortbillung, Casushildung der Nomiua (Nominalleklination), Pronumilla. gr. $8^{0}$. $38+\mathrm{S} .1891$. M. 10.-

- 2. Hialfe ischluss-jLiel. gr. 8. XIl und 502 S. 1892.

INHCJS (Wort, Sach- und Autorenindex) von Karl Brugmann. gr. 8. 1. 236 S. 1893.

M. $6-$.

111 lil: SYNTAX von B. Delbrück. 1. Teil. gr. 8". VIIl, $77.1 \leqslant 1893$.

II. 20.-.

Ein vierter Band, len 2. (Schluss-) 'leil ron Dellrücks vergl. Synt a enthaltend, wird das Werk abschliessen.

„.. . Brugunam's Werk gehör fortan zu dem unentbehrlichsten Rïstzoug eines jeden ludogermaniston; möge der zweite Band nicht allzu lange

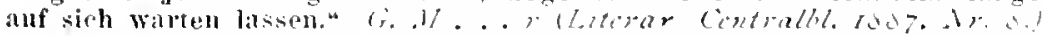

„.. . Nach meinem lirachten zenügt es, die Leser dieser Zeitschritt anf clio Bedrutung dow vorliegenden Werkes animerkan gemacht zu haben. und dass diese eine ausserordentliche ist, muss jeder unparteiseh uni billig Denkende mit lebhatcer freude ringestchen. Dass nuch gar manche Partie der Aufhellung bolarf, weiss obnehin jeder Einsichtige; aber wal mah fom gegenwïrtigen stanlpunkte des Wissens geboten werlen kann, bietet tas brugmannsehe Bueh in voll n Masse. Darum bedeutet es aunh einen Markstein in der Geschichte der indogermanisehen sprachnssonseluaft.

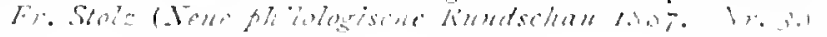




\title{
Griechische Geschichte
}

\author{
Julius Beloch.
}

Eriter Band: Bis auf die sophistische Bewegung und den peloponnesischen Krieg.

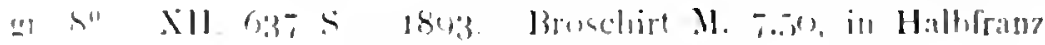 \\ enchuruen 11 10.-
}

1). LWeite biull: Vom peloponnesischen $K$ : jeg bis auf Aristoreles und die Eroberung Asjens int in Vorbereitming.

.. Das Ganze ist tliessent geschrieben, von durchsich. tiger Klarheit, gleich al,gerundet in Form und lassung. So tritt las Buch mit dem Anspruch auf, lem deutschen Publikum zu bieten. was es his jetzt noch nicht lesitzt: eine son wirklich historischem Geist getragene und \%ugleich leshare Geschichte Griechenlands. Kef. steht nicht an $\mathrm{zu}$ erliären, dass es diesen Anspruch in weitem l'mfang erfüllt. Durch einen freien und weiten Blick, durch umfassende historische Kenntnisse, durch gründliche Durcharbeitung des Materials war ler Verf. für seine Aufgabe vorlereitet. Von der Selhstindigkeit und der vor keiner Consequenz zuriickichreckenten Energie seines historischen $\mathrm{Cr}$ teils hat er schon früher vielfach Proben abgelegt....

(Edward Weyer im Litcrarischen Centrallatt rsat, $\mathrm{Nr}$.

Der eigentliche Vorzug des Werkes liegt a uf a e m Gebiete ler barstellunir ler wiltschaftlichen und socialen Giundlagen des lebens. in denen B. dic materiellen Cimmlagen erkennt. auf denen sich die grossirtigen Cmwälangen. auch der geistigen und politischen Entwickelung vollyogen. Da $B$. gerade in diesel Beriehung las Material beherrscht. Wie nicht leicht ein anderer Forscher. so durfte man hierin ron seiner Darstellung Ausführliches und Vorzügliches erwarten... Glanzpunkte ind der VIl. Abschnitt: Die Umwälsung im II irtschaftsleben (rom 7. sum 6. Jahh.) und der Xll.: Der wirtschaftliche Aufschwung mach den Perserkricgen .... [eher di* Berölkerungsverhältnisse. üher die Getreirlecinfubr, üher das Aufhören der Nitural- und den Beginn der Gelwirtschaft, die Futrignisse ler Industrie und des Han lels,

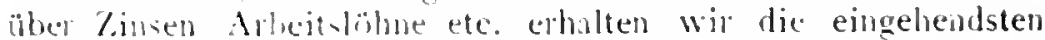
Aufschlüser unl wmitem uns. wie diese wichtigen Dinge bei ler loarstellung der griechischen Geschichte bisher unherüelisichtigt heibell lionntell.

1)ie Form der Darstellung ist eine ausserordentlich gewandte und fliessende.

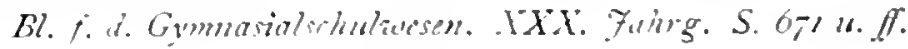




\title{
Geschichte der Griechischen Plastik
}

\author{
ron
}

Maxime Collignon

professor an der Faculte des Lettres in Paris.

Ins Inutsche ïbertrigen und init Anmerkungen hegleitet

Eduard Thraemer

Privalocent an der Universitat Strassburg

Erstes Band. Mit I2 Tafeln in Chromolithographie oder Heliogravure und 278 Abbildungen im Text.

Liste Licferung. Lexikon-Octav. 120 S. 1804. Preis Mark 4.-.

Der erste Band wird in 5 Lieferungen zu je M. 4.- in möglichst kurzen Zwischenräumen erscheinen.

"Collignou's IIistoire de la sculyture grectue, dereu erster uud bisher cinzigel Band 1892 erschien. hat mit Recht iiberall eine sph günstige Autnahme gefunden. Der Verf steht von vorn herein auf dem Boden, der turch die umwälzenden Entdeckmnsen der letzten Jabrzebnte geschaffen ist, nnd betrichtet von diesem ueu mewonnenen titandpun te aus aueh dic äleren Thatsachen und Forschungsergebnisse. Er beherrseht die einsehlägrige literatur, in der die deutsehe Forselıung eineu bedeutenden Platz cinuimint, und weiss die Streitfragen oder die Thatsuchen in geschmackvoller Form und olne ermüdende Breite darzustellen. Eine grosse Anzahl zut ausgefïhrter Texti]lustrationen, nach zum grössten Teil nè angefertigten Zeichuno en, dient dem Texte zu anschaulicher Belebung und hieter eine vornehme Zierde des Buches, sehr verschieden von jenen oft nichtssagenden Unrissen, welchen wir in ähnlichen Büchern so oft beyegneu. Su war es ein gliicklicber Gedanke, Collignon's Werk dem leutschen l'ublikum, nicht blos dem gelehrten, dureh cine deutsehe Ueber'setsung niher zu bringen. Von dieser liegt die erste Lieferung ror ; vier weitere von ungefähl gleichen Umfange sollen den ersten Band zu Endo bringen. Der Uebersetzer. Dr. Ed. Thracmer, hat seine nioht ganz einfacho Autgabe voltrefflieh gelöst: die Darstellung liest sich sehr gut und man wird nicht leicht larin erinnert, dass man eine Uebersctzung ror sieh liat. Hier und da ist ein leichtes thatsächliehes Versehen stillschweigend berichtigt, andel'swo dureh einen (als solcher bezeichneten) Zusatz ein Hinweis anf antgegenstehenle Auffassungen, auf neuerdings bekannt gewordene Thatsachen, af neu erschienene Literatur gegeben; besonders zallieich treteu solehe Besnerkungen be: der Kypseloslade auf (S. $97 \mathrm{fg}$.). Im Ganzen jedoch handelt es sich um eine Uebersetzung, nicht un eine durchgebeude llearbeitung les Originalwerkes, so dass Ier Leser überall Colliguon's Auffassungen olue fremde Aenderungen kennen lerut. Die Vorzüge des buches werden immer deutlicher hervortreteu, je festeren Grund die Dar. stellung vewinnt, je reieher und berlentender die Ionumeute zufliessen Ide crste Lietelung, die nabezu das erste Buch umfasst, hat es uur noch mit den "Anfäingen" zu thun'. Die aussere Iusstuttung jst in Papier und Druck der Originalausgabe mindestens ebenbürtig, die Abbilalungen sind z. T. noeh sehärfer als dort herausgekommen, und dabei ist der Preis gelinger , 20 Mark stutt 30 Franes für den Band). Die schönen Volltafelu, in Farbendruck oder Ileliogravure, werden je den betretfenden Licferungen beigegeben werden Wir kömnen dem Werke nur deu rasehen Fortgang wünsehen, len der Prospekt des Verlegers in Aussicht stellt."

$$
\text { s. (Liter. Centralblatt IS'g. N. 53.) }
$$




\section{Soiftorifde mo politifite 2haïüze and Reden

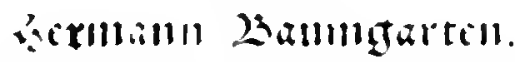

Wris ciller biographijd) (Fiuleituln! bon

\section{(Frid) Mllurks}

und cinem Rilanis de Rerfaijer:

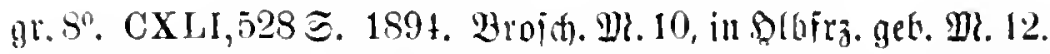

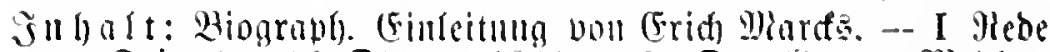

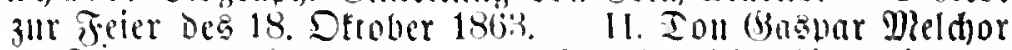
de jovellanos (1463). - III. Ier bellidie Qiberalismus. Gime Eelbitritif (1866). - IV. TRar Reifing eill eipriger Watriot? (1867). V. Sarl Wrater (1869). - VI WBie wir mieder ein 2olt geworoen find (1870). - VI1. 3ur

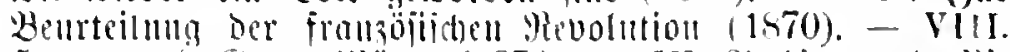

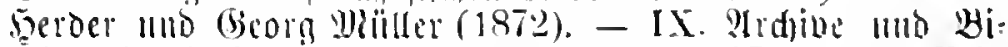

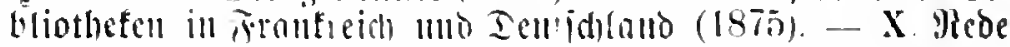
aui Jatob Etmin am 1. Whai 1876. - Xi. Strublumg vor der Yleformation ( 879$)$. - XII. Sunntills voll Qonota.

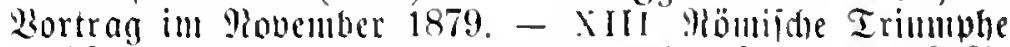
(1887). - XIV (Bcoüthtmisede nai Raije Friorid) (1888).

... Es bleibt uns wenig ütrig, als die Lexer zul dem Denkmal hinzufühen, das ihm, in Gemeinschaft und nmter thitiget Milwirkung Konrall Varentrapps, von einem seince jüngeren schüler in der ansprechendsten und wirrligsten Weise emichtel worden ist. Nachriem Erich Ma : cks schon in llerbst des vorigen Jahres in der Allgem. Zeitung eib L, ebenshild Baumgartens veröfentlicht hatte, ist dasselbe jetht in erweiterler Ungestaltung einer Sammlung historischer und politischer Aufsätze und Reden vorangestell worlen, die nach dem Urtcil der Herausgeher die sinnesweise, vielmeb die ganze Persönlichleil des Mannes zu zeichnen an geeignelsten er rchienen. Es wa die Mbscht. . Einen son dem Geschlechte festuuh, lten, hessen gesamtem Ringun wir las Reich verianken“. Indem in den mitgeteillen Stïcten aus den Jahren 186:3 - $1888 \mathrm{der}$ Mann sich selts ilarstell, wie er $\%$ verschiedenen Zeiten immer rerselbe war, inlem die hiographische Einleitung uns ras Werden des l'ublizisten und Ilistoriliers aufreigt und sein gesmmtes chriftstellerisches Wirken zu den inneren und äusseren Beziehungen zurïckserfolgt, atus lenen lasselbe entsprang, ist liese Absicht rorzüglich erreicht worden...."

$$
\text { Preussische Fahrbiucher, Bit. 76, Heft } 2 .
$$




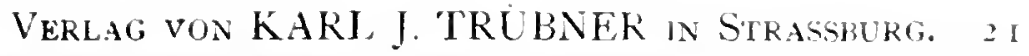

\section{Shafipere.}

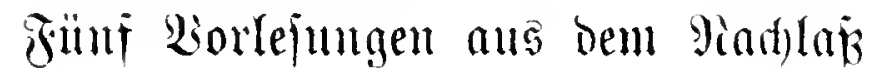

bon

Sernbard ten sirink.

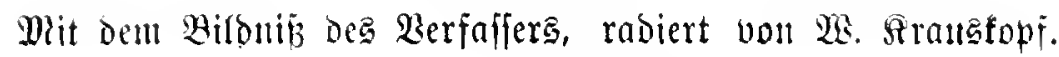

\section{(Erite und ziveite Pluflage.}

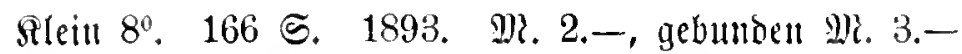

Suhalt: (5rite Borleima: Ier Didster und ber Menja. 3weite Dorlejullg: Die Beitjolge von Ghatipere马 2 serfen. - Dritte Siorlefung: Shafpere als Dramatifer. - Dierte Borleintug: Shafipere als fomifder Tidter. - Finfte Borleiung: Shatipere als Tragifer.

„... Es ist ein hoher und herrlicher Geist, der aus diesen Vorträgen spricht. Flammende Begeisterung, philosophische Bildung uml strenge $W$ issenschaftlichkejt, feinstes Verständniss und Nachfühlen des Dichters, das sind die Vorzüge, die sich hier miteinander vereinen."

\section{(Semanns Litterar. Fahreshoricht 9.3.)}

"Bedarf es eines Beispiels fül die Art von Wissenschaft, wie wir sie uns denken, so sei nur im Augenblick auf das köstliche Buch über "Shakespeare" verwiesen, das aus den Nachlasse von ten Brink, eines der hervorragendsten Gelehiten unserer Zeit, durch die Sorgfalt Edward Schröders zugänglich geworden ist. Was psychologische Synthese und nachfüblende Aesthetik zu leisten vermag, durüber belehı dieses kleine Werk besser, als es der weitläufigsten 'Theorie gelänge "

(Anton E. Schönbach in Irom Fols aum . Meer 19oj/ot Heft 1.)

Dieses Buch ten Brinks ist bei Schombach ( $\ddot{U} b e$. Lesen and Bildung, 4. Auh.) unter der besten deutschen Prosawerken genannt. 
22 Verlag vor K IRI I. TRLIBNER IN STKASBURG.

\title{
Sittlides Sein \\ unto
}

\section{Sittlithes Werden.}

(5) von

\section{Thcobals Jicgler.}

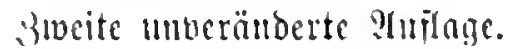

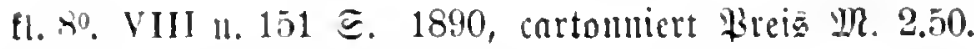

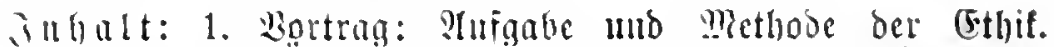
Sifitorifater llberbliaf. - 2. Mortrag: Die (Entitefung bes Sittliden. - 3. Mortrng: Das Mejen des Sittlident. 4. Bortrag: Biflidyt und Tugend. - 5. Bortrag: Giiter

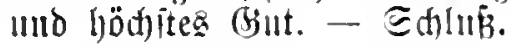

Diese Vorträge sind ebenfalls, wie die ten Brank schen ïber Shakspere. im freien leulschen Hochstift «u Frankfurt a. M. gehalten worden: infolge ihrer Bedeutung sind sie bereits ins Englische ühersetzt.

.Der Verfasser, dem wir bereits eime ausgezeichnete "Geschichte der christlichen Ehhik " rerdanken, hat in diesem Büchlein sechs ror cinem Kreise gebildeter Mämner und Frauen gehallene Vortrige vereinigt, die tr mil Fug und Recht als .Grundlinien eines Systems der Ethik" bezeichnen durfte. Nachdem et in der Einleitung lufgabe und Methode der Ethik präcisiert und einen historischen Übertlick gegeben hat, erörtert er die Entstehung und alsdann das Wesen des Sittlichen. um im Weiteren at die Pflichtenlehre sellst einzugehen. Die schwierigen Probleme sind mit einel solchen Feinheit und Frische behandelt, und man begegnet auf Schritt und Tritt so seistrollen Bemerkungen und neuen Gesichtspunkten. dass man lem Verfassel mil Fremle und Genuss Gefolgschaft leister und ihn fül die schöne Gibe zu auflichtigem Danke verptlichtet ist." .Magdeburgische Zeitung, 19. April 1890 . 
Soebell eriftien:

\section{Dex \\ ijraelitiide $\mathfrak{P r o p h e t i s n u s . ~}$}

Sin fïñ Borträgen fïr gebilote Senen gejchiloert volt

\section{Carl sinrids cornill,}

Der Theologie mo Rhilojophic Ioctor, orbentlichem Rrofeifor ber Theologie an Der lluiverjïtät fiönigh herg.

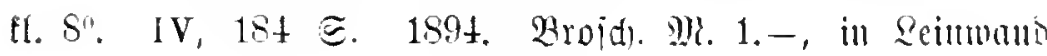
geb. 9 , 2...

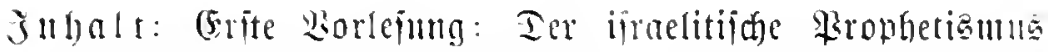

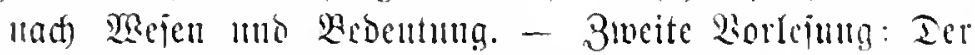

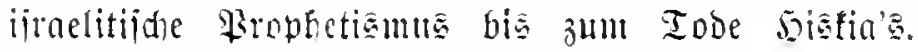

Dritte Rorleinng: Der ijraelitifde Prophetisums von Manafïe bis zur Berjtörung Jerujalems. - Bierte Bor= lejung: Der ifraelitifhe Brophetiomus währeno bes

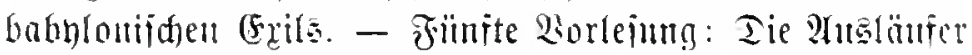
be iínaelitijden Prophetismm.

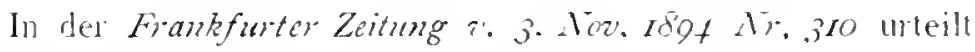
D. Ehlers über das Schriftchen wie folgt:

"Der Wahtheitsmuth. die geschichtliche Unbefangenheit. die lehendige Schilderung, die Schönheit der Form. hei allem Freimuth der Kritili die fromme ehrfurchtsvolle Schen ror den Heiligthümern des alten 'Testaments, welche die Cornill'schen Torträge auszeichnen, lassen den Wunsch entstehen. sie möchten von Tausenden und Tausenden gelesen werden: sie bieten verständigen Lesern für das Alte Testannent einen Schlüssel, der wirklich aufschliesst.» 


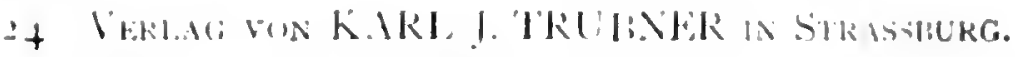

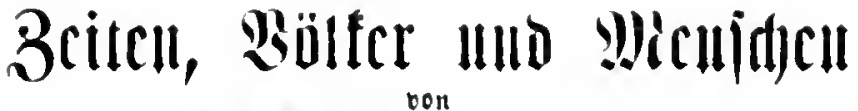 \\ Yiarl rjillebrand.}

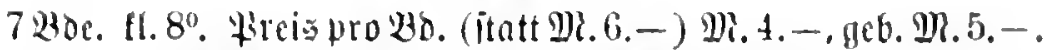

2b. 1. Frantreidy und die Granzoien.

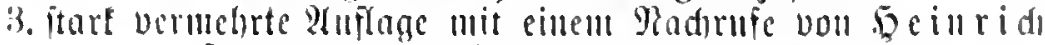

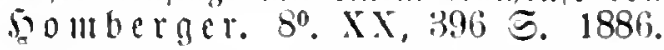

juhalt: Borrebe sur 2. Mno . Mulane. - Einleitendes. -

Die (Bejellidanit und Sitteratur. -- Bolitidaes Reben.

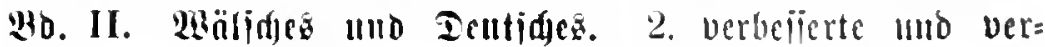
mehrte 2hiflage. 80. XIV 16.458 $\Xi$. 1892.

illbalt: Bormo t. - I. Bur Renaiijance. - II. Beitaenöiiiides

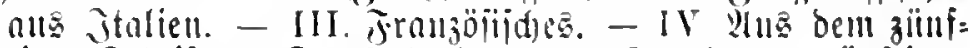

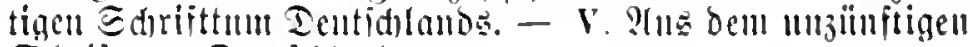
Edrifttun Dentidulands.

Bb. III. Hus und iiber England. 2. verbejerte mid vel: mehrte Ruflane. so. VIII u. 408 ङ. 1892.

Subalt: Borbemertmị. - I. Bricie ans England. - II. Tran=

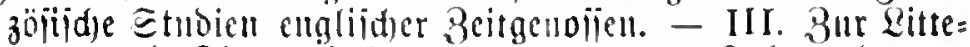

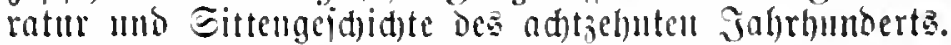

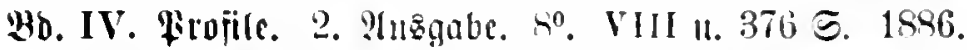

Bd. V. Pus bell Jahrhulaert ber Mevolution.

2. 2HEgabe. So. Vill, 366 ङ. 1896 .

Bd. VI. Beitgenoijen und Beitgenöijiidges.

2. 2lisgabe. so. VIII, 400 ङ. 1886 .

Bo. VII. (Eulturgeididytlides. So. XII, 335 ङ.

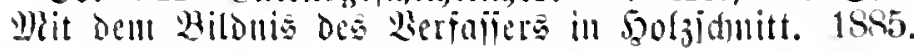

\section{Bülf Briefe eimes ätlietilfien dictgers volt \\ (siart gitlebrams.)}

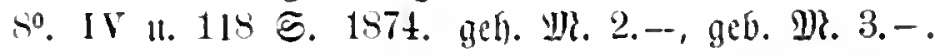

Die Selıriften Kurl llilluramb gehiirm mit mo dem Besten und Best-

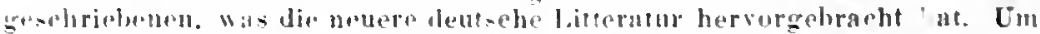

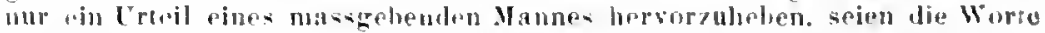

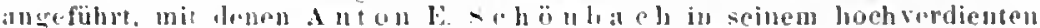

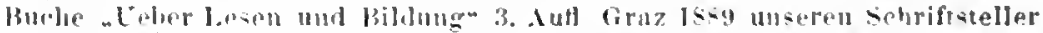

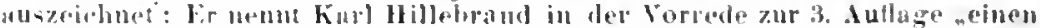

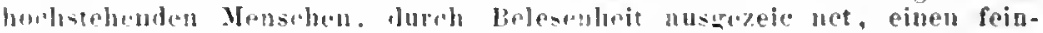
fïhligen krit ko: deusel von Gehure und lirziehung, infernational durch

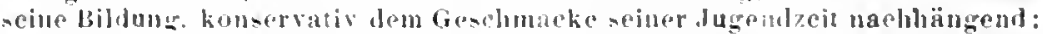

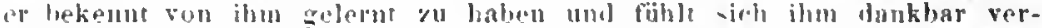

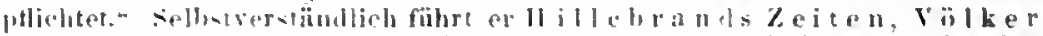

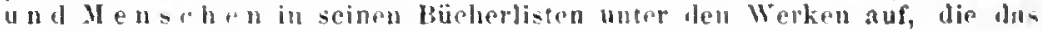

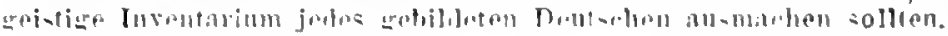




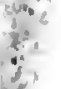

$y+a$

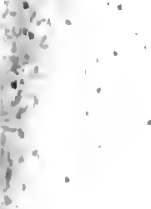

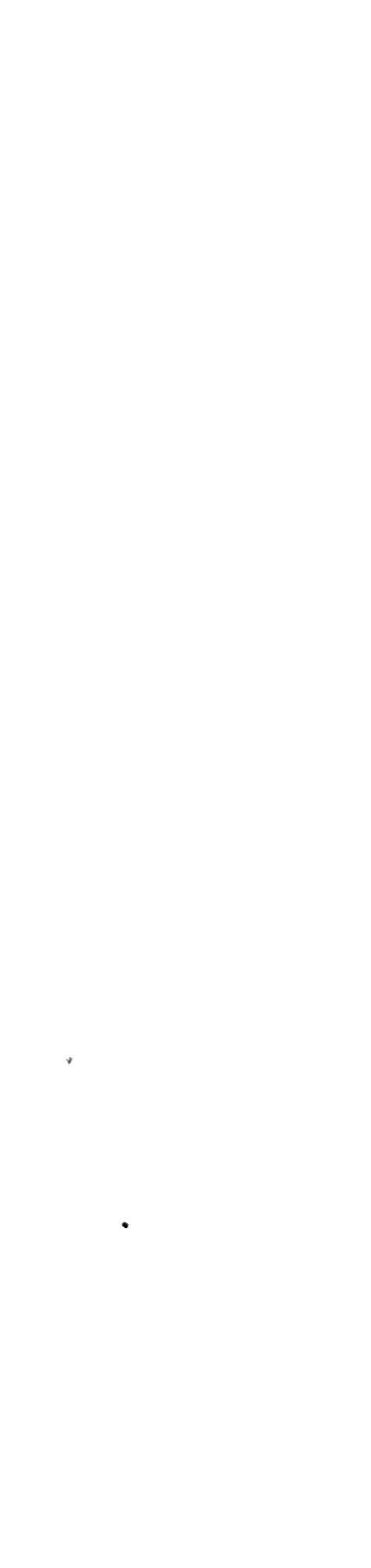
-

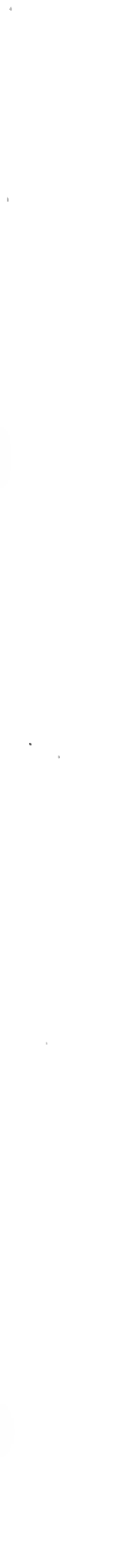

$+$ 
(3)

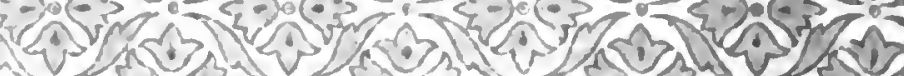

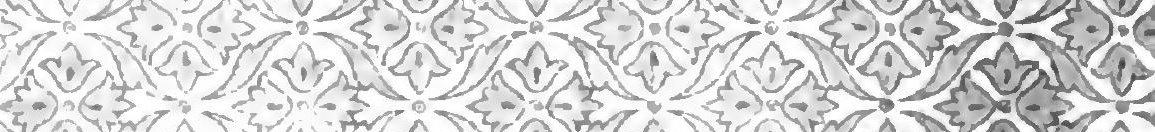

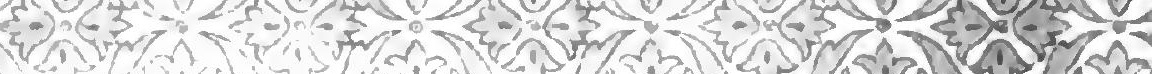

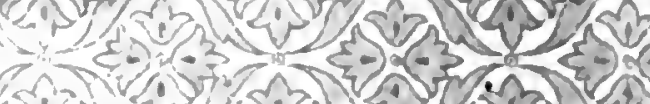

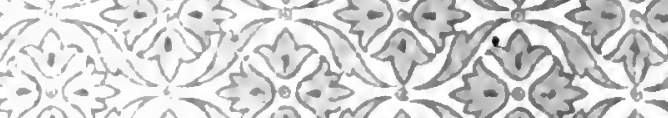

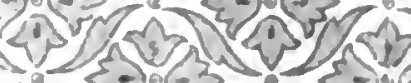

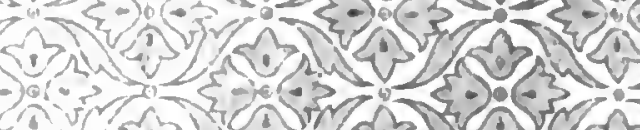

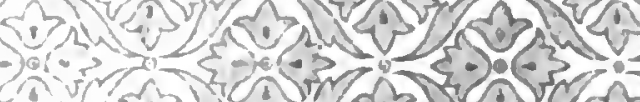

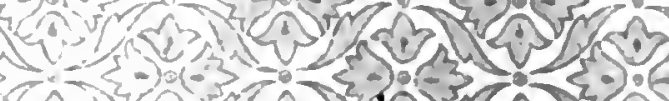

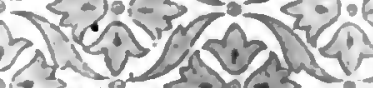
$-10-a-3=0$ a.j.

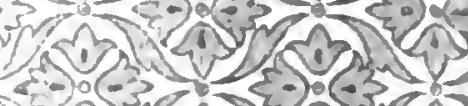

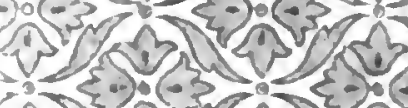

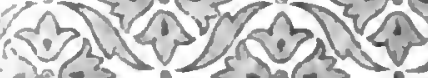
$7,2,030$ -

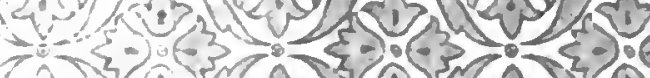
(for. i

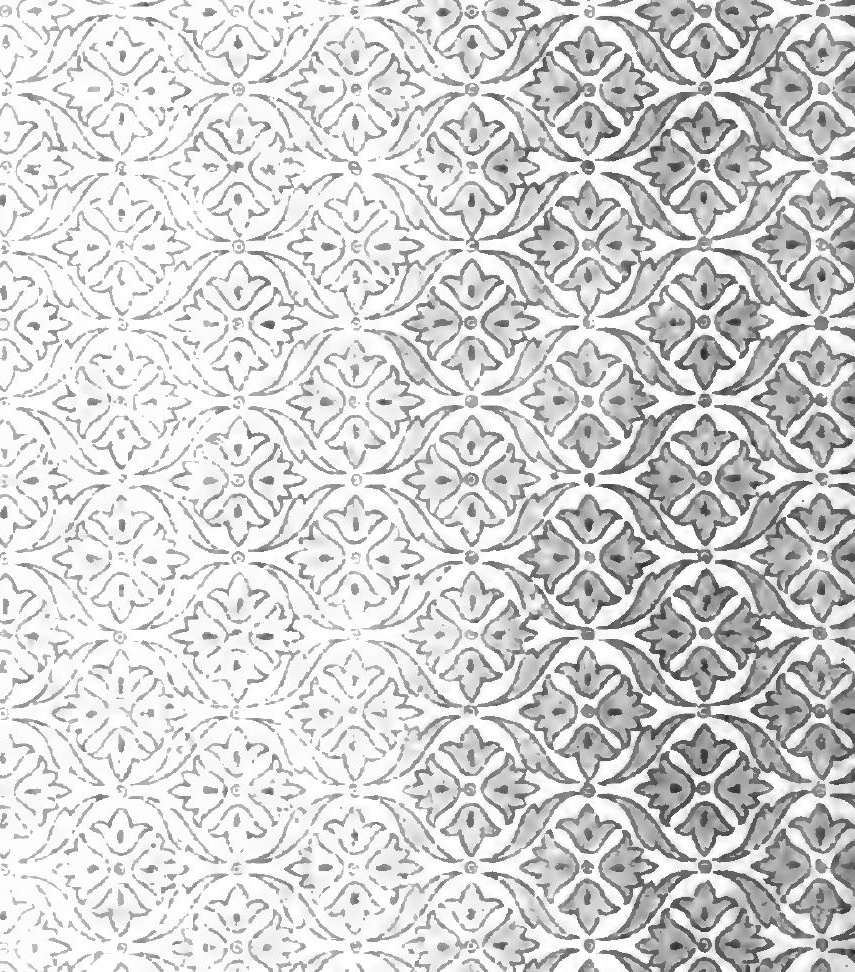
(0) $-\left\{\begin{array}{c}3 \\ 4\end{array}\right.$

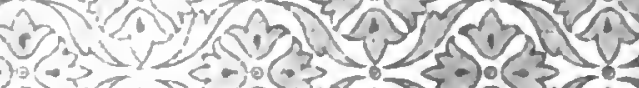

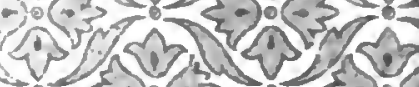

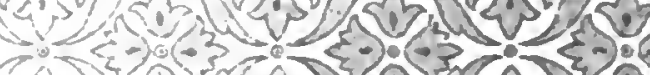
$-30(-2$ $-1 / 5$ 
